\title{
Qualitätsmanagement
}

\section{in der Jugend- und Sozialhilfe}

Literaturanalytische und empirische Studien

Dissertation

zur Erlangung des sozialwissenschaftlichen Doktorgrades der

Sozialwissenschaftlichen Fakultät

der Georg-August Universität Göttingen

vorgelegt

von

Klaus-Peter Gerull

aus Wülfrath

Göttingen 2004 
1. Gutachterin: $\quad$ Frau Prof. Dr. Margarete Boos

2. Gutachterin: $\quad$ Frau Prof. Dr. Ilona Ostner

Tag der mündlichen Prüfung: 11. Juli 2005 


\section{Erklärung}

Ich versichere, dass ich die eingereichte Dissertation

"Qualitätsmanagement in der Jugend- und Sozialhilfe

- Literaturanalytische und empirische Studien"

selbständig und ohne unerlaubte Hilfsmittel verfasst habe. Anderer als der von mir angegebenen Hilfsmittel und Schriften habe ich mich nicht bedient. Alle wörtlich oder sinngemäß den Schriften anderer AutorInnen entnommenen Stellen habe ich kenntlich gemacht. 


\section{Inhaltsverzeichnis}

A Qualitätsmanagement in der Jugend- und Sozialhilfe

- Kontext und methodischer Ansatz der Arbeit

1. Aktualität und Herkunft des Qualitätsdiskurses im Humandienstleistungsbereich 10

2. Der Qualitätsdiskurs in der sozialwissenschaftlichen und psychologischen Fachliteratur 16

3. Erkenntnisinteresse, Fragestellungen und Gliederung der Arbeit 24

$\begin{array}{ll}3.1 \text { Erkenntnisinteresse } & 24\end{array}$

$\begin{array}{ll}3.2 \text { Fragestellungen } & 26\end{array}$

$\begin{array}{ll}3.3 \text { Gliederung } & 27\end{array}$

4. Literaturanalytischer Teil der Arbeit (Abschnitte B und C) 29

4.1 Sozialpolitischer Kontext $\quad 29$

4.1.1 Struktur des deutschen Sozialsystems und Rolle freier Träger $\quad 29$

4.1.2 Ökonomisierung des Sozialen 32

4.1.3 Modernisierung der öffentlichen Verwaltung 38

4.1.4 Aufgaben der Jugend- und Sozialhilfe 42

4.1.5 Das Qualitätsthema in der Jugend- und Sozialhilfe 45

4.2 Methodik

$\begin{array}{ll}\text { 4.2.1 Literaturrecherchen: Suchbegriffe und -ergebnisse } & 50\end{array}$

4.2.1.1 Recherchen im sozialwissenschaftlichen Bereich $\quad 50$

4.2.1.2 Recherchen in psychologischen und allgemeinen
Datenbanken

4.2.1.3 Recherchen im betriebswirtschaftlichen Bereich $\quad 54$

4.2.2 Anmerkungen zur Literaturauswahl $\quad 55$

5. Empirischer Teil der Arbeit (Abschnitt D) 57

$\begin{array}{ll}5.1 \text { Vorbemerkungen } & 57\end{array}$

$\begin{array}{ll}5.2 \text { Hypothesen } & 58\end{array}$

5.2.1 Hypothesen zur Ausgestaltung des Qualitätsmanagements 58

5.2.2 Hypothesen zum Einsatz von Qualitätsbeauftragten 60

5.3 Methodik

5.3.1 Auswahl der Untersuchungsgruppen 63

5.3.2 Variablenauswahl und Untersuchungsplanung $\quad 65$

$\begin{array}{ll}\text { 5.3.3 Untersuchungsinstrumente } & 68\end{array}$

5.3.4 Umfang und Rekrutierung der Stichproben $\quad 72$

$\begin{array}{ll}\text { 5.3.5 Adressaten der Befragung } & 75\end{array}$

5.3.6 Durchführung der Befragung $\quad 76$

5.3.7 Einbeziehung von Vorstudien und Vergleichsuntersuchungen 77

B Qualitätsmanagement als interdisziplinäres Konzept

- Grundlagen und Schnittmengen

1. Qualitätsspezifische Grundlagen im Überblick 81

1.1 Kurze Geschichte der Qualität und ihres Managements $\quad 81$

$\begin{array}{ll}1.2 \text { Qualität } & 84\end{array}$

1.3 Qualitätsmodelle $\quad 86$

1.4 Qualitätsmanagement (QM)

1.5 QM-Modelle $\quad 95$

1.6 Qualitätstechniken und -instrumente $\quad 99$

$\begin{array}{ll}1.7 \text { QM-Kontextkonzepte } & 101\end{array}$

$\begin{array}{ll}1.8 \text { QM-Implementierungskonzepte } & 102\end{array}$

2. Verortung des Qualitätsmanagements in Wissenschaft und Praxis 106

3. Qualitätsmanagement und Konzepte der Organisationsforschung 110

$\begin{array}{ll}3.1 \text { Organisationsdiagnose und -analyse } & 110\end{array}$ 
$\begin{array}{lr}3.1 .1 \text { Organisationsstruktur } & 110\end{array}$

$\begin{array}{lr}3.1 .2 \text { Organisationskultur } & 112\end{array}$

$\begin{array}{ll}\text { 3.1.3 Organisationsklima } & 116\end{array}$

3.2 Organisationsentwicklung und Change Management 119

3.3 Personalentwicklung und Human Resource Management 123

3.4 Qualitätsmanagement und Evaluation $\quad 126$

3.5 Controlling und Balanced Scorecard 130

3.6 Mitarbeiterbeteiligung und Empowerment 135

3.7 Lernende Organisation und organisationales Lernen 141

3.8 Kritik des Qualitätsdiskurses und das Konzept des Sensemaking 147

4. Resümee: Zur Konvergenz und Integration der Ansätze 154

C Qualitätsmanagement in sozialen Handlungsfeldern

- Konzepte und Instrumente

1. Qualitäts(management)-Konzepte für Soziale Dienste im Überblick 161

1.1 Beschreibung und Bewertung von Qualitätskonzepten 161

1.2 Formale Systematisierung von Qualitätskonzepten 164

1.3 Ergebnisse der Literaturrecherchen zu Qualitätskonzepten 167

1.4 Darstellung und Kritik ausgewählter Universalkonzepte 171

1.4.1 Qualitätsmanagement nach DIN EN ISO $9000 \mathrm{ff.} \quad 171$

$\begin{array}{ll}\text { 1.4.1.1 Vorbemerkungen } & 171\end{array}$

$\begin{array}{ll}\text { 1.4.1.2 Darstellung des Ansatzes } & 172\end{array}$

1.4.1.3 Beurteilung des Ansatzes $\quad 175$

1.4.2 Das Konzept des Total Quality Management (TQM) 177

$\begin{array}{ll}\text { 1.4.2.1 Darstellung des Ansatzes } & 177\end{array}$

1.4.2.2 Beurteilung des Ansatzes 181

1.4.3 Das Konzept des Kontinuierlichen Verbesserungsprozesses
(KVP)

$\begin{array}{lr}\text { 1.4.3.1 Darstellung des Ansatzes } & 182\end{array}$

1.4.3.2 Beurteilung des Ansatzes $\quad 184$

1.4.4 Das Konzept der European Foundation for Quality Management
(EFQM)

$\begin{array}{ll}\text { 1.4.4.1 Darstellung des Ansatzes } & 185\end{array}$

1.4.4.2 Beurteilung des Ansatzes $\quad 188$

1.4.5 Integriertes/Integratives QM nach dem St. Galler Management
Konzept

$\begin{array}{ll}\text { 1.4.5.1 Darstellung des Ansatzes } & 190\end{array}$

1.4.5.2 Beurteilung des Ansatzes $\quad 194$

2. Instrumente des Qualitätsmanagements in Sozialen Diensten 197

2.1 Stellenwert der Technik im Qualitätsmanagement 197

2.2 Darstellung und Kritik ausgewählter Instrumente 199

$\begin{array}{ll}\text { 2.2.1 Qualitätsleitbild } & 199\end{array}$

2.2.2 Qualitäts(management)handbuch $\quad 203$

$\begin{array}{ll}2.2 .3 \text { Qualitätszirkel } & 205\end{array}$

2.2.4 Qualitätsbeauftragte (QB) 210

$\begin{array}{ll}2.2 .5 \text { Benchmarking } & 216\end{array}$

$\begin{array}{ll}\text { 2.2.6 Prozessmanagement } & 219\end{array}$

2.2.7 Ideenmanagement/Vorschlagswesen $\quad 223$

$\begin{array}{ll}2.2 .8 \text { Beschwerdemanagement } & 225\end{array}$

$\begin{array}{ll}\text { 2.2.9 Wissensmanagement } & 230\end{array}$

3. Resümee: Essentials eines Qualitätsmanagements in sozialen Handlungsfeldern 233

D Praxis des Qualitätsmanagements in der Jugend- und Sozialhilfe

- Empirische Befunde zur Umsetzung, Akzeptanz und Nutzenbewertung 241

1. Befunde aus Mitarbeiterbefragungen $\quad 241$

$\begin{array}{ll}1.1 \text { Vorbemerkungen } & 241\end{array}$ 
1.2 Einstellung zum Qualitätsmanagement 241

1.2.1 Angaben zur Methodik und Stichprobe $\quad 241$

$\begin{array}{ll}\text { 1.2.2 Ergebnisse } & 242\end{array}$

1.2.3 Zusammenfassung und Diskussion der Ergebnisse 244

1.3 Vorkehrungen zur Entlastung und Verbesserung der Arbeit /
Maßnahmen zur Qualitätsentwicklung (I)

$\begin{array}{ll}\text { 1.3.1 Angaben zur Methodik und Stichprobe } & 248\end{array}$

$\begin{array}{ll}\text { 1.3.2 Ergebnisse } & 250\end{array}$

1.3.3 Zusammenfassung und Diskussion der Ergebnisse $\quad 252$

1.4 Maßnahmen zur Qualitätsentwicklung (II) 254

1.4.1 Angaben zur Methodik und Stichprobe $\quad 254$

$\begin{array}{ll}\text { 1.4.2 Ergebnisse } & 254\end{array}$

1.4.3 Zusammenfassung und Diskussion der Ergebnisse 256

2. Befunde aus Leitungsbefragungen $\quad 259$

2.1 Maßnahmen zum Qualitätsmanagement 259

2.1.1 Angaben zur Methodik und Stichprobe $\quad 259$

$\begin{array}{ll}2.1 .2 \text { Ergebnisse } & 261\end{array}$

2.1.3 Zusammenfassung und Diskussion der Ergebnisse 262

2.2 Einstellung zum Qualitätsmanagement, Stellenwertzuschreibung
und Akzeptanz

2.2.1 Angaben zur Methodik und Stichprobe 264

$\begin{array}{ll}2.2 .2 \text { Ergebnisse } & 265\end{array}$

2.2.3 Zusammenfassung und Diskussion der Ergebnisse 268

3. Befunde aus Befragungen von Qualitätsbeauftragten $\quad 271$

3.1 Angaben zur Methodik und Stichprobe $\quad 271$

$\begin{array}{ll}3.2 \text { Ergebnisse } & 272\end{array}$

3.3 Zusammenfassung und Diskussion der Ergebnisse 274

4. Befunde einer Befragung von Einrichtungen und Diensten (Hauptstudie) 277

4.1 Bestimmung der Grundgesamtheiten und Quotierung der Stichproben 277

4.2 Verfahren der Stichprobenentnahme 283

$\begin{array}{ll}4.3 \text { Rücklauf } & 285\end{array}$

$\begin{array}{ll}\text { 4.3.1 Gesamtrücklauf } & 285\end{array}$

4.3.2 Rücklauf nach Faktoren $\quad 286$

4.3.3 Repräsentativität des Rücklaufs $\quad 290$

4.3.4 Interkorrelationen der Faktoren im Rücklauf 293

4.3.5 Mögliche Gründe für geringen Rücklauf 295

4.4 Auswertungsverfahren $\quad 297$

4.5 Behandlung von mehrfach vertretenen Einrichtungen 299

$\begin{array}{ll}4.6 \text { Ergebnisse } & 300\end{array}$

$\begin{array}{ll}\text { 4.6.1 Organisation des Qualitätsmanagements } & 300\end{array}$

4.6.1.1 Aufbau- und Ablaufbeschreibung $\quad 300$

$\begin{array}{ll}\text { 4.6.1.2 QM-System } & 301\end{array}$

4.6.1.3 Art des QM-Systems $\quad 304$

$\begin{array}{ll}\text { 4.6.2 Programmatik } & 305\end{array}$

4.6.2.1 Grundsätze zur Qualitätspolitik 305

4.6.2.2 Grundsätze zur Führung 306

4.6.2.3 Leitbild für die Gesamteinrichtung $\quad 307$

$\begin{array}{ll}\text { 4.6.3 Instrumente } & 307\end{array}$

$\begin{array}{ll}\text { 4.6.3.1 QM-Handbuch } & 307\end{array}$

4.6.3.2 Qualitätszirkel $\quad 308$

4.6.3.3 Vorschlagswesen und Ideenmanagement $\quad 309$

$\begin{array}{ll}\text { 4.6.3.4 Beschwerdemanagement } & 310\end{array}$

4.6.3.5 Personalentwicklungskonzept $\quad 311$

$\begin{array}{ll}\text { 4.6.3.6 Evaluation } & 311\end{array}$

$\begin{array}{ll}\text { 4.6.3.7 Controlling } & 312\end{array}$

$\begin{array}{ll}\text { 4.6.4 Beteiligung der MitarbeiterInnen } & 313\end{array}$

$\begin{array}{ll}\text { 4.6.5 Qualifizierung der MitarbeiterInnen } & 314\end{array}$

4.6.6 Signifikante Interkorrelationen der Ordinalvariablen 316

$\begin{array}{ll}\text { 4.6.7 Maßnahmen zur Qualitätsentwicklung } & 318\end{array}$

$\begin{array}{ll}\text { 4.6.7.1 Nutzenbewertung } & 318\end{array}$ 
4.6.7.2 Zusammenhänge zwischen UV und Nutzenbewertung

4.6.8 Qualitäts(management)beauftragte

4.6.8.1 Institutionalisierung des Instruments

4.6.8.2 Entscheidungsgründe gegen die Funktionsstelle QB

4.6.8.3 Zusammenhänge zwischen UV und Funktionsstelle QB

4.6.8.4 Strukturmerkmale der Funktionsstelle QB

4.6.8.5 Personen- und Ausbildungsmerkmale von QB

4.6.8.6 Tätigkeiten von QB

4.6.8.7 Typisierung von Tätigkeiten

4.6.8.8 Bewertung der Zweckmäßigkeit

4.6.8.9 Konfliktpotenziale in der Praxis

4.7 Prüfung der Hypothesen dieser Studie

4.7.1 Hypothesen zur Ausgestaltung des QM 346

4.7.2 Hypothesen zum Einsatz von QB 348

4.8 Zusammenfassung und Diskussion der Ergebnisse $\quad 350$

$\begin{array}{ll}\text { 4.8.1 Vorbemerkungen } & 350\end{array}$

4.8.2 Fragenkomplex "Umsetzung von QM" $\quad 350$

4.8.3 Fragenkomplex "Qualitätsentwicklungsmaßnahmen" 355

4.8.4 Fragenkomplex "Qualitätsbeauftragte/r" 357

5. Resümee: Folgerungen für die Praxis

1. Qualitätsmanagement und Organisationsforschung 368

$\begin{array}{ll}1.1 \text { Vorbemerkungen } & 368\end{array}$

1.2 Kontext, Grundlagen und thematische Schnittmengen 368

2. Qualitätsmanagement in sozialen Handlungsfeldern 370

$\begin{array}{ll}2.1 \text { Konzepte und Instrumente } & 370\end{array}$

2.2 Empirische Befunde zum QM in der Jugend- und Sozialhilfe $\quad 370$

$\begin{array}{ll}\text { 2.2.1 Einstellung zum Qualitätsmanagement } & 370\end{array}$

$\begin{array}{ll}\text { 2.2.2 Ausgestaltung des Qualitätsmanagements } & 371\end{array}$

2.2.3 Nutzenbewertung von Maßnahmen zur Qualitätsentwicklung 373

$\begin{array}{ll}\text { 2.2.4 Einsatz von Qualitätsbeauftragten } & 373\end{array}$

3. Ausblick: Zur Zukunft des Qualitätsmanagements 374

F Quellenverzeichnis 


\section{Vorwort}

Über Qualitätsmanagement zu dissertieren, ist nicht mehr besonders originell. Zu zahlreich sind die in unterschiedlichen Disziplinen und Branchen publizierten Studien, die sich mit Fragen der Ideologie, Implementation und Effektivität vor allem eines "Total Quality Management" befassen. Längst ist das Thema vom industriellen Produktionsbereich über den gewerblichen Dienstleistungssektor in soziale Handlungsfelder gelangt. Qualitätsmanagement, salopp: QM, ist zu einem boomenden Querschnittsfach geworden, zu dem Ingenieur-, Verwaltungs-, Arbeits-, Politik- und Rechtswissenschaften, Betriebswirtschaftslehre, Informatik, Organisationstheorie, Ethik und Pädagogik ihre Beiträge leisten. Allerdings dominieren die technischen und ökonomischen Disziplinen, während sich Psychologie und Sozialwissenschaften, zumal mit empirischer Forschung, bislang relativ zurückgehalten haben (und damit die Legitimationsbasis für vorliegende Arbeit liefern).

QM ist jedoch keineswegs der Dernier Cri der Modernisierungsdebatte. Die Ausweitung des Anwendungsbereichs auf den öffentlichen Sektor sozialer Dienstleistungen hat erheblich dazu beigetragen, das Konzept der Qualität und ihres Managements zu problematisieren und von seiner technizistischen Verhaftung zu lösen. Besonders AutorInnen sozialwissenschaftlicher Provenienz plädieren für eine postmoderne Überwindung des Qualitätsdiskurses. Die hier vorgelegte Studie greift diese Kritik punktuell auf, setzt jedoch empirisch am Status Quo an und befasst sich pragmatisch mit den derzeitigen Erscheinungsformen des QM in ausgesuchten sozialen Handlungsfeldern. Als stakeholder, also potenziell an den Ergebnissen dieser Organisationsdiagnose interessierte Gruppen, kommen sowohl die MitarbeiterInnen und das Management (z. B. hinsichtlich einer Positionierung der eigenen Organisation im Betriebsvergleich), als auch Trägergruppen und Externe (z. B. in Bezug auf Träger- und Arbeitsfeldvergleiche bei der Umsetzung von QM) in Betracht.

Die Studie entstand nicht als typische Postgraduierten-Arbeit, sondern fügt sich - wenn auch mit einem höheren theoretischen und methodischen Anspruch - nahtlos ein in eine Reihe praxisorientierter Untersuchungen im Feld der Kinder- und Jugendhilfe, die der Autor seit Mitte der Neunzigerjahre durchführen konnte. Parallel zur kontinuierlichen Arbeit an einem elektronischen Handbuch zum Qualitätsmanagement in sozialen Handlungsfeldern und der dazu erforderlichen permanenten Literaturrecherche, entstand das Bedürfnis, nicht nur zu sichten, zusammenzufassen und zu kommentieren, sondern empirisch unterbelichtete Fragestellungen selbst in Forschungs-Angriff zu nehmen. Ergebnis ist die hier vorgelegte Arbeit, in der sich beide Motive vereinen - einerseits den Stand der Diskussion und Theoriebildung des Qualitätsmanagements speziell unter Aspekten der Konvergenz mit anderen Disziplinen der Organisationsforschung darzustellen, andererseits eine konkrete und bislang vernachlässigte Fragestellung empirisch aufzugreifen: Umsetzung und Ausgestaltung des QM und des Instruments "Qualitätsbeauftragte/r" in der Jugend- und Sozialhilfe. 


\section{Danksagungen}

Für den externen Doktoranden mit festem Wohnsitz über $100 \mathrm{~km}$ vom Studienort entfernt war Selbständigkeit oberste Devise. Dennoch wäre die Studie nicht begonnen oder abgeschlossen worden, wenn nicht hilfreiche Geister ihre Unterstützung gewährt hätten, denen ich an dieser Stelle herzlich danken möchte:

- allen voran Frau Prof. Dr. Margarete Boos, Lehrstuhl für Sozial- und Kommunikationspsychologie an der Universität Göttingen, für ihre doktormütterliche Betreuung;

○ Frau Prof. Dr. Ilona Ostner, Lehrstuhl für Sozialpolitik an der Universität Göttingen, für Ihre spontane Bereitschaft, als Korreferentin zu fungieren;

- Frau Beate Irskens, wissenschaftliche Referentin beim Deutschen Verein für öffentliche und private Fürsorge, Berlin, (z. Zt. Bertelsmann Stiftung Gütersloh) für ihre tatkräftige Unterstützung bei Planung und Durchführung der Befragungsaktion;

○ Herrn Dr. Björn Hagen, Geschäftsführer des Evangelischen Erziehungsverbandes (EREV), Hannover, und seinen Mitarbeiterinnen für die freundliche Kooperation bei Literaturrecherchen und Stichprobengewinnung;

- Herrn Dipl.-Psych. Erich Hirschfeld, freier Heim- und Erziehungsberater, Detmold, für seine engagierte Rekrutierung zusätzlicher UntersuchungsteilnehmerInnen;

○ MitarbeiterInnen des Georg-Elias-Müller-Instituts für stets bereitwillige Gelegenheitshilfe;

- last, not least meiner Frau Siegrun Gerull, Lehrerin und Finanzier, Hessisch Oldendorf, ohne deren aktive Duldung das Projekt die Startlöcher nicht verlassen hätte.

Klaus-Peter Gerull

Hessisch Oldendorf, im November 2004 


\section{A Qualitätsmanagement in der Jugend- und Sozialhilfe \\ - Kontext und methodischer Ansatz der Arbeit}

\section{Aktualität und Herkunft des Qualitätsdiskurses im Humandienstleistungsbereich}

Neben dem Dauerbrenner-Thema der öffentlichen Finanznot und der aus dem Ruder gelaufenen Kosten ist im Humandienstleistungsbereich seit Anfang der Neunzigerjahre kaum ein Diskussionsgegenstand so prominent geworden wie Qualität und deren möglichst effektive und effiziente Steuerung durch Qualitätsmanagement. Die damit angeschnittenen Fragen haben angesichts veränderter Rechtsgrundlagen und verschärfter Wettbewerbssituation für viele sozialwirtschaftliche Organisationen existentielle Bedeutung erlangt.

Konzentrierten sich gesetzgeberische Reformaktivitäten zunächst auf den Gesundheitsbereich, gerieten nach und nach auch andere soziale Arbeitsfelder in den Sog dieser Entwicklungen. So sind die meisten Einrichtungen und Dienste inzwischen explizit verpflichtet - wenn auch in unterschiedlicher Diktion und Regelungstiefe - zu Maßnahmen

- der Qualitätsentwicklung und -bewertung (z. B. Achtes Buch Sozialgesetzbuch/SGB VIII - Kinder- und Jugendhilfe),

- der Qualitätssicherung (z. B. SGB V - Gesetzliche Krankenversicherung),

- des Qualitätsmanagements (z. B. SGB XI - Soziale Pflegeversicherung) und

○ der Qualitätsüberprüfung (z. B. SGB III - Arbeitsförderung).

Als exemplarisch für ein stark bundesgesetzlich normiertes Vorgehen kann das neue PflegeQualitätssicherungsgesetz (PQsG, Deutscher Bundestag, 2001) gelten, das die Träger von Pflegeeinrichtungen zur Qualitätsentwicklung und -sicherung verpflichtet und dazu neben einem umfassenden internen Qualitätsmanagement auch die Teilnahme an externen Prüfverfahren vorschreibt. Am wenigsten normiert ist dagegen der Bereich der Kinder- und Jugendhilfe. Hier gibt es lediglich auf Landesebene konkretere Vorgaben, die aber allenthalben stärker auf die Elemente Selbstbewertung und Dialog zwischen öffentlichen und freien Trägern abheben, als z. B. eine externe Prüfung oder Zertifizierung vorzusehen. 
(Landesjugendplan Nordrhein-Westfalen, vgl. Projektgruppe WANJA, 2000, S. 11) und künftig vorzulegende jährliche Qualitätsberichte (Beschluss der Gesundheitsministerkonferenz 1999, vgl. Fiene, Kirchner \& Ollenschläger, 2001, S. 132).

Wenngleich diese Entwicklung maßgeblich durch die Gesundheitsstrukturreform von 1989 und die Veröffentlichungen der Kommunalen Gemeinschaftsstelle für Verwaltungsvereinfachung (KGSt, 1993, 1994, 1995) beeinflusst wurde, so stellen diese ihrerseits eher Wirkungen als Ursachen in einem komplexen Bedingungsgefüge dar, in dem u. a. der Kostendruck der produzierenden Wirtschaft und die Finanzschwäche der öffentlichen Hand eine zentrale Rolle spielen.

Systematisch lässt sich dieses Bedingungsgefüge differenzieren in gesellschaftliche, volkswirtschaftliche, sozialpolitische, betriebswirtschaftliche sowie fachliche und berufspolitische Faktoren.

$\mathrm{Zu}$ den gesellschaftlichen Gründen - die hier nur im Rahmen dieser Einführung thematisiert werden - zählen ein Wandel der Erwartungen an die Leistungen des Bildungs, Gesundheits- und Sozialsystems (Stichworte "Individualisierung", "Selbsthilfe- und Bürgerrechtsbewegung", vgl. Schädler, 2001, S. 16 ff.) und eine zunehmende Sensibilität der Verbraucher, Klienten, Bürger und anderer "KundInnen" gegenüber Qualitätsfragen. Dies sind in erster Linie Fragen nach dem Gebrauchswert von Produkten und Dienstleistungen, häufig veranlasst durch wahrgenommene Mängel, Verdrossenheit über vorgefundene Zustände oder medienwirksam aufbereitete nationale Misserfolge (Stichwort "PISA-Studie").

Gewachsen z. B.

○ ist das Bedürfnis nach individuell zugeschnittenen und kundenfreundlich erbrachten Dienstleistungen auf Seiten selbstbewusster BürgerInnen,

$\circ \quad$ ist der Stellenwert von Mitbestimmung und Selbstverwirklichung im Beruf auf Seiten demokratiebewusster MitarbeiterInnen,

○ sind Erwartungen an eine möglichst hochwertige Gegenleistung bei zunehmender finanzieller Selbstbeteiligung auf Seiten anspruchsbewusster LeistungsempfängerInnen, 
○ ist nicht zuletzt die Abhängigkeit überforderter KonsumentInnen von - vermeintlich - objektiven Rationalitätskriterien zur Entscheidungsfindung in einer unübersichtlichen Informations- und Warenwelt.

$\mathrm{Zu}$ den volkswirtschaftlichen und sozialpolitischen Gründen gehören vor allem:

- die zur Beseitigung nichttarifärer Handelshemmnisse entwickelten internationalen Qualitätssicherungs-Standards, die zunehmend auch im Dienstleistungsbereich Verbreitung finden (s. Kap. B 1.1),

- die rezessionsbedingte Krise der öffentlichen Haushalte, stagnierende Sozialbudgets und der einsetzende Umbau bzw. Abbau sozialstaatlicher Leistungen nach Maßgabe zunehmend neoliberaler Wirtschaftspolitik, davon ausgehend, dass der Sozialstaat bisheriger Prägung zu teuer und dabei verhältnismäßig ineffektiv sei (s. Kap. A 4.1.2),

- die forciert betriebene Reorganisation der öffentlichen Verwaltungen nach Maßgabe betriebswirtschaftlicher Prinzipien (s. Kap. A 4.1.3),

○ die Stärkung der Position von Sozialleistungsträgern gegenüber den leistungserbringenden Einrichtungen, u. a. durch die Einführung leistungsbezogener Finanzierungssysteme und Legitimationsanforderungen (s. Kap. A 4.1.5),

○ die sich verschärfende Konkurrenz zwischen freigemeinnützigen und privatgewerblichen Anbietern sozialer Dienstleistungen durch Öffnung des Marktes, Niederlassungs- und Dienstleistungsfreiheit im Zuge des europäischen Einigungsprozesses (s. Kap. A 4.1.1).

Mitverantwortlich für den Boom des Qualitätsthemas waren insbesondere auch betriebswirtschaftliche Gründe:

- die Notwendigkeit der Kostensenkung durch Maßnahmen frühzeitiger Fehlererkennung und -vermeidung, Reduzierung von organisationsinternen Reibungsverlusten sowie von Ausschuss, Nacharbeiten und Reklamationen (s. Kap. B 1.1),

- die aus Japan importierte Erkenntnis der Bedeutung "verschlankter" Organisation, 
"beherrschter" Geschäftsprozesse, "kontinuierlicher" Verbesserung und verwandter Managementprinzipien für eine kunden- und zugleich erfolgsorientierte Unternehmenspolitik (s. Kap. B 1.1),

- die in bestimmten Branchen üblich gewordenen Kundenforderungen nach zertifizierter Qualitätsfähigkeit von Geschäftspartnern und deren regelmäßiger Überprüfung durch akkreditierte Stellen (s. Kap. B 1.1),

- die leichtere Abwehr von Produkthaftungsansprüchen durch Nachweis systematischer Maßnahmen zur Qualitätssicherung, analog die Senkung des Strafrechts- und Haftungsrisikos durch Organisationsverschulden und Behandlungsfehler (vgl. Böckels, 2002).

Wenngleich somit die wesentliche Dynamik des aktuellen Qualitätsthemas durch Impulse ausgelöst wurde, die nicht aus den sozialen Professionen selbst stammen, sondern auf primär wirtschaftlichen und gesetzgeberischen Druck zurückgehen, so ist doch die Behandlung von Qualitätsfragen jenseits aller Konjunkturen, sozialrechtlichen und betriebswirtschaftlichen Notwendigkeiten und Moden immer schon Bestandteil professioneller Arbeit gewesen (Gerull, 1999b, S. 13). Insofern müssen als viertes Einflussbündel auch fachliche und berufspolitische Gründe genannt werden, insbesondere:

○ die nicht nur nach außen, einer kritischen Öffentlichkeit gegenüber, sondern auch nach innen, an die Akteure gerichtete, verbesserungswürdige Legitimation als Profession, als ein Laienhandeln gegenüber fachlich überlegenes Handeln, welches einen individuellen und gesellschaftlichen Nutzen stiftet - den es im Zuge eines Qualitätsmanagements klarer zu beschreiben und nachzuweisen gilt (vgl. Engelhardt, 1999; König, 2000).

Sommerfeld \& Haller (2003, S. 65) sprechen von der Ablösung des Konzepts des "Vertrauens" durch das Konzept der "Accountability". Wendt (1999, S. 12) versteht darunter eine aktive Rechenschaftslegung, durch die ein Unternehmen gegenüber Partnern, Kunden und Öffentlichkeit die Werte ausweist, die es erzeugt oder erhält; darüber hinaus wird die Funktion der Selbstbestätigung und Explizierung des Wertes eigenen Tuns betont (S. 13). 
- die mit systematischer Qualitätsarbeit verbundenen Möglichkeiten, Akteure in einem durch Unsicherheit, Rollenkonflikte und "Technologiedefizit" (Luhmann \& Schorr, 1982) charakterisierten Handlungsfeld zu qualifizieren (Stichwort "Personalentwicklung"), indem z. B. mehr Klarheit über zielführende Arbeitsabläufe (Stichwort "Prozessmanagement"), mehr Rückmeldung über das eigene Tun (Stichwort "Selbst(Evaluation"), allgemein mehr Aufmerksamkeit für die Passung von Mensch und Organisation obwaltet (Stichwort "Organisationsentwicklung"), ohne die Besonderheiten Sozialer Arbeit aus dem Blick zu verlieren (vgl. Engelhardt, 1999);

○ der wachsende Widerstand gegen eine "Kolonialisierung" (Sommerfeld \& Haller, 2003, S. 84) der Sozialen Arbeit durch betriebswirtschaftliche Managementkonzepte, die sich einer technisch-formalisierenden Begrifflichkeit und Methodik bedienen und die sozialen Fachsprachen mit erheblichen Auswirkungen auf die kognitiven Strukturen der Akteure zu überwuchern drohen (vgl. Merchel, 2000; König, 2000);

○ eine verstärkte (Rück-)Besinnung auf genuin sozialwissenschaftliche Beiträge (z. B. Selbstevaluation, Supervision) zur Bearbeitung der Qualitätsfrage, namentlich zur Professionalisierung der Organisationsformen sozialer Dienstleistungen und zur Kompetenzsteigerung ihrer Akteure (vgl. B. Müller, 2000; von Spiegel, 2000a, 2002; Spreyermann, 1996).

Mit der ausdrücklichen Betonung der Qualitätskategorie richtet sich die Managementanforderung nicht mehr nur auf die "Hardware" sozialer Einrichtungen und Dienste, sondern ebenso auf die "Software", auf das sozialpädagogische, pflegerische, therapeutische, beratende Handeln. Das eigentlich Neue der aktuellen Qualitätsdiskussion zeigt sich danach vor allem in fünf Punkten (vgl. Merchel, 2001):

1. der Einbeziehung nicht nur strukturqualitativer Aspekte (z. B. in Entgeltverhandlungen) - früher zumeist mit fachlichen Standards (z. B. Fachpersonalquote) gleichgesetzt -, sondern ausdrücklich auch prozess- und ergebnisbezogener Kriterien;

2. der Forderung nach stärkerer Konkretisierung und Plausibilität der Standards und Konzepte, nicht zuletzt im Sinne einer Beschreibung des aktuellen state of the art, um fachliche Fehlervarianz zu reduzieren; 
3. der Integration strukturierter und kontinuierlicher Verfahren der Qualitätsbewertung in den Leistungsprozess;

4. der stärkeren Berücksichtigung des einrichtungsübergreifenden Kontextes im Sinne von Infrastrukturqualität;

5. der Verbindung fachlicher und sozialpolitischer Aspekte des Qualitätsthemas - in der Sozial- und Jugendhilfe durch die Verkoppelung der drei Vereinbarungsarten über Leistung, Qualität/Prüfung und Entgelt/Vergütung ausgestaltet (s. Kap. A 4.1.5).

Von der Qualitätsdiskussion werden mehrheitlich Impulse erhofft, die Professionalisierung der Hilfesysteme zum Nutzen der "VerbraucherInnen" voranzubringen (z. B. Späth, 1999; Merchel, 2001). Allerdings dürften die Erwartungen der verschiedenen Interessengruppen nicht alle miteinander zu vereinbaren sein (Gerull, 2000, S. 6f), was den diskursiven Charakter des Qualitätskonstrukts im Sozialbereich unterstreicht (s. Kap. B 3.8):

o Kostenträger wollen Ausgabenbegrenzung und Planungssicherheit,

o LeistungsempfängerInnen suchen bedürfnisgerechte und qualifizierte Unterstützung,

o die Öffentlichkeit erwartet zielgenaue und wirksame Aufgabenerledigung,

o Einrichtungsträger sind an Image und Auslastung ihrer Angebote interessiert,

o Fachdisziplinen an Leistungserbringung nach dem "Stand der Kunst" und

o MitarbeiterInnen an guten Arbeitsbedingungen. 


\section{Der Qualitätsdiskurs in der sozialwissenschaftlichen und psychologischen Fachliteratur}

Die beschriebenen Veränderungen der relevanten Umweltbedingungen für sozialwirtschaftliche Organisationen ${ }^{1}$ sind nicht auf nationale Kontexte beschränkt, sondern haben zumindest für die Industrieländer inter- bzw. transnationale Dimensionen erreicht (Köpp \& Neumann, 2002, S. 1). Die gemeinhin unter der Bezeichnung Qualitätsdebatte oder Qualitätsdiskurs erörterten Fragestellungen - in den USA auch als quality movement charakterisiert (vgl. Cole \& Scott, 2000) - sind zu einem zentralen Thema des zeitgenössischen Modernisierungsdiskurses geworden. Dessen Erscheinungsformen unterscheiden sich kaum: "Immer geht es um Fragen der Messung, Standardisierung und Kontrolle, um Effektivität und Effizienz sowie vor allem um Management" (Köpp \& Neumann, 2002, S. 11).

Welche Herausforderung die deutsche Qualitätsdebatte für die leistungserbringenden sozialen Einrichtungen und Dienste, ihre Träger, Interessenverbände und professionellen Kooperationspartner in Politik und Forschung bedeutete, lässt sich ermessen, wenn man sich die Entwicklung einschlägiger Fachpublikationen bibliometrisch vor Augen führt.

Wenngleich zu einem großen Teil von berufsmäßigen Profiteuren der Debatte (FachautorInnen, FortbildnerInnen, UnternehmensberaterInnen, FunktionärInnen, ReferentInnen u. a.) und Leitungskräften mit mehr oder minder großer Affinität zum Managerialismus ${ }^{2}$ verfasst, bringt die schiere Zahl von Buch- und Zeitschriftenbeiträgen, Positionspapieren und Erfahrungsberichten gut zum Ausdruck, welche materiellen und immateriellen Ressourcen in das Thema investiert wurden und immer noch werden.

Im Vordergrund vieler Veröffentlichungen stehen sozialrechtliche Regelungen, deren mögliche Folgen und betriebswirtschaftliche Anforderungen für Einrichtungen und Dienste

1 Der Begriff verdeutlicht, dass auch Anbieter sozialer Dienstleistungen "wirtschaften" müssen, dabei jedoch durch andere Zielsetzungen und Rahmenbedingungen geprägt sind als gewinnorientierte Unternehmen der Erwerbswirtschaft (Arnold \& Maelicke, 1998, S. 20). Der Begriff "Nonprofit-Organisation (NPO)" wird synonym verwendet.

2 "Leitidee des Managerialismus ist die Neuordnung und Standardisierung von Arbeitsprozessen, um diese effizienter gestalten und umstellen zu können. Diese Aufgabe umfasst auch die Anforderung eines ökonomischen Umgangs mit den Humanressourcen und dementsprechend konstituiert der Managerialismus auch eine Technik 'zeitgenössischer Menschenführung' ..." (Wohlfahrt, o. J., S. 6). Sommerfeld \& Haller (2003, S. 62) diskutieren den Begriff im Kontext der Befürchtung, dass die ohnehin fragile Professionalisierung der Sozialen Arbeit durch die "Subsumtion unter eine fremde Macht" - hier: der Betriebswirtschaft - ins Stocken gerät oder gar definitiv zerbricht. 
diskutiert und/oder durch praktische Empfehlungen und Handlungsanleitungen ergänzt werden, während grundlagentheoretische Beiträge eher selten anzutreffen sind (vgl. Köpp \& Neumann, 2002, S. 4).

Ohne inhaltlich und/oder arbeitsfeldspezifisch zu differenzieren, verdeutlicht nachstehende Abbildung den quantitativen Literatur-Output am Beispiel der Sozialen Arbeit und ihrer Grenzbereiche; dabei wird nicht einmal annähernd der Anspruch auf Vollständigkeit erhoben (vgl. Kap. A 4.2.1.1).

Abb. 1

\section{Das Qualitätsthema in Fachpublikationen der Sozialen Arbeit (incl. Grenzbereiche)}

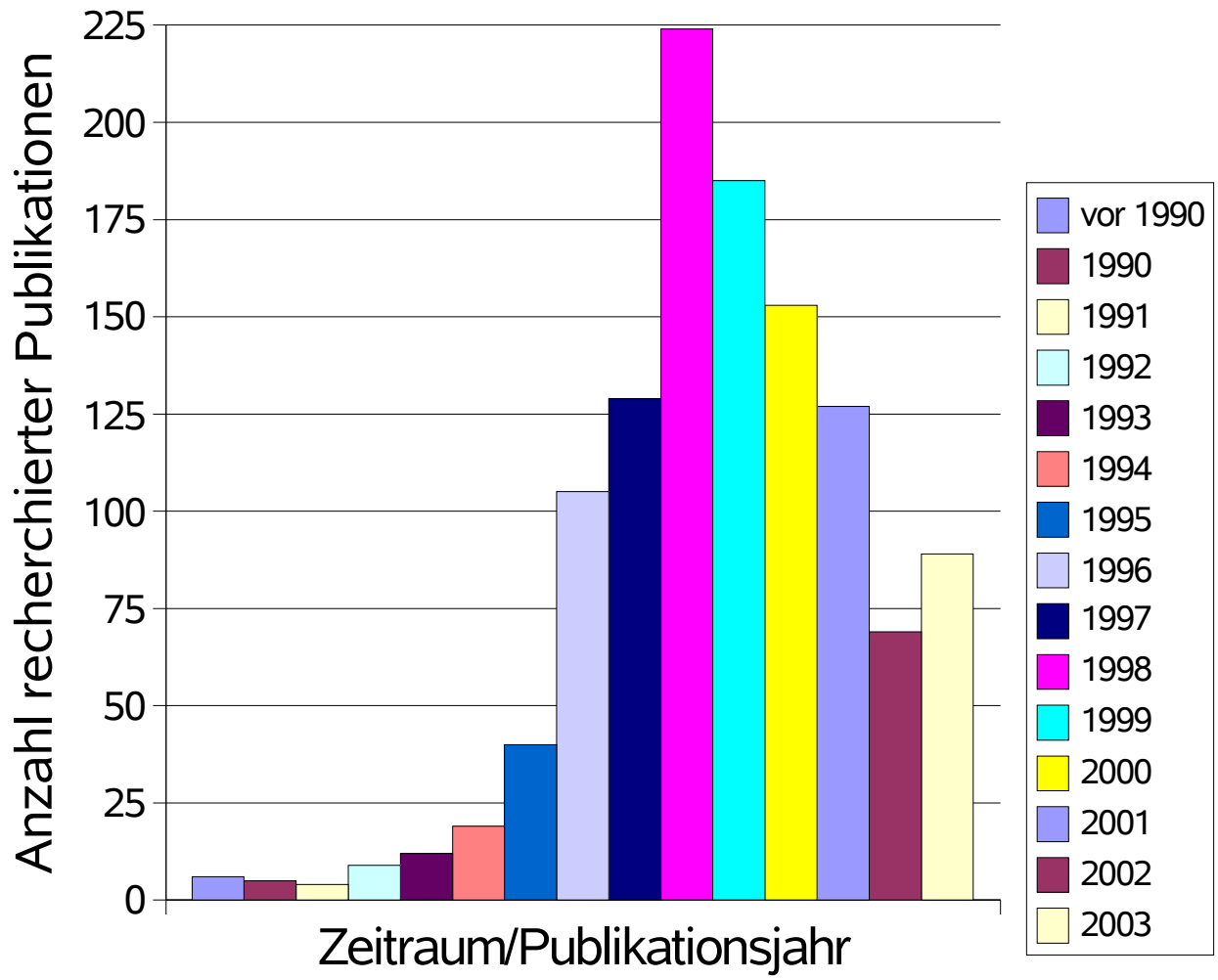

Quelle: eigene Recherchen

Qualitätsmanagement wird explizit bereits seit Längerem in Lehrbüchern der Sozialwirtschaft bzw. des Sozialmanagements (z. B. Arnold \& Maelicke, 1998; Badelt, 1997; Hauser, Neubarth, Obermair, 1997; Merchel, 2001) und in zahlreichen Monographien (z. B. Knorr \& Halfar, 2000; Maelicke, 1996; Müller-Kohlenberg \& Münstermann, 2000; 
Peterander \& Speck, 1999; Schubert \& Zink, 2001) behandelt, wenn auch überwiegend in Form theoretisch unverbundener Einzeldarstellungen, Erfahrungsberichte und Kongressbeiträge - was angesichts der Tatsache, dass Qualitätsmanagement kein einheitliches oder standardisiertes Phänomen darstellt (Pollitt, 2000, S. 68), nicht verwundern kann. Die Anzahl arbeitsfeldspezifischer Musteranleitungen und Lehrmaterialien, Hand- und Werkbücher geht darüber noch hinaus und macht einen beträchtlichen Teil der im Diagramm erfassten Beiträge aus.

Für den Bereich der Sozialwissenschaften lässt sich somit konstatieren, dass sich ein breiter, mit Modellen, Verfahrensvorschlägen und Konzepten angereicherter Publikationsmarkt entwickelt hat (vgl. Köpp \& Neumann, 2002, S. 3 ff.).

Ganz anders stellen sich die Verhältnisse im Bereich der Psychologie dar, die aufgrund des organisationsdiagnostischen - und damit stark Psychologie affinen (vgl. Büssing, 2004, S. 560) - Schwerpunktes der vorliegenden Arbeit (s. Kap. A 5.3.3) ausführlicher erörtert werden sollen.

$\mathrm{Zu}$ den laut Diagrammtitel einbezogenen Grenzbereichen gehören auch psychologische Beiträge aus der Kinder- und Jugendhilfe, Jugendpsychiatrie und Heilpädagogik. Aus dem Bereich der Erziehungsberatung wurde z. B. bereits 1998 ein zweibändiges Werk zum Qualitätsmanagement vorgelegt, das zahlreiche Aspekte der Diskussion thematisiert (Dietzfelbinger \& Haid-Loh, 1998). Klinisch tätige Psychologen waren ohnehin relativ früh von deren Auswirkungen im Gesundheitswesen tangiert und meldeten sich zu Wort (Schwarz, 2000).

Im Verhältnis zur Gesamtzahl der einschlägigen Publikationen muss jedoch festgestellt werden: Das Qualitätsthema scheint auf breiter Front noch nicht in der akademischen Psychologie angekommen zu sein!

Dieser Eindruck bestätigt sich besonders augenfällig in jener Teildisziplin, die sich mit "Zusammenhängen des Erlebens und Verhaltens bzw. Handelns des Menschen mit Struktur-, Prozess- und Zielcharakteristika von Organisationen" befasst (Schuler, 2004, S. 10) - die Organisationspsychologie. Ein Qualitätsmanagement im Sinne umfassender qualitätsbezogener Kontextsteuerung (vgl. Schiepek \& Bauer, 1998, S. 27) in einer 
Organisation ist - so sollte man meinen - genuiner Forschungsgegenstand einer zeitgemäßen Organisationspsychologie, die zunehmend auch den Einfluss von Makrovariablen auf Person, Gruppe und Organisationsverhalten in den Blick nimmt (Weinert, 1998, S. 61 ff.).

Bei Durchsicht aktueller Lehrbücher der Wirtschafts-, Arbeits- und Organisationspsychologie (z. B. Frieling \& Sonntag, 1999; Gebert \& Rosenstiel, 2002; Hoyos \& Frey, 1999; Kirchler, 2003; Rosenstiel, 2000; Schuler, 2004; Ulich, 2001; Weinert, 1998; Wiswede, 2000) fällt indes der geringe Anteil auf, der diesem Thema gewidmet ist.

Der Qualitätsbegriff als Index-Kategorie kommt nur in zusammengesetzten Termini vor wie "Qualität der Arbeit/des Lebens/des Arbeitslebens" (z. B. Weinert, S. 6, 57, 194 ff., 400 ff.; Schuler, S. 48, 161, 563f, 594; Ulich, S. 51), "Qualitätsaudit" (z. B. Frieling \& Sonntag, S. 65) oder "Qualitätszirkel" (z. B. Rosenstiel, S. 102 ff., 208, 280; Frieling \& Sonntag, S. 178, 435; Wiswede, S. 192; Weinert, S. 8, 20, 58, 182, 398ff., 493, 496; Schuler, S. 389, 402, 405, 446 ff., 563).

Qualitätsmanagement wird bei Wiswede, Kirchler, Frieling \& Sonntag, Rosenstiel und Gebert \& Rosenstiel gar nicht und bei Weinert nur in Verbindung mit der Entwicklung der Organisationsstruktur (S. 644f) und der Organisationsstrategie/-philosophie des Total Quality Management kurz erwähnt (S. 678f), ebenso bei Ulich (S. 359), während der Begriff bei Schuler immerhin elfmal auftaucht und im Rahmen der Diagnose von Qualität auf knapp vier Seiten exemplarisch erörtert wird (Büssing, in Schuler, S. 591 ff.).

Eine Ausnahme bildet das Kapitel "Qualitätssicherung und Qualitätsmanagement" von Schultz-Gambard, Lauche \& Hron in Hoyos \& Frey (1999), das sich unter den erwähnten Lehrbüchern als einziges systematisch mit dem Thema befasst und dessen psychologische Implikationen ansatzweise diskutiert (S. 94 ff.). Auch diese AutorInnen konstatieren jedoch: "Die Psychologie hat ... TQM nicht als wichtigen Forschungsgegenstand erkannt ..." (S. 94).

Recherchen im aktuellen Fachwortverzeichnis der American Psychological Association $(A P A)$ und in mehreren amerikanischen Hand- und Lehrbüchern der Industrie- und Organisationspsychologie ergeben ein ähnliches Bild.

In der jüngsten Auflage des Thesaurus of Psychological Index Terms (Walker, 2001) ist unter quality management oder quality assurance Fehlanzeige zu vermelden; lediglich quality circles, quality control, quality of care, quality of education, quality of life, quality of services und quality of work life kommen im 
Index vor.

Hellriegel, Slocum \& Woodman (1995), Aamodt (1996), Muchinsky (2000) und DuBrin (2002) verwenden den Begriff des quality management gar nicht bzw. nur in Verbindung mit Total Quality Management $(z . B$. DuBrin, S. 295f). Bei Hellriegel et al. wird TQM als Stichwort relativ häufig erwähnt und als eines von mehreren "organizational issues for the 1990s" (S.11f) auf gut einer Seite gewürdigt. Sogar die ISO 9000 findet als vor allem in Europa angewandtes Qualitätssicherungs-System bei den Autoren Beachtung (S. 211f). Ansonsten wird Qualität nur als quality of work life (Hellriegel et al., S. 659, 695; Aamodt, S. 270 ff.; Muchinsky, S. 6f, 453f), quality circles (Hellriegel et al., S. 701; Aaamodt, S. 445), und quality-enhancement strategy (Muchinsky, S. 173) thematisiert.

Dass die randständige Position des Qualitätsthemas in Lehrbüchern der Psychologie kein Charakteristikum dieser - naturgemäß nicht immer topaktuellen - Publikationsform ist, bestätigt sich bei Durchsicht namhafter psychologischer Datenbanken und Bibliotheksregister, auch unter Einschluss internationaler Zeitschriftenbeiträge (PSYNDEXplus, PsycInfo u. a., s. Kap. A 4.2.1.2).

Über die Gründe für die Vernachlässigung eines im Humandienstleistungsbereich derart breit diskutierten Gegenstandes durch die akademische Psychologie kann nur spekuliert werden:

- Ist das Thema seiner ingenieurwissenschaftlichen und betriebswirtschaftlichen Provenienz wegen (s. Kap. B 1.1) nicht "psychologisch" genug, um wenigstens im Rahmen der Organisationspsychologie eine bedeutendere Rolle zu spielen? Diese Annahme fällt schwer, weist doch die psychologische Forschung namentlich im Gewande ihrer früher so bezeichneten Teildisziplinen Angewandte Psychologie, Arbeits- und Betriebspsychologie seit jeher eine starke Affinität zur industriellen Arbeitswelt und anderen Großorganisationen auf.

"Insofern wurde der Taylorismus in Verbindung mit der Experimentalpsychologie zum 'Türöffner' in den Betrieben und der Erste Weltkrieg aufgrund entsprechender Nachfrage nach psychologischer Beratung zum 'Schrittmacher' der angewandten Wirtschaftspsychologie" (Rüegsegger, 1986, zit. nach Bungard, Holling \& Schultz-Gambard, 1996, S. 10).

- Ist das Thema mit seiner in weiten Teilen unspezifischen Substanz und den allenfalls punktuell originären Beiträgen seiner Propagandisten (s. Kap. B 3.) bereits hinlänglich 
von organisationspsychologischer Forschung absorbiert? Handelt es sich also in erster Linie um das Abfüllen alten Weins in neue Schläuche? Eine derart selbstbewusste Einstellung $\mathrm{zu}$ einem geradezu boomenden Markt neuer bzw. neu entdeckter Wissensprodukte sollte sich auf entsprechende Analysen gründen, die es jedoch in der psychologischen Fachliteratur nicht gibt - quod erat demonstrandum.

- Ist das Thema als aus der Praxis industrieller Produktion transportiertes Erfahrungswissen mit erst rudimentärer theoretischer Verankerung in den Bezugswissenschaften - namentlich der Betriebswirtschaftslehre (s. Kap. B 1.1, 2.) - nicht "würdig" oder ergiebig genug, um von der akademischen Psychologie als lohnenswertes Forschungsgebiet wahrgenommen zu werden? Dies wäre erstaunlich, wo sich doch die Organisationspsychologie ihrer Wurzeln im industriellen Fertigungsbereich und im Umfeld militärischer Verwertungsinteressen keineswegs zu schämen scheint.

"Die Psychodiagnostik konnte sich innerhalb weniger Jahrzehnte explosionsartig ausweiten, weil naheliegende Interessen beim Militär, im Arbeitsleben und im klinischen Bereich zu Forschungsaufträgen für Psychologen führten" (Bungard et al., 1996, S. 11).

- Ist das Thema wissenschaftlich bereits obsolet und für die Psychologie "erledigt", noch bevor die deutsche Forschung sich seiner angenommen hat? Spiegelt sich darin die Trendsetterfunktion amerikanischer AutorInnen wider, welche die mit dem Label "Total Quality Management" (TQM, s. Kap. C 1.4.2) assoziierte Qualitätsbewegung als passee betrachten (vgl. DuBrin, 2002, S. 296)? Dies wäre eine denkwürdige, volkswirtschaftlich u. U. hochrationale Variante der zuvor formulierten Option, eigene Forschungsbemühungen wegen mutmaßlicher Unergiebigkeit zu unterlassen.

- Oder ist das Thema bislang einfach "verschlafen" und in seiner Dringlichkeit unterschätzt worden? Ist die zögerliche Befassung womöglich darauf zurückzuführen, dass rechtlich verbindliche Vorgaben zur Qualitätssicherung bzw. -entwicklung (zur Nomenklatur s. Kap. C 1.1), wie sie die Praxisfelder Soziale Arbeit, Bildung und Gesundheitswesen seit Jahren kennen, erst allmählich ins Blickfeld der PsychologInnen geraten (s. Kap. A 4.1.5)? Dafür spricht, dass sich die bislang gründlichsten Bemühungen um das Thema im Bereich der Klinischen Psychologie abspielen, mithin in einem Fachgebiet, das von den rechtlichen Rahmenbedingungen - derzeit am 
deutlichsten in Form des Pflege-Qualitätssicherungsgesetzes (Deutscher Bundestag, 2001) ausgestaltet -, in zunehmendem Maße tangiert ist.

Dessen ungeachtet klagte der Vorsitzende der Fachgruppe Qualitätsmanagement in der Sektion Klinische Psychologie: "Wir müssen uns hierbei den Vorwurf gefallen lassen, dass wir durch mangelnde Kenntnis der Bedeutung der QM-Thematik für die Organisationsentwicklung ... eine historische Chance vertan haben, als im Bereich der stationären Versorgung diese Thematik auf unterschiedlichsten Ebenen diskutiert wurde" (Schwarz, 2000, S. 2).

Andererseits:

So wenig Qualität im Sinne des Bemühens um Fachlichkeit und Nutzenstiftung eine Erfindung des Qualitätsmanagements ist, so unstreitig dürfte sein, dass die Güte z. B. von unternehmerischen Problemlöse-, Führungs- und Entscheidungsprozessen sowie Strategien des Managements, durch geeignete Maßnahmen und Vorkehrungen solche Qualität zu optimieren, implizit schon lange - spätestens seit der humanistischen Neuorientierung der Disziplin in den Siebzigerjahren (vgl. Greif, 2004, S. 48) - im Zentrum organisationspsychologischen Interesses stehen. Stellvertretend seien hier nur die Bereiche der Personalauswahl und -entwicklung genannt, in denen es letztlich um qualitative Verbesserungen zugunsten von Organisationszielen und beteiligten Menschen ging und geht.

"Der Gefahr entgegenzutreten, dass Menschen allein als Produktionsfaktoren betrachtet werden, dass individuelle Interessen grundsätzlich der wirtschaftlichen und technischen Rationalität geopfert werden, bleibt ... im Berufsleben eine ubiquitäre Aufgabe" (Schuler, 2004, S. 12).

Dies könnte als Indiz dafür betrachtet werden, dass die unter dem Label "Qualitätsmanagement" subsumierten praktischen und wissenschaftlichen Fragestellungen doch mehr oder minder in allgemein organisationstheoretischen und speziell organisationspsychologischen Konzepten aufgehen, jedenfalls was ihre klassischen Psychologie affinen Themen anbelangt: z. B. Einstellungen, Interaktion, Selektion, Führung. Dass darüber hinaus andere Disziplinen maßgeblich tangiert sind, ändert daran nichts; denn keine Organisation agiert entlang der von wissenschaftlicher Systematik gezogenen Grenzen.

Organisationspsychologie bearbeitet das weite (Gegenstands)-"Feld zusammen mit vielen Nachbardiszi- 
plinen, wie der Betriebswirtschaft, den Ingenieurwissenschaften, der Arbeitsmedizin, der Betriebspädagogik oder der Organisationssoziologie sowie der interdisziplinär angelegten Arbeitswissenschaft und Ergonomie". "Das Besondere der Organisationspsychologie im Vergleich zu anderen Anwendungsfächern der Psychologie ... liegt in ihrer historischen, theoretischen und methodischen Vernetzung mit ihren Nachbardisziplinen" (Greif, 2004, S. 19).

Explizit werden die Fragestellungen des Qualitätsmanagements erst in jüngster Zeit als psychologische Themen begriffen (vgl. Schultz-Gambard et al., 1999, S. 94) und als Anwendungsgebiet organisationspsychologischer Forschung betrachtet. Erst vereinzelt taucht der Begriff des Qualitätsmanagements neben Themen wie Gruppenarbeit oder Organisationsentwicklung in Vorlesungsverzeichnissen und -unterlagen auf, z. B. auf der Website des arbeits- und organisationspsychologischen Bereichs der Universität Mannheim (www.psychologie.uni-mannheim.de/psychol/info). Insofern verwundert nicht, dass die systematische Behandlung des Themas selbst in aktuellsten Lehrbüchern zu wünschen übrig lässt. 


\section{Erkenntnisinteresse, Fragestellungen und Gliederung der Arbeit}

\subsection{Erkenntnisinteresse}

Von der zuvor geschilderten Situation ausgehend und im Anschluss an Vorüberlegungen zum literaturanalytischen und empirischen Teil der Studie (Kap. A 4. und 5.), will diese Arbeit vor allem Folgendes leisten:

1. soll das Qualitätsthema in Wissenschaft und Praxis verortet und der interdisziplinäre Charakter eines QM beleuchtet werden (Kap. B 1. - 4.). Grundlage bildet die Analyse einschlägiger Fachliteratur aus Sozialwissenschaften, Psychologie und Betriebswirtschaftslehre.

2. soll überprüft werden, inwieweit sich QM in wesentlichen Facetten mit organisationstheoretischen Konzepten vereinbaren lässt (Kap. B 3.). Dabei geht es um die Ermittlung relevanter Schnittmengen und die mögliche (Teil-)Transformation QM-typischer Ansätze in Sprache und Praxis der Organisationsforschung. Grundlage bildet wiederum die Analyse der recherchierten Quellen.

3. soll der Frage nachgegangen werden, ob und wie weit sich ein QM, dessen Konzeptualisierung den Ingenieurwissenschaften und der betriebswirtschaftlichen Praxis entstammt, auch für die Optimierung sozialer Dienstleistungen eignet. Dabei geht es um eine Klärung zentraler Begriffe sowie um eine Systematisierung, Darstellung und Bewertung der bekanntesten Konzepte aus Sicht der Praxis, soweit einschlägige Erfahrungen in der Fachliteratur berichtet sind (Kap. C 1. - 3.). Grundlage bildet die Literaturanalyse des Autors im Bereich der Sozialen Arbeit und relevanter Grenzbereiche (Gerull 1996 - 2004).

4. soll empirisch untersucht werden, welche Umsetzung, Akzeptanz und Nutzenbewertung durch professionelle Akteure ein QM im Allgemeinen und diverse Maßnahmen der Qualitätsentwicklung im Besonderen erfahren (Kap. D 1. - 4.). Dabei geht es vor allem um die aktuelle Praxis in sozialen Einrichtungen und Diensten, namentlich der Jugend- und Sozialhilfe, auch im Vergleich zu früheren Erhebungszeitpunkten (s. Punkt 6). Grundlage bildet eine bundesweite Befragung von Qualitätsbeauftragten und 
Leitungskräften aus Tätigkeitsfeldern der Sozialen Arbeit.

5. soll im Rahmen dieser Befragung speziell das Instrument des/der Qualitätsbeauftragten untersucht und hinsichtlich seiner konkreten Ausgestaltung in der institutionellen Praxis beleuchtet werden. Dabei geht es einerseits um die empirische Prüfung von Hypothesen über vermutete Korrelationsbeziehungen (Kap. D 4.7), andererseits um die beschreibende und explorierende Darstellung eines bislang weitgehend "unvermessenen" Werkzeugs des Qualitätsmanagements aus Sicht konkreter AnwenderInnen, einschließlich deren eigener Rollen- und Konfliktwahrnehmung (Kap. D 4.6.8).

6. soll zu weiteren, eher als Nebenprodukt zu betrachtenden, Fragestellungen (u. a. zur Nutzeneinschätzung bestimmter Maßnahmen der Qualitätsoptimierung, zur Einstellung sozialer Fachkräfte zum Qualitätsmanagement) auf empirisches Material des Autors teilweise aus Vorjahren - zurückgegriffen werden, das nach Möglichkeit zu den aktuellen Daten sowie zu Ergebnissen anderer Autoren (Wetzler, 2003; Peters, Dörfer \& Schollmeyer, o. J.) in Beziehung gesetzt werden kann (Kap. D 1. - 3.).

Der innovative Wert der literaturanalytischen Untersuchung besteht weniger in der Erörterung der sich abzeichnenden Integration des QM in einen größeren theoretischen Kontext, als vielmehr in der umfangreichen Recherche- und Systematisierungsarbeit, die Ausgangspunkt für weitere Konzeptualisierungen sein kann.

Im empirischen Teil der Untersuchung wird insofern Neuland betreten, als nicht auf ähnliche Studien rekurriert werden konnte. Wenngleich Verfahren der deskriptiven Statistik, Korrelationsmaße, Varianzanalysen und Signifikanztests, punktuell auch Klassifikationsverfahren (Faktorenanalyse) zur Anwendung gelangen, befasst sich die Arbeit nicht primär mit empirisch zu prüfenden Hypothesen zum untersuchten Gegenstandsbereich. Angesichts der skizzierten Enthaltsamkeit bisheriger organisationsdiagnostischer Forschung erscheint es legitim, zunächst einmal einen Teil des Feldes bestandsmäßig erfassen zu wollen und die Ergebnisse eher in einen "Entdeckungs-" als in einen "Begründungszusammenhang" zu stellen (vgl. Wiswede, 2000, S. 40). Insofern überwiegt der explorative und deskriptive, populationsbeschreibende Charakter der Untersuchung und das Analyseniveau ist eher molar als molekular. 


\subsection{Fragestellungen}

Die nachstehenden Fragen sind im Rahmen der Studien nur teilweise als Hypothesen (s. Kap. A 5.2) expliziert, implizit aber sämtlich in den zuvor formulierten Erkenntnisinteressen angesprochen. Die Aufzählung entspricht dabei grob der textlichen Gliederung der Arbeit (s. Kap. A 3.3) und verweist auf den Zusammenhang, in dem diesbezügliche Sachverhalte thematisiert und Fragen im Einzelnen erörtert werden.

1. Untersuchungsfragen zum Komplex "QM und Organisationsforschung":

○ In welchem Kontext steht die Befassung mit Qualität und QM?

- Welche Grundbegriffe gilt es zu klären?

- Wo lässt sich das Thema wissenschaftssystematisch und praktisch verorten?

- Wo liegen konzeptionelle Schnittstellen zwischen QM und Organisationsforschung?

- Lassen sich Ansätze des QM unter organisationspsychologische/-theoretische Labels subsumieren?

○ Welche Qualitäts- und QM-Konzepte werden diskutiert und wie sind sie bezüglich der Besonderheit sozialer Dienstleistungen zu bewerten?

- Welche Instrumente des QM spielen in der Praxis eine wichtige Rolle?

2. Untersuchungsfragen zum Komplex "QM in sozialen Einrichtungen und Diensten":

- Wie hat sich die Umsetzung von QM in der Praxis entwickelt?

- Nach welchen Konzepten organisieren soziale Einrichtungen und Dienste ihr QM?

- Welche Strukturelemente und Maßnahmen kommen dabei zur Anwendung?

- Kovariieren bestimmte Strukturen mit der Trägerschaft, Einrichtungsgröße und/oder dem Arbeitsfeld?

○ Wie wird der Nutzen bestimmter Maßnahmen zur Qualitätsentwicklung von PraktikerInnen eingeschätzt?

- Gibt es diesbezüglich Hinweise auf Unterschiede zwischen Leitung, Qualitätsbeauftragten und Mitarbeiterschaft?

○ Wie stehen soziale Fachkräfte generell zum QM? 
- Gibt es Hinweise auf einrichtungs-, gruppen- und/oder zeitpunktspezifische Unterschiede in dieser Frage?

3. Untersuchungsfragen zum Komplex "Erfahrungen mit dem Instrument des/der Qualitätsbeauftragten in sozialen Einrichtungen und Diensten":

- Welchen Stellenwert hat das Instrument in der Praxis?

- Wie ist das Instrument strukturell und personell ausgestaltet?

- Kovariieren Verbreitung und Ausgestaltung mit Strukturvariablen der Einrichtungen?

- Wie wird das Instrument von den Akteuren und ggf. von Nicht-Akteuren bewertet?

- Aus welchen Gründen wenden Einrichtungen das Instrument nicht an?

○ Welche Tätigkeiten und Rollen üben Qualitätsbeauftragte in der Praxis aus?

- Kovariieren die Tätigkeiten/Rollen mit Strukturvariablen der Einrichtung oder Personenmerkmalen?

- Wo liegen die wesentlichen Konfliktpotenziale bei der Anwendung des Instruments?

\subsection{Gliederung}

Der literaturanalytische Teil der Studie hat überwiegend synoptischen Charakter. Ausgehend von einer einleitenden Erörterung wesentlicher Bedingungsfaktoren des sozialpolitischen Kontextes (Kap. A 4.1), werden in prägnanten Übersichtskapiteln die spezifischen Grundlagen des Qualitätsmanagements (Kap. B 1.) und Schnittmengen zur Organisationsforschung (Kap. B 2. und 3.) dargestellt, bevor ein Resümee zur Konvergenz und Integration der Ansätze gezogen wird (Kap. B 4.). Nach einem systematischen Überblick zu Konzepten (Kap. C 1.) und Instrumenten (Kap. C 2.) steht die Frage nach Essentials eines Qualitätsmanagements in sozialen Handlungsfeldern (Kap. C 3.) im Mittelpunkt.

In ihrem empirischen Teil ist die Studie vorwiegend organisationsdiagnostisch ausgerichtet und fokussiert auf qualitätsbezogene Strukturen und Prozesse in sozialen Einrichtungen und Diensten. Die unter Einbeziehung von Vor- und Vergleichsstudien dargestellten 
Befunde sind nach den primären Zielgruppen bzw. Datenquellen gegliedert: Mitarbeiterbefragungen (Kap. D 1.), Leitungsbefragungen (Kap. D 2.), Befragungen von Qualitätsbeauftragten (Kap. D 3.) und schließlich die als Hauptstudie durchgeführte bundesweite Befragung von Einrichtungen und Diensten (Kap. D 4.). Als Resümee werden Folgerungen für die Praxis (Kap. D 5.) diskutiert.

Eine Zusammenfassung der wichtigsten Befunde und ein Ausblick auf den zukünftigen Stellenwert des Qualitätsmanagements im Allgemeinen und eines QM in sozialen Handlungsfeldern im Besonderen schließen den Kreis (Kap. E). 


\section{Literaturanalytischer Teil der Arbeit (Abschnitte B und C)}

\subsection{Sozialpolitischer Kontext}

\subsubsection{Struktur des deutschen Sozialsystems und Rolle freier Träger}

Die Bundesrepublik Deutschland ist nach ihrer Verfassung, dem Grundgesetz, ein demokratischer und sozialer Bundesstaat (Art. 20 Abs. 1 GG). Auch die verfassungsmäßige Ordnung der sechzehn Bundesländer muss den Grundsätzen des republikanischen, demokratischen und sozialen Rechtsstaates im Sinne dieses Grundgesetzes entsprechen (Art. 28 Abs. 1 Satz 1 GG). Der Sozialstaatsgrundsatz legitimiert den Gesetzgeber, leistend und gestaltend tätig zu werden, indem er sich um soziale Gerechtigkeit bemüht und die Sicherheit der BürgerInnen zu gewährleisten sucht.

Allerdings ist trotz bundesgesetzlicher Regelungen die inhaltliche Konkretisierung sozialer Dienstleistungen keineswegs einheitlich vorgegeben, sondern eine Angelegenheit sozialpolitischer Aushandlungsprozesse zwischen Bund, Ländern und Gemeinden unter Beteiligung freigemeinnütziger Wohlfahrtsverbände. Im Rahmen der bundesweit geltenden Normen, die im Sozialgesetzbuch I ausformuliert sind, verfügen namentlich die Kommunen über eigene Gestaltungsspielräume, z. B. was Menge und Qualitätsstandards von Leistungen, pluralistische Trägerlandschaft und Angebote von Diensten und Einrichtungen anbelangt (Backhaus-Maul, 1998, S. 26).

Dem Sozialstaatspostulat verpflichtet sind jedoch nicht allein der Bund und seine Verwaltungseinheiten; vielmehr verwirklichen sich entsprechende Grundsätze auch im solidarischen und sozialen Verhalten der BürgerInnen, in individueller und kollektiver Selbsthilfe sowie durch mitmenschlichen Beistand in Notsituationen (Wienand, 1999, S. 10). Ungeachtet dieser notwendigen "privaten Fürsorge", die in ihren unterschiedlichen Ausgestaltungen zum "Sozialkapital" einer Gesellschaft beiträgt (J. Müller, 2002), beruht das bundesdeutsche Netz der sozialen Sicherung im Wesentlichen auf den drei Säulen der

- Sozialversicherung (nach einer neueren Systematisierung auch als soziale Vorsorge bezeichnet, Schulin \& Igl, 2002) als einer weitgehend beitragsfinanzierten Solidargemeinschaft zur Absicherung des Lebensstandards gegen Risiken infolge von 
Krankheit, Pflegebedürftigkeit, Unfall, Berufs- und Erwerbsunfähigkeit, Erreichen der Altersgrenze oder Arbeitslosigkeit;

- Versorgung (auch als soziale Entschädigung bezeichnet, Schulin \& Igl, 2002) als einer aus allgemeinen Steuermitteln finanzierten Absicherung des Lebensstandards bestimmter Personengruppen - z. B. Beamte, Soldaten und Zivildienstleistende sowie zum begrenzten Ausgleich von Schäden, für die das Gemeinwesen gesteigerte Verantwortung trägt, z. B. Kriegsopfer, Opfer von Gewalttaten und Impfgeschädigte;

○ öffentlichen Fürsorge (bzw. soziale Förderung und Sozialhilfe, Schulin \& Igl, 2002) als einer aus überwiegend kommunalen Steuermitteln finanzierten und gegenüber anderen staatlichen Sozialleistungen und familiärem Unterhalt nachrangigen Hilfe bei individueller Bedürftigkeit. Die entsprechenden Leistungen lassen sich differenzieren in ein

1. besonderes Hilfe- und Fördersystem (Ausbildungs- und Berufsförderung, Kinderund Erziehungsgeld, Unterhaltsvorschuss, Wohngeld, Kinder- und Jugendhilfe) und ein

2. allgemeines Hilfe- und Fördersystem (Sozialhilfe zur Sicherung des Existenzminimums und zur Hilfe und Förderung in besonderen Bedarfslagen wie Krankheit, Behinderung, Pflegebedürftigkeit, vgl. Wienand, 1999, S. 13).

Leitziele dieses sozialen Sicherungssystems sind größtmögliche Gerechtigkeit, Solidarität, Bürgernähe, organisatorische Vielfalt (keine Einheitsversicherung), Effektivität und Effizienz. Sozialpolitischer Kristallisationspunkt für die Frage nach den richtigen Strukturen ist das Subsidiaritätsprinzip. Mit den Worten des Bundesverfassungsgerichts bedeutet dies, "dass in erster Linie die kleinere Gemeinschaft wirken soll und mit staatlichen Mitteln erst dann einzugreifen ist, wenn es unausweichlich wird." Kann der Einzelne sich nicht selbst helfen, sollen also zuerst die Familie, die Nachbarschaft, die Selbsthilfegruppe, die freie Wohlfahrtspflege, die Gemeinde und zuletzt die staatliche Institution helfend einspringen (a.a.O., S. 10f).

\footnotetext{
"Vor allem im Verhältnis von öffentlicher und freier Wohlfahrtspflege ist die Subsidiarität staatlichen, insbesondere kommunalen Handelns ein zentrales Thema. Die Träger der freien Wohlfahrtspflege machen
} 
für ihre soziale Arbeit einen bedingten Vorrang geltend, der auch in Grundnormen des Sozialrechts seinen Niederschlag gefunden hat, zumal freie Träger vielfach als Pioniere neu auftretende soziale Notlagen aufgreifen und in innovativer Weise überwinden helfen ....

Die Bedeutung des Subsidiaritätsprinzips wurde bei der Revision des Vertrages der Europäischen Union in Maastricht besonders unterstrichen und als übergreifendes politisches Prinzip für die Abgrenzung von Gemeinschaftszuständigkeit und Zuständigkeiten der Mitgliedstaaten anerkannt. Aus dem Subsidiaritätsprinzip wird auch der Vorrang nationaler Sozialpolitik abgeleitet ..." (a.a.O., S. 11).

Von einer grundsätzlichen Bedrohung des Subsidiaritätsprinzips als Folge des europäischen Einigungsprozesses kann somit keine Rede sein; vielmehr sieht der Entwurf für einen "Vertrag über eine Verfassung für Europa" eine konsequente Anwendung und Absicherung dieses Verfassungsprinzips ausdrücklich vor (Calliess, 2004, S. 24). Im Zuge eines Trends zu neoliberaler Wirtschaftspraxis und Gesetzgebung sehen sich die Verbände, Dienste und Einrichtungen der freien Wohlfahrtspflege, organisiert in den Spitzenverbänden

- der Arbeiterwohlfahrt,

- des Diakonischen Werkes der Evangelischen Kirche in Deutschland,

- des Deutschen Caritasverbandes,

○ des Deutschen Paritätischen Wohlfahrtsverbandes (seit 1990 "Der Paritätische"),

- des Deutschen Roten Kreuzes und

- der Zentralwohlfahrtsstelle der Juden in Deutschland

jedoch verstärkter Konkurrenz durch selbstorganisierte Gruppen und privat-gewerbliche Träger gegenüber. Fehlentwicklungen zu bürokratisierten Großorganisationen und die immer weniger tragende weltanschauliche Bindungsfähigkeit in dem "an Kleinstaaterei erinnernde[n] Wohlfahrtsverbandswesen" (Boeßenecker, 2001, S. 108) trugen hierzu entscheidend bei.

Inwiefern die weiterhin bestehende nationalstaatliche Privilegierung frei-gemeinnütziger Unternehmen in Deutschland - die sich vor allem in der Freistellung von diversen Steuerpflichten und in steuerlichen Anreizen für Dritte äußert, den frei-gemeinnützigen Trägern Geld-, Sach- und Zeitspenden zukommen zu lassen - mit europäischem Wettbewerbsrecht vereinbar ist (z. B. dem Verbot von Beihilfen), ist derzeit noch unsicher 
und Gegenstand juristischer Gutachten (Schruth, 2003).

Die Monopolkommission der Bundesregierung (12. Hauptgutachten, zit. nach Wetzler, 2003, S. 15f) verwies jedoch bereits 1998 darauf, dass die Stellung der freien Wohlfahrtspflege im sozialen Versorgungssystem eine Kartellbildung und die Bundesarbeitsgemeinschaft der freien Wohlfahrtspflege (BAG FW) ein staatlich unterstütztes Kartell sei, in dem die Koordination der Leistungen abseits der wettbewerblichen Ordnung in einem weitgehend gegen die Konkurrenz abgeschotteten System stattfinde. Die Steuerbefreiung im Zusammenhang mit dem Gemeinnützigkeitsstatus wird in diesem Gutachten als Diskriminierung privat-gewerblicher Anbieter betrachtet.

\subsection{2 Ökonomisierung des Sozialen}

Im internationalen Vergleich gilt das deutsche Sozialleistungssystem, seit Gründung der Bundesrepublik als umfassendes und lückenloses Netzwerk geknüpft, als "weder ausgeufert noch herausragend" (Alber, 1998, S. 225), aber auch als relativ unmodern, weil perspektivisch vergangenheits-, statt zukunftsorientiert (Schmid, o. J., S. 20f). Die desolate Situation der öffentlichen Haushalte - und hier im Besonderen die der kommunalen ${ }^{3}-$ ist unmittelbar verknüpft mit der Strapazierung dieses Netzes durch Wiedervereinigung, Wirtschaftskrise, hohe Arbeitslosigkeit und demographische Entwicklung. Die Zusammenhänge mit der aktuellen Debatte über die Grenzen des Sozial- und Wohlfahrtsstaates und dessen notwendiger Modernisierung sind evident.

Dass im Kontext der öffentlichen Haushaltssituation auch die Qualität der mit zunehmend verknappten Mitteln finanzierten Sozialleistungen auf den Prüfstand geriet, entbehrt insofern nicht der sachlichen Logik: Wenn schon nicht mehr alles Wünschbare geleistet werden kann/soll, gilt es, die Mittel dort einzusetzen, wo sie sozialpolitisch treffgenau denen zugute kommen, die ihrer am nötigsten bedürfen ("allokative Effizienz") und im Sinne inhaltlich-fachlicher Zielerreichung ("Effektivität") den größten Nutzen stiften (vgl.

3 Die Entwicklung der sozialpolitischen Kompetenzverteilung zwischen Bund und Ländern einerseits und Kommunen andererseits lässt sich als Zentralisierung von Entscheidungskompetenzen und Dezentralisierung der Aufgabenerbringung darstellen, wobei die Länder eine Vermittlungsposition einnehmen. Diese Aufgaben- und Kostenüberwälzung durch den Bundesgesetzgeber hat zur Folge, dass die kommunalen Gestaltungsspielräume vielerorts gegen Null tendieren (Backhaus-Maul, 1998, S. 39). 
Trube, Regus \& Depner, 2001, S. 229). Die dafür notwendigen Entscheidungen bedürfen möglichst rationaler, transparenter und verbindlicher Qualitätskriterien, um fachlich wie politisch legitimiert werden zu können. Darüber hinaus werden dem lange Zeit ungehindert expandierenden Sozialleistungssystem Ineffizienzen und erhebliche Rationalisierungsmöglichkeiten unterstellt (z. B. Schoch, 2000, S. 1), die zu beseitigen bzw. zu erschließen professionellere Formen des Managements, insbesondere des Qualitätsmanagements beitragen sollen.

Sozialrechtliche Änderungen der letzten Jahre stehen eindeutig in diesem Zusammenhang und führen in Richtung einer Ökonomisierung der Arbeit in Einrichtungen und Diensten. Diese kann als Prozess verstanden werden, "... in dem politisch vereinbarte Standards abgelöst werden durch eine stärkere 'Monetarisierung', d. h. Festlegung von output-Zielen, Controlling von input und output, Vergleichbarkeit von Produkten und Betonung von Effizienz als Kontrollkriterien" (Heinze, Schmid \& Strünck, 1997, S. 256).

Will man die Entwicklung dieser "Ökonomisierung des Sozialen" nachzeichnen, fallen mindestens zwei Begriffe ins Auge: Lean Management und Marktwirtschaft.

Das Konzept des Lean Management stammt aus der japanischen Erfolgswirtschaft der Achtzigerjahre und bezog sich als Lean Production zunächst auf die als "zweite Revolution in der Autoindustrie" bezeichnete Produktionsweise bei Toyota (Womack, Jones \& Ross, 1992). "Lean" steht symbolisch für alle Maßnahmen, die darauf abzielen, Überflüssiges aus der Betriebsführung zu entfernen und durch "Verschlankung" des Einsatzes von Personal, Sachmitteln, Produktionsflächen, Entwicklungszeiten und anderen Kostenfaktoren die Effizienz zu erhöhen.

Wesentliche Merkmale dieses Konzepts sind:

- Reduktion von Hierarchieebenen/Abflachung von Hierarchien,

○ Abbau von "nicht-produktivem" Personal, Priorität der Wertschöpfung,

○ Verlagerung von Autonomie auf kleine Einheiten, Teamarbeit,

○ Übertragung von Selbstverantwortlichkeit und Selbstkontrolle im Rahmen von Zielvereinbarungen,

○ differenziertes Controlling- und Berichtssystem als Steuerungsinstrument, 
○ hohe Kunden- und Marktorientierung,

○ kontinuierlicher Verbesserungsprozess im Sinne kleiner, beherrschter Schritte,

- strikte Orientierung an Qualität als strategischem Erfolgsfaktor im Sinne von erfüllten Kundenerwartungen und möglichst weitgehender Fehlervermeidung.

Waren für die Verbreitung des Lean Management in der Betriebswirtschaft vor allem die industriellen Erfolge dieses Produktions- und Betriebsführungssystems verantwortlich, so zeichnet sich seit den Achtzigerjahren auch auf der Ebene ganzer Volkswirtschaften ein Umdenken ab. Während die zumeist sozialdemokratisch geprägten Wohlfahrtsstaaten an die Grenzen ihres Wachstums stießen, verkündeten in den USA und Großbritannien so genannte "Monetaristen" im Gefolge Milton Friedmans eine neue Wirtschaftspolitik, die an die klassische Theorie von der Selbstregulierung einer Wirtschaft anknüpft und dem Staat nur ein sehr begrenztes Eingriffsrecht in die ökonomischen Abläufe und das "freie Spiel der Kräfte" zugesteht. Diese Wirtschaftspolitik wurde alsbald unter den Bezeichnungen "Reagonomics" und "Thatcherism" bekannt und propagiert eine am Modell der freien Marktwirtschaft orientierte Sozialpolitik (vgl. Kurzlechner, 1999).

Es wird von den Vertretern dieses so genannten "neoklassischen Paradigmas" (Etzioni, 1994, S. 20, zit. nach Kurzlechner, 1999, S. 46) angenommen, dass der Sozialstaat bisheriger Prägung teuer und dabei verhältnismäßig ineffektiv sei. Von der Einführung marktwirtschaftlicher Elemente wie Privatisierung, Deregulierung und Wettbewerb auf Seiten der Anbieter verspricht man sich mehr Effizienz und Qualität.

Diese Sichtweise, die auf der als "überheblich" kritisierten (Fukuyama, 1995, S. 33, zit. nach Kurzlechner, 1999, S. 49) Übertragung marktökonomisch durchaus erfolgreicher Prinzipien auf menschliches Verhalten im Allgemeinen beruht, ist stark inspiriert vom klassischen Liberalismus, Utilitarismus und Rationalismus, also von Weltanschauungen, die auf die individuelle Freiheit vernunftorientierter, nutzenmaximierender Bürger setzen. Die ausufernde Euro-Bürokratie und verkrustete Strukturen des Wohlfahrtsstaates haben mit einigungsbedingter Verspätung inzwischen auch in Deutschland die politische Großwetterlage in diesem neoliberalen Sinne bestimmt, allerdings auch heftige Kritik an einer zu weit gehenden Ökonomisierung des Sozialen hervorgerufen. 
Der enormen politischen Bedeutung wegen, die sich mit den hier nur anzudiskutierenden Fragen verknüpft und die auch Rolle und Stellenwert eines Qualitätsmanagements maßgeblich tangieren, sei ein Teil dieser Kritik kurz gewürdigt:

Maaser (2002, S. 135 ff.) konstatiert, dass sich im Gefolge des sozialstaatlichen Legitimationsdiskurses - Stichworte: Versorgungsstaat, Missbrauchsdebatte, Mitnahmeeffekte - ein neues Verständnis von Wohlfahrtskultur herausbilde, das als sozialethische Verlagerung von der Gerechtigkeits- auf die Barmherzigkeitsperspektive bezeichnet werden könne. Die Zurücknahme von selbstverständlich gewordenen Rechten und die stärkere Individualisierung gesellschaftlicher Risiken werden als ethisch zu reflektierende Veränderungen auf der strukturqualitativen Makroebene betrachtet.

B. Müller (1996) problematisiert die Übertragung von Marktmechanismen auf soziale Handlungsfelder und hält für deren Anwendbarkeit den ursprünglichen Charakter des Marktes als Ort des Austauschs für entscheidend: dass Partner im Bewusstsein gegenseitiger Abhängigkeit etwas voneinander wollen, sich jedoch nicht bedingungslos verpflichtet sind und nicht gegenseitig ihren Willen aufzwingen können, sich aber an bestimmte Spielregeln halten müssen (a.a.O., S. 76). Eine solche Dienstleistungsethik als Ethik fairer Zweckbündnisse wird sozialen Handlungsfeldern zwar als grundsätzlich angemessen betrachtet, jedoch nicht als hinreichend, weil sie immer funktionalen Charakter habe, also von einem Zweck-Mittel-Verhältnis her gedacht sei (S. 78). Darüber hinaus wird jedoch eine Ethik der Fürsorge und eine politische Ethik für notwendig gehalten, um dem Charakter sozialer Handlungsfelder gerecht zu werden (S. 77f).

Auch Depner \& Trube (2001, S. 233 ff.) betonen die Notwendigkeit extrafunktionaler Qualitätskriterien und verbinden damit die Frage nach einer universalistischen Ethik des Sozialen, damit Soziale Arbeit nicht zum willfährigen Handwerkszeug gesellschaftlicher Selektionsprozesse verkomme (S. 237). Die Autoren sehen einen Entwicklungstrend vom Wohlfahrtsstaat zum Wettbewerbs- bzw. zum "aktivierenden" Sozialstaat.

Kurzgefasst lautet ihre Analyse (S. 217f): Globalisierung und internationaler Wettbewerb führen auf der nationalen Ebene zunehmend zur Marginalisierung der quasi "konkurrenzuntüchtigen" Personen, deren typische Problemlagen allerdings zumeist individualisierend diagnostiziert werden. Sie sind deswegen Adressaten der Sozialen Arbeit (und nicht der Sozialpolitik), der jedoch immer weniger Ressourcen zur 
Verfügung stehen, da für den Nationalstaat aufgrund von internationalisierten Geld- und Güterströmen zunehmend Steuerabschöpfungsmöglichkeiten verloren gehen. Aufgrund dessen entwickelt sich zumeist zusätzlich noch ein Trend zur Senkung von Sozialabgaben, um in der weltweiten Standortkonkurrenz dem flexiblen Kapital die "besseren" Konditionen anzubieten. Dies verknappt wiederum die Geldmittel für soziale Leistungen, so dass die praktische Sozialarbeit unter noch größeren Effizienzdruck kommt. Sie hat nachzuweisen, dass sie die Modernisierungsopfer doch noch durch "qualitativ hochwertige" Interventionen aktivieren kann, um sie möglichst unabhängig von konsumtiven staatlichen Transferleistungen zu machen.

Ostner (2004) erkennt gleichfalls eine Veränderung in der Logik des sozialen Ausgleichs und der Bedeutung der Solidarität im neuen "Europäischen Sozialmodell". Die Verknüpfung von Sozialleistungen und Beschäftigungsbereitschaft bzw. das "Pochen auf strikte Reziprozität" (S. 53) werden als Anzeichen für eine "Amerikanisierung des Sozialen" bewertet.

Der Rückzug aus aktiver Sozialstaatlichkeit und die Hoffnung der Protagonisten einer ("europäischen") Zivilgesellschaft und des ("amerikanischen") Kommunitarismus, dass Bürger sich selbstbestimmt in einer demokratischen Wohlfahrtsgesellschaft ${ }^{4}$ (Keupp, 2000, S. 41) organisieren, statt sich vom Versorgungsstaat alimentieren zu lassen, verschleiert nach Depner \& Trube (a.a.O.) allerdings, wem diese Entwicklung letztlich nütze und schade:

Sie "schadet dem klassischen Klientel der Sozialen Arbeit, und von der Zivilgesellschaft profitieren die, denen es bereits jetzt schon materiell gut geht, die in ihrem sozialen Status und in ihrer personalen Identität nicht beschädigt sind und die die weiteren Modernisierungsschübe unserer Gesellschaft nicht nur nicht fürchten müssen, sondern denen sich hier neue Handlungsspielräume eröffnen" (S. 224).

Heiner (1996a, S. 28f) weist auf einen maßgeblichen Unterschied zwischen Humandienstleistungen und Güterproduktion hin:

"Die Qualität von Humandienstleistungen hängt entscheidend davon ab, welche Vorstellung eine Gesellschaft und dabei auch die zuständigen Berufe davon haben, wie man mit Menschen umgehen sollte, was man ihnen zumuten darf und was man ihnen schuldet. Diese ethische und normative Dimension des Qualitätsbegriffes ist für Humandienstleistungen zentral, während es bei der Produktion von Gütern nur um das Ethos der Zuverlässigkeit geht."

4 Die demokratische Wohlfahrtsgesellschaft ist geprägt von der "Rekonstruktion des Sozialen durch eine aktive und kompetente Gesellschaft." "Aufgabe der Politik ist es nicht, die Gesellschaft zu bedienen, sondern sie zu aktivieren" (Dettling, 1995, zit. nach Keupp, 2000, S. 41). 
Speck (2002, S. 6) betrachtet das Wirtschaftssystem als ein eigenes, selbstreferenziell konstituiertes System, das sich am eigenen Wachstum orientiere und zu dessen Aufgabe es nicht gehöre, sich an der Lösung anderer Probleme, zum Beispiel sozialer, zu beteiligen. Moralisches Handeln sei für die Wirtschaft nicht nur entbehrlich, sondern geradezu dysfunktional (Luhmann, 1987, zit. nach Speck, 1999, S. 21). Die fortschreitende Ökonomisierung teile die Gesellschaft immer deutlicher in "Gewinner" und "Verlierer" (Speck, 2002, S. 6ff.).

Speck (a.a.O.) warnt: "Die gegenwärtige Überhitzung des wirtschaftlichen Wettbewerbs forciert den Eigennutz, auch der verschiedenen Korporationen. Das Soziale droht dabei zu einem Epi-Phänomen zu degenerieren".

Nach Schmidt-Grunert (1997, S. 113) besteht ein offensichtlicher Zusammenhang zwischen der Expansion des sozialen Sektors und dem Wachstum von Armut und Verelendung in der Gesellschaft. Die zunehmend geforderte Rolle als Ausfallbürge für ein Versagen von Wirtschaft und Politik ist auch für Prölß (1999, S. 106) der Grund für den beschleunigten Professionalisierungsschub der sozialen Berufe seit den Siebzigerjahren.

Die im Gefolge der öffentlichen Haushaltskrise inzwischen betriebene staatliche Sparpolitik hält Schmidt-Grunert deshalb mitnichten einem überproportionalen Wachstum des sozialen Bereichs, "funktionalem Dilettantismus" oder Verschwendung geschuldet, sondern diese wird als Ausdruck eines politischen Willens bewertet, staatliche Leistungen abzubauen (a.a.O., S. 109 ff.). Während in der Tradition der Sozialen Arbeit stets die Auffassung vertreten worden sei, dass der soziale Sektor seiner Kompensationsaufgabe nur gerecht werden könne, wenn er nicht den Zwängen der freien Marktwirtschaft unterworfen sei, werde die Ökonomie nunmehr mit Hoffnungen belegt, "aus weniger mehr" zu machen (S. 115).

Die kritische Auseinandersetzung mit der "BWL-isierung" der Sozialen Arbeit (SchmidtGrunert, 1997) schließt jedoch eine "maßvolle und bedachte Ökonomisierung" (Hauser et al., 1997, S. III) und ein Lernen von der Wirtschaft ohne Berührungsängste nicht aus, z. B. im Hinblick auf ein besseres Marketing (Hütte, 1998, S. 120). Im Gegenteil: Die "Rückführung [hier: der Sozialpädagogik, P. G.] aus den subventionierten Kunstgärten in die ökonomischen Lebenswirklichkeiten" wird als nicht zu unterschätzender 
Qualitätsbeitrag bewertet (a.a.O., S. 121).

Die Befrachtung der Ökonomie mit überzogenen Heilserwartungen entspricht indes wohl mehr dem menschlichen Bedürfnis nach eindeutigen Auswegen aus einer Krise; denn

\begin{abstract}
"Krisenbeschreibungen und -beschwörungen gehören ... $\mathrm{zu}$ den Grundfigurationen der modernen Gesellschaftstheorie, die ja auch (fast) immer Theorie der Gesellschaftsreform sein will. Die selbstverständlich - wissenschaftliche Analyse der Krise geht einher mit einem Lösungsangebot, dem Aufzeigen eines Ausweges, dem Versprechen einer umfassenden Bewältigung und damit auch Beendigung der Krise. Die Einlösung des Versprechens ist freilich mit einem entscheidenden Akteurwechsel verbunden: Damit es zur (Er-)Lösung kommt, ist die strenge, 'orthodoxe', 'methodische' Befolgung des von den einen (der Avantgarde) gewiesenen Weges seitens der jeweils 'anderen' ... unbedingt notwendig; diese dürfen bzw. müssen dazu allerdings meist erst noch befähigt, sprich: erzogen werden" (Volz, 2000, S. 169).
\end{abstract}

Vieles spricht dafür, dass sich die Geschichte derartiger gesellschaftlicher Grundfigurationen auch gegenwärtig wiederholt. Ein markantes Beispiel hierfür liefert auch die angestrebte Modernisierung der öffentlichen Verwaltung.

\title{
4.1.3 Modernisierung der öffentlichen Verwaltung
}

Anfang der Neunzigerjahre begann das betriebswirtschaftliche Modell des Unternehmens die traditionelle Sichtweise öffentlicher Verwaltungen und ihrer Organisationsformen abzulösen. Das Neue Steuerungsmodell (NSM), namentlich propagiert von der Kommunalen Gemeinschaftsstelle für Verwaltungsvereinfachung (KGSt), der "Innovationsagentur des Deutschen Städte- und Gemeindetages" (B. Müller, 1996a, S. 8), begann seinen Siegeszug durch die bundesdeutschen Amtsstuben.

Das zentrale Motiv der Diskussionen um die Umstrukturierung der öffentlichen Verwaltungen "von der Behörde zum Dienstleistungsunternehmen" war bzw. ist die Hoffnung, die Handlungsfähigkeit des kommunalen Systems angesichts stagnierender oder rückläufiger Finanzmittel zu erhalten. Die Kritik richtete sich dabei insbesondere gegen die zentralen Organisationsstrukturen der Verwaltung, die großen Macht- und Entscheidungsbefugnisse der Querschnittsämter, die Orientierung am Input, ein veraltetes Finanzmanagementsystem und schwach ausgebildete Leistungsanreize im Vergütungssy- 
stem des öffentlichen Dienstes (Struck, 1995, S. 285). Den Kommunalverwaltungen wurde eine Management-, Attraktivitäts- und Legitimitätslücke attestiert. Defizite wurden in der effektiven Steuerung, der Motivierung von MitarbeiterInnen und der Rechtfertigung der Leistungen gegenüber den Bürgern ausgemacht (KGSt, 1993).

Als charakteristisch für die traditionelle Steuerung öffentlicher Verwaltungen gilt die Inputorientierung. Dabei wurde/wird der Verwaltung seitens der Politik ein Input in Form von Sach-, Finanz- und Personalmitteln zur Verfügung gestellt, ohne dass hinreichend deutlich war/ist, welche Leistungen im Einzelnen damit erbracht werden sollen. Demgegenüber betont das Konzept der Outputsteuerung, dass der gesamte Prozess von Planung, Durchführung und Kontrolle des Verwaltungshandelns strikt an den beabsichtigten und tatsächlichen Ergebnissen des Handelns ausgerichtet werden soll (Jordan \& Reisman, 1998, S. 60).

Die beschriebenen Probleme sollten durch Anleihen beim Konzept des Lean Management (s. o.) gelöst werden. Die wichtigsten Elemente dieses Neuen Steuerungsmodells (auch als New Public Management, NPM, diskutiert) sind (vgl. Struck, 1995; Kühn, 1995; Jordan \& Reisman, 1998):

○ die Übertragung betriebswirtschaftlicher Managementtechniken auf dafür geeignete Teile der Kommunalverwaltung ("Konzernmodell: Leitbild Dienstleistungsunternehmen"),

○ ein verändertes Verhältnis von Politik und Verwaltung (hier Zuständigkeit für strategische Planung und Kontrolle längerfristiger Richtungsentscheidungen, dort operative Umsetzung und Controlling der politischen Leitwerte, Steuerung mittels Kontraktmanagement),

○ die grundlegende Orientierung am Output der Verwaltung, d. h. ihren in Produktbeschreibungen $\mathrm{zu}$ definierenden Leistungen und den zuzuordnenden Kosten (Budgetierung als Teil des Kontraktmanagements zur Absteckung des Ressourcenrahmens, innerhalb dessen flexibler als bisher agiert werden kann), 
○ die Zusammenführung von Leistungs- und Budgetverantwortung auf der Basis der Selbststeuerung von Ämtern und Diensten sowie eine veränderte Darstellungsform kommunaler Haushalte (dezentrale Ressourcenverantwortung, Einführung des kaufmännischen Rechnungswesens statt der bisherigen Kameralistik),

○ die Einführung eines systematischen Controllings (strategisches und operatives, zentrales und dezentrales Steuerungsinstrument durch rechtzeitige Informationsbeschaffung und -verarbeitung, z. B. über Kennzahlen),

○ das Postulat der Orientierung an den Bedürfnissen der Abnehmer der kommunalen Dienstleistungen (Kundenorientierung),

○ die systematische Einführung von Markt- und Wettbewerbselementen in die Verwaltungsorganisation (Personalmanagement, Qualitätsmanagement, Benchmarking),

○ die Auslagerung von Leistungserbringungen auf preiswertere nichtöffentliche Anbieter (contracting out).

Für eine Bewertung des Neuen Steuerungsmodells muss maßgeblich sein, wie die neuen Steuerungselemente im Bereich der öffentlichen Verwaltung umgesetzt werden. Als Instrumente sind sie potenziell vernünftig und stellen gegenüber der bisherigen Praxis einen Fortschritt dar.

Doch auch der in den Siebziger- und Achtzigerjahren unternommene Versuch einer "Neuordnung der sozialen Dienste" im Zuge der kommunalen Gebietsreformen erfolgte in gut gemeinter Absicht ohne durchschlagenden Erfolg. Trotz zum Teil drastischer Veränderungen innerhalb des organisatorischen Gefüges blieben die beabsichtigten Effekte einer innovatorischen Praxis aus. Die seinerzeit stattgefundenen Verwaltungsreformen können jedoch als eine notwendige Voraussetzung für modernisierte Soziale Arbeit angesehen werden, wie sie im Zuge der Neuen Steuerungsmodelle nun vor allem über ein verbessertes Personalmanagement erreicht werden soll (Flösser \& Otto, 1992, S. 10 ff.).

Kritik am Ansatz des NPM ist vielfach geübt worden: an der vermeintlich dahinter stehenden Sozialabbaumentalität, an der Verbetriebswirtschaftlichung der Sozialen Arbeit, 
am vordergründigen Sparkonzept, an der Unangemessenheit für die freie Wohlfahrtspflege, an der Gefährdung gewachsener Partnerschaften zwischen öffentlichen und freien Trägern, an der Missbrauchsmöglichkeit von Instrumenten. Andere AutorInnen sehen dagegen Chancen, verkrustete Strukturen auch bei freien Trägern aufzubrechen und Angebotsformen kritisch zu revidieren. An dieser Stelle seien lediglich einige Aspekte im Zusammenhang mit Qualitätsmanagement aufgegriffen.

So bemängelt Klatetzki (1996) die Vernachlässigung der kundenorientierten Perspektive. Qualität werde in der Praxis letztlich immer durch Experten definiert. Deren Bestimmung der Qualitätseigenschaften anzubietender Dienstleistungen erfolge mit Hilfe von Konzepten, in denen mehr oder weniger plausible Begründungen dafür formuliert seien, für wen, warum, was, wie und mit welchen personellen, sachlichen und finanziellen Ressourcen zu tun sei. Eine direkte Qualitätsbestimmung durch Kinder, Jugendliche und Sorgeberechtigte gebe es bislang dabei nicht (a.a.O., S. 58). Dagegen hält Klatetzki (S. 60) die Orientierung des KGSt-Modells am Output, spezifiziert in der Produktbeschreibung und quantifiziert über Kennzahlen, für grundsätzlich sinnvoll. Während nämlich die Ergebnisqualität im Sinne von Outcome nie eindeutig dem Handeln der leistungserbringenden Organisation zuzuordnen sei, lenke die Outputorientierung den Blick auf die geeigneten Strukturen und Umsetzungsprozesse (hier: der Jugendhilfe).

Heiner (1996, S. 217) kritisiert dagegen, dass die KGSt mit ihren Produktbeschreibungen dazu verleite, geplante Aktivitäten bereits als "Erfolg" zu werten, wenn sie nur durchgeführt wurden, und andere Maßnahmen nicht zu entwickeln, weil sie in der offiziellen Produktbeschreibung nicht enthalten seien. Im Text der KGSt werde die im Titel anklingende programmatische Unterscheidung zwischen Output und Outcome nur am Rande erwähnt und die Grenzen dieser Outputorientierung würden nicht reflektiert. Diese Grenzen werden von Heiner vor allem in der Festschreibung und Normierung gesehen, die mit der präzisen Produktbeschreibung im Sinne von Tätigkeitsdokumentationen verbunden seien. Dies sei aber nur bei Mindeststandards vertretbar, während ansonsten fachliche Standards nur exemplarisch und niemals komplett konkretisiert werden könnten.

Inwieweit das durch das Neue Steuerungsmodell angeregte Instrument der Produkt- bzw. Leistungsbeschreibung die Praxis in sozialen Handlungsfeldern befruchtet, muss abgewartet werden. Jedenfalls dürfte der Gewinn für manche Einrichtung bereits darin bestanden haben, unter Beteiligung der Fachkräfte die eigenen Leistungstätigkeiten näher zu betrachten und zu systematisieren. Allerdings stellt sich die Frage, ob sich alle sozialen Dienstleistungen in Produkten erfassen und abbilden lassen (Meinhold \& Matul, 2003, S. 77).

Die mit den Instrumenten der Budgetierung, des Kontraktmanagements und des 
Controllings verknüpften Erwartungen stellen für die meisten freien Träger im Unterschied zu öffentlichen Verwaltungen kein Neuland dar. In der öffentlichen Verwaltungspraxis entzündet sich jedoch zunehmend Kritik an Ungereimtheiten im Zusammenhang mit Produktbudgets und Kontrakten (a.a.O., S. 77f).

Das ursprüngliche Vorbild all dieser Bemühungen, die Verwaltungsmodernisierung der niederländischen Stadt Tilburg ("Tilburger Modell"), gilt inzwischen im eigenen Land hinsichtlich der erhofften Einsparpotenziale als gescheitert (Boeßenecker, 2001, S. 104). Auch in Deutschland greift zunehmende Ernüchterung um sich (a.a.O.). Aus Sicht der BürgerInnen kann jedoch vielerorts konzediert werden, dass ein Stück Kundenorientierung in die Praxis der Amtsstuben unverkennbar Einzug gehalten hat.

Die Leitziele einer leistungsorientierten öffentlichen Verwaltung, wie sie sich unter dem Einfluss neuer Steuerungsmodelle auch im Sozialbereich zu etablieren beginnen, sind mithin keineswegs diskreditiert:

Verbesserung der Funktionsfähigkeit,

Vereinfachung der Organisation unter bestmöglicher Nutzung vorhandener Ressourcen,

mehr Flexibilität, Eigenverantwortung und Selbststeuerung,

mehr Bürger- bzw. Kundennähe, Transparenz und Akzeptanz in der Öffentlichkeit.

\subsubsection{Aufgaben der Jugend- und Sozialhilfe}

$\mathrm{Zu}$ den sozialstaatlichen Grundpositionen gehört die Überzeugung, dass jeder junge Mensch einen Anspruch darauf hat, in seinem Entwicklungsprozess so gefördert zu werden, dass er als Erwachsener zu einem autonomen und zugleich in die soziale Gemeinschaft eingebundenen Leben fähig ist. Diese Überzeugung spiegelt sich in den Leitparagraphen des Sozialgesetzbuches VIII (Kinder- und Jugendhilfegesetz, KJHG): Recht auf Erziehung, Elternverantwortung, Aufgaben der Jugendhilfe.

Der Begriff KJHG bezeichnet eigentlich nur das 1990 verabschiedete Reformgesetz, mit dem das (Reichs)Jugendwohlfahrtsgesetz von 1922 abgelöst wurde; darin sind neben der Neuordnung des Rechts der 
Kinder- und Jugendhilfe noch eine Anzahl weiterer Gesetzesänderungen, Übergangs- und Schlussvorschriften enthalten. Den Kern des KJHG bildet das Achte Buch Sozialgesetzbuch (SGB VIII), in dessen Paragraphen die jeweils gültigen Rechtsvorschriften für die Kinder- und Jugendhilfe als Teil des Sozialrechts formuliert sind (Wiesner, 2001, S. 330f).

$\mathrm{Zu}$ Garanten des Rechtsanspruchs junger Menschen bestimmt das Grundgesetz vorrangig die Eltern, daneben auch die Schule. Der Jugendhilfe kommt eine Unterstützungs- und Ergänzungsfunktion zu. Zur Erfüllung ihrer Präventions- und Schutzaufgaben hat Jugendhilfe ein breit gefächertes Leistungsangebot bereitzustellen, das für alle jungen Menschen und ihre Familien zugänglich sein soll. Dieses Leistungsangebot wird im KJHG unterteilt in institutionelle Angebote der allgemeinen Förderung (wie z. B. Jugendsozialarbeit, Kindertageseinrichtungen) und in individuelle Hilfen (z. B. Hilfen zur Erziehung, Eingliederungshilfe für seelisch behinderte Kinder und Jugendliche).

Während die Angebote allgemeiner Förderung ohne Prüfung einer besonderen Berechtigung in Anspruch genommen werden können, wird das Recht auf eine individuelle Hilfe davon abhängig gemacht, ob ein entsprechender Bedarf festgestellt werden kann. Vor der Leistungsgewährung obliegen dem Jugendamt als zuständigem öffentlichen Träger der Jugendhilfe somit entsprechende Ermittlungsaufgaben (Harnach-Beck, 1997).

Hilfe zur Erziehung wird bewilligt, wenn eine dem Wohl des Kindes oder des Jugendlichen entsprechende Erziehung nicht gewährleistet ist und die Hilfe für seine Entwicklung geeignet und notwendig ist. Hilfe zur Erziehung kommt also nicht erst in Betracht, wenn das Wohl des Kindes oder Jugendlichen gefährdet ist, sondern der Gesetzgeber stellt bewusst Maßnahmen zur Unterstützung der elterlichen Erziehungsverantwortung unterhalb der Eingriffsschwelle der $\S \S 1666$, 1666a des Bürgerlichen Gesetzbuchs (BGB) bereit, die das Familiengericht zu Eingriffen in das elterliche Sorgerecht befugen (Wiesner, 2001, S. 336). Die Leistungsberechtigten haben bezüglich der leistungserbringenden Einrichtungen und Dienste ein Wunsch- und Wahlrecht, dem seitens des Jugendamtes $\mathrm{zu}$ entsprechen ist, sofern nicht unverhältnismäßige Mehrkosten damit verbunden sind (§ 5 SGB VIII).

Die Gesamtverantwortung für die Bereitstellung eines bedarfsgerechten Angebots einschließlich der Planung liegt beim Träger der öffentlichen Jugendhilfe (§ 79 SGB VIII). 
Dessen zentrales Entscheidungsorgan - der Jugendhilfeausschuss - ist jedoch plural zusammengesetzt: zwei Fünftel seiner Mitglieder werden auf Vorschlag der anerkannten Träger der freien Jugendhilfe gewählt.

Die Sozialhilfe nach dem Bundessozialhilfegesetz (BSHG) ist das allgemeine Basissystem der sozialen Sicherung in Deutschland und funktioniert als "Netz unter dem Netz" der gehobenen Sicherungssysteme - Sozialversicherung und Versorgung (Wienand, 1999, S. $34)$.

"Wer nicht in der Lage ist, aus eigenen Kräften seinen Lebensunterhalt zu bestreiten oder in besonderen Lebenslagen sich selbst zu helfen, und auch von anderer Seite keine ausreichende Hilfe erhält, hat ein Recht auf persönliche und wirtschaftliche Hilfe, die seinem besonderen Bedarf entspricht, ihn zur Selbsthilfe befähigt, die Teilnahme am Leben in der Gemeinschaft ermöglicht und die Führung eines menschenwürdigen Lebens sichert" (§ 9 SGB I).

Auf Leistungen der Sozialhilfe hat der Hilfesuchende in der Regel einen Rechtsanspruch; über Art, Form und Maß entscheidet der Sozialhilfeträger nach der Besonderheit des Einzelfalls. Die Hilfe kann in persönlicher Hilfe (vor allem Beratung), in Geld- oder Sachleistungen bestehen und muss grundsätzlich nicht zurückgezahlt werden, es sei denn, die Hilfe wurde von vornherein als Darlehen gewährt oder die Bedürftigkeit wurde schuldhaft herbeigeführt (Wienand, 1999, S. 36).

Leistungen der Sozialhilfe werden unterteilt in Hilfe zum Lebensunterhalt und Hilfe in besonderen Lebenslagen. Erstere dient der Existenzsicherung; sie kann einmalig oder als laufende Leistung gewährt werden. Bei der Berechnung werden Einkünfte und Vermögen unter Anrechnung bestimmter Freibeträge berücksichtigt; auch wird geprüft, ob eventuell Dritte zu Unterhaltsansprüchen herangezogen werden können. Dies trifft gleichermaßen für die Hilfe in besonderen Lebenslagen zu, die vor allem Krankenhilfe (§ 37 BSHG), Eingliederungshilfe für Behinderte ( $\S 39$ ff. BSHG) und Hilfe zur Pflege (§§ 68 ff. BSHG) umfasst.

Sowohl für die Jugend- als auch die Sozialhilfe gilt, dass die öffentlichen Träger von der Schaffung eigener Einrichtungen absehen sollen, sofern geeignete Einrichtungen, Dienste und Veranstaltungen von anerkannten freien Trägern vorhanden sind, ausgebaut oder geschaffen werden können (sinngemäß § 93 Abs. 1 BSHG, § 4 Abs. 2 SGB VIII). 


\subsubsection{Das Qualitätsthema in der Jugend- und Sozialhilfe}

Eingebettet in die beschriebenen Bestrebungen, öffentliche Verwaltungen nach Maßgabe Neuer Steuerungsmodelle (KGSt, 1993) zu reformieren, kam die stark betriebswirtschaftlich geprägte Diskussion über z. B. Controlling, Produktbeschreibungen, Lean Management und Dienstleistungsorientierung mit der "Unwiderstehlichkeit einer Dampfwalze" (B. Müller, 1996a, S. 8) auch über die öffentlichen und freien Träger der Jugend- und Sozialhilfe. Mit der Novellierung einschlägiger Rechtsvorschriften - hier: $\S \S$ 78 ff. Achtes Buch Sozialgesetzbuch (KJHG) und $\S \S 93$ ff. Bundessozialhilfegesetz (BSHG) zum 01.01.1999 - wurden sozialrechtlich neue Weichen gestellt. Damit wurde analog zur Angebotssteuerung im Kranken- und Pflegeversicherungsbereich (SGB V und SGB XI) das bereits 1993 eingeführte Vereinbarungsprinzip zwischen Leistungsträgern und Leistungserbringern ausgebaut und verfeinert (Wiesner, 2001; Schoch, 2000).

Dem Gesetzgeber ging es vor allem darum, durch stärkere Markt- und Wettbewerbsorientierung eine Dämpfung der Kostenentwicklung zu erzielen, ohne die Qualität der erbrachten Leistungen $\mathrm{zu}$ Lasten der Berechtigten $\mathrm{zu}$ gefährden. Die traditionelle Bevorzugung der freigemeinnützigen Wohlfahrtsverbände wurde abgeschafft. § 93 BSHG stellt für den Abschluss von Vereinbarungen - bei Gleichheit von Inhalt, Umfang und Qualität der Leistungen - nunmehr allein auf die Höhe der Vergütung ab (Schoch, 2000, S. 7). Dies gilt entsprechend auch für $§ 78$ a SGB VIII.

Teuber, Stiemert-Strecker \& Seckinger (2000, S. 10) führen in diesem Zusammenhang aus: "Das alte korporatistische Arrangement ..., das quasi ein Monopol der Sozialverbände und -vereine für die Erbringung sozialer Leistungen beinhaltete, steht immer mehr in Frage, und es entwickelt sich ... eine Konkurrenz zwischen den unterschiedlichen Anbieterformen sozialer Dienste. Gilt es nun die Entscheidung für eines der Angebote zu begründen, wird Qualität zu einem herausragenden Kriterium, vor allem auch deshalb, weil in allen Sozialgesetzen die Verpflichtung zur Leistungserbringung an die reale Bedürftigkeit und nicht an die Finanzierbarkeit gekoppelt ist."

Die wesentlichen Neuregelungen in den Vereinbarungen zur qualitätsorientierten Arbeit in Einrichtungen der Jugend- und Sozialhilfe sind in nachstehenden Gesetzesauszügen expliziert. 


\section{§ 93 Abs. 2 BSHG: Einrichtungen}

Wird die Leistung in einer Einrichtung erbracht, ist der Träger der Sozialhilfe zur Übernahme der Vergütung für die Leistung nur verpflichtet, wenn mit dem Träger der Einrichtung oder seinem Verband eine Vereinbarung über

1. Inhalt, Umfang und Qualität der Leistungen (Leistungsvereinbarung),

2. die Vergütung, die sich aus Pauschalen und Beträgen für einzelne Leistungsbereiche zusammensetzt (Vergütungsvereinbarung) und

3. die Prüfung der Wirtschaftlichkeit und Qualität der Leistungen (Prüfungsvereinbarung) besteht.

Die Vereinbarungen müssen den Grundsätzen der Wirtschaftlichkeit, Sparsamkeit und Leistungsfähigkeit entsprechen.

\section{§ 93a Abs. 1 BSHG: Inhalt der Vereinbarungen}

Die Vereinbarung über die Leistung muss die wesentlichen Leistungsmerkmale festlegen, mindestens jedoch die betriebsnotwendigen Anlagen der Einrichtung, den von ihr zu betreuenden Personenkreis, Art, Ziel und Qualität der Leistung, Qualifikation des Personals sowie die erforderliche sächliche und personelle Ausstattung. In die Vereinbarung ist die Verpflichtung der Einrichtung aufzunehmen, im Rahmen des vereinbarten Leistungsangebotes Hilfeempfänger aufzunehmen und zu betreuen. Die Leistungen müssen ausreichend, zweckmäßig und wirtschaftlich sein und dürfen das Maß des Notwendigen nicht überschreiten.

\section{$\S 78 b$ SGB VIII: Voraussetzungen für die Übernahme des Leistungsentgelts}

(1) Wird die Leistung ganz oder teilweise in einer Einrichtung erbracht, so ist der Träger der öffentlichen Jugendhilfe zur Übernahme des Entgelts gegenüber dem Leistungsberechtigten verpflichtet, wenn mit dem Träger der Einrichtung oder seinem Verband Vereinbarungen über

1. Inhalt, Umfang und Qualität der Leistungsangebote (Leistungsvereinbarung),

2. differenzierte Entgelte für die Leistungsangebote und die betriebsnotwendigen Investitionen (Entgeltvereinbarung) und

3. Grundsätze und Maßstäbe für die Bewertung der Qualität der Leistungsangebote sowie über geeignete Maßnahmen zu ihrer Gewährleistung (Qualitätsentwicklungsvereinbarung) abgeschlossen worden sind.

(2) Die Vereinbarungen sind mit den Trägern abzuschließen, die unter Berücksichtigung der Grundätze der Leistungsfähigkeit, Wirtschaftlichkeit und Sparsamkeit zur Erbringung der Leistung geeignet sind.

(3) Ist eine der Vereinbarungen nach Absatz 1 nicht abgeschlossen, so ist der Träger der öffentlichen Jugendhilfe zur Übernahme des Leistungsentgelts nur verpflichtet, wenn dies insbesondere nach Maßgabe der Hilfeplanung (§ 36) im Einzelfall geboten ist.

Der Anwendungsbereich für diese bundesrechtlichen Rahmenvorschriften ist im BSHG (§ 93) auf Leistungen in voll- und teilstationären Einrichtungen (incl. ambulanter Dienste, Schoch, 2000, S. 6), im SGB VIII (§ 78a) auf teilstationäre und stationäre Leistungen 
bezogen.

Dazu zählen: Betreuung/Unterkunft in sozialpädagogisch begleiteten Wohnformen, Leistungen in gemeinsamen Wohnformen für Mütter/Väter und Kinder sowie zur Unterstützung bei notwendiger Unterbringung zur Erfüllung der Schulpflicht, Hilfen zur Erziehung in Tagesgruppen, Heimen/betreuten Wohnformen oder Einzelbetreuung außerhalb der eigenen Familie, Eingliederungshilfe für seelisch behinderte Kinder und Jugendliche in teilstationären und stationären Einrichtungen und Wohnformen, Hilfe für junge Volljährige sowie Leistungen zum Unterhalt, die im Zusammenhang mit den zuvor genannten Leistungen gewährt werden. Den Ländern wird die Möglichkeit eröffnet, weitere Leistungen in den Anwendungsbereich einzubeziehen.

Aus diesem Spektrum rekrutiert sich die erste der weiter unten beschriebenen Untersuchungsgruppen dieser Studie.

Repräsentativität im Hinblick auf die gesamte Angebotspalette der Einrichtungen und Dienste konnte dabei nicht angestrebt werden, wohl aber eine grobe Differenzierung der Aufgabenfelder nach den Schwerpunkten "Hilfe zur Erziehung" und "Hilfe in besonderen Lebenslagen".

Bedingt durch das in Deutschland obwaltende sozialpolitische Gestaltungsprinzip der Subsidiarität, befinden sich die weitaus meisten Anbieter von Leistungen im genannten Anwendungsbereich in Trägerschaft der freien Wohlfahrtspflege. Ihnen als Partner mitunter auch eher als Gegner, die fürchten, vom jeweils anderen "über den Tisch gezogen" zu werden (Hinte, in KGSt, 1998, S. 9) - stehen in dem gesetzlich geforderten Vereinbarungsverfahren über Leistungen, Qualität und Entgelte die leistungsgewährenden und -finanzierenden öffentlichen Jugend- und Sozialhilfeverwaltungen gegenüber, in der Regel also die kommunalen Jugendämter sowie die örtlichen und überörtlichen Träger der Sozialhilfe (Landes-, Sozialämter).

Vor dem erst 2001 kodifizierten Rehabilitationsrecht (SGB IX: Rehabilitation und Teilhabe behinderter Menschen) war SGB VIII (Kinder- und Jugendhilfegesetz) das bis dahin letzte der Sozialgesetze, in die das Qualitätsthema explizit aufgenommen wurde. Angesichts der spezifischen Schwierigkeit dieses Arbeitsfeldes, seine Prozesse im technisch-qualitätssichernden Sinne zu planen und zu steuern, wurde im Unterschied zur Sozialhilfe der Akzent weniger auf das administrativ-kontrollierende Motiv der Qualitätsprüfung gelegt, sondern der Impuls fachlicher Qualitätsentwicklung betont 
(Merchel, 2001, S. 23). Dem KJHG werden deshalb im Vergleich zum BSHG, aber auch zum SGB III (Arbeitsförderung), SGB V (Krankenversicherung) und SGB XI (Pflegeversicherung) die größten Potenziale für eine fachpolitisch offensive Verarbeitung des Qualitätsthemas attestiert (a.a.O., S. 24).

Während in weiten Teilen der Sozialhilfe der finanzielle und Sachleistungsaspekt gegenüber der persönlichen Hilfestellung dominiert - etwa in Form der regulären oder erweiterten Hilfe zum Lebensunterhalt - und die Leistungsgewährung der Logik des Verwaltungs-Verfahrensrechts (SGB $\mathrm{X}$ ) unterliegt, befindet sich die Kinder- und Jugendhilfe aufgrund ihres sozialpädagogischen Auftrags, wie er in den Leitparagraphen des KJHG zum Ausdruck kommt, in einer besonderen Situation. Speziell im Bereich der Hilfen zur Erziehung ist deshalb fachlich umstritten, ob die Regeln des Verwaltungsverfahrensrechts, das eine behördliche Entscheidung aus der Anwendung einer Rechtsnorm auf einen konkreten Sachverhalt ableitet, der Eigenart pädagogischer Entscheidungen überhaupt gerecht werden (Wiesner, 2001, S. 341f).

In der Fachdiskussion wird, ausgehend von einem Hilfeverständnis als personenbezogener sozialer Dienstleistung, wegen deren Prozesshaftigkeit, Vorläufigkeit und Interaktionsintensität ein kooperatives Verfahren gefordert, das auf einer vertrauensvollen Beziehung zwischen der zuständigen Fachkraft und den Leistungsadressaten basiert (a.a.O., S. 342). In diesem Verfahren kommt dem Hilfeplan nach § 36 SGB VIII eine entscheidende Rolle als Instrument der Prozesssteuerung zu.

Namentlich für den Jugendhilfebereich - grundsätzlich aber auch für die Sozialhilfe - lässt sich deshalb konstatieren, dass der Gesetzgeber mit dem verwendeten Begriff der Vereinbarung auf ein Verfahren abzielt, das dem diskursiven Charakter des Qualitätskonzepts in sozialen Handlungsfeldern, also seiner Relativität und Aushandlungsbedürftigkeit, angemessen ist. In diesem Diskurs nehmen die leistungserbringenden Einrichtungen einerseits und die (unter anderem) leistungsgewährenden Verwaltungen andererseits die Rollen von Kooperationspartnern ein.

Wie Münder im Frankfurter Kommentar zum KJHG betont, beinhaltet "...der Rechtsbegriff der Vereinbarung ... das Zustandekommen eines Vertrages durch zwei übereinstimmende Willenserklärungen über die wesentlichen Vertragsgegenstände. ... Wesentliche Voraussetzung für das Vorliegen einer Vereinbarung ist 
somit die Tatsache, dass keine der Parteien der Vereinbarung letztlich einseitig den Vertragsinhalt festlegt ..., sondern es zu zweiseitigen, ausgehandelten Ergebnissen kommt" (Münder, 2002).

Auf eine Erörterung des rechtlich komplexen Dreiecksverhältnisses zwischen Leistungsberechtigtem, Sozialhilfeträger und leistungserbringender Einrichtung - das in der Sozialhilfe auch zu einem Vier- oder gar Fünfecksverhältnis werden kann, wenn Renten- und Pflegeversicherungsansprüche auf den Sozialhilfeträger übergehen -, sei hier verzichtet (vgl. Schoch, 2000).

Legt man diese idealtypische Auffassung zugrunde, handelt es sich bei der Beziehung zwischen den öffentlichen Kostenträgern und leistungserbringenden Einrichtungen nicht um ein einseitiges Auftraggeber-Auftragnehmer-Verhältnis, in dem zunächst nur den Leistungsanbietern und ihren Verbänden die Aufgabe einer vereinbarungsgemäßen Qualitätsentwicklung obliegt (Wabnitz, 1999, S. 24). Vielmehr richten sich Erwartungen hinsichtlich eines zeitgemäßen Qualitätsmanagements auch an die öffentlichen Träger selbst.

Diese angestrebte, real allerdings in sehr unterschiedlichem Maße praktizierte "QualitätsPartnerschaft" (positive Beispiele sind beschrieben bei Link, Schöpflin \& Gruhler, 1999, sowie bei Koch, 2003) von Jugend- und Sozialhilfeverwaltungen einerseits und Leistungsanbietern in Einrichtungen und Diensten andererseits galt es, in der Untersuchung angemessen zu berücksichtigen.

Die zweite der weiter unten beschriebenen Zielgruppen rekrutiert sich deshalb aus dem Bereich der Jugend- und Sozialämter. Dabei stand die Frage im Vordergrund, ob sich die unterstellte Absicht des Gesetzgebers, beide Vereinbarungsparteien in Sachen Qualitätsentwicklung in die Pflicht zu nehmen, in entsprechenden Aktivitäten auf beiden Seiten der Praxis niederschlagen. 


\subsection{Methodik}

\subsubsection{Literaturrecherchen: Suchbegriffe und -ergebnisse}

\subsubsection{Recherchen im sozialwissenschaftlichen Bereich}

Im Online-Katalog der Niedersächsischen Staats- und Universitätsbibliothek (SUB) Göttingen sind in den Studienbereichen der Sozialwissenschaftlichen Fakultät (Soziologie, Politikwissenschaft, Medien- und Kommunikationswissenschaft, Ethnologie, Pädagogik und Sportwissenschaft) nur vereinzelte Einträge zum Stichwort "Qualitätsmanagement" zu finden.

Dass dies den tatsächlichen Publikationsstand nur sehr unzulänglich widerspiegelt und offenbar mehr über den Zustand der Bibliotheksbudgets aussagt als über die fachpublizistischen Aktivitäten der jeweiligen Forschungs- und Praxisbereiche, belegt eine Studie des Autors, die auch Grundlage der Ausführungen in Kap. A 2. gewesen ist.

Im Rahmen eines zweijährigen Forschungsprojektes zur Qualitätsentwicklung in Einrichtungen und Diensten der Erziehungshilfe (Gerull, 1998a) sichtete der Autor systematisch den gesamten Bibliotheksbestand des Evangelischen Erziehungsverbandes (EREV) mit über 50 Zeitschriften aus dem Sozialbereich sowie die Jahresregister bundesdeutscher Verlage mit dem Programmschwerpunkt Sozial- und Bildungswesen (vgl. Gerull, 2000, S. 8f). Die Recherche stützte sich auf Buch- und Zeitschriftenpublikationen, Verbandsperiodika, amtliche Mitteilungen und "graue Literatur" mit explizitem Bezug auf Qualität, Qualitätsmanagement, Qualitätsentwicklung o. Ä. im Haupt- oder Untertitel.

Wenngleich die Kinder- und Jugendhilfe den thematischen Fokus bildete, wurde wegen der zumeist arbeitsfeldübergreifenden und/oder interdisziplinären Konzeption zahlreicher Fachzeitschriften (z. B. SocialManagement, Theorie und Praxis der Sozialen Arbeit, Unsere Jugend, Zeitschrift für Jugendkriminalrecht und Jugendhilfe, Blätter der Wohlfahrtspflege, Neue Praxis, Kindheit und Entwicklung, Sozialmagazin, Sozialwirtschaft aktuell, Nachrichten des Deutschen Vereins) ein breiter Überblick zur Aktualität des Qualitätsthemas in sozialen Handlungsfeldern gewonnen. 
Die Recherche wird seither fortgeschrieben und umfasst aktuell die auf der beschriebenen Grundlage ermittelten Publikationen bis Anfang 2004 (s. Abb. 1, Kap. A 1.), vereinzelt bis Mitte 2004.

Wie aus Abbildung 1 ersichtlich, nahm die Zahl der in Printmedien veröffentlichten Beiträge zum Qualitätsthema in der Sozialen Arbeit und ihren Grenzbereichen (z. B. Behinderten- und Altenhilfe, Heilpädagogik, psychosoziale Beratung, Sozialpsychiatrie) seit Anfang der Neunzigerjahre stetig zu, kulminierte in 1998 und stieg nach mehrjährigem Rückgang in 2003 wieder an.

Möglicherweise zeichnet sich damit eine neuerliche Intensivierung des Qualitätsdiskurses ab, wobei sich die - hier nicht untersuchte - Frage nach inhaltlich veränderten Schwerpunkten stellt. Eindrucksmäßig scheinen nicht mehr die rechtlich-administrativen Aspekte (etwa zur Umsetzung der §§ 78a ff. SGB VIII) oder Fragen zur Wahl branchenübergreifender Qualitätsmanagementkonzepte (z. B. ISO 9000 ff., EFQM) im Mittelpunkt zu stehen, sondern praxisnähere Themen wie z. B. Kunden- und Mitarbeiterbefragungen, Beschwerdemanagement oder Partizipationsverfahren. Möglicherweise ist der ermittelte Anstieg in 2003 jedoch nur ein Artefakt aufgrund unwissentlich veränderter Auswahlstrategie des Autors und/oder eines erweiterten Bestandes der verwendeten Verbandsbibliothek.

Eine Web-Recherche im Social Sciences Citation Index (27.08.04) ergab zum Suchbegriff "quality management" 4.723 Treffer, darunter eine Vielzahl irrelevanter "exotischer" Beiträge, die im Kontext dieser Datenbank ungewöhnlich erscheinen (z. B. "QM in der türkischen Zementindustrie"). Die differenzierte Suche erzielte folgendes Ergebnis:

○ total quality management: 1.632 Treffer, nicht systematisch ausgewertet;

○ service quality: 1.578 Treffer, nicht systematisch ausgewertet;

- quality movement: 49 Treffer, davon 8 substantiell (zum Bewertungsmaßstab s. Kap. 4.2.1.2);

○ management representative: 2 Treffer, nicht substantiell (s. Kap. 4.2.1.2.);

○ social service management: 1 Treffer, nicht substantiell;

○ social + quality management: 0 Treffer;

- quality management representative: 0 Treffer;

○ social service quality: 0 Treffer;

○ total quality management + social services: 0 Treffer.

Zu den im Rahmen dieser Studie untersuchten Fragestellungen lieferte die SUB- und WebRecherche somit ein enttäuschendes Resultat. Ungeachtet der Möglichkeit einer effektiveren Suche unter anderen Begriffen und/oder in weiteren Datenbanken, basieren die Aus- 
führungen im literaturanalytischen Teil der Arbeit überwiegend auf kontinuierlichen Eigenrecherchen des Autors in Fachzeitschriften und Verlagskatalogen seit 1998.

\subsubsection{Recherchen in psychologischen und allgemeinen Datenbanken}

Die Recherche erfolgte Ende 2003/Anfang 2004 (Datum jeweils in Klammern angegeben) in den unten aufgeführten Datenbanken. Angegeben sind die Ergebnisse zu qualitätsbezogenen Suchthemen, die ggf. spezifiziert wurden, wenn die Zahl der Verweise zu groß war (Abbruchkriterium: mehr als 600 Treffer). In die anschließende Literaturauswertung einbezogen wurden alle Beiträge, deren Quellenbeschreibungen und/oder Abstracts substantielle Hinweise auf das zu untersuchende Thema lieferten und die im Rahmen der Fernleihe zugänglich waren.

Als "substantielle" Treffer zählen im Folgenden nur solche, die einen Titelbezug zu sozialen Dienstleistungen aufweisen oder - ungeachtet des genannten oder mutmaßlichen Anwendungsbereichs - eine inhaltliche (theoretische oder empirische) Auseinandersetzung mit dem Suchbegriff ankündigen, die für das Thema "interessant" sein könnte (z. B. "The Influence of Organizational Structure on the Effectiveness of TQM Programs"). ${ }^{5}$

Als "nicht substantielle" Treffer sind solche bewertet, in denen der Suchbegriff lediglich aufzählend (z. B. in Inhaltsverzeichnissen), Personen (z. B. AutorInnen) oder Veranstaltungen (z. B. Ausbildungskurse) bezeichnend vorkommt, eindeutig auf nichtsoziale Handlungsfelder zugeschnitten ist oder sehr spezifisch ohne übergreifende thematische Bedeutung zu sein scheint (z. B. "Relevanz rechtlicher Regelungen für die Qualitätssicherung der Weiterbildung auf Ebene der Länder in der Bundesrepublik Deutschland").

1. PSYNDEXplus (psychologische Literatur aus den deutschsprachigen Ländern ab 1977, 01.12.2003): Suchthemen Qualitätsmanagement -- Treffer: 0, Qualitaetsmanagement/quality management -- Treffer: 377 (überwiegend aus dem Gesundheitsbereich, selektiv einbezogen ab 1998, ansonsten 22 substantielle Treffer), Qualitaetsbeauftragte/Qualitätsbeauftragte -- Treffer: 2 (substantiell), Total Quality

5 Viele davon hielten bei Lektüre nicht, was sie im Titel zu versprechen schienen; allerdings dürften angesichts des recht subjektiven Auswahlkriteriums auch etliche Beiträge herausgefiltert worden sein, weil ihre Titel einen zu geringen Aufforderungscharakter hatten - quasi ein "Fehler zweiter Art", wenn man die Annahme der Irrelevanz als NullHypothese betrachtet. 
Management -- Treffer: 93 (überwiegend identisch mit Qualitaetsmanagement, 05.01.2004)

2. PsycInfo (internationale psychologische Literatur ab 1967, 08.12.2003):

Suchthemen quality management -- Treffer: 601 (weit überwiegend aus medizinischem Bereich und Schule, nur 12 substantielle Treffer ab 1995), quality management representative -- Treffer: 0 , management representative -- Treffer: 24 (1 substantieller Treffer), Total Quality Management -Treffer: 396 (überwiegend identisch mit quality management, 05.01.2004)

3. Metasuchmaschine der virtuellen Fachbibliothek Psychologie der Saarländischen Universitäts- und Landesbibliothek (01.12.2003):

Suchthemen Qualitaetsmanagement+Organisationspsychologie -- Treffer: 0, Qualitaetsmanagement -Treffer: 23 (nicht substantiell), quality management -- Treffer: 92 (weit überwiegend nicht substantiell, vereinzelte substantielle Treffer nicht verfügbar)

4. Niedersächsische Staats- und Universitätsbibliothek (SUB-Katalog ab 1994, 09.12.2003):

Suchthemen Qualitaetsmanagement/quality management -- Treffer: 572 (überwiegend aus Ingenieurund Wirtschaftswissenschaft sowie Medizin, substantielle Treffer: 42), Qualitaetsbeauftragte -- Treffer: 1 (substantiell)

5. Gemeinsamer Verbundkatalog der SUB (GVKplus, 08.12.2003):

Suchthema Qualitaetsmanagement/quality management -- Treffer: 717 (überwiegend aus Ingenieur- und Wirtschaftswissenschaft sowie Medizin, substantielle Treffer: 48)

6. altavista.com (internationale Suchmaschine, Suche weltweit englisch/deutsch, 02.12.2003): Suchthemen Qualitaetsmanagement+Psychologie -- Treffer: 0, Qualitätsmanagement+Psychologie -Treffer: 2 (nicht substantiell), Qualitätsmanagement+Organisationspsychologie -- Treffer: 0, Qualitätsbeauftragte -- Treffer: 2.602, Qualitätsbeauftragte+soziale Einrichtung -- Treffer: 200 (Fortbildungsangebote, zuständige Personen, Foren für Erfahrungsaustausch u. Ä., nicht substantiell), quality management representative -- Treffer: 347 (Fortbildungsangebote, zuständige Personen u. Ä., nicht substantiell), quality management representative+social services -- Treffer: 4 (nicht substantiell), quality management representative+psychology -- Treffer: 11 (nicht substantiell)

7. Google (internationale Suchmaschine, Suche weltweit englisch/deutsch, 02.12.2003): Suchthemen Qualitätsbeauftragte -- Treffer: 3540, Qualitaetsbeauftragte -- Treffer: 3530, Qualitaetsbeauftragte+soziale Einrichtung -- Treffer: 315 (weitgehend identisch mit altavistaRecherche), quality management representative -- Treffer: 554 (weitgehend identisch mit altavistaRecherche, weit überwiegend aus dem kommerziellen und medizinischen Bereich), quality management representative+social service -- Treffer: 1 (nicht substantiell), quality management representative+psychology -- Treffer: 12 (nicht substantiell), quality management+psychology -- Treffer: 17 (nicht substantiell) 
Die Rechercheergebnisse stützen den aufgrund von Lehrbuchanalysen gewonnenen Eindruck der Randständigkeit des Qualitätsthemas in der psychologisch relevanten Literatur. Zahlreiche Treffer zur thematischen Verknüpfung der Begriffe Qualität und Psychologie enthalten lediglich Hinweise auf die Qualität von Websites mit psychologischen Inhalten. Zum Vergleich: Unter dem relativ komplexen Deskriptor "Qualitätsmanagement in der sozialen Arbeit" lieferte die Suche bei Google bereits 815 deutschsprachige "hits" (23.09.2004) und die von Gerull (2004) erstellte Bibliographie zum gleichen Thema - Schwerpunkt "Kinder- und Jugendhilfe und Grenzbereiche" umfasst über 1.000 Titel aus dem Zeitraum 1990 bis 2003 (s. Abb. 1).

\subsubsection{Recherchen im betriebswirtschaftlichen Bereich}

Im zwölfseitigen Online-Katalog der SUB Göttingen findet sich das Stichwort "Qualitätsmanagement" unter 519 Gliederungspunkten zur systematischen Recherche im betriebswirtschaftlichen Literaturbestand explizit kein einziges Mal (!).

Implizit sind lediglich Teilfunktionen bzw. Schnittmengen unter Begriffen wie (in dieser Reihenfolge) "Philosophie und Ethik", "Verhalten/Management", "Controlling", "Unternehmensführung", "Strategische Planung", "Indikatoren", "Führungsleitbilder", "Lean Management", "Kontinuierlicher Verbesserungsprozess", "Corporate Identity", Innovationsmanagement", "Klima/Kultur", "Business Process Reengineering", "Organisationstheorie", "Humanisierung der Arbeitswelt", "Personalmanagement", "Vorschlagswesen", "Wege der Information/Kommunikation/Dokumentation" und "Marketing" subsumiert.

Dies belegt eindrucksvoll, dass auch die mutmaßlich "natürlichste" Bezugswissenschaft die Betriebswirtschaftslehre (s. Kap. B1.1 und 2.) - bislang noch keine Integration des Qualitätsmanagements als Teilfunktion der Unternehmensführung vollzogen hat. Vielmehr entwickelte sich das praktische und theoretische Rüstzeug quasi parallel zur akademischen Wirtschaftswissenschaft in der betrieblichen Praxis und unter Federführung namhafter "Gurus" der amerikanischen und japanischen Industrie (vgl. Seghezzi, 1996).

Die Recherche beschränkte sich auf Bestände der SUB Göttingen sowie mögliche Fernleihen im Rahmen des Gemeinsamen Verbundkatalogs (GVKplus). Unter den 717 Treffern zum Thema "Qualitätsmanagement/quality management" (08.12.03) ist eine Vielzahl dem betriebswirtschaftlichen Bereich zuzuordnen. Auf einen Teil dieser Beiträge 
wurde im Rahmen der Studie zurückgegriffen.

\subsubsection{Anmerkungen zur Literaturauswahl}

Wie ein Blick in das Quellenverzeichnis (Kap. F) zeigt, dominieren deutschsprachige Buch- und Zeitschriftenbeiträge aus den letzten zehn Jahren, darunter überproportional viele aus dem Bereich der Sozialen Arbeit. Genuin psychologische Beiträge zum Qualitätsmanagement sind - wie in Kap. A 2. ausgeführt - zwangsläufig recht spärlich vertreten (mangels Masse).

Die angloamerikanische Fachliteratur zum Qualitätsmanagement bzw. zum Organisationsverhalten und Dienstleistungsmarketing wurde zwar zahlreich (rund 70 Beiträge), aber nicht umfassend systematisch ausgewertet. Die durchgeführte Recherche in der englischsprachigen psychologischen und sozialwissenschaftlichen Fachliteratur ergab bekanntlich ein ähnlich spärliches Ergebnis wie die Suche im deutschen Sprachraum; die relativ geringe Anzahl inhaltlich substantieller Fundstellen, soweit zugänglich, wurde denn auch komplett berücksichtigt. Querverweise auf nichtpsychologische Quellen wurden jedoch nur punktuell einbezogen.

Ähnlich der bundesdeutschen Situation finden sich maßgebliche Veröffentlichungen überwiegend nicht in psychologischen Lehrbüchern und Periodika, sondern in Zeitschriften aus dem wirtschafts- und verwaltungswissenschaftlichen Bereich, z. B. "Journal of Accounting and Economics", "Administrative Science Quarterly", "Human Resource Management", "Academy of Management Executive" und "Harvard Business Review"; sie wurden zumeist nur aus Sekundärquellen erschlossen.

Nicht die Sprachgrenze als solche, auch nicht die erschwerte Zugänglichkeit dieser Quellen, sondern die begründete Vermutung, dass eine erhebliche Erweiterung des Literaturpools um englischsprachige Beiträge keine inhaltlich bedeutsame Verbesserung darstellen würde, ist neben der ohnehin enormen Menge verarbeiteten Materials Grund für diese Beschränkung:

- Die intensive Befassung deutscher Dienstleistungsexperten mit der zumeist amerikanischen Marketingliteratur gewährleistet, dass deren Substanz hinreichend präsent ist in der hiesigen Diskussion (z. B. Bruhn, 2003). 
"Gerade im Dienstleistungsbereich wird dies [die Orientierungsfunktion amerikanischer MarketingWissenschaft für deutsche Forscher, P. G.] besonders deutlich: Alle wichtigen Artikel im Zentralorgan des deutschen Marketing, der Zeitschrift 'Marketing', die in den 80er und zu Beginn der 90er Jahre zu diesem Thema erschienen sind, beschäftigen sich in erster Linie mit der Aufarbeitung der amerikanischen Entwicklungen" (Nerdinger, 1994, S. 266).

○ Die starke Ausrichtung der US-amerikanischen "Industrial and Organizational Psychology" auf marktpsychologische Aspekte in gewinnorientierten Unternehmen ließ soziale Dienstleistungen relativ unterbelichtet; allerdings waren diese auch in Deutschland lange Zeit ein absolutes Stiefkind der Psychologie (vgl. Nerdinger, 1994, S. $7 f f$.$) .$

○ Die zum Teil unkritische Rezeption angelsächsischer Ansätze in der Frühzeit der bundesdeutschen Qualitätsdebatte - zunächst im Pflegebereich (vgl. Ewers \& Schaeffer, 1998), danach auch in anderen sozialen Arbeitsfeldern (z. B. die breite Übernahme des Qualitätsmodells von Donabedian, 1966) - verweist auf die Problematik naiver Wissensimporte, namentlich auf

○ die Kulturspezifität von Einstellungen und Verhaltensmustern und die eingeschränkte Generalisierbarkeit von Modellen und Theorien, die im angloamerikanischen Kulturraum entwickelt wurden (z. B. kommunikative Darstellungsregeln im Rahmen des Impression Managements, vgl. Nerdinger, 1994, S. 124 ff.). Die praktische Verwertbarkeit von Qualitätskonzepten aus anderen Unternehmenskulturen stößt an Grenzen (z. B. "kaizen", s. Kap. C 1.4.3).

- Schließlich spielt auch eine Rolle, dass sich die amerikanische Fachliteratur vorwiegend auf das relativ diffuse TQM-Konzept (s. Kap. C 1.4.2) und seine Konkretisierung im Baldrige Award konzentriert. Auf die in Europa dominierenden Systeme ISO 9000 ff. (s. Kap. C 1.4.1) und EFQM (s. Kap. C 1.4.4) wird so gut wie kein Bezug genommen.

Hinsichtlich der Umsetzung der ISO 9000 ff. nehmen die Europäer, allen voran Briten, Deutsche und Italiener, eine Spitzenstellung weit vor Nordamerika ein (Ende 2000 ca. 54\% zertifizierte Unternehmen gegenüber ca. 12\%, Seghezzi, 2003, S. 287). 


\section{Empirischer Teil der Arbeit (Abschnitt D)}

\section{$5.1 \quad$ Vorbemerkungen}

Die als Teil dieser Dissertation durchzuführende schriftliche Befragung von sozialen Einrichtungen und Diensten ${ }^{6}$ konnte nicht am Reißbrett geplant werden, sondern war in mehrfacher Hinsicht thematisch und methodisch durch die Rahmenbedingungen festgelegt. Zum einen sollte in Fortsetzung und Ergänzung früherer Untersuchungen des Autors zur Qualitätsdebatte in der Sozialen Arbeit, denen der Status von Vorstudien eignet (s. Kap. A 5.3.7), aktuelles empirisches Material zu inhaltlich vergleichbaren Fragestellungen erhoben werden. Damit war der fachliche Schwerpunkt von vornherein in der Jugend- und Sozialhilfe angesiedelt. Zum anderen mussten sich Zielgruppe(n) und Verfahren für die Stichprobenentnahme nach der Kooperation kontaktierter Verbände und deren Bereitschaft richten, Adressverzeichnisse zugänglich zu machen oder Fragebögen an ausgewählte EmpfängerInnen weiterzuleiten. Die damit verbundenen Schwierigkeiten seien hier nur am Rande erwähnt.

Weder der Verband privater Träger der Kinder-, Jugend- und Sozialhilfe (VPK - Bundesverband e. V.) noch der Evangelische Erziehungsverband (EREV) als namhafte Vertreter privatgewerblicher und freigemeinnütziger Träger waren auf Anfrage bereit, die Studie offiziell zu fördern. Sie leisteten jedoch punktuelle Unterstützung in Form von Auskünften und/oder Weiterleitung von Fragebögen (EREV). Voranfragen bei den Spitzenverbänden der öffentlichen Träger ließen eine erhoffte Förderung als unrealistisch erscheinen; dies führte wiederum dazu, dass viele öffentliche Dienste eine Teilnahme an der Befragung mit der Begründung fehlender verbandsoffizieller Befürwortung ablehnten. Insbesondere zu Ämtern und Einrichtungen der Sozialhilfe war dadurch der Zugang erheblich erschwert. Maßgebliche Unterstützung leistete schließlich der Deutsche Verein für öffentliche und private Fürsorge, der ein umfangreiches Adressverzeichnis zur Verfügung stellte. Auch dadurch ließ sich insgesamt ein zahlenmäßiges Übergewicht der Jugendhilfe nicht verhindern.

6 Der Begriff "Soziale Dienste" umfasst sowohl die professionell, organisiert und entgeltlich erbrachten Dienstleistungen der sozialen, sozialpädagogischen, erzieherischen und gesundheitlichen Berufe und Einrichtungen, als auch solche Geldleistungen, die den Betroffenen dazu dienen, sich eigene Unterstützungsleistungen zu organisieren (Frerichs, Leichsenring, Naegele, Reichert \& Stadler-Vida, 2003, S. 11). 


\subsection{Hypothesen}

\subsubsection{Hypothesen zur Ausgestaltung des Qualitätsmanagements}

Im Zentrum der empirischen Untersuchungen stehen Fragen zur Umsetzung, Akzeptanz und Nutzenbewertung von Qualitätsmanagementsystemen und spezifischen Instrumenten. Diesbezüglich sind auf der Basis von Literaturanalyse und Vorstudien bestimmte Vorannahmen zu formulieren, die es statistisch zu prüfen gilt. Die in solchen Hypothesen geäußerten Vermutungen über Wirkungen und Effekte für "unabhängig" gehaltener Variablen (Faktoren) auf "abhängige" sind im vorliegenden Zusammenhang allerdings rein korrelativer Natur (s. Kap. A 5.3.2). Sie können nicht als kausale Beziehung zwischen verursachenden Faktoren und eintretenden Wirkungen interpretiert werden, sondern lediglich als "gemeinsam in Erscheinung tretend".

\section{Zusammenhänge zwischen Trägerschaft und Qualitätsmanagement:}

1. Freie Träger verfügen über ein signifikant elaborierteres Qualitätsmanagement als öffentliche Träger; Stand der Umsetzung und/oder Vielfalt des Instrumenteneinsatzes sind weiter entwickelt.

Begründung:

Freie Träger stehen als betriebliche ${ }^{7}$ Leistungserbringer unter stärkerem Markt- und Qualitätsdruck als die relativ wettbewerbs- und wagnisgeschützten öffentlichen Leistungsgewährer/Kostenträger. Für die Jugendhilfe wird aus Reihen der Einrichtungen vielfach konstatiert, dass entgegen der impliziten Absicht des Gesetzgebers, über die $\S \S 78$ a-g SGB VIII eine qualifizierte Kooperation öffentlicher und freier Träger im Rahmen der Leistungs-, Entgelt- und Qualitätsentwicklungsvereinbarungen zu erreichen, auf Seiten der Jugendämter relative Zurückhaltung dem Thema gegenüber vorherrscht und Qualitätsmanagement vielfach noch als einseitige

7 Ein Betrieb kann definiert werden als "organisierte Wirtschaftseinheit, in der verfügbare Mittel unter Wagnissen zur Erstellung von Leistungen und Abgabe dieser Leistungen an außenstehende Bedarfsträger eingesetzt werden" (Lechner, Egger \& Schauer, 1989, zit. nach Horak, 1995, S. 12). Nach dieser Definition sind auch NonprofitOrganisationen Betriebe mit den Spezifika der Zweckbindung und fehlender Gewinnausschüttung. Öffentliche Dienste setzen ihre Mittel zwar nicht unter dem hier "Wagnis" genannten wirtschaftlichen Risiko ein; allerdings geraten die Grenzen durch Umstrukturierungs- und Auslagerungsprozesse zunehmend ins Fließen. 
Bringschuld der freien Leistungsanbieter verstanden wird. Für die Sozialhilfe werden analoge Beobachtungen berichtet (Wetzler, 2003).

2. Nullhypothese:

Freie und öffentliche Träger unterscheiden sich in der Umsetzung systematischen Qualitätsmanagements und/oder in der Vielfalt des Instrumenteneinsatzes nicht signifikant.

\section{Zusammenhänge zwischen Arbeitsfeld und Qualitätsmanagement:}

Wegen der Dominanz freier Träger als Leistungserbringer und der unumkehrbaren Rolle der öffentlichen Träger als Leistungsgewährer, Kostenträger und Gesamtverantwortliche im Hilfeprozess ist der Faktor Arbeitsfeld stark mit dem Faktor Trägerschaft konfundiert. Jugend- und Sozialämter sind dementsprechend immer in öffentlicher, Erziehungshilfeeinrichtungen überwiegend in freier Trägerschaft. Die diesbezügliche Hypothese lautet demnach analog:

1. Betriebliche Einrichtungen der Kinder-, Jugend- und Sozialhilfe weisen ein signifikant elaborierteres Qualitätsmanagement auf als Einrichtungen und Dienste der Jugend- und Sozialhilfeverwaltung.

Die Begründung deckt sich mit der zum Faktor Trägerschaft formulierten.

2. Innerhalb der Gruppe öffentlicher Verwaltungen weisen Jugendämter eine signifikant stärkere Aktivität in Sachen QM auf als Sozialämter.

Begründung:

Der Bereich der Jugendhilfe wurde im Zusammenhang mit der Einführung Neuer Steuerungsmodelle früh exemplarisch diskutiert (KGSt, 1994) und vielfach zum Experimentierfeld kooperativer Qualitätsentwicklungsprojekte gemacht (z. B. Link et al., 1999; Koch, 2003; Schröer, Schwarzmann, Stark \& Straus, 2000). Dies sollte sich in einer relativ zu anderen öffentlichen Verwaltungen fortgeschrittenen Umsetzung von Qualitätsmanagement ausdrücken. 
3. Nullhypothesen:

(a) Zwischen den in der Studie eindeutig unterscheidbaren Arbeitsfeldern der Jugendund Sozialhilfeverwaltung einerseits und den leistungserbringenden Einrichtungen andererseits gibt es keine signifikanten Differenzen in Bezug auf Umsetzungsstand und Ausgestaltung des Qualitätsmanagements.

(b) Innerhalb der Gruppe der Jugend- und Sozialhilfeverwaltungen gibt es diesbezüglich keine Unterschiede.

\section{Zusammenhänge zwischen Einrichtungsgröße und Qualitätsmanagement:}

1. Einrichtungen und Dienste der Kinder-, Jugend- und Sozialhilfe weisen ein umso elaborierteres Qualitätsmanagement auf, je größer sie sind.

Begründung:

Qualitätsmanagement im Sinne der in dieser Studie erfragten systematisierten Weise ist eine organisationale Antwort auf die Frage nach der optimalen Koordination arbeitsteiliger Prozesse. Die Notwendigkeit, die Qualität dieser Prozesse zu managen, steigt mit zunehmender Organisationskomplexität, hier operationalisiert als wachsende Zahl beteiligter MitarbeiterInnen.

2. Nullhypothese:

Die in beschriebener Weise operationalisierte Größe der Einrichtungen und Dienste hat keinen signifikanten Einfluss auf Umsetzungsstand und Ausgestaltung des Qualitätsmanagements.

\subsubsection{Hypothesen zum Einsatz von Qualitätsbeauftragten}

Die Frage, ob die Umsetzung von Qualitätsmanagement und die Vielfalt in der Praxis angewandter Instrumente mit einem oder mehreren der untersuchten Faktoren (UV) korreliert, ist theoretisch unabhängig von der Frage zu stellen, ob eine unterschiedliche Präferenz für die Anwendung eines bestimmten Instruments, nämlich das des/der Qualitätsbeauftragten (QB), anzutreffen ist. Praktisch gibt es jedoch ein Abhängigkeits- 
verhältnis zwischen der Wahl eines bestimmten QM-Systems und der Institutionalisierung von QB: nur in der ISO 9000 ff. wird dieses Instrument obligatorisch gefordert. Seine Zweck- oder Unzweckmäßigkeit ist damit zwar nicht determiniert, wohl aber die flächendeckende Anwendung in Einrichtungen, die ein zertifizierungsfähiges Qualitätsmanagement nach ISO implementieren.

Sollte sich eine signifikante Bevorzugung der ISO in bestimmten Trägergruppen oder Arbeitsfeldern ergeben - was hypothetisch anzunehmen kein Anlass bestand -, wäre der Nachweis einer dort größeren Anwendungsfrequenz des Instruments QB trivial. Aus diesem Grunde wird auf die Formulierung eines hypothetischen Träger- oder Arbeitsfeldeffektes verzichtet. Als relevante Hypothese verbleibt ein vermuteter Zusammenhang mit dem Faktor Einrichtungsgröße.

\section{Zusammenhänge zwischen Einrichtungsgröße und Einsatz von Qualitätsbeauftragten:}

1. Das Instrument QB wird in sehr großen (Mitarbeiterzahl > 150) und großen Einrichtungen (Mitarbeiterzahl > 75) signifikant häufiger verwendet als in mittelgroßen (Mitarbeiterzahl > 30) und in diesen häufiger als in kleinen und kleinsten (Mitarbeiterzahl < 31) Einrichtungen. M. a. W., die Anwendungshäufigkeit steigt mit der Mitarbeiterzahl (linear?) an.

\section{Begründung:}

Ergänzend zur ansonsten gleichen Begründung wie weiter oben ist anzunehmen, dass sich mit zunehmender Einrichtungsgröße die Zweckmäßigkeit einer Delegation und Zentralisierung von Qualitätskompetenzen erhöht, zugleich das Erfordernis, dezentrale Prozesse zu steuern, zu moderieren oder flankierend $\mathrm{zu}$ unterstützen. Hierfür ist das Instrument des/der QB genuin vorgesehen.

2. Nullhypothese:

In der Anwendung des Instruments QB gibt es in der Stichprobe keinen signifikanten Unterschied zwischen Einrichtungen verschiedener Größe. 
Hypothesen zu Konfliktpotenzialen und zur Zweckmäßigkeit von Qualitätsbeauftragten:

Neben möglichen Einflüssen der Faktoren sind eine Reihe weiterer Fragen von Interesse, die sich auf die potenziell unterschiedliche Beurteilung des Instruments QB durch StelleninhaberInnen und leitende Nicht-StelleninhaberInnen beziehen. Im Falle ausreichenden Doppel-Rücklaufs aus Einrichtungen mit QB (Versionen 1 und 2 des Fragebogens, s. Kap. A 5.3.3) gilt es, folgende Annahmen zu überprüfen:

1. Das Konfliktpotenzial der Funktionsstelle QB bzw. wahrgenommene Hindernisse in der Rollenausübung werden von QB selbst stärker eingeschätzt als von Leitungskräften aus Einrichtungen mit QB.

Begründung:

Die QB-Stelle wird - so jedenfalls der Vorkenntnisstand zum Zeitpunkt der Untersuchungsplanung - in der Regel freiwillig angestrebt und nach eigener Bewerbung durch Leitungsentscheidung besetzt. Namentlich im Falle auftretender Widrigkeiten in der Praxis dürfte die Attribuierung rollen- und/oder umfeldbedingter Gründe psychologisch funktional sein, um das Selbstkonzept von QB zu schützen. Zur Reduzierung kognitiver Dissonanzen dürften Probleme weniger der eigenen Person zugerechnet, sondern externalisiert werden. Leitungskräfte - in der Regel für die Stellenbildung und -besetzung, zugleich aber oft auch für das Konfliktpotenzial mitverantwortlich - reduzieren ihrerseits kognitive Dissonanzen, indem sie auftretende Widrigkeiten, welche die Zweckmäßigkeit ihrer eigenen Entscheidung in Frage stellen können, in ihrer Bedeutung minimieren.

Nullhypothese: kein Unterschied.

2. Leitungskräfte beurteilen die Zweckmäßigkeit der Funktionsstelle QB signifikant positiver als Qualitätsbeauftragte selbst.

Die Begründung folgt der zuvor formulierten Argumentation:

Wenn QB in stärkerem Maße die Konflikthaftigkeit ihrer Rolle wahrnehmen, sollte dies mit häufigeren Zweifeln einhergehen, was die Zweckmäßigkeit der Stelle anbelangt - jedenfalls unter den gegebenen Bedingungen, $\mathrm{zu}$ denen auch 
Leitungsverhalten gehört. Für Leitungskräfte bedeuten solche Zweifel unter Umständen das Eingeständnis eigener Fehleinschätzung und/oder Konfliktbeteiligung, sofern sie - was hier unterstellt wird - selbst für die Ressourcenzuweisung und Stellenkonstruktion verantwortlich waren/sind. Auftretende Probleme dürften primär der Person des/der QB und weniger der Funktionsstelle selbst zugerechnet werden.

Nullhypothese: kein Unterschied.

\subsection{Methodik}

\subsubsection{Auswahl der Untersuchungsgruppen}

Aus Gründen forschungspraktischer Handhabbarkeit wurde unter Ausschluss von Leistungsanbietern in öffentlicher Trägerschaft die erste Untersuchungsgruppe auf teilstationäre und stationäre Einrichtungen der Kinder-, Jugend- und Sozialhilfe in freier Trägerschaft begrenzt.

Dieser Ausschluss erfolgte, um die unvermeidliche Konfundierung der Variablen "öffentliche Trägerschaft" und "Arbeitsfeld" (s. o.) wenigstens in Bezug auf die leistungserbringenden Einrichtungen zu vermeiden. Bei den abzuschließenden Vereinbarungen über Leistungen, Entgelte und Qualität sitzt der öffentliche Träger solcher Einrichtungen nämlich quasi mit sich selbst am Verhandlungstisch.

Auf Seiten der Jugendhilfe wurden somit Organisationen freigemeinnütziger und privatgewerblicher Träger einbezogen, die auf der Grundlage des Kinder- und Jugendhilfegesetzes (SGB VIII) Hilfen zur Erziehung insbesondere nach Maßgabe der $\S \S$ 28-35, Eingliederungshilfe für behinderte Kinder und Jugendliche nach § 35a sowie Hilfe für junge Volljährige nach $\S 41$ erbringen.

Im Einzelnen: Erziehungsberatung ( $§ \quad 28)$, Soziale Gruppenarbeit $(\S 29)$, Erziehungsbeistand, Betreuungshelfer ( $§ 30$ ), Sozialpädagogische Familienhilfe ( $\$ 31)$, Erziehung in einer Tagesgruppe ( 32 ), Vollzeitpflege ( $\$ 33)$, Heimerziehung, sonstige betreute Wohnform ( $\$ 34)$, Intensive Sozialpädagogische Einzelbetreuung ( $§ 35)$, Eingliederungshilfe für seelisch behinderte Kinder und Jugendliche ( $\$ 35 a)$. Letztere wurde mit der ersten Novellierung des SGB VIII 1993 in das Kinder- und Jugendhilferecht integriert. 
Auf Seiten der Sozialhilfe waren insbesondere Einrichtungen angesprochen, in denen Hilfe in besonderen Lebenslagen nach $\S 27$ ff. Bundessozialhilfegesetz (BSHG) gewährt wird, namentlich Eingliederungshilfe für behinderte Menschen nach $\S \S 39$ ff., Hilfe zur Pflege nach $\S 68$ ff. und Hilfe zur Überwindung besonderer sozialer Schwierigkeiten nach $\S 72$. Aufgrund eingeschränkter Zugangsmöglichkeiten zum Kreis der Sozialhilfe-Einrichtungen und Dienste (s. Kap. A 5.1) wurde allerdings mit einer nur schmalen empirischen Basis gerechnet. Den Kern der ersten Zielgruppe bildeten somit die Einrichtungen der Erziehungshilfe, Eingliederungshilfe für behinderte Kinder und Jugendliche sowie Hilfe für junge Volljährige.

Die zweite Untersuchungsgruppe der leistungsgewährenden, -kontrollierenden und finanzierenden Jugend- und Sozialhilfeverwaltungen wurde durch den Kreis der Jugendund Sozialämter zuzüglich der Jugendhilfeverwaltungen von Gemeinden und Gemeindeverbänden ohne Jugendamt als örtlichen Trägern der Jugend- bzw. Sozialhilfe definiert.

Auf die unterschiedliche Akzentuierung des Kontroll- bzw. Qualitätsentwicklungsaspekts in Sozial- und Jugendhilfe wurde bereits hingewiesen. Für die Sozialhilfe ist gleichsam bezeichnend, dass Qualität mit Prüfung konnotiert wird und nicht von Qualitätsentwicklungs-, sondern von Prüfungsvereinbarung die Rede ist. Fraglich ist, ob diese Art der Thematisierung von Qualität über die traditionelle Form der Heimaufsicht hinausführt (vgl. Merchel, 2001, S. 21f).

Beide Zielgruppen konnten sowohl als separate Grundgesamtheiten untersucht und unter den Aspekten der öffentlichen vs. freien Trägerschaft bzw. von Leistungsgewährern vs. Leistungsanbietern verglichen, im Sinne der gemeinsamen Verantwortung für den personenorientierten Hilfeprozess aber auch als eine Population (Total-Grundgesamtheit) betrachtet werden (s. Kap. D 4.1). Überdies war bei ausreichendem Rücklauf auch eine Binnendifferenzierung vorgesehen: Jugendamt vs. Sozialamt bzw. Erziehungshilfen vs. sonstige Aufgaben. 


\subsubsection{Variablenauswahl und Untersuchungsplanung}

Ob eine bestimmte Variable als unabhängige (UV) oder abhängige Variable (AV) fungiert, hängt vor allem von der Verwendung im jeweiligen Forschungskontext ab (Hager, 1987, S. 50). Die UV stellt die vermutete Einflussgröße (Faktor) dar, also eine mögliche Ursache für die Ausprägung der betrachteten AV, unabhängig davon, ob die UV vom Versuchsleiter systematisch, als von ihm herstellbare Bedingung ("Treatment"), variiert werden kann oder lediglich als eine von mehreren vorgegebenen Möglichkeiten ausgewählt und zugewiesen wird (z. B. Geschlecht, Intelligenzniveau, Firmenzugehörigkeit der ProbandInnen).

Im Falle der geplanten Befragung kamen nur solche zugewiesenen Variablen als UV in Betracht, und zwar folgende:

- das Arbeitsfeld der ProbandInnen, das möglicherweise durch unterschiedliche Rechtsnormen, Tätigkeitsmerkmale (z. B. eher verwaltungs- vs. klienten-orientiert) oder verbandliche Strategieempfehlungen mit differenten Positionierungen zum QMThema einschließlich des Stellenwertes von Qualitätsbeauftragten einhergeht;

- die Größe der (weitgehend) selbständig agierenden Organisationseinheit (Gesamtoder Teileinrichtung/Dienststelle), auf die sich die Angaben im Fragebogen beziehen sollten, und die aufgrund unterschiedlicher Anforderungen, z. B. an die Standardisierung von Prozessabläufen, möglicherweise Auswirkungen auf Umsetzungsstand und Wahl des verwendeten QM-Konzepts und die Institutionalisierung von speziell Beauftragten hat;

- die Trägerschaft der Einrichtung/des Dienstes, insofern diese - potenziell in Wechselwirkung mit den anderen Faktoren stehend - Umsetzung und Ausgestaltung des Qualitätsmanagements beeinflussen könnte.

Die Faktoren Arbeitsfeld und Trägerschaft sind qualitative Variablen mit mehreren Nominalstufen, während die Einrichtungsgröße ein quantitatives Merkmal darstellt.

Im Falle des Arbeitsfeldes sollte die zweckmäßige Stufenzahl im Nachhinein festgelegt 
werden, da das entsprechende Item des Fragebogens offen formuliert war und die in Population und Stichprobe repräsentierten Felder anteilsmäßig nicht bekannt waren (die Einrichtungs- oder Dienststellenadressen ließen zumeist keine eindeutigen Rückschlüsse auf den Arbeitsschwerpunkt zu). Deshalb schien eine Differenzierung aufgrund des tatsächlichen Rücklaufs am sinnvollsten. Diese Differenzierung umfasste schließlich folgende Kategorien: Jugend- und Sozialämter, Erziehungshilfeeinrichtungen, Kindertagesstätten/Fachberatungsdienste, Schule/Berufsbildung/Arbeitsförderung, Altenpflege/Behindertenhilfe/Sozialpsychiatrie. Bei zu geringen Zellenbesetzungen sollten diese Gruppen teilweise zusammengefasst werden.

Der (fixierte) Faktor Trägerschaft ging mit den drei möglichen Nominalstufen öffentlich, freigemeinnützig und privatgewerblich in den Versuchsplan ein; jede Kategorie war durch eine eigene Stichprobe repräsentiert. Für den Fall ausreichenden Rücklaufs war die zusätzliche Differenzierung der freien Träger nach Spitzenverbandszugehörigkeit vorgesehen; für den Fall nicht ausreichenden Rücklaufs aus dem privatgewerblichen Bereich konnten beide freien Trägergruppen zusammengefasst werden.

Die kontinuierliche Variable der Einrichtungsgröße wurde anhand des tatsächlichen Rücklaufs auf vier Ordinalstufen reduziert, um eine überschaubare Anzahl von Klassen gleichen Umfangs zu erhalten und mögliche Zusammenhänge kreuztabellarisch darstellen zu können. Die Ordinalstufen entsprachen den jeweiligen Quartilen der Verteilung und keinem inhaltlichen Zuordnungskriterium: kleinste und kleine Einrichtungen mit bis zu 30 MitarbeiterInnen, mittelgroße Einrichtungen mit 31 bis 75 MitarbeiterInnen, große Einrichtungen mit 76 bis 150 und sehr große mit 151 und mehr MitarbeiterInnen.

Die Frage der Sinnhaftigkeit dieser Größenkategorisierung stellt sich ungeachtet der statistisch zweckmäßigen Vorgehensweise. Es kann durchaus diskutiert werden, ob nicht eine stark dezentralisierte, überregional tätige Einrichtung mit 70 MitarbeiterInnen organisationspsychologisch "größer" ist als eine stationäre Einrichtung mit 150 Beschäftigten "unter einem Dach". Solche Fragen mussten hier jedoch ausgeklammert bleiben.

Da die Zugehörigkeit der verfügbaren Untersuchungseinheiten zu den verschiedenen Faktorstufen nicht vorab bekannt war, schon gar nicht durch Zufallsauswahl realisiert werden konnte, sondern sich allein aufgrund des Stichprobenrücklaufs ergeben würde, 
hatten wir es von vornherein nicht mit einem experimentellen, sondern allenfalls einem quasi-experimentellen Design (Cook \& Campbell, 1979) zu tun. Auch dieses setzt jedoch voraus, dass eine eindeutige zeitliche Abfolge (Sequenz) zwischen UV und AV besteht, dergestalt, dass zuerst die UV selegiert und variiert und anschließend die AV systematisch beobachtet/gemessen wird (Hager, 1987, S. 72).

Diese Voraussetzung war in der Studie nicht zu erfüllen, weil nicht mit einer Zufalls-, sondern einer Gelegenheitsstichprobe gearbeitet werden musste (s. Kap. A 5.3.4). Die Zielsetzung der Untersuchung bestand auch gar nicht darin, Einrichtungen und Dienste nach ihrer Zugehörigkeit zu einer der genannten Faktorstufen auszuwählen und dann auf die jeweilige Ausprägung der AV zu messen, um Einflüsse dieser Faktoren zu überprüfen. Möglicherweise unterschieden sich die solcherart ausgesuchten Einheiten ja bezüglich der AV schon, bevor sie einer bestimmten Faktorkombination zugehörig wurden, z. B. wenn eine Einrichtung sich erst zu einer bestimmten Größenstufe entwickelt hatte, nachdem bereits ein QM-System eingeführt worden war. Beobachtbare Zusammenhänge zwischen UV und AV wären somit nicht mit hinreichender Wahrscheinlichkeit der UV zuzuordnen gewesen, sondern hätten ebenso gut durch gruppenspezifische Merkmale "verursacht" worden sein können, die bereits vor der Untersuchung bestanden. Die Äquivalenz der zu vergleichenden Gruppen wäre mangelhaft gewesen und alternative Hypothesen über das Zustandekommen etwaiger Unterschiede in der AV hätten u. U. gleich große oder stärkere Plausibilität besessen.

Es war somit fraglich, ob im vorliegenden Kontext überhaupt von Variablen gesprochen werden konnte, die sich als UV und AV sinnvoll voneinander hätten unterscheiden bzw. trennen lassen (Hager, 1987, S. 50). Vielmehr haben wir es offenbar mit der Registrierung von Unterschieden und Zusammenhängen zu tun, die zwar möglicherweise regelhaft sind, aber keine Aussagen über Ursache-Wirkungs-Beziehungen implizieren. Solche Untersuchungen heißen gemeinhin Korrelationsstudien.

Damit ist auch die vorliegende Arbeit methodologisch korrekt charakterisiert: Es geht um die Bestandsaufnahme QM-bezogener Strukturen und Bewertungen an Personen, die Einrichtungen und Dienste unterschiedlicher Größe, Arbeitsfelder und Trägerschaften repräsentieren. Korrelative Zusammenhänge zwischen diesen Faktoren sollten zwar auf der Basis bestimmter Annahmen (Hypothesen, s. o.) untersucht werden, allerdings nicht im 
Rahmen systematischer Bedingungsvariation der unabhängigen Variablen.

Trotz dieser relativierenden Vorüberlegungen wird auf die sprachübliche Unterscheidung von UV und AV im Folgenden nicht verzichtet, zumal die Varianzanalyse als Auswertungsverfahren eine Rolle spielt und darin explizit diese Begriffe verwendet werden. Es geht jedoch lediglich darum, die qualitativen Variablen Arbeitsfeld, Einrichtungsgröße und Trägerschaft - ggf. auch nominalskalierte Charakteristika von Qualitätsbeauftragten (hier: Geschlecht) - hinsichtlich etwaiger Mittelwertsunterschiede auf den quantitativen Variablen sowie auf Kontingenzen zu anderen qualitativen Merkmalen zu untersuchen. Potenziell signifikante Differenzen bzw. Zusammenhänge sollten nicht als Folge eines Ursache-Wirkungs-Verhältnisses interpretiert werden, sondern als bloße Korrelationsbeziehung.

Auf eine rücklaufbedingt eingeschränkte Umsetzung des Untersuchungsplans sei bereits an dieser Stelle hingewiesen. Die hier als unabhängig deklarierten Variablen interkorrelieren mehr oder weniger stark, vor allem die Faktoren Trägerschaft und Arbeitsfeld (s. o.). Die durch diese Konfundierung schwer unterscheidbare "Wirkung" der einzelnen UV auf die AV hätte durch einen ausbalancierten komplexen Versuchsplan im Prinzip kontrolliert werden können, insofern bestimmte Wechselwirkungen sichtbar geworden wären. Selbst unter Reduzierung der möglichen Arbeitsfelder auf drei (Jugend-/Sozialhilfeverwaltungen, Erziehungshilfeeinrichtungen, Sonstige) hätte für ein solches $3 \times 3 \times 4$-Design jedoch eine wesentlich größere Stichprobe zur Verfügung stehen müssen, als sich im Rücklauf letztlich ergab. Stattdessen konnte nur durch Kreuztabellierung versucht werden, die jeweiligen Interaktionseffekte erkennbar $\mathrm{zu}$ machen und bei der Interpretation der Befunde $\mathrm{zu}$ berücksichtigen.

\subsubsection{Untersuchungsinstrumente}

Als unter den gegebenen Rahmenbedingungen (s. Kap. A 5.1) einzig realisierbare Methode - im Hinblick auf Verfahrenskontinuität zu den durchgeführten Vorstudien sowie Vergleichbarkeit zu anderen Untersuchungen jedoch ohnehin zweckmäßig und forschungsökonomisch sinnvoll - wurde eine anonyme postalische Befragung mit einem vollstandardisierten Fragebogen in drei Versionen gewählt. Um zum Zwecke eines 
möglichen und wünschenswerten Gruppenvergleichs auch Arbeitsfelder aus Grenzbereichen der Jugend- und Sozialhilfe anzusprechen, wurde das Untersuchungsinstrument neutral betitelt als Fragebogen zum Qualitätsmanagement/für Qualitätsbeauftragte in sozialen Einrichtungen und Diensten.

Der Fragebogen wurde vom Umfang und Layout so dimensioniert, dass er von einem sachkundigen Probanden in etwa 15 bis 30 Minuten vollständig zu bearbeiten sein sollte. Aus diesem Grunde wurden bis auf ein Item (Arbeitsfeld) alle Fragen geschlossen formuliert, bisweilen ergänzt um ein Antwortfeld "Sonstiges". Angehängt wurde eine offene Schlussfrage zu Fortbildungswünschen von Qualitätsbeauftragten; die Auswertung dieser Frage war allerdings nicht Bestandteil dieser Arbeit.

Um hinsichtlich der Angaben zum Instrument des/der QB mögliche Zusammenhänge zwischen Strukturvariablen der Einrichtungen, Personenvariablen der RolleninhaberInnen und Gruppenzugehörigkeit der InformantInnen prüfen zu können, wurde der Fragebogen von einem gemeinsamen Fragenpool zum Qualitätsmanagement abgesehen - in drei Versionen und für drei Personengruppen konstruiert:

1. Selbstbewertung durch Qualitätsbeauftragte (Version 1),

2. Fremdbewertung durch Leitungskräfte aus Einrichtungen mit QB (Version 2) sowie

3. Fremdbewertung durch Leitungskräfte von Einrichtungen ohne QB (Version 3, als Variante von 2 mit Filterfrage).

Die Auswahl der Items orientierte sich des deskriptiven Schwerpunktes der Studie wegen an den wichtigsten und am häufigsten verwendeten Grundkonzepten der Organisationsdiagnose (Scholl, 2004, S. 530). Solche sind im Sinne von Zustandsbeschreibungen:

○ Organisationsziele (angestrebte zukünftige Zustände): im Rahmen der Studie als Grundsätze zur Qualitätspolitik und als Leitbild einbezogen;

- Organisationsverfassung (gesetzliche, satzungsmäßige u. a. Regelungen zur Machtverteilung, Rechenschaftspflicht etc.): im Rahmen der Studie als Führungsgrundsätze und Trägerschaft einbezogen; 
- Organisationsstruktur (Regelsystem für Arbeitsteilung und Koordination): im Rahmen der Studie als Aufbau- und Ablaufbeschreibung sowie als Strukturmerkmale der Funktionsstelle Qualitätsbeauftragte/r einbezogen;

- Organisationsform (Einlinien- oder Mehrliniensysteme): im Rahmen der Studie nicht speziell, sondern als Aspekt der Organisationsstruktur betrachtet (hier: Größe der Einrichtung);

- Technologie (technische Hilfsmittel, Know-how, Arbeitsgestaltung etc.): im Rahmen der Studie als diverse Instrumente des Qualitätsmanagements einbezogen (QM-Handbuch, Qualitätszirkel u. a.);

- Organisationskultur und -klima (gemeinsame Werte und Normen, Wahrnehmung der Organisation durch die Mitglieder): im Rahmen der Studie als Konfliktpotenziale und Rollenwahrnehmung von Qualitätsbeauftragten einbezogen.

Obwohl es, wie Scholl (2004, S. 540) betont, schwierig ist, Zustandsbeschreibungen einer Organisation von den organisationalen Prozessen zu trennen, soll nachstehend versucht werden, auch bezüglich der häufigsten Prozesskonzepte eine Zuordnung zum Aufbau des Untersuchungsinstruments in der vorliegenden Studie vorzunehmen:

○ Entscheidungsprozesse: im Rahmen der Studie nicht explizit einbezogen;

- Machtausübung und Einflussnahme: im Rahmen der Studie nicht explizit einbezogen;

- Partizipation: im Rahmen der Studie als Beteiligung am QM-Prozess sowie implizit in Form diverser Instrumente des Qualitätsmanagements (z. B. Vorschlagswesen) einbezogen;

- Konflikt und Kooperation: im Rahmen der Studie als Konfliktpotenziale einbezogen;

- Information, Kommunikation und Wissen: im Rahmen der Studie als Qualifizierung der MitarbeiterInnen sowie in Form diverser QM-Instrumente (z. B. Evaluation) und Maßnahmen zur Qualitätsentwicklung einbezogen.

Zum Themenkomplex "QM in sozialen Einrichtungen und Diensten" sollte somit eine möglichst breite Bestandsaufnahme gewährleistet sein, indem die in der einschlägigen Fachliteratur diskutierten Standardkonzepte und Instrumente durch wenigstens ein Item repräsentiert waren.

Darüber hinaus galt es, zum Themenkomplex "Qualitätsbeauftragte/r" auf der Grundlage 
existierender Stellenbeschreibungen und Erfahrungsberichte die für relevant gehaltenen Aspekte anzusprechen.

Im Einzelnen enthielt der Fragebogen in seinen drei Versionen folgende Itemgruppen:

- 3 Items zu Arbeitsfeld, Trägerschaft und Größe der Einrichtung/des Dienstes (alle Versionen)

- 13 Items zur Struktur des Qualitätsmanagements (alle Versionen)

- 2 Items zur Beteiligung und Qualifizierung der MitarbeiterInnen (alle Versionen)

- 33 Items zum eingeschätzten Nutzen von Maßnahmen der Arbeitsoptimierung (alle Versionen)

- 65 Items zu Qualitätsbeauftragten (Version 1; Version 2 nur 37; Version 3 nur 1):

○ 7 Items zu Stellencharakteristika (Version 1 und 2),

- 6 Items zu Personencharakteristika (Version 1 und 2),

○ 3 Items zur Zweckmäßigkeit des Instruments (Version 1 und 2),

- 14 Items zu Konfliktpotenzialen (Version 1 und 2),

○ 7 Items zur Rollenwahrnehmung bzw. Rollenerwartung (Version 1 bzw. 2),

○ 28 Items zu Aufgaben und Tätigkeiten (Version 1),

○ 1 Item für Einrichtungen ohne QB (Version 3),

Insgesamt bestand Version 1 aus 117 (incl. offener Schlussfrage), Version 2 aus 88 und Version 3 aus 52 Items.

Die Items waren nicht dimensional geordnet, sondern inhomogen. Einige bezogen sich auf eher globale Aspekte betrieblicher Interaktion (z. B. "Ziehen an einem Strang"), andere auf eher spezifische Instrumente (z. B. Kundenpfadanalyse). Der Operationalisierungsgrad war unterschiedlich, ebenso die Differenziertheit der Antwortskalen sowie das Skalenniveau. Inhaltlich repräsentierten die Items zumeist singuläre Aspekte eines Themas und waren teils faktenorientiert, teils einstellungsbezogen. Insgesamt war das Untersuchungsinstrument als vorwiegend beschreibend und Hypothesen erkundend zu charakterisieren (vgl. Moser, 2004, S. 91).

Der Fragebogen wurde an fünf sachkundigen Einzelpersonen prä-getestet. Dies schien 
hinreichend, da die Mehrzahl der Items bereits in früheren Befragungen verwendet (und zuvor prä-getestet) worden war und für Vergleichszwecke ohnehin nicht substantiell verändert werden sollte. Für inhaltlich neue Aspekte war dessen ungeachtet zu überprüfen, ob wesentliche Gütekriterien erfüllt waren, vor allem Verständlichkeit, Eindeutigkeit, Relevanz und Eindimensionalität. Dies wurde im Prätest von allen Personen bestätigt; vorzunehmende Korrekturen waren minimal, itemkritische Kommentare im Rücklauf kein Thema. Dennoch erwies sich bei der Auswertung der eine oder andere Schwachpunkt, auf den im Text hingewiesen wird.

\subsubsection{Umfang und Rekrutierung der Stichproben}

Für verlässliche Aussagen zur Umsetzung und Ausgestaltung von Qualitätsmanagement in Einrichtungen und Diensten bundesdeutscher Jugend- und Sozialhilfe - als einem wesentlichen konzeptionellen Element Neuer Steuerungsmodelle - wäre eine Vollerhebung in den ausgewählten Zielgruppen mit offizieller Unterstützung durch die Spitzenverbände der öffentlichen und freien Wohlfahrtspflege wünschenswert gewesen. Sowohl vom Aufwand als auch von den Aussichten her, diese Unterstützung zu erhalten, war ein solches Vorhaben jedoch von vornherein illusorisch, zumal angesichts der Vielzahl empirischer Studien, die in diesem Arbeitsbereich durchgeführt werden.

In Frage kam somit nur eine Teilerhebung im Zielgruppenbereich. Hinsichtlich des Rücklaufs galt es aufgrund von Erfahrungen mit ähnlich angelegten Untersuchungen (Gerull, 1998a; Peters et al., o. J.; Wetzler, 2003), die Erwartungen mit etwa 20 bis 30\% nicht zu hoch zu schrauben.

Da in der Studie auch zufallskritische Entscheidungen auf der Basis statistischer Prüfverfahren vorgesehen waren (Signifikanzniveau $\alpha=.05$ ), wurde versucht, den optimalen Stichprobenumfang ungeachtet mutmaßlich verletzter parametrischer Voraussetzungen nach folgender Formel zu schätzen: $\mathrm{n}=[\mathrm{z}(1-\alpha)-\mathrm{z}$ $(\beta)]^{2} / \varepsilon^{2}$ (Cassel, 2004, Kap. 7.6, S. 50). Darin ist $\mathrm{n}$ der gesuchte Stichprobenumfang, $\alpha$ das gewählte Signifikanzniveau, $\beta$ die damit korrespondierende Wahrscheinlichkeit eines Fehlers der zweiten Art und $\varepsilon$ die Effektgröße (standardisierte Mittelwertsdifferenz), die ein Maß für die Trennschärfe einer Vergleichsmessung darstellt. Unter Berücksichtigung der zumeist verwendeten sechsstufigen Likertskalen wurde der kritische Wert für die Effektgröße mit 0,60 festgelegt, bei dessen Erreichen oder Überschreiten die zu testende Hypothese als bestätigt betrachtet werden sollte (hier nur zur Errechnung der optimalen 
Stichprobengröße verwendet).

Die so ermittelte Stichprobengröße von 29 musste wegen der vorgesehenen mehrstufigen Gruppenvergleiche - maximale Stufenzahl = 4 (Quartile der Einrichtungsgröße) - vervierfacht werden, um den optimalen Umfang der Untersuchungsstichprobe zu ergeben: 4 x $29=116$ (ohne Berücksichtigung von Interaktionseffekten). Bei einem erwarteten Rücklauf von ca. $25 \%$ resultierte daraus eine anzustrebende Gesamtstichprobe von $\mathrm{n}=116: 0,25=464$.

Als mutmaßlich ausreichende Stichprobengröße, um alle Fragestellungen der Studie auf statistisch hinreichender Grundlage zu untersuchen, wurde daher ein Pool von ca. 450 Einheiten erachtet, in dem die Zielgruppen möglichst proportional zu ihren jeweiligen Grundgesamtheiten vertreten sein sollten (s. Kap. D 4.3.3).

Die gesamte Gruppe der öffentlichen Träger konnte dabei nur mit Hilfe des Deutschen Vereins für öffentliche und private Fürsorge erschlossen werden.

Der Deutsche Verein ist der zentrale Zusammenschluss der öffentlichen und freien Träger sozialer Arbeit und repräsentiert bundesweit die gesamte Palette der öffentlichen und privaten Wohlfahrtspflege. Aus einer dankenswerterweise zur Verfügung gestellten Anschriftenliste mit rund 350 Organisationen bzw. Organisationsmitgliedern, die Anfang 2004 im Zusammenhang mit QM-bezogenen Anfragen, Anmeldungen oder Kursteilnahmen beim Deutschen Verein registriert waren, rekrutierten sich die im Folgenden Hauptstichprobe genannten Untersuchungseinheiten.

Diese Hauptstichprobe enthielt Personen, Einrichtungen und Dienste aus dem Sozial-, Gesundheits- und Bildungsbereich des gesamten Bundesgebiets, jedoch waren die einzelnen Arbeitsfelder und Trägerorganisationen sehr unterschiedlich repräsentiert. Mit einem Anteil von fast $70 \%$ dominierte die von öffentlichen Trägern geleistete Jugend- und Sozialhilfe, während freigemeinnützige und privatgewerbliche Träger nur mit knapp einem Drittel vertreten waren, weit überwiegend in der Kinder- und Jugendhilfe, zu einem geringen Anteil in anderen sozialen Handlungsfeldern tätig.

Die Dominanz des Jugend- und Sozialhilfebereichs in dieser Gelegenheitsstichprobe war an sich ein Glücksfall im Sinne der Zielgruppendefinition; auf diese Weise war nur eine geringfügige Bereinigung des Anschriftenpools um fachfremde AdressatInnen bei öffentlichen und freien Trägern erforderlich. Allerdings warf das trägerbezogene Ungleichgewicht die Notwendigkeit einer besseren Repräsentanz der verschiedenen freien 
Träger auf. Betroffen waren namentlich die in den Spitzenverbänden von Caritas, Diakonischem Werk, Paritätischem, Arbeiterwohlfahrt und Deutschem Roten Kreuz vertretenen Organisationen, die den weitaus größten Teil der leistungserbringenden Einrichtungen im Sozialwesen darstellen - in der Erziehungshilfe z. B. mit 4.751 von 7.346 Einrichtungen rund 65\% (s. Kap. D 4.1, Tab. 10).

Noch stärker unterrepräsentiert war der relativ stark expandierende Bereich der privatgewerblichen Träger; zweifelsfrei ließen sich von über 100 freien Einrichtungen in der Hauptstichprobe nur 5 diesem Sektor zuordnen. Durch Einbeziehung zusätzlicher Untersuchungseinheiten aus dem freigemeinnützigen und privatgewerblichen Bereich galt es deshalb, das Verhältnis der Trägergruppen auszutarieren und die formelmäßig ermittelte Stichprobengröße zumindest annähernd zu erreichen. Dies gelang durch Rekrutierung von Mitgliedseinrichtungen des Evangelischen Erziehungsverbandes (EREV) sowie des Bundesverbandes privater Träger der freien Kinder-, Jugend- und Sozialhilfe e. V. (VPK); darüber hinaus konnten mehrere Organisationen unterschiedlicher Träger im ostwestfälischen Raum über einen freiberuflichen Heimberater für die Befragung gewonnen werden.

Der EREV repräsentiert als evangelischer Fachverband auf Bundesebene mit über 500 Mitgliedern den nach Einrichtungszahl größten Anbieter von Erziehungshilfen in Deutschland, das Diakonische Werk der Evangelischen Kirche. Konkret handelte es sich bei der Stichprobe um die im Organ des EREV-Fachbeirats vertretenen rund 50 Einrichtungen, die ihrerseits einen nahezu kompletten Querschnitt der Jugendhilfeangebote freigemeinnütziger Träger überhaupt darstellen. Wenngleich somit in fachlicher Hinsicht Repräsentanz der Stichprobe für die Erziehungshilfe allgemein angenommen werden kann, geriet der Gesamtanteil diakonischer Einrichtungen dadurch fast doppelt so hoch, wie es dem relativen Anteil an Einrichtungen freier Träger entspricht. Im Rücklauf verringerte sich allerdings dieses Übergewicht (s. Kap. D 4.3.2).

Der VPK ist nach Verbandsangaben die einzige bundesweite Vertretung der privaten Träger der freien Kinder-, Jugend- und Sozialhilfe und zählte zum Untersuchungszeitpunkt 363 Mitgliedseinrichtungen. Die Frage, ob der Verband fachlich und strukturell die Grundgesamtheit aller Einrichtungen in privater Trägerschaft repräsentiert, kann nach Datenlage nicht entschieden werden, zumal die bundesamtliche Statistik (s. Kap. D 4.1) nicht ausweist, ob der VPK den Wirtschaftsunternehmen oder der Kategorie "Sonstige juristische Personen, andere Vereinigungen" zugeordnet wurde. Die Zuordnung des VPK zu den Wirtschaftsunternehmen wird jedoch für plausibel gehalten und im Folgenden unterstellt. Tangiert wäre im Falle einer Fehlzuordnung lediglich die Stichprobenquote an der Teilpopulation. 


\subsubsection{Adressaten der Befragung}

Als Adressaten der Befragung fungierten die Qualitätsbeauftragten und/oder Leitungskräfte mit Zuständigkeit für das Qualitätsmanagement der Einrichtungen und Dienste sofern überhaupt vorhanden oder vorgesehen.

Bei der Untersuchungsplanung wurde davon ausgegangen, dass auch Einrichtungen ohne ausgewiesene/n QB aufschlussreiche Informationen zum Instrument beizusteuern in der Lage wären. So konnte es Einrichtungen geben, die sich aufgrund gemachter Erfahrungen wieder von dem Instrument getrennt hatten, die mithin gerade sehr kompetent in dieser Frage sein durften. Des Weiteren mochte der Entscheidung, die Funktionsstelle nicht auszuweisen, ein sehr reflektierter Argumentationsprozess vorangegangen sein, in dem anderenorts gemachte Erfahrungen berücksichtigt wurden. Und schließlich konnte ein kompetentes Urteil auch auf rein analytischem Wege zustande gekommen sein.

Durch primäre Ansprache der QB galt es "zwei Fliegen mit einer Klappe" zu schlagen: Zum einen sollte differenziertes Material zum Instrument aus Binnensicht der Akteure, zum anderen zum Stand der Umsetzung von Qualitätsmanagement insgesamt gewonnen werden. Es konnte mit gutem Grund angenommen werden, dass die Bearbeitung beider Fragenkomplexe beim/bei der QB in den bestmöglichen Händen ruhte, fiel dies doch in dessen/deren genuinen Zuständigkeitsbereich; zudem konnte ein Interesse an empirischem (Vergleichs-)Material über die eigene Rolle und Funktion unterstellt werden, da in der Fachliteratur allenfalls anekdotische Beiträge dazu vorlagen. Beides wurde als gute Voraussetzung für eine ausreichende Rücklaufquote angesehen (eine Hoffnung, die sich nicht ganz erfüllte). Sofern ein/e QB als AnsprechpartnerIn nicht vorhanden war, sollte durch allgemeine Fragen zum Instrument gewährleistet werden, dass sich auch ein kompetenter Anderer zu einem Teil dieses Themenkomplexes aus der "Außensicht" äußern konnte. Auch in diesem Falle wäre ein substantieller Datenbeitrag zu erwarten gewesen, der bei ausreichender Stichprobengröße Vergleichsmöglichkeiten zur "Binnensicht" eröffnete.

Allein auf der Basis der einzuholenden Selbstauskünfte der QB und der Angaben von Leitungspersonen, die mutmaßlich an der Stellenbesetzung verantwortlich mitgewirkt haben dürften, waren naturgemäß keine bzw. nur eingeschränkt intersubjektive Aussagen 
zur tatsächlichen, "objektiven" Wirksamkeit und Zweckmäßigkeit des Instruments möglich. Vielmehr konnte von vornherein eine gewisse Reaktivität der Ergebnisse unterstellt werden, z. B. eine Schönfärbung zur Selbstbestätigung der eigenen Rolle und Entscheidung oder zur kognitiven Dissonanzreduktion.

Dies betont noch einmal den explorativen und deskriptiven Charakter der Untersuchung. Es ging im Rahmen des Designs nicht darum, die Überlegenheit des Faktors QB gegenüber einer Vergleichsgruppe ohne QB nachzuweisen, sondern zu beschreiben, welche Struktur-, Personen- und Tätigkeitsmerkmale, Gründe für und wider, Nutzeneinschätzungen und Konfliktpotenziale ohne Anspruch auf Repräsentativität im Feld vorkommen. Sofern es die rücklaufenden Daten erlauben würden, waren verschiedene Hypothesen testbar; anderenfalls würde die Untersuchung nur ihren explorativen Auftrag zu erfüllen in der Lage sein.

\subsubsection{Durchführung der Befragung}

Alle Einheiten der Gesamtstichprobe erhielten je einen Fragebogen der Version 1 und 2/3; insgesamt wurden somit mehr als 800 Fragebögen verschickt bzw. verteilt. Zur Unterstützung der Aktion und mutmaßlichen Erhöhung der Rücklaufquote wurde dem Fragebogen ein gemeinsames Anschreiben des Autors und einer wissenschaftlichen Referentin des Deutschen Vereins beigefügt, in dem das ausdrückliche Interesse des Verbands an den Ergebnissen betont wurde.

Zudem wurde als Anreiz angeboten, beim Autor ein Kapitel des Handbuchs auf CD-ROM "Qualitätsmanagement sozialer Dienstleistungen" (Gerull, 2004), und zwar die nach Stichworten und Arbeitsfeldern sortierte Bibliographie, als PDF-Datei abzurufen. (Davon machten allerdings nur 20\% der Rücklaufs Gebrauch, was nicht unbedingt für die Attraktivität des gewählten Anreizes spricht.)

Die Anschreiben und Fragebögen für die Hauptstichprobe wurden Anfang Februar 2004, diejenigen (Anschreiben leicht modifiziert) für die Zusatzstichprobe der privaten Träger Ende Februar auf dem Postwege zugestellt. Den VertreterInnen der evangelischen Erziehungshilfeeinrichtungen wurden die Bögen nebst (gleichfalls leicht modifiziertem) Anschreiben anlässlich einer Fachbeiratssitzung des EREV Ende März von einem Mitglied der Geschäftsstelle ausgehändigt. 
Deadline für den Rücklauf war zunächst Anfang April; aufgrund mehrerer Anfragen und des ohnehin schleppenden Eingangs der Fragebögen wurde diese Frist bis Ende Mai 2004 verlängert. Auf diese Weise konnte ein kleines Kontingent ostwestfälischer Einrichtungen noch zusätzlich in die Untersuchung einbezogen werden.

Die im Begleitschreiben begründete Bitte um Mitarbeit wandte sich zum Teil an einzelne Fachkräfte der Einrichtungen und Dienste - sofern namentlich bekannt - mit der Instruktion, den jeweils passenden Fragebogen (Version 1 oder 2/3) selbst zu beantworten - sofern zuständig - oder an den/die QB bzw. an die Leitung/Geschäftsführung weiterzugeben. Mit derselben Maßgabe wurden auch die nicht namentlich adressierten Bögen versehen. Ggf. sollten nicht benötigte Fragebögen der Version 1 und 2 (für Einrichtungen mit Qualitätsbeauftragten) ignoriert und nur die Version 3 (für Einrichtungen ohne Qualitätsbeauftragten) bearbeitet werden.

Einrichtungen mit QB wurden für den Fall einer Beantwortung der Versionen 1 und 2 durch verschiedene MitarbeiterInnen darum gebeten, die Bögen zusammen in dem beigefügten (adressierten, jedoch unfrankierten) Umschlag zurückzusenden. Die dadurch bedingte Doppelrepräsentanz dieser Einrichtungen wurde in der Auswertung berücksichtigt (s. Kap. D 4.5).

\subsubsection{Einbeziehung von Vorstudien und Vergleichsuntersuchungen}

Die vorliegende Studie steht in der Kontinuität größerer und kleinerer Untersuchungen, die der Autor zwischen 1997 und 2003 durchführen konnte. Dabei handelt es sich um standardisierte schriftliche Befragungen von Belegschaften, Leitungskräften und KursteilnehmerInnen, weit überwiegend aus dem Bereich der Erziehungshilfe. Wenngleich die eingesetzten Untersuchungsinstrumente nicht voll identisch sind, teilweise in verändertem Kontext eingesetzt wurden und Signifikanzprüfungen etwaiger Unterschiede wegen teils lückenhafter Kennwerte nur vereinzelt in Betracht kamen, ergaben sich potenziell interessante Vergleichsaspekte.

Nachfolgende Datenbestände und Kennwerte aus eigenen Vorstudien standen dafür zur Verfügung: 
1. Mitarbeiterbefragung in großer Einrichtung der Erziehungshilfe, April 1997, 80 MitarbeiterInnen, Rücklaufquote 81,2\% ( $\mathrm{n}=65)$; Häufigkeitsverteilungen und Mittelwerte der Gesamtstichprobe;

2. Mitarbeiterbefragung in sehr großer Einrichtung der Erziehungshilfe, Januar/Februar 1998, 216 MitarbeiterInnen, Rücklaufquote 86,1\% ( $\mathrm{n}=186$ ); Häufigkeitsverteilungen, Mittelwerte und Varianzanalysen;

3. verbandliche Mitgliederbefragung, August 1998, 393 Einrichtungsleitungen der Erziehungshilfe, Rücklaufquote 30,5\% ( $\mathrm{n}=120$ ); Häufigkeitsverteilungen, Mittelwerte und Varianzanalysen;

4. Mitarbeiterbefragung in großer Einrichtung der Erziehungshilfe, Januar 2000, 95 MitarbeiterInnen, Rücklaufquote 82\% ( $\mathrm{n}=78$ ); Häufigkeitsverteilungen, vollständige deskriptive Statistik und Varianzanalysen;

5. Mitarbeiterbefragung in mittelgroßer Einrichtung der Erziehungshilfe, Februar 2003, 44 MitarbeiterInnen, Rücklaufquote 95,3\% ( $\mathrm{n}=42)$; Rohdaten, Häufigkeitsverteilungen und vollständige deskriptive Statistik;

6. Teilnehmerbefragung eines Selbstevaluationskurses, August 2000, 18 Fachkräfte der Erziehungshilfe, Rücklaufquote 66\% ( $\mathrm{n}=12)$; Rang- und Mittelwerte;

7. Teilnehmerbefragung eines Akademiekurses für Qualitätsbeauftragte, Oktober 2002, 22 Fachkräfte der Jugend- und Sozialhilfe, Rücklaufquote 100\% ( $\mathrm{n}=22$ ); Rangwerte;

8. Teilnehmerbefragung eines Akademiekurses für Qualitätsbeauftragte, November 2003, 19 Fachkräfte der Jugend- und Sozialhilfe, Rücklaufquote 100\% ( $\mathrm{n}=19)$; Roh- und Rangwerte.

Die im Rahmen dieser Dissertation durchgeführte aktuelle Untersuchung (Hauptstudie) begründet sich vor allem darin, dass der Frage nach Umsetzungsstand und Ausgestaltungsformen eines sozialwirtschaftlichen Qualitätsmanagements bislang wenig empirische Aufmerksamkeit gewidmet wurde. Ungeachtet zahlreicher Evaluationen, wie sie auf der 
Ebene einzelner Einrichtungen, Verbände, Kommunen oder Forschungsgruppen durchgeführt wurden und werden, liegt nach eigenen Literatur- und Internet-Recherchen des Autors im Bereich der Sozialen Arbeit kein aktuelles Datenmaterial vor, das die Situation in der Jugend- und Sozialhilfe insgesamt beleuchtet. Zum viel diskutierten Instrument des/der Qualitätsbeauftragten ist - von eher anekdotischen und/oder programmatischen Beiträgen abgesehen - nahezu völlige Fehlanzeige zu vermelden.

Die von Berner \& Leisering (2003) publizierten Ergebnisse zur kommunalen Sozialhilfeverwaltung betreffen zwar unter anderem die Umsetzung des Neuen Steuerungsmodells an bundesdeutschen Sozialämtern, nehmen aber an keiner Stelle explizit Bezug auf Elemente des Qualitätsmanagements.

Die von Gerull (1998) im Rahmen einer Mitgliederbefragung des Evangelischen Erziehungsverbandes (EREV) erhobenen Daten dienten seinerzeit der ersten Orientierung in einem durch Veröffentlichungsflut und allgemeine Verunsicherung charakterisierten Praxisumfeld, dürften jedoch längst überholt sein.

Das von Peters geleitete Forschungsprojekt "Qualitätsentwicklung in der Jugendhilfe" - Datenerhebungszeitraum Ende 1999 bis Anfang 2000 - sollte hier teilweise Abhilfe schaffen können, harrt aber immer noch seiner vollständigen Publikation und fokussierte zudem auf eher allgemeine Fragestellungen der Qualitätsdebatte (www.fh-erfurt.de/so/projekte/qualitaet).

Eine Ende 2001 durchgeführte Untersuchung im Bereich der stationären Behindertenhilfe (Wetzler, 2003) liefert zwar ein informatives Schlaglicht, ist jedoch in weiten Teilen wenig differenziert und für die Jugendund Sozialhilfe nicht repräsentativ.

Von J. F. W. Müller (2004) im Rahmen einer Dissertation durchgeführte Fallstudien (n = 9) in Altenhilfeeinrichtungen erlauben aufgrund des qualitativen Untersuchungsansatzes keinerlei Vergleiche

Dass sich empirische Forschung im angesprochenen Bereich schwierig gestaltet, kann allen erwähnten Untersuchungen entnommen werden und blieb auch der vorliegenden Studie nicht erspart. Empfundener Zeitmangel angesichts des umfangreichen Fragebogens, Befürchtungen, sich durch allzu offenherzige Selbstauskünfte verbandspolitisch unzweckmäßig zu verhalten, Vermeidenwollen von Fehlanzeigen, Widerstände gegen vermeintliche Datensammelaktionen ohne erkennbaren praktischen Nährwert und ähnliche Befindlichkeitskonstellationen auf Seiten der potenziellen BefragungsteilnehmerInnen äußerten sich auch hier in einer eher unbefriedigenden Rücklaufquote (rund 21\%, s. Kap. D 4.3.1). 
Dennoch werden die Ergebnisse für aufschlussreich gehalten, um einen allgemeinen Überblick zur Umsetzung und Ausgestaltung von Qualitätsmanagement in der Jugend- und Sozialhilfe zu gewinnen, sei es zum Zwecke der eigenen Positionierung im Vergleich, sei es zur differenzierten Bestandsaufnahme speziell zum Instrument des/der QB.

Von den oben erwähnten Untersuchungen eröffnen vor allem die nachfolgenden zwei punktuell interessante Vergleichsmöglichkeiten:

- Peters et al. (o. J.): 110 nach Zufall ausgewählte Mitgliedseinrichtungen der Internationalen Gesellschaft für Heimerziehung (IGfH) und zusätzliche OnlineBefragung, Befragungszeitraum Januar bis April 2000, Rücklaufquote $30 \%$.

○ Wetzler (2003): rund 1.400 Wohneinrichtungen der Behindertenhilfe bundesweit, Befragungszeitraum Ende 2001, Rücklaufquote rund 16\% $(\mathrm{n}=216)$. 


\section{B Qualitätsmanagement als interdisziplinäres Konzept - Grundlagen und Schnittmengen}

\section{Qualitätsspezifische Grundlagen im Überblick}

\subsection{Kurze Geschichte der Qualität und ihres Managements}

Die Beschäftigung mit Qualität ist uralt. Gesetzlich vorgeschriebene Anforderungen an Bauwerke etwa sind schon aus babylonischen Zeiten überliefert (Codex Hammurabi) und im Mittelalter spielte der Qualitätsgedanke in den Regeln und Normen der Zünfte eine zentrale Rolle (Seghezzi, 1996, S. 5). Amtliche Beschauzeichen bestätigten die Qualität geprüfter Produkte, Meisterzeichen identifizierten den Hersteller. Aus ihnen entwickelten sich mit zunehmender Industrialisierung die Fabrik- und Qualitätsmarken (Wolters, Albrecht \& Schwabe, 1995, S. 5).

Die Herkunftskennzeichnung für Produkte, wie sie ein britisches Gesetz von 1887 aus protektionistischen Gründen vorschrieb, wandelte sich als "Made in Germany" vom Brandmal zum ausgesprochenen Qualitätsbegriff. Da die internationale Konkurrenz aufholte, verlor dieses ungewollt zum Gütesiegel gewordene Zeichen später einen erheblichen Teil seiner Wirkung. Es bedurfte neuer Methoden und Etikettierungen, um Qualität als kaufentscheidungsrelevantes Produktmerkmal $\mathrm{zu}$ entwickeln und nachzuweisen.

KundInnen wollen von einem Produkt in erster Linie einen Nutzen haben; sie wünschen sich jedoch auch einen angemessenen Preis und eine gute Verfügbarkeit in benötigter Menge, indem es z. B. zum gewünschten Zeitpunkt auf einfache Art beschafft werden kann (vgl. Seghezzi, 1994, S. 11). Um den Kundenbedürfnissen gerecht zu werden, müssen Anbieter von Produkten ihrerseits die Qualität, Kosten und Lieferbereitschaft bezüglich Menge und Termin optimal gestalten. In diesem unternehmerischen Spannungsviereck (Seghezzi, 2003, S. 20) aus Qualität, Geld, Quantität und Zeit sind die einzelnen Faktoren ständig gegeneinander abzuwägen, um den besten Erfolg zu erzielen.

Bis Anfang des 20. Jahrhunderts lag die innerbetriebliche Verantwortung hierfür in einer 
Hand. Mit Einführung der industriellen Arbeitsteilung, wie sie vor allem mit dem Namen des Amerikaners Frederick W. Taylor und dem von ihm begründeten scientific management ("Taylorismus") verbunden ist, wurde diese Verantwortung aufgespalten.

"Überspitzt ausgedrückt wurde die Arbeitsvorbereitung für die Kosten, die Fertigung für die Zeiten und die Qualitätskontrolle für die Qualität verantwortlich gemacht. Dadurch entwickelte sich in den Fertigungsabteilungen eine Art 'Schmugglermentalität'. Es kam nicht mehr darauf an, fehlerfreie Produkte zu fertigen, sondern 'durch die Kontrolle zu kommen'. Gleichzeitig führte dies zu einer Vielzahl von Qualitätsprüfern. In vielen Betrieben waren mehr als 10\% der Belegschaft in der Qualitätsprüfung beschäftigt" (Seghezzi, 1996, S. 5). Entsprach ein fertiges Produkt nicht den Anforderungen, wurde es aussortiert oder nachbearbeitet.

Spätestens seit den Sechzigerjahren steht nicht mehr diese Endkontrolle des Produkts im Mittelpunkt der Qualitätskontrolle. Solche Prüfungen erhöhen nämlich nicht die Qualität, sondern dienen nur zur Trennung von "gut" und "schlecht" (Daumenlang \& Palm, 1997, S. 356). Da Fehler sich umso kostspieliger auswirken, je später sie im Produktionsprozess auftreten oder auffallen, sind Endkontrollen letztlich teurer als Fehler vermeidende Maßnahmen (Wolters et al., 1995, S. 16). Aufgrund dieser Erkenntnis geriet zunehmend die vorbeugende Gestaltung und ständige Verbesserung der betrieblichen Prozesse ins Blickfeld, um möglichst gar keine Qualitätsfehler entstehen zu lassen und Qualität optimal zu bewirtschaften. Dabei veränderten sich auch die Rollen der MitarbeiterInnen, des Managements und der KundInnen, deren Einbeziehung in die Leistungserstellung zunehmend umfassender geriet.

Ein solches "Qualitätsmanagement" - lange Zeit unter Bezeichnungen wie Qualitätskontrolle oder Qualitätssicherung firmierend - entstand in der Praxis auf pragmatische Art (Seghezzi, 1994, S. 11); seine Umsetzung in theoretische Konzepte und die Gestaltung als eigene Disziplin im Rahmen der Unternehmensführung entwickelten sich erst viel später (vgl. Kap. B 2.). In Europa wurde Qualität zwar traditionell groß geschrieben, jedoch nicht im eigentlichen Sinne systematisch bewirtschaftet wie die übrigen Faktoren des unternehmerischen Spannungsvierecks im Rahmen von Logistik, Materialwirtschaft, Finanz- und Rechnungswesen (a.a.O., S. 12). Es verwundert daher nicht, dass diese Entwicklung sich in Ländern vollzog, die keine lange Tradition mit hoher Produktqualität haben, nämlich in Japan und in den USA (S. 2). 
Eine Rolle für die seither nicht mehr nachlassende Aktualität des Qualitätsthemas in der Wirtschaft spielt die empirisch gesicherte Erkenntnis, dass zwischen der Qualität von Produkten bzw. Dienstleistungen und dem wirtschaftlichen Erfolg von Unternehmen ein positiver Zusammenhang besteht; Qualität ist ein entscheidender Erfolgsfaktor (Eversheim, 1997, S. 4 ff.). Dementsprechend wird der Frage zunehmende Bedeutung beigemessen: Wie kann eine Organisation/ein Projekt/ein Prozess/ein Produkt oder - um die Terminologie der internationalen Normungsbehörde ISO aufzugreifen - eine Einheit so gesteuert werden, dass Qualität systematisch optimiert wird und keine "Glückssache" ist (Gerull, 2000, S. 1-9). Qualitätsmanagement ist jener Teil der Gesamtführungsaufgabe, der dieser Frage gewidmet ist und Antworten in Form von Konzepten und Werkzeugen zu geben verspricht, die sich u. a. in den Bereichen Marketing, Organisation und Psychologie bewährt haben; Qualitätsmanagement "erfindet" das Qualitätsthema nicht neu, sondern "bringt es auf den Punkt" (Bretzke, 1995, S. 424).

Mit zunehmender Globalisierung der Märkte wurde es wichtig, einheitliche Anforderungen an Qualitätssysteme zu formulieren, die weltweit Akzeptanz finden (Seghezzi, 1996, S. 204). Diese Entwicklung wurde durch die hohen Qualitätsforderungen im militärischen Bereich und in der Luft- und Raumfahrt nachhaltig beeinflusst. Die aus den Forderungen der militärischen Beschaffungsstellen entstandenen Systeme bildeten den Ursprung der späteren DIN EN ISO 9000-Normenfamilie (s. Kap. C 1.4.1), die bereits in etwa 130 Ländern anerkannt wird (Zollondz, 2002, S. 46) und in über 80 Ländern institutionalisiert ist. Sie ist weltweit das angeblich in kürzester Zeit verbreitete Normenwerk überhaupt (Wolters et al., 1995, S. 3).

Mit der Normenreihe ISO 9000 wurden erstmals Unternehmensführungssysteme in Normen aufgenommen, während diese sich zuvor auf Maße, technische Eigenschaften und ähnliche Sachverhalte beschränkten (Seghezzi, 1996, S. 205). Inzwischen sind eine Vielzahl von branchenübergreifenden oder branchenspezifischen, mehr oder minder umfassenden, originären oder adaptierten Systemen und Verfahren auf dem Markt, die mit oder ohne Möglichkeit zur Zertifizierung, intern oder extern ausgerichtet, mit diagnostischer oder Prozess entwickelnder Fokussierung, in Selbstführung oder mit externer Begleitung implementiert werden können. Sie werden in Kap. C 1. systematisiert und teilweise ausführlicher erörtert. 


\subsection{Qualität}

Qualitätsmanagement setzt eine Vorstellung von Qualität voraus, auch wenn diese nicht immer explizit formuliert sein muss. Es ist deshalb zweckmäßig, zunächst solche Vorstellungen zu untersuchen.

Qualität kann allgemein definiert werden als die Beschaffenheit eines Produkts, einer Dienstleistung, eines Unternehmens oder einer anderen Einheit (s. o.), gemessen an den Bedürfnissen der Anspruchsgruppen (Seghezzi, 1996, S. 17), das sind alle an dem Produkt interessierten bzw. davon betroffenen Personen (auch stakeholder genannt), vor allem die KundInnen.

Qualität lässt sich aber nicht nur neutral als Beschaffenheit einer Einheit im Hinblick auf gestellte Anforderungen definieren, sondern wird häufig auch wertend als Güte im Sinne von Zweckerfüllung verstanden. Einem solchen Verständnis von Qualität folgend, kann man unterschiedliche Anspruchsklassen bilden, wie etwa die Sterne-Kategorisierung im Hotelbereich. Dabei wird festgelegt, welche Eigenschaften jeweils zu erfüllen sind, um einer bestimmten Qualitätsklasse oder -stufe zugeordnet werden zu können. Beschaffenheit und Anspruchsklasse sind Kernbegriffe modernen Qualitätsmanagements (Zollondz, 2002, S. 145).

\section{Definitionen von Qualität - eine Auswahl}

- Qualität ist die Beschaffenheit (eines Produkts), gemessen an den Bedürfnissen der Anspruchsgruppen (Seghezzi, 1996).

- Qualität ist die Relation zwischen einem Ist-Zustand und einer Soll-Forderung (Daumenlang \& Palm, 1997).

- Qualität ist die Gesamtheit von Eigenschaften und Merkmalen eines Produkts oder einer Tätigkeit, die sich auf deren Eignung zur Erfüllung gegebener Erfordernisse beziehen (DIN 55 350).

- Qualität ist der Übereinstimmungsgrad zwischen versprochener und erbrachter Leistung (Gerull, 2001). 
- Qualität: Beschaffenheit, Güte oder Wert einer Sache oder Dienstleistung (Kommerell, 2000).

- Qualität ist realisierte Beschaffenheit einer Einheit bezüglich Qualitätsforderung an diese (Geiger, 1998).

- Qualität: Grad, in dem ein Satz inhärenter Merkmale (An)forderungen erfüllt (ISO 9000:2000).

Von allen Versuchen, den Qualitätsbegriff so allgemein wie möglich zu bestimmen, ist die konzept- und branchenneutrale Definition der ISO 9000:2000 die einzige international normierte; sie entspricht inhaltlich voll derjenigen von Geiger (siehe Kasten), ist sprachlich allerdings so abstrakt formuliert, dass die ISO es für nötig hielt, der Definition zwei Anmerkungen hinzuzufügen:

"Qualität: Grad, in dem ein Satz inhärenter Merkmale (An)forderungen erfüllt" (ISO 9000:2000)

Anmerkung 1: Die Benennung "Qualität" kann zusammen mit Adjektiven wie schlecht, gut oder ausgezeichnet verwendet werden.

Anmerkung 2: "Inhärent" bedeutet im Gegensatz zu "zugeordnet" "einer Einheit innewohnend", insbesondere als ständiges Merkmal.

Mit Einheit ist dabei der Bezugspunkt für die Qualitätsbetrachtung gemeint, also das, was einzeln beschrieben und betrachtet werden kann: das Ergebnis einer Tätigkeit, diese Tätigkeit selbst, eine Person, ein System oder eine Kombination daraus (Geiger, 1998, zit. nach Zollondz, 2002, S. 155). Von Bedeutung ist diese Definition nicht allein deshalb, weil sie international abgestimmt ist und damit als Grundlage verschiedenster Qualitätsmanagement-Konzepte dienen kann, sondern auch, weil sie die Brücke von einer nur produktbezogenen Qualitätsbetrachtung zum umfassenden Qualitätsverständnis des Total Quality Management (s. Kap. C 1.4.2) schlägt. Da Einheiten beliebig gewählt und kombiniert werden können, sind alle denkbaren zusätzlichen Einheiten möglich (Zollondz, 2002, S. 158). Während die "inhärenten" Merkmale der Einheit für die Qualität des Angebotsprodukts (z. B. Pflege bei Dekubitus) direkt maßgeblich sind, also unmittelbaren Qualitätsbe- 
zug aufweisen (z. B. die fachlich einwandfreie einzelne Pflegehandlung), lassen sich einem Vorschlag von Geiger entsprechend "zugeordnete" Einheiten mit mittelbarem Qualitätsbezug (z. B. die Freundlichkeit der Krankenschwester) sowie ohne direkten Qualitätsbezug zum Angebotsprodukt (z. B. das Image des Krankenhauses) unterscheiden (Zollondz, 2002, S. 158; Beispiele P. G.).

Der erweiterte Qualitätsbegriff im TQM wäre somit wie folgt zu definieren:

"Im TQM ist Qualität die realisierte Beschaffenheit von Einheiten mit unmittelbarem, mittelbarem und keinem direkten Qualitätsbezug bezüglich Qualitätsforderung und anderer Forderungen an diese Einheit" (Zollondz, a.a.O.).

\subsection{Qualitätsmodelle}

Modelle sind "analoge Realitätsausschnitte" (Schlottke, 1998, S. 543f) zum Zwecke der Veranschaulichung oder Ableitung damit zusammenhängender Fragestellungen (Drever \& Fröhlich, 1968, S. 152). Diesem Zweck dienen auch Qualitätsmodelle. Sie setzen zumeist einen Begriff von Qualität voraus, ohne ihn immer zu explizieren. Zollondz (2002, S. 163 ff.; 2001, S. 589 ff.) beschreibt eine Auswahl allgemeiner und spezieller Qualitätsmodelle, die nachstehend in Grundzügen aufgeführt, kurz kommentiert und um Bemerkungen zu weiteren Modellen ergänzt werden:

- Qualitätskreis-Modell von Masing (1990, nach Zollondz, 2002, S. 164 ff.):

Dieses Modell war Gegenstand der inzwischen nicht mehr gültigen Empfehlungsnorm ISO 9004:1994. Ausgehend von den Kundenforderungen, sind den Phasen der Planung, Realisierung und Nutzung von Produkten verschiedene Unternehmensfunktionen wie Marktforschung, Beschaffung, Fertigung, Prüfung usw. zugeordnet. In jeder Phase müssen die Verantwortlichen qualitätssichernde Maßnahmen treffen. Als logische Folge der Qualitätsarbeit aller Beteiligten ergibt sich das Qualitätsprodukt. Das Modell lässt sich ohne weiteres auf (standardisierbare) Dienstleistungen übertragen, stößt aber bei interaktiven Leistungsprozessen rasch an Grenzen. 
- Qualitätsregelkreis-Modell von Pfeifer (1996, nach Zollondz, 2002, S. 167 ff.):

Es handelt sich um eine auf Qualität bezogene Anwendung des kybernetischen Modells mit den Elementen Regelgröße (hier: Qualität), Störgröße (z. B. ungeplante Einwirkungen), Stellgröße (z. B. durchgeführte Maßnahme), Sollgröße (hier: Qualitätsforderung), Regelstrecke (z. B. Prozess) und Regler (z. B. Qualitätstechnik). Das Modell ist stark von ingenieurwissenschaftlichem Denken geprägt und stößt seines technischen Charakters wegen im Sozialbereich auf Vorbehalte.

- Qualitäts-Termin-Kosten-Kreis von Geiger (1998, nach Zollondz, 2002, S. 170 ff.):

Das Modell geht über die Qualitätsbezogenheit des Qualitätskreises (s. o.) hinaus und beschreibt das Zusammenwirken der Aspekte Zeit, Qualität und Kosten. Dem Modell wird ein universeller Charakter im Managementdenken zugeschrieben, weil es die Parallelität der jeweiligen Tätigkeiten betont. Daraus lässt sich ableiten, dass ein Qualitätsmanagement nicht - wie z. B. eine Vertriebsabteilung - einer eigenen Aufbauorganisation bedarf, sondern eine integrale Funktion darstellt. Die Grundgedanken des Modells finden sich bereits in dem erwähnten unternehmerischen Spannungsviereck wieder und spielen vor allem im Konzept des Integrierten Qualitätsmanagements (Seghezzi, 1994, 1996, 2003) eine zentrale Rolle (s. Kap. C 1.4.5). Im sozialen Dienst-leistungsbereich ist es erst vereinzelt aufgegriffen worden, obwohl es grundsätzlich geeignet erscheint.

- Wertschöpfungsanalyse von Weth (nach Zollondz, 2002, S. 173 ff.):

Wertschöpfung entsteht aus der Differenz zwischen dem Wert eines Produkts vor und nach der Verarbeitung. Dabei spielt Verschwendung eine entscheidende Rolle. Die Wertschöpfungsanalyse prüft, welchen Beitrag die jeweiligen Prozesselemente zur Wertschöpfung beitragen. Das Modell wurde von Weth auf das Qualitätsmanagement übertragen. Die Relevanz für soziale Dienstleistungen ist fraglich. 
- Partialanalytisches Qualitätsmodell von Garvin (1984, nach Zollondz, 2001, S. 589f):

Das für die Diskussion der Dienstleistungsqualität sehr einflussreich gewordene Modell differenziert nach folgenden Qualitätsansätzen: absolute (transcendent) Qualität, Qualität des Produkts (product-based), Qualität für den Kunden (user-based), Qualität der Herstellung (manufacturing-based) und Qualität als Wert (value-based). Im Sozialbereich werden analoge Bezugsgrößen u. a. als sozialtechnologischer, expertokratischer und adressatenorientierter Qualitätsansatz diskutiert (Piel, 1996; Gerull, 2000). Keiner wird allein der Komplexität sozialer Dienstleistungen gerecht.

- Dienstleistungsqualitätsmodell von Donabedian (1966, nach Zollondz, 2002, S. 147 ff.):

Das ursprünglich auf medizinische Pflegeleistungen bezogene Qualitätsmodell unterscheidet drei Dimensionen: Strukturqualität (structure), Prozessqualität (process) und Ergebnisqualität (outcome). Structure umfasst vor allem die zur Dienstleistungserstellung notwendigen fachlichen, personellen und sächlichen Ressourcen; process bezeichnet die Gesamtheit der Aktivitäten im Verlauf der Dienstleistungserbringung; outcome steht für eine Änderung des Patientenzustandes, sofern diese sich auf die erbrachte Leistung zurückführen lässt (Meyer \& Westerbarkey, 1995, S. 86). Das Modell wurde, ausgehend vom Pflegebereich, in weiten Teilen der deutschen Sozialarbeit übernommen, obwohl es die Rolle der AdressatInnen nicht explizit berücksichtigt (zur Kritik s. Gerull, 2004, Kap. 1.4.6).

Gelegentlich werden einzelne Kategorien anders benannt - z. B. Produkt- statt Ergebnisqualität (Trube, Regus \& Depner, 2001), Potenzial- statt Strukturqualität (Eversheim, 1997) -, kontextspezifisch ersetzt z. B. Orientierungs- statt Ergebnisqualität (Tietze, Schuster \& Rossbach, 1997) - oder um eine vierte und fünfte ergänzt - Konzeptqualität (von Spiegel, 1994), Normqualität (Vilain, 2003), Procederequalität (Trube et al., 2001), Beziehungs- und Erlebnisqualität (Ackermann, 2003). Die Reihe ließe sich fortsetzen (technische, Rahmen-, Handlungs-, Wirkungsprozess-, Verfahrens-, Verrichtungsqualität u.a.m.).

- Dienstleistungsqualitätsmodell von Grönroos (1984, nach Zollondz, 2002, S. 174 ff.): 
Das kundenorientierte Qualitätsmodell geht von einem abwägenden Beurteilungsprozess beim Nachfrager (Kunde, Adressat usw.) aus, in welchem dieser seine Erwartungen an das Dienstleistungsergebnis (Soll-Wert) mit der tatsächlichen Leistung (IstWert) vergleicht (Meyer \& Westerbarkey, 1995, S. 86). Unterschieden wird eine eher objektiv zu bestimmende technische Qualität ("Was" erhält der Nachfrager?) und eine eher subjektiv wahrgenommene funktionale Qualität ("Wie" wird dem Nachfrager die technische Qualität dargeboten?); für das Gesamtqualitätsurteil bzw. die Zufriedenheit des Kunden wird die funktionale Qualität für bedeutsamer gehalten. Der Ansatz dürfte im Sozialbereich als zu "konsumeristisch" auf Vorbehalte stoßen.

- Dienstleistungsqualitätsmodell von Meyer \& Mattmüller (1987, nach Zollondz, 2002, S. $176 f f$.$) :$

Das Modell erweitert das Qualitätsmodell von Donabedian und verbindet es mit dem zuvor beschriebenen kundenorientierten Ansatz von Grönroos. Unterschieden werden die Dimensionen der Potenzialqualität des Anbieters und des Nachfragers, der Prozess- und Ergebnisqualität. Bei der Potenzialqualität des Anbieters wird zwischen dem Spezifizierungspotenzial (individuelle und spezifizierte Problemlösungen) und dem Kontaktpotenzial differenziert; die Potenzialqualität des Nachfragers wird in Integrations- und Interaktivitätspotenziale unterteilt (Einbringungsbereitschaft und Auswirkungen von Kundenkontakten untereinander). Das Modell wird im Sozialbereich trotz seiner Vorteile gegenüber dem Donabedian-Modell kaum verwendet.

- Dienstleistungsqualitätsmodell von Parasuraman, Zeithaml \& Berry (1985, nach Zollondz, 2002, S. $178 f f$.$) :$

Dieses, in der deutschen Fachliteratur breit rezipierte, Modell der Servicequalität wurde im Rahmen empirischer Studien entwickelt und benennt fünf Gruppen von Merkmalen, welche die Qualität von Dienstleistungen bestimmen: Annehmlichkeiten des tangiblen Umfeldes, Verlässlichkeit der Leistungsausführung, Reagibilität bei Problemen, Leistungskompetenz, Einfühlungsvermögen. Messinstrument für diese Merkmale ist ein spezieller Fragebogen ("ServQual", Zeithaml, Parasuraman \& Berry, 1992). Im Sozi- 
albereich stößt eine direkte Übertragung des Modells an Grenzen, für die auch das "Hilfe-Kontrolle-Dilemma" verantwortlich ist (vgl. Meinhold \& Matul, 2003, S. 41f).

Auch Zollondz (2002, S. 183) resümiert, dass keines dieser Qualitätsmodelle völlig befriedigen könne, vor allem im Hinblick auf soziale Dienstleistungen. In der sozialen, pädagogischen und pflegerischen Praxis hat sich dessen ungeachtet das Modell von Donabedian als geläufigstes Gliederungssystem (z. B. für Leistungsbeschreibungen) durchgesetzt. Vereinzelt wird auch das Modell von Meyer \& Mattmüller verwendet (Lemme \& Ochs, 1998; Gerull, 2004) oder unter Einbeziehung zusätzlicher bzw. anders benannter Dimensionen (Heiner, 1996; von Spiegel, 1994; Trube et al., 2001) zu einem eklektischen Qualitätsmodell sozialer Dienstleistungen integriert (Gerull, 2004, Kap. 1.4.6.3).

Ein von Zollondz (2001, 2002) nicht berücksichtigtes Qualitätsmodell, das nach Nerdinger (1994, S. 206 ff.) einen völlig neuen Gedanken in die Diskussion einbringt, stammt von Klaus (1991), der Qualität als Epiphänomen der Interaktion zwischen Dienstleister und Bedientem im Sinne einer gemeinsam geteilten Erfahrung der Zielerfüllung versteht. Diese Qualität könne nicht direkt gestaltet und kontrolliert werden, wohl aber Elemente der "Konfiguration" (Klaus, a.a.O., S. 259).

Wie Klaus (S. 261f) empirisch bestätigen konnte, entsteht "gute" Bedienungsqualität aus dem kumulativen Zusammentreffen von drei Aspekten: Kongruenz der wechselseitig aufeinander bezogenen Verhaltensweisen der Interaktionspartner (z. B. Beachtung von Umgangsnormen), Grad an sachlicher Aufgabenerfüllung, der erzielt und von den Interaktionspartnern wahrgenommen wird (Erreichen des Sachzwecks der Interaktion) und Grad an emotionaler Zufriedenheit, der von den Interaktionspartnern empfunden wird (z. B. Gefühl der Einbeziehung).

Von den in diesem Kapitel kurz dargestellten Qualitätsmodellen scheinen jene von Klaus und Meyer-Mattmüller am besten geeignet, den Charakteristika sozialer Dienstleistungen gerecht zu werden. Anstatt Qualität einseitig aus der Zufriedenheit der "Bedienten" abzuleiten - unter dem Stichwort Konsumerismus ein auch in Psychotherapie und Sozialarbeit viel diskutiertes Thema (z. B. Piel, 1996; Fourali, 1999) -, oder sie "expertokratisch" definieren zu lassen, wird Qualität bei Klaus als Merkmal einer gemeinsam geteilten Erfahrung 
beider Interaktionspartner betrachtet, während Meyer \& Mattmüller den notwendigen Eigenbeitrag der "KundInnen" über die Potenzialqualität der Nachfrager thematisieren (ausführlicher diskutiert bei Gerull, 2004, Kap. 1.4.6).

Nerdinger (1994, S. 206) kritisiert allerdings am Ansatz von Klaus, dass eine so verstandene Qualität der empirischen Erfassung nicht zugänglich sei und schlägt ergänzend das Konzept des Dienstleistungsklimas vor, das die Organisation mit den Bedienten über die Wahrnehmungen und das Erleben der Organisation durch die Dienstleister verknüpft ( $S$. 219). In dieser Triade kommt der Organisation die Aufgabe zu, u. a. durch umweltpsychologische Gestaltung des Erlebens von Raum und Zeit ein unterstützendes Dienstleistungsklima zu fördern, in dem das dienstleistende Organisationsmitglied durch geeignete "instrumentelle" und "sozio-emotionale" Handlungen gemeinsam mit dem Bedienten eine Leistung erstellt, die als qualitätsvoll erlebt werden kann.

\subsection{Qualitätsmanagement (QM)}

Der Management-Begriff wird in zahllosen Wortverbindungen verwendet, um die planvolle Bewältigung spezieller Aufgaben oder Problemfelder zu kennzeichnen (Greif, 1998c, S. 515). Je nach Zielbereich entstehen so Umwelt-, Kosten-, Wissens-, Arbeitsschutz- und andere Managementsysteme, die es im hochaktuellen Ansatz des "Integrierten Managementsystems" (vgl. Initiative Qualitätssicherung NRW, o. J.) wieder zusammenzuführen gilt.

Während Management auf zielgerichtete, koordinierte, kontrollierte und effiziente Tätigkeiten fokussiert, werden spontane, ungeplante oder chaotische Strukturierungen organisationaler Prozesse eher unter Selbstorganisation subsumiert (vgl. Greif, a.a.O.). Das mit dieser Unterscheidung implizierte Organisationsverständnis wird im Rahmen einer kritischen Auseinandersetzung mit dem Qualitätsdiskurs noch zu thematisieren sein (s. Kap. B 3.8).

Qualitätsmanagement heißt, funktionsbereichsübergreifend Qualität zu planen, zu steuern und zu überwachen. Die ISO 9000:2000 definiert Qualitätsmanagement sinngemäß als 
"aufeinander abgestimmte Tätigkeiten zum Leiten und Lenken einer Organisation bezüglich Qualität."

\section{Definitionen von Qualitätsmanagement (QM) - eine Auswahl}

- QM ist Teil der Gesamtführungsaufgabe eines Unternehmens zur Entwicklung, Aufrechterhaltung und Gewährleistung von Qualität (versch.).

- QM ist die Gesamtheit der Aktivitäten zur Erreichung und Förderung der Qualität von Leistungen (Hollerith, 1994).

- $\mathrm{QM}$ ist die Summe aller qualitätsbezogenen Zielsetzungen, Vorkehrungen und Maßnahmen in einer Organisation (versch.).

- QM wird verstanden als das Herstellen günstiger Struktur-, Rahmen und Prozessbedingungen, unter denen sich Systeme selbst steuern, organisieren und entwickeln können (Schiepek \& Bauer, 1998).

- QM ist Kontextsteuerung zur Optimierung der Leistungspotenziale, -prozesse und -ergebnisse einer Organisation (Gerull, 2001).

- QM: aufeinander abgestimmte Tätigkeiten zum Leiten und Lenken einer Organisation bezüglich Qualität (ISO 9000:2000).

Basis des QM ist das Prozessmanagement. Damit ist ein Führungskonzept gemeint, in dem Hierarchie und Bereiche nicht mehr streng gegen- und untereinander abgegrenzt sind, sondern "bereichs- und funktionsübergreifend ganzheitlich synergetisch zusammenwirkend einen Kundennutzen erzeugen" (Zollondz, 2002, S. 200).

Ein Prozess ist nach ISO 9000:2000 ein Satz von in Wechselbeziehung oder Wechselwirkung stehenden Tätigkeiten, der Eingaben in Ergebnisse umwandelt (a.a.O., S. 205, siehe auch Kap. C 2.2.6). Ziel der Prozessorientierung ist es, die integrationshemmende Funktionsoptimierung durch die integrationsförderliche Flussoptimierung abzulösen. Nur so könne eine Schnittstellenreduzierung erfolgen und der künstlichen Zersplitterung des 
Unternehmens entgegengewirkt werden (a.a.O., S. 208).

Trotz seines integralen, querschnitthaften Charakters wird das operative Qualitätsmanagement in vielen betriebswirtschaftlich orientierten Darstellungen in Teilfunktionen gegliedert:

- Qualitätsplanung,

- Qualitätslenkung (-regelung, -steuerung),

- Qualitätssicherung (-prüfung, -kontrolle) und

- Qualitätsförderung (-verbesserung), bisweilen ergänzt um die

- Qualitätsdarlegung oder Qualitätsdokumentation

(vgl. Arnold, 1998; Seghezzi, 1994, 1996). Dabei wird jedoch betont, dass es sich um miteinander zusammenhängende, in einem kontinuierlichen Rückkopplungsprozess wiederholt zu durchlaufende Stadien handele ("Qualitätskreis", s. o.).

Die Qualitätsplanung bezweckt, die Produkte und Herstellungsprozesse bedarfsgerecht und mit geringem Fehlerrisiko zu gestalten; dazu wird häufig zunächst der Soll-Zustand eines Produkts/einer Dienstleistung ermittelt und als Bündel von Qualitätszielen formuliert, für deren Erreichung geeignete Umsetzungsstrategien entwickelt werden.

Mit Qualitätslenkung wird versucht, die Leistungen so zu erbringen, dass sie mit den Anforderungen (Spezifikationen, im Dienstleistungsbereich zumeist Standards genannt) konform sind; wirksame Mittel sind neben Vorbeugung, Überwachung und Korrektur des Ist-Zustandes z. B. Maßnahmen zur Qualifizierung der MitarbeiterInnen, zur Integration des QM in die betriebliche Organisationsstruktur und zum Aufbau geeigneter Informations- und Kommunikationsformen.

Trotzdem gibt es im Leistungsprozess Stellen erhöhter Fehleranfälligkeit, zu deren Begrenzung Qualitätssicherung im engeren Sinne eines aktiven Risikomanagements beitragen soll; dazu sind in der Regel Überwachungsmaßnahmen zur Ermittlung von Soll/Ist-Abweichungen erforderlich, aus denen wiederum Maßnahmen im Rahmen der Qualitätsplanung oder -lenkung abzuleiten sind. 
Qualitätssicherung wurde im deutschen Sprachraum lange Zeit mit Qualitätsmanagement gleichgesetzt, ist aber nach aktueller Auffassung nur ein instrumenteller Teil desselben.

\section{Definitionen von Qualitätssicherung - eine Auswahl}

- Qualitätssicherung: systematische Maßnahmen und Vorkehrungen zur Beherrschung arbeitsteilig organisierter Herstellungsprozesse für ein Produkt (N. N.)

- Qualitätssicherung: Summe aller organisierten verbindlichen Vorkehrungen, Dienstleistungen so zu erbringen, wie sie als fachlich richtig erkannt und anderen versprochen wurden (Tornow, 1999).

- Qualitätssicherung: Alle geplanten und systematischen Tätigkeiten, die innerhalb des QM-Systems verwirklicht sind, und die wie erforderlich dargelegt werden, um angemessenes Vertrauen zu schaffen, dass eine Einheit die Qualitätsforderung erfüllen wird (ISO 9000:1994).

- Qualitätssicherung und methodisches Arbeiten sind weitgehend identische Prozeduren (von Spiegel, 1997).

- Interne Qualitätssicherung: Anweisungen zur Herstellung der innerbetrieblichen Voraussetzungen für die Förderung und Sicherung von Qualität (Schild, 1996).

- Externe Qualitätssicherung: Beurteilung und Kontrolle der Produkt- oder Dienstleistungsqualität und ihrer Entstehungsvoraussetzungen durch den Abnehmer oder durch befugte Stellen (Schild, 1996).

Qualitätsförderung hat die Aufgabe, eine Unternehmenskultur der kontinuierlichen Verbesserung zu schaffen und zu pflegen.

Bei der Qualitätsdarlegung schließlich geht es um die Abbildung des betrieblichen QM für Zwecke der Innen- und Außendarstellung, zumeist in Form eines Qualitäts(management)Handbuchs (siehe Kap. C 2.2.2), spezieller Qualitätsaufzeichnungen und statistischer Kennzahlen.

Im Bereich sozialer Dienstleistungen werden die Teilfunktionen eines QM zumeist nicht so 
differenziert und in anderen Begriffen verwendet. Allein aufgrund der eingeschränkten Möglichkeit, Leistungsprozesse zu standardisieren und nach präziser Planung und Lenkung normkonform umzusetzen, wird z. B. im Kinder- und Jugendhilfegesetz (SGB VIII) der Terminus "Qualitätssicherung" wegen seiner technischen Assoziationen vermieden. Vielmehr ist hier wie auch anderenorts die Rede von Qualitätsentwicklung und - im Zusammenhang mit Fragen der Fremd- oder Selbstüberprüfung - von Qualitätsbewertung.

\subsection{QM-Modelle}

Wie schon bezüglich des Begriffs der Qualität und der darin zum Ausdruck kommenden Vorstellungen, so existieren auch hinsichtlich des Managements von Qualität mehr oder minder systematische Versuche, dessen zweckmäßige Elemente zu konzeptualisieren. Trotz der Verschiedenheit dieser Ansätze konstatiert Zollondz (2002), dass alle QM-modelle eine Reihe von Voraussetzungen als gemeinsamen Nenner aufweisen.

Dieses "Conditio-Sine-Qua-Non-Modell" (a.a.O., S. 189f) enthält folgende sechs Elemente, denen bestimmte Anforderungen zugeordnet werden können:

\section{Prozesse}

Kern-, Führungs- und Supportprozesse sind zu identifizieren und ihre Qualitätsfähigkeit ist zu bestimmen.

\section{Management}

Es muss u. a. die Qualitätspolitik und die daraus abgeleiteten Qualitätsziele formulieren. Das Management ist in das QM einzubinden und hat es uneingeschränkt vorzuleben.

\section{Ressourcen}

Materielle und immaterielle Ressourcen sind vom Management bereitzustellen. 


\section{Mitarbeiter}

MitarbeiterInnen sind zu qualifizieren, Qualitätsbewusstsein und mitunternehmerisches Denken sind zu fördern.

\section{Kunden}

Aus deren Erwartungen sind Qualitätsforderungen abzuleiten.

6. Verbesserungen

Kontinuierlicher Verbesserungsprozess ist integraler Bestandteil des QM-Systems; Ziel ist es, Verschwendung zu beseitigen.

Jedes QM hat sich mit diesen sechs Elementen als zentralen Erfolgsfaktoren auseinanderzusetzen. Diese Auffassung gilt sowohl für die branchenunabhängigen Modelle als auch für branchenspezifische Adaptationen, Verkürzungen und Eigenentwicklungen.

Zollondz (2002, S. 222 ff.) differenziert QM-Modelle in allgemeine Kernmodelle, Branchenmodelle und Kontextkonzepte. Die prominentesten Konzepte werden in Kap. C 1.4 und 2. näher beschrieben und beurteilt, allerdings unter einer anderen Systematik (Kap. C 1.3). An dieser Stelle soll ein erster Überblick nach der Zuordnung von Zollondz (a.a.O.) erfolgen, ausgenommen die Kategorie der Kontextkonzepte (s. Kap. B 1.7).

\section{Allgemeine Kernmodelle des QM}

- QM vom Elektrizitätstypus (Seghezzi, 1996, S. 200):

Es handelt sich nicht um ein explizit formuliertes QM-Modell, sondern eher um einen charakteristischen Handlungstypus von Organisationen, mit der Qualitätsfrage umzugehen: "Steht Elektrizität (= Qualität, P. G.) zur Verfügung, fällt dies niemandem auf. Fällt sie dagegen aus, so geht die Beleuchtung aus, die Heizung oder Lüftung steht still ... Gute Elektrizitätswerke haben ein System, um solche Pannen in kürzester Zeit zu beheben" (a.a.O.). 
- QM vom Kulturtypus (Seghezzi, 1996, S. 200 f):

Dieses Konzept lässt sich durch vier Merkmale charakterisieren, die den eigenen Anspruch der Organisation verkörpern: gute fachliche Qualifikation der Führungskräfte und Mitarbeiter, qualitätsorientierte Unternehmenskultur, Unterstützung durch die oberste Leitung und gute technische Ausstattung. Obwohl oftmals ohne transparente Qualitätsplanung oder explizites QM-System, werden in solchen Organisationen Leistungen von hoher Qualität erbracht.

- Kontinuierliches Verbesserungsmanagement (KVM, Zollondz, S. 225 ff.):

Der Ansatz wird sowohl als eigenständiges Managementkonzept (z. B. Kaizen von Imai, s. Kap. C 1.4.3) oder als in QM eingebettetes Instrument praktiziert. Die internationale Normung zum QM hat weder den - umfassenderen - Begriff des Kaizen, noch die bekannte Bezeichnung "kontinuierlicher Verbesserungsprozess (KVP)" aufgenommen, sondern sich für "Qualitätsverbesserung" entschieden. Diese ist nach ISO 9000:2000 jener "Teil des Qualitätsmanagements, der auf die Erhöhung der Fähigkeit zur Erfüllung der Qualitäts(an)forderungen gerichtet ist" (zit. nach Zollondz, S. 230).

- QM-Systeme (hier: ISO 9000:2000-Normenreihe, Zollondz, S. 242 ff.):

Alle Aufgaben, Strukturen, Organisationselemente und Maßnahmen, die zur Bewirtschaftung von Qualität eingesetzt werden, bilden ein Qualitäts(management)system. Dieses ist also ein qualitätsbezogenes, die Erfüllung von Qualitätsanforderungen betreffendes Managementsystem (S. 251). Entgegen einem häufigen Missverständnis gibt es keine genormten QM-Systeme, sondern nur Normen zum QM, auf deren Grundlage dann ein je spezifisches QM-System der Organisation aufzubauen ist (S. 245). ISO 9001:2000 ist ein solches QMSystem zur externen Darlegung (auch als Darlegungs-, Nachweis- oder Forderungsnorm bezeichnet).

ISO 9000:2000 ist die Begriffsnorm, d. h. darin sind die Grundlagen und Begriffe definiert. ISO 9004:2000 (auch als Leitfaden, Empfehlungs- oder Leistungsnorm bezeichnet) schließt die Darlegungsnorm 9001 ein, 
bezieht sich aber nicht nur auf die Produktqualität, sondern auf die relevanten Prozesse der Organisation insgesamt. Während die ISO 9001:2000 auf den Kunden fokussiert, hat die ISO 9004:2000 alle Stakeholder im Blick und ist somit ein Modell für Verbesserungen in Richtung TQM (s. Kap. C 1.4.2).

Neben den potenziellen Vorteilen für die Kunden, deren Kaufrisiko durch ein nach ISO 9001:2000 zertifiziertes QM-System gemindert werden soll, ergeben sich aus der Anwendung von QM-Systemen auch potenzielle Vorteile für den Lieferanten: Schaffung von Vertrauen zwischen ihm und den Kunden, Verbesserung der betrieblichen Abläufe und ihrer Dokumentation, Schaffung von Vertrauen der Organisation in die eigenen Geschäftsprozesse, Entlastungsmöglichkeit im Produkthaftungsfall.

- TQM-Modelle (Zollondz, S. 261 ff.):

Ein QM-Modell, das im Begriff ist, sich zu einer übergeordneten Unternehmensstrategie zu entwickeln, stellt das Total Quality Management (TQM) dar. Es wird in verschiedenen Ausgestaltungen praktiziert. Als besonders wichtige Elemente von TQM gelten (S. 265): Kundenorientierung, partnerschaftliche Kunden-Lieferanten-Beziehungen, Integration und Partizipation der Mitarbeiter aller Hierarchieebenen, Gruppenarbeit und Mitwirkung unterstützende Arbeitsbedingungen, Qualitätsorientierung, Qualifizierung der Mitarbeiter, Anerkennung guter Leistungen, Berücksichtigung von Humanität und sozialen Komponenten, ständiger Verbesserungsprozess, Wertschöpfungskonzentration und Abbau nicht kundenrelevanter Tätigkeiten, Anwendung moderner Methoden und Techniken des QM, Betonung Fehler vermeidender Maßnahmen, top-down-Ansatz mit missionarischer Einbringung der obersten Leitung, partizipatives und zugleich straffes Management.

Die bekanntesten TQM-Modelle sind: Deming Application Prize, Malcolm Baldrige National Quality Award, EFQM-Modell als Basis des European Quality Award (s. Kap. C 1.4.4), Ludwig-Erhard-Preis.

- Integratives/integriertes QM (Zollondz, S. 296 ff.):

Es handelt sich um die Umsetzung des anspruchsvollen Modells von K. Bleicher (St. Galler Management Konzept) durch Zink (Integratives QM) und Seghezzi (Integriertes QM, s. 
Kap. C 1.4.5). Beiden Modellen wird nur begrenzte Fachresonanz attestiert, verknüpft mit der Hoffnung, dass sich dies zukünftig ändern möge (a.a.O., S. 305 f.).

- Integrierte Managementsysteme (IMS, Zollondz, S. 306 ff.):

IMS berücksichtigen, dass Organisationen sich neben qualitätsbezogenen Forderungen auch noch mit anderen Anforderungen zu befassen haben: z. B. Umwelt, Arbeitsschutz, Anlagensicherheit, Notfall, Hygiene. Idee ist, die Integration der verschiedenen Managementsysteme voranzutreiben, statt sie isoliert aufzubauen.

Branchenbezogene QM-Regelwerke (Zollondz, S. $311 \mathrm{ff.}$.)

Sie setzen entweder die Forderungen der ISO 9001:2000 voraus und erweitern sie um branchenspezifische Aspekte (z. B. in der Automobilbranche: Norm QS-9000) oder sie haben eigenständigen Charakter mit und ohne Bezug auf die ISO 9000-Familie oder andere Referenzsysteme (z. B. KTQ: Kooperation für Transparenz und Qualität im Krankenhaus).

\subsection{Qualitätstechniken und -instrumente}

Qualitätstechniken (auch als Werkzeuge oder tools bezeichnet) verkörpern die genuin praktisch-methodische Seite des QM auf der operativen Ebene. Qualität entsteht aus dem Einsatz von "Technik" und "Geisteshaltung" (Kamiske, zit. nach Zollondz, 2002, S. 318).

K. Frey (1997, S. 14) bezeichnet den Ansatz des Total Quality Management (TQM, s. Kap. C 1.4.2) seiner fehlenden Theorie oder Systematik wegen als "Ansammlung von über 200 Werkzeugen".

Viele dieser Werkzeuge gehören zum traditionellen Rüstzeug von z. B. FortbildnerInnen, UnternehmensberaterInnen, GruppentherapeutInnen und LehrerInnen und wurden lediglich für Zwecke des QM instrumentalisiert. Sie kennzeichnen einerseits bestimmte Organisationsformen und soziale Settings für Lernprozesse (z. B. Open Space), andererseits vor allem 
Formen der Strukturierung (z. B. Mind Mapping), Visualisierung (z. B. Histogramm), Medienunterstützung (z. B. Präsentationstechnik), Problembearbeitung (z. B. UrsacheWirkungs-Diagramm), Ideengenerierung (z. B. Brainstorming), Datenverdichtung, -auswertung und -dokumentation (vgl. Gerull, 2000, S. 3-2 ff.).

$\mathrm{Zu}$ den Qualitätstechniken im engeren Sinne zählt Zollondz (2001, S. 1006) neben Verfahren wie Quality Function Deployment (QFD), Fehlermöglichkeits- und Einflussanalyse (FMEA) und Statistische Prozessregelung (SPR) die namentlich von den Japanern Imai und Ishikawa zusammengestellten "sieben Qualitätswerkzeuge" (Q7: seven tools of quality) und "sieben Managementwerkzeuge" (M7: new seven tools for quality control) sowie die von Shainin entwickelten "seven tools" der statistischen Versuchsplanung (DoE: design of experiments).

Als Qualitätstechniken im weiteren Sinne bezeichnet Zollondz (a.a.O.) die als D7 bekannt gewordenen tools für Dienstleister (in Klammern: primärer Einsatzzweck):

- Vignettentechnik (Design/Entwicklung/Prävention/Ermittlung der Qualitätsmerkmale)

- Service-Blueprinting (Prozessdarstellung)

- Sequentielle Ereignis-Methode (Ermittlung der Qualitätsmerkmale)

- ServQual (Qualitätsmessung)

- Beschwerdemanagement (Beschwerden)

- FRAP: Frequenz Relevanz Analyse von Problemen (Analyse)

- Service-FMEA (Prävention und Verbesserung),

sowie die unter $K 7$ firmierenden Kreativitätstechniken (u. a. Brainstorming, DelphiMethode, morphologischer Kasten, Synektik) und bestimmte Elemente des Produktionssystems und der Arbeitsorganisation: Kaizen, Poka-Yoke, Gruppenarbeit, Qualitätszirkel, Audits und Just-in-time (JIT).

Die auffallend häufige Beschränkung auf sieben hat im Übrigen keine eindeutig belegbare Ursache, sondern ist wohl eher zahlenmythologischer Natur (Zollondz, 2001, S. 779).

Auf eine Beschreibung der genannten und weiterer Techniken muss mit Rücksicht auf den Umfang dieser Arbeit verzichtet werden. Nähere Ausführungen finden sich u. a. bei Theden \& Colsmann (1996), Seghezzi (2003), Zollondz (2001, 2002) und Gerull (2000, 2004). 
Von den Qualitätstechniken im bislang beschriebenen Sinne abzugrenzen sind jene Werkzeuge, die nicht auf einzelne Fragestellungen zugeschnitten sind, sondern umfangreichere betriebliche Funktionen erfüllen und mehr oder weniger tragende Praktiken des QM darstellen. Sie sollen im Folgenden Qualitäts-Instrumente genannt werden, lassen sich teilweise aber auch problemlos den weiter unten erörterten QMKontextkonzepten zuordnen (z. B. Benchmarking):

- Qualitätsleitbild,

- QM-Handbuch,

- Qualitätszirkel,

- Qualitätsbeauftragte,

- Kunden- und Mitarbeiterbefragungen,

- Prozessanalyse,

- Benchmarking,

- Ideen-, Wissens- und Beschwerdemanagement.

Die meisten dieser Instrumente sind Gegenstand ausführlicher Betrachtung in Kap. C 2.; auf die wenig trennscharfe, aus pragmatischen Gründen dennoch vorgenommene, Unterscheidung von Techniken und Instrumenten wird in Kap. C 2.1 noch einmal eingegangen.

\subsection{Qualitätsmanagement-Kontextkonzepte}

Wie Hoerner \& Vitinius (1997, S. 14) in einem kritischen Führer durch die Managementtheorien sarkastisch betonen, steht der Nachfrage verunsicherter Manager nach Erfolgsrezepturen ein breitgefächertes Angebot der UnternehmensberaterInnen an Methoden, Konzepten und Strategien gegenüber. Viele davon lassen sich nicht ohne weiteres dem Qualitäts-Label zu-, unter- oder überordnen und bisweilen ist es eine willkürliche Entscheidung, ein Konzept als eigenständigen methodischen Ansatz oder als Element des QM zu betrachten. Um zu verdeutlichen, dass es sich um Verfahren oder Modellvorstellungen handelt, die im Zusammenhang mit der Qualitätsdebatte (wieder) Aktualität erlangt haben, zum Teil aber in einer anderen Wissenschaftstradition stehen und als selbständige Ansätze (ko)existieren können, wird in Anlehnung an Zollondz (2002, S. 316 ff.) im Folgenden der Begriff des QM-Kontextkonzepts verwendet.

Dazu können vor allem gezählt werden: 
Lean Management,

Business Reengineering,

Evaluation,

Controlling,

Balanced Scorecard,

Benchmarking,

Organisationsentwicklung (OE),

Change Management,

Lernende Organisation,

○ Unternehmensphilosophie, Corporate Identity u. Ä.,

- Personalentwicklung (PE),

○ Human Resource Management (HRM),

- Partizipation und Empowerment,

- Gruppenarbeit und Qualitätszirkel,

○ Arbeitsgestaltung (job design),

- Betriebliches Vorschlagswesen (BVW),

- Beschwerdemanagement,

$\circ \quad$ Wissensmanagement und Management-Informationssysteme (MIS).

Einige dieser Konzepte (z. B. Benchmarking, MIS) lassen sich auch unter Qualitätstechniken oder -instrumenten subsumieren, andere (z. B. Lernende Organisation, Empowerment) stellen mehr oder minder umfassende Ansätze zur Veränderung ganzer Organisationskulturen dar. Auf die meisten der genannten Konzepte wird in Kap. B 3. oder Kap. C 2. ausführlicher eingegangen.

\subsection{QM-Implementierungskonzepte}

Implementierung meint den Prozess der Umsetzung einer Idee von der Theorie in die Praxis. Im vorliegenden Zusammenhang kann Implementierung definiert werden als Summe aller Maßnahmen, die notwendig sind, um ein QM-System in einer Organisation wirksam werden zu lassen (Zollondz, 2002, S. 325). Weder die ISO-Normen noch TQMModelle oder andere QM-Konzepte sind direkt für die Umsetzung gedacht und enthalten oft wenige bis gar keine Hinweise auf Implementierungsfragen. Es verwundert deshalb nicht, dass viele gut gemeinte Systeme an der betrieblichen Wirklichkeit scheitern.

Neben der Bedeutung einer für alle sichtbaren Einbeziehung des Managements und dessen klar formulierter Qualitätspolitik spielen häufig institutionelle Absicherungen des Implementierungsprozesses in Form von Steuerungsorganen (Lenkungskreis o. Ä.), Qualitätskoordinatoren oder -beauftragten, Qualitätszirkeln oder anderen Teamkonzepten 
eine große Rolle. Einführungspläne mit Prioritäten, Projekten und Zwischenzielen, Schulungsmaßnahmen, Belohnungssysteme und Fortschrittskontrollen in Form von Reviews, Audits und Evaluationen vervollständigen den Implementierungsprozess. Nicht zuletzt ist jedes QM selbstreferenziell einzubetten in einen Kreislauf der kontinuierlichen Verbesserung.

Im Folgenden wird kurz auf einige der bekannteren Implementierungskonzepte eingegangen, um den eher betriebswirtschaftlich orientierten Überblick zu Qualitäts- und QM-Modellen in diesem Kapitel zu vervollständigen.

- Implementierungskonzept von Illi (nach Zollondz, 2002, S. 325 ff.):

Illi betont die Notwendigkeit, alle Bereiche des Unternehmens einzuschließen und Insellösungen zu vermeiden. Neben den erforderlichen Rahmenbedingungen personeller, struktureller, technischer und methodischer Art werden zeitlich und inhaltlich aufeinanderfolgende Phasen unterschieden (a.a.O., S. 326f). Hingewiesen wird auf besondere Problemzonen.

- Implementierungskonzept von Malorny (1996, nach Zollondz, 2002, S. 328):

Das Business Excellence Modell gilt als anspruchsvollster und umfassendster Implementierungsansatz; er berücksichtigt in hohem Maße sozialpsychologische Aspekte. Bezugsmodell ist das EFQM-Konzept (s. Kap. C 1.4.4). Unterschieden werden eine Sensibilisierungs-, Realisierungs-, Stabilisierungs- und Excellence-Phase. Zollondz ( $S$. 330) wirft die Frage auf, ob dem Modell nicht ein zu idealisiertes Menschenbild zugrunde liege.

- Das Berliner TQM-Umsetzungsmodell von Radtke (1999):

Das Berliner Umsetzungsmodell zerlegt TQM in handliche Module und will so eine unternehmensspezifische und pragmatische Implementierung ermöglichen. Arbeitspa- 
kete des Startmoduls sind u. a. die Information der Führungskräfte und die umfassende Vermittlung der TQM-Geisteshaltung. Weitere Module sind Führung, Politik und Strategie, Mitarbeiterorientierung, Ressourcen, Prozesse, Kunden- und Mitarbeiterzufriedenheit, Einfluss auf Gesellschaft und Geschäftsergebnisse. Diese, ebenfalls dem EFQM-Modell entsprechenden, Kriterien bilden einen geschlossenen Kreislauf, wobei die Geisteshaltung im Zentrum steht. Den einzelnen Modulen des Bereichs "Mittel und Wege" sind Aufgaben (z. B. Führung: Grundwerte erarbeiten, kommunizieren, vorleben), den Modulen des Ergebnisbereichs sind Indikatoren (z. B. Kundenzufriedenheit: Befragung, Beobachtung, Datenanalyse) zugeordnet.

Fraglich ist, ob sich die Prozesse der Entwicklung, Beschreibung und Einführung von QMSystemen im Humandienstleistungsbereich so trennen lassen, wie es die Unterscheidung von Phasen und Modulen insinuiert. So wird einerseits betont, dass Implementierung ein schwieriger und langwieriger Vorgang sei, der ein Umdenken aller Organisationsmitglieder und eine Veränderung der Organisationskultur erfordere (Zollondz, 2001, S. 352). Andererseits besteht die Wirkung eines nach Gestaltungsparametern des TQM entwickelten Qualitätsmanagements ja gerade darin, ein entsprechendes Bewusstsein im Unternehmen zu fördern. Bereits im Prozess des betrieblichen Qualitätsdiskurses sind somit Implementierungsaspekte stets präsent und keine Schritte, die es erst nach Abschluss dieses Diskurses zu unternehmen gilt.

Sinngemäß äußert sich Bleicher (1996, zit. nach Zollondz, a.a.O., S. 324): "Die postulierte Unterscheidung von strategischem und operativem Management ist ... gefährlich, denn sie widerspricht der Notwendigkeit, dass Strategien im Unternehmensalltag leben müssen."

In den meisten Qualitätskonzepten, die im sozialen Dienstleistungsbereich verwendet werden, wird deutlich, dass dort die Implementierungsfrage offenbar eine sehr viel größere Rolle spielt, als Bleicher (a.a.O.) dies für den gewerblichen Bereich zu konstatieren Anlass sah. Unter den als "formale und materiale Branchenkonzepte" etikettierbaren Ansätzen (s. Kap. C 1.3) finden sich zahlreiche, die sich primär als Verfahrensstrategie verstehen oder bei denen sich methodisches Procedere und inhaltliche Fokussierung mehr oder weniger miteinander verschränken. Darin äußert sich die Erkenntnis, dass für Organisationen, in denen zentral personenbezogene Dienstleistungen erbracht werden, die Einbeziehung der sozialpsychologischen Dimension selbstverständlich sein muss. Dass dies grundsätzlich 
auch für den Profit-Bereich gilt, belegen die elaborierten Implementierungskonzepte etwa von Radtke oder Malorny (s. o.).

Die meisten Handlungsanleitungen und Empfehlungen zum QM in der Sozialen Arbeit thematisieren denn auch die Art und Weise, wie Einrichtungen ein QM-System bei sich einführen (sollten). Im Vordergrund steht aber auch dabei eine Abfolge von Schritten oder Phasen, mit denen die Anforderungen des Systems abzuarbeiten sind. Zumeist wird dazu eine Projektorganisation für erforderlich gehalten, mit der sichergestellt werden soll, dass innerhalb eines überschaubaren zeitlichen und finanziellen Rahmens bestimmte Arbeitsergebnisse vorliegen (vgl. Gerull, 2001). 


\section{Verortung des Qualitätsmanagements in Wissenschaft und Praxis}

Qualitätsmanagement bezieht sich auf die Planung, Steuerung, Kontrolle und Optimierung von Leistungen, die von Betrieben, Unternehmen, Einrichtungen, Diensten und anderen Organisationen erbracht werden. QM ist insofern (auch) Gegenstand der Organisationsforschung.

"Eine Organisation ist ein soziales Gebilde, das bestimmte Ziele verfolgt und formale Regelungen aufweist, mit deren Hilfe die unter die Mitgliedschaftsbedingungen fallenden Aktivitäten der Mitglieder auf diese Ziele ausgerichtet werden sollen" (Kieser \& Kubicek, 1992, S. 1).

In vielen Themenfeldern der Organisationsforschung, wie z. B. Strategische Planung, Führung und Motivation, ging die Entwicklung der theoretischen Grundlagen der praktischen Anwendung von Konzepten voraus. Bezüglich der amerikanischen "quality movement" - weitgehend identisch mit TQM - wird eine umgekehrte Richtung konstatiert (Knouse, Smith \& Smith, 2001, S. 757). Hier waren es vor allem PraktikerInnen und UnternehmensberaterInnen, welche die Qualitätsbewegung dominierten (Scott \& Cole, 2000, S. xxii).

Auch in Europa vollzog sich die Entwicklung eines systematischen Managements zur optimalen Bewirtschaftung von Qualität nicht im Rahmen der akademischen Managementlehre, sondern weitgehend parallel und separat in der industriellen Praxis (vgl. Seghezzi, 1994, Vorwort). Jahrzehntelang übten amerikanische und japanische "Qualitätsgurus" aus dem Industriebereich wie Deming, Juran, Feigenbaum, Crosby, Ishikawa, Imai und andere größten Einfluss auf die Entwicklung aus. Der japanische Deming-Prize und der amerikanische Malcolm Baldrige National Quality Award dienten als Vorbilder für europäische Bemühungen, Unternehmen zur Business Excellence zu führen.

Während QM in der Praxis bereits seit den Achtzigerjahren eine Rolle spielt, werden erst seit Mitte der Neunzigerjahre Beiträge registriert, eine Organisationstheorie des QM zu formulieren (Knouse et al., a.a.O.). Gründe dafür werden einerseits in der ingenieurwissenschaftlichen Provenienz der meisten Qualitätspraktiken vermutet, andererseits in der anfänglichen Skepsis der Organisationstheoretiker, ob es sich bei der Qualitätsbewegung um mehr als eine zeitweilige Modeerscheinung handele (a.a.O.). Ungeachtet der zweifellos 
auch von einem "faddish element" (Dean \& Bowen, 2000, S. 4) und Partikularinteressen der Beratungsbranche durchwirkten Debatte (vgl. Shapiro, 1998; Hoerner \& Vitinius, 1997; Knorr, 1999), muss inzwischen allerdings eine bemerkenswerte Langlebigkeit dieser Qualitätsbewegung festgestellt werden, dominiert sie doch angeblich seit einem halben Jahrhundert die Aufmerksamkeit des Managements (Cole \& Scott, 2000, S. xxi).

Die empirische Organisationsforschung ist eines der wenigen wirklich interdisziplinären Gebiete (Scholl, 2004, S. 515). Konzepte der Organisation - häufig als Organisationstheorien firmierend - wurden von soziologischer, betriebswirtschaftlicher, psychologischer, politologischer, anthropologischer und ökonomischer Seite entwickelt und rücken jeweils verschiedene Aspekte in den Vordergrund. Diese Konzepte gewinnen ihre Überzeugungskraft aus integrativen Denkfiguren bzw. Metaphern, die das unübersichtliche organisatorische Geschehen ordnen und begreifbar machen sollen: z. B. die Ausbeutungs-, Maschinen-, Bedürfnis-, Problemlösungs-, Politik-, Organismus-, Kultur-, Kosten- und Netzwerkmetapher (a.a.O., S. 520 ff.).

Ohne die Analyse an dieser Stelle vertiefen zu wollen, kann eine besondere Nähe des QM zur Kulturmetapher der Organisationsforschung festgestellt werden (vgl. Kap. B 3.1.2). Deren Wert liegt vor allem darin, die "Organisationsrealität" auch als Deutungsprodukt der Akteure (vgl. Kap. B 3.8) und damit als Kritik an mechanistischen Forschungskonzeptionen zu analysieren. Dabei wurden qualitative Ansätze und intensive Fallstudien als legitime Forschungsmethoden etabliert und weiterentwickelt (Scholl, 2004, S. 527).

Weinert (1998, S. 69 ff.) konstatiert in der Organisationsforschung das Fehlen einer einheitlichen Theorie und Handlungsebene und fordert die Schaffung einer interdisziplinären Organisationswissenschaft. Eine solche ist mittlerweile zwar ausgewiesen im Rahmen universitärer Ausbildungsangebote (z. B. Fakultät der Wirtschafts- und Organisationswissenschaft an der Universität der Bundeswehr München), wird aber eher als Addition aus verschiedenen Teildisziplinen abgehandelt (z. B. als soziologische, wirtschaftswissenschaftliche und angewandte Organisationswissenschaft plus ergänzende Gebiete an der Universität Köln), denn als integratives Rahmenwerk für die Forschungsergebnisse der Einzeldisziplinen (vgl. Weinert, 1998, S. 70).

Dennoch liegt es nahe, QM wissenschaftssystematisch in einer solchen interdisziplinären Organisationswissenschaft zu verorten. Danach gehört QM keiner etablierten akademischen Bezugsdisziplin an, sondern erfährt je nach thematischer Fokussierung und Art 
der Organisation (z. B. Industrie, Bildungsinstitut, Bank, Arztpraxis) eine unterschiedliche Ausgestaltung (z. B. was den Stellenwert technischer Prozessparameter, standardisierter Handlungsvollzüge oder situativer Ermessensspielräume anbelangt). QM stellt somit die qualitätsbezogene Schnittmenge der tangierten Teildisziplinen dar.

Einen anderen Ansatz wählt Seghezzi (1996, 2003), der die Bewirtschaftung des Unternehmensfaktors Qualität (= Qualitätsmanagement) gleichberechtigt mit den Teilkonzepten des Kosten- und Zeitmanagements in ein allgemeines Managementmodell zu integrieren und damit in die Betriebswirtschaftslehre einzubetten versucht (vgl. St. Galler Management Konzept, Kap. C 1.4.5). Allerdings wurden seine durchaus überzeugenden Bemühungen in weiten Teilen der wirtschaftswissenschaftlichen Standardliteratur bislang nicht aufgegriffen. So wird weder in dem weitverbreiteten Lehrbuch von Wöhe (2002), noch bei Bofinger (2003) oder Mankiw (2004) der Begriff QM überhaupt erwähnt; selbst im Katalog der SUB Göttingen (s. Kap. A 4.2.1.3) findet sich nicht ein einziger Hinweis darauf, dass QM inzwischen Eingang in die betriebswirtschaftliche Systematik gefunden hätte.

"Heute bietet man zwar Kurse in Qualitätsmanagement an, meistens aber in Ergänzung und ohne engen Bezug zur bestehenden Betriebswirtschaftslehre" (Seghezzi, 2003, S. 4).

Man mag dieses Phänomen der Nichterwähnung in Lehrbüchern publikationsspezifischen "Trägheitsmomenten" zurechnen, zumal in aktuellen Vorlesungsverzeichnissen bundesdeutscher Wirtschafts- und Verwaltungsfakultäten (Quelle: eigene Internet-Recherchen) durchaus Veranstaltungen dem QM-Thema gewidmet werden. Berücksichtigt man die Geschichte des QM (s. Kap. B 1.1) und die inhaltliche Affinität zur Betriebswirtschaftslehre und deren "Hilfswissenschaften" (vgl. Wöhe, 2002), so erscheint eine entsprechende Zuordnung des QM legitim und sinnvoll, auch wenn gerade in Nonprofit-Organisationen die Rolle dieser Hilfswissenschaften - namentlich der Psychologie und Soziologie - darin besteht, den ökonomischen Blickwinkel der Betriebswirtschaft wesentlich, um nicht zu sagen: um das Wesentliche, zu erweitern.

Versuche wie die von J. F. W. Müller (2004), ausgehend von einer vorgeblichen Ablösung herkömmlicher Konzepte wie Organisations- und Personalentwicklung durch Qualitätsmanagement das TQM-Konzept als organisationstheoretischen Oberbegriff zu diskutieren, können dagegen nicht so recht überzeugen. Gerade der Bereich des Human Resource 
Management (s. Kap. B 3.3) widmet sich dezidiert auch solchen Fragen, die im QM bislang chronisch zu kurz gekommen sind, z. B. Personalselektion und -platzierung. Ein proaktives Personalmanagement, das in seiner Ausgestaltung weit über das traditionelle betriebswirtschaftliche Personalwesen hinausgeht, ist konzeptionell ebenso gut geeignet, zentrale Anliegen eines QM aufzunehmen, wie dies umgekehrt der Fall ist. Dies gilt gleichermaßen für die Frage, ob Organisationsentwicklung im Sinne eines ganzheitlichen Veränderungsprozesses die aktuellen QM-Konzepte integrieren kann oder QM besser als eine besondere, nämlich auf die Qualität des Angebotsprodukts fokussierende, Form des Change Management aufgefasst wird (s. Kap. B 3.2). Die jeweilige Zu-, Über- oder Unterordnung scheint eher eine Frage des Blickwinkels als der wissenschaftssystematischen Logik zu sein.

QM als organisationstheoretisches "Superkonzept" zu betrachten, an dem alle Betriebsbereiche auszurichten sind und das letztlich mit der Gesamtführungsaufgabe der Organisation identisch wird ("Total" QM, vgl. Kap. C 1.4.2), hieße aus betriebswirtschaftlicher Sicht, die anderen Faktoren des unternehmerischen Spannungsvierecks (Seghezzi, 2003, S. 19f, s. Kap. B 1.1) zu vernachlässigen. Damit würde jedoch die historische Bedeutung des Qualitätsdiskurses - genannt seien hier vor allem die Fokussierung auf Kundenbedürfnisse, partizipative ("mitunternehmerische") Organisationsstrukturen, Orientierung am Stakeholder-Value-Prinzip - durch Überdehnung des Konzepts diskreditiert.

Die immer noch stiefmütterliche Behandlung des Themas in prominenten Lehrbüchern der Betriebswirtschaft, Psychologie und Soziologie stellt vielleicht sogar eine Art Widerstand oder Korrektiv der "Etablierten" gegen die rhetorische Aufblähung und Selbstüberschätzung einer "Parvenü"-Disziplin dar, die bei näherer Betrachtung kaum originäre und empirisch alles andere als überzeugend fundierte Beiträge einzubringen hat.

Diese nüchterne Einschätzung entbindet jedoch nicht davon, im Folgenden möglichst unvoreingenommen der Frage nachzugehen, wie diese Beiträge aussehen und in mindestens welchen Fällen QM und ausgewiesene Bereiche der Organisationsforschung sich überschneiden bzw. identische Probleme bearbeiten und gegenseitig befruchten können. 


\section{Qualitätsmanagement und Konzepte der Organisationsforschung}

\subsection{Organisationsdiagnose und -analyse}

Die systematische und wissenschaftlich fundierte Zustands- und Prozessbeschreibung von Organisationen kann je nach Zielsetzung und Betrachtungsebene als Organisationsdiagnose oder Organisationsanalyse bezeichnet werden. Während Erstere einen vornehmlich psychologischen Ansatz verkörpert, bei dem das Erleben und Verhalten der Organisationsmitglieder im Vordergrund steht, ist die Organisationsanalyse bislang eine Domäne der Betriebswirtschaft, Organisationssoziologie und Verwaltungswissenschaft und fokussiert auf bedingungsbezogene Aspekte wie Ordnungen und Regeln der Organisation (Büssing, 2004, S. 559). Die Grenzen sind jedoch fließend; speziell für die nordamerikanische Organisationsforschung wird die Durchlässigkeit zwischen den beteiligten Disziplinen als charakteristisch bezeichnet (a.a.O., 2004, S. 561).

Von den wichtigsten Grundkonzepten der Organisationsdiagnose/-analyse (Scholl, 2004, S. 530, vgl. Kap. A 5.3.3) werden nachstehend Organisationsstruktur, Organisationskultur und Organisationsklima erörtert. Die Ausführungen werden ergänzt durch ausgewählte empirische Befunde zum Zusammenhang dieser Organisationsvariablen mit dem Erfolg von TQM-Implementierungsprojekten. Damit soll verdeutlicht werden, dass TQM (s. Kap. C 1.4.2) trotz seines Anspruchs, ein integratives Gesamtkonzept der Unternehmensführung zu sein, in der Organisationspraxis vielfach "nur" als eine komplexe Form der Intervention, als ein Aktionsprogramm behandelt wird, dessen Effekte zumindest teilweise von jenen Variablen abhängig ist, die zu fördern TQM sich anheischig macht. Wie schon in Kap. A 5.3.2 betont, hängt es vom Forschungskontext ab, ob eine Variable - hier: TQM - als "unabhängig" oder "abhängig" betrachtet wird.

\subsubsection{Organisationsstruktur}

Als Organisationsstruktur bezeichnen Kieser \& Kubicek (1992, S. 22) das "System von geltenden Regelungen für die Steuerung von Leistung und Verhalten der Organisationsmitglieder". Bemühungen, primäre Dimensionen für die Synthese verschiedener Strukturvariablen zu bestimmen, konnten bislang nur teilweise empirisch verifiziert werden (Wei- 
nert, 1998, S. 608). Ungeachtet der hierfür verantwortlichen methodischen Problematik (a.a.O., S. 611), erörtert Weinert (S. 612) folgende Strukturvariablen als die am häufigsten analysierten Organisationsdimensionen:

1. für die Gesamtorganisation:
a) Größe,
b) Konfiguration (steil oder flach),
c) Form (Zentralisiertheit - Dezentralisiertheit; Lokus der Autorität für das Fällen von Entscheidungen)

2. für die Unter- oder Teilsysteme einer Organisation:

d) Organisationsebene (in der ein/e MitarbeiterIn tätig ist),

e) Hierarchiesystem (Führungskräfte und Untergebene),

f) Kontrollspanne (Anzahl der einem Vorgesetzten untergebenen MitarbeiterInnen),

g) Größe der Unter- oder Teilsysteme.

Tata, Prasad \& Thorn (1999) beziehen den Begriff der Organisationsstruktur auf die Art und Weise,

○ wie Arbeitsaufgaben formal geteilt, gruppiert und koordiniert werden (S. 442),

○ wie Menschen miteinander interagieren,

○ wie die Kommunikation fließt und

○ Machtbeziehungen definiert sind (S. 441).

Die Organisationsstruktur reflektiert den Autoren zufolge die wertbasierten Entscheidungen des Unternehmens. Solche Werte seien z. B. "Kontrolle" versus "Flexibilität" und korrespondierten mit hierarchisch-zentralisierten bzw. organisch-dezentralen Strukturen $(S$. 442). Mehrere Studien werden als Beleg dafür angeführt (z. B. Harris \& Purdy, 1998; Johannesson \& Ritchie, 1997; Crom \& France, 1996; Whalen \& Rahim, 1994), dass partizipative und teamorientierte Strukturen - charakterisiert durch Involvement, Empowerment und Verantwortung - die Ergebnisse von TQM verbessern können. 
Ungeachtet der Popularität des TQM-Konzepts wird auf Berichte in der Fachliteratur Bezug genommen, wonach nur ein Drittel bis die Hälfte der untersuchten Organisationen signifikante Verbesserungen dadurch erzielt haben (Burdett, 1994; Garvin, 1986; Grant, Shani \& Krishnan, 1994). Betont wird, dass dieser Mangel an Erfolg möglicherweise nicht dem Konzept, sondern der zu geringen Beachtung des organisationalen Kontextes zuzuschreiben sei. Da TQM eine veränderte Art des interaktiven Umgangs und Arbeitens in Organisationen bedeute, sei es ein kontextabhängiges Programm, dessen Erfolg zu einem großen Anteil von Faktoren wie Organisationskultur, Marktstruktur, Managementstil und Organisationsstruktur abhänge (Tata et al., 1999, S. 440f).

Tata et al. (a.a.O.) testeten empirisch die Hypothese, dass die Effektivität von TQM-Programmen in Unternehmen mit flexibilitätsorientierten, organischen Strukturen höher sei als in Unternehmen mit kontrollorientierten, mechanistischen Strukturen.

Als UV fungierte die Organisationsstruktur, als AV die über sechs Items ermittelte TQM-Effektivität; als Kontrollvariablen wurden mehrere Qualitätspraktiken erhoben, die sich an den Kriterien des Baldrige Awards - eines amerikanischen Qualitätspreises - ausrichten (z. B. Kundenorientierung, Qualitätsbewusstsein, Personalentwicklung), des Weiteren biographische Variablen, um nur jenen Varianzanteil herauszufiltern, der ggf. der UV zuzurechnen war. Die Stichprobe bestand aus 89 Managern/Supervisoren verschiedener Industriebetriebe.

Die Ergebnisse bestätigten den Zusammenhang zwischen Organisationsstruktur und TQM-Effektivität (Interkorrelation .55); der zusätzlich erklärte Varianzanteil betrug $\Delta \mathrm{R}^{2}=.11(S .447)$.

Tata et al. (S. 450f) schlussfolgern, dass Unternehmen vor Einführung von TQM-Programmen ihre Organisationsstruktur überprüfen und ggf. verändern sollten, um die Effektivität des Programms zu erhöhen.

\subsubsection{Organisationskultur}

Unter Organisationskultur werden die von den Mitgliedern geteilten Grundannahmen, Werte und Normen in der Organisation verstanden, von denen angenommen wird, dass sie die Gestaltung und Wahrnehmung von Prozeduren, Strategien und Strukturen beeinflussen (Scholl, 2004, S. 538). Organisationskultur äußert sich in verschiedensten Phänomenen und wird vor allem durch qualitative, interpretative Methoden rekonstruiert. 
Seitens der Unternehmensberatungsbranche wurden Hoffnungen geweckt, durch geeignete Gestaltung der Organisationskultur einen Schlüssel zum wirtschaftlichen Erfolg in der Hand zu haben (a.a.O., S. 526); entsprechend vielfältig sind Bemühungen, durch Maßnahmen des Change Management einen Kulturwandel zu vollziehen. Dabei wird allerdings verkannt, dass die Essenz einer Organisationskultur, zu der auch Rituale, Firmenjargon, die Organisationsgeschichte, Beförderungspraktiken, Sicherheitsstandards u. a. Faktoren gehören, zu einem erheblichen Teil nicht bewusst sind (a.a.O., S. 527). "Organisationskulturen sind zwar Konstrukte, nicht aber konstruierbar" (Türk, 1989, S. 110). Seit Ende der Achtzigerjahre verstärkte Forschung mündete denn auch in der Zurïckweisung der (Wunsch-)Vorstellung einer einheitlichen Organisationskultur (Scholl, a.a.O.).

Hamada (2000, S. 296 ff.) unterscheidet drei aktuelle Modellvarianten von Organisationskultur, die er durch eine anthropologische Sichtweise ergänzt und im Hinblick auf den Qualitätsdiskurs erörtert:

1. Das Integrationsmodell repräsentiert eine managementzentrierte Perspektive und betrachtet die Qualitätskultur als Teil der Organisationskultur, die es durch Förderung gemeinsamer Werte und Normen vor allem mittels geeigneten Führungsverhaltens zu entwickeln gilt. Es wird ein gesamtorganisationaler Konsens angestrebt, der sich in Bezug auf Qualität als gemeinsame Orientierung artikuliert ("sharing a cognitive view of quality", $S$. 296). Qualität wird dabei als ein objektivierbares Phänomen verstanden, das zu managen ist (S. 299).

2. Das Differenzierungsmodell betont dagegen die innerorganisatorischen "inconsistencies" und die Möglichkeit, nur innerhalb subkultureller Grenzen Konsens herzustellen. Die Machbarkeit einer qualitätsorientierten Organisationskultur durch das Top Management wird als eingeschränkt betrachtet, weil der gesellschaftliche Kontext starken Einfluss ausübt:

"Current total quality management (TQM) statements fail to argue persuasively why workers should share their ideas with managers if the adoption of innovation and new methods are likely to threaten a worker's job" (S. 297). 
Qualität und Macht werden in dieser soziozentrischen Sichtweise als objektiv gegeben und Qualität als erkämpfbar betrachtet (S. 299).

3. Das Fragmentierungsmodell geht noch über diesen Ansatz hinaus und sieht Ambiguität und Mangel an gemeinsamen Überzeugungen als Wesen der Organisationskultur an, die demzufolge stark durch Unsicherheit und Flüssigkeit charakterisiert wird. Während das Differenzierungsmodell die subkulturellen Grenzen zwischen organisationalen Gruppen betont, verweist das Fragmentierungsmodell auch auf die gruppeninternen Ambivalenzen. Die Bedeutung von Qualität kann danach nur verstanden werden, wenn Position und Geschichte der agierenden Individuen und Gruppen einbezogen werden; Qualität wird als verhandelbar und in ihren Bedeutungen als subjektiv betrachtet (S. 299).

4. Das anthropologische Modell schließlich sieht die Organisation als einen contextualizing process an. Kultur ist dabei eher als "root metaphor" zu verstehen. Danach hat eine Organisation keine Kultur, die es zu verändern gilt, sondern ist eine Kultur im Sinne eines emergenten, kollektiven Prozesses der Sinngebung (meaning configuration, S. 300). In diesem Prozess internalisieren Subjekte eine soziale Grammatik, die es ihnen ermöglicht, Realität als bedeutungsvoll zu erleben. Bezogen auf den Qualitätsdiskurs, führt eine solche Internalisierung dazu, dass Menschen sich bereitwillig dem Qualitäts-Kanon fügen "commitment to institutionalized ideas (such as quality management)" -, wenn dieser ihrem persönlichen Habitus entspricht; anderenfalls sind Formen der Noncompliance, Sabotage und heimlicher bis offener Widerstand die mögliche Folge (S. 301).

Nerdinger (1994, S. 305 ff.) thematisiert in ähnlicher Weise die von Vertretern des Kulturansatzes intendierte Verhaltenskontrolle durch Identifikation und berichtet über entsprechende Maßnahmen von Unternehmen, die jedoch nicht verhinderten, dass sich ein gewisses Widerstandspotenzial seitens der MitarbeiterInnen erhalte. Würden die konkreten strukturellen Bedingungen des Unternehmens dabei ausgeblendet, komme es alsbald zu Wider-sprüchen zwischen Kulturrhetorik und Praktiken und Regeln des Arbeitsvollzugs; die Folge sei Zynismus.

Der Ansatz von Hamada ist dem sozialkonstruktivistischen Konzept des Sensemaking (Weick, 2000) oder Meaning Making (Dahlberg, Moss \& Pence, 1999, s. Kap. B 3.8) verwandt und betont stärker als die anderen drei Kulturmodelle die kontextuellen, ganzheitlichen und longitudinalen Aspekte. 
Am Beispiel der Entwicklung der japanischen Managementphilosophie illustriert Hamada (S. 302 ff.) einen Bedeutungswandel hin zur "ökologischen Exzellenz":
○ conformance to requirements
1950-1960,
$\circ$ customer satisfaction
1970-1980,
○ environmentalism
1990-2000.

Hamada (S. 305) weist darauf hin, dass in diesem Zusammenhang neue Begriffe (z. B. kyosei, zu übersetzen als living together) entstanden sind, die eine bestimmte Ideologie transportieren sollen - zero waste, zero emission, flow analysis, sustainable development $\mathrm{u}$. a. - und sich stärker biologischer als ingenieurwissenschaftlicher Analogien bedienen. Abzuwarten bleibe, ob diese neue Managementrhetorik zur ökologischen Qualität von anderen Organisationsmitgliedern geteilt werde.

Über eine Strategie, durch Veränderungen der Organisation auf lange Sicht Veränderungen der Organisationskultur zu bewirken, berichtet Ho (1999). Während traditionelle Strategien für Veränderungsprozesse durch die Schrittfolge

\section{Vision Mission Verhalten Aktion $>$ Kultur}

gekennzeichnet seien, gehe ein neues Paradigma von der Abfolge aus:

Aktion Verhalten Mission $>\operatorname{Vision} \longrightarrow \operatorname{Kultur}($ S. 170).

Diese - in erfolgreichen Organisationen häufig anzutreffende - Strategie (Peters \& Waterman, 1982, zit. nach Ho, 1999, S. 170) impliziere, dass Aktionen zu Verhaltensänderungen der Beschäftigten führen, weil durch Aktionen Lernprozesse stattfinden. Solche Änderungen könnten z. B. in den Bereichen Wissen, Selbstvertrauen, Flexibilität und Offenheit auftreten und die Organisation insgesamt auf ein dynamischeres und Herausforderungen suchendes Niveau führen. Dies wiederum beeinflusse das Top Management dahingehend, seine Mission und Vision zu definieren und so letztlich eine neue Kultur innerhalb der Organisation zu begründen.

Als bekanntestes Beispiel hierfür wird das japanische Kaizen (s. Kap. C 1.4.3) herangezogen. Die Bedeutung des "close to the scene", des "offenen Büros", des "management by 
walking around" und der "gläsernen Küche" werden ebenfalls in diesem Zusammenhang betont (a.a.O., S. 170).

Laut Deming (1986) sind 94\% aller Qualitätsprobleme durch das Management und das von ihm geschaffene System bedingt (zit. nach Ho, 1999, S. 172). Aus diesem Grunde sei das Commitment des Managements von primärer Bedeutung. Kontinuierliches Lernen in einer lernenden Organisation schaffe hierfür die besten Voraussetzungen.

\subsubsection{Organisationsklima}

Organisationskultur und Organisationsklima sind nicht klar voneinander abzugrenzen und nehmen beide auf Merkmale Bezug, die auf Kommunikation und Kooperation aufbauen (Moran \& Volkwein, 1992, zit. nach Büssing, 2004, S. 589). Während Organisationskultur jedoch auf das Besondere und Typische einer Organisation fokussiert, das oft nicht explizit benannt und reflektiert wird (Scholl, 2004, S. 539), ist Organisationsklima als Wahrnehmung wichtiger Facetten einer Organisation durch ihre Mitglieder operational definiert und wird quantitativ durch dimensional vorstrukturierte Fragebögen erfasst (a.a.O.).

Emery, Summers \& Surak (1996, S. 485) verstehen Organisationsklima als ein relativ stabiles Charakteristikum von Organisationen, das sich in den Einstellungen und Beschreibungen von Beschäftigten niederschlägt, wie sie die Politik, Praxis und Bedingungen ihrer Arbeitsumgebung erleben. Danach kann ein solches Klima betrachtet werden "as a measure of whether people's expectations about what it should like to work in an organization are being met" (Schwartz \& Davis, 1981, S. 31). Klima wird somit als eine Erscheinungsform von Kultur aufgefasst, wobei Letztere gewöhnlich als ein tiefergehendes, weniger bewusst empfundenes Set von Bedeutungen definiert wird (Reichers \& Schneider, 1990, zit. nach Emery et al., 1996, S. 485).

Das in der Fachliteratur berichtete häufige Scheitern von TQM-Programmen (z. B. Erickson, 1992; Fuchsberg, 1992; Kendrick, 1993; Doyle, 1992) - die Rede ist von bis zu zwei Dritteln Misserfolge - wird gewöhnlich Defiziten in folgenden Bereichen zugerechnet (Emery et al., 1996, S. 484f):

- gemeinsame Vision (shared vision), 
- Anwendungsplanung (application planning),

- organisationales Commitment,

- Training,

- Belohnungssysteme (reward systems),

○ Empowerment,

○ interfunktionelle Integration (cross-functional integration).

Ungeachtet der Bedeutung dieser Faktoren für eine Internalisierung des TQM-Konzepts, halten Emery et al. (a.a.O.) es für möglich, dass ihnen eine fundamentale Determinante zugrunde liegt: das Bedürfnis nach einem förderlichen Organisationsklima. Während die Bedeutung einer TQM-typischen Kultur (z. B. Kundenorientierung) vielfach diskutiert worden sei, habe niemand die Effekte präimplementationeller klimatischer Faktoren empirisch untersucht. Bezug nehmend auf Schwartz \& Davis (1981) wird betont, dass ein Klima, welches mit den angestrebten Veränderungen inkompatibel ist, ein hohes Maß an Widerstand auslösen und noch so gut geplante Veränderungsprozesse zum Entgleisen bringen könne. Die Literatur zum Change Management belege ebenfalls, dass eine erfolgreiche Implementation von TQM von einem innovationsförderlichen Arbeitsklima abhänge (z. B. Kim, 1989; Senge, 1990; Glover, 1993), welches den notwendigen atmosphärischen Rahmen für Lernprozesse abgebe. Hierfür müssten Beschäftigte jedoch ein Klima des Vertrauens spüren, bevor sie maximales Commitment für TQM entwickeln können (Emery et al., 1996, S. 485f).

Auf diesem Literatur-Review basierend, untersuchten Emery et al. die Hypothesen,

1. dass die Wahrnehmung des Organisationsklimas durch Beschäftigte in Unternehmen mit erfolgreicher TQM-Implementierung günstiger ist als bei nicht erfolgreichen TQMImplementierern;

2. dass die Wahrnehmung des Organisationsklimas durch Beschäftigte sich während der TQM-Implementierung verbessert.

Anhand einer Sekundäranalyse eines an 15.722 Beschäftigten verschiedener Unternehmen der amerikanischen Luftfahrtindustrie erhobenen Datenpools (1. Messung vor, 2. Messung acht Monate nach Beginn der TQM-Implementierung) wurden 12 von insgesamt 50 Items als relevant für die Fragestellung ausgewählt (co-workers' level of commitment to success, organization's respect for the individual, level of interdepart- 
mental cooperation, quality of training and development, company's usage of employee skills, rewarding of good performance, quality of direct supervision, clarity of company goals and objectives, willingness of employees to reveal problems, upward communication/company's willingness to listen and take action, fair application of organizational policies, flow of information within the company).

Es zeigten sich im Sinne der Hypothese 1 hochsignifikante Zusammenhänge zwischen der Güteeinschätzung des jeweiligen Klimafaktors durch die Beschäftigten und der Zugehörigkeit zur Gruppe der erfolgreichen bzw. erfolglosen TQM-Implementierer (Chi-Quadrat-Tests, p. <0.001).

Ebenso bestätigte sich die in Hypothese 2 formulierte Erwartung, wonach sich im Verlaufe des TQM-Prozesses die Einschätzung des Klimas durch die Beschäftigten verbessert (t-Tests für abhängige Stichproben, $\mathrm{p}<$ 0.001). In 11 von 12 Items lagen die Klimaeinschätzungen bei den letztlich erfolgreichen TQM-Implementierern jedoch schon vor der Implementierung höher als bei den Erfolglosen (aus statistischen Gründen waren dort auch die Zuwachsraten relativ größer als bei den Erfolgreichen). Dies wird als Hinweis darauf gewertet, dass Organisationen einen bestimmten "Zündpunkt" (flashpoint) besitzen, ab dem TQM-Programme ihre Wirksamkeit entfalten können (S. 493).

Die Ergebnisse stützen nach Ansicht der Autoren die Annahme, dass der Wahrnehmung interner Trainings- und Entwicklungsmaßnahmen durch die Beschäftigten und einem Klima hohen Vertrauens Schlüsselfunktionen für nachhaltiges organisationales Lernen zu$\operatorname{kommt}(S .489 f)$.

Wenngleich bereits die bloße Implementierung von TQM Klimaverbesserungen zur Folge haben könne, hätten Organisationen mit schlechtem Klima ein hohes Misserfolgsrisiko angesichts der Zeit und Mühen, die mit der Internalisierung von TQM verbunden seien. Vorgeschaltete Maßnahmen zur Klimaverbesserung seien unter Umständen notwendig, um die Erfolgswahrscheinlichkeit zu erhöhen (S. 493). Hierfür empfehle sich der Einsatz von Klima-Fragebögen, Teamentwicklungsmaßnahmen, Problemlöse-Seminaren und verstärkter interfunktioneller Integration in der Präimplementationsphase (S. 494).

Nerdinger (1994, S. 318) betont im Zusammenhang mit der Herstellung eines "Dienstleistungsklimas", dass hierfür an den Praktiken und der Struktur der Organisation angesetzt werden müsse, um die Arbeit der Dienstleister zu unterstützen. Der Autor hält ein solches Verständnis von Dienstleistungsklima für besser geeignet als die Steuerung von Verhalten über die Manipulation von Symbolen - wie es das Konzept der Dienstleistungskultur (keine Hervorhebung im Original, P. G.) nahe lege -, um bloßes organisationales "Impression Management" zu überwinden. 


\subsection{Organisationsentwicklung und Change Management}

Während der Begriff der Organisationsdiagnose oder -analyse auf die Beschreibung von Strukturen, Prozessen und Ergebnissen, mithin auf die Erhebung und Darstellung quantitativer und qualitativer Befunde abzielt, stehen bei der Organisationsentwicklung Fragen umfassender und bewusst gesteuerter Veränderungen durch methodisch angelegte Interventionen im Vordergrund (vgl. Gebert, 2004, S. 601).

Organisationsentwicklung (OE) ist ein Oberbegriff für geplante Maßnahmen mit dem Ziel verbesserter Anpassung an sich wandelnde Aufgaben und Umweltbedingungen, z. B. rechtlicher, sozialer und technologischer Art. OE ist oft mit einer Neudefinition des Unternehmensleitbildes und administrativer Umstrukturierung verbunden (Flösser \& Otto, 1992, S. 9 ff.). Es handelt sich zumeist um längerfristig angelegte, oft mehrjährige Veränderungsprozesse, die neben den Personen die sie umgebende Situation zu integrieren versuchen und häufig extern begleitet werden (Greif, 1998, S. 600).

Kennzeichnend für $\mathrm{OE}$ ist, dass Veränderungen der ganzen Organisation und nicht nur einzelner Abteilungen oder Gruppen angestrebt werden. Rationalisierungsprojekte, die ausschließlich auf eine Erhöhung der Produktivität abzielen, zählen gemeinhin nicht dazu. Vielmehr soll durch die Konzipierung und Implementation geeigneter Arbeits-, Führungsund Kooperationsformen ein hohes $\mathrm{Ma}$ an Commitment sowie eine hinreichende Effektivität aller Abläufe sichergestellt werden. Darüber hinaus geht es darum, die Lernfähigkeit einer Organisation bzw. ihre Flexibilität und Innovationsbereitschaft zu stärken (Gebert, 2004, S. 601). Nach diesem Verständnis zielt OE sowohl auf mitarbeiterbezogene Ziele (Humanität, Verbesserung der Arbeitssituation, Partizipation), als auch auf organisationsbezogene Wirkungen (Leistungsfähigkeit, Effektivität, vgl. J. F. W. Müller, 2004, S. 80f).

Die im Rahmen der OE durchgeführten Interventionen sind am Modell der Aktionsforschung orientiert und insofern eine spezifische Form des Change Management (Gebert, a.a.O., S. 602), der zielgerichteten Steuerung und Bewältigung von Veränderungen durch Konzepte, Prozesse und Werkzeuge (vgl. Czichos, 1990). Dabei lassen sich die verschiedenen Ansätze grob klassifizieren in einen personalen und strukturalen. Während jener die angestrebten Organisationsveränderungen vor allem über eine Steigerung der sozialen und fachlichen Kompetenz der Akteure zu erreichen versucht (z. B. durch 
gruppendynamische Trainings und Weiterbildung), bestehen die wesentlichen Strategien des strukturalen An-satzes in Formen der Arbeitsgestaltung (z. B. job enrichment, Installierung teilautonomer Arbeitsgruppen). Organisationen werden dabei als soziotechnische Systeme betrachtet, in denen technologische, arbeitsorganisatorische und soziale Prozesse vernetzt sind (Gebert, a.a.O.).

Die Grenzen zwischen OE und Personalentwicklung (PE) sind fließend; auch die personalen und strukturalen Ansätze innerhalb der OE sind komplementär zu verstehen, nicht alternativ. Leitungs- und Führungskräften kommt in OE-Prozessen vor allem eine Multiplikationsfunktion zu, "... indem sie - mit neuen Kompetenzen ausgerüstet - durch verbesserte Kommunikations- und Kooperationsstrukturen $\mathrm{zu}$ einer aktivierenden Organisationskultur beitragen sollen" (Flösser \& Otto, 1992, S. 12).

Greift die Organisation im Rahmen ihres geplanten Entwicklungsprozesses auf externe Berater (change agents) zurück, so vollzieht sich dies zweckmäßigerweise nicht in Analogie zum Arzt-Patienten-Verhältnis, in dem der Experte präzise inhaltliche Empfehlungen ausspricht. Vielmehr wird eine diskursähnliche Kommunikationsstruktur zwischen Beratern und dem zu beratenden System für angemessen gehalten, die sich am Modell der Tat- bzw. Aktionsforschung orientiert und die Expertenrolle eher als Prozessberatung in teilnehmender und moderierender Funktion akzentuiert (Gebert, 2004, S. 603). Allerdings gerät ein derartiges Selbstverständnis häufig in Konflikt mit konkreten Lösungserwartungen der Auftrag gebenden Organisation, so dass zu Beginn des Beratungsverhältnisses eine allseitige Interessenpräzisierung unabdingbar ist.

Im Zusammenhang mit der Gefahr, dass langfristig und partizipativ angelegte Veränderungsprozesse versanden, kristallisierte sich in den Neunzigerjahren eine alternative Veränderungsstrategie des Change Managements heraus, die keinen allmählichen und inkrementellen, sondern einen radikalen und irreversiblen Wandel anstrebt (Business Reengineering). Dieser Ansatz, der zugleich eine völlig andere Rolle des Beraters erfordert, verlässt allerdings den Boden der Aktionsforschung (a.a.O., S. 604).

Unabhängig von der Frage, ob der Veränderungsprozess mit Hilfe externer Beratung eingeleitet werden soll, bedarf es innerorganisatorischer Vorkehrungen, diesen Prozess zu steuern. Paritätisch besetzte Lenkungskomitees unterstreichen in der Regel den auch 
politischen Charakter einer OE und signalisieren wirkungsvoll die Einbeziehung des Topmanagements. Die auf Kurt Lewin zurückgehende Datenrückkopplung des survey feedback, bei dem Analyseergebnisse über den Status quo der Organisation allen MitarbeiterInnen zugänglich gemacht werden, ist eine verbreitete Methode zur Integration der Betroffenen (Greif, 1998, S. 600), an die sich Teamentwicklungsprozesse und Aktionsplanungen anschließen lassen. Eine zeitlich oder inhaltlich forcierte Partizipation der Belegschaft setzt mitunter allerdings eine Veränderungsdynamik frei, die aus Sicht der Führungsspitze als bedrohlich empfunden werden kann und nicht selten Strategien des Machteinsatzes herausfordert (Gebert, a.a.O., S. 605f).

Die gerade in differenzierten Organisationen notwendige Integration der verschiedenen Subsysteme weist der Kommunikation eine zentrale Rolle im OE-Prozess zu. Leitlinien für Führung und Zusammenarbeit sollen vielerorts eine an gemeinsamen Grundüberzeugungen orientierte Organisationskultur manifestieren, in der Vertrauen neben einer normativen zunehmend auch zu einer ökonomischen Kategorie avanciert: Vertrauen stellt "Sozialkapital" dar und erleichtert die Ausschöpfung des "Humankapitals" (a.a.O., S. 607).

Grenzen der OE ergeben sich aus der beschränkten Planbarkeit und Steuerbarkeit betrieblicher Wandlungsprozesse in einer zunehmend turbulenten Organisationsumwelt. Häufig werden Effekte der Partizipation überschätzt, da Veränderungen stets nicht nur Verbesserungen, sondern auch Verluste darstellen (z. B. führen "Öffnungsprozesse" wie Dezentralisierung zu einem Verlust an Sicherheit und Ordnung). Vor diesem Hintergrund kommt es darauf an, mit widersprüchlichen Organisationsmustern und Paradoxien leben zu lernen. Eine wichtige Voraussetzung hierfür liegt darin, valide über diesen Sachverhalt miteinander zu kommunizieren, um zumindest die vorhandenen Steuerungspotenziale ausschöpfen zu können (a.a.O., S. 615).

Zollondz (2001, S. 323 ff.) betont die Relevanz von OE-Ansätzen für die Implementation von QM und führt die weitgehende Abstinenz in Theorie und Praxis des QM auf die Dominanz der Ingenieurinteressen und der ihnen affinen Umsetzungsstrategien zurück. In der Tat ist $\mathrm{OE}$ bis heute eine Domäne der Psychologie und Soziologie, während umgekehrt QM vorwiegend in den Fachdisziplinen der Ingenieur-, Arbeits- und Wirtschaftswissenschaften angesiedelt ist. 
Der Entwicklungsaspekt von Unternehmen spielt in allen universellen QM-Systemen (s Kap. C 1.3) eine zentrale Rolle. So betont das graphische Modell der ISO 9001:2000 (Deutsches Institut für Normung, 2000) die Einbettung der Strukturelemente ManagementVerantwortung, Ressourcen-Management, Prozessmanagement und Bewertung und Analyse in einen Regelkreis aus Kundenanforderungen, ständiger Verbesserung des QMSystems und Kundenzufriedenheit. Das EFQM-Excellence-Modell (Kirstein, 2000) verdeutlicht den gleichen Sachverhalt durch ein Innovation und Lernen genanntes Systemelement, welches beim Integrierten Qualitätsmanagement nach dem St. Galler Konzept (Seghezzi, 1996) als Unternehmensentwicklung firmiert. Das Konzept des Kontinuierlichen Verbesserungsprozesses schließlich rekurriert explizit auf diesen Aspekt.

Bereits 1989 schlug Maelicke (S. 67 ff.) eine Vorgehensweise für ganzheitliche und sozialökologische Organisationsentwicklung in Non-Profit-Organisationen vor, bei der er folgende Aufgaben unterschied:

1. Entwicklung eines Leitbildes, einer Unternehmensphilosophie, einer corporate identity,

2. Zielfindung,

3. Aufgabendefinition,

4. Aufbau- und Ablauforganisation,

5. Führungs- und Mitarbeiterverhalten,

6. Soziale Infrastrukturentwicklung, Vernetzung,

7. Projektmanagement,

8. Sozial-Marketing,

9. Evaluation und Fortschreibung,

10. Förderung der Selbstorganisation.

Bemerkenswert ist dabei, dass dieser Katalog nicht nur die dem oben genannten Verständnis von OE entsprechenden Interventionen aufnimmt, sondern sich darüber hinaus wie ein Aufgabenmodell der kunden-, mitarbeiter- und gesellschaftsorientierten Unternehmensführung schlechthin liest, sieht man einmal von der vernachlässigten Ressourcen-Kategorie ab.

Ein späteres Resümee teilweise vorwegnehmend, soll das Beispiel verdeutlichen, dass QM - sei es als Partialkonzept innerhalb der Unternehmensführung oder als integratives Gesamtkonzept für alle Managementbereiche im Sinne des TQM (s. Kap. C. 1.4.2) verstanden -, von seiner Fokussierung auf Qualität und der Verbreitung einschlägig verwendbarer Instrumente abgesehen, keine wesentlich neuen Impulse in die Organisationsforschung einbringt, sondern Ansätze "nur" eklektisch in qualitätsspezifischer Weise konfiguriert. 


\subsection{Personalentwicklung und Human Resource Management}

Personalentwicklung (PE) wird als Oberbegriff für ein breites, schwer abgrenzbares Spektrum von Maßnahmen zur Analyse, Planung, Förderung und Evaluation des personellen Potenzials einer Organisation verstanden (Greif, 1998b, S. 623). In einer Zeit beschleunigten technologischen und ökonomischen Wandels soll PE berufliche Handlungskompetenzen erweitern bzw. verbessern und zur Qualifikationsanpassung an gegenwärtige und zukünftige Anforderungen beitragen. Insofern stellt PE eine strategische Komponente des Human Resource Management dar (Cascio, 1992), dem als übergeordnetem Begriff auch diverse Schlüsseltechniken eines QM-Systems zugeordnet werden können (Ichniowski \& Shaw, 2000, S. 364). Holling \& Liepmann (2004, S. 349) betonen, dass insbesondere Veränderungen des Wissens sowie kognitive und interpersonale Kompetenzen Gegenstand der gegenwärtigen PE sind.

Die Entwicklung des betriebswirtschaftlichen Personalwesens zum aktiven Personalmanagement als integrativem Bestandteil eines gesamtorganisatorischen Unternehmensführungsmodells kennzeichnet den Ansatz des Human Resource Management, zu dessen Handlungsfeldern Personalführung, -motivation, -beschaffung und -entwicklung gezählt werden (J. F. W. Müller, 2004, S. 128 ff.).

Zur PE gehören vor allem betriebliche Weiterbildung im Sinne von Anpassungsqualifikation - während die Ausbildung als Erstqualifikation eine Domäne pädagogischer Disziplinen ist (a.a.O.) -, Trainee- und Feedback-Programme, Supervision, Coaching und Formen der Beratung, aber auch Maßnahmen der Arbeitsgestaltung, Einführung, Einsatz- und Karriereplanung der MitarbeiterInnen. Von PE abgegrenzt werden von den meisten AutorInnen Prozesse ungeplanten Lernens und beruflicher Sozialisation sowie Ansätze, die darauf abheben, Ziele durch Personalselektion, technologische oder organisationsstrukturelle Veränderungen zu erreichen (Staufenbiel, 1999, S. 510). Stehen die gesamte Organisation oder größere Einheiten im Blickpunkt, spricht man zumeist von Organisationsentwicklung (OE, s. o.).

Wenngleich in der Praxis häufig punktuell und nicht im Rahmen einer abgestimmten Konzeption betrieben, gehören unter systematischen Gesichtspunkten folgende Phasen unabdingbar zu einer PE dazu (Staufenbiel, 1999; Holling \& Liepmann, 2004):

1. Bedarfsanalyse: 
- Organisationsanalyse (u. a. Festlegung der Grobziele, Prüfung der Indikation und alternativer Maßnahmen);

- Analyse des Soll-Zustandes (u. a. Analyse der Aufgaben- und Qualifikationsanforderungen, individuelle Laufbahnberatungen);

- Analyse des Ist-Zustandes (u. a. Eignungsdiagnostik, Leistungsbeurteilung, Assessment Center, $360^{\circ}$-Feedback);

- Soll-Ist-Vergleich und ggf. Interventionsplanung;

2. Interventionen (u. a. Durchführung von Maßnahmen, s. u.; Gestaltung der Kontextbedingungen);

3. Evaluation (u. a. Festlegung von Erfolgskriterien, Versuchsplanung und Datenauswertung).

\section{Methoden der Personalentwicklung (Holling \& Liepmann, 2004, S. 359)}

- traditionelle Unterrichtsformen (Frontalunterricht, Vortrag) mit oder ohne Einbezug von Gruppendiskussionen und/oder Übungsaufgaben in Form von Einzel- oder Kleingruppenarbeit,

- $\quad$ individuelle Aneignung von Wissen und Fertigkeiten (z. B. Studium von Fachliteratur),

- computergestütztes Training (mit unterschiedlicher Interaktivität - vorwiegend für die Vermittlung von Wissensinhalten und kognitiven Fertigkeiten eingesetzt),

- Vier-Stufen-Methode (Vorbereitung, Vorführung, Ausführung und Abschluss - vorwiegend zum Erwerb einfacher Tätigkeiten eingesetzt),

- Cognitive Apprenticeship (interaktive Lernmethode zwischen Experten und Novizen - vorwiegend zum Erwerb kognitiver Strategien eingesetzt),

- Lernen anhand heuristischer Regeln (spezielle Methode des selbstgesteuerten Lernens - vorwiegend für den Erwerb allgemeiner Problemlösestrategien eingesetzt),

- Fallstudien, Rollen- und Planspiele (Simulationsverfahren zur Ausarbeitung realitätsnaher Lösungsvorschläge, Einstellungs- und Verhaltensänderung),

- Sensitivitätstraining (gruppendynamisches Verfahren zur Förderung sensiblerer Selbst- und Fremdwahrnehmung),

- Verhaltensmodellierung (mit den Komponenten Modellierung, Behaltensprozess, Verhaltenswiederholung, Feedback und Trainingstransfer - hochwirksame Methode zum Erlernen neuer Verhaltensweisen),

- Coaching und Mentoring (aufgabenbezogene bzw. generelle Kompetenzentwicklung auf der Basis persönlicher Unterstützung durch Coach bzw. Mentor). 
Angesichts der abnehmenden Halbwertzeit von Wissensinhalten kommt dem Lernen des Lernens und kognitiven Heuristiken oder anderen generellen Strategien der Personalentwicklung immer größere Bedeutung zu. Dabei besteht jedoch in der entsprechenden Grundlagenliteratur mittlerweile Einmütigkeit darüber, dass das Erlernen effektiver Problemlösestrategien nur domänenspezifisch, d. h. anhand bestehender Wissensbestände auf einem bestimmten Gebiet erfolgen kann (a.a.O., S. 354). Inhaltsleere Trockenübungen zur Vermittlung bestimmter Strategien oder Techniken zur Problemlösung sind demzufolge relativ nutzlos.

Betont werden muss auch, dass es sich bei den so genannten Schlüsselkompetenzen, deren Entwicklung zahlreiche PE-Veranstaltungen explizit gewidmet sind, um populärwissenschaftlich umbenannte generelle Persönlichkeitsmerkmale handelt, die zu verändern im Rahmen zeitlich beschränkter Fördersettings für relativ aussichtslos gehalten wird (a.a.O., S. 352f). Personen, die nur über Minimalausprägungen dieser Merkmale verfügen, sind möglicherweise mit Selektions- und Platzierungsinterventionen besser bedient.

Metaanalysen zur Evaluation von PE-Maßnahmen belegen im Übrigen eine relativ hohe Wirksamkeit, namentlich von Trainings- und Anleitungsmaßnahmen. Effektive und nachhaltige PE bedarf jedoch einer Einbettung in ein umfassendes Personalmanagement, um sicherzustellen, dass erworbene Kompetenzen auch im beruflichen Alltag abgerufen werden können.

Die Affinität von PE-Konzepten zum Qualitätsmanagement ist außerordentlich stark und wird auch in der produzierenden Erwerbswirtschaft anerkannt:

\footnotetext{
"Je weiter sich ... das Produkt der Arbeit vom Rohstoff entfernt, je höher also sein Veredelungsgrad ist, desto ausschließlicher bestimmt die Güte der Arbeit die Güte des Resultats. In einem rohstoffarmen, hochindustrialisierten Land wie Deutschland ... wird Qualitätsmanagement folglich immer Personalmanagement sein. Die Qualifikation des einzelnen ist es, die hier in hohem Maße über den Erfolg des Gesamten bestimmt" (Ehrhart, o. J., S. 4).
}

Personalentwicklung bzw. Human Resource Management als übergeordneter Begriff, der auch Maßnahmen wie Selektion und Platzierung umfasst (s. o.), gehört damit zu den Kernbereichen eines QM, zumal in sozialwirtschaftlichen Organisationen. 


\subsection{Qualitätsmanagement und Evaluation}

Unter dem Begriff der Evaluation werden ziel- und zweckorientierte Verfahren zur Beschreibung und Bewertung von Gegenständen der sozialen Wirklichkeit (z. B. Ferienfreizeiten, Unterrichtsprogramme, Projekte) zusammengefasst (vgl. Beywl \& ScheppWinter, 2000, S. 17). Dabei kommen quantitative und qualitative Methoden der empirischen Sozialforschung zum Einsatz.

Schwerpunktmäßig soll Evaluation der Optimierung des untersuchten Gegenstandes dienen, z. B. eines laufenden Programms (formative oder Gestaltungsevaluation) oder der Berichterstattung und Entscheidung über diesen Gegenstand (summative oder Bilanzevaluation).

Evaluationen werden mithin zum Zwecke der

o Planungsverbesserung (z. B.: "Was müssen wir noch berücksichtigen?"),

o Entscheidungsfindung (z. B.: "Welche von zwei Alternativen ist die zweckmäßigere?"),

o Praxisoptimierung (z. B.: "Was können wir anders und besser machen?") und/oder

o Rechenschaftslegung (z. B.: "Was haben wir getan und wie ist es angekommen?") durchgeführt.

Beteiligte und Betroffene, Anlass, Zweck, Fragestellung, Auftraggeber, Umfang, Anspruchsniveau, Art der Datenerfassung und -auswertung sowie Darstellung und Verwendung der Ergebnisse beeinflussen den Charakter der Evaluation (vgl. Gerull, 1999a, 2000).

Sie kann als Fremdevaluation von Personen betrieben oder in Auftrag gegeben werden, die nicht mit der evaluierten Maßnahme selbst befasst sind, z. B. Vorgesetzte. Solche Evaluationen lösen oftmals Ängste und Widerstände aus, weil sich davon Betroffene ausgeforscht und von negativen Konsequenzen bedroht fühlen. Hier sind sensibles und faires Vorgehen gefordert (Datenschutz, Transparenz der Absichten).

Interne Evaluationen sind solche Beschreibungen und Bewertungen, die von Mitgliedern der Organisation selbst durchgeführt werden. Die Ergebnisse sind zunächst für interne Zwecke vorgesehen, können aber auch publiziert werden, z. B. im Rahmen von Sach- oder 
Rechenschaftsberichten.

Sind die evaluierenden Personen mit jenen identisch, die das zu evaluierende Programm durchführen, sprechen wir von Selbstevaluation; diese ist also eine Sonderform der internen Evaluation. Dabei liegt die Kontrolle über Planung, Durchführung und Nutzung bei den Fachkräften selbst und wird auf freiwilliger Basis wahrgenommen.

Interne Fremdevaluationen werden von eigens beauftragten Mitarbeiter(inne)n oder Vorgesetzten durchgeführt, die hinreichende Distanz und Unabhängigkeit zur evaluierten Maßnahme haben.

Die verschiedenen Ansätze lassen sich auf einem Kontinuum zwischen Fremdbewertung (externer Evaluation) und Selbstbewertung (interner Evaluation) positionieren: Evaluation zwischen kritischem Experten-Blick von außen und Selbstbeobachtung (vgl. Beywl, 1999).

Von Supervision, kollegialer Beratung, Fallkonferenzen und anderen Formen der Praxisreflexion und Qualitätsentwicklung unterscheidet sich Evaluation durch das Ausmaß empirischer Datenbasierung. Auch die schriftliche Fixierung und Aufbereitung der Daten in Berichtsform ist für Evaluationen kennzeichnend.

Die Entscheidung für externe, interne Fremd- oder Selbstevaluation hängt unter anderem von der Zielsetzung, aber auch von den verfügbaren Ressourcen ab. Für Evaluationen allgemein gilt, dass sie nur dann zu rechtfertigen sind, wenn sie keiner pseudowissenschaftlichen Selbstgewissheit Vorschub leisten, sondern methodische Mindestansprüche erfüllen (zu Evaluationsstandards s. Sanders, 1999). Sowohl der Fremd- als auch der Selbstevaluation sind spezifische Vor- und Nachteile zu Eigen (vgl. Gerull, 2000; Koch, 2000; von Spiegel, 2001).

Um den Primärprozess in den Einrichtungen selbstevaluativ zu begleiten, ohne die Fachkräfte der zusätzlichen Belastung durch Entwicklungsarbeiten auszusetzen, sind Checklisten und strukturierte Fragebögen zweckmäßig, die auf der Ebene der MitarbeiterInnen ausgewertet werden können und Selbststeuerungsprozesse unterstützen. Dem Vorteil größerer Ökonomie steht allerdings das Risiko einer möglichen Deprofessionalisierung gegenüber, wenn Fachkräfte sich des eigenen Reflexionsaufwandes durch 
Abarbeiten vorgefertigter Checklisten entledigen können (von Spiegel, Sturzenhecker \& Deinet, 2002, S. 251).

In der gängigen Evaluationsforschung ist - wie R. Lang (o. J., S. 48) kritisch anmerkt - die in den Sozialwissenschaften verbreitete fortschrittsoptimistische Vorstellung enthalten, dass "die Entwicklung individuellen und gemeinschaftlichen Lebens in Quantitäten kodifizierbar und i. S. unbegrenzter Machbarkeit in Richtung auf einen Idealzustand wissenschaftlich zu planen und zu optimieren sei." Dagegen charakterisieren Wottawa \& Thierau (1990, S. 16) Evaluationen vielmehr als "Übelminimierung statt Ideallösung":

"Die Evaluation kann dazu dienen, innerhalb eines wissenschaftsexternen, vorläufigen und in gewissen Grenzen willkürlichen Rahmens die Wahrscheinlichkeit für die Auswahl einer besonders guten Verhaltensalternative zu erhöhen und analog dazu die Wahl einer besonders schlechten Alternative zu verringern" (a.a.O.).

R. Lang (a.a.O., S. 54) plädiert für eine Evaluationsforschung, die nicht Hypothesen und Wirkungen im Sinne der Produktorientierung prüft, sondern ihr Augenmerk im Sinne einer Prozessorientierung auf qualitative Veränderungen in der Ausführung einer Maßnahme und im Verhalten der Zielpopulation innerhalb ihres jeweiligen lebensweltlichen Kontextes richtet und untersucht, was im Untersuchungsfeld passiert.

"Dadurch können Eingriffs- und Handlungsmöglichkeiten von ProgrammitarbeiterInnen und TeilnehmerInnen qualifiziert und Zusammenhänge zwischen institutionellen Rahmenbedingungen, vorhandenen Ressourcen, sozialpädagogischem Konzept und tatsächlicher Umsetzung ebenso transparent werden, wie die Unterscheidung von Handeln und Verantwortung der Professionellen bzw. der Träger einerseits und strukturbedingten Wirkungen von Programmen und Maßnahmen andererseits."

In diesem Kontext rücken so genannte responsive (Müller-Kohlenberg, 1997), experimentierende (Heiner, 1998) oder nutzenfokussierte (Beywl, 1999) Verfahren der Evaluation, welche die Beteiligtengruppen einbeziehen, in den Mittelpunkt. Dabei geht es mehr um Ziele wie Qualitätsentwicklung, Praxisoptimierung und Innovation statt um Kontrolle, Legitimationsnachweis und Selektion.

Auch Patton beklagt, dass meistenteils nur die Ergebnisse im Vordergrund stehen, wenn von Evaluation und deren Nutzen die Rede sei (1998, S. 56). Dabei habe Evaluation 
vielfach einen ganz anderen Nutzen, den Patton als Prozessnutzen bezeichnet. Während nämlich Ergebnisse eine sehr kurze Halbwertzeit und nur ein schmales "Zeitfenster der Relevanz" besäßen, könne das während der Evaluation bei den Beteiligten und Betroffenen angeregte Lernen, evaluativ zu denken, nachhaltigen Einfluss auf ihr Handeln ausüben und Organisationen dabei helfen, das zu werden, was populär "lernende Organisation" genannt werde (S. 57).

Wenngleich in QM-Systemen ingenieurwissenschaftlicher Provenienz dem Bereich quantitativer Messung und Analyse traditionell besondere Aufmerksamkeit geschenkt wird - dort allerdings nicht "Evaluation" genannt -, sind es die zuletzt betonten Entwicklungen im Bereich der Evaluationsforschung, die eine zunehmende Konvergenz mit QMKonzepten im Sozialbereich erkennen lassen.

Explizit vergleicht Pollitt (2000, S. 62 ff.) die beiden Ansätze und betont folgende Ähnlichkeiten zwischen Evaluation und Qualitätsmanagement:

o Beide Verfahrensweisen sind amerikanischen Ursprungs.

o So wie viele Versuche zur Einrichtung von Qualitätsverbesserungssystemen mehr oder weniger fehlschlagen, ermangelt es auch an Beweisen, dass Evaluationen einen wirksamen Einfluss auf Schlüsselentscheidungen ausüben.

o Die Ausrichtung auf Kunden, Interessenpartner oder stakeholder ist beiden gemeinsam.

o Häufig sind in beiden Bereichen externe Beratungsagenturen tätig, obwohl die Aufgaben auch von eigenem Personal der betreffenden Organisation ausgeführt werden könnten.

o Sowohl Evaluation als auch Qualitätsmanagement stehen in einem potenziellen Spannungsverhältnis zur beruflichen Autonomie der Betroffenen.

o Beide Ansätze sind zu einem Symbol der Modernisierung geworden und schließen eine starke rhetorische und rituelle Dimension mit ein.

Es werden jedoch auch Unterschiede verdeutlicht (a.a.O., S. 65 ff.):

o Qualitätsverbesserungsansätze entwickelten sich hauptsächlich im privatwirtschaftlichen Sektor; Evaluationsverfahren hatten stets ein öffentliches Grundprinzip. 
o Qualitätsmanagement ist prinzipiell ein kontinuierlich ablaufendes, integriertes Programm; Evaluation ist eine periodische Aktivität, die von routinemäßiger Kontrolle zu unterscheiden ist.

o Qualitätsverbesserung findet innerhalb der Organisation statt; Evaluation wird zumeist in Form einer externen Visitation durchgeführt, zumindest aber mit notwendiger Distanz und Neutralität.

o Qualitätsverbesserung ist integraler Bestandteil des Managements; Evaluationen nehmen eine andere Perspektive ein, die auch andere Gruppen von Betroffenen einbezieht.

o Qualitätsverbesserung ist eng mit dem Begriff der Innovation verknüpft; diese Beziehung besteht bei traditionellen Evaluationsformen nur indirekt.

Pollitt (S. 68) moniert, dass es kein einheitliches oder standardisiertes Phänomen gebe, das man entweder als Evaluation oder Qualitätsmanagement bezeichnen könne. Zwischen den Vertretern traditioneller, wissenschaftsorientierter Evaluation und den Propagandisten kooperativer, auf den Nutzen der Betroffenen fokussierender Evaluationsformen (s. o.) werden mitunter "heiße Kämpfe" konstatiert, wie sie - mutatis mutandis - auch zwischen den unterschiedlichen Systemen des Qualitätsmanagements stattfänden.

\section{5 Controlling und Balanced Scorecard}

Unter dem Begriff des Controlling wird allgemein die von der Unternehmensführung ausgeübte Steuerungsfunktion verstanden (Dudenredaktion, 2000). Bis in die Sechzigerjahre waren Budgetkontrolle und Soll-Ist-Vergleiche im Rahmen des Rechnungswesens eine Domäne des Controllings. Danach setzte jedoch ein Wandel im Aufgabenverständnis ein, indem auch Planung, Zukunftssteuerung und Strategieentwicklung einbezogen wurden. Controlling in diesem Sinne ist ein Instrument der zielorientierten und vorausschauenden Unternehmensführung (Matul, 1998, S. 4f).

Neben Planungsaufgaben und Fragen der Zielvereinbarung hat Controlling auch die Zielerreichung zu überwachen und im Abweichungsfalle Maßnahmen zur Abhilfe vorzuschlagen. Insofern ist Controlling auch Kontrolle und prüft unter anderem den wirtschaftlichen Einsatz der Ressourcen. Es geht jedoch stets weniger um nachträgliche 
Rechenschaftslegung als vielmehr darum, dem Management erforderliche Korrekturmaßnahmen anzuzeigen und die Koordination des Leistungsgeschehens auf die angestrebten Ziele hin zu verbessern. So verstanden, ist Controlling "Selbststeuerung der Organisation durch systematische Differenzbeobachtung" (Halfar, 2000, S. 71).

Controlling bedeutet zum einen Informationsbeschaffung (z. B. durch Ist-Soll-Vergleiche, Kennzahlen, Rechnungswesen, Leistungserfassung, Aktenauswertung, Organisations- und Marktanalyse), zum anderen Informationsaufbereitung und -verwendung (z. B. Berichterstattung, Planung, Beratung). Die systematische, verständliche und zeitnahe Zusammenstellung von steuerungsrelevanten Daten soll Transparenz und Vertrauen schaffen.

Unterschieden werden die Ebenen des

- $\quad$ normativen $(\Rightarrow$ Unternehmensethik),

- $\quad$ strategischen $(\Rightarrow$ Unternehmensentwicklung) und

- operativen $(\Rightarrow$ Unternehmenseffizienz)

Controllings (vgl. Wieneke, 1993). Normatives Management wirkt begründend; es befasst sich mit Sinn und Zweck der Organisation und hat ihre Lebens- und Entwicklungsfähigkeit zum Ziel (Seghezzi, 2003, S. 66). Aufgaben des Controllings betreffen hier Aspekte der Organisationskultur und -politik, wie sie häufig in einem Leitbild formuliert sind. In der Praxis kaum davon zu trennen ist das strategische Controlling, das sich mit langfristigen Umsetzungsprogrammen für die normativen Ziele beschäftigt. Dabei ist es notwendig, mögliche Entwicklungen im Umfeld zu berücksichtigen und potenzielle Chancen rechtzeitig in das unternehmerische Handeln zu integrieren. Für wichtig wird auch befunden, die Kernkompetenz einer Organisation zu klären und die Unterschiede zu anderen Leistungsanbietern herauszuarbeiten (Matul, 1998, S. 6). Operatives Controlling fordert soziale Dienstleister auf, die strategischen Ziele zu operationalisieren, d. h. in konkrete Sachverhalte und Handlungen umzumünzen. Es sind Produktentscheidungen zu treffen, welche Leistungen in welcher Qualität zu welchem Preis welchen KlientInnengruppen angeboten werden (a.a.O., S. 7). 
( $\Rightarrow$ Leistungsvereinbarung), einer Ermittlung der zur Verwirklichung benötigten Ressourcen und Infrastrukturen $(\Rightarrow$ Entgeltvereinbarung) und einer Konzeption über die Mittel und Wege, die versprochenen Qualitätsstandards zu gewährleisten ( $\Rightarrow$ Qualitätsentwicklungsvereinbarung).

Obwohl in größeren Betrieben das Controlling in der Regel als Stabsstelle der Geschäftsführung angesiedelt ist, geht es im weiteren Sinne darum, Controlling als Denkhaltung in die Handlungsprozesse zu integrieren (Matul, 1998, S. 7f). Controlling im Rahmen des QM will programmatisch möglichst alle MitarbeiterInnen zum verantwortungsvollen und effektiven Umgang mit knappen Ressourcen motivieren und die angelegten Qualitätsmaßstäbe auf breiter Front verwirklichen. Controlling vollzieht sich deshalb in regelmäßigen Datenerhebungen und Auswertungen und nicht in "Feuerwehreinsätzen" (Schramm, 1994, S. 27).

In der Betriebswirtschaft werden Instrumente zur Deckung des entsprechenden Informationsbedarfs unter den Stichworten Kennzahlen und Frühwarnsysteme behandelt. Analog zu der geläufigen Praxis, finanzwirtschaftliche Kennzahlen zur Abbildung der Vermögens- und Kapitalstruktur einer Einrichtung zu verwenden, sind zunehmend auch im Bereich der "weichen Daten" Indikatoren gefragt, die einen betriebsinternen und -externen Vergleich, z. B. verschiedener Geschäftsperioden, ermöglichen sollen.

Aus dem Gesamtkonzept des Controllings lassen sich die Teilbereiche Personal-, Leistungs- (incl. Qualitäts-) und Finanzcontrolling differenzieren. Personalcontrolling bedeutet im Wesentlichen Führung durch Zielvereinbarung. Die gesamtorganisatorischen Ziele sind so "herunterzubrechen", dass sich spezifische Zieldefinitionen bzw. Aufgabenprofile für jede Abteilung und jede Stelle ergeben. Anzustreben sind verbindliche Verhaltensregeln und Ziele, die ein regelmäßiges Feedback über den Erfolg der eigenen Arbeit ermöglichen und damit eine Standortbestimmung und Selbstkontrolle für die Mitarbeiter(innen) (Matul, 1998, S. 10f). Aufgabe des Leistungscontrollings ist es, Wirkungsziele, also beabsichtigte Ergebnisse, festzulegen und mit Hilfe geeigneter Indikatoren überprüfbar zu machen. Analoge Aufgaben stellen sich im QM: Welche fachlichen Qualitätsziele sind vereinbart (z. B. "Verselbständigung") und woran ist zu erkennen, ob und in welchem Maße die Ziele erreicht sind?" Das Finanzcontrolling schließlich befasst sich mit der Ermittlung angefallener und geplanter Kosten, differenziert nach Kostenstellen, -arten und -trägern. Des Weiteren dient das Finanzcontrolling der Sicherstellung der Liquidität, der Optimierung der Kredit- und Darlehensstruktur und eines 
effizienten Forderungsmanagements. Wichtig ist, dass entsprechende Daten zeitnah zurückgemeldet werden, um Steuerungseffekte möglich zu machen (a.a.O.).

Auf eine Begrenzung des Controlling-Konzepts sei ausdrücklich hingewiesen: Die in diesem Rahmen ermittelten und verwendeten Daten bilden eine notwendige und gute empirische Basis für kognitives Lernen der Organisationsmitglieder. Eine für deren Verhalten oftmals bedeutsamere Grundlage für "emotionales Lernen" und Einstellungsänderungen bietet das Konzept zumeist nicht bzw. nur in geringem Maße (Schöpflin, 2000, Kap. 4.2.12). Controlling bedient primär die rationale Ebene der Unternehmensführung und bildet im Rahmen des komplexen Organisationsgeschehens nur einen Teilaspekt ab. Anforderungen an eine lernende Organisation und eine von allen stakeholdern getragene Unternehmenskultur gehen über die Leistungsfähigkeit eines strategischen und operativen Controllings noch hinaus und beziehen normative Aspekte mit ein.

Diesem Erfordernis will das Konzept der Balanced Scorecard (BSC) stärker Rechnung tragen.

Der Begriff der Balanced Scorecard wurde Anfang der Neunzigerjahre von amerikanischen Wissenschaftlern (Kaplan \& Norton, 1997) zur Beschreibung eines mehrdimensionalen Kennzahlensystems geprägt. Ausgangspunkt war die Überlegung, wie ein Unternehmen möglichst zweckmäßig zu bewerten sei und mit wenigen, aber sehr aussagekräftigen Kennzahlen beschrieben und gesteuert werden könne.

Die BSC beruht auf der Annahme, dass eindimensionale Bewertungen - wie sie in Unternehmen traditionell vor allem auf der Basis von finanziellen Messgrößen stattfinden - der Realität nicht gerecht werden. Deshalb werden weitere Dimensionen in die Beschreibung und Bewertung einbezogen und in Form einer Karte mit allen wesentlichen Kennzahlen und Zusammenhängen schriftlich aufbereitet. Balanced Scorecard lässt sich daher am besten mit "ausgewogener Auswertungsbogen" oder "austariertes Zielsystem" übersetzen.

Daten aus vier Bereichen werden als besonders relevant für diesen Bogen ausgewählt und dienen als Frühindikatoren für Entwicklungstendenzen, aber auch als Anstöße für Veränderungen (vgl. Kirstein, o. J.): 
- finanzielle/wirtschaftliche Daten,

- interne Prozessdaten/Geschäftsprozesse,

- Daten aus Kundensicht und

- Informationen über Innovations- und Lernfähigkeit des Unternehmens.

Während die finanzielle Sicht vor allem die Interessen der Aktionäre (bei NPO sind analog primär die Träger zu nennen) berücksichtigt, steht bei der internen Sicht die Frage im Mittelpunkt: Welche Prozesse sind wichtig? Die Kunden-Sicht vermittelt Daten darüber, wie die Leistungen des Unternehmens bei den Adressaten ankommen und wahrgenommen werden. Der Aspekt Innovation und Lernen schließlich repräsentiert die Kreativität und das Verbesserungspotenzial des Unternehmens bzw. der sozialen Einrichtung.

In einer strategischen Ausrichtung des Unternehmens werden die wesentlichen Kenngrößen auf diesen vier Gebieten ausgewählt und als verbindlich festgelegt. Danach werden zu jedem Gebiet entsprechende Messgrößen erarbeitet, die es gestatten, Ergebnisse und Fortschritte zu ermitteln. Durch die Messung werden die Kenngrößen quantifiziert, so dass sich Anhaltspunkte für die Steuerung des Unternehmens ergeben (a.a.O.). Diese strategische Ausrichtung ist der zentrale Handlungsschritt bei Einführung der BSC. Eine Organisation gilt dann als "ausgerichtet", wenn die Ziele aller Arbeitsprozesse klar mit den gemeinsamen Zielen gemäß der Unternehmens-Mission verbunden sind. Die BSC stellt dabei ein wichtiges Kommunikationsmittel innerhalb des Unternehmens dar, um die Ausrichtung aller Abteilungen auf die gemeinsamen Ziele zu befördern. Im Rahmen dieser Ausrichtung kommt es besonders auf die Vermittlung der strategischen Ziele und ihre Übersetzung in Kenndaten für jede Ebene an. Der Prozess, diese Daten regelmäßig zu allen erfolgsrelevanten Schlüsselgrößen des Unternehmens zu erheben und in Form einer Scorecard darzustellen, ist damit von entscheidender Bedeutung.

Die Entwicklung einer Balanced Scorecard erfolgt in der Praxis häufig in sechs Stufen:

(1) partizipative Formulierung der Unternehmensvision,

(2) Ableitung der strategischen Ziele für die vier Kennzahlenbereiche (Perspektiven),

(3) Abbildung der strategischen Ziele in einem Ursache-Wirkungs-Modell (Wirkungskette), 
(4) Entwicklung einer Messgröße für jedes strategische Ziel,

(5) Festlegung der operativen Ziele,

(6) Entwicklung von Initiativen.

Einführungsdauer und Kosten hängen von den betrieblichen Voraussetzungen ab, z. B. der Existenz eines Leitbildes und der Vertrautheit mit strategischer Planung und finanziellem Controlling (C. Koch, 2003, S. 20f). Im Gegensatz zum Benchmarking (s. Kap. C 2.2.5), bei dem die Einrichtungen Daten erheben und für einen Vergleich mit anderen Betrieben oder Betriebsteilen zur Verfügung stellen, sind BSC-Daten auf das Unternehmen selbst und dessen individuelle Intentionen ausgerichtet (Offermann, 2001a, S. 110). BSC können deshalb auch nicht standardisiert erstellt werden (Eisenreich, 2000, S. 4-197).

Der Ansatz der BSC wird als besonders interessant für Unternehmen eingeschätzt, die nicht mit herkömmlichen finanziellen Verfahren angemessen bewertet werden können, also Unternehmen, deren Erfolg auf intensiver Kundenbindung beruht, die besonders innovativ sind und bei denen das Know-how der MitarbeiterInnen ein entscheidender Produktionsfaktor ist (vgl. Friedag, o. J.). C. Koch (2003, S. 18) weist jedoch darauf hin, dass auch mit BSC das Problem bestehen bleibe, Indikatoren für nicht metrische Eigenschaften zu finden und zu diesen Datenmaterial zu beschaffen.

Ob die BSC im Übrigen als Qualitätsmanagement-Kontextkonzept (Zollondz, 2002, S. 316 ff.), Qualitätstechnik oder eigenständiger Managementansatz betrachtet wird, dem sich Elemente des QM zu- und unterordnen lassen, hängt vom Standpunkt ab.

\subsection{Mitarbeiterbeteiligung und Empowerment}

Das Konzept der Mitarbeiterbeteiligung ist in der Organisationsforschung wie kaum ein anderes mit normativen, moralischen und ideologischen Ansprüchen belastet (Antoni, 1999, S. 569). Programmatisch als Kriterium humaner Arbeit gefordert und rechtlich verbrieft im Rahmen des Betriebsverfassungs- und Mitbestimmungsgesetzes, wurde Mitarbeiterbeteiligung auch als eines der "Geheimnisse" japanischen Wirtschaftserfolgs entdeckt und als Mittel zur Erhöhung unternehmerischer Wertschöpfung propagiert (a.a.O.). 
Im organisationspsychologischen Kontext wird das Konzept vor allem unter den Begriffen Delegation und Partizipation untersucht. Delegation wird in der Regel als Übertragung von Zuständigkeiten, Leistungen, Befugnissen und Entscheidungskompetenzen verstanden; Partizipation meint die Teilhabe, Teilnahme oder Beteiligung an Problemlösungs- und Entscheidungsprozessen. Partizipation impliziert nach diesem Verständnis eine Einfluss- bzw. Machtteilung, während Delegation eine einseitige Einflussnahme und Machtausübung vorsieht (Leana, 1987). Die Begriffe sind häufig nicht klar voneinander abzugrenzen, z. B. dann, wenn Entscheidungsbefugnisse an eine Gruppe delegiert werden. Bezugspunkt der Delegation ist dann die Gruppe, Subjekt der Partizipation das Gruppenmitglied (Antoni, 1999, S. 571).

Zur Veranschaulichung eines Kontinuums von Mitwirkungsmöglichkeiten bezieht sich Antoni (S. 570) auf eine Abstufung von Dachler \& Wilpert (1978):

- keine Mitsprachemöglichkeiten,

- Informationsrechte,

- Vorschlagsrechte,

- Mitbestimmungsrechte,

- Vetorechte,

- völlige Autonomie.

Wenngleich die empirischen Befunde zu den Auswirkungen von Entscheidungsdelegation und autonomieorientierter Arbeitsgestaltung keineswegs einheitlich sind, zieht Antoni (a.a.O., S. 574) zu den Effekten und Wirkungsmechanismen von Mitarbeiterbeteiligung das folgende positive Fazit:

"... höhere Arbeitsleistung und Mitarbeiterzufriedenheit ... sind insbesondere bei komplexen Arbeitsaufgaben zu erwarten, wenn sich die Mitarbeiter an der Entwicklung der Bearbeitungsstrategien beteiligen und eigene Informationen einbringen können. Dies wird mit einem verbesserten vertikalen Informationsfluss, einer besseren Ausnutzung, Integration und letztlich einer Weiterentwicklung von Wissen sowie einem größeren Problem- bzw. Arbeitsverständnis seitens der Mitarbeiter erklärt ... Die Einbindung von Mitarbeitern in Planungsprozesse erleichtert die Entwicklung aufgabenangemessener Bearbeitungsstrategien und Handlungsschemata. Diese ermöglichen es den Mitarbeitern, auf unerwartete Störungen oder eine Veränderung der Rahmenbedingungen frühzeitig (antizipativ) und adäquat zu reagieren und gegegebenfalls alternative Bearbeitungswege zu entwickeln und einzuschlagen, ohne ständig Rücksprache mit dem Vorgesetzten nehmen zu müssen. Damit sind auch wesentliche Voraussetzungen für die effektive Delegation von Aufgaben mit Planungs-, Entscheidungs- und Kontrollkompetenzen gegeben." 
Wie Scholl (2004, S. 546) betont, sind die Vorteile partizipativer Entscheidungen für die Beteiligten und die Organisation seit Langem bekannt, so dass es zunächst verwunderlich erscheint, warum diese Vorteile immer wieder neu entdeckt werden müssen und in der Praxis so oft vernachlässigt werden. Im Hinblick auf die erforderlichen Veränderungen von Machtstrukturen in Organisationen kann man allerdings vermuten, dass die Propagierung solch anspruchsvoller Konzepte oft nur ein zeitgeistiges Lippenbekenntnis ist und Führungskräfte nicht wirklich an einer Teilung ihrer Macht interessiert sind. Auf dem Verordnungswege und im Rahmen eines technizistischen Verständnisses von Führung lassen sich jedenfalls entsprechende Veränderungen der Organisationskultur nicht bewerkstelligen; vielmehr scheint es sinnvoll, Konzepte der Mitarbeiterbeteiligung auch partizipativ zu entwickeln und umzusetzen (Antoni, 1999, S. 581).

Auf Grenzen der Partizipation, was die Kompensierung potenziell mit Organisationsveränderungen einhergehender Verluste anbetrifft, wurde bereits beim Stichwort "Organisationsentwicklung" hingewiesen (s. Kap. B 2.1).

Welch entscheidende Rolle die Mitarbeiterbeteiligung für die erfolgreiche Umsetzung von QM-Systemen spielt, verdeutlicht ein Kommentar von Zollondz,(2002):

Mit Bezug auf die Kybernetik und ihre Unterscheidung von wohl-definierten (trivialen) und schlechtdefinierten (nicht-trivialen) Systemen wird betont ( $S .351$ ff.), dass Menschen schlecht-definierte Systeme seien. QM sei nun vermeintlich das ideale Werkzeug, die [aus diesem Grunde, P. G.] zahllosen Fehlerquellen zu eliminieren. Mittels TQM wohl-definierte, rational reagierende Systeme zu schaffen und nichts dem Zufall überlassen zu wollen, wird jedoch nicht für erstrebenswert gehalten. Zwar würden viele Organisationen genau dies intendieren, müssten aber nach der Zertifizierung merken, dass nicht alles "wie geschmiert" laufe, weil die Menschen nicht mitspielten. MitarbeiterInnen wollten nämlich nicht wohl-definiert sein, sondern herausgefordert werden, sie wollten selbst mitdefinieren, mitentscheiden, mitverantworten.

Mit dieser Bemerkung zu den Grenzen vorgegebener, standardisierter Handlungsabläufe im Rahmen des QM (s. auch Kap. C 3.) ist eine Brücke zu einem Konzept geschlagen, das sich in Profit- und Nonprofit-Organisationen gleichermaßen großer Aktualität erfreut: Empowerment.

Mit dem Begriff des Empowerment wird allgemein die Er- oder Bemächtigung bzw. Befähigung von Menschen bezeichnet. Es handelt sich einerseits um ein Konzept zur 
Beschreibung psychologischer Konstrukte (z. B. Gefühle und Einstellungen gegenüber einer Organisation oder Rolle), andererseits um ein Set von Maßnahmen, diese Gefühle und Einstellungen zu fördern (vgl. Wall, Cordery \& Clegg, 2002, S. 147). Durch Einführung solcher Maßnahmen (z. B. job enrichment, Teamarbeit, TQM-Projekte) sollen Partizipation, Verantwortungsübernahme und Selbstbefähigung als wichtige humane Ressourcen erschlossen werden, um letztlich im Interesse optimierter Systemfunktionalität Ergebnisse und Zufriedenheit zu verbessern.

Wenn auch der Begriff selbst relativ neu ist, so sind wesentliche Bestandteile des Empowerment-Konzepts "alte Bekannte" der Organisationspsychologie und Betriebssoziologie (z. B. Quality of Work Life-Bewegung in den USA, Forschungen zur Arbeitszufriedenheit und zum Transparenzerleben, Mitarbeiterbeteiligung, s. o.). Nerdinger (1994, S. 271f) versteht darunter im Kern die Übertragung des herkömmlichen arbeitspsychologischen Konzepts des Handlungsspielraums auf die Situation von Dienstleistern. Dem nunmehr stark popularisierten Ansatz wird im Wirtschaftsbereich ein großes Potenzial unterstellt, mitunternehmerisches Denken und Handeln auf allen betrieblichen Ebenen zu entwickeln und damit den Herausforderungen einer sich ständig wandelnden Organisationsumwelt zu begegnen.

Auch im Sozialbereich wurde das Empowerment-Konzept von der professionellen Kultur wohlwollend aufgenommen,

- um Selbstkontrolle kranker Menschen und Unabhängigkeit von professionellen Versorgern zu fördern (Hellerich \& White, 2003, S. 36),

- burning out von SozialarbeiterInnen vorzubeugen (Flösser \& Otto, 1992, S. 13; bezogen auf Transparenz und Mitwirkung an der organisationellen Entscheidungsbildung),

- Potenziale der Selbstorganisation und des gemeinschaftlichen Handelns zu fördern (Stark, 1996, S. 159), 
- bürgergesellschaftliche Antworten auf neue soziale und demokratische Fragen zu liefern (Keupp, 1998),

- Qualitätsmanagement in den Einrichtungen und Diensten lebendig umzusetzen (Bobzien, Stark \& Straus, 1996, S. 24).

Kritik am Ansatz des Empowerments wird zum einen aus Sicht der Praxis geübt (Hellerich \& White, 2003, S. 37), die auf zahlreiche Umsetzungsprobleme und Widersprüchlichkeiten hinweist, darunter auch im Zusammenhang mit dem Compliance genannten Sachverhalt, bei dem sich der Anspruch auf Selbstbefähigung an moderner professioneller Rationalität stoße (hier: Befolgung ärztlicher Anweisungen). Zum anderen wird Kritik am methodischen Individualismus des Empowerment-Konzepts geübt, in dem sich ein Wandel in den Strategien der Macht und Mächtigen widerspiegele (a.a.O., S. 37).

Unter Bezug auf Foucault (1976), der vom Wandel einer repressiven zu einer produktiven Machtstrategie spricht, konstatieren Hellerich \& White (S. 38): "Die Machtsuchenden werden durch EmpowermentStrategien kontrolliert, die ihre machtbetonten Bestrebungen in die gesellschaftlich akzeptablen Formen der Selbstverwirklichung und steigender Arbeitsproduktivität kanalisieren."

Am Beispiel der Psychiatrie-Selbsthilfebewegung wird zu zeigen versucht, wie stattdessen ein postmodernes Verständnis von Empowerment als Modell einer selbstbestimmten Subjektivität und sozialen Ökologie der Beziehungen funktionieren könnte (a.a.O., S. 40f).

Wall et al. (2002) weisen in einer theoretischen Untersuchung nach, dass die universalistische Annahme einer Leistungssteigerung durch Empowerment nicht zutrifft und die Effektivität von Empowerment-Maßnahmen kontextabhängig und kontingent zum Ausmaß der bestehenden "operational uncertainty" ist. (Nerdinger, 1994, S. 274, spricht in gleichem Zusammenhang von "riskanten" Dienstleistungen.) Solche Unsicherheit resultiert in Variabilität und Mangel an Vorhersehbarkeit in den Arbeitsvollzügen und Anforderungen, einschließlich der Frage, was zu tun ist und auf welche Weise (Wall et al., a.a.O., S. 151). Angesichts der noch fehlenden empirischen Evidenz formulieren die Autoren ein vorsichtiges Fazit:

\footnotetext{
"Where the focus is on performance, initiatives to empower employees with respect to the execution of their core task are likely to be effective given a high level of operational uncertainty, but will be of much less value (and perhaps none) where work processes are more predictable and well understood. In contrast,
} 
initiatives to empower employees with regard to wider role responsibilities are likely to yield productivity benefits irrespective of the prevailing levels of operational uncertainty" (a.a.O., S. 164).

Ähnlich argumentiert Argyris (1998). Ausgehend von einer begrifflichen Unterscheidung von außen- und innengeleitetem Engagement, betont er, dass äußeres Engagement als vertraglich verabredete Pflichterfüllung eine Form des Anpassungsverhaltens darstelle, mit dem viele Mitarbeiter in den meisten Arbeitsumgebungen bestens zurechtkommen (a.a.O., S. 12). Am Beispiel des Reengineerings, das sich programmatisch zumeist dem Empowerment verwandter Floskeln bedient, weist Argyris (S. 9) darauf hin, dass Forschung und Praxis schließen lassen, diese Form des Transformationsmanagements sei dann am erfolgreichsten, wenn Aufgaben genauestens vorgegeben werden und nicht, wenn einzelne Mitarbeiter die Freiheit haben, sie selbst zu bestimmen. Empowerment impliziere dagegen ein hohes Maß an Partizipation und innengeleitetem Engagement, das als Ziel für Organisationen von hohem Wert sei, ohne je ganz erreicht werden zu können (S. 10).

Ungeachtet aller "schönen Worte über Wandel und Organisationsentwicklung" (S. 15), wird die Praxis der Change-Programme insgesamt als trübe bezeichnet. In Bezug auf Empowerment-Projekte belegt Argyris an zahlreichen Beispielen, dass die Idee eines inneren, intrinsisch geleiteten Engagements keineswegs auf ungeteilte Gegenliebe bei MitarbeiterInnen und Vorgesetzten stößt. Für jene verbinde sich damit neben der Aussicht auf mehr Verantwortung und Entscheidungsfreiheit auch Mehrarbeit und Rechenschaftspflicht, diese vertrauten häufig doch lieber dem altbekannten Führungsmodell samt seinen Anweisungen und Kontrollen. Vor allem in Unternehmen, in denen langjährig äußeres Engagement praktiziert und durch verschiedenste Anreizsysteme (z. B. höhere Vergütung, bessere Aufstiegsmöglichkeiten, "Mitarbeiter des Monats") begünstigt wurde, werden er-hebliche Schwierigkeiten konstatiert, Eigeninitiative und Sinn für persönliche Verantwor-tung zu entwickeln. Auch die Rolle von Beratern für OE (change agents) wird in diesem Zusammenhang problematisiert. Dadurch, dass die Grenzen und Widersprüchlichkeiten des Ansatzes nicht offen eingeräumt würden und Berater Managern nicht mit praktischen Ratschlägen zur Seite stünden, aus den resultierenden Zwickmühlen herauszukommen, würden viele Projekte nach anfänglicher Begeisterung in Schwierigkeiten geraten (S. 14).

Argyris (S. 15) beklagt, dass Maßnahmen zu mehr Empowerment allzu häufig in den Dunstkreis politischer Korrektheit gerieten und dann niemand mehr offen ausspreche, was er denke. Stattdessen sollten die Grenzen des Ansatzes und die Koexistenz äußeren und inneren Engagements bedacht werden. Argyris (S. 15f) plädiert deshalb ähnlich wie Wall et al. (2002, s. o.) dafür, nach Tätigkeiten zu differenzieren, ob eine stärkere Ermächtigung der MitarbeiterInnen vorteilhaft oder nicht erforderlich wäre (z. B. bei vielen 
Routinetätigkeiten). Nerdinger (1994, S. 274) teilt diese Auffassung und betont, dass nicht alle MitarbeiterInnen dem nach Selbstverwirklichung strebenden Menschenbild entsprächen.

\subsection{Lernende Organisation und organisationales Lernen}

Lernen ist in der modernen Gesellschaft, die u. a. von Ungewissheit und Turbulenz gekennzeichnet ist, ein überaus positiv besetzter Begriff.

"Er verspricht nicht nur die Bewältigung des immer rascheren sozialen Wandels, er erweckt außerdem Assoziationen von Selbständigkeit, Eigeninitiative und Eigenverantwortung ... In der Informationsgesellschaft ist Lernen der Weg, eine der entscheidenden Steuerungsressourcen ... zu vermehren: das Wissen (Heiner, 1998, S. 11).

Diese positive Konnotation des Lernbegriffs machen sich neuere Organisationskonzepte zunutze, die weniger den Stellenwert formaler Strukturen, rationaler Zweckorientierung und generalisierter Handlungsmuster für die Charakterisierung des "Organisation" genannten sozialen Gebildes betonen, sondern mehr die Bedeutung der beteiligten Akteure, kognitiven Prozesse und Selbststeuerungspotenziale in den Mittelpunkt stellen. Diese Konzepte der "lernenden Organisation" stellen organisationstheoretisch ein Bindeglied zwischen Handlungs- und Akteurstheorien einerseits und Strukturtheorien andererseits dar und erscheinen besonders geeignet, moderne Institutionalisierungsprozesse mit ihrer starken Veränderungsdynamik zu erfassen (a.a.O., S. 15).

Der dem Direktor des Center for Organizational Learning am Massachusetts Institute for Technology (MIT), Peter Senge (1990), zugeschriebene - in Ansätzen jedoch bereits im scientific management eines $F$. W. Taylor und später vor allem in den Arbeiten von $H$. A. Simon (1960) und C. Argyris \& D. A. Schön (1978) anklingende - Begriff der "lernenden Organisation" ist deshalb zu einem viel benutzten Schlagwort geworden, wird aber keineswegs in einheitlichem Sinne verwendet. Luthans, Rubach \& Marsnik (1995, S. 26) weisen überdies darauf hin, dass auch zwischen lernender Organisation und organisationalem Lernen unterschieden werden könne. Letzteres sei als Theorie jedoch erst in seiner "embryonic phase" (a.a.O.). 
Das Konzept lässt sich, plakativ und stark simplifiziert, unter Verwendung originärer Schlüsselbegriffe ("core competencies") von Senge (1992) wie folgt charakterisieren:

"In learning organizations, managers should put aside their old ways of thinking (mental models), learn to be open with others (personal mastery), understand how their company really works (systems thinking), form a plan everyone can agree on (shared vision), and then work together to achieve that vision (team learning)" (Dumaine, 1994, zit. nach Luthans et al., 1995, S. 25).

- Persönliche Entwicklung (personal mastery) bezieht sich auf die Schnittstelle zwischen organisationalem und individuellem Lernen - das eine ist ohne das andere nicht zu realisieren.

○ Eine Veränderung der mentalen Modelle (mental models) bezieht sich auf oftmals ungeschriebene Spielregeln, die den notwendigen Wandel behindern: Menschen halten gern an vertrauten Denk- und Handlungsmustern fest, auch wenn diese längst dysfunktional geworden sind.

- Gemeinsame Visionen (shared visions) beziehen sich auf erstrebenswerte Zukunftsbilder, denen sich die Organisationsmitglieder verpflichtet fühlen und für die sie sich einsetzen.

○ Teamlernen (team learning) bezieht sich auf die Notwendigkeit, Problemlösekompetenzen und Dialogfähigkeit zu entwickeln.

○ Systemisches Denken (systems thinking) - als die zentrale, "fünfte Disziplin" im gleichnamigen Buch von Senge (1990) - bezieht sich auf die zirkuläre Verknüpfung von Ursachen und Wirkungen, von Verzögerungs- und Beschleunigungsmomenten in den Rückkopplungsschleifen komplexer Systeme (Kolhoff, 2003, S. 59f).

Leitbild der lernenden Organisation sind zu solcherart vernetztem, systemischem Denken fähige MitarbeiterInnen, die bewusst, freiwillig, intrinsisch motiviert Verantwortung übernehmen und mitunternehmerisch handeln. Eine Unternehmensverpflichtung zur Qualität, die nicht auf intrinsischer Motivation gründet, ist nach Senge (1992, S. 31) auf Sand gebaut. 
Führungskräfte lernender Unternehmen werden als Designer von Lern- und Veränderungsprozessen verstanden (Boxberg, 2003, S. 9); ihre Schlüsselaufgabe besteht darin, einen Interpretationsrahmen zu schaffen, auf den sich die einzelnen Organisationsmitglieder oder Organisationsteile beziehen können - Kontexte für Wirklichkeitsinterpretationen, die für jene Realitätskonstruktionen anschlussfähig sind, auf deren Grundlage die Organisationsmitglieder und -einheiten als sich selbst steuernde Systeme handeln (Heiner, 1998, S. 14).

Kumuliertes Lernen der MitarbeiterInnen macht dabei noch kein lernendes Unternehmen aus, obwohl naturgemäß nicht die Organisation als solche lernt, sondern ihre Mitglieder. Deren erworbenes und in Gruppen ausgetauschtes Wissen ist gleichfalls kein hinreichendes Charakteristikum für eine lernende Organisation. Die besondere Qualität ergibt sich neben der Kumulation des Wissens aller Organisationsmitglieder und der Möglichkeit, durch dessen Nutzung individuelle Lernprozesse abzukürzen, aus der Aufnahme, Archivierung und Weitergabe dieses Wissens (a.a.O., S. 44).

Allerdings ist damit noch keineswegs gewährleistet, dass die organisationale Wissensbasis qualitativ immer mehr ist als die Summe ihrer Teile, wie programmatisch gern behauptet wird. Organisationsmitglieder können Informationen zurückhalten, Kommunikationskanäle verstopfen oder Falschmeldungen streuen, wenn ihnen dies strategisch günstig erscheint, und bestimmte Organisationsstrukturen können ein solches Verhalten durchaus befördern (a.a.O.). Die heimlichen Machenschaften in Organisationen, Vorteile zugunsten einzelner Mitglieder oder Gruppen zu erlangen, werden als Mikropolitik bezeichnet (Blickle, 2004, S. 149).

Wissen als eine zentrale Steuerungsgröße in Organisationen muss deshalb im Kontext materieller Strukturen und Machtdimensionen analysiert werden. Ohne Berücksichtigung der Prozesse, wie Wissen z. B. verfügbar gemacht und in die organisationale Wissensbasis aufgenommen oder von ihr ausgeschlossen wird, gerät die einseitige Fokussierung auf diese kognitive Ressource und die damit verknüpften Lernprozesse zu einem idealistischen und harmonistischen Organisationsverständnis (Heiner, 1998, S. 44). In solchen Organisationen wäre jedoch ein Lernen im umfassenden Sinne nicht mehr möglich; denn dazu gehört ein Mindestmaß an Herausforderung und Dissens (vgl. Heiner, a.a.O.; Klatetzki, 1998, S. 61 ff.). 
Ohne an dieser Stelle zugrunde gelegte Konzeptionen von Lernen und die nur scheinbar einfache Frage "Wer lernt, wer lehrt und was wird gelernt?" zu erörtern (dazu Heiner, 1998, S. 15 ff.), sei auf einige Aspekte näher eingegangen, um die Verwandtschaft mit Ansätzen des Qualitätsmanagements zu verdeutlichen.

Senge äußert in einem 1992 erschienenen Aufsatz die Befürchtung, dass die Qualitätsbewegung in den USA Gefahr laufe, ohne einigendes Rahmenkonzept in isolierte Initiativen und Slogans zu zerfallen. Angesichts der zahlreichen und theoretisch unverbundenen Ansätze wird es als nicht überraschend bezeichnet, dass viele PraktikerInnen darin jeweils nicht mehr sähen als "das Thema des Monats", das es auszuhalten gelte, bis die nächste Mode Einzug hält. Gesucht werde ein vereinigender konzeptioneller Rahmen, der berücksichtige, dass Total Quality keine geschlossene Methodologie sei, sondern eine offene, die sich mit den Bedürfnissen der Gesellschaft entwickele. Diesen Rahmen soll nunmehr die Konzeption der lernenden Organisation darstellen (Senge, 1992, S. 30).

Senge nimmt an, dass die Qualitätsbewegung nichts anderes ist als die erste Welle in der Entwicklung solcher lernenden Organisationen, verstanden als Organisationen, die kontinuierlich ihre Fähigkeit erweitern, die eigene Zukunft zu gestalten. In der ersten Welle lag seiner Ansicht nach der Fokus auf den "frontline workers" (wahrscheinlich ist damit das "Kundenkontaktpersonal" gemeint, P. G.). Der Job des Managements habe darin bestanden, kontinuierliche Verbesserungen zu erkämpfen, bürokratische Hindernisse zu beseitigen, welche das Personal demotivieren und neue Methoden einzuführen, um die Prozesse zu verbessern (a.a.O., S. 31).

Die zweite Welle kennzeichnet nach Senge ein Fokuswechsel von der Prozessverbesserung zur Verbesserung der Art, wie wir arbeiten, denken und interagieren; im Mittelpunkt stehen die Manager selbst. Diese zwei Wellen werden nach Auffassung von Senge in eine dritte münden, in der Lernen als unausweichliche Anforderung für Manager und MitarbeiterInnen gleichermaßen institutionalisiert sein werde (a.a.O.).

Das auf Leistung, statt auf Lernen aufgebaute Erziehungssystem wird von Senge dafür verantwortlich gemacht, dass der natürliche Trieb zu lernen, der früher auftrete und länger anhalte als der Sexualtrieb, unterdrückt werde und Menschen Wissen stets als etwas betrachteten, das andere haben und man selbst nicht. Diese Haltung münde in die Figur des Bosses, des Lehrers, des Experten, der die Antworten hat und unsere 
Leistung beurteilt, statt dass wir uns als Lernende empfinden, die ihre Fähigkeiten entfalten. Eine radikale Reform des Schulsystems wird für notwendig gehalten, um das Arbeitsleben im Sinne der Vision der lernenden Organisation zu revolutionieren (a.a.o., S. 38).

Senge (zit. nach Dumaine, 1994, S. 154) soll auf die Frage, ob die lernende Organisation tatsächlich die nächste Stufe nach TQM sei, geantwortet haben: "If it isn't working, we should stop and do something else." Dem Konzept ist offenbar die Selbstreflexion und Veränderung im Falle der Nichtbewährung immanent. Um hierüber ein Urteil fällen zu können, bedarf es nach Luthans et al. (a.a.O.) jedoch neben traditionellen Maßzahlen (z. B. aus Befragungen) innovativer Evaluationskriterien zur Feststellung, ob Lernen tatsächlich stattfindet und die Organisation effektiver als zuvor arbeitet.

Die Modelle lernender Organisationen und die Vorstellungen von den Möglichkeiten, die organisatorische Wissensbasis produktiv zu nutzen, variieren - wie schon betont - erheblich (Heiner, 1998, S. 42). Gemeinsam ist ihnen, dass Wissen als zentrale Steuerungsressource betrachtet wird. Die Nähe des Ansatzes zu Konzepten des Kontinuierlichen Verbesserungsprozesses, des Wissensmanagements und betrieblichen Vorschlagswesens ist evident.

Cameron \& Barnett (2000, S. 288) betonen die Bedeutung von "information-gathering activities focused on quality in the organization" und "balance between using standardized quality tools ... and encouraging and rewarding innovation, creativity, new ideas, originality, and invention in producing quality improvements" als Prädiktor für "high levels of effectiveness and improvement in quality performance".

Luthans et al. (1995, S. 27 ff.) fassen die allgemein akzeptierten Charakteristika lernender Organisationen in drei Merkmalsgruppen zusammen:

$\circ$ presence of tension,

$\circ$ presence of systems thinking,

$\circ$ a culture which facilitates learning.

Die Autoren konzedieren, dass diese Charakteristika idealistisch und vage formuliert sind und nur von wenigen in Frage gestellt würden. Die Herausforderung bestehe jedoch darin zu bestimmen, welche operationalen Techniken eingesetzt werden müssen, um die Lernprozesse der Organisation zu befördern, m. a. W.: Was können Organisationen tun, um sich zu lernenden zu transformieren (a.a.O., S. 31f)? Als spezifische Techniken, die 
sich bei der Entwicklung organisationalen Lernens als hilfreich herauskristallisiert haben sollen, werden aufgeführt:

○ learning laboratories/managerial micro-worlds,

- scenario planning,

○ experimentation,

- learning from the past,

- learning from others,

o systemic problem solving,

$\circ$ active learning.

In diesem Zusammenhang entscheidend ist die Frage nach der Qualität der organisationalen Lernfähigkeit. Regelgetreue Anwendung des bestehenden Wissens und Korrekturen als Reaktion auf Probleme, von Argyris (1992) "single loop learning" genanntes, adaptives Lernen wird als nicht hinreichend betrachtet. Für bedeutsamer werden generative Lernprozesse gehalten ("double-loop learning"), bei denen kreativ nach besten Lösungen gesucht wird, ggf. auch unter Infragestellung der Zielvorgaben. Reflexionsprozesse über diese Vorgänge werden schließlich als "triple-loop learning" bezeichnet. Sie erfolgen selbstorganisiert und selbstverständlich, ohne dass jeweils erst eine Aufforderung notwendig wäre (Heiner, a.a.O., S. 45). Auch Senge (1990) unterscheidet ähnlich Argyris zwei Arten des Lernens: adaptive (coping and accomodating) und generative (creative and innovative).

Angesichts des historischen Abstiegs von Firmen wie IBM oder General Motors trotz Umsetzung von TQM und des gleichzeitigen Erfolgs anderer Unternehmen wie Wal-Mart oder Motorola konstatieren Luthans et al. (1995, S. 25): "There now seems little question, on both an intuitive and empirical basis, that the ability of these organizations to learn, not just react to change but anticipate change, has much to do with their success or failure."

Der spezifische Beitrag des Konzepts der lernenden Organisation zur Erweiterung der "QM-Toolbox" besteht in der Betonung einer Steuerung durch Selbstreflexion, wie sie im Wechsel von zielbezogenem Vorgehen und reflexiven Schleifen, von Erkunden und Plädieren, von single und double loop learning, von Dialog und Diskussion zum Ausdruck kommt (Rappe-Giesecke, 2003, S. 11f). 


\subsection{Kritik des Qualitätsdiskurses und das Konzept des Sensemaking}

Ungeachtet aller positiven Konnotationen, die sich mit "Qualität" verbinden und auch den systematischen Bemühungen, sie möglichst wirkungsvoll zu steuern - Qualitätsmanagement genannt - zugute kommen, bietet der Qualitätsdiskurs Angriffsflächen, an denen sich Kritik im Detail oder im Grundsätzlichen entzündet. Einige Aspekte sollen im Folgenden erörtert werden. Dabei wird besonders Bezug genommen auf ein Konzept, das den Anspruch erhebt, über den Qualitätsdiskurs hinauszuweisen: Sensemaking.

Zunächst jedoch sei ein Sachverhalt problematisiert, der auf den ersten Blick spezifisch für den Profit-Bereich zu sein scheint, aber auch in Non-Profit-Organisationen eine erhebliche Rolle spielt - die Frage, wem QM auf lange Sicht nützt.

So äußert z. B. Kottmann (2002, S. 194) im Kontext einer Stellenbeschreibung für Qualitätsbeauftragte im Pflegebereich: "Es erfordert Geschick und Beharrlichkeit, die Skepsis der Mitarbeiter gegenüber Qualitätsmanagement auszuräumen. Ziel hierbei ist es, dass die Mitarbeiter Qualitätsmanagement nicht als Kontrolle, sondern als Chance zur Verbesserung und zur Erhöhung der eigenen Arbeitszufriedenheit ansehen."

Anders als seinerzeit in Japan ist in den westlichen Industrienationen eine fatale Gleichzeitigkeit des Aufkommens der Qualitätsbewegung mit dem Anstieg der Massenarbeitslosigkeit (Stichwort "Downsizing") zu konstatieren. Auch in der amerikanischen Fachliteratur wird nicht verhehlt, dass die rhetorische Beschwörung einer "shared vision" und organisationalen Commitments häufig in Widerspruch gerät zur praktizierten Fokussierung des Managements auf die Interessen der "stockholders" (vgl. Cole \& Scott, 2000, S. xvi).

Die Frage, wie weit unter den Bedingungen gewinnorientierten kommerziellen Wettbewerbs die viel zitierten organisationskulturellen Erfolgsfaktoren Vertrauen, Fehlerfreundlichkeit, Offenheit u. a. m. sich mit betrieblichen Realitäten vereinbaren lassen (vgl. Kap. B 3.1.2: Fragmentierungsmodell), sei hier allerdings nur rhetorisch gestellt; sie lässt sich nicht "dekontextualisiert" (s. u.) beantworten.

Ein Nebenaspekt dieser urpolitischen Frage betrifft den Stellenwert von Anreizsystemen. Während z. B. der Nestor der Qualitätsbewegung, W. E. Deming (1986), und einer der Väter der "lernenden Organisation", Argyris (1998), die Bedeutung intrinsischer Motivation betonen und extrinsische Belohnungen für problematisch erachten, konstatieren Hackman \& Wageman (2000, S. 43) nüchtern: 


\begin{abstract}
"When workers perceive that they are contributing more to the organization than they did previously, their initial response may be pride and pleasure. That may suffice for a while. Eventually, however, members of profit-making firms will realize that somebody is making more money as a result of their greater contributions, and it is not them. At that point, they may begin to withdraw their commitment to the enterprise, and signs of a motivational backlash may even be seen."
\end{abstract}

Auch Levine \& Shaw (2000, S. 383) argumentieren pragmatisch: "Some respond well to intrinsic incentives, but others respond better to the extrinsic incentives of pay increases - they want to be paid for their extra effort and for the resulting improvements."

Dass Organisationskonzepte immer auch die Machtdimension tangieren, wurde bereits im Kontext von Mitarbeiterbeteilung, Empowerment und lernender Organisation thematisiert. Im Folgenden soll versucht werden, einen anderen Aspekt der Kritik ausführlicher zu erörtern, der sich mit "postmoderner Perspektive" umschreiben lässt. Dabei wird im Wesentlichen auf Beiträge von Dahlberg, Moss \& Pence (1999) sowie Weick (2000) zurückgegriffen, deren sozialkonstruktivistische Argumentation sich - ohne aufeinander Bezug zu nehmen - weitgehend deckt.

Nach Dahlberg et al. (1999) gibt es keinen Zweifel daran, dass das Konzept der Qualität inzwischen eine dominante Rolle in unserem Denken, unserer Sprache und unseren Handlungen spielt: "The 'age of quality' is now well and truly upon us ..." (a.a.O., S. 4). Die AutorInnen plädieren jedoch am Beispiel der Elementarerziehung dafür, den Qualitätsdiskurs in Richtung auf einen discourse of meaning making zu überwinden. Nach ihrer Auffassung ist der Qualitätsdiskurs fest eingebettet in die Tradition und Erkenntnistheorie des logischen Positivismus, der seinerseits tief im Projekt der Moderne verwurzelt sei. Der Qualitätsdiskurs stelle in gesellschaftstheoretischer Hinsicht die qualitätsspezifische Variante des modernistischen Aufklärungskonzepts dar. Durch Standardisierung, Quantifizierung, Objektivierung, Varianzreduzierung, Globalisierung usw. solle erreicht werden, Produkte definierter Qualität herzustellen und Kundenbedürfnisse optimal zu befriedigen. Dabei spiele auch der demokratische Wunsch nach unparteiischen und transparenten Methoden der Bewertung an Stelle von persönlicher Willkür eine Rolle. Das Konzept der Qualität befasse sich primär damit, durch die Spezifikation von Kriterien einen generalisierbaren Standard zu definieren, gegen den ein Produkt verglichen und somit sicher bewertet werden könne. Qualität in diesem Sinne sei ein dekontextualisiertes Konzept (a.a.O., S. 93f). 
Die zunehmende Erkenntnis, dass Qualität jedoch ein subjektives, wertbasiertes, relatives, dynamisches, multiperspektivisches, kontext- und prozessabhängiges Konzept sei, das sich nicht in Einklang bringen lasse mit Diversität, Multiperspektivität, Kontextspezifität und Subjektivität, erfordere ein Hinausgehen über dieses Konzept. Ein solches neues Konzept wird meaning making genannt (a.a.O.).

Statt von sozialen Einrichtungen wie früher von services zu sprechen, benutzen die AutorInnen nunmehr den Begriff der institution, um zu betonen, dass ein Wechsel von der Sprache der Anbieter und Nachfrager, Geber und Nehmer, Produzenten und Konsumenten zur Sprache der öffentlichen Foren, Plazas und Arenen mit kultureller und symbolischer Bedeutung sinnvoll sei, die in der Zivilgesellschaft lokalisiert sei und diese mitkonstituiere. In diesem Zusammenhang wird auch die Rolle der (vor allem amerikanischen) Entwicklungspsychologie problematisiert, die von universellen alterstypischen Entwicklungsstufen ausgeht.

Die AutorInnen argumentieren, dass der modernistische Qualitätsdiskurs die dekontextualisierte Suche nach Sicherheit durch die losgelöste und objektive Anwendung universeller und zeitloser Kriterien darstelle (S. 106). Dahlberg et al. halten das Konzept des "meaning making" für geeigneter, wenngleich Kontinuitäten zwischen den beiden Diskursen betont werden. Diese beträfen besonders den Wunsch, die Frage zu beantworten, was eigentlich vor sich gehe und was gute Arbeit ausmache. In postmoderner Sicht wird "gut" jedoch nicht verstanden als inhärente, substantielle und universelle Kategorie, sondern als Produkt einer Diskurspraxis, die immer zeitlich und räumlich kontextgebunden und oftmals der Unstimmigkeit und Verhandlung unterworfen sei. Während der Qualitätsdiskurs wertfreie technische Auswahlmöglichkeiten im Sinn habe, erfordere der Diskurs des meaning making explizit ethische und philosophische Entscheidungen mit Bezug auf weiterreichende Fragen wie die nach dem "guten Leben". Dieser Diskurs nehme nicht nur eine sozialkonstruktivistische Perspektive ein, sondern knüpfe an ein Verständnis von Lernen als Prozess der Ko-Konstruktion an, in dem Menschen in Beziehung zu anderen stehen und sich ein Bild von der Welt machen. Demgegenüber basiere der Qualitätsdiskurs auf einem Lernverständnis der Reproduktion eines festgelegten Wissensumfangs mit dem Experten als Wissensvermittler (S. 107).

\footnotetext{
"From a postmodern perspective, there is no absolute knowledge, no absolute reality waiting 'out there' to be discovered. There is no external position of certainty, no universal understanding that exists outside history or society that can provide foundations for truth, knowledge and ethics. Instead, the world and our knowledge of it are seen as socially constructed and all of us, as human beings, are active participants in this process ..., engaged in relationship with others in meaning making rather than truth finding ... For these reasons, knowl-
} 
edge and its construction is always context-specific and value-laden, challenging the modernist belief in universal truths and scientific neutrality" (Dahlberg et al., 1999, S. 23).

Während mit diesen Bemerkungen die postmoderne Perspektive relativ deutlich charakterisiert wird durch Aufweis der Schwächen eines zu statischen, präskriptiven, universalistischen und vermeintlich "objektiven" Qualitätskonzepts, ist die Beschreibung dessen, was die AutorInnen eigentlich unter "meaning making" verstehen, wesentlich schwieriger. Es handelt sich aber offenbar um sinngemäß das gleiche Konzept, welches von Weick (1995, 2000) "sensemaking" genannt wird. Auch das Sensemaking-Konzept ist ein Plädoyer wider technokratischen Glauben an die Beherrschbarkeit der Geschäftsprozesse zugunsten einer dynamischen Offenheit gegenüber den diversen organisationalen Umwelten. Sensemaking oder meaning making soll hier übersetzt werden mit "(soziale) Sinngebung" (vgl. Sommerfeld \& Haller, 2003, S. 67f) und impliziert die immer subjektive Konstruktion von Bedeutung, Plausibilität und Wirklichkeitsverständnis.

Die aus dieser Perspektive gemachten Ausführungen von Weick (2000, S. 155-171) zur amerikanischen TQM-Bewegung sind geprägt vom Konstruktivismus. Dieser erkenntnistheoretische Ansatz betont die Abhängigkeit von Wahrnehmung und Handeln von "Vorgängen im Kopf". Erkenntnis beruht danach nicht auf einer Korrespondenz mit der externen Wirklichkeit, sondern auf Konstruktionen eines Beobachters; nur durch Konsens mit anderen wird Wirklichkeit "erfunden". Die Welt wird in Form von sozialen Artefakten verstanden und mit Hilfe von Begriffen, die in historisch bestimmten Austauschprozessen zwischen Personen gebildet worden sind (Bergius, 1998, S. 456). Vor allem in sozialen Handlungssystemen spielen sprachliche Symbole (Wörter) eine herausragende Rolle; sie repräsentieren Reize, die Reaktionen auslösen und erlangen dadurch selbst die Fähigkeit, Reaktionen hervorzurufen ("symbolischer Interaktionismus", Bergius, 1998b, S. 852). Im Wechselspiel von symbolischen und materiellen Aktivitäten wird soziale Realität konstruiert (Weick, 1995, zit. nach Scholl, 2004, S. 527).

Weick (2000, S. 158) äußert im Zusammenhang mit einer Kritik am TQM-Label: "However, if one grants that words are all we have, that words gain their meaning from their connections with other words rather than with external objects (a correspondence theory of truth is untestable and therefore of little help), and that people learn what they think by seeing what they say, and learn what they want by seeing what they do, then it makes sense to linger over words, their connotations, and their surplus meanings that are taken for granted." 
Weick betont, dass menschliches Verhalten vielfach in Begriffen des individuellen Bedürfnisses erklärt werden könne, einer Situation Bedeutung zu verleihen ("making sense") und als kompetenter Akteur darin $\mathrm{zu}$ erscheinen. Mit zunehmender Institutionalisierung der Situationen akzeptierten Menschen existierende soziale Skripte, statt originäre Skripte zu entwerfen. Deshalb folgten viele Individuen Routinen und Leistungsprogrammen für Alltagsaktivitäten, statt neue Handlungspläne zu entwickeln (zit. nach Hamada, 2000, S. 300).

Die Konstruktion solcher Sinn- und Deutungsmuster, Sensemaking, wird als eine Schlüsselaktivität von Menschen verstanden, die eine Welt gestalten wollen, in der Qualität eine wichtige Rolle spielt (Weick, 2000, S. 155). Mit Bezug auf Heidegger ("Geworfenheit"), Quanten- und Chaostheorie wird die Welt als prinzipiell unerkennbar und unvorhersehbar bezeichnet. Sensemaking wird als ein Steuern mehr mit Kompass als mit Landkarte betrachtet. Während Landkarten Bekanntheit voraussetzen, führe ein Kompass auch durch unbekanntes Gelände und sei daher eine Säule für Lernen und Erneuerung (Weick, a.a.O., S. $161 \mathrm{ff}$.$) .$

Hamada (2000, S. 300) verdeutlicht den Prozess des individuellen sensemaking durch Analogie: Eine Person der Gegenwart, die Jazzmusik höre, könne diese sofort als solche identifizieren; Johann Sebastian Bach würde dagegen dieselbe Musik vermutlich nur als einen Mix aus Geräuschen bezeichnen. Um an bedeutungshaltigen sozialen Interaktionen teilnehmen und soziale Realität verstehen zu können, müsse der Mensch eine soziale und symbolische "Grammatik" erlernen (a.a.O., vgl. Kap. B 2.3.2).

Weick (2000, S. 170f) fasst die Essenz seiner perspektivischen Überlegungen zum Sensemaking in 12 Punkten zusammen und formuliert praktische Implikationen für das Qualitätsmanagement (quality improvement):

"... if one wanted to improve quality, then one would socialize people to make do, be resilient, view constraints as of their own making, value quests, strive for plausibility, treat past experience with ambivalence, keep showing up, use retrospect to get a sense of direction, craft descriptions that energize, ground identity in a protean rather than singular self-image, and be wary of using rational accounts as job descriptions ....

If a team enacted those 12 practices, ... there would be continuous updating and co-evolution of producercustomer relationships ..., whether it was called quality improvement or not."

Das Verhalten von Menschen in Organisationen wird von Weick und anderen VertreterInnen des Konstruktivismus bevorzugt als ein sich selbst regulierendes System 
betrachtet, das durch organisationspsychologische Interventionen nur unterstützt, aber nicht von außen umstrukturiert oder durch Input wissenschaftlicher Informationen verändert werden kann (Holling \& Kanning, 2004, S. 83). Dem konstruktivistischen Ansatz werden wertvolle Anregungen zum Organisationsverständnis konzediert (a.a.O., S. 84). Allerdings wird es für möglich gehalten, die von den Konstruktivisten präferierten Maßnahmen - z. B. verstärkte Partizipation, selbstorganisierte Lernprozesse - auch ohne den "ideologischen Überbau" zu begründen, etwa durch Bezug auf Motivationstheorien oder die Forschung zur hypothesengeleiteten Wahrnehmung (a.a.O.).

Diese Auffassung wird geteilt. Bei aller grundsätzlichen Berechtigung der konstruktivistischen Kritik ist nicht zu übersehen, dass sie sich - jedenfalls bei den zitierten AutorInnen - an einem Qualitäts- und Managementverständnis entzündet, das aus sozialwissenschaftlicher Sicht inzwischen als überholt gelten kann (vgl. Cole \& Scott, 2000). Bezogen auf die Qualitätsrhetorik der Achtziger- und frühen Neunzigerjahre und die auch bei Angehörigen sozialer Berufe (meistens Leitungspersonal mit Sozialmanagement-Ambitionen, vgl. Gerull, 2001, Vorwort) zeitweilig anzutreffende naive Begeisterung für "Qualitäts-Managerialismus", mag die von Dahlberg et al. und Weick kritisierte Sichtweise auf Qualität legitim und notwendig (gewesen) sein. Den aktuellen Qualitätsdiskurs in sozialen Handlungsfeldern kennzeichnet sie jedoch nicht (mehr) korrekt.

So kommt bspw. Gerull (2004) im Zusammenhang mit der Frage, welche allgemeinen oder spezifischen Qualitätsmerkmale für soziale (zentral personenbezogene, Beziehungs-) Dienstleistungen in der einschlägigen Fachliteratur diskutiert werden, zu folgendem Resümee:

o Qualität sozialer Dienstleistungen ist nichts unmittelbar Gegebenes, sondern muss kontextbezogen bestimmt und kommunikativ validiert werden ( $\rightarrow$ "Konstruktcharakter").

o Qualität sozialer Dienstleistungen wird durch multiple Anspruchsgruppen definiert und muss diskursiv verhandelt werden ( $\rightarrow$ "Aushandlungscharakter"). 
o Qualität sozialer Dienstleistungen wird nicht technisch erzeugt, sondern muss durch interaktives Handeln realisiert werden ( $\rightarrow$ "Koproduktionscharakter").

o Qualität sozialer Dienstleistungen muss mehrdimensional und multiperspektivisch betrachtet werden ( $\rightarrow$ "Komplexitätscharakter").

Diese Bemerkungen mögen hier genügen, um zu verdeutlichen, dass der Qualitätsdiskurs in sozialen Handlungsfeldern keineswegs (mehr) so geführt wird, wie Dahlberg et al. (1999) dies zur Begründung ihrer postmodernen Perspektive zu insinuieren für notwendig hielten.

Im Zusammenhang mit einer Konzeptualisierung von Qualität als interaktives Epiphänomen (s. Kap. B 1.3) formulierte Klaus (1991, S. 256) in einem ansonsten betriebswirtschaftlich orientierten Beitrag bereits vor über einem Jahrzehnt:

"Das Dienstleistungs-Qualitätsmanagement wird ... gezwungen, die Grundlagen objektiver und absoluter Standards und Werte zu verlassen, wie sie im industriellen Qualitätsmanagement gefordert und genutzt werden. Stattdessen muß es sich in einer noch wenig erforschten Welt flüchtiger, subjektiver und relativer Qualitätsgrößen zurechtfinden." 


\section{Resümee: Zur Konvergenz und Integration der Ansätze}

Kundenorientierung (customer focus), kontinuierliche Verbesserung (continuous improvement), umfassende Beteiligung (total participation) und gesellschaftliche Vernetzung (societal networking): nach Ansicht von Shiba \& Walden (2001) - beides Professoren am Massachusetts Institute for Technology (MIT) und Autoren eines in den USA führenden Management-Lehrbuchs - sind dies die vier in der Praxis sich auswirkenden Revolutionen, die das heutige Management auszeichnen. Mit ihrer Hilfe soll es bei konsequenter Anwendung möglich sein, eine einzigartige organisationale Fähigkeit zu schaffen.

Die Nähe dieser Ansätze zu zentralen Konzepten des QM liegt auf der Hand. Zollondz (2002, Vorwort) sieht darin eine Bestätigung dafür, dass QM das gesamte Wirtschaftsleben seit über 50 Jahren nachhaltig geprägt habe wie keine andere Managementkonzeption und keinesfalls eine totgesagte Mode sei.

"Bei den Systemen des Qualitätsmanagements handelt es sich nicht um Kopfgeburten. Sie basieren auf jahrzehntelangen Erfahrungen anderer Unternehmen, stammen aus Beratungsleistungen von Experten und aus den Untersuchungen und Theoriebildungen angewandter Wissenschaften" (a.a.O., Hervorhebungen im Original, P. G.).

Dabei werden die Effekte dieser Bewegung durchaus unterschiedlich eingeschätzt. Während auf akademischer Seite Skepsis überwiegt und empirische Forschung - wenngleich mit erheblichen methodologischen Schwierigkeiten behaftet (vgl. Hackman \& Wageman, 2000, S. 33f) - häufig auf Lücken zwischen Qualitätsrhetorik und Realität verweist (Cole \& Scott, 2000, S. xxi, vgl. auch Kap. B 3.1), betonen Manager gern die positiven, vorwiegend prozessualen Veränderungen in größerem Kontext, z. B. die verbesserte Kooperation zwischen Design und Produktion oder die erhöhte Aufmerksamkeit gegenüber externen Kunden (a.a.O., S. xxii; Hackman \& Wageman, 2000, S. 35).

Wenn somit auch hinsichtlich der empirischen Wirksamkeit des Qualitätsmanagements viele offene Fragen bleiben und unklar ist, ob Annäherungen zwischen "PraktikerInnen" und "TheoretikerInnen" mehr die eine oder andere Seite befruchten können, ist die Tatsache einer verstärkten Befassung der wissenschaftlichen Disziplinen mit dem Phänomen "QM" unverkennbar; sie ist auch überfällig, wenn man sich die in den Kapiteln zuvor er- 
örterten Grundlagen und thematischen Schnittmengen von Qualitäts- und Organisationslehre bewusst macht.

In nahezu allen diesen Konzepten - seien sie eher betriebswirtschaftlich oder sozialwissenschaftlich akzentuiert - fehlt es nicht an Hinweisen auf die Bedeutung von systemischen Aspekten (Vermeiden von "Insel-Lösungen"; "Einbettung" in ein Bündel von Maßnahmen; struktureller, kultureller und klimatischer "Kontext") und Soft-Faktoren Vertrauen, Offenheit, Fehlerfreundlichkeit, Kooperativität, Beteiligung, Commitment, Kommunikation, Lernen, Teamwork, evaluatives Denken. Dies offenbar sind die maßgeblichen Ingredienzen für effektive und nachhaltige Organisationsgestaltung. Dabei insinuiert der Gestaltungsbegriff ein Potenzial an aktiven Zugriffsmöglichkeiten, das von namhaften Organisationstheoretikern für gar nicht existent gehalten wird: Grenzen der Planbarkeit, Betonung von Anpassungs-, Lern- und Widerstandsfähigkeit ("resilience") in einer prinzipiell nicht vorhersagbaren Welt und ähnliche postmoderne Warnungen vor Überschätzungen eines Managerialismus sind hier zu nennen (vgl. Kap. B 3.8).

Die unterschiedlichen Perspektiven eher "universalistisch" argumentierender VertreterInnen des Qualitätsmanagements und eher "adaptationistisch", kontingenztheoretisch orientierter OrganisationswissenschaftlerInnen finden sich auch im bislang ambitioniertesten Beitrag zu einer Integration von QM und Organisationstheorie wieder: in dem von Cole \& Scott (2000) herausgegebenen Sammelband "The Quality Movement and Organizational Theory".

Die Editoren diskutieren eingangs die amerikanische Qualitätsbewegung im zeit- und raumübergreifenden Kontext der komplexen Fragen, wie und warum sich Organisationen verändern (S. xiii). QM wird als rezentes und wichtiges Beispiel für einen solchen Wandel verstanden, der - ungeachtet der langjährigen Vernachlässigung durch die Organisationstheorie - eine Einbettung dieses Themas in einen größeren theoretischen Zusammenhang erfordere. Die Autoren würdigen Dean \& Bowen (1994, Reprint 2000), die vornehmlich aus Sicht der Managementtheorie wichtige Beiträge zu dieser Frage leisteten, indem sie das Qualitätsthema in Verbindung brachten mit Führungstheorien, Human Resource Management, Strategischer Planung sowie Informations- und Analyseansätzen.

Die Überlegungen von Dean \& Bowen (2000, S. 16f) lauten zusammengefasst: 
1. Das Konzept der Total Quality (TQ) ist konsistent mit der Managementtheorie in folgenden Bereichen: Führung, Human Resource Practices (Mitarbeiterbeteiligung, Teamwork, Bedarfsanalyse und Evaluation von Trainingsmaßnahmen, Karrieremanagement).

2. Das TQ-Konzept muss durch Beiträge der Managementtheorie ergänzt oder korrigiert werden in folgender Hinsicht: Vermeiden einer Überschätzung der formalen Analyse von Informationen, besonders in uneindeutigen und politischen Settings; Berücksichtigung nicht nur von Kundenerwartungen, sondern auch von organisationalen Stärken und Schwächen bei der Strategieformulierung; höherer Stellenwert von Selektionsverfahren, besonders im Hinblick auf die Passung von Personen und Organisation; Verwendung eines kontingenztheoretischen Zugangs statt universalistischer Ansätze zur Gestaltung der internen und externen Beziehungen (Kunden-Lieferanten-Beziehungen, Mitarbeiterbeteiliung, Empowerment).

3. Das TQ-Konzept kann der Managementtheorie wichtige Forschungsimpulse vermitteln in folgenden Bereichen: Informationsverarbeitung, Strategieimplementation, Prozessverbesserung, Kundenorientierung und -zufriedenheit.

Scott \& Cole (S. xiv) ergänzen diese Ausführungen um weitere Beiträge der Organisationstheorie: Contingency Theory, Resource Dependence, Evolutionary Economics, Organizational Learning, Organizational Ecology, Institutional Theory. Auf zwei der genannten Aspekte soll etwas näher eingegangen werden (zur Lernenden Organisation s. Kap. B 3.7):

\section{○ Kontingenztheorie}

Die im Rahmen der vergleichenden Organisationsforschung entwickelte Theorie besagt, dass organisationale Strukturen und Prozesse maßgeblich durch Umwelteinflüsse geformt werden und beobachtbare Strukturdifferenzen auf unterschiedliche Situationen zurückzuführen sind, in denen sich die jeweiligen Organisationen befinden (vgl. Büssing, 2004, S. 566). Dementsprechend wird betont, dass es nicht den einen besten Weg des Organisierens gebe, sondern das Organisationsdesign situativ abhängig (kontingent) sei bzw. sein sollte von der Art und Umgebung der auszuführenden Arbeit 
(Scott \& Cole, a.a.O.).

Auf der Basis dieser allgemeinen Theorie wurden spezifische Kontingenzmodelle entwickelt, um z. B. die Effektivität verschiedener Formen von Fertigungs- und Montageteams (Cummings \& Blumberg, 1987, nach Bungard \& Antoni, 2004, S. 460) oder die Beziehung zwischen Dimensionen des Führungsverhaltens und Merkmalen von Gruppenaufgaben zu optimieren (Fiedler, 1964, nach Bergius, 1998a, S. 458).

Kontingenztheoretiker betonen die Bedeutung der Produktionsprozesse und der zur adäquaten Bewältigung der Arbeitsaufgaben benötigten Informationen. Je größer die Unsicherheit, Komplexität und Interdependenz der Aufgaben und Arbeitsprozesse ist, desto größer sei das Informationsbedürfnis und der Aufwand für Beschaffung und Vermittlung von Informationen (Scott \& Cole, a.a.O.).

Bezogen auf QM, wird dessen analoger Beitrag vor allem darin gesehen, die Rolle der Organisationsumwelt aus Sicht des Managements neu zu definieren. Sei es früher darum gegangen, durch Vereinfachung und Routinisierung der Arbeitsprozesse den Umwelteinfluss möglichst zu minimieren, um nach internen Maßstäben qualitativ hochwertige Produkte herzustellen, fokussiere QM auf den externen Kunden als Maßstab für zufrieden stellende Produkte und Serviceleistungen: "products were to satisfy consumers, not engineers" (a.a.O.).

Während die Kontingenztheorie primär auf die interne Struktur und aufgabenbezogene Eignung einer Organisation abhebt, steht bei diesem Ansatz die Interdependenz einer Organisation mit anderen Organisationen der relevanten Umwelt im Vordergrund der Aufmerksamkeit. Um diese Abhängigkeit und die daraus resultierende Unsicherheit zu reduzieren, entwickeln Organisationen z. B. Allianzen, joint ventures und andere Formen der Koordination.

Cole \& Scott (S. xvi) stellen zwar keine direkte Kommunikation zwischen Vertretern des QM und Theoretikern des resource-dependence-Ansatzes fest, konstatieren jedoch 
offenkundige Ähnlichkeiten, z. B. die beiderseitige Annahme der willkürlichen Natur von Organisationsgrenzen, aus der sich eine erweiterte Sicht auf Produktions- und Marketingfunktionen ergebe. Konsumenten werden nicht als passive LeistungsempfängerInnen betrachtet, sondern als unersetzliche Informationsquellen, deren Bedürfnisse Grundlage für neue Produkte und Dienstleistungen sein können ("fitness to latent requirements", Winter, 2000, S. 69). Enge Arbeitsbeziehungen zu urteilsfähigen

Kunden und kompetenten Lieferanten lägen demzufolge im langfristigen Eigeninteresse des Unternehmens.

Resümee:

Ungeachtet der forschungsthematischen Schnittmengen und des offensichtlichen theoretischen Annäherungsprozesses zwischen QM, Betriebswirtschaftslehre, Organisationspsychologie und anderen betroffenen Wissenschaftsfeldern, wie sie in den Kapiteln des Abschnitts B 3. exemplarisch erörtert wurden, ist der gegenwärtige Status dieses Prozesses als fragmentarisch zu bezeichnen. Dabei ist auch keineswegs auszumachen, unter welchem wissenschaftssystematischen Dach sich die verschiedenen Ansätze integrieren lassen oder angesiedelt sein sollten.

So werden einerseits Hoffnungen auf eine im Entstehen begriffene Qualitätswissenschaft artikuliert. Diese wird als interdisziplinäre Querschnittsverbindung verstanden, in der die traditionell bis heute dominierenden - Ingenieurwissenschaften den technischen Anteil, die Sozialwissenschaften den humanen, die Umweltwissenschaften den ökologischen und die Wirtschaftswissenschaften den ökonomischen Aspekt repräsentieren (Kamiske \& Brauer, 1999, S. 239 ff.). Hinzu werden mindestens noch Rechtswissenschaft, Informatik und Statistik gezählt (Zollondz, 2002, S. 22f).

Weinert (1998, S. 69 ff.) fordert dagegen eine interdisziplinäre Organisationswissenschaft, um die für ein tieferes Verständnis von Organisationen hemmende Filterfunktion der einzelwissenschaftlichen Paradigmen zu überwinden. Es sei noch keine interdisziplinäre Wissenschaftsdisziplin geboren, "wenn die mikrotheoretischen Probleme von Psychologen, die makrotheoretischen von Soziologen, Politologen, Wirtschaftswissenschaftlern, Historikern und von Leuten aus dem Management- und Human-Relations-Bereich behandelt werden und man wechselseitig die Forschungsergebnisse austauscht" (S. 69). 
Seghezzi (1994, 1996, 2003) schließlich betont die Zuordnung der entstehenden Querschnittsdisziplin QM in die Wirtschaftswissenschaften, insbesondere in die Betriebswirtschaftslehre. Systematik und Struktur des St. Galler Management-Konzepts dienen ihm dazu, QM in die allgemeine Managementlehre zu integrieren (Seghezzi, 2003, S. VII). Wenngleich dieser Ansatz am weitesten gediehen ist und durchaus überzeugen kann, ist ihm eine größere fachliche Resonanz bislang vorenthalten geblieben.

Ein Resümee zur Konvergenz und Integration der verschiedenen Konzepte kommt deshalb nicht umhin, dem von Weinert (1998, S. 70) in Bezug auf die gegenwärtige Organisationsforschung geäußerten Eindruck zuzustimmen: verwirrend, diffus und ungeordnet, ohne integratives Rahmenwerk für die Forschungsergebnisse der einzelnen Disziplinen.

Ob die von Senge (1992, S. 30) propagierte learning organization die Funktion des "unifying conceptual framework" erfüllen kann, erscheint mehr als fraglich.

Kühl (2000) setzt sich kritisch mit Widersprüchen und Aberglauben im Konzept der lernenden Organisation auseinander. Er zitiert ethnologische und sozialanthropologische Erkenntnisse, wonach Rituale wie das des Regenmachers in traditionellen Kulturen latent funktional, nämlich gemeinschaftsfördernd sind, auch wenn sie ihren vorgeblichen Zweck - hier: Regen zu produzieren - gar nicht erfüllen. Diese Einsicht lässt sich nach Kühl auf das Konzept der "lernenden Organisation" bzw. auf Managementkonzepte allgemein übertragen. Ähnlich dem "Regenmacher-Phänomen" werden vermeintlich rationale Prinzipien für einen erfolgreichen Unternehmenswandel entwickelt, die zwar nicht zum Erfolg führen, aber dennoch einen versteckten, nicht sofort sichtbaren Nutzen stiften, nämlich den MitarbeiterInnen in einer Situation hoher Verunsicherung Orientierung zu geben. Statt die Rationalität der zugrunde gelegten Prinzipien in Frage zu stellen, werden in der Regel Erklärungsmuster für Misserfolge bereitgehalten, die nur auf Umsetzungsprobleme fokussieren.

Die konstruktivistische Skepsis in Bezug auf den Traum vom geplanten Wandel und die akkurate Beantwortbarkeit wissenschaftlicher Fragestellungen bei gleichzeitiger Betonung des "discovering rather than discoveries" (Weick, 2000, S. 171) ist vermutlich eine angemessene Reaktion auf eine Welt, die sich den objektivierenden Bemühungen positivistischer Wissenschaft gegenüber allzu sperrig verhält.

Zugleich erhärtet sich der Verdacht, dass an die Stelle einer Qualitätsbewegung mit ausgeprägt rhetorischen, rituellen, ja quasi-religiösen Zügen eine nicht minder ideologisierte Sichtweise tritt, für die nicht mehr der moderne Glaube an die Gestaltbarkeit der Organisation durch Management kennzeichnend ist, sondern Vertrauen auf die Selbstregulierungs- 
kräfte eines kontinuierlich lernenden organisationalen Systems. Beide Pole des Ansichtenspektrums repräsentieren Formen säkularisierter Religiosität, zwischen denen sich dienstleistende Einrichtungen und praktische Organisationsforschung irgendwo positionieren müssen. 


\section{Qualitätsmanagement in sozialen Handlungsfeldern - Konzepte und Instrumente}

\section{Qualitäts(management)konzepte für Soziale Dienste im Überblick}

\subsection{Beschreibung und Bewertung von Qualitätskonzepten}

Qualität

- auf der Basis ausgehandelter Ziele der Anspruchsgruppen zu planen und im angestrebten Sinne zu lenken, also mit geeigneten Methoden "herzustellen" ( $\Rightarrow$ Qualitätsentwicklung),

○ zuverlässig auf dem versprochenen Niveau zu gewährleisten, also mit zweckmäßigen Vorkehrungen gegen Fehlerrisiken "abzusichern" ( $\Rightarrow$ Qualitätssicherung) und

○ den Stand der Umsetzung regelmäßig zu evaluieren, also möglichst objektiv zu "messen" und zu beurteilen ( $\Rightarrow$ Qualitätsbewertung),

○ um Ansatzpunkte für gezielte Korrekturen und Innovationen zu gewinnen, also Praxis zu "optimieren" ( $\Rightarrow$ Qualitätsverbesserung),

sind die wesentlichen operativen Aufgaben eines Qualitätsmanagements, das sich als Querschnitts- und Daueraufgabe qualitätsbezogener Kontextsteuerung versteht (Gerull, 2004, Kap. 2.1.1).

Die Besonderheiten eines solchen Qualitätsmanagements in sozialen, pädagogischen und therapeutisch-pflegerischen Handlungsfeldern sind durch die in Anführungszeichen gesetzten Begriffe "herstellen", "sichern", "messen" und "optimieren" angedeutet und führen zu spezifischen Anforderungen und Beschränkungen, was das "Machen", "Interpretieren" und "Verändern" der Phänomene anbelangt, mit denen es Akteure in diesen Handlungsfeldern zu tun haben. Zugleich muss stets die Kontextgebundenheit jener 
Maßstäbe bedacht werden, nach denen eine Intervention oder Entwicklung als "Verbesserung" bewertet wird.

Alle Systeme, Modelle, Ansätze und Verfahren, die sich schwerpunktmäßig mit Aspekten der Qualitätsentwicklung, -sicherung, -bewertung und/oder -verbesserung befassen, sollen im Folgenden unter dem Begriff Qualitätskonzept subsumiert werden; dieser wird somit als unspezifische Sammelbezeichnung verwendet.

In der sozialwissenschaftlichen Fachliteratur sind erst vereinzelt Versuche unternommen worden, das mittlerweile fast unüberschaubare Feld solcher Qualitätskonzepte beschreibend, bewertend und/oder vergleichend darzustellen (in Ansätzen z. B. Merchel, 2001; Boeßenecker, Vilain, Biebricher, Buckley \& Markert, 2003; Kämmer, Hennecke, Trapp \& Bruns-Waigand, 2001; Gerull, 2000). Der bislang umfangreichste Überblick wurde vom Autor in Form eines elektronischen Handbuchs erarbeitet (Gerull, 2004), dem Teile der folgenden Ausführungen mit geringen Änderungen entnommen sind.

Die ursprüngliche Absicht, sämtliche Konzepte nach einem einheitlichen Kriterienraster zu beschreiben und zu bewerten, ließ sich dabei nicht durchhalten. Zwar sind in den allermeisten Darstellungen Angaben zu nachstehenden Aspekten enthalten:

o förmliche Bezeichnung des Konzepts,

o Autor, Herausgeber, Bezugsquelle,

o Ersterscheinungsjahr, Revisionen,

o Entstehungshintergrund, fachlicher Kontext,

o Einsatzspektrum,

o inhaltlicher Aufbau (möglichst mit Beispielen),

o methodische Umsetzung (Anwendungsschritte, Beteiligte, Einbettung in Organisationsstruktur),

o Aufwand (Anschaffungskosten, externe Unterstützung, Implementierung, Zertifizierung),

o Erfahrungen mit dem Konzept (Akzeptanz, Ergebnisse, Vergleiche, Spezifika).

Diese Angaben sind jedoch mehr oder minder ausführlich, teilweise lückenhaft oder mangels besserer Quellen recht vage formuliert. Auch musste davon abgesehen werden, die verschiedenen Konzepte nach einem begründeten Schema miteinander zu vergleichen, wie es Kämmer et al. (2001) und Boeßenecker et al. (2003) versucht haben. 
So verdienstvoll solche Bemühungen sein mögen - sie täuschen ein Ausmaß an Objektivität vor, das nicht gerechtfertigt erscheint. Weder lässt sich z. B. angesichts unterschiedlicher Ausgangsbedingungen der AnwenderInnen ein ggf. erforderlicher interner Schulungsaufwand ermessen, noch können die Prozess auslösenden Wirkungen eines Verfahrens pauschal beurteilt oder gar in Folgekosten beziffert werden.

Der aufwändige Versuch von Kämmer et al. (2001), für den Bereich der Altenhilfe einen "Weg zum besten System" zu weisen, ist zweifellos von hohem Informationswert. Angesichts der Tatsache, dass die AutorInnen ihr Urteil überwiegend auf Erfahrungsberichte von Propagandisten ("Experten-Interviews") des jeweiligen Konzepts stützen und trotz einheitlichen Gliederungsrasters keineswegs gleichwertige Angaben zu wesentlichen Kriterien machen können (teilweise Fehlanzeige oder nur Schätzungen), verwundert jedoch nicht, dass Kämmer et al. trotz eindrucksvoller "Punktetabelle" und Balkendiagramme (S. 152 ff.) keine Empfehlungen aussprechen und die Entscheidung über die Wahl eines Konzepts den einzelnen Organisationen überlassen.

Dies scheint in der Tat vernünftig, denn nur die Einrichtungen und Dienste können beurteilen, worauf es ihnen schwerpunktmäßig ankommt: z. B. auf Optimierung ihrer Geschäftsprozesse, auf öffentlichkeitswirksame Qualitätsdarlegung, auf nachhaltige Organisationsentwicklung oder fachliche Qualifizierung ihrer MitarbeiterInnen.

Der Versuch einer vergleichenden Synopse über gängige Qualitätskonzepte von Boeßenecker et al. (2003) wird dem Anspruch an Objektivität und Aussagekraft noch weniger gerecht als die Arbeit von Kämmer et al. (2001). Zwar bemühen sich die AutorInnen um eine einheitliche Gestaltung und Berücksichtigung von Kriterien wie "Genese", "Philosophie", "Qualitätsdimensionen", "organisationale Relevanz", "Ressourcen", "Umsetzungsdauer" und "Anwendungsrelevanz". Mehr als eine grobe Orientierung, die dazu noch von jeweils verschiedenen AutorInnen aus ihrem je spezifischen Blickwinkel heraus vorgenommen wird, kann das Buch indes nicht leisten.

Wie bei den meisten Konzepten und Werkzeugen kommt es letztlich darauf an, zu welchem Zweck sie von welchen Personen in welchen Organisationen wie oft und sorgfältig angewandt werden. Ansprüche und Kontextbedingungen vor Ort sind viel zu unterschiedlich, als dass sich Qualitätskonzepte über einen einheitlichen Leisten schlagen ließen - so reizvoll dies auch wäre. Der Autor nimmt in dieser Hinsicht einen "kontingenztheoretischen" Standpunkt (vgl. Kap. B 3.) ein: Entscheidend ist nicht das gewählte Konzept, sondern das, was man unter bestimmten Situationsbedingungen daraus macht (vgl. Gerull, 1999, S. 12). 
Schließlich ist es nicht das Qualitätskonzept, welches Qualität erzeugt oder verbessert, sondern diejenigen sind es, welche das Instrument sinnvoll benutzen. Qualitätsmanagement kompensiert nicht mangelnde Professionalität der Fachkräfte, sondern setzt diese voraus und die Professionalität der Anwender von Systemen der Qualitätsentwicklung ist entscheidend dafür, dass "etwas Gescheites dabei herauskommt" (B. Müller, 2000, S. 142).

\subsection{Formale Systematisierung von Qualitätskonzepten}

Bei der Durchsicht veröffentlichter Qualitätskonzepte fällt ins Auge, dass sich einige Ansätze als rein formal verstehen, d. h. als inhaltlich offene Handlungsstruktur zur Optimierung dessen, was die jeweiligen Akteure unter Qualität verstehen. Andere Konzepte betonen dagegen ihren fachlich-wertorientierten Hintergrund und markieren ein inhaltliches Spektrum, innerhalb dessen die einrichtungsspezifische Qualität zu definieren ist. Teilweise sind diese Vorgaben als "beste Fachpraxis" (z. B. Kronberger Kreis für Qualitätsentwicklung in Kindertageseinrichtungen, 2000) so konkret formuliert, dass sie als Messlatte für die eigene Statusbewertung dienen können.

Die von Gerull (2004) verwendete Systematik orientiert sich an den jeweils vorherrschenden Merkmalsprofilen der Konzepte und gliedert sich in fünf Kategorien:

1. Formale Universalkonzepte (branchenunabhängige QM-Modelle und allgemeine Strategien)

Hierzu werden QM-Modelle mit sehr breitem Einsatzspektrum sowie generelle Strategien zur Qualitätsoptimierung gezählt. Sie beanspruchen, in Form von Anforderungen und Empfehlungen alle qualitätsrelevanten Gestaltungsfaktoren zu berücksichtigen und wollen den einrichtungsinternen Weg zu umfassendem QM strukturieren helfen. Die inhaltliche Definition dessen, was jeweils "gute Qualität" sein soll, wird allein den Einrichtungen und ihren KundInnen überlassen. 
2. Formale Branchenkonzepte (adaptierte Universal-Modelle und feldspezifische Strategien)

Es handelt sich um Branchen angepasste Derivate von Universal-Modellen sowie um bereichsbezogen generalisierbare Strategien zur Qualitätsoptimierung. Neben modifizierten Metakonzepten finden sich in dieser Kategorie methodische Leitfäden und strukturierte Arbeitshilfen, teilweise mit exemplarischen fachlichen Konkretisierungen. Die inhaltliche Definition dessen, was jeweils "gute Qualität" sein soll, wird jedoch den internen und externen Akteuren überlassen.

3. Materiale Branchenkonzepte (feldspezifische Anforderungskataloge und Regelwerke)

$\mathrm{Zu}$ dieser Kategorie zählen mehr oder weniger umfassende Manuale zum Aufbau eines feldspezifischen QM und/oder Leitfäden zur Selbst- und Fremdüberprüfung. Die inhaltliche Definition dessen, was jeweils "gute Qualität" sein soll, wird auf der Basis eines breit akzeptierten fachlichen Modells vorgegeben und in Form von zu beachtenden Zielen und Kriterien konkretisiert, zum Teil auch als Indikatoren operationalisiert. Einrichtungsspezifische Variationen und dialogische Verständigung über die Qualitätskriterien sind jedoch vorgesehen.

4. Materiale Branchenkonzepte (operationalisierte Prüfkriterien und standardisierte Bewertungsverfahren)

Diese Kategorie stellt eine Teilgruppe der vorherigen dar. Kennzeichnend für die hier subsumierten Ansätze ist, dass es sich um strukturierte (Selbst-)Prüfverfahren und/oder standardisierte diagnostische Hilfsmittel handelt. Diese können für die Statusermittlung, für Verbesserungsplanung, Vorbereitung auf eine Zertifizierung sowie zur möglichen Positionierung im internen Zeit- und externen Betriebsvergleich (Benchmarking) eingesetzt werden. Die inhaltliche Definition dessen, was jeweils "gute Qualität" sein soll, wird auf der Basis eines breit akzeptierten fachlichen Modells vorgegeben und in Form von Indikatoren operationalisiert. Modifikationen sind zwar möglich, gehen aber zu Lasten potenzieller Vergleichbarkeit der Ergebnisse. Wenn auch die diagnostische Funktion im 
Vordergrund steht, sind Prozess auslösende Wirkungen schon im Verfahren angestrebt (Qualitätsdiskurse, Wirksamkeitsdialoge u. a.).

5. QM-Kontextkonzepte und Qualitätstechniken (formalisierte Verfahren und Instrumente)

In dieser Kategorie sind einerseits formalisierte Verfahren zusammengefasst, die im Kontext der Qualitätsdebatte (wieder) Aktualität erlangt haben, jedoch in einer anderen Wissenschaftstradition stehen; zum Teil werden sie als eigenständige Managementansätze behandelt. Andererseits sind hier jene Teilkonzepte und instrumentellen Hilfsmittel gebündelt, die in der Fachliteratur als Qualitätstechniken firmieren. In beiden Fällen handelt es sich um zumeist unspezifische - also auch in anderen Zusammenhängen verwendbare - Modelle und Instrumente zur Bearbeitung von qualitätsrelevanten Fragestellungen. Die inhaltliche Definition dessen, was jeweils "gute Qualität" sein soll, wird den Akteuren überlassen; dies kann auch eine externe Anspruchsgruppe sein, z. B. im Falle einer Fremdevaluation.

Im Rahmen der vorliegenden Arbeit kann nur ein Teil dieser Qualitätskonzepte behandelt werden, darunter die im Mittelpunkt der empirischen Hauptstudie stehenden Instrumente (s. Kap. C 2.). Sie sind ebenso branchenunspezifisch einsetzbar wie die als formale Universalkonzepte bezeichneten QM-Modelle der ersten Kategorie (s. o.). 


\subsection{Ergebnisse der Literaturrecherchen zu Qualitätskonzepten}

Die in Kap. A 4.2 beschriebenen Literaturrecherchen liefern den Fundus, aus dem im Folgenden zunächst die als Qualitätskonzepte (s. o.) etikettierten Ansätze zusammengestellt sind. Als Gliederungskriterium wird dabei die zuvor erörterte formale Systematik verwendet, ergänzt um Angaben zum primär vorgesehenen Einsatzbereich. Sofern die Konzepte eindeutig AutorInnen oder offiziellen Informationsquellen zugeordnet werden können, sind diese aufgeführt, ansonsten wird auf kompetente andere Quellen verwiesen.

QM-Kontextkonzepte und Qualitätstechniken im Sinne der fünften Kategorie (s. o.) sind in der folgenden Aufstellung nicht berücksichtigt; sie wurden bereits in Kap. B 1.7, teilweise auch in Kap. B 3. erörtert. Praktische Instrumente für die Umsetzung von Konzepten werden in Kap. C 2. behandelt. Die Grenzen zwischen solchen Instrumenten und dem jeweiligen, als Qualitätskonzept bezeichneten, "theoretischen Überbau" oder den als Qualitätstechniken etikettierten Werkzeugen sind fließend und über manche Zuordnung wird man geteilter Meinung sein können. Im Vordergrund der getroffenen Unterscheidung von Konzepten und Instrumenten steht der eher technische Charakter der Letzteren: organisatorische Maßnahmen und soziale Settings, Dokumentationsformen und funktionale Gliederungen im Arbeitsablauf u. dgl. mehr.

Warum diese Phänomene nicht pauschal zu den Qualitätstechniken gezählt werden, sondern hier als

"Instrumente" firmieren, wird am Beispiel des/der Qualitätsbeauftragten deutlich: Die Funktionsstelle ist sowohl Bestandteil eines (organisatorischen) Konzepts, als auch praktisches Hilfsmittel bei der Umsetzung von QM - aber ist sie auch eine Qualitätstechnik?

Manche AutorInnen verwenden zur Differenzierung der gleichen Sachverhalte die Begriffe "Prinzipien", "Praktiken" und "Techniken" (z. B. Dean \& Bowen, 2000, S. 4 ff.).

In der Kategorie der materialen Branchenkonzepte sind auch verbandsspezifische, vorwiegend dem Gesundheitswesen zuzurechnende Modelle der Qualitätsprüfung aufgeführt, die primär im Zeichen der Zertifizierung bzw. Gütesiegelvergabe stehen. 
1. Formale Universalkonzepte (branchenunabhängige QM-Modelle und allgemeine Strategien):

○ ISO-Normenreihe 9000 ff. (Deutsches Institut für Normung, 2000),

○ Total Quality Management (TQM - Zink, 1995; Zollondz, 2002)

○ Kontinuierlicher/s Verbesserungsprozess/management (KVP/KVM, Kaizen - Imai, 1994),

○ Excellence Modell der European Foundation for Quality Management (EFQM) und Derivate (European Quality Award EQA, Ludwig-Erhard-Preis - www.deutscheefqm.de),

- Integriertes QM nach dem St. Galler Managementkonzept (Seghezzi, 1994, 1996, 2003),

- Freiburger Management-Modell für Nonprofit-Organisationen (Schwarz, Purtschert, Giroud, 1999),

○ Service Assessment (ServAs - Eversheim, 1997).

2. Formale Branchenkonzepte (adaptierte Universalmodelle und feldspezifische Strategien):

○ QAP (Qualität als Prozess, versch. Branchen - www.freyakademie.ch)

○ CAF (Common Assessment Framework, europäisches Qualitätsbewertungssystem für öffentliche Verwaltungen - www.caf-netzwerk.de; Deutsche Bundesregierung, 2003),

○ 2Q-System (Qualität und Qualifizierung, versch. Branchen - K. Frey, 1997, 1997a),

○ GAB-Verfahren (soziale Dienstleistungen - Brater \& Maurus, 1997),

- CBO (Dezentrale Qualitätsentwicklung in der Interkollegialen Methode, Pflege, Altenhilfe - Giebing, 1991),

○ Münchner Modell (Partizipatives QM, Gesundheitsbereich und Jugendhilfe - Bobzien et al., 1995),

○ Internes QM (versch. Ansätze, Soziale Arbeit - Gerull, 2004; Meinhold, 1996; Burmeister \& Lehnerer, 1996),

- QM als organisationale Lernstrategie (Kriterien- und Verfahrensmodell, Jugendhilfe/Soziale Arbeit - Merchel, 2001), 
○ QQS (Qualitätsentwicklung, Qualitätssicherung und Selbstevaluation, Jugendhilfe/Soziale Arbeit - von Spiegel, 2002),

○ AZOMP (praxisorientiertes Verfahrensmodell, Jugendhilfe/Soziale Arbeit - Hekele, 1999),

○ QUIND (Selbstevaluation mit Qualitätsindikatoren, Schule/Weiterbildung $w w w . l s w . n r w . d e)$,

○ LQW (Lernerorientiertes Qualitätsmodell Weiterbildung, Erwachsenenbildung www.artset-lqw.de),

- QUI (Entwurf eines integrativen Qualitätskonzepts, Soziale Arbeit - Boeßenecker et al., 2003).

3. Materiale Branchenkonzepte (feldspezifische Anforderungskataloge und Regelwerke):

○ Ziel- und kriterienbezogenes QM (Soziale Arbeit - Heiner, 1996),

- Wege zur Qualität (Heilpädagogik und Sozialtherapie - Herrmannstorfer, 1999),

○ Qualitäts-Check PQ-Sys (Sozialwirtschaft - Paritätische Gesellschaft für Qualität mbH, 1999, s. auch Wanke, 2003),

○ Dormagener Qualitätskatalog der Jugendhilfe (Stadt Dormagen, 2001),

○ Qualitätshandbuch Wohnen im Heim (Altenhilfe - Kuratorium Deutsche Altershilfe, $w w w . k d a . d e)$,

○ Qualitätssiegel Seniorengerechtes Leben und Wohnen (Altenhilfe - Bundesarbeitsgemeinschaft der Seniorenorganisationen, www.bagso.de),

○ TÜV-Siegel ambulante Pflegedienste und Pflegeheime (Reck-Hog \& Klink, 1998),

- Diakonie-Siegel Pflege (Diakonisches Werk Hamburg, 2000),

○ Qualitätsgemeinschaft Pflege (QgP - LIGA der Freien Wohlfahrtspflege im Land Brandenburg; J. F. W. Müller, 2002),

- Pflege-TÜV (Bundesverband privater Anbieter sozialer Dienstleistungen, 1998; Seits \& Tews, 1999),

- Gütegemeinschaft Pflege (Der Paritätische Wohlfahrtsverband, Landesverband Hamburg, 1996),

○ Heime zum Leben (Alten- und Behindertenhilfe - Harris, Klie \& Ramin, 1995),

○ Kooperation für Transparenz und Qualität im Krankenhaus (www.ktq.de), 
- MUM (Monitoring Evaluation Management, Pflegebereich - Roes, François-Kettner, Schmälzle \& Lehmann, 2000),

○ AMIQ (QMK: Qualitätsmodell Krankenhaus - Paeger, Ortega-Suhrkamp, Hofmann, 1998),

○ QM-System der DEGEMED (Deutsche Gesellschaft für medizinische Rehabilitation e. V., 1998),

○ Lörracher Qualitätskonzept für die Krankenhaus-Sozialarbeit (Trost \& Marquart, 2002),

○ AQUA-FUD/FED (Arbeitshilfe zur Qualitätsentwicklung für Familienunterstützende und Familienentlastende Dienste - McGovern, Oberste-Ufer, Rohrmann, Schädler \& Schwarte, 2000),

○ PPQ (Pro Psychiatrie Qualität - Sozialpsychiatrie, Bundesverband Evangelische Behindertenhilfe (BEB), Fachverband Psychiatrie in der Caritas (PiC), 1998),

○ IQUE (Integrierte Qualitäts- und Personalentwicklung in Kindertageseinrichtungen Ziesche, 2001).

4. Materiale Branchenkonzepte (operationalisierte Prüfkriterien und standardisierte Bewertungsverfahren):

○ Selbstbewertungssystem für stationäre Einrichtungen der Altenhilfe (SEA - BETTAL Quality Consultancy, Fraunhofer-Institut für Arbeitswirtschaft und Organisation IAO, Gemeinschaftsprojekt Handbuch zur Qualitätssicherung, Pilota - Mobiles Fortbildungsteam Altenhilfe, 1998),

- Standardisiertes Instrumentarium zur Evaluation von Einrichtungen der stationären Altenhilfe (SIESTA - Berger, 1998),

○ Lebensqualität in Wohnstätten für erwachsene Menschen mit geistiger Behinderung (LEWO - Schwarte \& Oberste-Ufer, 1997),

○ Verfahren zur EDV-gestützten Gestaltung der Betreuung für Menschen mit Behinderungen (GBM - Haisch, 1998),

○ System der Leistungsbeschreibung, Qualitätsbeschreibung, Qualitätsprüfung und Entgeltberechnung in der Behindertenhilfe (SYLQUE - Verband katholischer Einrichtungen und Dienste für lern- und geistigbehinderte Menschen e. V., 1996), 
○ Qualität im Dialog entwickeln (Kronberger Kreis für Qualitätsentwicklung in Kindertageseinrichtungen, 1998),

○ Kindergarten-Einschätzskala (KES/KES-R - Tietze, Schuster \& Rossbach, 1997; Tietze, Schuster, Grenner \& Rossbach, 2001),

○ Kieler Instrumentarium für Elementarpädagogik und Leistungsqualität (K.I.E.L., Kindertagesstättenbereich - Krenz, 2001),

○ WANJA-Instrumentarium (Selbstbewertung in der Jugendhilfe - Projektgruppe WANJA, 2000),

○ Selbstbewertung des Qualitätsmanagements in der Jugendhilfe (SQ-J - Gerull, 2000a; Biebricher, 2003),

○ Evaluations-Instrumentarium kommunaler Arbeitsmarktpolitik (EIKAP - www.achimtrube.de).

\subsection{Darstellung und Kritik ausgewählter Universalkonzepte}

\subsubsection{Qualitätsmanagement nach DIN EN ISO 9000 ff.}

\subsubsection{Vorbemerkungen}

Als Mitte der Achtzigerjahre ein erster DIN-Entwurf über ein Qualitätssicherungssystem vorgelegt wurde - ein Vorläufer der ISO 9000 -, war sich die deutsche Industrie noch in dessen Ablehnung einig. So etwas verteuere nur die ohnehin unter Preisdruck stehenden Produkte; deutsche Anbieter könnten sich angesichts ihres weltweit anerkannten Qualitätsstandards auch ohne solche bürokratischen Regularien durchsetzen (Wolters et al., 1995, S. 3).

Diese Einschätzung hat sich inzwischen grundlegend verändert. "Made in Germany" reicht nicht mehr als Gütesiegel. Systematisches Qualitätsmanagement wurde als erfolgreiches Instrument zur Förderung von Wettbewerbsfähigkeit, Kunden- und Mitarbeiterzufriedenheit anerkannt und europaweit vereinheitlicht. Die ISO 9000 ff. gilt längst nicht mehr nur für den Produktionsbereich, sondern gleichermaßen für die Dienstleistungsbranche. Dabei handelt es sich keineswegs um ein starr anzuwendendes 
Regelwerk, sondern um eine Reihe von Empfehlungen hochkarätiger internationaler Wirtschaftsfachleute zum Aufbau und Ablauf eines Betriebs, um ein bestimmtes Niveau zu erreichen, zu halten und zu verbessern. Normiert werden Mindestanforderungen an die qualitätsrelevanten unternehmerischen Prozesse und deren Dokumentation (Gerull, 2004, Kap. 2.2.1).

\subsubsection{Darstellung des Ansatzes}

ISO (International Organization for Standardization) ist die internationale Dachorganisation praktisch aller bedeutenden nationalen Normenverbände mit Sitz in Genf. Normenorganisationen veröffentlichen Vorschriften für unterschiedlichste Anwendungsbereiche. Die meisten Normentexte von ISO (International), EN (Europa) und DIN (Deutschland) sind identisch. Da jede Norm nummeriert ist und die bekanntesten Normen zur Qualität von Produkten und Dienstleistungen die Nummern 9001 bis 9004 tragen, spricht man von Normen der ISO-9000-Familie. Die zunächst für die produzierende Industrie entwickelte Norm wurde 1988 vom internationalen Dachverband ISO übernommen und seither mehrfach neu aufgelegt.

Die Normen der ISO-9000-Familie entstanden aus dem Bedürfnis, betriebliche Maßnahmen zur Sicherung von Qualität zu standardisieren, um das Vertrauen von Kunden bzw. Auftraggebern in die Leistungsfähigkeit ihrer Lieferanten bzw. Auftragnehmer zu gewinnen. Vor allem die Hersteller von Produkten, die aus zahlreichen Zulieferteilen gefertigt werden, sind auf solches Vertrauen angewiesen. Da es weder technisch möglich noch wirtschaftlich sinnvoll ist, jedes zugelieferte Teil vor der Weiterverarbeitung darauf zu überprüfen, ob es den Anforderungen entspricht, leuchtet ein, wie wichtig für einen Kunden die Überzeugung ist, dass der Lieferant selbst bereits Maßnahmen der Qualitätssicherung und Fehlervermeidung im Herstellungsprozess angewandt hat.

Die ISO 9000 ff. war seinerzeit der erstmalige Versuch, für Aspekte der Betriebsführung verbindliche - wenngleich sehr allgemein formulierte und in unterschiedlichen Branchen verschiedenartig auszugestaltende - Vorgaben zu machen, um diese "Prozessqualität" im Sinne möglichst fehlerfreier Verfahren zu gewährleisten. Die Vorgaben der ISO 9000 ff. waren bis zu ihrer Revision 1999 als 20 Elemente (u. a. Verantwortung der Leitung, 
Prozesslenkung, Korrektur- und Vorbeugungsmaßnahmen) formuliert, die den vollständigen Leistungserstellungsprozess abdecken sollten. Soweit betriebsrelevant, waren diese Elemente anforderungsgerecht zu bearbeiten und in einem QM-Handbuch (s. Kap. C 2.2.2) darzustellen. Damit waren die Normforderungen hinsichtlich der Darlegung des QM erfüllt und eine Zertifizierung des Betriebs nach Maßgabe der Bezugsnorm war möglich.

Eine Zertifizierung dient dem Nachweis, dass das QM-System des Betriebes von einer kompetenten und neutralen Stelle umfassend auf seine Norm entsprechende Installation und Funktionsfähigkeit geprüft ("auditiert") und laufend überwacht wird; bei erfolgreicher Prüfung wird ein Zertifikat mit befristeter Gültigkeit erteilt. Dieses macht jedoch lediglich Aussagen über die Qualitätsfähigkeit einer Organisation, nicht aber über die Produktqualität selbst und deren Übereinstimmung mit den Erwartungen der Kunden. M. a. W., nicht die Qualität des herzustellenden Produkts oder der zu erbringenden persönlichen Dienstleistung wird bewertet, sondern lediglich das im Sinne der ISO-Norm vorschriftsmäßige System aufbau- und ablauforganisatorischer Maßnahmen, um Qualität und ihre Sicherung möglich zu machen (Gerull, 2001).

Die zum Jahreswechsel 2000/2001 veröffentlichte Revisionsfassung der ISO 9001 behandelt nicht mehr nur die Qualitätssicherung/QM-Darlegung als Mittel zur Erzielung fehlerfreier Produkte, sondern schließt in die Forderungen an das QM-System auch die auszuweisende Fähigkeit der Organisation ein, Kundenzufriedenheit zu erreichen. Damit bewegt sich das ISO-QM-System inhaltlich auf die umfassenderen Konzepte eines Total Quality Management (TQM, s. Kap. C 1.4.2) zu.

Die 20 Elemente der Vorgängernorm wurden gegen acht Prinzipien ausgetauscht, in denen sich die Elemente wiederfinden (Zollondz, 2002, S. 259; Kämmer et al., 2001, S. 54):

o Kundenorientierung,

o Führung,

o Beteiligung der Mitarbeiter,

o prozessorientierter Ansatz (Tätigkeiten und zugehörige Ressourcen werden als Prozess geleitet und gelenkt), 
o systemorientierter Managementansatz (in Wechselbeziehung stehende Prozesse werden als System verstanden und gelenkt),

o ständige Verbesserungen,

o sachbezogener Ansatz zur Entscheidungsfindung (auf der Analyse von Daten und Informationen beruhend),

o Lieferantenbeziehungen zum gegenseitigen Nutzen (Beachtung der gegenseitigen Abhängigkeit).

Während für Zertifizierungszwecke weiterhin die revidierte ISO 9001 verwendet wird, enthält die neue ISO 9004 unter dem Titel "Qualitätsmanagementsysteme - Leitfaden zur Leistungsverbesserung" Anregungen $\mathrm{zu}$ allen Aspekten eines TQM-Systems. ISO 9004:2000 ist somit wie ihre Vorgängerin (ISO 9004:1994) keine eigentliche Norm ("Darlegungsnorm"), also verbindliche Vorschrift, sondern eine "Empfehlungsnorm". Die früheren Normen 9002 und 9003 wurden bei Veröffentlichung der ISO 9001:2000 zurückgezogen und durch die Möglichkeit ersetzt, bestimmte Forderungen an die Produktrealisierung auszuschließen, die für das Unternehmen nicht relevant sind.

Mit der Überarbeitung der Normen sollten offenkundig gewordene Schwächen der bisherigen ISO 9000 ff. beseitigt werden: die stark industrielastige Sprache, die willkürlich erscheinende Festlegung von 20 Normelementen, die Vernachlässigung kundenorientierter und dynamischer Aspekte - um nur einige zu nennen. Als wesentliches Merkmal der neuen Normen wird die weitgehende Annäherung an ein Prozessmodell des Qualitätsmanagements betrachtet. Darin wird die systematische Erkennung und Beherrschung der organisationsinternen Prozesse und die Einbeziehung maßgeblicher externer Schnittstellen in die Prozessgestaltung im Sinne eines Regelkreises der Verbesserung begriffen.

Das Prozessmodell der ISO 9001:2000 enthält folgende Strukturelemente:

- Management-Verantwortung (z. B. Politik, Ziele, Planung),

- Ressourcen-Management (z. B. Personalentwicklung, Informationsmanagement, Ausstattung),

- Prozess-Management (z. B. Entwicklung, Beschaffung, Dienstleistungserbringung), 
- Bewertung und Analyse (z. B. Audits, Evaluation, Verbesserungen).

Dieses Gerüst ist eingebettet in die Elemente "Kundenanforderungen" und "Kundenzufriedenheit", die den Regelkreis auf der Input- und Output-Seite vervollständigen.

\subsubsection{Beurteilung des Ansatzes}

Grundsätzliche Kritik an der ISO-9001:1994 (vgl. Bretzke, 1995) galt ihrer mangelhaften Vollständigkeit und Relevanz (wesentliche Qualitätselemente fehlten, andere waren übererfüllt), ihrer geringen Präzision (branchenspezifisches Organisationswissen war nicht enthalten) und problematischen Wirksamkeit (Überfixierung auf formale Aspekte und quasi-mechanisches Qualitätsverständnis). Kritisiert wurde auch der statische Blickwinkel (Beherrschung der Prozesse, Fehlerfreiheit des Produkts, Normkonformität des QMSystems), der Anwender dieses Systems dazu verführen konnte, nur auf die zertifizierbaren Anteile eines QM zu achten, wohingegen dynamische Faktoren wie Mitarbeiter- und Kundenorientierung sowie die Frage, worin "Qualität des Produkts" denn eigentlich bestehe, gar nicht von der Norm erfasst wurden (Gerull, 1999b).

Ein Teil dieser Kritik dürfte nach der Revision der Norm obsolet sein; die dynamischen und kundenbezogenen Elemente des Qualitätsmanagements wurden in der ISO 9001:2000 erheblich aufgewertet. Defizite werden allenfalls noch im Bereich der Personalentwicklung ausgemacht (Offermann, 2001, S. 60).

Die ISO 9000 ff. eignet sich nach Auffassung vieler Autoren gut zur Herstellung von Transparenz und zur Optimierung der Aufbau- und Ablaufstrukturen einer Organisation $(z$. B. K. Frey, 1997a, S. 32f). Die Einführung eines QM-Systems nach Gestaltungsparametern der ISO 9000 ff. kann insofern einen Strukturgewinn für die Stabilität und Nachhaltigkeit einer QM-Kultur bedeuten (Gerull, 2000).

Offermann (2001, S. 59) hebt die Klärung der Prozesse und Verantwortlichkeiten als positiv hervor und sieht in der Bündelung aller Systembeschreibungen in einem zentralen Dokument, dem QM-Handbuch, einen besonderen Vorzug des Ansatzes gegenüber anderen Modellen. Allerdings verführe die geforderte Dokumentation zu Überre- 
gulierungen (a.a.O., S. 59f). Vorbehalte gibt es auch gegen die Anwendung in sehr kleinen Einrichtungen, die mehr den Charakter sozialer Gruppen haben (S. 57f).

Nach Auskunft eines TÜV-Chefauditors (Wiegelmann, 1999, mdl. Mitteilung) finden sich in der Praxis häufige Schwachstellen, die nicht primär dem System, sondern Anwendern anzulasten sind:

- bürokratisch-perfektionistische Interpretation der Norm statt unternehmensspezifisch flexibler Auslegung;

- Missverhältnis von Dokumentationsaufwand und praktischem Nutzen: detaillierte Verfahrensanweisungen zu irrelevanten Prozessen, komplizierte Ausdrucksweise statt Wahrung des Prinzips der Einfachheit und Verständlichkeit;

- Verstaubenlassen von Qualitätshandbüchern statt lebendiger Integration in die Unternehmenskultur, Vorschriften für die Schublade;

- Überbetonung des formalen und statischen Charakters von Qualität (Zertifizierung).

Merchel (2001, S. 69f) betont die Gefahr, dass durch den systembedingten Ansatz der Standardisierung und Kontrolle von Verfahren Tendenzen unterstützt werden können, das Qualitätsthema zu bürokratisieren. Außerdem stehe der Qualitätssicherungsgedanke der Prozesshaftigkeit und Kontextgebundenheit sozialer Dienstleistungen entgegen.

Im Profit-Bereich kann ein ISO-Zertifikat angesichts der großen Bekanntheit des Systems einen gewissen Wettbewerbsvorteil erbringen, zumal dann, wenn Geschäftskunden danach verlangen. Im sozialen Dienstleistungsbereich sprechen jedoch viele Gründe dafür, statt einer Zertifizierung nach ISO branchenspezifische Formen eines punktuellen oder umfassenden Qualitätsmanagements zu entwickeln und primär auf interne Steuerungsinstrumente zu setzen, statt auf die kostspielige Expertise neutraler und häufig feldunkundiger Dritter (Gerull, 1999b).Hinzu kommt, dass Hoffnungen auf eine Verbesserung der Marktposition durch Zertifizierung in der Regel frustriert werden; Belegungsprobleme von stationären Einrichtungen etwa lassen sich damit nicht bewältigen. Vielfach bleibt "allein die bürokratische Last - und das Problem, wie man sich der nur für die Außendarstellung erworbenen und dort verwendeten Zertifizierungen ohne Marketing-Probleme wieder entledigen kann" (Severing, 1999, zit. nach Schädler, 2001, S. 33f). 
Grundsätzlich ist es jedoch möglich, sich an der ISO als Einstiegs- und Strukturierungshilfe für ein betriebliches Qualitätsmanagement zu orientieren. Wen die immer noch industrielastige Nomenklatur nicht abschreckt, wird in der Norm und im Leitfaden eine Vielzahl nützlicher Anregungen finden, das eigene Betriebssystem zu optimieren, ohne an eine vollständige Übernahme des Systems oder eine Zertifizierung zu denken. Auch auf die Norm entsprechende Installierung des keineswegs unumstrittenen Systemelements "Qualitätsbeauftragter" (s. Kap. C 2.2.4 und D 4.6.8) oder die Erarbeitung eines alle Systemelemente umfassenden QM-Handbuchs kann gegebenenfalls zugunsten anderer Lösungen des Koordinations- und Dokumentationsproblems verzichtet werden.

Zwar stellen die Einarbeitung in die textlichen Grundlagen des Systems, die Übersetzung der formalen Sprache in Begriffe und fachliche Inhalte des eigenen Berufsfeldes und die zu leistende Überzeugungsarbeit gegenüber ISO-skeptisch eingestellten MitarbeiterInnen eine erhebliche Ressourcenbindung dar. Soziale Einrichtungen, welche sich des "Werkzeugs ISO" bedient haben, berichten jedoch überwiegend positive Erfahrungen, ohne aus dem erwähnten Übersetzungsaufwand einen Hehl zu machen (z. B. Maaß, 1999).

Inzwischen liegt eine Reihe von Veröffentlichungen für verschiedene Branchen vor ( $\mathrm{g} l$. Gerull, 2004), die den Einführungsprozess erheblich erleichtern, darunter eine von Rugor \& Studzinski (2003) erarbeitete Praxisanleitung für soziale Einrichtungen auf der Basis der revidierten Normen. Die Autorinnen konstatieren eine Präferenz der großen Wohlfahrtsverbände für die ISO und betonen, dass die Anforderungen der Kostenträger an die Qualitätsentwicklung sich in weiten Teilen mit den Anforderungen der ISO-Norm deckten (a.a.O., S. 94).

\subsubsection{Das Konzept des Total Quality Management (TQM)}

\subsubsection{Darstellung des Ansatzes}

Qualität wurde lange Zeit lediglich als Übereinstimmung eines fertigen Produkts mit Anforderungen des Kunden verstanden. Diese eher statische, zustandsbezogene Bedeutung erweiterte sich nach und nach auf die "Gesamtheit von Eigenschaften und Merkmalen ei- 
nes Produkts oder einer Tätigkeit, die sich auf deren Eignung zur Erfüllung gegebener Erfordernisse beziehen" (DIN 55 350).

Dadurch wurde die Dienstleistung in den Qualitätsbegriff miteinbezogen und das Spektrum der Anspruchsgruppen erweitert, die über Qualität oder Nicht-Qualität entscheiden (Horvath, 1997). Außer den Kunden im engeren Sinne beteiligen sich daran auch die MitarbeiterInnen (interne Kunden), die Geschäftsführung, die Öffentlichkeit und ggf. weitere Interessen- oder Anspruchsgruppen (stakeholder), da sie alle an die Dienstleistungen und Produkte bestimmte Erwartungen stellen. Das Ausmaß, in dem diese Erwartungen erfüllt werden, stellt die Unternehmensqualität dar, die Total Quality; sie ist Bezugspunkt für den Ansatz des Total Quality Managements (TQM).

Der konzept- und branchenneutrale Qualitätsbegriff der ISO ("unmittelbarer Qualitätsbezug" nach Geiger, 1998, zit. nach Zollondz, 2002, S. 158) wird im TQM ergänzt durch Einheiten mit mittelbarem sowie ohne direkten Qualitätsbezug. Im Qualitätsbegriff des TQM sind somit neben den "inhärenten" auch "zugeordnete" Merkmale einbezogen (s. Kap. B 1.2).

Neu am Konzept des TQM ist folglich die dynamische Erweiterung des Verständnisses von Qualität von der reinen Produktbezogenheit auf das "Drumherum", auf die Qualität der Prozesse, die zu diesem Produkt beitragen, vor allem die Dienstleistungsqualität (sachlich, persönlich, sozial) und die Sozialverträglichkeit (gesellschaftlicher Aspekt).

\footnotetext{
"Entgegen dem traditionellen Verständnis von Qualität als technischer Perfektion orientiert sich TQM nicht am denkbar Besten, sondern zielt auf die realen Wünsche der potentiellen Käufer ab ... Durch [die Kombination von Produkt- und Prozessqualität, P. G.] wird gewährleistet, dass der angestrebte Kundennutzen nicht auf unwirtschaftliche Weise erzielt wird" (Schultz-Gambard et al., 1999, S. 92).
}

Die Bezeichnung "Total Quality" geht vermutlich auf das Buch von A. Feigenbaum "Total Quality Control" (1961) zurück, in dem das klassische industrielle Kontrollkonzept Prüfung des Endprodukts - auf den gesamten Herstellungsprozess und alle Unternehmensteile bezogen wurde. In Japan war es Ishikawa, der, darauf aufbauend, das Konzept der "Company-Wide Quality Control" entwickelte. Darin spielt die Beteiligung aller Arbeitsgruppen über die populär gewordenen Qualitätszirkel eine wesentliche Rolle $(K$. 
Frey, 1997, S. 12f).

Aus Japan quasi reimportiert, wurde das Total Quality-Konzept in den USA mit dem betriebswirtschaftlichen Management-Begriff verknüpft und zu TQM umdefiniert, einem "strategischen, integrierten Managementsystem zur Erreichung zufriedener Kunden. Das Managementsystem bezieht alle Vorgesetzten und Mitarbeiter/-innen mit ein. Man benützt qualitative Methoden, um die Prozesse im Unternehmen dauernd zu verbessern" (Federal Quality Institute, USA, zit. nach K. Frey, 1997, S. 13).

TQM wird somit als relativ unspezifisches Konzept betrachtet, als ein Konglomerat aus Haltungen, Prinzipien und sehr unterschiedlichen Instrumenten. Die in der Praxis verwendeten, inzwischen über 200 Werkzeuge (tools, vgl. Kap. B 1.6), von denen Qualitätszirkel und Brainstorming mit die bekanntesten sind, entstammen vorwiegend dem Marketing, der Organisations- und Personalentwicklung sowie der Sozialpsychologie. Dahinter steht laut K. Frey keine geschlossene Theorie oder Systematik, sondern reiner Pragmatismus; empirische Forschung zu den Prozessen und Effekten begann erst Mitte der Neunzigerjahre (a.a.O.).

Zollondz (2002, S. 264f) betont, dass in TQM keine revolutionären oder unbekannten Elemente enthalten seien. Vielmehr handele es sich um die systematische und konsequente Anwendung einiger Methoden innerhalb einer auf Qualität und Kundenzufriedenheit ausgerichteten Unternehmenskultur. Easton \& Jarrell (2000, S. 90) würdigen genau diese integrative Funktion des TQM-Konzepts:

"Because most of the themes and approaches that make up TQM were not originated as a part of TQM, but, rather, adopted by TQM, much of the contributions of TQM has to do with the integration of the various themes into a coherent management system. More than any of TQM's specific aspects, it is this integration of technical, cultural, behavioural, and organizational facets that makes TQM a distinct phenomenon."

Noch dezidierter äußern sich Dean \& Bowen (2000); sie konstatieren zwar, dass das TQMKonzept in der Fachliteratur uneindeutig und wie eine Art Rorschach-Test verwendet werde, auf den Menschen gemäß ihren Überzeugungen und Erfahrungen unterschiedlich reagieren (S. 4). Die Autoren halten jedoch die Verknüpfung von Kundenorientierung, kontinuierlicher Verbesserung und Teamwork für TQM-charakteristisch: 
"Thus, TQ is not simple a hodgepodge of slogans and tools; it is a set of mutually reinforcing principles, each of which is supported by a set of practices and techniques, and all of which are ultimately based on fulfilling customers’ needs" (S. 6).

In der Mehrheit der einschlägigen Publikationen werden die grundlegenden Konzepte des TQM - in der ISO 8402:1994 erstmals als Umfassendes Qualitätsmanagement übersetzt unterschiedlich akzentuiert. Während sich die abstrakten Leitsätze weitgehend ähneln, bleiben die konkreten Inhalte in der Regel offen und sollen von den einzelnen Einrichtungen selbst festgelegt werden (Regus, 2001, S. 86).

Eine komprimierte und systematisierte Übersicht zu den wichtigsten Aspekten des TQM vermittelt der folgende Kasten (Gerull, 2000).

\section{Total Quality Management (TQM) -}

ein ganzheitlicher Ansatz zur Erzielung von Unternehmensqualität

Vier konzeptionelle Säulen:

- Kundenorientierung

(Erwartungen/Bedürfnisse der Adressaten stehen im Mittelpunkt: Beteiligung, Kundenzufriedenheit als Ergebnisziel, internes Kunden-Lieferanten-Verhältnis)

- Prozessorientierung

(Regelmechanismen und Lernfähigkeit sind wichtiger als statische Strukturen: kontinuierliche Verbesserung, Selbststeuerung, fehlerfreundliche und lernende Organisation)

- $\quad$ Mitarbeiterorientierung

(MitarbeiterInnen werden als zentrale Ressource für Unternehmensqualität gewürdigt: Beteiligung, Teamarbeit, Vorrang von Selbstbewertung vor Fremdbewertung, kooperative Führung, Personalentwicklung)

- Ergebnisorientierung

(Zielvereinbarungen und deren Umsetzung stehen im Vordergrund: Kunden- und Mitarbeiterzufriedenheit, finanzielle und nichtfinanzielle Geschäftsergebnisse, Image) 
Zollondz (2002, S. 265) betont weitere Gesichtspunkte, u. a.:

- Aufbau eines Netzwerkes von partnerschaftlichen Kunden-Lieferanten-Beziehungen, wobei jeder nachfolgende Prozess als Kunde zu betrachten ist;

- Konzentration auf die Wertschöpfungskette und Abbau nicht kundenrelevanter Tätigkeiten;

- top-down-Ansatz mit missionarischer Einbringung der obersten Unternehmensleitung;

- partizipatives und zugleich straffes Management.

\subsubsection{Beurteilung des Ansatzes}

Das TQM-Konzept ist sicherlich die umfassendste Qualitätsstrategie, die für ein Unternehmen denkbar ist; QM-System und Organisationsstruktur gehen quasi ineinander über. An die Stelle einer inhaltlich von anderen Managementaufgaben abgrenzbaren Funktion tritt eine ganzheitliche Unternehmensphilosophie, in der eine spezifische, nämlich kunden- und qualitätsorientierte Haltung zum Ausdruck kommt. Überlegene Produkte, fähige Prozesse und motivierte MitarbeiterInnen stellen die übergeordneten Ziele dar (Zollondz, 2002, S. 262).

Als Sammelbegriff für grundlegende Gestaltungsprinzipien zeitgemäßer Betriebsführung gerät das Konzept allerdings in Gefahr, als Sprachhülse verwendet zu werden.

\footnotetext{
"Wenn alles zur Qualitätsfrage wird, verliert das Qualitätsthema seine spezifischen Herausforderungen; es gerät faktisch aus dem Blick, obwohl alle das Wort 'Qualität' im Munde führen" (Merchel, 2001, S. 113).
}

Dennoch eignet sich TQM nach Meinung vieler AutorInnen als Führungskonzept zur Verbreitung einer qualitätsorientierten Unternehmenskultur (z. B. Bläsing, 2001). Die in der Praxis entwickelten Verfahren, etwa für die Moderation von Arbeitsgruppen, können mit Gewinn eingesetzt werden, um Problemlösungen $\mathrm{zu}$ entwickeln, Schulungen durchzuführen und MitarbeiterInnen auf allen betrieblichen Ebenen einzubeziehen.

Schwerpunkt von TQM ist somit die Qualitätsentwicklung. Für eine auch extern nachvollziehbare Qualitätssicherung eignet sich der Ansatz weniger, da er keine eigene 
Systematik und keinen expliziten Normenbezug enthält, so dass Einrichtungen, die nach Prinzipien des TQM arbeiten, nicht ohne weiteres miteinander verglichen werden können.

Aus diesem Grunde wird die Philosophie des TQM zwar fast einhellig propagiert; die praktische Umsetzung lehnt sich jedoch meistens an standardisierte Bewertungssysteme bzw. -normen an (z. B. EFQM, Service Assessment, ISO 9001).

\subsubsection{Das Konzept des Kontinuierlichen Verbesserungsprozesses (KVP)}

\subsubsection{Darstellung des Ansatzes}

Verfolgt eine Organisation eine ganzheitliche Führungsstrategie, bei der es um die dynamische Weiterentwicklung zu immer besserer Qualität geht, wird oft auf den Ansatz des Japaners Masaaki Imai (1994) zurückgegriffen. Das Konzept wurde unter dem Namen "Kaizen" bekannt und zumeist - wenn auch verkürzend - mit "kontinuierlicher Verbesserungsprozess (KVP)" oder umfassender mit "kontinuierliches Verbesserungsmanagement $(\mathrm{KVM}) "$ übersetzt. Darin spielt ein von allen MitarbeiterInnen getragener aktiver Prozess der undramatischen, kleinschrittigen Optimierung von Produkten und Tätigkeiten die zentrale Rolle.

Laut Yasuda (1994, zit. nach D. Frey, Brodbeck \& Schulz-Hardt, 1999, S. 124) setzt sich der japanische Begriff Kaizen aus den beiden Silben Kai (= Veränderung) und Zen (= das Gute zum Besseren) zusammen und heißt so viel wie "langsame, nie endende Vervollkommnung in allen Bereichen des Lebens."

Wenngleich Imai seine Managementkonzeption ursprünglich an den typisch japanischen Sozialcharakter gebunden hatte, wird der Ansatz längst auf andere Kulturkreise übertragen, allerdings ohne expliziten Bezug auf eine bestimmte Lebensweise. Vielmehr umfasst der Begriff eine Vielzahl von Führungspraktiken, die unter dem "Kaizen-Schirm" gebündelt werden: u. a. Kundenorientierung, Qualitätszirkel, Vorschlagswesen, Null-Fehler-Ansatz, Kanban und Just-in-Time (beides Begriffe aus dem Bereich des Toyota Production Systems - lean production). 
Der von Walter Edwards Deming formulierte PDCA-Zyklus (Deming-Kreis) wird häufig verwendet, um Kerngedanken des Kaizen-Ansatzes zu visualisieren. Der Deming-Zyklus verkörpert einen systematischen Ansatz zur Problemlösung und gehört zum StandardDenk- und Handlungsschema jeglicher Verbesserungsaktivitäten (Zollondz, 2002, S. 237):

o Planen (plan - P),

o Ausführen (do - D),

o Überprüfen (check - C),

o Verbessern $($ act $-\mathrm{A})$.

Der PDCA-Zyklus und andere Werkzeuge des Kontinuierlichen Verbesserungsmanagements werden heute überwiegend gruppenorientiert in Qualitätszirkeln angewandt. Neben gruppenorientiertem Kaizen werden von Imais Firma "Kaizen Support Services, London" auch management- oder personenorientierte Programme vermarktet (a.a.O.).

Kämmer et al. (2001, S. 82) fassen den Zyklus der Kaizen-Aktivitäten wie folgt zusammen:

1. Mitarbeiter motivieren, sich aktiv an Problemlösungen zu beteiligen und ihre täglich anfallenden Arbeiten zu erledigen.

2. Mitarbeiter motivieren, ihre Vorschläge zu Papier zu bringen.

3. Vorschläge prüfen, auswerten, Anleitung und Hilfestellung geben.

4. Leistung anerkennen und vergüten.

Der Prozess wird über Push- und Pull-Strategien gesteuert: zum einen durch motivationsfördernde Hilfen, z. B. durch Zielvereinbarungen, zum anderen durch Anreize auf Unternehmensebene, z. B. Prämien.

Zollondz (2002, S. 239f) berichtet folgendes Procedere bei der Implementierung von Kaizen:

1. Festlegung eines Bereichs für den Start.

2. Mitarbeiter des Bereichs ausführlich informieren.

3. Verbesserungsgruppen bilden. 
4. Methoden und Werkzeuge trainieren.

5. Verschwendung mit Hilfe von Prüfbogen erkennen.

6. Verbesserungsmöglichkeiten erkennen.

7. Maßnahmen diskutieren und auswählen.

8. Maßnahmen durchführen.

9. Auswirkungen messen.

10. Erfolgreiche Maßnahmen generell einführen.

11. Gute Ideen und Leistungen sofort anerkennen und belohnen.

12. Situation erneut analysieren und neue Verbesserungsansätze finden. Wenn der betreffende Bereich von selbst läuft, wird ein neuer Bereich festgelegt.

\subsubsection{Beurteilung des Ansatzes}

Im Grunde handelt es sich bei Kaizen nur um eines von verschiedenen TQM-Konzepten. Als Bindeglied fungieren die Axiome der Problem-, Kunden-, Prozess- und Mitarbeiterorientierung. Besonderes Gewicht wird allerdings den MitarbeiterInnen verliehen; sie werden nicht als nur ausführende Akteure gesehen, sondern als selbstverantwortliche Individuen, denen Instrumente zur Verfügung gestellt werden, die es ihnen ermöglichen, als kritische Beobachter am Unternehmensgeschehen teilzunehmen (Zollondz, 2002, S. 241). Die Affinität des Ansatzes zu Konzepten eines partizipativen QM im Allgemeinen und des betrieblichen Vorschlagswesens im Besonderen liegt auf der Hand.

Wie Zollondz (a.a.O., S. 225) betont, wird das KVM entweder als eigenständige Managementpraxis verstanden oder als eingebettet in das QM. In jedem Falle gehört Kaizen zu den dezentralen internen Modellen. Die Abgrenzung zu anderen TQM-Ansätzen fällt schwer, weil Begriffe unspezifisch und wenig trennscharf verwendet werden. Nicht einmal die "Erfindung" des Konzepts scheint authentisch japanisch; ein betriebliches Vorschlagswesen als Vorläufer entwickelte sich zuerst in den USA (a.a.O., S. 231). Unzweifelhaft ist jedoch die erfolgreiche Vermarktung mittels Kaizen ${ }^{\circledR}$ als eingetragenem Warenzeichen: "Imais KAIZEN entpuppt sich als geschickt positioniertes TQM-Berater-Konzept" ( $S$. 228).

Im Vergleich mit zwölf anderen QM-Ansätzen (Kämmer et al., S. 152f) wird dem Kaizen- 
Konzept eine relativ hohe Mitarbeiterbeteiligung, Transparenz, Verständlichkeit und Praxisbezogenheit attestiert. Einen Spitzenplatz in der Bewertung durch die AutorInnen nimmt das Verfahren in puncto Prozess- und Strukturoptimierung ein (z. B. optimale Erfüllung bezüglich Messung von Effektivität und Effizienz). Dagegen werde die für soziale Dienstleistungen besonders wichtige externe Kundenorientierung nur partiell berücksichtigt (a.a.O.).

\subsubsection{Das Konzept der European Foundation for Quality Management (EFQM)}

\subsubsection{Darstellung des Ansatzes}

In der bis 2000 gültigen Fassung der ISO 9001:1994 wurden 20 obligatorische Elemente eines QM-Systems beschrieben, von denen 12 mehr oder weniger explizit Dokumentationstätigkeiten einschlossen, während in keinem Element gefordert wurde, inhaltliche Standards zu erarbeiten (Gerull, 2000, S. 2:14). Diese formale Schwerpunktsetzung der ISONormenreihe gab den Anlass, stärker an konkreten Qualitätskriterien orientierte Systeme zu entwickeln, die ein weniger statisches Verständnis von Qualität zugrunde legen und auf einer umfassenden Qualitätsphilosophie beruhen (Total Quality Management, s. o.).

Ein solches TQM-Konzept stellt das Modell für Business Excellence der European Foundation for Quality Management (in der Version 2000 "EFQM Excellence Modell" genannt) dar, in dem Qualitätsmanagement als breit angelegter Organisationsentwicklungsprozess verstanden wird. Dieser beruht auf den "acht Eckpfeilern der Excellence" (www.deutsche-efqm.), die allerdings nicht EFQM-spezifisch sind, sondern generell TQM-Ansätze charakterisieren (Gerull, 2004, Kap. 2.2.4).

o Ergebnisorientierung,

o Kundenorientierung,

o Führung und Zielkonsequenz,

o Management mit Prozessen und Fakten,

o Mitarbeiterentwicklung und -beteiligung,

o kontinuierliches Lernen, Innovation und Verbesserung, 
o Aufbau von Partnerschaften,

o Verantwortung gegenüber der Öffentlichkeit.

EFQM ist das europäische Pendant zum amerikanischen Baldrige-System, benannt nach dem von Staatssekre tär Baldrige ausgeschriebenen Qualitätspreis (Baldrige Award). Mit diesem reagierte der Kongress der USA 1988 auf die qualitative Überlegenheit japanischer Importgüter, um über ein nationales Wettbewerbsprogramm für Unternehmen verschiedenster Bereiche die Qualität der eigenen Produkte zu verbessern und Marktanteile zurückzuerobern. In Europa, das sich den gleichen wirtschaftlichen Problemen gegenübersah, oblag die Initiative nicht nationalen Regierungen, sondern 14 international tätigen Großunternehmen (u. a. Bosch, Fiat, KLM, Philips, VW).

Das EFQM Excellence Modell (EFQM, 1998, 1998a) ist ein Schema, nach dem Unterneh men sich selbst evaluieren (Self Assessment) und/oder durch externe ExpertInnen bewerten lassen können (Externes Assessment). Die EFQM-Organisation in Brüssel vergibt jährlich den European Quality Award (EQA). Dieser Preis steht aber nicht im Mittelpunkt der Systemanwendung, sondern ist eher als eine Form der Motivierung von Unternehmen zur öffentlichen Darstellung und damit als eine den Wettbewerbsgedanken symbolisierende Zugabe zu verstehen (Merchel, 2001, S. 71). "Herzstück" des Modells und wichtigstes Hilfsmittel im Rahmen eines systematischen Kontinuierlichen Verbesserungsprozesses ist die Selbstbewertung der Unternehmensqualität nach Maßgabe der Modellkriterien (Langnickel, 2003, S. 40f). Das Modell stützt sich auf neun solcher Bewertungsbereiche (Kriterien), welche jene Schlüsselmerkmale widerspiegeln, nach denen hervorragende Organisationen geführt werden: "Exzellente Ergebnisse im Hinblick auf Leistung, Kunden, Mitarbeiter und Gesellschaft werden durch eine Führung erzielt, die Politik und Strategie, Mitarbeiter, Partnerschaften, Ressourcen und Prozesse auf ein hohes Niveau hebt" (www.deutsche-efqm.de).

\section{Bewertungskriterien des EFQM Excellence Modells}

Potenzialfaktoren ("Befähiger"-Kriterien, bezogen auf das "Wie?" der Unternehmensleistungen):

- Führung: z. B. Verhalten der Leitungskräfte zur Erreichung des Zieles "Total Quality",

- Politik und Strategie: z. B. Unternehmenskultur, Ziele und strategische Ausrichtung,

- Mitarbeiter: z. B. Personalentwicklung, Schulung, Motivation,

- Partnerschaften und Ressourcen: z. B. externe Kooperationspartner, Finanzen, Information, Technik, 
- Prozesse: z. B. Betriebsabläufe, Wertschöpfungsprozesse im Unternehmen;

Ergebnisfaktoren ("Ergebnis"-Kriterien, bezogen auf das "Was?" der Unternehmensleistungen):

- Mitarbeiter Ergebnisse: z. B. Beurteilung des Unternehmens durch die Mitarbeiter,

- Kunden Ergebnisse: z. B. Beurteilung der Dienstleistungen durch externe Kunden,

- Gesellschaft Ergebnisse: z. B. öffentliche Meinung und Image des Unternehmens hinsichtlich Funktion, Qualität und Sozialverträglichkeit,

- Schlüsselleistungen Ergebnisse: z. B. finanzielle und nichtfinanzielle Ergebnisse im Vergleich zu gesteckten Zielen.

Die einzelnen Kriterien werden für die Gesamtbeurteilung unterschiedlich gewichtet, so dass Unternehmen, die in marktstrategisch besonders relevanten Bereichen Stärken aufweisen (z. B. Kunden Ergebnisse: 20\% des Gesamtpunktwertes), der Maximalpunktzahl näher kommen als solche, die in nachgeordneten Bereichen (z. B. Mitarbeiter Ergebnisse: 9\%) vorn liegen. Die maximal erreichbare Punktzahl wurde bislang noch von keinem Preisträger erreicht (Langnickel, 2003, S. 39).

$\mathrm{Zu}$ allen neun (Haupt-)Kriterien gibt es Unterkriterien (insgesamt 32), die im Detail bezeichnen, was bei Total Quality beachtet werden sollte. Dennoch ist die Standardversion des EFQM-Modells recht allgemein gehalten, so dass branchen- und unternehmensspezifische Konkretisierungen (Spezifikationen) erforderlich sind, um aussagekräftige Informationen über das betriebliche Qualitätsprofil zu liefern.

Inzwischen liegen Versionen für den öffentlichen Sektor (Gesundheits-, Erziehungs- und Bildungswesen, Verwaltungen) sowie für kleine und mittelgroße Unternehmen (KMU) vor. Adaptationen des Modells werden auch im sozialen und pflegerischen Bereich eingesetzt (z. B. Vogt, 2000). Das EFQM-Modell 2000 sieht ohnehin vor, von den verschiedenen Versionen wieder abzugehen, um die Vergleichbarkeit der Kriterien zu vereinfachen. Durch "Öffnungsklauseln" ist jedoch gewährleistet, dass jede Organisation die Behandlung der Kriterien ihren spezifischen Bedürfnissen gemäß vornehmen kann.

Um speziell kleineren Organisationen die Arbeit mit dem Modell zu erleichtern, wurden 
zwei unterstützende Hilfsmittel entwickelt: die RADAR-Bewertungsmatrix und die Pathfinder-(Wegweiser)Karte. Trotzdem dürften die meisten Einrichtungen, die mit dem EFQM-Modell arbeiten wollen, auf eine externe Begleitung nicht verzichten können, vor allem in der Anfangsphase und in Verbindung mit periodischen externen Bewertungen (Langnickel, 2003, S. 43). Die Ausbildung von erfahrenen MitarbeiterInnen zu EFQMAssessorInnen ist denn auch oft der erste Schritt im Rahmen der Modelleinführung.

Der finanzielle Aufwand für die Organisationsentwicklung mittels des EFQM-Modells ist - abgesehen von den Kosten für externe Begleitung und Ausbildung von Mitarbeiterlinnen

- einrichtungsindividuell steuerbar und letztlich vom Ausgangsstatus und angestrebten Qualitätsniveau abhängig.

\subsubsection{Beurteilung des Ansatzes}

Auch das EFQM-Modell gehört $\mathrm{zu}$ den allgemeinen Referenzmodellen des Qualitätsmanagements, ist also branchenunspezifisch konzipiert und deshalb wenig konkret in den ausformulierten Bewertungskriterien. Diese stellen keine empirischen Indikatoren dar, sondern nur Orientierungspunkte, die es ans jeweilige Praxisfeld anzupassen und inhaltlich auszufüllen gilt. Das Anspruchsniveau des Modells ist dabei sehr hoch; EFQM setzt auf nachhaltige Entwicklung und kontinuierliche Verbesserung.

Das Konzept ist im Grunde ein Praktiker-Modell und kein nach wissenschaftlichen Kriterien entwickeltes Managementsystem (Zollondz, 2002, S. 291). Es beansprucht, alle wesentlichen Bestandteile aus den bekannten Denk- und Managementschulen zu enthalten, ohne es mit dem Lehrgebäude der einzelnen Schulen zu überfrachten (Bergbauer, 1998). Das Konzept will TQM aus dem "Himmel wolkiger Verkündigungen auf den Boden der Unternehmensrealität" herunterholen, indem es Qualität systematisch auf Fakten und Faktenüberprüfung stützt (Langnickel, 2003, S. 39f). Dieser Grundansatz verdeutlicht eine starke Verwandtschaft zur Balanced Scorecard (s. Kap. B 2.5), mit der das EFQM-Modell häufig kombiniert wird (Gerull, 2000, S. 2:113).

Qualitätsmanagement nach EFQM vollzieht sich primär auf dem Wege der Selbstbewertung. Externe Fachleute (EFQM-Assessoren/-Auditoren) können jedoch 
einbezogen werden, um die Gefahr betriebsblinder Fehleinschätzungen zu reduzieren.

Das Modell ist nach K. Frey (1997a, S. 33f) gut zur Erfassung des momentanen Qualitätsstandes einer Einrichtung geeignet, weil es - je nach Spezifizierungsgrad der angewendeten Kriterien - ein weitreichendes Profil der Leistungsstärken und -schwächen vermittelt. Eine im Abstand von ein bis zwei Jahren durchgeführte Selbstbewertung kann wichtige Anhaltspunkte liefern, Schwachstellen zu entdecken und Maßnahmen zur Qualitätsverbesserung durchzuführen. Frey (a.a.O.) hält das System auch für sehr geeignet, wenn ein Verband einen gemeinsamen minimalen Standard mit Profilierungsmöglichkeiten für die Mitgliedsinstitutionen anstrebt.

Das EFQM-Modell gibt allerdings kein System an die Hand, wie bzw. mit welchen Methoden die Qualität verbessert werden kann; auch fehlen Vorschläge für die Implementation. Eindeutiger Schwerpunkt ist somit die Qualitätsbewertung und Selbstdiagnose, welche den Handlungsbedarf einer Organisation markiert und Grundlage für die Planung von Verbesserungsmaßnahmen darstellt. Eine solche Selbstbewertung nach EFQM ist insofern lediglich Teil des Qualitätsmanagements, allerdings handelt es sich um ein Instrument mit Prozess auslösender Wirkung (Merchel, 2001, S. 80).

Wunderer (1998, zit. nach Zollondz, 2002, S. 290f) kritisiert am EFQM-Modell, dass es ein geschlossenes System sei, welches dazu verleite, unreflektiert angewandt zu werden. Das Konzept beschränke sich auf neun Komponenten und erwähne die Faktoren Unternehmenskultur und Organisation nur in den Unterkriterien; auch sei das Punktverteilungsmodell willkürlich und betone die Ergebnisse mit 50\% übergebührlich. Plädiert wird dafür, die Prozessdimension von den Befähiger-Kriterien zu trennen (a.a.O.).

Grundsätzliche Kritik an der Eignung des EFQM-Modells für soziale Dienstleistungen gilt der eindeutigen Anbieterzentrierung. Trotz kontinuierlich ermittelter Kundenansprüche und -interessen, welche in die Planung und Gestaltung der Produkte eingingen, sei eine aktive Beteiligung der Nachfragenden im Produktionsprozess selbst, der Interaktion, nicht vorgesehen (Flösser, 2000, S. 167). "Nicht die Binnenstrukturen des Aushandlungsprozesses zwischen Anbietern und Nachfragenden, sondern die strikte Einhaltung standardisierter Produktionsweisen wird diesen Konzepten zufolge als Qualitätsnachweis betrachtet" (a.a.O.). Damit gerate das EFQM-Modell in Konflikt mit situativen, flexiblen 
und kontextgebundenen Handlungsanforderungen im Feld der Sozialen Arbeit (S. 168).

Auch die Problematik des Kundenbegriffs, spezielle Messprobleme bezüglich der Erfassung von Kundenerwartungen und -zufriedenheit sowie eine andere Gewichtung ökonomischer Faktoren und Gestaltungsspielräume sozialer Einrichtungen und Dienste etwa bei personalpolitischen oder strategischen Entscheidungen - werden als Einschränkungen des EFQM-Modells thematisiert (Langnickel, 2003, S. 45f).

Struck (2000, S. 191 ff.) kritisiert dogmatische, nicht näher oder nur zirkulär begründete Setzungen sowie "eine Pseudo-Exaktheit und Pseudo-Objektivität ..., die modellimmanent nur durch die trainierte Intersubjektivität der EFQM-Auditoren aufhebbar" sei.

In einem für den Altenhilfe- und stationären Pflegebereich vorgenommenen Vergleich von dreizehn Qualitätskonzepten attestieren Kämmer et al. (2001, S. 152f) dem EFQM-Modell eine relativ gute Mitarbeiterbeteiligung, Motivationsförderung und Praxisrelevanz; auch die externe Kundenorientierung wird vergleichsweise hoch eingestuft. Noch deutlich besser bewertet wird ein Konzept, das auf der Grundlage von EFQM feldspezifisch angepasst wurde (QAP, vgl. Gerull, 2004, Kap. 2.3.2). Das formalen Universalkonzepten wie ISO 9000 ff. oder EFQM immanente Problem einer wirtschaftslastigen Sprache lässt sich durch angemessene Übersetzungen für die MitarbeiterInnen offenkundig reduzieren.

\subsubsection{Integriertes/Integratives Qualitätsmanagement nach dem St. Galler Management Konzept}

\subsubsection{Darstellung des Ansatzes}

Das in Wissenschaft und Wirtschaft stark beachtete St. Galler Management Modell (Ulrich/Krieg, 1972, nach Seghezzi, 1994, S. 65), seit 1991 in modifizierter Form St. Galler Management Konzept genannt (Bleicher, 1991) erhebt den Anspruch, ein ganzheitliches und neutrales Ordnungsgerüst zu sein, in das sich Konzepte und Managementsysteme in universeller Art einfügen lassen (Seghezzi, 1996, S. 231). 
Im Rahmen dieses ganzheitlichen Unternehmensmodells entwickelte Seghezzi (1994, 1996) das Konzept des Integrierten Qualitätsmanagements. Aus dem allgemeinen Managementmodell werden deduktiv - "top-down" - alle Teilkonzepte (Zeit, Qualität und Kosten) abgeleitet. Qualitätsmanagement wird danach zwar als eigenständige Disziplin, jedoch nicht als selbständige Funktion betrachtet (Seghezzi, 1996, S. 48).

Das Modell integriert diese Teilkonzepte und will drei wesentliche Funktionen erfüllen:

- Kompatibilität von Qualitäts- und Managementkonzepten,

- Integration von Qualitätsmanagement in die Betriebswirtschaftslehre und

- Berücksichtigung des Paradigmenwechsels im Management (Seghezzi, 1994, S. 65).

Zink (1995) stellte ein QM-Modell vor, das ebenfalls auf dem St. Galler Management Konzept aufbaut und sich nur geringfügig vom Ansatz Seghezzis unterscheidet, indem es stärker auf arbeitswissenschaftliche Erkenntnisse rekurriert (Zollondz, 2001, S. 232). Das Modell firmiert unter "Integratives Qualitätsmanagement" und hat wie das "Integrierte QM" in der Fachöffentlichkeit nur begrenzte Resonanz gefunden (a.a.O., S. 305f). Eine ausführliche Würdigung findet der Ansatz bei C. Bauer (1996).

Der formale Ordnungsrahmen des St. Galler QM-Konzepts besteht wie das allgemeine Managementmodell aus einem dreidimensionalen Gebilde mit drei Ebenen (normativ, strategisch und operativ) als erster Dimension, drei Säulen (Strukturen, Aktivitäten, Verhalten) als zweiter Dimension und der Unternehmensentwicklung in der Zeit als dritter Dimension (Seghezzi, 1996, S. 48f). Ebenen und Säulen definieren eine Matrix, an deren Schnittstellen so genannte Managementmodule gebildet werden, z. B. die Module Qualitätsverfassung (Schnittstelle: normative Ebene/Struktur), Qualitätsstrategie (Schnittstelle: strategische Ebene/Aktivität) und Qualitätsverbesserung (Schnittstelle: operative Ebene/Verhalten). Die Module beeinflussen sich in vertikaler und horizontaler Richtung (vielfältige Vor- und Rückkopplungsprozesse). Dies macht den integrativen Charakter des Konzepts aus, das durch Einbeziehung der dritten Dimension, der Unternehmensentwicklung, dynamisiert wird (in der 2. Auflage ergänzt durch die Entwicklung der Qualitätsfähigkeit, Seghezzi, 2003, S. 7).

Die 3x3-Matrix des St. Galler Management Konzepts definiert neun Managementmodule. Da das Konzept Integriertes QM beim Übergang von der strategischen Ebene in den 
operativen Bereich durch Einfügung des Moduls "Qualitätsplanung" eine Erweiterung gegenüber dem allgemeinen Modell vornimmt (Seghezzi, 1996, S. 51), enthält das Integrierte QM insgesamt 10 Module, je drei normative und strategische sowie vier operative:

- Qualitätsverfassung, -politik und -kultur (normatives Management),

- QM-Systeme/-Strukturen, Qualitätsstrategie und qualitätsbezogene Verhaltensentwicklung (strategisches Management),

- Qualitätsplanung, -lenkung, -sicherung und -verbesserung (operatives Management).

Die einzelnen Module werden durch jeweils vier bipolare Achsen (Einflussgrößen) veranschaulicht, die durch ein Gegensatzpaar charakterisiert sind.

Dazu ein Beispiel aus dem Aufgabenbereich der qualitätsbezogenen Verhaltensentwicklung, die im graphischen Modell der strategischen Ebene und der Verhaltens-Säule entspricht (Seghezzi, 1996, S. 187194).

Strategische Ebene: Qualitätsbezogene Verhaltensentwicklung (ausgerichtet auf Ziele und Maßnahmen zur Beeinflussung von Wahrnehmungs- und Verhaltensmustern der Führungskräfte und Mitarbeiter):

a) Führungsstil: "Befehlen" versus "Coachen";

b) Einbindung der Mitarbeiter: "Direktiven" versus "Partizipation";

c) Autorität und Verantwortung: "institutionalisiert" versus "tätigkeitsbezogen";

d) Lernverhalten: "instruktiv" versus "prozessual".

Aus den zehn Managementmodulen mit ihren jeweils vier Gestaltungsfaktoren (bipolare Achsen) werden somit vierzig Kontinua von Qualitätsmerkmalen definiert. Diese sind bei Seghezzi (1996) durch mehrere Beschreibungen operationalisiert.

So wird in dem erwähnten Beispiel der Führungsstil "Befehlen" wie folgt charakterisiert: Der Vorgesetzte gibt detaillierte Aufträge und Arbeitsanweisungen und kontrolliert deren Ausführung. Er trifft die Entscheidungen und gibt sie von oben nach unten weiter. Informationen werden oft als Machtinstrument eingesetzt (a.a.O., S. 189).

Der Nutzen einer solchen Darstellung des Konzepts wird zum einen darin gesehen, dass sie einen Überblick über die Gestaltungsfaktoren und die Spannweite möglicher 
Gestaltungsfelder des jeweiligen Moduls gibt; zum anderen kann das einzelne Unternehmen auf den vier Achsen jedes Moduls sein eigenes Profil festlegen und sichtbar machen, wodurch es sich von Konkurrenten unterscheiden will (a.a.O., S. 71f). Das Konzept hat somit eine analytische, strategische und diagnostische Funktion: Es lassen sich maßgebliche Handlungsfelder markieren, auf denen sich eine Organisation strategisch positionieren oder mittels Assessment selbst bewerten kann.

- Soll-Profil: In welche Richtung will sich das Unternehmen entwickeln?

- Ist-Profil: Wo befindet sich das Unternehmen zur Zeit?

Die Differenzen zwischen Soll- und Ist-Profilen markieren den jeweiligen Handlungsbedarf für die einzelnen Managementmodule im Rahmen der Gesamtunternehmensentwicklung.

Wenngleich betont wird, dass die Gegensatzpaare keine Bewertung zum Ausdruck bringen sollen, wird eine "äußere" Positionierung (d. h. gemäß der graphischen Modelldarstellung außen liegende; im Beispiel ist dies der rechte Achsenpol) für diejenige gehalten, die der Philosophie des Integrierten Managements nach dem St. Galler Konzept besser entspricht (a.a.O., S. 237).

Das Konzept versteht sich als "Lebenshilfe" bei der Bewältigung des Führungsproblems in Theorie und Praxis. Situationsbezogene Problemkenntnisse sollen vermittelt und Wege zur Lösung aufgezeigt werden (Loh, 1999a, S. 69). Dabei wird die überragende Bedeutung einer normativen Orientierung für alle Einzelmaßnahmen betont. Wichtiger Bestandteil des St. Galler Ansatzes ist deshalb der partizipativ vollzogene Lernprozess der Leitbilderarbeitung, der für die Implementation des Qualitätsmanagements gute Voraussetzungen schaffen soll (a.a.O., S. 71).

Praktischer Ausgangspunkt bei der Anwendung des Konzepts ist demzufolge eine gemeinsam zu erarbeitende Unternehmensphilosophie bzw. eine Vision, die sich als Leitbild eignet. Dieses stellt für alle Beteiligten die integrierende Klammer für ihr berufliches Handeln dar und ermöglicht eine Identifikation (C. Bauer, 1996, S. 28). Auf der Ebene des strategischen Managements sind dann die Organisationsstrukturen, langfristigen Programme, personellen Konzepte und Verhaltensanforderungen zu bestimmen, und zwar so, dass sie den normativen Vorgaben entsprechen. Auf dieser Ebene 
sind auch die Instrumente zur Steuerung des Gesamtprozesses angesiedelt. Aufgabe des Controllings ist es, allen Beteiligten einen möglichst umfassenden Über- und Einblick zu verschaffen, Vernetzung herzustellen und Mitbestimmung zu ermöglichen (a.a.O., S. 31). Auf der operativen Ebene werden schließlich die konkreten Alltagsaufgaben beschrieben. Größtmögliche Kongruenz zu den strategischen und normativen Vorgaben wird gefordert (S. 32).

Von den Autoren des Konzepts wird betont, dass der St. Galler Ansatz helfen könne, komplexe Strukturen besser zu bewältigen und mehrdimensionale Ziele anzustreben. Damit werde der klassische Rahmen des Komplexitätsmanagements - Arbeitsteilung, Spezialisierung, Arbeitsgruppen - erweitert (Seghezzi, 1994, S. 67). Seghezzi hebt zudem die Bedeutung hervor, die der richtigen Passung von Qualitätsmanagementsystem und Gesamtmanagement zukommt:

\footnotetext{
"Ein Qualitätskonzept kann nicht unabhängig vom allgemeinen Unternehmensführungskonzept, sondern nur als dessen Bestandteil erfolgreich sein. Deshalb muss ein Qualitätskonzept in das Unternehmensführungskonzept integriert werden, was nur möglich ist, wenn beide miteinander verträglich sind" (Seghezzi, 1994, S. $63)$.
}

Der Vorbildfunktion und Glaubwürdigkeit der Leitungskräfte werden dabei eine besondere Rolle zugemessen.

\subsubsection{Beurteilung des Ansatzes}

Das St. Galler Management Konzept beansprucht nicht weniger, als alle bekannten Organisationsmodelle aufzunehmen und in einem umfassenden Konzept zu integrieren. Das heißt auch, dass Ansätze wie TQM, ISO 9000 ff., EFQM und andere im Modell des Integrierten Qualitätsmanagements ihren Platz finden (Seghezzi, 1996, S. 232). Damit gebührt dem Modell in der Rangreihe der universellen Metakonzepte eigentlich die erste Position. Der hier nur in Grundzügen beschriebene Ansatz ist anspruchsvoll und komplex und besticht durch formale Brillanz.

Seghezzi (1996, Vorwort) betont, dass mit der Integration der Querschnittsdisziplin Qualitätsmanagement in die allgemeine Managementlehre jede Führungskraft Wissen und 
Instrumentarium bezüglich des Faktors "Qualität" anwenden könne, so wie dies in Bezug auf die Faktoren "Kosten" und "Zeit" bereits der Fall sei. Insofern wird QM als genuine Aufgabe des Managements betrachtet, die in die Unternehmenspraxis zu integrieren ist.

Wie, mittels welcher Strategie, ob mit oder ohne externe Unterstützung, binnen welcher Zeiträume und zu welchen Kosten dies geschehen soll, wird nur sehr allgemein und kurz thematisiert (a.a.O., S. 231-239). Die auf den ersten Blick komplizierte Modellstruktur muss in der Praxis jedoch nicht in einem Stück abgearbeitet werden. Welche Module und Einflussgrößen betrachtet werden, hängt von der jeweiligen Interessenpriorität und den verfügbaren Ressourcen ab. Entscheidend ist der normative Ausgangspunkt und die konsequente Anpassung der Managementmodule an die Soll-Werte des Modells. Der Ansatz des Integrierten QM ist dezidiert "top-down" angelegt und lebt von der glaubwürdigen Entschlossenheit der Leitungsebene, sich in den organisationalen Lernprozess einzubringen. Inwieweit dazu eine Strategie vonnöten ist, die Senge (zit. nach Loh, 1999b, S. 78) als "Verkünderstrategie" bezeichnet hat, wird nicht ganz klar. Jedenfalls gehen dabei die Vorteile eines kraftvollen, verbindlichen Vorgehens mit den Nachteilen möglicher Widerstände und geringer Identifikation auf Seiten der Mitarbeiter einher.

Über die pragmatische Adaptation des St. Galler Ansatzes im Rahmen eines einrichtungs- und trägerübergreifenden Projekts im Bereich der Erziehungshilfe berichtet Loh (1999a, 1999b). Darin wird der formale Ordnungsrahmen ("Neun-Felder-Matrix") als geeigneter methodischer Raster und gutes Diagnoseund Analyseinstrument bezeichnet, um unterschiedliche Praxisbereiche auf ihre innere Kongruenz von Strukturen, Instrumenten und Verhalten auf allen Ebenen zu untersuchen.

C. Bauer (1996) bezieht sich in einem theoretischen Beitrag zur Organisationsentwicklung von Trägern der Jugendhilfe auf den eng verwandten Ansatz des Integrativen QM nach Zink (1995).

Von diesen Beispielen abgesehen, ist dem Autor im Rahmen umfangreicher Literaturrecherchen kein Fall bekannt geworden, in dem das Konzept Seghezzis in der Praxis der Sozialen Arbeit und verwandter Berufsfelder als Referenzsystem bei der Einführung von QM verwendet wurde.

Ins Internet gestellte Beiträge, in denen die Bezeichnung "Integriertes QM" vorkommt, ohne jedoch auf das St. Galler Konzept Bezug zu nehmen, entstammen überwiegend dem Klinikbereich. Auch J. F. W. Müller (2004), der das St. Galler Management-Konzept im Übrigen als weitverbreitet bezeichnet und relativ ausführlich würdigt (S. 286 ff.), weist in seinem Fallstudien-Sample keine Einrichtung auf, die nach dem 
Modell arbeitet.

Wahrscheinlich ist das St. Galler Konzept potenziellen Anwendern zu kompliziert oder nicht feldspezifisch operationalisiert genug, was in dieser Form aber auch für Konzepte wie EFQM oder ISO 9000 ff. gilt. Angesichts der formalen Brillanz und theoretischen Stringenz sowohl des integrierten als auch des integrativen Modells und der von den Autoren vertretenen Managementphilosophie ist dies zu bedauern. Auch Zollondz (2002, S. 305f) wünscht den Ansätzen eine breitere Resonanz. 


\section{Instrumente des Qualitätsmanagements in Sozialen Diensten}

\subsection{Stellenwert der Technik im Qualitätsmanagement}

Eversheim (1997, S. 15) formuliert als Leitgedanken eines umfassenden Qualitätsmanagements: "Hohe Qualität lässt sich nur durch die Kombination eines systematischen Ansatzes mit einer Qualitätskultur erreichen." Anders ausgedrückt: Qualität entsteht aus dem Einsatz von "Technik" und "Geisteshaltung" (Kamiske, zit. nach Zollondz, 2002, S. 318).

Die Einführung eines QM-Systems nach Gestaltungsparametern z. B. der ISO 9000 ff. kann demnach zwar einen Strukturgewinn für die Stabilität und Nachhaltigkeit einer Qualitätskultur bedeuten (Gerull, 2000, S. 2.1-9), damit allein ist eine solche Kultur jedoch nicht hervorzubringen. Der "technische" Teil eines QM muss in einen umfassenden Organisationsentwicklungsprozess eingebettet sein, damit das operative QM seine Wirkung zur Geltung bringen kann (a.a.O.).

Ein vernünftig dimensioniertes QM zu betreiben heißt letztlich, sich bei der Verfolgung der Qualitätsziele in angemessenem Umfang verfügbarer Planungs-, Entscheidungs-, Moderations-, Dokumentations-, Evaluations-, Controllingverfahren und sonstiger rationaler Instrumente $\mathrm{zu}$ bedienen, sich der Grenzen dieser Rationalität im sozialen Dienstleistungsbereich jedoch bewusst zu bleiben (Gerull, 2001, S. 12).

Wenngleich die Verwendung von Techniken und Instrumenten in der Praxis des QM eine große Rolle spielt und diese Werkzeuge sich bei der Aufgabenbewältigung als hilfreich und nützlich erweisen, ist die Gefahr nicht $\mathrm{zu}$ unterschätzen, damit eine "ingenieurwissenschaftliche Aura" (a.a.O.) zu erzeugen, die ebenso als Transport- und Verstärkungsmedium für sozialtechnische Einstellungsmuster wirken kann wie ein unkritischer Umgang mit dem Begriff "Qualitätssicherung" (Merchel, 1998, S. 35).

Nicht alles, was die Qualität von Leistungen mit hohem Interaktivitätsanteil beeinflusst, ist methodisierbar. Die Qualität sozialer Dienstleistungen lässt sich nicht durch sorgfältigen Instrumenteneinsatz "in den Griff" bekommen (a.a.O.) oder durch planmäßig umgesetzte Wirkungszusammenhänge "herstellen" (von Spiegel, 2000, S. 191). Die Komplexität des 
Bedingungsfeldes sozialer Dienstleistungen weist subjektiven Faktoren wie persönlichen Haltungen zur Aufgabe und zum Beruf entscheidende Bedeutung zu. Diese Haltungen lassen sich mit Begriffen wie Commitment, Engagement, Charisma oder "Liebe zur Sache" umschreiben (vgl. Merchel, 1998, S. 33).

"... Liebe zur Sache und Qualität (sind) der innere und äußere Aspekt ein und derselben Sache ..." (Pirsig, zit. nach Merchel, a.a.O.).

Engel-Kemmler (1996, S. 7) äußert in ähnlichem Zusammenhang: "Die Rede von Qualität und ihrem 'Management' hat in ... Bereichen intersubjektiver Arbeit an der Humanität ihren lebendigen Sinn erst im ganz persönlichen Durchgang durch die eigene Identität und im glaubwürdigen Bemühen um Qualität in der Führung der eigenen Person. Letztere mit dem Begriff des 'Self-Managements' ... zu bezeichnen, weigere ich mich weiterhin. Ich markiere eine Differenz, die praktisch zu leben etwa so schwer ist, wie immer an das Ganze (das Totum) zu denken und dabei nicht totalitär zu werden".

Die berechtigte Kritik an der Konstruktion von Technologien für soziale Dienstleistungen darf allerdings nicht dazu führen, auf Planung zu verzichten (von Spiegel, a.a.O.) oder den methodischen Einsatz von Werkzeugen des QM über Gebühr zu problematisieren. Die angesprochenen Grenzen der Rationalität verweisen zwar auf die Bedeutung emotionaler, intuitiver und subjektiver Faktoren, machen rationale Strategien und Techniken jedoch nicht verzichtbar.

Die Notwendigkeit, beide Aspekte in ihrer Polarität zu begreifen und zu berücksichtigen, wird in folgendem Zitat deutlich, das auf die Situation der offenen Jugendarbeit gemünzt ist:

"Ohne den Aspekt des produktiven Chaos würde das reine Organisieren zu einem technischen Durchstrukturieren, das jedoch die Komplexität und Potenziale dieses erzieherischen Feldes nicht aufnehmen, sondern eher verhindern würde. Ohne Planung jedoch entstünde die Gefahr, dass die reine Anarchie ein Handlungsfeld produzieren würde, in dem professionelles Handeln nicht mehr mit Kategorien begründbar wäre und methodisches Handeln und Reflektieren in einem 'Wursteln' unterging, das nicht mehr fachlich und produktiv genannt werden könnte" (Sturzenhecker, 2000, S. 160).

Techniken, Werkzeuge, tools und Instrumente - diese wenig trennscharfen Begriffe wurden in Kap. B 1.6 verwendet, um Hilfsmittel des QM zu beschreiben. Sie entstammen zu einem großen Teil nicht diesem selbst, sondern wurden und werden auch in anderen 
Bereichen entwickelt und verwendet. Sind solche Techniken oder Instrumente mit dem weitergehenden Anspruch verbunden, einen spezifischen Managementansatz zu verkörpern (z. B. Balanced Scorecard) oder einen breiten Ausschnitt der relevanten betrieblichen Wirklichkeit zu repräsentieren (z. B. Personalentwicklung), wurde der Begriff des Kontextkonzeptes gewählt. Dabei hängt es vom Standpunkt des Betrachters ab, solche Kontextkonzepte den Ansätzen des QM über- oder unterzuordnen; zumeist dürften sie sich in einem eher komplementären Verhältnis zueinander befinden.

Die im Folgenden erörterten Instrumente lassen diesbezüglich kaum Zweifel aufkommen. Es handelt sich um mehr oder weniger eindeutige Hilfsmittel des QM mit definierter Funktion. Sie lassen sich unabhängig voneinander einsetzen, werden in der Praxis aber häufig zu spezifischen Modulen eines QM-Systems kombiniert.

\subsection{Darstellung und Kritik ausgewählter Instrumente}

\subsubsection{Qualitätsleitbild}

Schlagworte wie "normatives Management", "visionäre Führung", "Unternehmensmissionen" und "Leitbilder" kennzeichnen das Bedürfnis von Organisationen nach mitreißenden Zielvorgaben für die Zukunft und die Hoffnung, dadurch erfolgreich aktuelle Probleme lösen zu können (Gerull, 2000b, S. 263).

"Gesucht ist der große, visionäre Wurf eines Zukunftsbildes, das durch die Missionen der Unternehmenspolitik generell ausformuliert und durch die Strukturen der Unternehmensverfassung eingerahmt wird. Er zeichnet sich eher durch seine begeisterungsfähige Sogwirkung in der Schaffung einer neuen und andersartigen Zukunft als durch operationale Definition von unmissverständlichen Details aus" (Bleicher, 1994, S. 485).

Ein Leitbild ist ein Katalog normativer Vorstellungen darüber, wie sich eine Organisation selbst versteht und entwickeln möchte. Ein gutes Leitbild enthält neben fachlichen und ethischen Prinzipien "Bilder" (Visionen), in denen sich die Mitglieder der Organisation wiederfinden können. Bilder sind vor allem dann wertvoll, wenn sie Originale sind. 
"Abgekupfert" sind sie nicht mehr wert als eine "Kunst-Postkarte" (B. Müller, 2000, S. 139f).

"Leitbildentwicklung, wie prozesshaft und 'original' sie immer vorangetrieben werden mag, erzeugt kein realistisches Selbstbild einer Organisation. Das ist auch nicht ihre Aufgabe. (Sie) soll eher ein Idealbild erzeugen, eine gemeinsame Vorstellung von dem, was man sein möchte und unter guten Bedingungen auch glaubt sein zu können" (a.a.O., S. 140).

Dies führt allerdings häufig dazu, dass formulierte Leitbilder "... nahezu die Abstraktionsebene der Menschenrechte erreichen. Es entstehen Schriften, die fast selbstverständlich auf allgemeine Zustimmung angelegt sind" (Lachnit, 2003, S. 178).

Damit Leitbilder nicht zu Phrasen werden, bedürfen sie der Konkretisierung, z. B. in pädagogische Konzeptionen und Zielvereinbarungen auf der Ebene des Alltagshandelns (vgl. Lachnit, 2003), allgemein der Übersetzung in "Tätigkeitsworte" (B. Müller, 2000, S. 141). Leitbilder sollten deshalb eher in Form von Verfahrensregeln, denn als abstrakte Wertmaßstäbe formuliert werden. Solche Leitbilder sind keine statischen Produkte für die Ewigkeit, sondern als nie abgeschlossene Kernprozesse einer lernenden Organisation mittels geeigneter Methoden der Selbst- und Fremdbeobachtung auf ihre Realitätstüchtigkeit zu überprüfen (a.a.O.).

Ein Leitbild kann Aussagen treffen über

- das Selbstverständnis der Einrichtung,

- ihre obersten Ziele und Aufgaben ("Missionen"),

- die dafür einzusetzenden Leistungsarten und

- Grundsätze über die Organisationspotenziale: Mitglieder, MitarbeiterInnen, Finanzen, Organisation (Gerull, 2000, S. 4:10).

Exemplarisch seien die Elemente des Leitbildes einer diakonischen Einrichtung beschrieben (Ringwald, 2000, S. 4:12-13):

(1) Aufgaben und Ziele, Selbstverständnis (wer wir sind)

(2) Unsere Wurzeln (woher wir kommen)

(3) Unser Umfeld/Markt (wo wir arbeiten)

(4) Unsere Leistungen (was wir tun) 
(5) Unsere Organisation/unser Unternehmen

(6) Unsere Mitarbeiter/-innen

Für die Erarbeitung eines Leitbildes empfiehlt sich ein Projektmanagement (vgl. Ringwald, 2000, S. 4:14 ff.). Funktionen und Bedeutung eines partizipativ entwickelten und schriftlich formulierten Leitbildes sind vor allem (vgl. Achberger \& Wittig-Koppe, 1998):

o Harmonisierung (Klärung von Erwartungen und Austragung von Konflikten),

o Aktivierung (Willensbildungsprozess und Beteiligung),

o Orientierung (Grundsatzdokument als Basis und Maßstab für Entscheidungen),

o Information (für Organisationsmitglieder und Umwelt),

o Motivation (durch Ausrichtung auf Zielerreichung).

Bezüglich der Leitbildentwicklung muss deutlich gemacht werden, dass es sich nicht um eine demokratische Ermittlung des "kleinsten gemeinsamen Nenners" der Beschäftigten handelt, sondern um ein intensives Erkenntnisbemühen um das "Wesen" der Einrichtung, wozu auch Ziele hinsichtlich der zu gestaltenden Zukunft gehören (Brater \& Maurus, 1997, S. 32). Eine Orientierung an der Biographie der Einrichtung, am Selbst- und Fremdbild der darin Tätigen sowie an den äußeren Erscheinungsformen wird als hilfreich betrachtet (Maurus, o. J., S. 3f). Da die Leitbildarbeit folglich zu weiten Teilen ein Erkenntnisprozess ist und nicht die Summe der individuellen Grundwerte und Arbeitsziele der Organisationsmitglieder, müssen bei der Erarbeitung des Leitbildentwurfs auch nicht alle MitarbeiterInnen beteiligt sein. Für ausreichend wird eine Gruppe von ca. acht bis zwölf, maximal fünfzehn Personen mit möglichst unterschiedlichem Erfahrungshintergrund gehalten: langjährige und neue MitarbeiterInnen, Kontaktpersonen zu externen Kooperationspartnern, Organisationsmitglieder, die nach Auffassung der Belegschaft besonders charakteristisch für die Einrichtung sind (vgl. Maurus, o. J.).

Im Qualitätskonzept des GAB-Verfahrens (Brater \& Maurus, 1997) wird beispielsweise das Leitbild als eine tragende Selbstverständigung der Einrichtung nach innen aufgefasst, während die Arbeitskonzepte die besonderen inhaltlichen Ansätze der einzelnen Arbeitsbereiche darstellen. Leitbild und Arbeitskonzepte werden auf der Handlungsebene in Handlungsleitlinien konkretisiert.

Allgemein dienen Leitbilder sowohl der Sinngebung und Sinnvermittlung als auch der Sinnfindung (Zollondz, 2002, Anhang). Als spezielles Leitbild, mit dem die 
Qualitätspolitik einer Organisation nach innen und außen transparent gemacht werden soll, haben auch Qualitätsleitbilder diese Funktion.

Skeptischer formuliert, sind Leitbilder - und Qualitätsleitbilder machen da vermutlich keine Ausnahme "selbstproduzierte Glaubensprodukte, deren Schubkraft für die erste Stufe eines Organisationsprozesses ausreichen mag, aber bei konkreten Umsetzungen regelmäßig verpufft" (Knorr \& Halfar, 2000, S. 231).

Auch Qualitätsleitbilder erhalten eine größere Verbindlichkeit nach innen und bessere Glaubwürdigkeit nach außen, wenn sie unter Beteiligung der MitarbeiterInnen beschlossen und eingeführt werden. Die Inhalte orientieren sich an dem durch Satzung, Unternehmensrichtlinien, gesetzliche Vorgaben, einbezogene Stakeholder und/oder bereichsspezifische Besonderheiten vorgegebenen Rahmen. Partizipativ entwickelte, inhaltlich konkrete und verständlich formulierte Qualitätsleitbilder sind dokumentierter Teil der betrieblichen Kommunikationspolitik. Sie können als gemeinsames und gepflegtes Orientierungssystem für qualitätsbezogene Tätigkeiten gelebter Bestandteil guter Organisationskultur sein. Sie können aber auch zu hohlen Instrumenten der Selbstdarstellung degenerieren, wenn sie der betrieblichen Praxis in keiner Weise (mehr) entsprechen. Wenngleich Leitbilder die Entwicklung und Aufrechterhaltung einer qualitätsorientierten Unternehmenskultur somit nicht gewährleisten können, stellen sie dennoch ein potenziell überaus wichtiges Element dar (z. B. auf der normativen Ebene des St. Galler Management Konzepts, Seghezzi, 2003). In vielen Vorschlägen für die Implementierung eines Qualitätsmanagements steht der Leitbildprozess denn auch dezidiert am Anfang. Andererseits gibt es Einrichtungen, die aus guten Gründen ein eher induktives Vorgehen bei der Qualitätsentwicklung bevorzugen (z. B. mit der Beschreibung von Schlüsselprozessen beginnen) und den Leitbildprozess mehr im Sinne einer verdichteten Zusammenschau dessen verstehen, was an "guter Praxis" vorhanden und angestrebt ist.

Ein implizites Leitbild haben letztlich alle Organisationen; denn sie entscheiden sich nach irgendwelchen Kriterien, z. B. für die Arbeit nach einem bestimmten Qualitätskonzept - und diese Kriterien sind ihr Leitbild. "Wer Normen so hoch aufhängt, dass keiner dran kommt, definiert damit sein Leitbild; und wer sagt, wichtig ist nur, dass die Kohle rüberwächst ebenfalls" (B. Müller, 2000, S. 142). 


\subsubsection{Qualitäts(management)-Handbuch}

Das QM-System eines Betriebs wird üblicherweise in einem zentralen Dokument beschrieben: dem Qualitäts-, Qualitätssicherungs- oder Qualitätsmanagement-Handbuch, bisweilen auch schlicht "Qualitäts-Ordner" genannt (GAB, 2003). Dabei liegt das Prinzip zugrunde, dass qualitätsrelevante Maßnahmen nicht nur durchgeführt, sondern stets auch nachvollziehbar dokumentiert werden müssen.

Im QM-Handbuch werden Konzept und Grundzüge des betriebsspezifischen QM-Systems dargestellt und ggf. nach den Anforderungen der gewählten Bezugsnorm strukturiert. Die derzeit verbreitetste Bezugsnorm ist die Norm ISO 9001:2000 (s. Kap. C 1.4.1). Betriebe, die ihr QM-System nach Maßgabe dieser Norm darlegen und sich von einer dazu befugten Stelle die Ordnungsmäßigkeit der Darlegung bestätigen (zertifizieren) lassen wollen, müssen ein Handbuch erstellen, das den Anforderungen der Norm entspricht. Neben dem eigentlichen QM-Handbuch spielen dabei die so genannten Verfahrens-, Arbeits- und ggf. Prüfanweisungen eine wesentliche Rolle (s. u.).

Da jedes QM-System nur so umfassend sein sollte, wie dies zum Erreichen der Qualitätsziele notwendig ist, bedarf es in vielen Organisationen keiner Berücksichtigung aller Normelemente der ISO 9001. Auch die Reihenfolge ist nicht zwingend vorgegeben. Viele Betriebe bauen ihr Handbuch prozessorientiert auf, d. h. die Gliederung erfolgt nicht nach Funktionen und Abteilungen, sondern in Übereinstimmung mit den wertschöpfenden und sonstigen Unternehmensabläufen. Für zahlreiche Arbeitsfelder liegen inzwischen Muster-Handbücher vor, an denen sich Einrichtungen und Dienste orientieren können ( $\mathrm{gl}$. Gerull 2000, 2004).

Nur die unternehmensspezifisch relevanten Informationen gilt es, in kompakter und leicht zu aktualisierender Form im QM-Handbuch zu beschreiben. Beobachtbare Auswüchse, mit hohem bürokratischen Aufwand alle Geschäftsprozesse minutiös darzustellen, entspringen zumeist einem statischen Qualitätsverständnis; die Installierung des QM-Systems wird mit seiner perfektionistischen Darstellung verwechselt. Formal ist das QM-Handbuch häufig in drei Teile gegliedert: 
- Teil 1 enthält Hinweise zur Organisation (z. B. Unternehmenszweck, Organigramm), zur Herausgabe und Pflege sowie zum Gebrauch der QM-Dokumentation.

- Teil 2 beschreibt die eigentlichen Elemente des QM-Systems (z. B. "Prozesse": Hilfeplanung, Aufnahmeverfahren u. a.).

- Teil 3 verweist als Anhang auf mitgeltende Unterlagen (z. B. Verfahrensanweisungen, Protokollbögen), deren Ausformulierung den Zweck des Handbuchs als Kommunikationsmittel sprengen würde.

Das QM-Handbuch enthält in der Regel nur organisatorisches Betriebs-Know-how und kann aus geschäftlichen Gründen an externe Dritte ausgegeben werden, z. B. für Zwecke der Werbung und Vertrauensbildung.

Im Unterschied zu den eher allgemeinen Ausführungen im QM-Handbuch beschreiben Verfahrensanweisungen (VA) detailliert, wie die qualitätssichernden Maßnahmen durchgeführt bzw. in die Unternehmensprozesse integriert werden sollen. Nach Maßgabe der ISO handelt es sich um Beschreibungen von Regeln, die abteilungsübergreifend gelten und somit Schnittstellen enthalten.

VA werden unternehmenseinheitlich gestaltet und verwendet. Sie verkörpern organisatorisches und technisches Betriebs-Know-how und sind deshalb nur intern zu benutzen. Die Abläufe können verbal und/oder graphisch (z. B. in Form von Flussdiagrammen) dargestellt werden.

Eine weitere Differenzierung stellen so genannte Arbeitsanweisungen (AA) dar. Sie beziehen sich nach ISO auf eine in sich abgeschlossene konkrete Tätigkeit, die in der Regel an einem Arbeitsplatz oder einem Gerät von einer einzelnen Person vollständig ausgeführt wird. Zu den AA gehören auch Prüfanweisungen, Formulare und Checklisten. AA enthalten detaillierte Angaben und technisches Betriebs-Know-how; sie sind deshalb ausschließlich intern zu verwenden.

Unabhängig davon, ob eine Einrichtung beabsichtigt, ihr QM-System nach den Vorgaben der ISO 9001:2000 darzustellen und zertifizieren zu lassen, können die Elemente der Norm als Leitfaden verwendet werden, ein Qualitäts-Handbuch für den internen und externen 
Gebrauch zu erstellen. Dies erscheint nicht nur zweckmäßig im Hinblick auf eine gründliche und übersichtliche Dokumentation der Betriebsstruktur und -organisation, sondern auch wegen der alltagspraktischen Verwendbarkeit als umfassendste Darstellung des betrieblichen Leistungsgeschehens (Nachschlagewerk, Einarbeitungshilfe u. a.). Die Gliederung eines Handbuchs kann sich aber auch an anderen QM-Systemen orientieren (z. B. EFQM, ServAs) oder ganz auf die Eigenheiten des Arbeitsfeldes abgestellt sein.

Bereits der Prozess der Handbucherstellung schafft vielfältige Gelegenheiten, die MitarbeiterInnen inhaltlich einzubeziehen, z. B. bei der Erarbeitung von so genannten Kundenpfaden und daraus abzuleitenden Verfahrensanweisungen (vgl. Gerull, 2000, 2004). Darüber hinaus empfiehlt sich eine Lose-Blatt-Dokumentation, die von Zeit zu Zeit zu aktualisieren ist und vermeiden hilft, ein allzu statisches Verständnis von Qualität zu etablieren. Außerdem eignet sich eine solche Art der Dokumentation dazu, die prozesshafte Arbeit am QM-Handbuch sichtbar zu machen, indem das Werk allmählich "wächst" (Meinhold, 1999).

Mindestens folgende Funktionen kann ein solcherart aufgebautes Qualitäts-Handbuch erfüllen:

- Dokumentation der betrieblichen Aufbau- und Ablauforganisation nach zweckrationalen Kriterien für die Innen- und Außendarstellung,

- Beteiligungsinstrument für MitarbeiterInnen,

- Nachschlagewerk und Strukturierungshilfe in Entscheidungssituationen,

- Führungsinstrument (Kontraktmanagement, Controlling).

\subsubsection{Qualitätszirkel}

Eine eindeutige Definition dessen, was ein Qualitätszirkel (QZ) ist und wie er am zweckmäßigsten zu organisieren sei, gibt es nicht. In der Organisationspsychologie werden QZ unter Oberbegriffen wie "gruppenorientierte Interventionstechniken" (Bungard \& Antoni, 2004) und "Mitarbeiterbeteiligung" (Antoni, 1999) thematisiert. Moore \& Kelly 
(1996, S. 35) verstehen darunter eher eine organisationale Orientierung als eine spezifische Technik.

\section{Qualitätszirkel (QZ) - eine Auswahl unterschiedlicher Auffassungen}

- Bungard \& Antoni (2004, S. 447) verstehen unter QZ "kleine Gruppen von Mitarbeitern der unteren Hierarchieebene, die sich regelmäßig auf freiwilliger Basis treffen, um selbstgewählte Probleme aus ihrem Arbeitsbereich zu bearbeiten."

- Schreyer-Schubert, Hanselmann \& Friz (2000, S. 87) beschreiben QZ als in der Regel bereichsund/oder hierarchieübergreifende Gesprächsgruppen.

- Eversheim (1997, S. 39) spricht von "Qualitätszirkel" nur im Zusammenhang mit abteilungsinternen Teams und verwendet ansonsten den Begriff "Qualitätsgruppe".

- $\quad$ Merchel (2001, S. 164) definiert QZ allgemein als "Arbeitsgruppen, die innerhalb eines umgrenzten Zeitraums im Rahmen eines Projektes ein bestimmtes Qualitätsproblem bearbeiten und dafür Lösungsvorschläge erarbeiten sollen."

- Demgegenüber betont Zollondz (2001, S. 1025), dass QZ "an den Schnittstellen von Arbeitsbereichen auf Dauer angelegte Gesprächsgruppen" seien.

- Knorr \& Halfar (2000, S. 102) empfehlen, QZ nach den allgemeinen Prinzipien des Projektmanagements durchzuführen.

- Im Qualitätskonzept der Gesellschaft für Ausbildungs- und Berufsforschung (GAB-Verfahren, Brater \& Maurus, 1997) ist dagegen die Einbindung der gesamten Mitarbeiterschaft in Qualitätszirkeln als "natürlichen" Arbeitseinheiten vorgesehen.

Ihren geistigen Ursprung haben die QZ in den USA; praktische Umsetzung in großem Stil erfuhren sie jedoch erst ab 1962 in Japan (Zollondz, 2001, S. 1025). Mit Hilfe der QZ sollten Defizite der traditionell hierarchischen, bürokratischen und tayloristischen Arbeitsorganisation ausgeglichen werden (a.a.O.). 
Seitens der Gewerkschaften wurden QZ anfangs abgelehnt, weil sie keine echte Mitbestimmung, sondern Ausbeutung der intellektuellen Fähigkeiten der ArbeitnehmerInnen zugunsten unternehmerischer Rationalisierungsambitionen mit sich brächten und die Position der Betriebsräte unterminierten (laut einer Stellungnahme der IG Metall, 1984, zit. nach Zollondz, 2001, S. 1027).

Inzwischen hat sich allgemein eine wohlwollende Einstellung zu diesem Instrument der Partizipation, Ressourcenorientierung und Qualifizierung entwickelt.

"Qualitätszirkelarbeit ist kreative Entwicklungsarbeit und ein gutes Stück innerbetriebliches Verbesserungswesen. Hier wird ergebnisorientiert gearbeitet, aber mit Spaß und mit Interesse!" (SchreyerSchubert et al., 2000, S. 87).

An Stelle einer Definition folgt eine Auflistung jener Merkmale, die am häufigsten für die Charakterisierung von Qualitätszirkeln verwendet werden.

\section{Qualitätszirkel (QZ) - eine Auswahl charakteristischer Merkmale}

- $\quad$ sind speziell eingerichtete oder aus "natürlichen" Teams bestehende,

- drei bis zehn Personen umfassende,

- $\quad$ paritätisch von einem Mitglied (seltener: extern) moderierte

- Arbeits-/Gesprächs-/Problemlösegruppen,

- die mit Unterstützung durch die Einrichtungsleitung

- freiwillig gebildet werden

- $\quad$ zur Behandlung von Qualitätsfragen und Lösung von Qualitätsproblemen;

- QZ sind autonom in der Wahl ihrer Themen und Moderatoren,

- auf eine bestimmte Dauer oder unbefristet konstituiert,

- $\quad$ regelmäßig und kontinuierlich im Rahmen der Dienstzeit tagend;

- $\quad$ sie arbeiten ziel- und ergebnisorientiert,

- dokumentieren ihre Vorschläge schriftlich und berichten darüber,

- agieren hierarchieübergreifend und gleichberechtigt,

- $\quad$ sind bereichsspezifisch oder -übergreifend zusammengesetzt,

- beziehen bedarfsweise und/oder an geeigneter Stelle "KundInnen" ein,

- bestehen auch in Form interdisziplinär besetzter und

- $\quad$ einrichtungsübergreifender externer Arbeitskreise. 
Eine erfolgreiche Arbeit von QZ bedarf eines organisationskulturellen Rahmens, der durch partizipative und kooperationsorientierte Leitungsstile geprägt ist (Merchel, 2001, S. 165). Von besonderer Bedeutung ist die Kompetenz der Moderation. Schreyer-Schubert et al. (2000, S. 89) betonen, dass ModeratorInnen nicht als FachexpertInnen zum jeweiligen Thema fungieren, sondern "nur" den Prozess in Gang halten und die Ergebnissicherung leisten sollten. Wichtig seien auch Aktionen, mit denen der Mitarbeit in QZ Wertschätzung und Respekt erwiesen werde und nicht beteiligte KollegInnen seriös informiert würden über das, was im QZ erarbeitet und vorgeschlagen wird (a.a.O.).

Ein QZ ist kein "Diskussionsclub", sondern eine methodisch vorgehende moderierte Gruppe, die in der Regel nach folgendem Schrittmuster arbeitet (vgl. Bobzien et al., 1996, S. 105; Schnoor, 2003, S. 74):

(1) Wahl und Abgrenzung des Themas (möglichst konkret, praxisrelevant, überschau- und bearbeitbar),

(2) Bestandsaufnahme der aktuellen Situation, Dokumentation der Realität,

(3) Bestimmung der Problempunkte und Defizite, Analyse der Realität,

(4) Bestimmung eines Katalogs von Anforderungen, Formulierung von Zielvorstellungen,

(5) Vorstellungen über die angestrebte Situation, Planung von Veränderungen,

(6) Formulierung von Vorschlägen zur Erreichung der angestrebten Situation, Umsetzung von Veränderungen,

(7) Evaluation der Veränderungen, Erstellen eines Qualitätsprofils (Überprüfung des Vorgehens, verständliche und widerspruchsfreie Darstellung der Ergebnisse).

Zu der Frage "Was macht eine gute Sitzung aus?" formulieren Schreyer-Schubert et al. (2000, S. 33f) "zehn Sterne der Moderation" und eine "Checkliste zum Ablauf" (S. 35). Die Verhaltensempfehlungen an ModeratorInnen lauten:

o Organisieren Sie das Treffen.

o Leiten Sie die Sitzung.

o Achten Sie auf die Zielorientierung der Arbeit.

o Gestalten Sie den Arbeits- und Gruppenprozess.

o Fördern und fordern Sie die Teilnehmer(innen).

o Schützen Sie die Teilnehmer(innen) und setzen Sie Grenzen.

o Sprechen Sie Konflikte an und klären Sie diese.

o Sorgen Sie dafür, dass Entscheidungen getroffen werden. 
o Organisieren Sie die Dokumentation.

o Stellen Sie die Verbindung zum Gesamtprozess her (Information von Projektgremien, Qualitätsbeauftragten u. a.).

Teilnehmer an QZ sehen in der Regel ihre Erwartungen an deren Nutzen bestätigt und behalten eine positive Haltung zu dieser Arbeitsweise (Schnoor, 2003, S. 80). Problematisch ist jedoch, dass NichtteilnehmerInnen sich häufig distanzieren und eine wesentlich negativere Einschätzung der QZ und ihrer Effekte abgeben, was einer Fraktionsbildung in der Belegschaft Vorschub leisten kann (vgl. Schnoor, a.a.O.). Für wichtig wird gehalten, die Frage der Entscheidungskompetenz von QZ im Vorfeld zu klären (a.a.O.).

Bungard \& Antoni (2004, S. 452) ziehen auf der Basis von Umfragen und Fallstudien ein überwiegend positives Fazit zu QZ, berichten aber auch von häufig genannten Problemen (mangelnde Unterstützung durch das mittlere Management, fehlende Zeit für die QZArbeit, lange Verzögerungen bei der Rückmeldung auf Verbesserungsvorschläge und deren Umsetzung). Zur Lösung dieser Probleme bedarf es nach Ansicht der AutorInnen der Einbettung der QZ-Einführung in umfassende Organisationsentwicklungsmaßnahmen.

"Embeddedness of quality practices" wird auch von Kochan \& Rubinstein (2000, S. 389f) als entscheidendes Erfolgsmerkmal für organisationale Transformationen betont und am Beispiel des "rise and fall of quality circles" in der amerikanischen Autoindustrie illustriert.

Schultz-Gambard et al. (1999, S. 101) berichten über eine kontrollierte Langzeitstudie von Griffin (1988), wonach QZ die arbeitsbezogenen Einstellungen und Verhaltensweisen der MitarbeiterInnen sowie die Effektivität der Produktion verbesserten, nach einiger Zeit jedoch zu Ritualen erstarrten, nicht mehr effizient waren und sich häufig auflösten.

In der Tat dürfte das größte Problem von dauerhaft installierten QZ darin bestehen, sie zeitlich, personell und thematisch so interessant zu konfigurieren, dass sie sich nicht totlaufen. 


\subsubsection{Qualitätsbeauftragte (QB)}

Hinsichtlich der Frage, wie ein Qualitätsmanagement am zweckmäßigsten in einer Einrichtung verankert werden kann, gibt es zwei Extrempositionen. Die eine geht davon aus, dass für die sorgfältige Beachtung der Qualitätsaufgaben eine spezifische Struktur erforderlich ist, weil "Qualitätsmanagement nicht von alleine funktioniert" (Seghezzi, 2003, S. 191). Die QM-Aufgaben werden von einer oder mehreren speziellen Fachstellen wahrgenommen, denen Aufbau, Pflege und Überwachung des Systems obliegen (Typus "Kontrolle", a.a.O., S. 190f). Auf diese Weise soll verhindert werden, dass das Qualitätsthema aus dem Blick gerät und schlechte Leistungen den Kunden erreichen.

Die andere Extremposition betrachtet Qualitätsmanagement als Querschnittsaufgabe, die von allen wahrgenommen werden muss. Die Verantwortung für die Leistungsqualität wird direkt von den wertschöpfenden MitarbeiterInnen ohne Mitwirkung spezifischer Fachstellen getragen; diesen kommen allenfalls unterstützende Funktionen zu (Typus "Dienstleister", a.a.O., S. 191). Besonders im Bereich sozialer Dienstleistungen gilt: Die Zuständigkeit für Qualität obliegt denjenigen, die sie "im Augenblick der Wahrheit" erbringen sollen. Umtausch, Nachbesserungen, Aussortieren der fehlerhaften Produkte sind infolge der Gleichzeitigkeit von Produktion und Konsumtion nicht möglich.

Auch wer das Kontroll-Modell favorisiert, weiß, dass es die MitarbeiterInnen vor Ort sind, die Qualität produzieren, und dass Qualität nicht in Produkte hineingeprüft werden kann. Andererseits ist auch beim Dienstleister-Modell zu berücksichtigen, dass durch explizite Delegation von Verantwortung ein verbindliches Element ins Spiel kommt (Kontraktmanagement) und nachhaltige Prozesse vielfach der strukturellen Absicherung bedürfen, um nicht zu versanden. Ohnehin sind auch Zwischenformen denkbar und praktiziert (z. B. Bündelung von Zuständigkeiten in der zentralen Funktionsstelle des/der Qualitätsbeauftragten, s. u., ohne zusätzliche Strukturelemente).

Wird Qualitätsmanagement als Projekt eingeführt, bedarf es notwendigerweise einer besonderen Organisationsstruktur, deren einzelne Elemente variieren und verschieden benannt werden: z. B. Steuerungsgruppe, Lenkungskreis, (externer) Moderator, Qualitätsbeauftragter, interner Prozessbegleiter, Qualitätskoordinator, Qualitätszirkel, Verbesserungsprojektgruppen. Diese Projektarchitektur ist aber zumeist vorübergehender Natur, auch wenn einzelne Elemente dauerhaft installiert werden können. 
Am häufigsten dürfte davon neben den schon länger etablierten Qualitätszirkeln die Position des/der Qualitätsbeauftragten $(Q B)$ betroffen sein, die inzwischen in zahlreichen Einrichtungen und Diensten offiziell ausgewiesen ist und einen blühenden Fortbildungsmarkt unterhält.

Ungeachtet der sachlichen Notwendigkeit oder Zweckmäßigkeit dieses Instruments spielt dabei eine Rolle, dass die Einsetzung eines QB neben der Einrichtung von Qualitätszirkeln und der Mitwirkung in externen Qualitätskonferenzen am häufigsten in den einschlägigen Gesetzen und Kommentierungen genannt wird, wenn potenziell geeignete Maßnahmen der Qualitätssicherung exemplarisch aufgelistet werden (z. B. Bundesempfehlung nach $\S 93 \mathrm{~d}$ Abs. 3 BSHG vom 15.02.99, zit. nach Wetzler, 2003, S. 23). Mit der Ausweisung eines QB meint denn auch manche Organisationsleitung, den größten Handlungsdruck in Sachen interner QM-Maßnahmen erst einmal abgearbeitet zu haben.

Obligatorisch ist die Installierung von QB für Einrichtungen, die sich an den Normen und Empfehlungen der ISO 9000 ff. orientieren und eine Zertifizierung ihres QM-Systems anstreben. Die ISO 9001:2000 (Deutsches Institut für Normung, 2000) sieht in ihrem Element 5.5.2 ("Beauftragter der Leitung") vor, dass die oberste Leitung ein Leitungsmitglied benennen muss, das unabhängig von anderen Verpflichtungen bestimmte qualitätsbezogene Verantwortungen und Befugnisse hat.

Seghezzi (1996, S. 178) betont, dass davon jedoch nur ein Teilbereich des Qualitätsmanagements betroffen sei und die übrigen Leitungsmitglieder nicht von eigenen qualitätsrelevanten Verantwortungen entbunden seien. Es bestehe aber immer die Gefahr, die eigene Verantwortung an den QB abzuschieben. Seghezzi hält diese Gefahr für am geringsten dort, wo der Vorsitzende der Geschäftsleitung die Aufgabe des QB selbst wahrnimmt (a. $a$. O.).

Diese Lösung der Zuständigkeitsfrage entspricht allerdings nicht der üblichen Praxis. In der recherchierten sozialwirtschaftlichen Fachliteratur finden sich ausnahmslos Empfehlungen und Berichte, die im Zusammenhang mit QB oder analogen Konstruktionen von einzurichtenden Stabsstellen ausgehen (z. B. Arnold \& Maelicke, 1998, S. 298). SchreyerSchubert et al. (2000, S. 11f) halten diese Stabsstelle für ein "Muss" in jeder Organisation, die Qualitätsmanagement entwickelt. Dabei wird in größeren Einrichtungen eine Person 
der mittleren Leitungsebene favorisiert, die in der Hierarchie Vertrauen nach unten und oben genießt.

Exemplarisch werden im Folgenden drei Rollen- und Aufgabenbeschreibungen für Qualitätsbeauftragte in verschiedenen Praxisfeldern dargestellt, bevor die damit verbundenen Hoffnungen und Enttäuschungen thematisiert werden.

Im TQM-Ansatz der Evangelischen Heimstiftung Pfalz, einem großen Einrichtungsverbund des Sozial- und Gesundheitssektors, wird die Rolle des QB folgendermaßen skizziert (Hollerith, 1995, 1996):

Der QB ist das Bindeglied zwischen Steuerungsgruppe und Qualitäts-Verbesserungsgruppen (QVG) in der Einrichtung. Er fungiert als Multiplikator der TQM-Philosophie auf allen Ebenen, aber auch als Ansprechpartner und Berater der QVG sowie der Steuerungsgruppe, für die er Servicefunktionen erfüllt (Sichtung von Literatur, Umsetzung von Beschlüssen usw.).

Der QB sollte die Einrichtung gut kennen und hohe Akzeptanz genießen, jedoch nicht selbst der Leitung angehören; er muss theoretisch und methodisch gut vertraut sein mit TQM und fähig, heterogene Arbeitsgruppen zu moderieren. Zu den Aufgaben des QB gehören u. a.:

- "Sekretär" der Steuerungsgruppe,

- Detailplanung des Einführungsprogramms,

- Beratung der Führungskräfte und Anleitung von QVG,

- Beachtung des TQM-Prozesses und Aufzeigen von Schwachstellen,

- Controlling der Verbesserungsprojekte,

- Erschließung von Fortbildungsmaterial und Vorbereitung von Informationsveranstaltungen.

In der Funktionsbeschreibung der Qualitätsmanagementbeauftragten (QMB) des Allgemeinen Sozialdienstes Kiel (Amt für Soziale Dienste, 2001, S. 12) heißt es:

Die QMB

- übernehmen die Leitung der Steuerungsgruppe sowie deren Vor- und Nachbereitung.

- $\quad$ strukturieren den Qualitätsmanagementprozess.

- kontrollieren die Terminierung für zu erledigende Aufgaben.

- entwickeln Verfahrensabläufe zur Umsetzung.

- $\quad$ erledigen im Auftrage der Steuerungsgruppe bestimmte Aufgaben und setzen geplante Abläufe um.

- sind AnsprechpartnerInnen für sämtliche Belange im Qualitätsmanagementprozess sowie für die Qualitätszirkel und Internen Prozessbegleiter. 
- übergeben den Qualitätszirkeln den Auftrag bzw. Überarbeitungsauftrag.

- stehen in engem Kontakt zur Fortbildungsabteilung des Personalamtes, um notwendige Fortbildungen und Fachtage zu organisieren.

- halten alle relevanten Ergebnisse schriftlich fest und koordinieren den Transfer von Informationen.

- haben die Verantwortung für die Pflege, Registrierung und Verteilung der QM-Dokumente.

- entwickeln das QM-System weiter.

Schreyer-Schubert et al. (2000, S. 14) zählen in ihrem Leitfaden für Qualitätsbeauftragte zu den Aufgaben des QB die Organisation, Koordination, Aufrechterhaltung und Weiterentwicklung des Qualitätssystems. Diese Aufgaben werden in verschiedene Tätigkeiten aufgefächert:

- QB als Geschäftsführerin der Steuerungsgruppe

- QB als Beraterin der Qualitätszirkel

- QB als Fortbildnerin in Sachen Qualität

- QB als Organisatorin der Dokumentation

- QB als Verantwortliche für Information

- QB als Koordinatorin von Audits und Zertifizierungen.

Der Deutsche Verein für öffentliche und private Fürsorge, der wie viele andere Verbände Ausbildungskurse für QB durchführt, begründet dieses Angebot unter anderem damit, dass es wichtig sei, das Qualitätsthema in den Einrichtungen und Diensten durch die Institutionalisierung von QB strukturell abzusichern (Irskens, 2002, mdl. Mitteilung). Auch Nüßle (1999, S. 106 ff.) betont den Aspekt der Nachhaltigkeit bei der Etablierung von Qualitätsmanagement und plädiert für eine Strategie, möglichst viele MitarbeiterInnen durch Fortbildung zu befähigen, qualitätsbezogene Funktionen wahrzunehmen. Der QB wird dabei als Motor und Mentor der Qualitätsentwicklung betrachtet.

Um diese Rolle optimal ausfüllen zu können, benennt Nüßle Voraussetzungen auf der persönlichen, strukturellen und Beziehungsebene: fundierte feldspezifische Fachlichkeit, Planungs-, Gestaltungs- und Moderationskompetenz, Freiräume für Fortbildung und QMMaßnahmen, kontinuierlicher Austausch mit anderen QB (a.a.O., S. 116f). Schreyer-Schubert et al. (a.a.O., S. 13) zählen ähnliche Fähigkeiten auf, die QB mitbringen und/oder durch Weiterbildung lernen und durch Erfahrung entwickeln müssen. Dazu gehören überdies Präsentations-, Konflikt- und Überzeugungsfähigkeit, Belastbarkeit, Lernbereitschaft und Vertrauen in das Potenzial der MitarbeiterInnen. 
Lo \& Sculli (1998, S. 83f) kommen in einer Untersuchung zur Rolle von QB in ISO 9000 orientierten QMProjekten zu den Schlussfolgerungen, dass QB

- autorisiert sein sollten, wichtige Projektmitarbeiter vorzuschlagen,

○ über ein hohes Maß an Ansehen und Erfahrung in der Organisation verfügen sollten,

○ commitment und Pflichtbewusstsein mitbringen müssen,

○ Fähigkeiten zur effektiven Arbeitskoordination und Beziehungspflege innerhalb des Teams besitzen müssen,

○ über hinreichendes technisches Wissen zur Unterstützung der Teammitglieder in arbeitsbezogenen Entscheidungen verfügen und

○ verantwortlich für die gesamte Ressourcen- und Ablaufplanung des Projekts sein sollten.

Wegen der Verzahnung von Qualitätsmanagement mit strategischen Planungen steht der/die QB in engem Kontakt mit geschäftsführenden Aufgaben, woraus Konfliktfelder zwischen QB und Leitung erwachsen können (Nüßle, a.a.O.). Schreyer-Schubert et al. (a.a.O., S. 15) skizzieren eine Reihe möglicher Konstellationen:

- schwache Leitung - starke QB,

- nicht interessierte Leitung - engagierte QB,

- die (im Alleingang) pflegende QB,

- die alleingelassene QB,

- die QB ohne oder mit zu wenig Zeit.

Merchel (2001, S. 159 ff.) setzt sich eher skeptisch mit dem "Implementierungsinstrument QB" auseinander. Er sieht einerseits dahinter die Hoffnung, dem Qualitätsthema einen organisatorisch sichtbar verankerten und methodisch kompetent ausgefüllten Stellenwert innerhalb der Organisation zu verschaffen, namentlich durch

- Unterstiutzung der Mitarbeitergruppen durch spezifische methodische Kompetenz des $\mathrm{QB}$,

- bessere Motivierung der Mitarbeiter durch QB als durch Leitung, der möglicherweise Kontrollabsichten unterstellt werden,

- höhere Akzeptanz von QB und geringeres Maß an Überlagerung der Qualitätsentwicklung durch Auseinandersetzung mit Leitungspersonen,

- Zusammenführung der Qualitätsentwicklungsprozesse einzelner Organisationssegmente durch besondere Zuständigkeit des QB, 
- spezifische personelle Verankerung des Qualitätsthemas als wirksamer Motor für Qualitätsentwicklung.

Andererseits werden Risiken betont, die sich aus der Positionierung von QB ergeben und eine Prozessdynamik in Gang setzen können, an dessen Ende das Qualitätsthema nicht, wie beabsichtigt, zu einem Lernthema der Organisation, sondern zu einem Thema mit künftig negativen Konnotationen geworden ist (a.a.O., S. 161):

- Besetzung des Qualitätsthemas durch QB bei nur abstrakt behaupteter Letztverantwortung der Leitung,

- Verführung der Leitung zur "Entsorgung" des Qualitätsthemas durch Delegation an Beauftragte,

- implizite Konkurrenz zwischen Leitung und QB infolge von Übernahme "informeller" Leitungsfunktionen,

- Rollenunsicherheit von QB infolge der strukturellen Konfliktanfälligkeit von Stabsfunktionen.

Merchel (a.a.O., S. 163) resümiert:

\begin{abstract}
"Insgesamt gesehen scheinen Qualitätsbeauftragte ein nicht unproblematisches Instrument bei der Implementation von Qualitätsmanagement in Einrichtungen der Sozialen Arbeit zu sein. Sicherlich bieten sie auch Chancen, das Qualitätsthema in einer Organisation zu verankern und es kompetent zu bearbeiten, aber gleichzeitig stehen die Gefahren einer schleichenden Verantwortungsdiffusion bei der Leitung. Leitung darf sich des Qualitätsthemas wegen seiner immensen strategischen Bedeutung für die Organisation nicht entledigen und darf die eigene Verantwortung für dieses Thema weder proklamatorisch noch in den faktischen Handlungsvollzügen in Zweifel ziehen."
\end{abstract}

Die Installierung von QB - wenn sie denn binnenorganisatorisch oder aus Gründen der externen Darlegung für zweckmäßig gehalten wird - sollte also in jedem Falle problemsensibel erfolgen. Es handelt sich um eine zweifellos schwierige Rollenkonstellation, der gerecht $\mathrm{zu}$ werden jedoch "nicht mehr an psychischen und sozialen Energien sowie an zeitlichen Ressourcen in Anspruch nehmen (sollte) als man als Nutzen in Aussicht stellen und kalkulieren kann" (a.a.O.).

In der deutschsprachigen Fachliteratur sind Stellenbeschreibungen (Kottmann, 2002) und programmatische Erörterungen zur Funktion und/oder zum Anforderungsprofil (Hollerith, 1995, 1996; Nüßle, 1999), mitunter auch Erfahrungsberichte (Sörgel \& Merkl, 2000; 
Thorwart, 2000) zum Instrument des/der QB in sozialen Einrichtungen und Diensten zu finden, jedoch keine empirischen Studien zur konkreten Ausgestaltung und Bewährung in der Praxis. Auch eine auf den angloamerikanischen Bereich erweiterte Literaturrecherche zeitigte nur ein spärliches Ergebnis (Lo \& Sculli, 1998; Mclaughlin \& Herr, 1999) mit geringer Relevanz für sozialwirtschaftliche Unternehmen.

In welchem Umfange das Instrument QB in den einschlägigen Organisationen implementiert wurde und welcher Stellenwert ihm seitens der Akteure und KooperationspartnerInnen beigemessen wird, kann daher nur gemutmaßt werden.

An diesem Punkt setzt ein Teil der in Kap. D 4. dargestellten Studie an.

\subsubsection{Benchmarking}

Als Benchmark bezeichnet man einen Ziel- oder Orientierungspunkt, an dem sich die eigene Position bestimmen lässt. Benchmarking ist analog ein strukturierter Prozess des Lernens aus der Praxis anderer (Interner oder Externer), die als Führer (Beste) bezüglich dieser Praxis anerkannt sind (Burmeister, 2000, S. 78). Ziel ist es, Leistungsfähigkeit und Wissen durch planmäßige Informationsgewinnung und offenen Erfahrungsaustausch nach fairen Regeln zu steigern. Im Rahmen der Betriebsführung erweitert ein Benchmarking vorhandene Ansätze der Qualitätsentwicklung um die systematische Kommunikation zwischen verschiedenen Einrichtungen und/oder Abteilungen ("vergleichende Betriebsanalyse").

Untersuchungseinheit beim Benchmarking ist also nicht der Einzelfall (wie z. B. bei individuellen Evaluationsstudien erzieherischer Verläufe). Es können eigene Produkte und Dienstleistungen mit denen von Wettbewerbern (kompetitives Benchmarking) oder einzelne betriebliche Prozesse und Methoden (funktionelles Benchmarking) verglichen werden. Aus ermittelten Unterschieden lassen sich deren Ursachen und Möglichkeiten zur Verbesserung erschließen. Vergleiche können sowohl inner- als auch außerbetrieblich erfolgen (internes/externes Benchmarking), wobei das Ziel darin bestehen kann, die jeweils besten Lösungen zu bestimmen und umzusetzen (Best Practice Benchmarking). 
Die praktische Anwendung des Benchmarking-Ansatzes - Metzen (1994, S. 38f) spricht von "erlaubter Werksspionage" - ist im Bereich der Wirtschaft weit fortgeschritten. Aber auch im Bildungsbereich und zunehmend im Sozial- und Gesundheitswesen wachsen die Anwenderzahlen.

In Datenbanken werden betriebswirtschaftliche, personalwirtschaftliche und zielgruppenbezogene Informationen gesammelt, aufbereitet und ausgewertet. Die Einrichtungen, die sich am Benchmarking-Projekt beteiligen, verwenden Checklisten und Fragebögen, die der Datenbankbetreiber zur Verfügung stellt bzw. die zuvor von den TeilnehmerInnen entwickelt und ausgewählt wurden. Die Datenerhebung erfolgt in den Einrichtungen durch beauftragte und geschulte MitarbeiterInnen; das Material wird von der Datenbank anonymisiert, statistisch ausgewertet und den Benchmarking-Partnern in Form eines Profils zurückgeschickt. Dieses kann mit Profilen anderer Leistungsanbieter verglichen werden, um eigene Stärken, Schwächen und Verbesserungsbedarf zu erkennen. Bei wiederholter Anwendung lassen sich Entwicklungsprozesse verfolgen und Eigenvergleiche anstellen.

Das Konzept des anonymisierten Betriebsvergleichs basiert auf der Annahme, dass Qualität im Sozial- und Gesundheitswesen nicht als feste Größe messbar ist, sondern sich nur aufgrund von Selbstevaluation und Vergleichen mit anderen erschließen sowie durch Entwicklung des eigenen Potenzials verbessern lässt. Beim Benchmarking werden deshalb keine Vorgaben und Normen gesetzt, sondern Qualität wird als relational, einrichtungsund kontextabhängig definiert. Die Devise lautet: voneinander lernen. Dabei ist es Sache der Benchmarking-Partner, sich auf gegenseitige Verpflichtungen, zum Beispiel Regeln der Datengewinnung und -verwendung, zu verständigen und diese vertraglich zu fixieren. Trotz grundsätzlich vereinbarter Anonymität wird zumeist der "Klassenbeste" mit dessen Zustimmung identifiziert, um einen Know-how-Transfer zu ermöglichen. Dabei wird zu Recht unterstellt, dass auch dieser ein Eigeninteresse hat, sich kontinuierlich zu vergleichen, sei es, um sich der eigenen Spitzenposition zu vergewissern und sie aufrechtzuerhalten, sei es, um jene Teilbereiche $\mathrm{zu}$ optimieren, in denen andere Leistungsanbieter "die Nase vorn" haben.

Benchmarking stellt im Rahmen eines prozessorientierten Qualitätsmanagements einen instrumentellen Teilausschnitt dar und ist somit als Ergänzung, nicht als Alternative zu anderen Verfahren der Qualitätsentwicklung, -sicherung und -bewertung zu betrachten. Benchmarking kann Selbstbewertungsprozesse unterstützen, indem es Maßstäbe für Vergleiche liefert. Benchmarking setzt aber die Einführung qualitätsorientierter 
innerbetrieblicher Strukturen und Prozesse in gewissem Umfange voraus, da es auf Vertrauen in die Seriosität des Verfahrens und seiner Propagandisten angewiesen ist. Ein Qualitätsmanagement mit Benchmarking $\mathrm{zu}$ beginnen, ohne zuvor den betriebsklimatischen Boden dafür bereitet zu haben, dürfte deshalb problematisch sein (Gerull, 2004, Kap. 2.6.5).

Auf bereits vorzufindende Missbräuche des Konzepts weisen u. a. von Eiff (2000) und Spindler (2001) hin. Es sei ein Irrtum, durch bloßes Übernehmen erfolgreicher Konzepte könnten Organisationen (hier: Krankenhäuser, Eiff, a.a.O., S. 97) sich notwendige eigene Innovationen ersparen; Kreativität und Einfallsreichtum seien Voraussetzung für einen Erfolg versprechenden Benchmarking-Prozess. Spindler (a.a.O., S. 145) kritisiert die falsche Anwendung des Benchmarkings im Rahmen der Kostensteuerung in der Sozialhilfe und fragt, von welchem "Besten" die sich vergleichenden Kommunen eigentlich lernen möchten. Mitverantwortlich für die Nichtberücksichtigung des Bedarfsdeckungsprinzips und des "Kunden" der Sozialhilfe (S. 152) werden auch Beraterfirmen gemacht, welche die Geldleistungen der Sozialhilfe lediglich als Kosten betrachteten und eine Produktverschlechterung fälschlicherweise zur Kostensenkung umdefinierten (S. 146).

Freigang (2001) sieht die Vorteile des Benchmarkings darin, dass es keine normativen Vorgaben mache oder moralisch apelliere, sondern realistisch und pragmatisch vom Ist ausgehe. Grenzen werden darin gesehen, dass die Daten interpretationsbedürftig seien. Fraglich sei auch, wie sich so etwas wie Beziehungsqualität abbilden lasse, wohingegen technokratische Kategorien (z. B. Anzahl von Therapiestunden) leichter zu operationalisieren seien. Betont wird, dass das Verfahren mit anderen kombiniert werden müsse.

Löwenhaupt (1999) betont die Anschlussfähigkeit zu anderen Verfahren (z. B. EFQM, ISO 9000 ff., Selbstevaluation) und hält die flexible Einsatzmöglichkeit des Benchmarkings für vorteilhaft.

Grundsätzliche Kritik am Benchmarking äußern Hoerner \& Vitinius (1997, S. 175 ff.). Die in der Theorie unterstellte und in der Praxis kaum gegebene Übertragbarkeit von einem Unternehmen zum anderen sei das Grundproblem, an dem Benchmarking unheilbar kranke. "Best-Practice"-Unternehmen lägen deshalb vorn, weil die jeweilige Vergleichsdisziplin (z. B. Anzahl der Fehler bei Rechnungsstellung) nahe an ihrem 
Kerngeschäft läge (z. B. bei Kreditkartenunternehmen im Vergleich mit Computerherstellern).

"Gehört der Versand von Ersatzteilen oder dergleichen aber zu den Nebensächlichkeiten im Geschäft, dann macht es keinen Sinn, hier mit übertriebenem Aufwand an Verbesserungen zu arbeiten" (a.a.O.).

Ungeachtet berechtigter Warnungen vor Überschätzung ist dem Ansatz jedoch ein erhebliches Potenzial zu attestieren, Qualitätsentwicklungsprozesse einrichtungsübergreifend in Gang zu setzen bzw. zu unterstützen. Die erforderliche Verwendung eines standardisierten Sets von Datenerfassungsinstrumenten dürfte die Kooperation zwischen Einrichtungen, die sich am Benchmarking beteiligen wollen, stark befördern. So könnten sich z. B. regionale oder überregionale Gütegemeinschaften herausbilden, die ein gemeinsam entwickeltes Inventar feldspezifischer und aussagekräftiger Qualitätsindikatoren benutzen. Je nach Anlage des Benchmarking-Prozesses können Einrichtungen in einen Lern- und Problemlösungsdialog eintreten, der den Übergang von der lernenden Organisation zum lernenden Organisationsverbund kennzeichnet (Tornow, 1999).

Als Nachweis für die Selbstverpflichtung zur empirisch gestützten Reflexion der betrieblichen Qualitätsfähigkeit kann die Teilnahme an regelmäßigen BenchmarkingProzessen auch eine positive Außenwirkung erzielen. Damit ist Benchmarking eine vielversprechende Alternative zur Zertifizierung (vgl. Halfar, 1996a; Burmeister, 1997, 1999, 2000).

\subsubsection{Prozessmanagement}

Ein Prozess ist ein Satz von in Wechselbeziehung oder Wechselwirkung stehenden Tätigkeiten, der Eingaben (Input) in Ergebnisse (Output) umwandelt (DIN EN ISO 9000:2000). (Geschäfts-)Prozesse stellen Vorgehensweisen zur Lösung von Aufgaben ("Kundenproblemen") dar und kombinieren funktionales Know-how im Hinblick auf die Bedürfnisse der Kunden mit den Interessen des Unternehmens (Haselbacher, Prax, Vogt \& Wuksch, 2001, S. 23). 
Die meisten Prozesse sind mit vor- oder nachgeschalteten Prozessen, an denen andere Personen beteiligt sind, verknüpft ("vernetzt"). So ist oft der Output eines Prozesses der Input eines daran anschließenden Prozesses. Solche Übergänge von einem Prozess oder Teilprozess zum anderen heißen Schnittstellen. Sie entstehen da, wo die Tätigkeit einer Person, einer Abteilung usw. die Tätigkeit von anderen berührt.

$\mathrm{Zu}$ Schnittstellen gehören immer mindestens zwei Beteiligte; Schnittstellen machen also Kooperation erforderlich und sind daher prinzipiell störanfällig. Es gibt innerbetriebliche (interne) und außerbetriebliche (externe) Schnittstellen. Beiden gebührt im Rahmen eines Qualitätsmanagements starke Beachtung; die meisten Verbesserungsvorschläge beziehen sich auf solche Schnittstellen. Schnittstellen, die einen hohen Stellenwert für gelingende Prozesse haben, werden "sensible Schnittstellen" genannt, weil ihre Gestaltung weitreichende Konsequenzen für Erfolg und Misserfolg bzw. Zufriedenheit oder Unzufriedenheit der (internen und externen) Kunden hat ("Schlüsselsituationen", vgl. von Spiegel, 2000, S. 188).

Als einleuchtend hat sich herausgestellt, diejenigen, welche einen Output erhalten, als "Kunden" zu bezeichnen und diejenigen, die einen Input einbringen, als "Lieferanten". Dies gilt auch für innerbetriebliche Schnittstellen; sinngemäß wird dann von internen Kunden und Lieferanten gesprochen. Einrichtungen funktionieren am besten, wenn sie sowohl an den äußeren als auch inneren Schnittstellen nach dem Prinzip der Kunden- und Lieferantenbeziehungen aufgebaut sind, ohne diese Begriffe explizit verwenden $\mathrm{zu}$ müssen.

Mit dem Begriff des Prozessmanagements werden Versuche bezeichnet, ein neues Paradigma der Organisationsform zu begründen, bei dem die Hierarchie einerseits und die Bereiche andererseits nicht mehr streng gegen- und untereinander abgegrenzt sind, sondern bereichs- und funktionsübergreifend ganzheitlich synergetisch zusammenwirkend einen Kundennutzen erzeugen (Zollondz, 2001, S. 739). Prozessmanagement heißt also, die zur Erstellung der Produkte und Dienstleistungen benötigten operativen Aktivitäten so zweckmäßig wie möglich, nämlich prozesshaft zu gestalten. Dabei ist von zentraler Bedeutung, dass die zahlreichen Tätigkeiten nicht abteilungs- oder funktionsorientiert ausgerichtet, sondern zu sinnvollen Abläufen verknüpft werden, welche die internen und externen Schnittstellen zu Verbindungs- oder Nahtstellen machen sollen. Alle Prozesse 
dienen letztlich der Erfüllung von Kundenerwartungen und sind kein Selbstzweck, den es abteilungsegoistisch zu perfektionieren gelte.

Grundidee des Prozessmanagements ist die kontinuierliche Verbesserung der Betriebsabläufe, jedoch mit völliger Neugestaltung einzelner Prozesse, wenn die Notwendigkeit hierzu durch eine Prozessanalyse (s. u.) erkannt werden sollte. Prozessmanagement verbindet somit den Ansatz des kontinuierlichen Verbesserungsprozesses mit dem Konzept des Business (Process) Reengineering (Haselbacher et al., 2001, S. 20; Zollondz, 2001, S. 742). Oberster Grundsatz des Prozessmanagements ist, dass für jeden Prozess eine Person verantwortlich sein muss (a.a.O., S. 746).

Grundlage des Prozessmanagements ist eine Prozessanalyse. Bumbacher \& Kaufmann (1998, zit. nach Knorr \& Halfar, 2000, S. 271) empfehlen, die wichtigsten Prozesse inklusive ihrer Schnittstellen nach folgendem Frageschema zu analysieren:

(1) Was tun wir? (Erhebung der Aktivitäten)

(2) Warum tun wir es? (Ziele/Zweckerhebung)

(3) Wie tun wir es? (Erhebung der Prozesse)

(4) Wie gut tun wir es? (Messung, Auswertung, Controlling)

(5) Wie wissen wir, dass wir es gut tun? (Betriebsvergleich, Befragungen)

Um zu verhindern, dabei "strukturkonservativ" in der Logik der beobachteten Gegenwart zu verbleiben, schlagen Knorr \& Halfar (S. 271) als denkbare Alternative den "sollzustandsorientierten Ansatz" von Heine (1995) vor, in dem zuerst eine quasi-optimale Prozessarchitektur entwickelt und diese dann mit dem Ist-Prozessverlauf verglichen wird.

Ein solches Vorgehen ist auch in einem "Grundschema des Prozessmanagements" in mehreren Arbeitsschritten enthalten, das von Haselbacher et al. beschrieben wird (2001, S. 21f):

o umfassende Beschreibung der im Unternehmen auftretenden Prozesse (Ist-Erhebung),

o Zusammenfassung und klare Gliederung der erhobenen Prozesse (Strukturierung),

o Erkennung und Dokumentation von Gemeinsamkeiten der unterschiedlichen Prozesse (Analyse), 
o Soll-Modell-Erstellung unter Zuhilfenahme von Benchmarking oder Referenzmodellen,

o Simulation der Prozesse zur Klärung möglicher Auswirkungen geänderter Prozesse,

o Auswahl des den Unternehmenszielen am besten entsprechenden Soll-Modells (Selektion),

o Einführung der gewünschten Prozesse in das Unternehmen (Implementierung),

o laufende Überwachung der tatsächlichen Prozesse (Monitoring).

Dieses Grundschema wird in der Praxis teilweise noch erheblich differenziert; allerdings besteht die Gefahr, durch zu viele Detaillierungen eine Informationsflut auszulösen, die nicht mehr verarbeitet werden kann. Prozessanalysen sollten deshalb nicht am "grünen Tisch", sondern unter Einbeziehung der betroffenen MitarbeiterInnen durchgeführt werden. Qualitätszirkel sind oft der zweckmäßigste Ort, solche Analysen, z. B. von Schlüsselprozessen, zu erarbeiten (exemplarisch hierzu Amann \& Straus, 2000, S. 135 147).

Ein Prozessmodell ist der Versuch, die wertschöpfenden unternehmensspezifischen Abläufe klar, einfach und übersichtlich darzustellen. Während das Prozessmodell z. B. der ISO 9001 branchenneutral ist, liefern Referenzmodelle branchentypische Beispiele für Prozesse, die als Basis für die eigenen Abläufe dienen können und erheblichen eigenen Modellierungsaufwand ersparen können (Haselbacher et al., 2001, S. 25). Innerhalb des Prozessmodells können die einzelnen Prozesse hinsichtlich ihres Einflusses auf die Erfüllung des Unternehmenszwecks gewichtet werden: Haupt-, Kern- oder Schlüsselprozesse, Unterstützungs- (Support-)Prozesse, Teil- und untergeordnete Prozesse. Ein Hauptprozess ist z. B. die Einstellung neuer MitarbeiterInnen; ein wichtiger Teilprozess ist dann deren Einarbeitung (Gerull, 2004, Kap. 3.3.7). Tätigkeiten, die in besonderer Weise maßgeblich sind, werden als Schlüsselprozesse bezeichnet.

Die entsprechenden Bezeichnungen sind nicht genormt; mitunter ist zusätzlich oder stattdessen von Hebelprozessen, Primär-, Sekundär- und Tertiärprozessen, strategischen und operativen Kernprozessen die Rede.

Die "Beherrschung" von Schlüsselprozessen ist das Kernanliegen aller Qualitätsmanagementsysteme. Durch die Beschreibung dieser Prozesse werden die Arbeitsziele für 
die Prozessgestaltung in Standardsituationen operationalisiert, es wird also konkret angegeben, was zu tun ist, um die Qualitätsmerkmale (Kriterien) der Situation zu erfüllen und an welchen Sachverhalten (Indikatoren) dies ggf. erkannt werden kann. Die Beschreibung von Schlüsselprozessen erfasst gewissermaßen das durchschnittlich erwartbare Verhalten der Fachkräfte in Bezug auf wiederkehrende Situationen (von Spiegel, 2000, S. 188).

Prozesse werden oft in Form von Ablaufdiagrammen, z. B. als Flussdiagramm dargestellt. Auch werden in der Bezeichnung verschiedener Prozessphasen und unterschiedlicher Rollen der an Prozessen beteiligten oder von ihnen betroffenen Personen häufig Begriffe wie Prozessbeginn und -ende, Prozesseigentümer, -benutzer, -kunde und -experte verwendet (Bobzien et al., 1996, S. 59). Allerdings stößt solches "Einsickern von formalisierender, betriebswirtschaftlich induzierter Sprache" in soziale Handlungskontexte auf erhebliche Bedenken (Merchel, 2000, S. 166f).

"Das Arbeitsfeld und die darin enthaltenen eigentlichen (hier: sozialpädagogischen, P. G.) Aufgaben werden unkenntlich. Dies erzeugt zum einen emotionale Differenzen bei denjenigen, die ohne eine Identifikation mit dem Arbeitsfeld ihre Aufgaben gar nicht angemessen bewältigen können, und zum anderen ruft es die kritische Frage nach dem Verhältnis formaler Verfahren und spezifischen Inhalten hervor" (a.a.O.).

\subsubsection{Ideenmanagement/Vorschlagswesen}

Im Zusammenhang mit prominent gewordenen Instrumenten des Qualitätsmanagements namentlich Qualitätszirkeln und dem Konzept des kontinuierlichen Verbesserungsprozesses - ist auch das altehrwürdige Betriebliche Vorschlagswesen (BVW) wieder in den Blickpunkt geraten. Bereits Ende des 19. Jahrhunderts bei Siemens und Bosch als zentraler Bestandteil der Unternehmenskultur gewürdigt, werden dem traditionellen Vorgehen vieler Firmen allerdings erhebliche Schwachstellen attestiert, vor allem der lange Weg durch die Instanzen, die geforderte Schriftform, schleppende Umsetzung und fehlende Mitwirkung der Ideengeber (D. Frey et al., 1999, S. 123). Verstärkt stehen deshalb Bemühungen im Vordergrund, das Verfahren zu entbürokratisieren und $\mathrm{zu}$ einem dynamischen, innovativen Ideenmanagement umzugestalten, um die "Unternehmensressource Kreativität" optimal zu nutzen. 
Thom (1991) unterscheidet dabei zwischen Produkt- und Dienstleistungsinnovation, Sozialinnovation (z. B. Partizipationsprozesse) und Verfahrensinnovation (= Prozessinnovation). Um diese häufig miteinander verschränkten Innovationsarten zu "managen", sind folgende Aufgaben zu beachten (vgl. Hentze, Kammel \& Schwager, 2000; D. Frey et al., 1999, S. 127 ff.):

- Voraussetzungen für eine Ideengenerierung schaffen,

- Problemstellungen, die einer Lösung bedürfen, identifizieren,

- Ideen in betriebliche Zusammenhänge einordnen,

- Verbesserungsvorschläge konzipieren und bewerten,

- als nutzbringend bewertete Vorschläge in das betriebliche System umsetzen.

Techniken wie Tages-, Wochen- und Monatsreflexion, Mängelliste, Kunden-, Lieferantenund Wissenschaftsforen, Aufdecken geheimer Spielregeln, Brainstorming, Brainwriting u. a. m. unterstützen den Prozess der Problemidentifikation und Ideenfindung. Sind zudem entsprechende Strukturbedingungen gegeben, lässt sich die Anzahl eingehender Vorschläge um ein Vielfaches erhöhen und ein neues Vertrauen in die Institution des Vorschlagswesens begründen (D. Frey et al. 1999, S. 123f).

Solche Strukturbedingungen und Erfolgsfaktoren sind vor allem:

- Förderung von organisationalem Lernen, innovativem Teamklima und "Implementierungskultur",

- Honorierung aller Ideen, ungeachtet ihrer Umsetzung,

- kontinuierliche Verbesserung, Partizipation, Delegation und eine Politik der "offenen Tür" als zentrale Führungsaufgaben,

- dezentrale Umsetzung der Ideen durch einreichende Mitarbeiter/Teams,

- rasche Begutachtung der Vorschläge,

- Transparenz und Visualisierung der Verbesserungsideen (a.a.O.).

Während das Betriebliche Vorschlagswesen in der Regel so aufgebaut ist, dass ein zentrales Organ aufgrund von Gutachterempfehlungen beurteilt, wie Vorschläge zu handhaben sind, wird beim Kontinuierlichen Verbesserungsprozess (KVP) von vornherein 
dezentral vorgegangen. Ausgehend von konkreten Problemen werden Lösungen vor Ort initiiert und umgesetzt (a.a.O., S. 124).

Wenn auch je nach Sichtweise das KVP-Konzept als Pfeiler der Unternehmensphilosophie oder als Bestandteil und Ergänzung des Betrieblichen Vorschlagswesens betrachtet werden kann und in vielen Firmen beide Konzepte koexistieren, wird eine Integration der Ansätze in Richtung eines Ideenmanagements für sinnvoll gehalten. D. Frey et al. (1999, S. 126) bezeichnen es als ideal, wenn Verbesserungsvorschläge in jedem Fall durch ein paritätisch besetztes Lenkungsteam dezentral abgewickelt würden.

\subsubsection{Beschwerdemanagement}

Beschwerdemanagement ist der systematische und zielorientierte Umgang mit Beschwerden und umfasst die Planung, Durchführung und Kontrolle aller Maßnahmen, die ein Unternehmen im Zusammenhang mit Kundenbeschwerden ergreift (Zollondz, 2001, S. 59). Beschwerden sind artikulierte Unzufriedenheit mit einer erhaltenen Leistung, oftmals verbunden mit Forderungen nach Wiedergutmachung oder Änderung des kritisierten Verhaltens. Reklamationen als Teilmengen von Beschwerden bezeichnen solche Beanstandungen in der Nachkaufphase, die mit einer juristisch durchsetzbaren Forderung verbunden sind (a.a.O.).

Beschwerdemanagement nimmt eine Schlüsselstellung im Kundenbeziehungs- bzw. Qualitätsmanagement ein, weil sich gerade in Beschwerdesituationen entscheidet, ob ein Kunde den Dienstleister wechselt oder auf Dauer gebunden werden kann (Stauss \& Seidel, 2002). Als aktive Reaktion auf Unzufriedenheit mit einem Produkt, das seinen Erwartungen nicht entspricht, kann ein Kunde $\mathrm{zu}$ einem anderen Leistungsanbieter abwandern ("exit"), negative "Werbung" betreiben oder sich gegenüber dem Unternehmen bzw. Drittinstitutionen (z. B. Verbraucherorganisationen, Schiedsstellen) durch Widerspruch beschweren ("voice", Hirschman, 1974, zit. nach Engel, Flösser \& Gensink, 1996, S. 61).

Solche Beschwerdefälle betreffen im sozialen Dienstleistungsbereich zumeist fachliche 
Entscheidungen, organisatorische Abläufe und persönliches Verhalten von MitarbeiterInnen. Daneben gibt es mitunter politisch motivierte Beschwerden, einfache Unmutsäußerungen sowie formlose und förmliche Rechtsbehelfe, auf die seitens der Einrichtung zu reagieren ist (Gerull, 2004, Kap. 3.3.8).

Was die aktive Reaktion des Kunden anbelangt, schlägt Oppen (1993, zit. nach Engel et al., S. 62) in Abgrenzung von Hirschman vor, die Option des "exit" durch die der "choice" zu ersetzen, mit der ein unzufriedener Kunde den Dienstleistungsanbieter wechselt. Dieses Modell wird von Engel et al. (a.a.O.) für soziale Dienstleistungen für attraktiver gehalten, da für einzelne Arbeitsbereiche die Möglichkeit des Verlassens der Organisation nicht gegeben sei. Die Option der "voice" wird im demokratietheoretischen Sinne als effektive Partizipation und individuelle Interessenbekundung verstanden, die über eine Äußerung von Unzufriedenheit hinausgehe (a.a.O., S. 62). AdressatInnen sozialer Dienstleistungen sollten nicht erst im Falle von Leistungsminderung oder -versagen in den Genuss kommen, mit eigener Stimme für ihre Bedürfnisse einzutreten; vielmehr müsse "voice" zum konstitutiven Bestandteil der Leistungsproduktion selbst werden und sich auf alle Phasen während der gesamten Dauer des Hilfeprozesses erstrecken (a.a.O.). Auch die Option der "choice" beinhalte nicht nur die Möglichkeit des Anbieterwechsels bei bereits erlebtem Organisationsversagen, sondern versetze AdressatInnen in die Lage, Leistungsangebote unter Berücksichtigung ihrer Interessen und Präferenzen frei zu wählen (a.a.O.).

Die allgemeinen Ziele des Beschwerdemanagements liegen darin, Kundenzufriedenheit wiederherzustellen, die negativen Auswirkungen von Kundenunzufriedenheit auf das Unternehmen zu minimieren und die in Beschwerden enthaltenen Hinweise auf betriebliche Schwächen zu identifizieren (Zollondz, 2001, S. 61). Auch bietet ein systematisches Beschwerdemanagement als Teil eines Marketing-Konzepts die Möglichkeit, sich verändernde Wünsche und Bedürfnislagen von z. B. Heimbewohnern wahrzunehmen und für die Weiterentwicklung der Dienstleistung zu nutzen (Titz \& Paratsch, 2001, S. 27).

Um diese Ziele zu erreichen, müssen für unzufriedene Kunden leicht zugängliche Beschwerdekanäle geschaffen werden. Darüber hinaus muss eine sach- und problemgerechte Beschwerdereaktion und -bearbeitung erfolgen sowie eine systematische Auswertung des informatorischen Gehalts von Beschwerden. Die entsprechenden Aufgaben lassen sich wie folgt umreißen (Zollondz, a.a.O., S. 61 ff.):

o Beschwerdestimulierung: Diese Aufgabe knüpft an die vielfach nachgewiesene Beobachtung an, dass es einen hohen Anteil unterbliebener Beschwerden trotz Unzufriedenheit gibt. $\mathrm{Zu}$ den Determinanten des Beschwerdeverhaltens werden u. a. die mate- 
riellen, zeitlichen und psychischen Kosten gezählt, die dem Kunden entstehen, wenn er sich beschwert. Dementsprechend müssen leicht zugängliche Beschwerdewege eingerichtet werden, die dem Kunden in angemessener Form mitzuteilen sind.

o Beschwerdeannahme: Diese Aufgabe umfasst den Erstkontakt mit dem unzufriedenen Kunden und die inhaltliche und formale Dokumentation der Beschwerdeinformation, z. B. mittels Formblättern oder PC-Eingabemasken. Besondere Bedeutung kommt der sozialpsychologisch angemessenen Reaktion auf die vorgebrachte Beschwerde zu.

o Beschwerdebearbeitung und Beschwerdereaktion: Hierbei geht es um die Gestaltung der internen Bearbeitungsprozesse, die Festlegung von Verantwortlichkeiten, die Definition von Bearbeitungsterminen und die Überwachung der Termineinhaltung. Außerdem sind Entscheidungen über Umfang und zeitliche Gestaltung der Kommunikation mit dem unzufriedenen Kunden sowie über die anzubietende Lösung zu treffen. Um Kunden das Erlebnis zu ersparen, von Mitarbeitern, die sich für unzuständig halten, an andere Personen weiterverwiesen zu werden, ist ein "Empowerment" des Kundenkontaktpersonals zweckmäßig (Verlagerung von Entscheidungsrechten und Übertragung autonomer Handlungsspielräume auf Mitarbeiter unterer Hierarchiestufen, vgl. Seidel \& Stauss, 2002, S. 136f).

o Beschwerdeauswertung: Neben einer quantitativen Auswertung des Beschwerdeaufkommens, differenziert nach festzulegenden Merkmalen (z. B. Abteilungen, Problemarten, Kundengruppen), gilt es, durch qualitative Beschwerdeauswertung eine systematische Ursachenanalyse für betriebliche Schwachstellen zu betreiben.

o Beschwerdecontrolling: Im Rahmen des Aufgabencontrollings wird überwacht, inwieweit die zuvor beschriebenen Aufgaben erfüllt werden, z. B. im Hinblick auf termingerechte Bearbeitung. Dazu sollten entsprechende Leistungsindikatoren formuliert werden, die laufend zu überprüfen sind. Beim Kosten-Nutzen-Controlling geht es um die Abschätzung der Auswirkungen eines Beschwerdemanagementsystems, soweit diese sich quantifizieren lassen. 
Als Möglichkeiten für leicht zugängliche Ideen- und Beschwerdewege in der öffentlichen Verwaltung benennt H. Lang (2002):

- zentrale Anlaufstelle zur Beschwerdeannahme,

- zentrales Bürgertelefon (Telefon-Hotline),

- Internet (Beschwerdeformular, besondere mail-Adresse),

- Beschwerdebriefkästen zur schriftlichen Befragung im Foyer oder Sachgebiet,

- Beigabe von Rückmelde-Vordrucken bei schriftlichem Kontakt zum Bürger/Kunden (Meinungskarten),

- $\quad$ persönliche Befragung.

Hinsichtlich der Beschwerdebearbeitung und -reaktion wird eine zentrale Verantwortlichkeit für die Art und Weise empfohlen, wie die Beschwerden bearbeitet werden, während die inhaltliche Bearbeitung dezentral erfolgen sollte (a.a.O.).

Krause (2003, S. 208f) diskutiert folgende methodischen Ansätze des Beschwerdemanagements und berichtet über gute Erfahrungen im Erziehungshilfebereich:

- "Telefonnummer nach draußen" (z. B. vom Träger unabhängige Beratungsstelle),

- Beschwerdegremien (z. B. Sprecherrat),

- öffentliche Sprechstunde (z. B. der Leitung),

- $\quad$ persönliche Befragung (z. B. durch gruppenübergreifend tätige MitarbeiterInnen),

- Befragung per Fragebogen (z. B. anonym in regelmäßigen Abständen).

Wenngleich viele dieser Ansätze gar nicht oder nur selten von Kindern und Jugendlichen genutzt würden, trügen sie dazu bei, ein Klima der Offenheit, Sicherheit und Akzeptanz zu fördern (a.a.O.).

Fast alle AutorInnen betonen, dass ein Beschwerdemanagement nicht primär ein instrumentelles Verfahren sein dürfe, sondern in eine Haltung der Offenheit und Lernbereitschaft eingebettet sein müsse. Beschwerdemanagement lässt sich deshalb nicht auf dem Anordnungswege einführen, sondern bedarf der umfassenden Beteiligung der Mitarbeiterschaft, auch im Sinne eines "Mitarbeiter-Beschwerderechts" über Fehlverhalten von Leitung (Hiller, 2003, S. 198). Partizipation und Umgang mit Beschwerden stehen in einem wechselseitigen Verhältnis zueinander (Hemker, 2003, S. 215). Darüber hinaus bedarf die Installierung eines systematischen Beschwerdemanagements einer Schulung und Beteiligung der MitarbeiterInnen, um eine positive Beschwerdekultur zu fördern, in der nicht unprofessionell auf berechtigte und unberechtigte Beschwerden reagiert wird. 
Titz \& Paratsch (2001, S. 27) geben folgende Beispiele für Reaktionen und Empfindungen von Mitarbeiter(inne)n auf Beschwerden:

o Sich angegriffen fühlen und Verteidigungshaltung einnehmen.

o Rechtfertigung der Situation und dabei die Seite des Beschwerdeführers aus den Augen verlieren.

o Sich persönlich missverstanden fühlen.

o Das Gefühl haben, die Arbeitsleistung werde nicht anerkannt.

o Hilflosigkeit empfinden, die Beschwerden nicht lösen zu können.

o Überforderung und Druck empfinden.

o Den eigenen Ansprüchen nicht gerecht werden.

o Angst vor dem Vorgesetzten.

o Irritiert sein über die Beschwerde: Ich meine es doch nur gut.

o Sich ertappt fühlen, nicht korrekt gearbeitet zu haben.

o Für andere den Kopf hinhalten müssen.

Seidel \& Stauss (2002, S. 136) empfehlen u. a. folgende Reaktionen für den Umgang mit unzufriedenen Kunden:

- Verstehen Sie Beschwerden als einen normalen Teil Ihrer Arbeit und als Chance, Kundenunzufriedenheit abzubauen und Kundenbindung zu sichern.

- $\quad$ Sprechen Sie den Kunden mit Namen an.

- Signalisieren Sie Gesprächsbereitschaft.

- Hören Sie gut zu.

- Wählen Sie eine ruhige und höfliche Gesprächsart.

- Stellen Sie inhaltliche Fragen, bis die Situation geklärt ist.

- Versetzen Sie sich in die Lage des Kunden.

- Leiten Sie sofort die Bearbeitung der Beschwerde ein.

- Bieten Sie eine faire Lösung an. Erkundigen Sie sich, ob der Kunde mit der Regulierung einverstanden ist.

- Sind Sie nicht zuständig oder können Sie nichts tun, leiten Sie die Beschwerde eigenhändig weiter.

- Beenden Sie das Gespräch mit einer positiven Formulierung.

Gerade deshalb, weil Beschwerden für MitarbeiterInnen einen enormen Stressfaktor darstellen, kommt einem Management, das offen mit Beschwerden umgeht und den Umgang damit zuverlässig regelt, entlastende und die Arbeitszufriedenheit fördernde Bedeutung zu. MitarbeiterInnen fühlen sich nicht mit Beschwerden allein gelassen und sehen Verhaltensmöglichkeiten auch dann, wenn sie für die Problemlösung nicht verantwortlich sind (Titz \& Paratsch, 2001, S. 28). 


\subsubsection{Wissensmanagement}

Vorbemerkungen: Daten sind Darstellungen von Zahlen, Mengen, Variablen und Fakten durch Symbole und enthalten keine Aussagen über deren Bedeutung. Sie bilden die Grundlage für Informationen, die dadurch entstehen, dass Daten in einen Kontext gebracht werden. Wissen hingegen wird generiert, wenn Informationen in spezifischen Situationen angewendet und produktiv genutzt werden.

Wissen bezeichnet die Gesamtheit der Kenntnisse und Fähigkeiten, die zur Lösung von Problemen eingesetzt werden können und umfasst theoretische Erkenntnisse und praktische Handlungsanweisungen (Probst, Raub \& Romhardt, 1999).

Produktivität und Innovationsfähigkeit hängen maßgeblich davon ab, wie Wissen im Unternehmen und in den Köpfen der MitarbeiterInnen mobilisiert und genutzt wird. Dies gilt in besonderem Maße für die Sozialwirtschaft, in der sämtliche Dienstleistungen von Menschen für Menschen erbracht werden (Peschke, 2003, S. 31). Durch Fluktuation, Ausscheiden erfahrener MitarbeiterInnen (z. B. durch Frühpensionierung), erhöhte Mobilität und seltener werdende langjährige Bindung an ein Unternehmen geht ständig betriebsrelevantes Wissen verloren.

Wie man Information und Kommunikation so anregen, strukturieren und steuern kann, dass möglichst umfassendes und valides Wissen zur Fundierung organisatorischer Entscheidungen und Handlungen entsteht, ist deshalb zum Kernproblem von Organisationen in einer sich ständig wandelnden Welt geworden. Menschen und Organisationen so mit Informationen zu versorgen und ihre Kommunikation zu unterstützen, dass der Aufbau von Wissen gefördert und rationalisiert wird und zugleich Ursachen für "Informationspathologien" abgebaut werden - das sind Beeinträchtigungen der Information, Kommunikation und Wissensgewinnung, z. B. durch übermäßige Konformität in Entscheidungsgruppen, Beschönigung und Verschleierung -, wird als Wissensmanagement bezeichnet (Scholl, 2004, S. 552f). Als wichtigster Erfolgsfaktor für ein solches Wissensmanagement wird eine geeignete Organisationskultur betrachtet, in der eigenverantwortliches Handeln und Lernen, Risikobereitschaft, Fehlertoleranz und Wissensaustausch ermutigt werden (a.a.O.).

Wissensmanagement hat das Ziel, die erfolgskritische Ressource "Wissen" zur Effizienzsteigerung und Qualitätserhöhung einzusetzen. Zum Konzept der lernenden Organisation und zentralen Anliegen eines TQM besteht somit große Nähe. In Analogie dazu spricht 
North (1999) denn auch vom "Total Knowledge Management". Büssing (2004, S. 594) betont die Verknüpfung von QM und Wissensmanagement, indem er auf typische gemeinsame Fragestellungen hinweist: Wer sind die Wissensträger? Wie gelange ich an das Wissen der Wissensträger? Und wie schöpfe ich aus dem erworbenen Wissen neues Wissen? Dazu wird Bezug genommen auf die geläufige Unterteilung des Wissens in explizites Wissen, das artikulier-, übertrag- und beschreibbar ist (Erkenntnis, "Wissen über") und implizites Wissen (tacit knowledge), das subjektive Fähigkeiten beinhaltet und nur schwer artikulier- und dokumentierbar ist (Erfahrung, "Wissen wie") (Peschke, 2003, S. 32; Scholl, 2004, S. 550 ff.). Durch Überführung impliziten Wissens in explizites wird neues Wissen möglich. Dieser Prozess wird als das zentrale Problem des Wissensmanagements in Organisationen bezeichnet (Wilpert, 2004, S. 654).

Wissensmanagement als systematischer Prozess beginnt mit der Frage, welches Wissen überhaupt geschäftsrelevant ist und von den potenziellen EmpfängerInnen gesucht wird. Im nächsten Schritt müssen für jede relevante Wissenskategorie vorhandene Inhalte identifiziert und bewertet werden: Fakten, Erfahrungen, beste Verfahren und Ideen, aber auch "negatives Wissen" über Fehlschläge und Irrtümer (Simon, 1999, S. 307). Dabei ist die Explizierung von implizitem Wissen, beispielsweise dem Erfahrungswissen langjähriger MitarbeiterInnen, die wichtigste Form des Wissenstransfers. Die Bereitschaft zur Wissensabgabe kann durch Förderung des Qualitätsbewusstseins, intelligente Anreizsysteme und Einbettung des Wissensmanagements in eine allgemeine Kultur von Vertrauen, Transparenz und Partizipation erhöht werden (Peschke, 2003, S. 32f).

Ermittelte Defizite in den vorhandenen Wissensfeldern müssen durch Aufbau und/oder Einkauf entsprechenden Praxiswissens geschlossen werden. Von zunehmender praktischer Bedeutung sind Intranetportale, die sich auch extern beschaffter Informationen bedienen (a.a.O.).

Die Grundidee und zentrale Funktion solcher Managementinformationssysteme (MIS) besteht darin, dem Management die richtigen Informationen zum gewünschten Zeitpunkt zur Verfügung zu stellen, um anstehende Entscheidungen rationaler gestalten zu können (Knorr \& Halfar, 2000, S. 224). Die Affinität des Begriffs zum Konzept des Controllings liegt damit auf der Hand. Maelicke (1998, S. 595) zählt MIS denn auch zu den Bausteinen des operativen Controllings - neben weiteren Modulen wie Basisdatensysteme, Kosten- 
und Leistungsrechnung, Budgetierung und Soll-Ist-Vergleiche.

Im Grunde ist es jedoch Ansichtssache, ob eine solche Differenzierung überhaupt erforderlich ist. Ein substantieller Unterschied zwischen einem MIS und den Aufgaben eines zweckmäßigen Informations- und Berichtswesens herkömmlicher Art besteht allenfalls in der zeitgemäßen Nutzung elektronischer Datenverarbeitungssysteme. Als MIS wird deshalb auch häufig eine spezielle Softwarelösung bezeichnet, die den Führungskräften einer Organisation Zugriff auf alle benötigten Unternehmensdaten erlauben soll (vgl. Knorr \& Halfar, 2000, S. 224).

Kritische Faktoren für die Effektivität und Effizienz eines MIS sind zum einen die Verlässlichkeit, Konsistenz und Dichte der verfügbaren Informationen sowie die Breite, in der relevante Dimensionen der Organisation abgebildet werden (a.a.O.). Zum anderen sind die Intervalle von Bedeutung, in denen Informationen erhoben werden; je nach Art des Unternehmens stehen Aspekte der laufenden Kontrolle von Prozessen oder die systematische Aufbereitung von Informationen aus mehr oder weniger langen Phasen der Geschäftsperiode im Mittelpunkt. Als besondere Stärke eines MIS wird die Möglichkeit betrachtet, mittels geeigneter Instrumente neben Standard-Datenabfragen eine Analyse von Zusammenhängen und Mustern vornehmen zu können und auf diese Weise neues Wissen zu kreieren (a.a.O., S. 225).

Knorr \& Halfar (S. 222-286) beschreiben ein Qualitäts-Managementinformationssystem (Q-MIS), das auf einem anonymisierten datenbankgestützten Betriebsvergleich (Benchmarking) basiert. Im Rahmen dieses Betriebsvergleichs werden Daten für umfangreiche Struktur-, Prozess- und Ergebnisanalysen über alle Teilbereiche der Organisation erhoben, die zunächst im Rahmen von Stärken- und Schwächen- sowie Prozessanalysen untersucht und anschließend im Sinne von Qualität interpretiert werden. Im Ergebnis dieser Interpretationen können dann Aussagen und Vereinbarungen darüber getroffen werden, wie sich die Organisation gegenüber anderen Einrichtungen positionieren möchte und welche Qualitätsstandards in Zukunft bindend sein sollen. Danach können Überlegungen dahingehend angestellt werden, wie die entwickelten Qualitätsstandards im Rahmen einer systematischen Qualitätssicherung überprüft und dokumentiert werden können. Wird dieser Prozess mehrfach durchlaufen oder automatisiert, ist Qualitätsmanagement als Daueraufgabe angelegt (S. 222f). 


\section{Resümee:}

\section{Essentials eines Qualitätsmanagements in sozialen Handlungsfeldern}

Ohne die in den vorausgegangenen Kapiteln geäußerten Kommentare zur Zweckmäßigkeit bestimmter Konzepte, Instrumente und Methoden des QM für soziale Handlungsfelder zu wiederholen, soll im Folgenden versucht werden, Essentials eines sozialwirtschaftlichen QM abzuleiten. Sie sind nicht als methodische Empfehlungen oder gar als Implementierungskonzept zu verstehen, sondern dienen im vorliegenden Zusammenhang dazu, in Abgrenzung von QM in erwerbswirtschaftlichen Profit-Organisationen die grundsätzlichen Besonderheiten eines Managements sozialer Dienstleistungsqualität in den Mittelpunkt zu stellen, bevor im empirischen Teil der Studie der Blickwinkel wieder pragmatisch verengt wird.

Schild (1999, S. 69 ff.) betont, dass QM-Systeme aus der Wirtschaft sich auf die Optimierung betrieblicher Abläufe (namentlich durch Standardisierung), Verbesserung des Kundenbezugs (Kundenzufriedenheit als Mittel der Kundenbindung) und die Erreichung wirtschaftlicher Ziele (Gewinn) auf einem Dienstleistungsmarkt konzentrieren. Damit würden sie der Komplexität sozialer Hilfesysteme nicht gerecht. Weder seien viele Leistungen standardisierbar, noch gehe es um Kundenbindung, noch könnten Marktprinzipien umstandslos auf den Sozialbereich übertragen werden.

Diese Einwände gegen naiven "Technologie-Transfer" sind zutreffend und angesichts der Avancen geschäftstüchtiger QM-Vermarkter (Gerull, 2001, S. 7f) allzu notwendig. Ein Qualitätsmanagement allerdings, das den Besonderheiten von sozialwirtschaftlichen (Nonprofit-)Organisationen angemessen ist, kann auch soziale Einrichtungen und Dienste in ihrem Qualifizierungsprozess voranbringen. Jedenfalls sprechen dafür die Erfahrungen von Trägern, die sich mit systematischen Ansätzen der Qualitätsverbesserung auf den Weg begeben haben (vgl. Straus, 1998; Gerull, 2000).

Dass aufgrund solcher Selbstreporte nicht beurteilt werden kann, ob sich positive Effekte dabei oder dadurch eingestellt haben, mithin die Wirkung bestimmten Qualitätskonzepten, der methodisch geschickten Implementierung und/oder unspezifischen Prozessvariablen zuzurechnen ist (ein Aspekt der internen Validität), sei ausdrücklich betont. Der größte 
Teil der Qualitätsdiskussion spielt sich nicht auf solider Forschungsbasis ab, sondern auf der Ebene programmatischer Behauptungen, anekdotischer Fallberichte und empirischer Schlicht-Designs (Evaluationen vom Typ "vorher-nachher", vgl. Hackman \& Wageman, 2000, S. 45).

Dies kann allerdings nicht einer Praxis angelastet werden, deren Aufgabe darin besteht, ihre Arbeit angesichts neuer organisationaler Herausforderungen zu professionalisieren und/oder besser darzustellen, nicht aber zugleich auch den "Kausalbeweis" zu liefern, dass die dabei verwendeten Methoden verfügbaren anderen unter äquivalenten (Ceteris-paribusdistributionibus-)Bedingungen (vgl. Hager, 2004, S. 25) überlegen sind. M. a. W.: Im außerwissenschaftlichen Kontext muss es genügen, dass eine Organisation relevante eigene Kriterien erfüllt, ihre Bemühungen um Verbesserung oder Sicherung von Qualität erfolgreich zu gestalten. Eine andere Frage ist allerdings, ob z. B. eine kritische Öffentlichkeit diese Kriterien zu teilen gewillt ist.

Diese Bemerkung verweist darauf, dass eine Besonderheit sozialwirtschaftlicher Organisationen darin besteht, es mit mehreren Anspruchsgruppen zu tun zu haben, deren teils sehr unterschiedliche Erwartungen sie "bedienen" sollen (vgl. Kap. A 1.). Dabei können sich völlig differente Bewertungen ergeben; denn nur wenige Qualitätsmerkmale lassen sich eindeutig ("objektiv") messen und sind zwischen den Anspruchsgruppen unumstritten. Da sich zudem deren Bedürfnisse dynamisch entwickeln, ändern sich auch die Ansprüche an Qualität und machen neue "Aushandlungsprozesse" erforderlich.

Auch in einem sozialwirtschaftlichen Qualitätsmanagement geht es somit nicht nur darum, Fehler bei der Leistungserstellung zu vermeiden oder schlechte Produkte zu identifizieren und nachzubessern - was bei Dienstleistungen wegen des obwaltenden Uno-Actu-Prinzips ("Produktion" und "Konsumtion" fallen raumzeitlich zusammen, vgl. Groß, 1983) ohnehin kaum möglich ist. Es geht auch um die rechtzeitige Anpassung der Leistungseigenschaften an sich wandelnde Anforderungen des "Marktes" bzw. der maßgeblichen Anspruchsgruppen, vor allem der externen KundInnen (LeistungsempfängerInnen und Kostenträger).

Das in Kap. B 1.1 erwähnte unternehmerische Spannungsviereck aus Qualität, Kosten, Quantität und Zeit (Seghezzi, 2003, S. 20) lässt sich - mutatis mutandis - auf Anbieter des 
immateriellen Produkts "soziale Dienstleistungen" übertragen. Auch dort gilt es, überzeugende Leistungen $\mathrm{zu}$ branchenüblichen Entgelten in erforderlicher Menge innerhalb vertretbarer Zeiträume realisieren zu können. M. a. W.: Die Einrichtungen und Dienste müssen bedarfsgerechte Angebote mit erwartbarem Nutzen ( $\rightarrow$ Qualität) zu angemessenem Aufwand ( $\rightarrow$ Kosten) in hinreichender Menge ( $\rightarrow$ Quantität) bereitstellen und innerhalb akzeptabler Fristen $(\rightarrow$ Zeit) auf Leistungsanfragen reagieren können (oder ggf. Mithilfe bei der Suche nach einer Alternative leisten - eine besondere Form von Servicequalität).

Beim Aufbau eines betrieblichen Qualitätsmanagements in sozialen Einrichtungen kann die Orientierung an einem der in Kap. C 1.1 erwähnten oder dargestellten Konzepte hilfreich sein, um wichtige Bereiche zu identifizieren, in denen eine Organisation aktiv werden und bleiben muss. Aber wenn auch in diese Konzepte umfangreiches Organisationswissen aus vielen Branchen eingeflossen ist, so geben sie doch nur Hinweise auf Anforderungen, denen sich die Einrichtungen und Dienste zu stellen haben. Die Art und Weise der Umsetzung wird aufgrund des branchenübergreifenden Geltungsbereichs vieler Systeme stets der einzelnen Organisation überlassen, die auch entscheiden muss, welche Bestandteile überhaupt für die eigene Leistungstätigkeit relevant sind. Allen QMSystemen mit allgemeinem Gültigkeitsanspruch ermangelt es zudem an konkreten Hinweisen darauf, wie die Qualität entwickelt werden kann.

Die ISO 9001 etwa fordert zwar Verfahrens-, Arbeits- und Prüfanweisungen für alle als qualitätskritisch erkannten Teilprozesse, liefert aber kein fachliches Know-how dazu; das EFQM-Modell erlaubt eine Bewertung der bereits erreichten Qualität und markiert Stärken und Schwachstellen des Betriebs, enthält aber keine methodischen Hilfen zur Verbesserung.

Die ISO betonte schon in ihren über die früheren Normen 9001-9003 hinausgehenden Empfehlungen zum Qualitätsmanagement (ISO 9004/2:1994), dass die Anwendbarkeit bei zunehmender Prozesskomplexität beschränkt sei (wie sie gerade für soziale Dienstleistungen kennzeichnend ist):

\footnotetext{
"Je genauer ein Prozess entweder durch Mechanisierung oder durch detaillierte Verfahren festgelegt werden kann, desto größer ist die Möglichkeit, strukturierte und disziplinierte Grundsätze des Qualitätssicherungssystems anzuwenden" (Deutsches Institut für Normung, 1992, S. 11).
} 
Zwar gibt es auch bei sehr kundenindividuellen Dienstleistungen (z. B. im Pflegebereich) stets einen Anteil immer wiederkehrender, also standardisierbarer Teilprozesse (z. B. Aufnahmevorbereitung, Dokumentation), deren systematische Planung $\mathrm{zu}$ einer professionelleren Leistungserbringung insgesamt beitragen kann. Allgemein gilt jedoch für den Dienstleistungsbereich, dass sich hohe Qualität nicht allein durch Anwendung systematischer Ansätze zur Prozesssteuerung erreichen lässt, sondern der Kombination mit einer Qualitätskultur bedarf (Eversheim, 1997, S. 15). Für den Bereich sozialer Dienstleistungen ist daher nur ein QM-Ansatz angemessen, der die herausragende Bedeutung einer normativen, auf Leitungsebene vorgelebten Qualitätskultur und der Mitarbeiterschaft als entscheidende Ressource im Leistungsprozess berücksichtigt.

Bereits in Kap. B 3.6 war Zollondz (2002, S. 352) zitiert worden, der Menschen und Organisationen im Sinne der Kybernetik als eher schlecht-definierte, nicht-triviale Systeme betrachtet. Solche Systeme seien einerseits instabil und ungewiss, andererseits neigten sie dazu, Vorurteile zu bilden und hartnäckig daran festzuhalten (a.a.O.). Demzufolge wäre QM das vermeintlich ideale Managementwerkzeug, um die zahllosen Fehlerquellen zu eliminieren und potenzielle Organisationszustände durch Regeln festzulegen (S. 353). Seit Taylor's Zeiten sei es Absicht der Qualitätsexperten gewesen, solche rationalen Systeme zu schaffen, um den irrtumsanfälligen Menschen so weit wie möglich in die Klammern und Leitplanken eines QM einzubinden und nichts dem Zufall zu überlassen (a.a.O.). Zollondz (S. 353 ff.) konstatiert jedoch, dass diese Ansätze nicht funktionieren und plädiert für eine Balance zwischen wohl- und schlechtdefinierten Zuständen im Sinne von Flexibilität und organisationalem Lernen.

Ähnlich äußert sich Klatetzki (1998, S. 65 ff.): Interpretationsvielfalt, Verfügbarkeit unterschiedlicher Wissensbestände und ein mittleres Maß an Dissens werden als Qualitätsmerkmale von Organisationen betrachtet, die sich als organische Systeme verstehen und im Unterschied zu mechanischen Systemen durch eine hohe Komplexität, geringe Formalisierung und Zentralisierung sowie durch einen netzwerkartigen Aufbau statt hierarchischer Anordnung charakterisiert werden.

Für die praktische Arbeit wichtiger als die Wahl eines mehr oder weniger formalen QMBezugssystems ist die Befassung mit den inhaltlich-fachlichen Fragen eines arbeitsfeldspezifischen Qualitätsmanagements. 


\section{Kernfragen praktischer Qualitätsentwicklung}

- Welche Ziele gilt es für welche Zielgruppe zu verfolgen?

- $\quad$ Welche Mittel und Wege sind erforderlich und zielführend?

- An welchen Qualitätskriterien sollen die Ziele bemessen werden?

- Welche Indikatoren zeigen an, ob ein Kriterium erfüllt ist?

- Welche Prüfinstrumente erlauben, die Indikatoren $\mathrm{zu}$ erfassen und Qualitätsaussagen über die geleistete Arbeit zu treffen?

- Welche Verbesserungsmaßnahmen sollen daraus abgeleitet und umgesetzt werden?

- Wie können einrichtungsübergreifende Effekte zur besseren Vernetzung und Optimierung der Infrastruktur erzielt werden.

Qualitätsmanagement versucht, diese und andere im Sozialbereich schon bislang unternommenen und weiterzuentwickelnden Bemühungen um Qualität in einen systematischen Zusammenhang zu bringen. Über eine "qualitätsstimmige" Gestaltung betrieblicher Einflussfaktoren und wichtiger Schnittstellen zur Umwelt soll letztlich die fachliche Qualität im Sinne guter Arbeit (aus Sicht der AnbieterInnen) und guter Dienste (aus Sicht der NutzerInnen) optimiert werden.

Ungeachtet der Zweckmäßigkeit eines Vorgehens, in einer suboptimal strukturierten Organisation durch z. B. ISO-orientierte Prozessbeschreibungen, Verfahrens- und Arbeitsanweisungen die Funktionalität betrieblicher Abläufe zum Nutzen der internen und externen KundInnen zu verbessern, muss dem klassischen Kontroll-Ansatz eines QM und dessen Vorliebe für "beherrschte Geschäftsprozesse" und "minimierte Varianz" für soziale Dienstleistungen attestiert werden, nicht geeignet zu sein, Qualität zu gewährleisten.

"Public organizations demonstrate to private organizations that in situations, where goals are ambiguous, technologies uncertain, and environments complex and dynamic, quality methods are unlikely to be very useful" (Kaboolian, 2000, S. 132).

Weick (2000) weist in einer Kritik an der Sprache des TQM darauf hin, dass neben den Unzulänglichkeiten der Begriffe "total" und "quality" - Qualität könne z. B. niemals total sein (a.a.O., S. 156) -, auch "Management" keineswegs ein neutraler Deskriptor sei, son- 
dern vielmehr den Glauben impliziere, Fortschritt sei durch Perfektionierung von Strukturen zur Verhaltenskontrolle möglich (S. 157). Ein Qualitätsmanagement, das auf Kontrolle fokussiere, schenke dem Organisieren von Lernen, Führung und Widerstandsfähigkeit (resilience) jedoch zu wenig Aufmerksamkeit (a.a.O.).

Klaus (1991, S. 256) resümiert nach einer Differenzierung verschiedener Dienstleistungsarten: "Die Anwendung von Instrumenten und Theorien zum Qualitätsmanagement, die in der Welt 'trivialer Maschinen' bei industrialisierbaren Dienstleistungen hervorragende Erfolge bringen mögen, wird im Bereich zwischenmenschlich-interaktiver Dienstleistungen nicht funktionieren."

B. Müller (2000, S. 137f) spricht von QM als Kampf gegen die "dumme Organisation" und betont an anderer Stelle die unterschiedlichen Voraussetzungen eines QM in der Industrie und in sozialen Diensten:

\footnotetext{
"In der Industrie setzen ... die fortgeschrittenen Formen des Qualitätsmanagements bei dem Problem an, wie rational durchkonstruierte, klug geplante Organisationsformen sich durch Elemente der Eigenverantwortung der Mitarbeiter steigern lassen - statt in Kauf $\mathrm{zu}$ nehmen, dass die Mitarbeiter wegen der perfekt rationalisierten Organisation verblöden. In den sozialen Diensten besteht das umgekehrte Problem, wie für immer schon ihrer Eigenverantwortung überlassene MitarbeiterInnen Organisationsformen gefunden werden können, die halbwegs rational sind, d. h. diese Eigenverantwortung an die Organisationsverantwortung anzukoppeln, statt die Arbeit der Einzelnen im Ergebnis schlechter aussehen zu lassen, als sie eigentlich ist, weil sie in einer 'dummen Organisation' stattfindet" (B. Müller, 1998, S. 49).
}

Rudolf (2003, S. 385 ff.) beschreibt am Beispiel der Altenhilfe die charakteristischen Anforderungsmerkmale dieser Form der "Interaktionsarbeit": dialogisch-explorative Vorgehensweise, assoziatives und erlebnisbezogenes Denken, ganzheitliche Wahrnehmung und persönliche Nähe. Qualität in sozialer und pädagogischer Arbeit wird deshalb nicht als eine Frage der präzisen Umsetzung klar dokumentierter Handlungsanweisungen bzw. der Einhaltung vorgegebener Standards betrachtet, sondern als Frage der möglichst situationsangemessenen, kreativen Gestaltung offener, das heißt immer wieder sich wandelnder Situationen (S. 385). Bezug nehmend auf den Koproduktionscharakter sozialer Dienstleistungen wird deutlich gemacht, dass sich ein mechanistisch orientiertes Arbeitsmodell und ein darauf beruhendes QM mit starren Prozessbeschreibungen schwer mit professioneller Arbeit vereinbaren lasse (S. 386). Dementsprechend rückt bei Rudolf nicht der Kontroll-, sondern 
der Verantwortungsaspekt einer Qualitätssicherung in den Vordergrund (S. 388). Wolle man die Qualität sozialer Dienstleistungen verbessern, so führe der Weg über die MitarbeiterInnen und die Entwicklung ihrer Fähigkeiten (S. 386).

Auch die bereits in den Ausführungen zum Empowerment-Konzept (s. Kap. B 3.6) verdeutlichten Unterschiede zwischen sicheren und riskanten Tätigkeiten sind hier zu berücksichtigen. Sutcliffe, Sitkin \& Browning (2000, S. 319f) differenzieren analog zwischen sicheren, analysierbaren, routinehaften und vorhersehbaren Situationen einerseits und deren Gegenteil; sie sprechen denn auch an anderer Stelle von Total Quality Control und Total Quality Learning als jeweils angemessenen Strategien des TQM (Sitkin, Sutcliffe \& Schroeder, 1994).

Jedes Qualitäts- und QM-Konzept modelliert die berufliche Wirklichkeit in spezifischer Weise und reduziert damit die vorhandene Komplexität. Was vom gewählten Modell nicht abgebildet wird oder werden kann, ist aber dessen ungeachtet wirksam. So wie zwischenmenschliche Kommunikation nur teilweise durch (bewusste) verbale und vokale Signale, sondern mindestens gleichermaßen durch (unbewusste) Körpersprache gesteuert wird, so ist auch betriebliches Geschehen mehr als das, was sich mittels Verfahrensanweisungen und definierter Handlungsspielräume oder in den Kategorien von "Struktur", "Prozess" und "Ergebnis" darstellen lässt.

Augenmaß, Bescheidenheit, Bodenhaftung, Erdung, Kontinuität, Kleinschrittigkeit - diese und andere Begriffe werden deshalb von unterschiedlichen AutorInnen und BerichterstatterInnen mal mehr, mal weniger dezidiert verwendet, um der falschen Erwartungshaltung zu begegnen, mit Qualitätsmanagement seien die Widrigkeiten des betrieblichen Alltags wundersam aus der Welt zu schaffen (vgl. z. B. Kämmer et al., 2001, S. 15; Zollondz, 2002, S. 353 ff.; Gerull, 2001, S. 12).

Jenseits aller "faddish elements" (Dean \& Bowen, 2000, S. 4) und überzogenen Verheißungen ist Qualitätsmanagement jedoch eine seriöse Art und Weise, sich "ewiger" Fragen der Organisationspraxis anzunehmen:

- Wie macht eine Organisation ihre Arbeit gut (d. h., wie werden in Abstimmung mit relevanten Anspruchsgruppen Kriterien für Fachlichkeit, Nutzen und Qualität definiert)?

- Wie können alle MitarbeiterInnen in die Lage versetzt werden, ihre Arbeit gut zu 
machen (d. h., wie kann die Organisation anforderungsgerechte Strukturen, Informationen, Fähigkeiten, Lerngelegenheiten usw. gewährleisten)?

- Wie kann erreicht werden, dass alle MitarbeiterInnen verantwortungsvoll und qualitätsorientiert handeln (d. h., wie schafft die Organisation günstige Rahmenbedingungen für Motivation, Commitment, Zuverlässigkeit, Verantwortungsbereitschaft usw.)?

- Wie kann vorgesorgt werden, dass die Organisation merkt, wenn eines oder mehrere dieser Ziele verfehlt werden (d. h., wie können Formen der Selbststeuerung, Fremdkontrolle und Rechenschaftslegung sinnvoll miteinander verknüpft werden)?

Das damit skizzierte Arbeitspensum ist auf Dauer angelegt und kann im Rahmen von Projekten allenfalls schwerpunktmäßig angegangen werden. Einen solchen Schwerpunkt stellt für viele Einrichtungen der Aspekt des organisationalen Lernens dar:

"Strukturiertes Qualitätsmanagement lässt sich in diesen Kontext einer systematischen Reflexionsförderung in Organisationen einordnen. Qualitätsmanagement trägt dazu bei, die Selbststeuerungspotenziale von Organisationen zu verbessern, indem Möglichkeiten der strukturierten Selbstbeobachtung eröffnet werden" (Merchel, 2001, S. 118).

Stark (2000, S. 265) hebt hervor: "Qualitätsmanagement darf nicht bei der aktuellen Konzeption und dem vorhandenen Methodenkanon stehen bleiben. Ein modernes Qualitätsmanagement lernt selbstreferentiell und nutzt den kontinuierlichen und beteiligungsorientierten Verbesserungsprozeß wiederum zur Weiterentwicklung von Konzept und Methoden von Qualitätsmanagement."

Ob diese Bemühungen auch zukünftig "Qualitätsmanagement" oder gar "TQM" genannt werden, ist keineswegs ausgemacht, allerdings auch irrelevant (vgl. Kap. E). 


\section{Praxis des Qualitätsmanagements in der Jugend- und Sozialhilfe - Empirische Befunde zur Umsetzung, Akzeptanz und Nutzenbewertung}

\section{Befunde aus Mitarbeiterbefragungen}

\subsection{Vorbemerkungen}

Auf die Beschränkungen der nachfolgend erörterten Untersuchungen (Vorstudien) wurde bereits in Kap. A 5.3.7 hingewiesen. Die Angaben zu Methodik und Stichproben sind deshalb auf das Nötigste reduziert. Im Ergebnisteil sind die numerischen Daten tabellarisch aufbereitet und bedarfsweise verbal erläutert; ihre Zusammenfassung und Diskussion erfolgt anschließend in gemeinsamer Darstellung. Angesichts partiell eingeschränkter Vergleichbarkeit der jeweiligen Untersuchungsbedingungen und zur Vermeidung von Überkomplexität sind überdies nur die eindeutigsten Befunde betont und interpretiert.

\subsection{Einstellung zum Qualitätsmanagement}

\subsubsection{Angaben zur Methodik und Stichprobe}

Die Daten entstammen breit angelegten Mitarbeiterbefragungen in den Einrichtungen A (1998), B (1997) und C (2003); die Reihenfolge der Ergebnisdarstellung richtet sich dabei nicht nach der Chronologie der Untersuchungen, sondern nach der Größe der Einrichtungen (operationalisiert als Zahl der Beschäftigten).

Items zum Qualitätsmanagement waren als Zusatzfragen gekennzeichnet, um sie von den übrigen, stärker betriebs- und arbeitsplatzbezogenen Fragen abzuheben. Im Vordergrund stand nicht die Konstruktion einer homogenen Skala zur Messung des Merkmals "Einstellung zum Qualitätsmanagement", sondern die Meinungsabfrage zu verschiedenen Facetten der Qualitätsdiskussion: grundsätzliche Haltung, Bereitschaft zu aktiver Mitarbeit, Ausmaß vorhandener Skepsis und Zuschreibung von erwarteten Wirkungen. 
seinerzeit irrelevant und wurde nicht überprüft (z. B. über Cronbach's $\alpha$ ). Statt einer mehrstufigen Skala wurden die Antwortkategorien "stimme zu/stimme nicht zu/weiß nicht" vorgegeben.

Die drei untersuchten Stichproben seien kurz charakterisiert:

- $\mathrm{A}=$ sehr große dezentrale Einrichtung der Jugendhilfe (216 MitarbeiterInnen), Befragungszeitpunkt Januar/Februar 1998, Rücklaufquote 86,1\% ( $=186)$;

- $\mathrm{B}=$ große dezentrale Einrichtung der Jugendhilfe (80 MitarbeiterInnen $)$, Befragungszeitpunkt April 1997, Rücklaufquote 81,2\% ( $\mathrm{n}=65)$;

○ $\mathrm{C}=$ mittelgroße, überwiegend stationäre Einrichtung der Jugendhilfe (44 MitarbeiterInnen), Befragungszeitpunkt Februar 2003, Rücklaufquote 95,3\% ( $\mathrm{n}=42)$.

\subsubsection{Ergebnisse}

Im Folgenden sind die absoluten Antwortverteilungen zu vorgegebenen Aussagen zum Qualitätsmanagement pro befragter Einrichtung aufgeführt sowie die relative Gesamtverteilung über alle drei Einrichtungen (in \%). Weitere Kennwerte der deskriptiven Statistik wurden des rein betrieblichen Verwendungskontextes wegen nicht berechnet; die Rohdaten liegen nicht mehr vor. Bei B sind wegen teils abweichender ItemFormulierungen einige Daten nicht aufgenommen (keine Angabe: k. A.).

Tab. 1: Bewertung von Aussagen zum Qualitätsmanagement durch Fachkräfte der Jugendhilfe

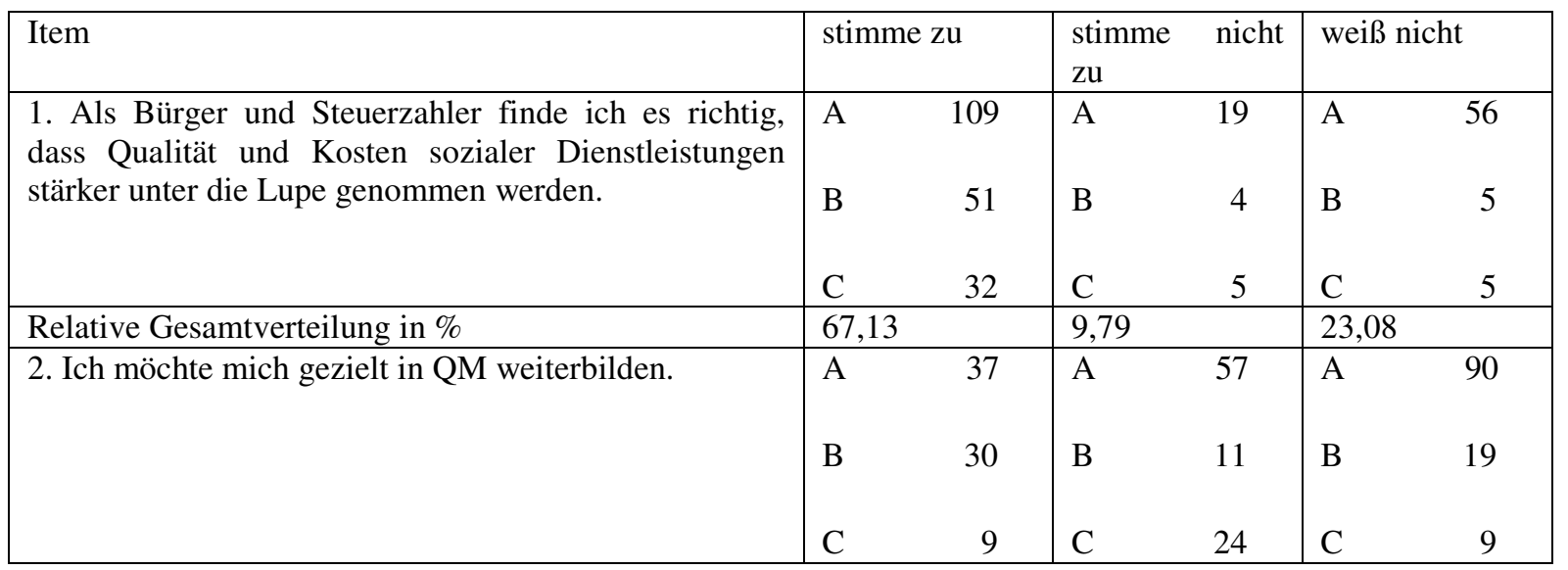




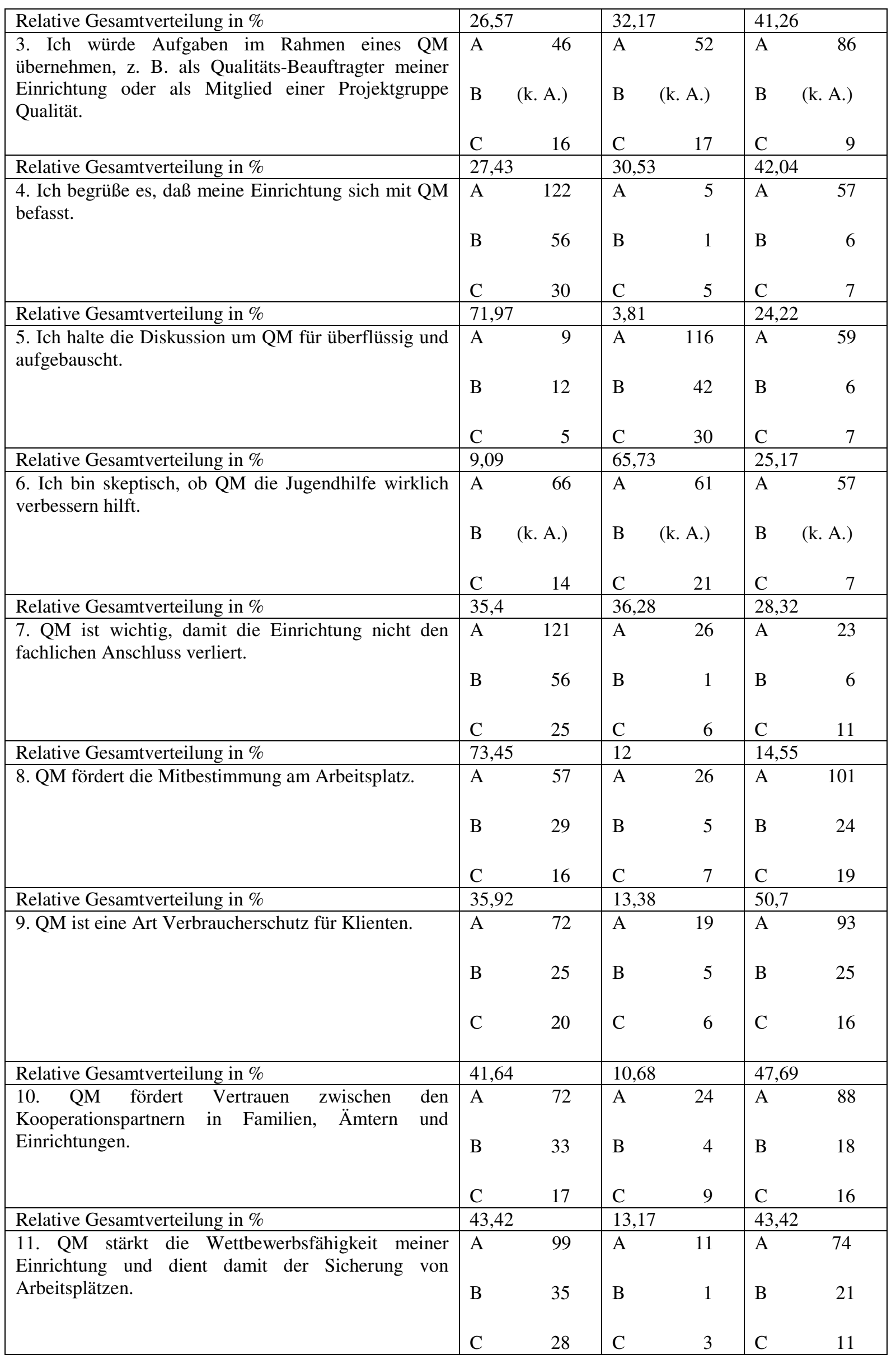




\begin{tabular}{|c|c|c|c|c|c|c|}
\hline Relative Gesamtverteilung in \% & 57,24 & & 5,3 & & 37,46 & \\
\hline \multirow{3}{*}{$\begin{array}{l}\text { 12. QM fördert das berufliche Selbstbewußtsein der } \\
\text { MitarbeiterInnen. }\end{array}$} & A & 61 & A & 30 & A & 93 \\
\hline & $\mathrm{B}$ & 29 & $\mathrm{~B}$ & 4 & $\mathrm{~B}$ & 25 \\
\hline & $\mathrm{C}$ & 21 & $\mathrm{C}$ & 9 & $\mathrm{C}$ & 12 \\
\hline Relative Gesamtverteilung in \% & 39,08 & & 15,14 & & 45,77 & \\
\hline \multirow{3}{*}{$\begin{array}{l}\text { 13. QM hilft, die Arbeitsbedingungen, -abläufe und - } \\
\text { ergebnisse zu verbessern. }\end{array}$} & A & 94 & A & 10 & A & 80 \\
\hline & B & 41 & B & 2 & B & 16 \\
\hline & $\mathrm{C}$ & 30 & $\mathrm{C}$ & 3 & $\mathrm{C}$ & 9 \\
\hline Relative Gesamtverteilung in \% & 57,89 & & 5,26 & & 36,84 & \\
\hline \multirow{3}{*}{$\begin{array}{l}\text { 14. QM unterstützt mich dabei, meine Arbeit fachlich } \\
\text { gut zu leisten. }\end{array}$} & A & 65 & A & 18 & A & 101 \\
\hline & $\mathrm{B}$ & 30 & $\mathrm{~B}$ & 1 & B & 27 \\
\hline & $\mathrm{C}$ & 19 & $\mathrm{C}$ & 10 & $\mathrm{C}$ & 13 \\
\hline Relative Gesamtverteilung in \% & 40,14 & & 10,21 & & 49,65 & \\
\hline \multirow[t]{3}{*}{ 15. QM fördert die Arbeitszufriedenheit. } & A & 53 & A & 21 & A & 110 \\
\hline & $\mathrm{B}$ & 24 & $\mathrm{~B}$ & 3 & $\mathrm{~B}$ & 31 \\
\hline & $\mathrm{C}$ & 21 & $\mathrm{C}$ & 9 & $\mathrm{C}$ & 12 \\
\hline Relative Gesamtverteilung in \% & 34,51 & & 11,62 & & 53,87 & \\
\hline \multirow{3}{*}{$\begin{array}{l}\text { 16. QM fördert die fachliche Gründlichkeit in der } \\
\text { Kinder- und Jugendhilfe. }\end{array}$} & A & 79 & A & 16 & A & 89 \\
\hline & $\mathrm{B}$ & 43 & B & 2 & $\mathrm{~B}$ & 16 \\
\hline & $\mathrm{C}$ & 23 & $\mathrm{C}$ & 7 & $\mathrm{C}$ & 12 \\
\hline Relative Gesamtverteilung in \% & 50,52 & & 8,71 & & 40,77 & \\
\hline Relative Gesamtverteilung über alle Items in \% & 48,06 & & 14,14 & & 37,80 & \\
\hline
\end{tabular}

\subsubsection{Zusammenfassung und Diskussion der Ergebnisse:}

○ Die Einstellung zum Qualitätsmanagement ist mit einem durchschnittlichen Anteil (= relative Gesamtverteilung über alle Items) von rund $48 \%$ zustimmender Antworten (darunter zwei Items mit negativer Polung: Nrn. 5 und 6) auf den ersten Blick zwiespältig: Nur jeder zweite Proband betont die möglichen Vorteile.

- In diesen durchschnittlichen Grad allgemeiner Zustimmung geht allerdings auch die relativ geringe Bereitschaft ein, sich aktiv in Qualitätsmanagement weiterzubilden oder entsprechende Aufgaben in der Einrichtung selbst wahrzunehmen (Items 2 und 3, jeweils rund 27\%). Bei Reduktion der Gesamt-Itemzahl um diese zwei auf 14 resultiert 
eine mittlere Zustimmung von rund 51\%.

Der Anteil nicht zustimmender Antworten an der relativen Gesamtverteilung (darunter zwei Items mit positiver Polung: Nrn. 5 und 6) fällt mit durchschnittlich rund 14\% deutlich geringer aus; entsprechend groß ist mit rund 38\% der Anteil unentschiedener Antworten an der Gesamtverteilung.

- Insgesamt kann konstatiert werden, dass unter den MitarbeiterInnen, die sich in der Lage sahen, eine zustimmende oder nicht zustimmende Bewertung zum Qualitätsmanagement abzugeben, eine positive Einstellung im Verhältnis von etwa 3,5 zu 1 die ablehnende Haltung überwiegt; der Anteil Unentschiedener ist jedoch sehr hoch - teilweise über $50 \%$ - und sinkt bei keinem Item unter $23 \%$.

- Die Feinanalyse macht deutlich, dass die insgesamt positivsten Bewertungen zu jenen Items abgegeben werden, die sich auf die eher allgemeine Einstellung zur Qualitätsdiskussion (Item 1: ca. 67\% Zustimmung) und die Befassung mit Qualitätsmanagement auf Einrichtungsebene beziehen (Items 4, 7 und 11: ca. 72\% bzw. 73\% und 57\% Zustimmung), ohne die persönliche Eigenbeteiligung (Items 2 und 3) oder konkrete bzw. programmatisch behauptete Auswirkungen von Qualitätsmanagement anzusprechen (alle übrigen Items). Letztere Items weisen einen besonders hohen Anteil Unentschiedener auf - man könnte in diesem Fall auch sagen: skeptisch Abwartender.

- Allerdings zeigen sich zwischen den Einrichtungen deutliche Unterschiede. Bei nahezu allen 14 Items, zu denen komplette Datensätze vorliegen (alle außer Items 3 und 6), weichen die beobachteten Häufigkeitsverteilungen hochsignifikant von jenen Werten ab, die bei Annahme einer gemeinsamen Population zu erwarten wären. Dies spricht für die Bedeutung von Kontextvariablen, wie sie z. B. in Form unterschiedlicher einrichtungshistorischer Ausgangssituationen in der Befassung mit dem Thema trotz einheitlicher Rechtslage (alle Einrichtungen liegen im Geltungsbereich des nordrheinwestfälischen Rahmenvertrages nach $\S 78$ f SGB VIII) zum Ausdruck kommen.

Im vorliegenden Befragungskontext erweisen sich die augenfälligsten Bewertungsunterschiede durchaus als schlüssig interpretierbar: 
Der Anteil Unentschiedener geht bei allen Items überwiegend auf das Konto der größten Einrichtung A. Hier war die Mitarbeiterbefragung (Anfang 1998) quasi die erste konkrete Begegnung mit dem Thema Qualitätsmanagement nach einem Zusammenschluss bis dato unabhängiger Einzeleinrichtungen $\mathrm{zu}$ einem Organisationsverbund und traf auf eine eher skeptisch abwartende Belegschaft, was den Nutzeffekt dieser Aktion anbelangte.

Die seinerzeit durchgeführte Analyse zur Überprüfung möglicher Einflüsse der Beschäftigtengruppe ergab im Übrigen, dass bei insgesamt recht homogener Antwortverteilung die Gruppe der Leitungs- und Beratungskräfte die höchste Bereitschaft zur Weiterbildung in Qualitätsmanagement und zur Übernahme entsprechender Funktionen aufwies. Die Antwortverteilungen für diese Bereitschaft lagen bei den MitarbeiterInnen des ambulanten Bereichs signifikant $(\mathrm{p} \leq .05)$ niedriger, noch mit jeweils deutlichem Abstand gefolgt vom stationären, teilstationären sowie hauswirtschaftlich-technischen und Verwaltungsbereich $(\mathrm{p} \leq .01)$.

In der 1997 befragten Einrichtung B ist der Anteil Unentschiedener erheblich niedriger als in A und sogar etwas (insgesamt nicht signifikant) niedriger als in C, obwohl die Untersuchung ein knappes Jahr bzw. sechs Jahre früher erfolgte und somit nicht auf einen allgemeinen Faktor "Bekanntheit des Themas in der Fachöffentlichkeit" rekurriert werden kann. Vielmehr dürfte auch hier der jeweilige Einrichtungskontext verantwortlich gewesen sein, konkret die Pionierrolle der seinerzeitigen Heimleitung, die das Thema nicht erst im Zusammenhang mit der Befragung betriebsintern vorantrieb.

- Auch die Bereitschaft der MitarbeiterInnen, sich - ganz im Sinne der Leitungserwartung - weiterzubilden, ist in B vergleichsweise hoch: 30 von 60 BefragungsteilnehmerInnen $=50 \%$ gegenüber $20 \%$ in $\mathrm{A}$ und $21,4 \%$ in $\mathrm{C}$ (Chi-Quadrat 35,61; df $=4 ; \mathrm{p} \leq .01)$. Dies gilt allerdings auch für den Anteil jener, welche die Diskussion um QM für überflüssig und aufgebauscht halten: $20 \%$ gegenüber $4,9 \%$ in A und $11,9 \%$ in $\mathrm{C}$ (Chi-Quadrat 22,53; df $=4 ; \mathrm{p} \leq .01$ ). Dabei handelt es sich möglicherweise um einen Polarisierungseffekt aufgrund der forcierten innerbetrieblichen Schwerpunktsetzung durch die Leitung. 
- Die positivste Einstellung insgesamt und speziell zu den intendierten Auswirkungen von Qualitätsmanagement auf fachlicher Ebene (z. B. Items 7, 10 und 16) wird somit in Einrichtung $\mathrm{B}$ abgegeben, also zum frühesten Befragungszeitpunkt der Untersuchungsreihe (1997). Mögliche Gründe dürften im zuvor erörterten Kontext zu suchen sein. Auf die besondere Situation der Einrichtung A wurde bereits hingewiesen.

- Was die Belegschaft in Einrichtung $\mathrm{C}$ anbelangt, so hatte diese zum Befragungszeitpunkt 2003 im Vergleich zu A und B ein halbes Jahrzehnt länger die Qualitätsdiskussion und die Umsetzung erster Maßnahmen und Vereinbarungen zum Qualitätsmanagement verfolgen können. Dass dies nicht mit einem höheren Anteil zustimmender Antworten (= positive Einstellung zum QM) einhergeht, ist ohne Kenntnis des betrieblichen Kontextes nicht interpretierbar.

Es handelte sich um eine Auftrags-Auswertung der Daten, die mit einem Mitarbeiter-Fragebogen des Autors (MFEE) erhoben wurden; die Einrichtung sowie die näheren Umstände der Befragung waren nicht bekannt.

Allerdings befand sich die Qualitätsdiskussion in der Jugendhilfe um 1998 auf dem bislang relativen Höhepunkt, wenn man die Zahl der einschlägigen Veröffentlichungen (s. Abb. 1), Tagungen und Arbeitsgruppen zugrunde legt. Die wohlwollende, jedoch keineswegs einhellig positive Haltung der MitarbeiterInnen aus Einrichtung C in 2003 dürfte ein Hinweis darauf sein, dass die seitherige Umsetzungsphase des Qualitätsmanagements vielfach (noch) nicht das erbracht hat, was ihre Propagandisten sich einst erhofften, allerdings wohl doch mehr, als nur die von vielen befürchtete "heiße Luft" (Hoerner \& Vitinius, 1997). 


\subsection{Vorkehrungen zur Entlastung und Verbesserung der Arbeit / Maßnahmen zur Qualitätsentwicklung (Teil I)}

\subsubsection{Angaben zur Methodik und Stichprobe}

Das Thema "Maßnahmen zur Qualitätsentwicklung" wurde im Rahmen von Mitarbeiterbefragungen in mehreren Einrichtungen der Jugendhilfe (A - C: s. o.; D: s. u.) sowie punktuell in der Teilnehmerbefragung eines Selbstevaluationskurses (E: s. u.) angeschnitten. Es wurden 15 - im Falle E mehr, zudem überwiegend andere Möglichkeiten vorgegeben, die Arbeit zu erleichtern oder Arbeitsergebnisse zu verbessern.

Bewertet werden sollte mit $1=$ sehr hilfreich, $2=$ hilfreich, $3=$ teils-teils, $4=$ wenig hilfreich, $5=$ nicht hilfreich.

Die zusätzlichen Stichproben waren:

○ $\quad \mathrm{D}=$ große stationäre Einrichtung der Jugendhilfe (Kinderdorf mit 95 MitarbeiterInnen), Befragungszeitpunkt Januar 2000, Rücklaufquote 82,1\% ( $\mathrm{n}=78)$;

○ $\quad \mathrm{E}=$ Selbstevaluationskurs/Erziehungshilfe (18 TeilnehmerInnen), Befragungszeitpunkt August 2000, Rücklaufquote 66\% ( $\mathrm{n}=12)$.

Die angebotenen Verbesserungsmaßnahmen waren zumeist im Komparativ formuliert (z. B. mehr innerbetriebliche Fortbildung), bezogen sich also auf qualitative oder quantitative Veränderungen des wahrgenommenen betrieblichen Ist-Zustandes im Sinne eines "mehr desselben" (teilweise Ausnahme: Einrichtung C sowie KursteilnehmerInnen E, s. u.). Aus den Bewertungen sollte der individuell eingeschätzte Handlungsbedarf in den einzelnen Einrichtungen abgeleitet werden, um Anhaltspunkte für gezielte Maßnahmen gewinnen und damit verbundene Optimierungsressourcen ausschöpfen zu können.

An dieser Stelle ist jedoch ein methodenkritischer Exkurs angezeigt:

Fraglich ist nämlich, ob in den Bewertungen tatsächlich die jeweilige Ausgangssituation der Einrichtungen maßgeblich war oder ob mehr die allgemeine Wertschätzung der 
Maßnahme - ungeachtet ihres betrieblichen Realisierungsgrades - zum Ausdruck kommt. Hinweise geben die Vergleiche der Einrichtungen A, B und D mit jenen der Gruppen C und E, die teilweise mit neutralen Formulierungen - also ohne Zusatz von "mehr" oder "besser" - befragt wurden. (Leider liegen keine Standardabweichungen mehr vor, so dass eine statistische Prüfung der Mittelwertsunterschiede nicht möglich ist, sondern nur eine Inspektion nach Augenschein.)

Um das Ergebnis dieses Vergleichs vorwegzunehmen: Es zeigt sich von fünf in Frage kommenden Items (Supervision, inner- und außerbetriebliche Fortbildung, Zusatzausbildung und Beratung durch externe Fachleute) nur bei einem Item (Beratung durch externe Fachleute) ein durchgängiger Unterschied. Während die Mittelwerte der Einrichtungen mit komparativer Item-Formulierung bei 2,24 (A), 2,48 (B) und 2,41 (D) liegen, betragen die Werte für die Gruppen mit neutraler Item-Formulierung 1,97 (C) und $2,10(\mathrm{E})$.

Mit Vorbehalt erscheint die Folgerung zulässig, dass in der Bewertung der Maßnahmen vermutlich beide oben genannten Aspekte eine Rolle spielen: MitarbeiterInnen beurteilen Vorkehrungen zur Entlastung und Verbesserung der Arbeit nach ihrer subjektiv eingeschätzten Nützlichkeit ("hilfreich") und begrüßen es, wenn "mehr derselben" in der Praxis zur Verfügung stünden, relativ unabhängig davon, wie ausgiebig die eigene Einrichtung bereits von diesen Maßnahmen Gebrauch macht.

Trifft diese Schlussfolgerung jedoch zu, ist der Spielraum für sinnvolle Handlungsoptionen unter Umständen eingeschränkt: Wenn MitarbeiterInnen sich mehr oder bessere Maßnahmen wünschen, obwohl das betrieblich Leistbare bereits ausgeschöpft wird und die MitarbeiterInnen dies vermutlich auch konzedieren würden, gerät die Befragung zu diesen Themen zur reinen Affirmation der Praxis bzw. zur Vorgaukelung möglicher "SchlaraffenArbeitsplätze".

Aus vorstehenden - etwas spekulativen - Überlegungen ergibt sich eine sinnvolle methodische Konsequenz: Trotz des hier nicht nachgewiesenen Einflusses der ItemFormulierung auf die Bewertungsergebnisse scheint es zweckmäßig zu sein, die Items neutral zu gestalten, um die grundsätzliche Wertschätzung der MitarbeiterInnen für die angesprochene Maßnahme zu eruieren. In einem zusätzlichen Schritt sollte dann - wie im 
Übrigen auch in den durchgeführten Befragungen geschehen - nach den Bereichen mit größtem und dringlichstem Handlungsbedarf in der eigenen Einrichtung gefragt werden. Ein Vergleich der Mitarbeiterurteile mit der betrieblichen Praxis ergibt dann durchaus nützliche Hinweise auf Bewährtes oder Vernachlässigtes.

\subsubsection{Ergebnisse}

Tabellarisch aufgeführt sind im Folgenden die Items, die Stichprobengrößen (n), die Mittelwerte (M), die jeweiligen Bewertungsränge (R) der Items in den einzelnen Gruppen sowie Gesamtmittelwerte und Gesamtränge der Items über alle Gruppen. Die Standardabweichungen wurden zwar seinerzeit berechnet, liegen jedoch - wie erwähnt nicht mehr vor, weshalb keine Signifikanzprüfungen vorgenommen werden können. Bei B und E findet sich teilweise keine Angabe (k. A.) wegen abweichender Item-Formulierung und fragwürdiger Vergleichbarkeit.

Die Reihenfolge der Einrichtungen A bis E entspricht auch diesmal nicht dem chronologischen Verlauf der Befragungen, sondern dem Grad der Ähnlichkeit des Arbeitsfeldes sowie der Einrichtungsgröße. Die Einrichtung D fällt wegen ihrer flächendeckend besonderen Arbeitsweise (Familienprinzip) aus diesem Rahmen, die Gruppe E aufgrund des speziellen Kontextes. 
Tab. 2:Bewertung von Maßnahmen der Qualitätsentwicklung durch Fachkräfte der Jugendhilfe

\begin{tabular}{|c|c|c|c|c|c|c|c|}
\hline Item & $\mathrm{A}$ & B & $\mathrm{C}$ & $\mathrm{D}$ & $E$ & $\begin{array}{l}\text { Gesamt-n } \\
\text { und -M }\end{array}$ & $\begin{array}{l}\text { Gesamt- } \\
\text { Rang }\end{array}$ \\
\hline $\begin{array}{l}\text { bessere } \\
\text { Zusammenarbeit/Zie- } \\
\text { hen an einem Strang }\end{array}$ & $\begin{array}{lr}\mathrm{n} & 132 \\
\mathrm{M} & 1,88 \\
\mathrm{R} & 4\end{array}$ & $\begin{array}{l}69 \\
1,53 \\
1\end{array}$ & $\begin{array}{l}36 \\
1,46 \\
1\end{array}$ & $\begin{array}{l}50 \\
1,96 \\
3\end{array}$ & k. A. & $\begin{array}{l}287 \\
1,71\end{array}$ & 1 \\
\hline $\begin{array}{l}\text { mehr } \\
\text { außerbetriebliche } \\
\text { Fortbildung }\end{array}$ & $\begin{array}{lr}\mathrm{n} & 162 \\
\mathrm{M} & 1,78 \\
\mathrm{R} & 2\end{array}$ & $\begin{array}{l}67 \\
2,24 \\
9\end{array}$ & $\begin{array}{l}38 \\
1,61 \\
2\end{array}$ & $\begin{array}{l}58 \\
1,86 \\
2\end{array}$ & $\begin{array}{l}12 \\
1,91 \\
1\end{array}$ & $\begin{array}{l}337 \\
1,88\end{array}$ & 2 \\
\hline $\begin{array}{l}\text { mehr Klarheit über } \\
\text { die Kriterien fachlich } \\
\text { guter Arbeit }\end{array}$ & $\begin{array}{lr}\mathrm{n} & 129 \\
\mathrm{M} & 1,89 \\
\mathrm{R} & 5\end{array}$ & $\begin{array}{l}71 \\
2,07 \\
4\end{array}$ & $\begin{array}{l}37 \\
2,03 \\
6\end{array}$ & $\begin{array}{l}50 \\
1,84 \\
1\end{array}$ & k. A. & $\begin{array}{l}287 \\
1,96\end{array}$ & 3 \\
\hline $\begin{array}{l}\text { bessere personelle } \\
\text { Ausstattung }\end{array}$ & $\begin{array}{lr}\mathrm{n} & 137 \\
\mathrm{M} & 1,86 \\
\mathrm{R} & 3\end{array}$ & $\begin{array}{l}72 \\
1,78 \\
2\end{array}$ & $\begin{array}{l}38 \\
1,79 \\
3\end{array}$ & $\begin{array}{l}48 \\
2,69 \\
10\end{array}$ & k. A. & $\begin{array}{l}295 \\
2,03\end{array}$ & 4 \\
\hline $\begin{array}{l}\text { mehr } \\
\text { innerbetriebliche } \\
\text { Fortbildung }\end{array}$ & $\begin{array}{lr}\mathrm{n} & 160 \\
\mathrm{M} & 1,92 \\
\mathrm{R} & 6 \\
\end{array}$ & $\begin{array}{l}69 \\
2,20 \\
8\end{array}$ & $\begin{array}{l}37 \\
1,80 \\
4\end{array}$ & $\begin{array}{l}57 \\
2,37 \\
5\end{array}$ & $\begin{array}{l}12 \\
2,00 \\
3\end{array}$ & $\begin{array}{l}335 \\
2,06\end{array}$ & 5 \\
\hline $\begin{array}{l}\text { mehr Supervision } \\
\text { (Einzel-, Gruppen-) }\end{array}$ & $\begin{array}{lr}\mathrm{n} & 157 \\
\mathrm{M} & 1,73 \\
\mathrm{R} & 1\end{array}$ & $\begin{array}{l}68 \\
2,16 \\
6\end{array}$ & $\begin{array}{l}38 \\
2,11 \\
7\end{array}$ & $\begin{array}{l}52 \\
2,38 \\
6\end{array}$ & $\begin{array}{l}12 \\
2,00 \\
3\end{array}$ & $\begin{array}{l}327 \\
2,08\end{array}$ & 6 \\
\hline $\begin{array}{l}\text { mehr Mitbestimmung } \\
\text { bei der Auswahl der } \\
\text { Betreuten }\end{array}$ & $\begin{array}{lr}\mathrm{n} & 91 \\
\mathrm{M} & 2,15 \\
\mathrm{R} & 9\end{array}$ & $\begin{array}{l}58 \\
1,97 \\
3\end{array}$ & k. A. & $\begin{array}{l}38 \\
2,53 \\
9\end{array}$ & k. A. & $\begin{array}{l}187 \\
2,22\end{array}$ & 7 \\
\hline $\begin{array}{l}\text { mehr Beratung durch } \\
\text { externe Fachleute (z. } \\
\text { B. Jugendpsychiater) }\end{array}$ & $\begin{array}{lr}\mathrm{n} & 151 \\
\mathrm{M} & 2,24 \\
\mathrm{R} & 11\end{array}$ & $\begin{array}{l}66 \\
2,48 \\
12\end{array}$ & $\begin{array}{l}36 \\
1,97 \\
5\end{array}$ & $\begin{array}{l}52 \\
2,41 \\
7\end{array}$ & $\begin{array}{l}12 \\
2,10 \\
5\end{array}$ & $\begin{array}{l}317 \\
2,24\end{array}$ & 8 \\
\hline $\begin{array}{l}\text { mehr Unterstützung } \\
\text { durch Vorgesetzte }\end{array}$ & $\begin{array}{lr}\mathrm{n} & 96 \\
\mathrm{M} & 2,19 \\
\mathrm{R} & 10\end{array}$ & $\begin{array}{l}66 \\
2,14 \\
5\end{array}$ & k. A. & $\begin{array}{l}34 \\
2,44 \\
8\end{array}$ & k. A. & $\begin{array}{l}196 \\
2,26\end{array}$ & 9 \\
\hline $\begin{array}{l}\text { mehr Zusatzaus- } \\
\text { bildung (z. B. Heilpä- } \\
\text { dagogik, Sozialther.) }\end{array}$ & $\begin{array}{lr}\mathrm{n} & 151 \\
\mathrm{M} & 2,12 \\
\mathrm{R} & 7\end{array}$ & $\begin{array}{l}58 \\
2,36 \\
10\end{array}$ & $\begin{array}{l}36 \\
2,29 \\
8\end{array}$ & $\begin{array}{l}46 \\
2,31 \\
4\end{array}$ & $\begin{array}{l}12 \\
1,91 \\
1\end{array}$ & $\begin{array}{l}303 \\
2,27\end{array}$ & 10 \\
\hline $\begin{array}{l}\text { mehr Entlastung von } \\
\text { bestimmt. Aufgaben } \\
\text { (z. B. Verwaltung) }\end{array}$ & $\begin{array}{lr}\mathrm{n} & 99 \\
\mathrm{M} & 2,31 \\
\mathrm{R} & 12 \\
\end{array}$ & $\begin{array}{l}56 \\
2,29 \\
9 \\
\end{array}$ & $\begin{array}{l}15 \\
2,93 \\
11 \\
\end{array}$ & $\begin{array}{l}36 \\
3,11 \\
13\end{array}$ & k. A. & $\begin{array}{l}206 \\
2,57\end{array}$ & 11 \\
\hline $\begin{array}{l}\text { mehr pädagogische } \\
\text { Angebote von dritter } \\
\text { Seite }\end{array}$ & $\begin{array}{lr}\mathrm{n} & 147 \\
\mathrm{M} & 2,51 \\
\mathrm{R} & 13\end{array}$ & $\begin{array}{l}62 \\
2,42 \\
11\end{array}$ & k. A. & $\begin{array}{l}49 \\
2,89 \\
11\end{array}$ & k. A. & $\begin{array}{l}258 \\
2,61\end{array}$ & 12 \\
\hline $\begin{array}{l}\text { bessere finanzielle } \\
\text { Ausstattung }\end{array}$ & $\begin{array}{lr}\mathrm{n} & 157 \\
\mathrm{M} & 2,12 \\
\mathrm{R} & 8\end{array}$ & $\begin{array}{l}64 \\
2,78 \\
13\end{array}$ & $\begin{array}{l}38 \\
2,30 \\
9\end{array}$ & $\begin{array}{l}47 \\
4,01 \\
15\end{array}$ & k. A. & $\begin{array}{l}306 \\
2,80\end{array}$ & 13 \\
\hline $\begin{array}{l}\text { mehr } \\
\text { Leistungsanreize }\end{array}$ & $\begin{array}{lr}\mathrm{n} & 80 \\
\mathrm{M} & 2,85 \\
\mathrm{R} & 14\end{array}$ & $\begin{array}{l}66 \\
3,11 \\
14\end{array}$ & $\begin{array}{l}36 \\
2,77 \\
10\end{array}$ & $\begin{array}{l}28 \\
3,01 \\
12\end{array}$ & k. A. & $\begin{array}{l}210 \\
2,94\end{array}$ & 14 \\
\hline $\begin{array}{l}\text { bessere } \\
\text { Arbeitszeitregelungen }\end{array}$ & $\begin{array}{lr}\mathrm{n} & 88 \\
\mathrm{M} & 3,55 \\
\mathrm{R} & 15\end{array}$ & $\begin{array}{l}54 \\
4,04 \\
15\end{array}$ & k. A. & $\begin{array}{l}27 \\
3,93 \\
14\end{array}$ & k. A. & $\begin{array}{l}169 \\
3,84\end{array}$ & 15 \\
\hline
\end{tabular}




\subsubsection{Zusammenfassung und Diskussion der Ergebnisse:}

- Als hilfreichste Maßnahmen zur Entlastung und Optimierung werden insgesamt bessere Zusammenarbeit/Ziehen an einem Strang, mehr außerbetriebliche Fortbildung und mehr Klarheit über die Kriterien fachlich guter Arbeit bewertet.

- Deutlich ab fallen mehr pädagogische Angebote von dritter Seite, bessere finanzielle Ausstattung, mehr Leistungsanreize und bessere Arbeitszeitregelungen.

- Die Mittelwerts-Abstände zwischen den ersten zehn Rangplätzen sind jeweils gering; danach klafft eine größere Lücke, auch zwischen den meisten nachfolgenden Rängen.

- Die inhaltliche Analyse ergibt nur wenige interpretationsbedürftige Differenzen zwischen den Einrichtungen. Die Ergebnisse sind bemerkenswert homogen. Vor allem die Mittelwerte differieren nur geringfügig, wogegen die Rangplätze sich teilweise etwas stärker unterscheiden.

- Der Aspekt der besseren personellen Ausstattung spielt in der Kinderdorf-Einrichtung D - deren Wohngruppen nach dem Familienprinzip strukturiert sind - eine relativ untergeordnete Rolle im Vergleich zu den anderen Einrichtungen.

- Dies gilt auch für den Aspekt der besseren finanziellen Ausstattung (Kinderdorf: keine Trennung von Privat- und Dienstbereich) sowie - weniger ausgeprägt - für die Entlastung von bestimmten Aufgaben/z. B. Verwaltung (Kinderdorf: Zentralverwaltung).

- Zwischen Einrichtungsgröße und Befragungsergebnissen treten keine systematischen Zusammenhänge in Erscheinung. Sehr große, große und mittelgroße Einrichtungen unterscheiden sich im Hinblick auf die Bewertungen der MitarbeiterInnen nicht regelhaft.

Bemerkenswert ist, dass der hohe Stellenwert einer guten bzw. noch zu verbessernden Zusammenarbeit im Sinne eines Ziehens an einem Strang sowie die gleichfalls hohe Bedeutung (Rangplatz 3) von mehr Klarheit über die Kriterien fachlich guter Arbeit gut 
zur Deckung zu bringen sind mit zentralen Anliegen eines Qualitätsmanagements: nämlich unerwünschte Varianz in der Fertigung/Behandlung/Betreuung zu minimieren, Teamverantwortung $\mathrm{zu}$ fördern, Qualitätskriterien $\mathrm{zu}$ operationalisieren und implizites Wissen zu explizieren. Diese Konvergenz individueller fachlicher Prioritäten und programmatischer Rhetorik gilt es bei der Implementation von Qualitätsmanagement zu nutzen! 


\subsection{Maßnahmen zur Qualitätsentwicklung (Teil II)}

\subsubsection{Angaben zur Methodik und Stichprobe}

Beurteilt werden sollte wiederum der praktische Nutzen verschiedener Maßnahmen und Vorkehrungen zur Qualifizierung der Leistungsprozesse und Akteure.

Verwendet wurde folgende Skala:

$0=$ kann ich nicht beurteilen - Maßnahme wird bei uns nicht praktiziert (zu "missing data" umcodiert, um die Mittelwerte nicht zu verfälschen, s. Kap. D 4.6.7.1), $1=$ sehr hoch, $2=$ hoch, $3=$ eher hoch als gering, 4 $=$ eher gering als hoch, $5=$ gering, $6=$ sehr gering.

Die Daten sind teilweise der in Kap. D 4. dargestellten Hauptstudie (F) entnommen. Die Vergleichswerte für $\mathrm{E}$ und $\mathrm{C}$ entstammen den mit einer etwas anderen Bewertungsskala arbeitenden Befragungen von 2000 (E) bzw. 2003 (C) und sollen hier lediglich der Tendenz nach interpretiert werden.

○ $\mathrm{F}=102$ TeilnehmerInnen an der Hauptstudie 2004 (s. Kap. A 5.3.5, Qualitätsbeauftragte und Leitungskräfte);

- $\quad \mathrm{E}=12$ TeilnehmerInnen eines Selbstevaluations-Kurses (Arbeitsfeld Erziehungshilfe, s. Kap. D 1.3.1.);

- $\quad \mathrm{C}=42$ MitarbeiterInnen einer mittelgroßen Einrichtung der Erziehungshilfe (s. Kap. D 1.2.1).

\subsubsection{Ergebnisse}

Aufgeführt sind die Stichprobengrößen (n), die Mittelwerte (M), die Rangplätze (R) der Items innerhalb der einzelnen Gruppen sowie die Gesamt-Mittelwerte und -Rangplätze über alle Gruppen hinweg. Auf die Angabe der Standardabweichungen für F wird hier verzichtet, da für E und C keine Vergleichsdaten vorliegen. (Die vollständigen Daten für F werden in Kap. D 4.6.7 weiterverarbeitet.) 
Tab. 3: Bewertung von Maßnahmen zur Qualitätsentwicklung durch Fachkräfte der Jugend- und Sozialhilfe

\begin{tabular}{|c|c|c|c|c|c|}
\hline Item & $\begin{array}{l}\text { QB/Leitung } \\
\text { F }\end{array}$ & $\begin{array}{l}\text { Fachkräfte } \\
\text { E }\end{array}$ & $\begin{array}{l}\text { Einrichtung } \\
\mathrm{C}\end{array}$ & $\begin{array}{l}\text { Gesamt- } \\
\text { M }\end{array}$ & $\begin{array}{l}\text { Gesamt- } \\
\text { rang }\end{array}$ \\
\hline $\begin{array}{l}\text { innerbetriebliche Fortbildung durch } \\
\text { interne Leitungs- und Beratungskräfte }\end{array}$ & $\begin{array}{lc}\mathrm{n} & 87 \\
\mathrm{M} & 2,54 \\
\mathrm{R} & 10\end{array}$ & $\begin{array}{l}12 \\
2,90 \\
14\end{array}$ & $\begin{array}{l}38 \\
2,59 \\
14\end{array}$ & 2,68 & 14 \\
\hline $\begin{array}{l}\text { innerbetriebliche Fortbildung durch } \\
\text { externe Beratungskräfte ("Inhouse"- } \\
\text { Seminare) }\end{array}$ & $\begin{array}{lc}\mathrm{n} & 88 \\
\mathrm{M} & 2,28 \\
\mathrm{R} & 6\end{array}$ & $\begin{array}{l}12 \\
2,00 \\
7\end{array}$ & $\begin{array}{l}37 \\
1,80 \\
4\end{array}$ & 2,03 & 7 \\
\hline $\begin{array}{l}\text { außerbetriebliche Fort- und } \\
\text { Weiterbildung }\end{array}$ & $\begin{array}{lc}\mathrm{n} & 100 \\
\mathrm{M} & 2,33 \\
\mathrm{R} & 8 \\
\end{array}$ & $\begin{array}{l}12 \\
1,91 \\
3\end{array}$ & $\begin{array}{l}38 \\
1,61 \\
1\end{array}$ & 1,95 & 4 \\
\hline Team-/Gruppensupervision & $\begin{array}{lc}\mathrm{n} & 87 \\
\mathrm{M} & 2,15 \\
\mathrm{R} & 4 \\
\end{array}$ & $\begin{array}{l}12 \\
2,00 \\
7\end{array}$ & $\begin{array}{l}38 \\
2,11 \\
9\end{array}$ & 2,09 & 8 \\
\hline Einzelsupervision & $\begin{array}{lc}\mathrm{n} & 82 \\
\mathrm{M} & 2,28 \\
\mathrm{R} & 6 \\
\end{array}$ & $\begin{array}{l}12 \\
2,00 \\
7\end{array}$ & $\begin{array}{l}38 \\
2,30 \\
11\end{array}$ & 2,19 & 9 \\
\hline $\begin{array}{l}\text { Zusatzausbildung (Heilpädagogik, } \\
\text { Sozialtherapie u. Ä.) }\end{array}$ & $\begin{array}{lc}\mathrm{n} & 81 \\
\mathrm{M} & 2,36 \\
\mathrm{R} & 9\end{array}$ & $\begin{array}{l}12 \\
1,91 \\
3\end{array}$ & $\begin{array}{l}36 \\
2,29 \\
10\end{array}$ & 2,19 & 9 \\
\hline $\begin{array}{l}\text { Fachberatung durch Externe } \\
\text { (PsychiaterInnen, PsychologInnen u. } \\
\text { a.) }\end{array}$ & $\begin{array}{lc}\mathrm{n} & 77 \\
\mathrm{M} & 2,70 \\
\mathrm{R} & 11 \\
\end{array}$ & $\begin{array}{l}12 \\
2,10 \\
11 \\
\end{array}$ & $\begin{array}{l}36 \\
1,97 \\
6 \\
\end{array}$ & 2,26 & 11 \\
\hline $\begin{array}{l}\text { bedarfsgerechte personelle Ausstattung } \\
\text { oder Dienstorganisation (z. B. } \\
\text { "Doppeldienst" zu bestimmten Zeiten) }\end{array}$ & $\begin{array}{l}\mathrm{n} \\
\mathrm{M} \\
\mathrm{R}\end{array}$ & $\begin{array}{l}12 \\
1,54 \\
1\end{array}$ & $\begin{array}{l}38 \\
1,79 \\
3\end{array}$ & 1,67 & 1 \\
\hline $\begin{array}{l}\text { Selbstevaluation (u. a. Datenerhebung } \\
\text { und Auswertung durch Beteiligte) }\end{array}$ & $\begin{array}{lc}\mathrm{n} & 64 \\
\mathrm{M} & 2,70 \\
\mathrm{R} & 11\end{array}$ & $\begin{array}{l}12 \\
2,27 \\
12\end{array}$ & $\begin{array}{l}36 \\
2,34 \\
12\end{array}$ & 2,44 & 13 \\
\hline $\begin{array}{l}\text { kollegiale Beratung (in einem } \\
\text { systematischen Setting, also nicht } \\
\text { beiläufig) }\end{array}$ & $\begin{array}{lc}\mathrm{n} & 92 \\
\mathrm{M} & 2,18 \\
\mathrm{R} & 5 \\
\end{array}$ & $\begin{array}{l}12 \\
1,91 \\
3\end{array}$ & $\begin{array}{l}37 \\
1,83 \\
5\end{array}$ & 1,97 & 6 \\
\hline $\begin{array}{l}\text { Fallkonferenzen, systematische } \\
\text { Erziehungsplanung u. ä. Formen } \\
\text { einzelfallbez. interner Fachgespräche }\end{array}$ & $\begin{array}{lc}\mathrm{n} & 92 \\
\mathrm{M} & 1,79 \\
\mathrm{R} & 1 \\
\end{array}$ & $\begin{array}{l}12 \\
1,91 \\
3\end{array}$ & $\begin{array}{l}38 \\
1,69 \\
2\end{array}$ & 1,80 & 2 \\
\hline $\begin{array}{l}\text { Qualifiz. des Hilfeplanprozesses und } \\
\text { der Kooperation (bessere Einbezieh. } \\
\text { von Klientel, Jugendamt usw.) }\end{array}$ & $\begin{array}{ll}\mathrm{n} & \\
\mathrm{M} & \mathrm{k} . \mathrm{A} . \\
\mathrm{R} & \\
\end{array}$ & $\begin{array}{l}12 \\
1,63 \\
2\end{array}$ & $\begin{array}{l}37 \\
2,06 \\
8\end{array}$ & 1,85 & 3 \\
\hline $\begin{array}{l}\text { zielorientierte } \\
\text { Konzeptionsentwicklung (Zielfindung } \\
\text { und Operationalisierung) }\end{array}$ & $\begin{array}{lc}\mathrm{n} & 98 \\
\mathrm{M} & 1,82 \\
\mathrm{R} & 2\end{array}$ & $\begin{array}{l}12 \\
2,00 \\
7\end{array}$ & $\begin{array}{l}36 \\
2,03 \\
7\end{array}$ & 1,95 & 4 \\
\hline $\begin{array}{l}\text { Beschreibung von Schlüsselprozessen } \\
\text { (methodische Gestaltung von } \\
\text { Schlüsselsituationen) }\end{array}$ & $\begin{array}{lc}\mathrm{n} & 91 \\
\mathrm{M} & 2,01 \\
\mathrm{R} & 3 \\
\end{array}$ & $\begin{array}{ll}12 \\
2,36 \\
13 \\
\end{array}$ & $\begin{array}{l}36 \\
2,49 \\
13 \\
\end{array}$ & 2,29 & 12 \\
\hline $\begin{array}{l}\text { Fremdevaluation (u. a. Datenerhebung } \\
\text { und Auswertung durch Dritte) }\end{array}$ & $\begin{array}{lc}\mathrm{n} & 43 \\
\mathrm{M} & 3,60 \\
\mathrm{R} & 15 \\
\end{array}$ & $\begin{array}{l}12 \\
3,22 \\
16 \\
\end{array}$ & $\begin{array}{l}35 \\
3,37 \\
16 \\
\end{array}$ & 3,40 & 17 \\
\hline $\begin{array}{l}\text { interne Qualitätskontrollen durch } \\
\text { Leitungskräfte und/oder beauftragte } \\
\text { KollegInnen (so gen. "interne Audits") }\end{array}$ & $\begin{array}{lc}\mathrm{n} & 56 \\
\mathrm{M} & 2,82 \\
\mathrm{R} & 13\end{array}$ & $\begin{array}{l}12 \\
3,00 \\
15\end{array}$ & $\begin{array}{l}38 \\
3,07 \\
15\end{array}$ & 2,96 & 15 \\
\hline $\begin{array}{l}\text { externe Qualitätskontrollen durch } \\
\text { beauftragte Stellen (so gen. "externe } \\
\text { Audits" mit oder ohne Zertifizierung) }\end{array}$ & $\begin{array}{lc}\mathrm{n} & 42 \\
\mathrm{M} & 3,19 \\
\mathrm{R} & 14 \\
\end{array}$ & $\begin{array}{l}12 \\
3,55 \\
17\end{array}$ & $\begin{array}{l}36 \\
3,44 \\
17 \\
\end{array}$ & 3,39 & 16 \\
\hline
\end{tabular}




\subsubsection{Zusammenfassung und Diskussion der Ergebnisse}

Wegen der eingeschränkten Vergleichbarkeit in Bezug auf Skalenbeschriftung und exakten Wortlaut einiger Items werden Mittelwertsdifferenzen nicht interpretiert, sondern nur die eindeutigsten Rangunterschiede.

- Insgesamt zeigt sich ein sehr homogenes Urteilsbild bei den meisten Items, wie es auch in den zuvor erwähnten Mitarbeiterbefragungen der Fall war. Trotz der vergleichsweise größeren Heterogenität der Gesamtgruppe (Fach- und Leitungskräfte aus unterschiedlichen sozialen Arbeitsfeldern öffentlicher und freier Träger) sind sich die Befragten relativ einig in der Nutzenbewertung verschiedener Maßnahmen der Qualitätsentwicklung.

- Lässt man die fehlenden Angaben in Stichprobe F zu zwei sehr erziehungshilfespezifischen Items außer Betracht (in F nicht erhoben), so sind lediglich die folgenden Rang- (und Mittelwerts-)unterschiede auffällig und erklärungsbedürftig: Beschreibung von Schlüsselprozessen (Rang 3 in F vs. 13 in $\mathrm{E}$ und C) und - wenngleich weniger eklatant - außerbetriebliche Fort- und Weiterbildung (Rang 8 in F vs. 3 in E und 1 in C).

Im ersteren Falle ist anzumerken, dass in Studie F die exakte Formulierung des Items lautete "Beschreibung und fachgerechte Gestaltung von Schlüsselprozessen", während in E und C die Formulierung in Tab. 3 verwendet worden war. Möglicherweise ist durch die additive Nennung von Beschreibung und fachgerechter Gestaltung in F ein stärkerer Handlungsbezug hergestellt als in der anderen Formulierung, die lediglich mit einer Klammerbemerkung darauf aufmerksam machen soll, dass nicht eine kunstgerechte Beschreibung für die "pädagogische Galerie", sondern die Konkretisierung fachlichen Know-hows gemeint ist.

Im zweiten Fall waren die Itemformulierungen identisch. Anhaltspunkte für den auch numerisch recht großen Unterschied liefern die Daten aus der Hauptstudie (s. Kap. D 4.6.7.2). Danach besteht ein auf dem 5\%-Niveau signifikanter Unterschied in der Nutzenbewertung außerbetrieblicher Fort- und Weiterbildung durch öffentliche und freigemeinnützige Träger (hier weitgehend identisch mit Jugendund Sozialhilfeverwaltungen einerseits und Erziehungshilfeeinrichtungen andererseits). Öffentliche Träger geben eine mittlere Bewertung von 2,53 ab, freigemeinnützige Träger eine von 2,05. Dies hätte im hier vorgenommenen Vergleich einen Rangplatz 4 bedeutet und keiner speziellen Interpretation bedurft. 
- Es lässt sich deshalb konstatieren, dass die ermittelten größeren Unterschiede in der Nutzenbewertung zum einen mit Bedeutungsdifferenzen des verwendeten Items und zum anderen mit Stichprobenmerkmalen erklärbar sind. Innerhalb des Erziehungshilfebereichs, wie er hier durch Einrichtungen in freigemeinnütziger Trägerschaft repräsentiert ist, besteht bemerkenswerte Einmütigkeit in der Priorisierung der vorgegebenen Maßnahmen.

○ Die größte Bedeutung für die Qualifizierung der Leistungsprozesse und Akteure wird eher "klassischen" Interventionen zugemessen, die auch schon vor Aufkommen der Qualitätsdebatte fachlich en vogue waren:

1. bedarfsgerechte personelle Ausstattung oder Dienstorganisation (z. B. "Doppeldienst" zu bestimmten Zeiten) - zentrales Thema in Kostenverhandlungen und Betriebsführung;

2. Fallkonferenzen, systematische Erziehungsplanung u. ä. Formen einzelfallbezogener interner Fachgespräche - als interne Fallreflexion auch ein Favorit in vielen Jugendämtern ( $M=2,2$; s. Kap. D 4.6.7.2);

3. Qualifizierung des Hilfeplanprozesses und der Kooperation (bessere Einbeziehung von Klientel, Jugendamt, Angehörigen, anderen Institutionen usw.) - immer schon Kennzeichen sozialraum- und ressourcenorientierter Erziehungshilfe;

4. zielorientierte Konzeptionsentwicklung (Zielfindung und Operationalisierung) - Dauerbrenner der ambulanten wie stationären Kinder- und Jugendhilfe;

5. außerbetriebliche Fort- und Weiterbildung - seit jeher Inbegriff prophylaktischer Maßnahmen gegen Betriebsblindheit und qualitätsfeindliche Routinisierung beruflichen Handelns.

○ Dagegen finden sich auf den hinteren Plätzen der Rangordnung eher "moderne" und namentlich durch den Qualitäts-Zeitgeist prominent gewordene Instrumente, denen mit 
deutlichem Abstand geringerer Nutzen attestiert wird:

1. interne Qualitätskontrollen durch Leitungskräfte und/oder beauftragte KollegInnen (so genannte interne Audits) - immerhin noch mit "numerischer Tuchfühlung" zu vorgelagerten Rängen;

2. externe Qualitätskontrollen durch beauftragte Stellen (so genannte externe Audits mit oder ohne Zertifizierung/Gütesiegel) - ob aus Überzeugung der relativen Nutzlosigkeit oder aus Widerstand gegen "fremde Blicke von außen", sei dahingestellt;

3. Fremdevaluation (u. a. Datenerhebung und Auswertung durch Dritte) unter ähnlichen Vorbehalten zu interpretieren.

- Im Zusammenhang mit den allgemein eher positiven Einstellungswerten gegenüber einem Qualitätsmanagement (s. Kap. D 1.2.2) müssen die zurückhaltenden Urteile über den praktischen Nutzeffekt QM-spezifischer Instrumente wie Audits und Evaluationsverfahren nicht zwangsläufig als Beleg für Widersprüchlichkeit oder klammheimliche Abwehr interpretiert werden. Vielmehr könnte sich darin auch die nachvollziehbare Skepsis gestandener PraktikerInnen gegenüber Kontrollmotiven einer weniger an Fachlichkeits- als an Kostenkriterien interessierten Sozialadministration ausdrücken.

Hinzu kommt, dass weite Bereiche der Praxis noch gar keine oder erst geringe Erfahrung mit den genannten Instrumenten sammeln konnten und von daher ohnehin eher defensiv reagiert haben dürften. $\mathrm{Ob}$ sich durch zunehmend häufigere und vor allem sach- und zielgerechte Anwendung der entsprechenden Verfahren größeres Vertrauen in ihren Nutzen aufbauen kann, muss sich noch erweisen. Auch in der Hauptstudie (s. Kap. D 4.6.7.1) kommen die genannten Maßnahmen über letzte Ränge nicht hinaus. 


\section{Befunde aus Leitungsbefragungen}

\subsection{Maßnahmen zum Qualitätsmanagement}

\subsubsection{Angaben zur Methodik und Stichprobe}

Im Rahmen einer 1998 vom Autor durchgeführten Mitgliederbefragung des Evangelischen Erziehungsverbandes (EREV), einem bundesweiten Zusammenschluss evangelischer Einrichtungen und Dienste der Jugendhilfe, wurden Daten zum Stand der Qualitätsmanagement-Aktivitäten und zur Haltung der Leitungskräfte erhoben (Gerull, 1998). Diese Daten - soweit vergleichbar - in Beziehung zu setzen zu Ergebnissen späterer Erhebungen, erscheint aus mehreren Gründen interessant:

1. lassen sich möglicherweise Umsetzungstendenzen ermitteln, also Entwicklungen in der Anwendung oder Priorisierung bestimmter Qualitätsmanagement-Instrumente;

2. lassen sich möglicherweise Einstellungsänderungen ermitteln, also Entwicklungen in der Haltung von Leitungskräften gegenüber Qualitätsmanagement;

3. lassen sich möglicherweise Unterschiede zwischen beruflichen Handlungsfeldern ermitteln, sofern hinreichend differente Stichproben zur Verfügung stehen.

Während für die ersten beiden Aspekte auf die Hauptstudie sowie Teile einer ähnlich angelegten Untersuchung der Fachhochschule Erfurt (Peters et al., o. J.) zurückgegriffen werden kann, ergibt sich für den dritten Gesichtspunkt eine Vergleichsmöglichkeit mit einer Befragung in Wohneinrichtungen der Behindertenhilfe (Wetzler, 2003) sowie mit jenem Anteil der in Kap. D 4.6 dargestellten Resultate der Hauptstudie, der eine Differenzierung nach Handlungsfeldern zulässt.

Die in den Vergleich einbezogenen Stichproben sind:

- $\mathrm{G}=404$ Mitgliedseinrichtungen des Evangelischen Erziehungsverbandes (EREV), Vollerhebung, Befragungszeitraum August 1998, Rücklaufquote rund 30\% ( $\mathrm{n}=123$ ); 
○ $\quad H=110$ nach Zufall ausgewählte Mitgliedseinrichtungen der Internationalen Gesellschaft für Heimerziehung (IGfH) und zusätzliche Online-Befragung, Befragungszeitraum Januar bis April 2000, Rücklaufquote rund 30\% ( $\mathrm{n}=79)$;

○ $\quad \mathrm{I}=$ rund 1.400 Wohneinrichtungen der Behindertenhilfe bundesweit, Befragungszeitraum Ende 2001, Rücklaufquote rund 16\% ( $\mathrm{n}=216$ );

- $F=413$ Einrichtungen und Dienste der Jugend- und Sozialhilfe bundesweit, Befragungszeitraum Februar bis Mai 2004, Rücklaufquote rund 21\% ( $\mathrm{n}=85$, s. Kap. D 4.3.1).

Berücksichtigt sind lediglich die im vorliegenden Untersuchungszusammenhang relevanten Daten, also solche, die sich auf die Einstellung zum Qualitätsmanagement, die Umsetzung bestimmter Maßnahmen sowie speziell den Einsatz von Qualitätsbeauftragten beziehen. Die Formulierungen der entsprechenden Items sind nicht identisch, beziehen sich aber auf hinreichend ähnliche Sachverhalte, so dass im Interesse eines überhaupt möglichen Vergleichs Nuancierungen vernachlässigt werden.

So wird z. B. in der 1998 durchgeführten EREV-Befragung (Gerull, 1998) als eine von mehreren Möglichkeiten zur Qualitätsentwicklung/zum Qualitätsmanagement die Organisationsanalyse/Aufbau/Ablauforganisation angesprochen, während in der Befragung von 2001 (Wetzler, 2003) das entsprechende Item lautet: funktionale Aufbaubeschreibung durch Organigramm. Dieses Beispiel repräsentiert zugleich den semantisch größtmöglichen Abstand zwischen den Formulierungen, während die übrigen der insgesamt 9 vergleichbaren Items stärker übereinstimmen (Leitbild/Qualitätsgrundsätze - in G zusammengefasst - vs. Leitbild/Qualitätskonzept; Einsatz von umfassenden QM-Systemen vs. Entscheidung für bestimmtes QMSystem; Anwendung von Evaluationsverfahren vs. Einsatz spezieller Verfahren zur Messung der Ergebnisqualität; Aufbau interner Qualitätszirkel vs. Einsatz von Qualitätszirkeln; Beteiligung der MitarbeiterInnen vs. Einbeziehung Mitarbeiterschaft) bis zur Identität der Formulierung (QM-Handbuch, Qualitätsbeauftragte, Zertifizierung).

Leider sind auch die verwendeten Antwortkategorien nicht identisch, so dass ein weiterer Anpassungsschritt erforderlich war. Während Gerull 1998 eine fünfstufige Skala ( 1 = kein Thema, 2 = noch nicht begonnen/keine Planung, $3=$ in Vorbereitung/geplant, $4=\mathrm{im}$ Gange/in 1998 abgeschlossen und 5 = weitgehend abgeschlossen) und 2004 vier Kategorien (vorhanden/in Ansätzen vorhanden/geplant/weder vorhanden noch geplant) 
formulierte, arbeitete Wetzler 2001 mit den Kategorien "ja", "nein" und "geplant"; Peters et al. benutzten 2000 zum Teil eine dreistufige Skala (ja/nein/teilweise).

Die im Folgenden aufgeführten Vergleichsdaten sind nach der Kategorisierung von Wetzler (2003) codiert; z. B. wurden die Skalenstufen 1 und 2 bei Gerull (1998) in "nein", die Stufen 4 und 5 in "ja" transformiert; "in Arbeit/in Ansätzen vorhanden" aus Studie F wurde als "geplant" zusammengefasst.

\subsubsection{Ergebnisse}

Angegeben sind die vergleichbaren Items, die Größe (n) der vier Stichproben (hier chronologisch geordnet: G, H, I, F) sowie die relativen Antwortverteilungen in jeder der drei Kategorien (in \%: ja/geplant/nein). Auf eine statistische Unterschiedsprüfung mittels Chi-Quadrat-Test wird wegen der Heterogenität der Stichproben und eingeschränkter Vergleichbarkeit der Items und/oder Antwortkategorien verzichtet. Nur herausragende Befunde werden interpretiert.

Die Untersuchung von Wetzler (I) konnte bezüglich der Beteiligung der Mitarbeiterschaft nicht in den Vergleich einbezogen werden (k. A.), da dort nach dem Anteil der MitarbeiterInnen mit QM-Fortbildung gefragt wurde, der einen deutlich abweichenden Sachverhalt repräsentiert; auch Studie F lässt sich nur bedingt in diesen Vergleich einbringen; die verwendeten Antwortkategorien weichen erheblich vom Schema "ja/geplant/nein" ab. Beim Item "Qualitätsbeauftragte" entspricht die angegebene Kategorie "geplant" der Kategorie "noch nicht entschieden" in Studie F. Bezüglich der Studie von Peters et al. ist häufiger Fehlanzeige zu vermelden (k. A.), weil die Untersuchung auf andere Themen fokussierte. Die Prozentangaben in Studie G wurden gerundet. 
Tab. 4: Umsetzung ausgewählter Maßnahmen zum Qualitätsmanagement in sozialen Einrichtungen

\begin{tabular}{|c|c|c|c|c|c|}
\hline Item & $\mathrm{n} /$ Antwort $\%$ & G (1998) & $\mathrm{H}(2000)$ & I (2001) & $\mathrm{F}(2004)$ \\
\hline $\begin{array}{l}\text { Organigramm/ } \\
\text { Aufbau- } \\
\text { /Ablauforganisation }\end{array}$ & $\begin{array}{l}\text { n } \\
\text { ja } \\
\text { gepl. } \\
\text { nein }\end{array}$ & $\begin{array}{l}114 \\
43,0 \\
28,0 \\
29,0\end{array}$ & k. A. & $\begin{array}{l}216 \\
82 \\
4,2 \\
13,8\end{array}$ & $\begin{aligned} 85 \\
83,5 \\
14,1 \\
2,4\end{aligned}$ \\
\hline $\begin{array}{l}\text { Leitbild/ } \\
\text { Qualitäts-grundsätze }\end{array}$ & $\begin{array}{l}\mathrm{n} \\
\text { ja } \\
\text { gepl. } \\
\text { nein }\end{array}$ & $\begin{array}{l}116 \\
55,0 \\
35,0 \\
10,0\end{array}$ & $\begin{array}{r}74 \\
59,5 \\
1,3 \\
38,0\end{array}$ & $\begin{array}{l}213 \\
66,2 \\
15,0 \\
18,8\end{array}$ & $\begin{array}{l}85 \\
69,4 \\
20,0 \\
10,6\end{array}$ \\
\hline $\begin{array}{l}\text { Evaluationsverfahren/ } \\
\text { Messung } \\
\text { Ergebnisqualität }\end{array}$ & $\begin{array}{l}\mathrm{n} \\
\text { ja } \\
\text { gepl. } \\
\text { nein }\end{array}$ & $\begin{array}{l}98 \\
16,0 \\
26,0 \\
58,0\end{array}$ & $\begin{array}{l}79 \\
29,1 \\
\text { k.A. } \\
70,9\end{array}$ & $\begin{array}{l}206 \\
48,5 \\
16,0 \\
35,5\end{array}$ & $\begin{array}{r}82 \\
69,6 \\
28,0 \\
2,4 \\
\end{array}$ \\
\hline $\begin{array}{l}\text { Einsatz von QM- } \\
\text { Systemen }\end{array}$ & $\begin{array}{l}\mathrm{n} \\
\text { ja } \\
\text { gepl. } \\
\text { nein }\end{array}$ & $\begin{array}{l}107 \\
18,0 \\
17,0 \\
65,0\end{array}$ & k. A. & $\begin{array}{l}210 \\
30,0 \\
17,1 \\
52,9\end{array}$ & $\begin{array}{l}78 \\
35,9 \\
52,6 \\
11,5\end{array}$ \\
\hline Zertifizierung & $\begin{array}{l}\mathrm{n} \\
\text { ja } \\
\text { gepl. } \\
\text { nein }\end{array}$ & $\begin{array}{r}112 \\
4,0 \\
8,0 \\
88,0 \\
\end{array}$ & k. A. & $\begin{array}{r}204 \\
12,3 \\
7,8 \\
79,9 \\
\end{array}$ & $\begin{array}{l}78 \\
9,0 \\
\text { (ja/geplant) } \\
91,0 \\
\end{array}$ \\
\hline QM-Handbuch & $\begin{array}{l}\mathrm{n} \\
\text { ja } \\
\text { gepl. } \\
\text { nein }\end{array}$ & $\begin{array}{l}113 \\
14,0 \\
35,0 \\
51,0\end{array}$ & k. A. & $\begin{array}{l}203 \\
32,0 \\
33,0 \\
35,0\end{array}$ & $\begin{array}{l}85 \\
29,4 \\
44,7 \\
25,9\end{array}$ \\
\hline $\begin{array}{l}\text { Beteiligung } \\
\text { Mitarbeiterschaft }\end{array}$ & $\begin{array}{l}\mathrm{n} \\
\text { ja } \\
\text { gepl. } \\
\text { nein }\end{array}$ & $\begin{array}{l}116 \\
45,0 \\
42,0 \\
13,0\end{array}$ & $\begin{array}{l}52 \\
32,9 \\
\text { k. A. } \\
32,9\end{array}$ & k. A. & $\begin{array}{l}85 \\
63,5 \\
18,8 \\
17,7\end{array}$ \\
\hline $\begin{array}{l}\text { Aufbau/Einsatz von } \\
\text { Qualitätszirkeln }\end{array}$ & $\begin{array}{l}\mathrm{n} \\
\text { ja } \\
\text { gepl. } \\
\text { nein }\end{array}$ & $\begin{array}{c}111 \\
2,0 \\
27,0 \\
41,0\end{array}$ & k. A. & $\begin{array}{l}207 \\
65,7 \\
10,1 \\
24,2\end{array}$ & $\begin{array}{r}85 \\
72,9 \\
18,8 \\
8,3 \\
\end{array}$ \\
\hline $\begin{array}{l}\text { Benennung/Einsatz } \\
\text { von } \\
\text { Qualitätsbeauftragten }\end{array}$ & $\begin{array}{l}\mathrm{n} \\
\text { ja } \\
\text { gepl. } \\
\text { nein }\end{array}$ & $\begin{array}{l}114 \\
27,0 \\
13,0 \\
60,0\end{array}$ & k. A. & $\begin{array}{r}210 \\
52,3 \\
6,7 \\
41,0 \\
\end{array}$ & $\begin{array}{l}85 \\
34,1 \\
15,3 \\
50,6\end{array}$ \\
\hline
\end{tabular}

\subsubsection{Zusammenfassung und Diskussion der Ergebnisse}

- Gegenüber der EREV-Befragung von August 1998 zeigt sich in den späteren Untersuchungen fast durchgängig und linear eine deutliche Zunahme in der Anwendung der erfragten QM-Maßnahmen und Instrumente. Dies gilt vor allem für den Einsatz von QM-Systemen, Evaluationsverfahren und Qualitätszirkeln. Diese "tools" werden offenbar in steigendem Maße von der Praxis genutzt. 
Ob der diesbezügliche Vorsprung der Jugend- und Sozialhilfe (Studie F) vor der Behindertenhilfe (Studie G) der größeren Untersuchungsaktualität oder arbeitsfeldspezifischen Bedingungen zuzuschreiben ist, lässt sich dabei nicht entscheiden.

○ Während in 1998 die funktionale Aufbau- und Ablaufbeschreibung mittels Organigramm erst von knapp der Hälfte der Einrichtungen geleistet bzw. für zweckmäßig befunden wurde, gehört dieses Instrument schon 2001, mehr noch 2004 zum Standardrepertoire.

- Stetig zunehmend ist auch die Zahl der Einrichtungen, die über ein Leitbild bzw. fixierte Grundsätze zur Qualitätspolitik verfügen. Vielfach dürften 1998 geplante Aktivitäten inzwischen realisiert worden sein.

- Was den Einsatz von QM-Handbüchern anbelangt, ist ein deutlicher Zuwachs in den Kategorien "ja" und "geplant" zu verzeichnen. Unterschiede zwischen Behindertenhilfe und Jugend-/Sozialhilfe sind relativ gering; die größere Aktualität der Studie F spielt anscheinend keine Rolle. Möglicherweise liegen Arbeitsfeldeffekte vor, die mit dem nächsten Befund zusammenhängen.

- Der Anteil der Einrichtungen mit zertifiziertem Qualitätsmanagementsystem nach ISO 9000 ff. wird nämlich im Behindertenbereich mit immerhin 12,3\% angegeben (Ende 2001). Wenngleich auch dort mit rund $80 \%$ die "Nicht-Zertifizierer" dominieren, ist die Ablehnung der in Fachkreisen umstrittenen Zertifizierung (Gerull, 2001, S. 35 ff.) in der Jugend- und Sozialhilfe noch deutlicher ausgeprägt. Da die ISO 9000 ff. die Dokumentation des QM-Systems in einem Handbuch ebenso zwingend vorsieht wie die Ernennung eines "Beauftragten der Leitung", lässt sich die relativ stärkere Verbreitung dieser beiden Instrumente des Qualitätsmanagements in der Behindertenhilfe damit in plausible Verbindung bringen.

Die Annahme einer relativ stärkeren Affinität der Behindertenhilfe zur ISO 9000 ff. bzw. zur Zertifizierung hat im Übrigen damit zu tun, dass Werkstätten für Behinderte sich vielfach als Zulieferer für die Industrie betätigen und von dort einem gewissen Marktdruck ausgesetzt sind, ihre Qualitätsfähigkeit per dargelegtem QM-System nach ISO 9001 bestätigen zu lassen.

○ Während in der Behindertenhilfe - genauer: in den hier repräsentierten 
Wohneinrichtungen der Studie I - Qualitätsbeauftragte in jeder zweiten Einrichtung institutionalisiert sind, verfügt in der Jugend- und Sozialhilfe auch drei Jahre später nur ein Drittel über diese Funktionsstelle. Gegenüber 1998 ist überdies kein spektakulärer Zuwachs zu verzeichnen; bereits damals gab es in 27\% der Fälle eine/n QB. Dies spricht dafür, dass im Unterschied zu etwa Qualitätszirkeln und Evaluationsverfahren das Instrument sein Akzeptanzpotenzial in der Praxis bereits weitgehend ausgeschöpft hat, obwohl alle Einrichtungen mit QB in Studie F die Funktionsstelle mindestens als "eher zweckmäßig" beurteilen (s. Kap. D 4.6.8.8).

Wie a. a. O. dargestellt, haben gut 35\% der befragten Einrichtungen das Thema QB überhaupt noch nicht diskutiert und weitere $15 \%$ noch keine Entscheidung in dieser Frage getroffen; $15 \%$ entschieden sich dagegen.

\subsection{Einstellung zum Qualitätsmanagement, Stellenwertzuschreibung und Akzeptanz}

\subsubsection{Angaben zur Methodik und Stichprobe}

$\mathrm{Zu}$ diesem Fragenkomplex sind die hier einbezogenen Untersuchungen nicht bzw. nur sehr eingeschränkt vergleichbar, da sie auf je spezifische Aspekte zielen. Die Ergebnisse sind deshalb nicht in Form einer Zusammenstellung, sondern getrennt aufgeführt.

In der EREV-Studie von 1998 (Studie G, s. Kap. D 2.1.1) wurde z. B. gefragt: "Wie stehen Sie persönlich zur Diskussion um ein Qualitätsmanagement in der Erziehungshilfe"? Bewertet werden sollten sieben Aussagen auf einer fünfstufigen Skala: 1 = ja, ganz genau, $2=$ stimme weitgehend zu, $3=$ teils-teils, $4=$ stimme überwiegend nicht zu, 5 = nein, ganz und gar nicht.

Peters et al. fokussierten in ihrer 2000 durchgeführten Untersuchung (H, s. Kap. D 2.1.1) auf die Handlungsebene der MitarbeiterInnen und ihre Sicht (Grad der Zustimmung/Ablehnung) der Entwicklungen und Veränderungen im Laufe der Qualitätsdebatte. Weitere Fragen richteten sich auf die Kenntnis und Anwendung von Methoden und Verfahren zur Qualitätsentwicklung.

Bei Wetzler (I, s. Kap. D 2.1.1) standen Ende 2001 "einrichtungsspezifische Bewertungen über den 
Stellenwert des QM-Prozesses und die Bewertung der Einführung von Qualitätsmanagement" (Wetzler, 2003, S. 79) im Blickpunkt. Beurteilt werden sollte nach den Kategorien "hoher/steigender/geringer Stellenwert" bzw. "hohe/steigende/verhaltene Akzeptanz".

\subsubsection{Ergebnisse}

Aus der Studie an Erziehungshilfe-Einrichtungen (Gerull, 1998) sind im Folgenden die Items in Kurzform, die Stichprobengröße (n) sowie die Skalenmittelwerte (M) und absoluten Antwortverteilungen angegeben, geordnet nach dem Grad der Zustimmung zu den Items auf einer fünfstufigen Skala (s. o.).

Tab. 5: Einstellung zum Qualitätsmanagement bei Leitungskräften der Erziehungshilfe (1998)

\begin{tabular}{|l|l|l|l|l|l|l|l|}
\hline Item & $\mathrm{n}$ & $\mathrm{M}$ & $\begin{array}{l}\text { ja, } \\
\text { ganz } \\
\text { genau }\end{array}$ & $\begin{array}{l}\text { weit- } \\
\text { gehend } \\
\text { ja }\end{array}$ & $\begin{array}{l}\text { teils- } \\
\text { teils }\end{array}$ & $\begin{array}{l}\text { über- } \\
\text { wiegd. } \\
\text { nein }\end{array}$ & $\begin{array}{l}\text { nein, } \\
\text { gar } \\
\text { nicht }\end{array}$ \\
\hline $\begin{array}{l}\text { wohlwollend und aufgeschlossen, weil } \\
\text { fachlichen Gewinn und mehr Klarheit für alle } \\
\text { Beteiligten erwartend }\end{array}$ & 115 & 1,94 & 45 & 42 & 21 & 4 & 3 \\
\hline $\begin{array}{l}\text { fühle mich von der Vielfalt der Ansätze, } \\
\text { Begriffe und Konzepte überfordert und } \\
\text { wünsche dringend Orientierungshilfe }\end{array}$ & 112 & 2,59 & 29 & 24 & 34 & 14 & 11 \\
\hline $\begin{array}{l}\text { eher skeptisch und vorsichtig; möchte nicht } \\
\text { vorschnell Zeit und Geld investieren }\end{array}$ & 110 & 3,3 & 13 & 22 & 25 & 19 & 31 \\
\hline $\begin{array}{l}\text { neutral und abwartend; möchte erst erfahren, } \\
\text { welche Maßnahmen sich anderenorts als } \\
\text { zweckmäßig erweisen }\end{array}$ & 112 & 3,4 & 13 & 23 & 18 & 22 & 36 \\
\hline $\begin{array}{l}\text { fühle mich nicht informiert genug, um Sinn } \\
\text { und Notwendigkeit eines QM in der } \\
\text { Erziehungshilfe beurteilen zu können }\end{array}$ & 112 & 3,54 & 8 & 17 & 25 & 31 & 31 \\
\hline $\begin{array}{l}\text { halte Diskussion für zu aufgebläht und } \\
\text { theoretisch; Praxis hat weder Zeit noch } \\
\text { Veranlassung, sich damit intensiv zu befassen }\end{array}$ & 112 & 3,83 & 5 & 7 & 29 & 32 & 39 \\
\hline $\begin{array}{l}\text { ablehnend; "viel Lärm um nichts"; Sach- und } \\
\text { Personalmittel können sinnvoller eingesetzt } \\
\text { werden }\end{array}$ & 111 & 4,29 & 3 & 7 & 15 & 16 & 70 \\
\hline
\end{tabular}

In der Untersuchung von Peters et al. ${ }^{7}$ wurden Leitungskräfte von Einrichtungen nach dem Grad ihrer Zustimmung/Ablehnung zu QM-bezogenen Statements befragt (sehr zutreffend - zutreffend - weniger zutreffend - gar nicht zutreffend). Die Ergebnisse in absoluten und relativen Zahlen sind in nachstehender Tabelle zusammengefasst.

7 Rohunterlagen wurden freundlicherweise von Peters zur Verfügung gestellt vor Veröffentlichung des ProjektAbschlussberichts im Internet. 
Tab. 6: Einstellung zu QM bezogenen Statements bei Leitungskräften der Erziehungshilfe (2000)

\begin{tabular}{|c|c|c|c|c|c|c|c|c|}
\hline \multirow[t]{2}{*}{ Item } & \multicolumn{2}{|c|}{ sehr zutreffend } & \multicolumn{2}{|c|}{ zutreffend } & \multicolumn{2}{|c|}{ weniger zutreffd. } & \multicolumn{2}{|c|}{ gar nicht zutreffd. } \\
\hline & $\mathrm{n}$ & $\%$ & $\mathrm{n}$ & $\%$ & $\mathrm{n}$ & $\%$ & $\mathrm{n}$ & $\%$ \\
\hline $\begin{array}{l}\text { Möglichkeit des } \\
\text { Missbrauchs für } \\
\text { Fremdkontrolle }\end{array}$ & 7 & 8,9 & 15 & 19 & 47 & 59,5 & 10 & 12,7 \\
\hline $\begin{array}{l}\text { schafft Klima des } \\
\text { Vertrauens zwischen } \\
\text { Leitung, Auftraggebern } \\
\text { und Kunden }\end{array}$ & 31 & 39,2 & 35 & 44,3 & 11 & 13,9 & 2 & 2,5 \\
\hline $\begin{array}{l}\text { übertriebene } \\
\text { Datensammlung führt } \\
\text { zu gläsernen } \\
\text { Institutionen }\end{array}$ & 11 & 13,9 & 28 & 35,4 & 32 & 40,5 & 7 & 8,9 \\
\hline $\begin{array}{l}\text { Quantifizierungstendenz } \\
\text { führt zur Dominanz } \\
\text { betriebswirtschaftlicher } \\
\text { Interessen }\end{array}$ & 12 & 15,2 & 27 & 34,2 & 32 & 40,5 & 7 & 8,9 \\
\hline $\begin{array}{l}\text { Fachlichkeit wird durch } \\
\text { Qualitätsentwicklung } \\
\text { erhöht }\end{array}$ & 27 & 34,2 & 41 & 40,5 & 10 & 12,7 & 7 & 8,9 \\
\hline $\begin{array}{l}\text { Qualitätsstandards } \\
\text { verkennen die } \\
\text { Nichtnormierbarkeit } \\
\text { pädagogischer Prozesse } \\
\end{array}$ & 5 & 6,3 & 15 & 19 & 48 & 60,8 & 11 & 13,9 \\
\hline $\begin{array}{l}\text { Ziele von QM führen zu } \\
\text { mehr Sozialqualität }\end{array}$ & 24 & 30,4 & 41 & 51,9 & 14 & 17,7 & 0 & 0 \\
\hline $\begin{array}{l}\text { QM führt zu besserem } \\
\text { Image }\end{array}$ & 20 & 25,3 & 42 & 53,2 & 13 & 16,5 & 3 & 3,8 \\
\hline $\begin{array}{l}\text { Beurteilung von } \\
\text { Pädagogik durch } \\
\text { Kennziffern weitgehend } \\
\text { unmöglich }\end{array}$ & 9 & 11,4 & 28 & 35,4 & 36 & 45,6 & 5 & 6,3 \\
\hline $\begin{array}{l}\text { QM erhöht } \\
\text { Wirtschaftlichkeit und } \\
\text { Effektivität }\end{array}$ & 15 & 19 & 41 & 51,9 & 20 & 25,3 & 3 & 3,8 \\
\hline $\begin{array}{l}\text { QM führt zu mehr } \\
\text { Transparenz und } \\
\text { Verbindlichkeit }\end{array}$ & 25 & 31,6 & 45 & 57 & 6 & 7,6 & 3 & 3,8 \\
\hline $\begin{array}{l}\text { QM führt zu mehr } \\
\text { Konkurrenz und lenkt } \\
\text { ab von pädagogischen } \\
\text { Fragen }\end{array}$ & 4 & 5,1 & 14 & 17,7 & 46 & 58,2 & 15 & 19 \\
\hline $\begin{array}{l}\text { QM führt zu Absicherg. } \\
\text { und Verbesserung der } \\
\text { Wettbewerbs- und } \\
\text { Marktsituation }\end{array}$ & 13 & 16,5 & 46 & 58,2 & 20 & 25,3 & 0 & 0 \\
\hline
\end{tabular}

Durchgängig zeigt sich in der Studie von Peters et al. eine deutliche Mehrheit zustimmender Antworten auf jene Items, die Qualitätsmanagement/Qualitätsentwicklung positiv charakterisieren: 

zutreffend),

○ Vertrauensbildung: $83,5 \%$,

- mehr Sozialqualität: $82,3 \%$,

- besseres Image: $78,5 \%$,

- bessere Wettbewerbs- und Marktsituation: 74,7\%,

○ erhöhte Fachlichkeit: 74,7\%,

○ erhöhte Wirtschaftlichkeit und Effektivität: 70,9\%,

○ Quantifizierungstendenz: 49,4\%,

○ übertriebene Datensammlung: $49,3 \%$

○ Kennziffern: $46,8 \%$,

- Fremdkontrolle: $27,8 \%$,

○ Nichtnormierbarkeit päd. Prozesse: 25,3\%,

○ mehr Konkurrenz: 22,8\%.

Die Ergebnisse aus den Wohneinrichtungen der Behindertenhilfe (Wetzler, 2003, S. 79 ff.) liegen lediglich als Prozentangaben vor:

- Aus Sicht der Leitungen sprechen $63,4 \%$ der befragten Einrichtungen Qualitätsmanagement einen hohen Stellenwert innerhalb der Organisation zu, insbesondere zur Weiterentwicklung der fachlichen wie strukturellen Standards.

- 25,4\% der Einrichtungsleitungen weisen dem QM-System einen steigenden Stellenwert zu; die Möglichkeiten würden erst sukzessive erkannt.

- Lediglich 7,1\% der Antworten lassen sich der Kategorie "geringer Stellenwert" zuordnen, da die bisherigen Anstrengungen keinen erkennbaren Effekt auf das Tagesgeschäft aufweisen und lediglich auf die veränderten gesetzlichen Rahmenbedingungen reagiert worden sei.

- 4,1\% der Einrichtungen nehmen weder eine konstruktive noch kritische Position ein, sondern antworten undifferenziert. 
45,6\% der Einrichtungsleitungen sprechen nach der Einführung des QM-Systems von "hoher Akzeptanz" auf Seiten der Mitarbeiterschaft.

○ Weitere 35,5\% der befragten Einrichtungen sprechen von einer "steigenden Akzeptanz" nach eher skeptischem Abwarten zu Beginn, wobei Rationalisierungsängste, Zeitaufwand und Sorge um Formalismus und bürokratischen Aufwand genannt werden.

- 19,1\% der Einrichtungen verweisen im Zusammenhang mit den zuvor erwähnten Faktoren auf eher "verhaltene Akzeptanz"; vor allem kleinere Einrichtungen bringen das Argument von erheblichem Aufwand und geringem Effekt ins Spiel.

○ Durchgängig von den Einrichtungen der Behindertenhilfe bemängelt werden fehlende Kooperation mit den Kosten- bzw. Leistungsträgern und deren offensichtliches Desinteresse, abgesehen von vermeintlichen Kosteneinsparungen.

\subsubsection{Zusammenfassung und Diskussion der Ergebnisse}

○ Die Einstellung von Leitungskräften sowohl aus dem Jugendhilfe- als auch dem Behindertenhilfebereich gegenüber einem Qualitätsmanagement lässt sich als weit überwiegend positiv charakterisieren.

Lediglich 7,1\% von 211 Wohneinrichtungen der Behindertenhilfe (also 15, Wetzler gibt keine absoluten Zahlen an), äußern sich aufgrund der gemachten Erfahrungen kritisch zum Verhältnis von Aufwand und Ertrag. In der gut drei Jahre zuvor durchgeführten Befragung von Leitungskräften aus Erziehungshilfeeinrichtungen stimmen nur 10 Personen (von $111=9 \%$ ) der Aussage völlig oder weitgehend zu, dass "viel Lärm um nichts" gemacht werde und "Sach- und Personalmittel sinnvoller eingesetzt" werden könnten (Gerull, 1998, S. 281). In der Studie von Peters et al. werden zwar durchaus Risiken betont - vor allem eine Dominanz betriebswirtschaftlicher Interessen und eine "gläserne Institution" infolge übertriebener Datensammlung -, insgesamt ist das Thema QM jedoch eindeutig positiv konnotiert. 
- Vergleicht man diese Zahlen mit den Ergebnissen aus Mitarbeiterbefragungen (siehe Kap. D 1.), so lässt sich zu allen Untersuchungszeitpunkten eine erheblich positivere Einstellung der Leitungskräfte zum Qualitätsmanagement im Vergleich zur "Basis" konstatieren.

So stimmen in den durchgeführten Studien von Gerull bzw. Peters et al. durchschnittlich nur 43\% der MitarbeiterInnen der Aussage zu, dass QM das Vertrauen zwischen den Kooperationspartnern befördere, während dieser Anteil unter Leitungskräften bei über $83 \%$ liegt. Eine Stärkung der Wettbewerbssituation erwarten im Mittel immerhin 57\% der MitarbeiterInnen, jedoch fast 75\% der Leitungskräfte; eine Erhöhung der Fachlichkeit durch Qualitätsentwicklung/Qualitätsmanagement wird von gut $50 \%$ der MitarbeiterInnen und rund $75 \%$ der Leitungskräfte angenommen.

- Einerseits dürfte sich darin die Affirmation einer Praxis widerspiegeln, welche ja nicht zuletzt durch Initiative von Leitungskräften zustande kam und kommt, in deren Zuständigkeitsbereich derartige Strategieentscheidungen naturgemäß fallen. In diesem Zusammenhang ist vermutlich auch die größere Bereitschaft von Leitungs- und Beratungskräften $\mathrm{zu}$ bewerten, sich aktiv in Sachen QM weiterzubilden und entsprechende Funktionen auszuüben (s. Kap. D 1.1.3).

- Andererseits kommt darin der Sachverhalt zum Ausdruck, dass in vielen Einrichtungen die Beteiligung der Mitarbeiterschaft im Sinne eines "Sich-gemeinsam-auf-den-WegMachens" nicht annähernd so gut zu gelingen scheint wie die vollmundige Verkündigung hehrer Ziele des Qualitätsmanagements seitens der Leitungsorgane (vgl. Gerull, 2001, S. 23ff.). Auch Wetzler (2003, S. 91f) moniert eine große "Lücke im Informationsmanagement" und betont die Einbeziehung aller Beteiligten: "Denn: Qualität lässt sich nicht verordnen." Peters et al. berichten, dass in den von ihnen befragten Einrichtungen Klienten/Betroffene bei der Erarbeitung des Qualitätsentwicklungskonzepts überhaupt keine Rolle zu spielen scheinen.

- Sowohl in der Stichprobe von Wetzler als auch in der von Gerull waren die größeren bzw. volldifferenzierten Einrichtungen überrepräsentiert. Darin spiegelt sich die Tatsache, dass dort die Bemühungen um Qualitätsmanagement zunehmend "zum 
Standardprogramm gehören" (Wetzler, 2003, S. 36), während kleinere Einrichtungen eher zurückhaltend sind und in der Regel geringere Ressourcen einsetzen können. 


\section{Befunde aus Befragungen von Qualitätsbeauftragten}

\subsection{Angaben zur Methodik und Stichprobe}

Im Rahmen von Akademiekursen des Deutschen Vereins für öffentliche und private Fürsorge, die 2002 und 2003 für werdende oder bereits aktive Qualitätsbeauftragte (QB) in der Jugend- und Sozialhilfe veranstaltet wurden, führte der Autor jeweils Befragungen zur Rollenwahrnehmung als Funktionsträger durch. Sieben vorgegebene Typisierungen sollten in eine Rangordnung gebracht werden, die dem eigenen Selbstverständnis als QB am besten entsprach. Auch in der Hauptstudie von 2004 spielten diese Fragen eine Rolle. Zum Zwecke des Vergleichs werden die entsprechenden Ergebnisse bereits an dieser Stelle beschrieben. Ausführlichere Befunde zum Fragenkomplex QB sind in Kap. D 4.6.8 dargestellt.

Die in 2002 und 2003 verwendeten Items lauteten:

"Als Qualitätsbeauftragter verstehe ich mich in erster Linie als ..." (Bitte Rangplätze von 1 bis 7 zuordnen!):

○ Koordinator und Beobachter in Sachen Qualität (Typus Controller)

- Treiber und Werber in Sachen Qualität (Typus Promotor)

- Informationsdienstleister und Protokollführer in Sachen Qualität (Typus Sekretär)

- Prozessbegleiter und Verfahrenshüter in Sachen Qualität (Typus Moderator)

- Prüfer und Beurteiler in Sachen Qualität (Typus Auditor)

- Berater und Fortbildner in Sachen Qualität (Typus Experte)

- Anlaufstelle und Vertrauensperson in Sachen Qualität (Typus Delegierter)

$\mathrm{Zu}$ ansonsten inhaltlich unveränderten Items wurde in der Studie von 2004 (F, s. Kap. D 1.4.1) abweichend gefragt: "Wie gut charakterisieren die folgenden Typisierungen Ihre eigene Rollenwahrnehmung, also die Art, wie Sie Ihre Rolle verstehen?" Die Antworten waren auf einer sechsstufigen Skala zu differenzieren:

$1=\mathrm{ja}$, ganz genau charakterisiert; 2 = ja, ziemlich genau charakterisiert; 3 = eher zutreffend charakterisiert; 4 = eher unzutreffend charakterisiert; $5=$ nein, ziemlich unzutreffend charakterisiert; $6=$ nein, völlig 
unzutreffend charakterisiert.

Analog wurden in derselben Studie Leitungskräfte aus Einrichtungen mit QB gefragt: "Wie können die Erwartungen, die in Ihrer Einrichtung an die Rolle des/der QB gestellt werden, am besten charakterisiert werden?"

Die Stichproben und Datenbestände zu diesem Teilkomplex der Studie umfassen somit:

○ 22 TeilnehmerInnen eines Akademiekurses für Qualitätsbeauftragte, Befragungszeitpunkt Oktober 2002, Fachkräfte der Jugend- und Sozialhilfe, Rücklaufquote $100 \%$; Rangwerte;

- 19 TeilnehmerInnen eines Akademiekurses für Qualitätsbeauftragte, Befragungszeitpunkt November 2003, Fachkräfte der Jugend- und Sozialhilfe, Rücklaufquote $100 \%$; Roh- und Rangwerte;

- 31 (davon zwei angehende) Qualitätsbeauftragte aus 85 Einrichtungen und Diensten der Jugend- und Sozialhilfe, Befragungszeitraum Februar bis Mai 2004; Rücklaufquote nicht ermittelbar, da QB-Anteil an der Stichprobe nicht bekannt; Roh-, Rang- und Mittelwerte, Standardabweichungen;

○ 13 Leitungskräfte aus 17 Einrichtungen der Jugend- und Sozialhilfe mit Qualitätsbeauftragten, Befragungszeitraum Februar bis Mai 2004, Rücklaufquote nicht ermittelbar, da QB-Anteil an der Stichprobe ( $\mathrm{n}=85$, s. o.) nicht bekannt; Roh-, Rangund Mittelwerte, Standardabweichungen.

\subsection{Ergebnisse}

Dargestellt sind zunächst die Gesamt-Rangwerte der Items in den verschiedenen Stichproben von Qualitätsbeauftragten und Leitungskräften sowie die mittleren Ränge (Gesamtränge) über alle Stichproben. Für die Stichproben von 2004 wurden dazu die Mittelwerte (s. Tab. 8) in Rangwerte verwandelt. 
Tab. 7: Qualitätsbeauftragte: präferierte Rollenwahrnehmungen (Selbstbild) und Rollenerwartungen (Fremdbild) in verschiedenen Gruppen (nach Rangwerten)

\begin{tabular}{|l|l|l|l|l|l|}
\hline Typus & $\begin{array}{l}\text { Gruppe } \\
(\text { Selbstbild) } \\
(2002, \mathrm{n}=22)\end{array}$ & $\begin{array}{l}\text { Gruppe } \\
(\text { Selbstbild) } \\
(2003, \mathrm{n}=19)\end{array}$ & $\begin{array}{l}\text { Gruppe } \\
(\text { Selbstbild) } \\
(2004, \mathrm{n}=31)\end{array}$ & $\begin{array}{l}\text { Gruppe } \\
(\text { Fremdbild) } \\
(2004, \mathrm{n}=13)\end{array}$ & Gesamt-Rang \\
\hline Controller & 5 & 3 & 5 & 2 & 4 \\
\hline Promotor & 2 & 4 & 3 & 5 & 3 \\
\hline Sekretär & 6 & 7 & 7 & 6 & 7 \\
\hline Moderator & 3 & 1 & 1 & 1 & 1 \\
\hline Auditor & 7 & 5 & 6 & 7 & 6 \\
\hline Experte & 1 & 2 & 2 & 3 & 2 \\
\hline Delegierter & 4 & 6 & 4 & 4 & 5 \\
\hline
\end{tabular}

Ein zusätzlicher Vergleich (Studie F) der Rollenwahrnehmungen von QB (Selbstbild) und Rollenerwartungen von Leitungskräften (Fremdbild) anhand der Mittelwerte und Häufigkeitsverteilungen in den verschiedenen Antwortkategorien ist nachstehend dargestellt. Die Signifikanzprüfung wurde dabei auf der Basis der Häufigkeiten vorgenommen, also nicht der Mittelwerte (Chi-Quadrat-Tests, $\mathrm{p}=$ Signifikanz). In Tab. 9 ist zusätzlich der Kontingenzkoeffizient angegeben, der als nichtparametrisches Korrelationsmaß die Stärke der Wechselbeziehung zwischen zwei mehrklassigen qualitativen Variablen und die relative Größe der gemeinsamen Varianzanteile ausdrückt.

Tab. 8: QB-Rollentypisierungen im Selbst- und Fremdbild (Mittelwerte)

\begin{tabular}{|l|l|l|l|l|l|l|l|}
\hline Stichprobe & $\begin{array}{l}\text { Con- } \\
\text { troller }\end{array}$ & Promotor & Sekretär & $\begin{array}{l}\text { Mode- } \\
\text { rator }\end{array}$ & Auditor & Experte & $\begin{array}{l}\text { Dele- } \\
\text { gierter }\end{array}$ \\
\hline QB $(\mathrm{n}=31)$ & 2,97 & 2,58 & 3,81 & 2,26 & 3,48 & 2,57 & 2,94 \\
\hline Leitung $(\mathrm{n}=13)$ & 2,42 & 2,85 & 3,15 & 1,85 & 3,62 & 2,54 & 2,62 \\
\hline $\mathrm{p}$ & .049 & .608 & .149 & .038 & .338 & .423 & .700 \\
\hline
\end{tabular}

Anmerkung:

Die angegebenen Signifikanzen beziehen sich auf zweiseitige Abweichungswerte und sind bei einseitiger Hypothesenprüfung zu halbieren. Ungeachtet dessen sind auf dem 5\%-Niveau die Häufigkeitsunterschiede in den Antworten zu Controller und Moderator signifikant. 
Tab. 9: QB-Rollentypisierungen im Selbst- und Fremdbild (Häufigkeitsverteilungen und Zusammenhangsmaß)

\begin{tabular}{|c|c|c|c|c|c|c|c|c|c|c|c|c|c|}
\hline \multirow{3}{*}{ Typus } & \multicolumn{6}{|c|}{$\mathrm{QB}(\mathrm{n}=31 / 30)$} & \multicolumn{6}{|c|}{ Leitung $(n=13 / 12)$} & \multirow{2}{*}{$\begin{array}{l}\text { Kontin- } \\
\text { genzko- } \\
\text { effizient }\end{array}$} \\
\hline & \multicolumn{6}{|c|}{ Antworten pro Skalenstufe } & \multicolumn{6}{|c|}{ Antworten pro Skalenstufe } & \\
\hline & 1 & 2 & 3 & 4 & 5 & 6 & 1 & 2 & 3 & 4 & 5 & 6 & \\
\hline Controller & 0 & 13 & 10 & 5 & 2 & 1 & 3 & 4 & 4 & 0 & 0 & 1 & $.453 \mathrm{~s}$. \\
\hline Promotor & 4 & 9 & 15 & 2 & 1 & 0 & 3 & 5 & 3 & 1 & 1 & 0 & .241 \\
\hline Sekretär & 2 & 4 & 8 & 5 & 8 & 4 & 0 & 5 & 5 & 1 & 0 & 2 & .395 \\
\hline Moderator & 5 & 15 & 9 & 2 & 0 & 0 & 7 & 3 & 2 & 0 & 1 & 0 & $.432 \mathrm{~s}$. \\
\hline Auditor & 0 & 6 & 10 & 10 & 4 & 1 & 1 & 1 & 2 & 7 & 2 & 0 & .338 \\
\hline Experte & 3 & 1 & 13 & 2 & 1 & 0 & 4 & 4 & 3 & 1 & 1 & 0 & .288 \\
\hline Delegierter & 5 & 5 & 14 & 2 & 4 & 1 & 4 & 3 & 3 & 1 & 1 & 1 & .253 \\
\hline$\sum$ Antworten & 19 & 53 & 79 & 28 & 20 & 7 & 22 & 25 & 22 & 11 & 6 & 4 & \\
\hline
\end{tabular}

Anmerkung:

s. $=$ signifikant $(\alpha=.05)$

Zwischen den qualitativen Variablen des QB-Rollentypus (sieben Klassen) und der Personengruppe (zwei Klassen: QB und Leitung) bestehen punktuell signifikante Zusammenhänge: Die Typen Controller und Moderator kovariieren mit der Personengruppe. Die relativen Anteile gemeinsamer Varianz betragen über 40\% (Kontingenzkoeffizienten .453 bzw. .432, s. Tab. 9).

Zusätzlich geprüft wurde an der Stichprobe von Qualitätsbeauftragten, ob die Rollenwahrnehmungen geschlechtsspezifisch differieren. Auf der Basis der Skalenmittelwerte wurde dabei ein Einfluss des Geschlechts nur hinsichtlich des Typus Delegierte/r festgestellt. Während weibliche QB im Mittel einen Wert von 2,4 zuweisen, urteilen Männer mit 3,3 deutlich ablehnender $(\mathrm{t}=-2,114 ; \mathrm{df}=28 ; \mathrm{p}=.044)$.

\subsection{Zusammenfassung und Diskussion der Ergebnisse}

- Die Rangordnungen der Rollenwahrnehmungen und -erwartungen fallen insgesamt recht homogen aus. Sowohl Qualitätsbeauftragte als auch Leitungskräfte charakterisieren den Aufgabentypus der Funktionsstelle QB in ähnlicher Weise. Augenfällige Unterschiede in den Rangordnungen betreffen die höhere Gewichtung des Controller-Typus seitens der Leitungskräfte und deren etwas geringere Bewertung für die Typen Promotor und Experte. 
○ Signifikant auf dem 5\%-Niveau sind die höheren Gewichtungen des Controller- und des Moderator-Typus seitens der Leitungskräfte. Bezüglich des Ersteren bestätigt sich damit die zuvor erwähnte Rangdifferenz. Eine solche tritt in Bezug auf den ModeratorTypus nicht auf, da beide Gruppen diesem den ersten Rang zuweisen; der Mittelwertsvergleich (Tab. 8) und die Antwortverteilungen (Tab. 9) enthüllen allerdings, dass diese Positionierung auf Seiten der Leitungskräfte pointierter ausfällt.

- Nicht signifikant und im Rangvergleich auch nicht augenfällig ist die deutliche Mittelwertsdifferenz bezüglich des Typus Sekretär. Die Inspektion der Antwortverteilungen zeigt ein Übergewicht der Qualitätsbeauftragten in den Kategorien "ziemlich/völlig unzutreffend". Während Leitungskräfte eher moderate Zustimmung ausdrücken, verteilen sich die Reaktionen der QB über die gesamte sechsstufige Skala.

- Deutlich wird, dass sich das favorisierte Selbstverständnis der aus unterschiedlichen Praxisfeldern der Sozialen Arbeit rekrutierten Qualitätsbeauftragten weniger als "Mandatsträger" mit den Funktionen Zuarbeiten/Prüfen/Koordinieren/Berichten, sondern eindeutig als "Qualitäts-Profi" mit Funktionen wie Moderieren/Beraten/Promoten charakterisieren lässt. Die Annahme liegt nahe, dass diese mit fachlicher Autonomie assoziierte Rollenzuschreibung dem professionellen Selbstwertgefühl dienlicher ist als die Attribuierung stärker instrumentell ausgerichteter Funktionen.

$\mathrm{Ob}$ es sich hierbei um eine Idealisierung handelt oder eine Einschätzung, die dem realen Handlungsspielraum und Tätigkeitsprofil angemessen ist oder nicht, kann auch aufgrund von weiteren Ergebnissen aus der Hauptstudie nicht klar beurteilt werden. Auf den ersten Tätigkeitsrängen liegen jedenfalls überwiegend Dokumentationsaufgaben (s. Kap. D 4.6.8.6).

- Allerdings sind die Reaktionen sehr individuell, wie sich an der Antwortverteilung zum Typus Sekretär erweist (s. o.). Während Leitungskräfte mehrheitlich ihre Rollenerwartungen auch durch diesen Typus eher zutreffend charakterisiert sehen, antworten Qualitätsbeauftragte disparat, mit einem Übergewicht ablehnender Antworten. Vermutlich drückt sich darin die verschieden ausgeprägte Konnotation des Sekretär-Begriffs mit subalternen Assistenzfunktionen aus, mit deren Zuschreibung Leitungskräfte mutmaßlich weniger Probleme haben als die Rollenträger selbst. 
- Der signifikante geschlechtsspezifische Unterschied in der Bewertung des DelegiertenTypus kann mit gebotener Vorsicht so interpretiert werden, dass Frauen sich mit der entsprechend charakterisierten Rolle, Anlaufstelle und Vertrauensperson in Sachen Qualität zu sein, leichter anfreunden können als Männer.

○ Im Übrigen zeigen die Ergebnisse, dass sowohl QB als auch Leitungskräfte die Rolle als "multifunktional" beschreiben. Die Aufgaben von QB werden nicht eindimensional charakterisiert - z. B. schwerpunktmäßig als Controller oder als Auditor -, sondern durch Zuschreibungen, die komplementär, nicht exkludierend verstanden werden. Dass auf diese Weise z. B. der (prozessorientierte) Moderator-Typus im Durchschnitt favorisiert wird, zugleich aber die mittlere Wertschätzung für den (ergebnisorientierten) Experten kaum geringer ausfällt, ist deshalb kein Widerspruch. Die verwendeten Typisierungen wurden ja auch auf der Basis existierender Stellenbeschreibungen konstruiert und spiegeln insofern die Vielfalt der in praxi vorhandenen und nicht selten konfligierenden Anforderungsmuster wider. 


\section{Befunde einer Befragung von Einrichtungen und Diensten (Hauptstudie)}

\subsection{Bestimmung der Grundgesamtheiten und Quotierung der Stichproben}

Zielgruppen und verfügbare Untersuchungseinheiten, Fragestellungen und Hypothesen wurden bereits in Kap. A 5.3 beschrieben. An dieser Stelle sollen ergänzend forschungspraktische Aspekte erörtert werden, welche die Übertragbarkeit oder Generalisierung der Ergebnisse von den befragten Stichproben auf die jeweilige Grundgesamtheit, m. a. W. die externe Validität ${ }^{8}$ der Studie tangieren.

Um gemäß der klassischen Konzeption von Campbell \& Stanley (1973) extern valide zu sein, bedarf es zufälliger Stichproben aus realen oder fiktiven Populationen, so dass jedes Element der Grundgesamtheit die gleiche Wahrscheinlichkeit aufweist, in die Stichprobe aufgenommen zu werden. Hager (2004, S. 31f) weist jedoch darauf hin, dass in der Forschung kaum mit Zufalls-, sondern zumeist mit Gelegenheitsstichproben operiert werde, weil es selten gelinge, alle MerkmalsträgerInnen einer wohl-definierten Population zur Mitarbeit an einem Versuch zu bewegen. Der Aspekt der externen Validität wird als häufig überbewertet bezeichnet (Hager \& Spies, 1991, S. 40).

Einige Beschränkungen, was die angestrebte Repräsentativität der untersuchten Stichproben anbetrifft, waren in der vorliegenden Studie unvermeidlich. Die reale Population, aus der ggf. eine Zufallsstichprobe hätte gezogen werden können, war nämlich nur teilweise bekannt. So lagen z. B. keine Informationen darüber vor, wie viele Einrichtungen und Dienste der jeweiligen Zielgruppe die Funktionsstelle des/der Qualitätsbeauftragten überhaupt besetzt hatten. Auch gab es keine zugänglichen Unterlagen darüber, wie sich die einbezogenen Arbeitsfelder und Träger hinsichtlich der für hypothesenrelevant gehaltenen Variable der Einrichtungsgröße zusammensetzten.

Im Vorfeld war deshalb nicht für alle unabhängigen Variablen festzulegen, wie viele der

8 Wie Hager (2004, S. 31) unter Bezugnahme auf Westermann (2000) betont, ist die externe Validität kein Gütemerkmal des psychologischen Experiments mehr, weil sie nicht nur von den methodischen Untersuchungseigenschaften selbst abhänge, sondern auch von den empirischen Regelmäßigkeiten im betrachteten Phänomenbereich und den intendierten Auswirkungen, auf die generalisiert werden solle. Während in der klassischen Sichtweise der Signifikanztest beim Vorliegen von Zufallsstichproben als Mittel zum Inferenzschluss (Generalisierung) von wenigen Stichprobendaten auf die vielen nichtuntersuchbaren Daten einer oder mehrerer Population/en fungiere, verneint Hager (a.a.O., S. 32) die Frage, ob die psychologische Forschung überhaupt Zufallsstichproben brauche. 
Untersuchungseinheiten welcher Faktorstufe und -kombination zugehörig sein mussten, um auf statistisch verlässlicher Grundlage beurteilen zu können, ob die Stichprobe für die Population und der Rücklauf für die Stichprobe bezüglich dieser Variablen repräsentativ war. Als maßgebliches Kontrollmerkmal, hinsichtlich dessen die Untersuchungsstichprobe so gut wie möglich zu quotieren war, kam allein die Trägerschaft in Betracht.

Bei der geschichteten Stichprobe (Quotaverfahren) wird die Grundgesamtheit in möglichst homogene Merkmalsgruppen (hier: Trägerschaften) aufgeteilt, die entweder gemäß ihrem Anteil an der Grundgesamtheit in der Stichprobe vertreten sind (feste Anteile) oder je nach Homogenität der betreffenden Schicht mit variablen Anteilen (Häcker \& Stapf, 1998, S. 838). Der diesbezüglichen Planbarkeit waren in der vorliegenden Studie praktische Grenzen gesetzt.

In nachstehender Tabelle ist zunächst die Verteilung bundesdeutscher Einrichtungen der Kinder- und Jugendhilfe nach Art und Trägerschaft zum Ende 2002 dokumentiert. Auf der Grundlage dieser und weiterer Zahlen (Tab. 11) war es möglich, für die maßgeblichen (Teil-)Populationen der Untersuchung die Frage zu prüfen, inwieweit die Stichproben die Verhältnisse in den (Teil-)Grundgesamtheiten angemessen widerspiegeln. 
Tab. 10: Einrichtungen der Kinder- und Jugendhilfe in Deutschland zum 31.12.2002

\begin{tabular}{|c|c|c|}
\hline Einrichtungsart & $\begin{array}{l}\text { Gesamtzahl der } \\
\text { Einrichtungen }\end{array}$ & $\begin{array}{l}\text { Einrichtungen der } \\
\text { Erziehungshilfe }^{9}\end{array}$ \\
\hline Kinderkrippen & 798 & \\
\hline Kindergärten & 27830 & \\
\hline Horte & 3469 & \\
\hline $\begin{array}{l}\text { Tageseinrichtungen mit alterseinheitlichen } \\
\text { tersgemischten Gruppen }\end{array}$ & 15182 & \\
\hline Tageseinrichtungen insgesamt & 47279 & \\
\hline $\begin{array}{l}\text { Sonstige Einrichtungen (Heimerziehung, Jugendarbeit, } \\
\text { Frühförderung, Familienferienstätten, Jugendherbergen, } \\
\text { Beratungsstellen } ш . \text { _.. ohne Jugendhilfeverwaltung) }\end{array}$ & 28286 & 7346 \\
\hline freie Träger & 20818 & $6796(100 \%)$ \\
\hline Arbeiterwohlfahrt & 1170 & $441(6,49 \%)$ \\
\hline Deutscher Paritätischer WV & 2792 & $1363(20,06 \%)$ \\
\hline Deutsches Rotes Kreuz & 439 & $203(2,99 \%)$ \\
\hline Diakonisches Werk/sonst. ev. Träger & 4846 & $1827(26,88 \%)$ \\
\hline Caritasverband/sonst. kath. Träger & 4435 & $917(13,49 \%)$ \\
\hline Wirtschaftsunternehmen & 813 & $613(9,02 \%)$ \\
\hline davon VPK (private Träger, Stand 2/2004) & $(363)$ & $(5,34 \%)$ \\
\hline Sonst. jur. Personen, andere Vereinigungen & 4563 & $1380(20,31 \%)$ \\
\hline Sonstige & 1760 & $52(0,77 \%)$ \\
\hline öffentliche Träger & 7468 & $550(100 \%)$ \\
\hline $\begin{array}{l}\text { Einrichtungen/Geschäftsstellen } \\
\text { der Jugendhilfeverwaltung }\end{array}$ & 3010 & \\
\hline Gemeinden/Gemeindeverbände ohne Jugendamt & 206 & \\
\hline Jugendämter $^{10}$ & 749 & \\
\hline Landesjugendämter & 16 & \\
\hline Oberste Landesjugendbehörde(n) & 12 & \\
\hline Geschäftsstellen eines Trägers der freien Jugendhilfe & 1711 & \\
\hline $\begin{array}{l}\text { Arbeitsgemeinschaften und sonstige Zusammenschlüsse } \\
\text { von Trägern der Jugendhilfe }\end{array}$ & 316 & \\
\hline
\end{tabular}

Anmerkung:

Unterstellt wird, dass die Zahlen für den VPK in den Wirtschaftsunternehmen enthalten sind.

Quellen:

Statistisches Bundesamt, Statistik der Kinder- und Jugendhilfe, Teil III.2, Pressemitteilungen vom 18.12.2003 und 28.01.2004; VPK-Bundesgeschäftsstelle, Mitteilung vom 06.02.2004; eigene Internet-Recherchen

Die Grundgesamtheit der freien Träger der Erziehungshilfe umfasst nach vorstehender Tabelle 6.796 Einheiten (613 Wirtschaftsunternehmen, 6.183 freigemeinnützige und sonstige).

9 Hier definiert als stationäre und teilstationäre Leistungen nach § 27ff. SGB VIII, also ohne ausschließlich ambulante Leistungen nach $\S$ 28-31 (Erziehungsberatung, Soziale Gruppenarbeit, Erziehungsbeistand/Betreuungshelfer,Sozialpädagogische Familienhilfe). Die Zahlen stimmen aufgrund unterschiedlicher Systematik der Einrichtungsarten nur näherungsweise überein mit eigenen Angaben der Verbände.

10 Ziegenhorn (2004) beziffert die Zahl der Jugendämter in Deutschland mit 938 (www.ziegenhorn.com); allerdings dürften darunter auch die Gemeinden/Gemeindeverbände ohne Jugendamt subsumiert worden sein, welche die Jugendhilfeverwaltung anders organisieren. Die Summen beider Kategorien aus der Tabelle entsprechen jedenfalls mit 955 nahezu den Zahlen von Ziegenhorn. 
Zur Grundgesamtheit der örtlichen Jugendhilfeverwaltungen gehören 749 Jugendämter und 206 Gemeinden/Gemeindeverbände ohne Jugendamt.

Die entsprechenden Zahlen über die Grundgesamtheit der örtlichen Sozialhilfeverwaltungen entstammen einer von Berner \& Leisering (2003) durchgeführten Vollerhebung. Als erste Befragung ihrer Art vermittelt diese Untersuchung ein genaues Bild der Trägerstruktur und Delegation der Sozialhilfe. Danach gibt es in Deutschland 2.132 Sozialämter, die sich aus 36 Bezirksämtern der Stadtstaaten (allein 23 in Berlin), 115 Sozialämtern in kreisfreien Städten, 322 Kreissozialämtern und 1.659 so genannten Delegationssozialämtern zusammensetzen.

Dabei handelt es sich um kreisangehörige Gemeinden oder Gemeindeverbände, die vom jeweiligen Kreis mit der Wahrnehmung von Aufgaben der Sozialhilfeverwaltung beauftragt wurden und dazu in der Regel ein eigenes Sozialamt betreiben, in dem der Publikumsverkehr und die Sachbearbeitung stattfinden.

Der besseren Übersicht wegen sind in der nachstehenden Tabelle die Jugendhilfeverwaltungen noch einmal mit aufgeführt.

Tab. 11: Örtliche Träger der Jugend- und Sozialhilfe in Deutschland

\begin{tabular}{|l|l|l|l|l|l|l|}
\hline Träger & Jugendämter & $\begin{array}{l}\text { Gemeinden, } \\
\text { Gemeinde- } \\
\text { verbände } \\
\text { ohne } \\
\text { Jugendamt }\end{array}$ & $\begin{array}{l}\text { Bezirks- } \\
\text { (sozial)ämter } \\
\text { (Berlin, } \\
\text { Hamburg, } \\
\text { Bremen) }\end{array}$ & $\begin{array}{l}\text { Sozialämter } \\
\text { (kreisfreie } \\
\text { Städte) }\end{array}$ & $\begin{array}{l}\text { Kreis- } \\
\text { sozialämter } \\
\text { (Landkreise) }\end{array}$ & $\begin{array}{l}\text { Delegations- } \\
\text { sozialämter } \\
\text { (kreisangeh. } \\
\text { Gemeinden/- } \\
\text { verbände) }\end{array}$ \\
\hline Anzahl & 749 & 206 & 36 & 115 & 322 & 1659 \\
\cline { 2 - 7 } & 955 & 2132 & \\
\cline { 2 - 7 } & 3087 &
\end{tabular}

Quellen:

Jugendhilfe: Statistisches Bundesamt, 2004 (Stand: 31.12.2002); Sozialhilfe: Berner \& Leisering, 2003 (Erhebungszeitpunkt: Herbst 2000)

Aufschlussreich ist in der Untersuchung von Berner \& Leisering, dass die Zahl der Delegationssozialämter - als Indikator von Bürgernähe und Dezentralisierung - ein deutliches Nord-Süd-Gefälle aufweist mit den Extremfällen Schleswig-Holstein 
(flächendeckende Delegation) und Bayern (keine Delegation). Im vorliegenden Kontext relevanter ist indessen der Befund, dass insgesamt nur 18,3\% der - vergleichsweise kleinen - Delegationssozialämter das Neue Steuerungsmodell oder Komponenten davon eingeführt hatten (Städte: 61,4\%, Landkreise: 33,3\%), jedoch mehr als die Hälfte der Delegationssozialämter mit mehr als 20 MitarbeiterInnen.

Bezüglich der Umsetzung von Qualitätsmanagement in bundesdeutschen Sozialämtern durfte man auf dieser Basis begründet annehmen, dass auch hier ein deutlicher Zusammenhang mit der Größe des Amtes besteht. Allerdings war eine repräsentative Prüfung dieser Annahme nicht möglich, da über die Größe der Einrichtungen und Verwaltungen keine Populationskennwerte vorlagen; allenfalls ließ sich bei ausreichender Zellenbesetzung im Rücklauf ein entsprechender Zusammenhang der Stichprobenvariablen untersuchen. Dabei war auch die Frage interessant, ob sich zwischen Jugend- und Sozialhilfeverwaltungen diesbezügliche Unterschiede zeigen würden. Leider erfüllte der geringe Rücklauf aus den Sozialämtern die Hoffnung auf Beantwortung dieser Fragen nicht (s. Kap. D 4.3.2).

Wie aus den Tabellen 10 und 11 ersichtlich, umfasst die Total-Grundgesamtheit ${ }^{11}$ dieser Studie 9.883 Einrichtungen der Erziehungshilfe, Jugend- und Sozialhilfeverwaltung und stellt somit eine bedeutende Teilpopulation sozialwirtschaftlicher Organisationen insgesamt in Deutschland dar.

Auf der Basis der erweiterten Hauptstichprobe (s. Kap. A 5.3.4, im Folgenden Gesamtstichprobe genannt) sollen im Rahmen statistischer Inferenz empirische Aussagen zum Qualitätsmanagement dieser Total-Grundgesamtheit und der darin enthaltenen Teilpopulationen gemacht werden. Neben der deskriptiv-populationsbeschreibenden Funktion dieser Aussagen sind dabei auch bestimmte Vorannahmen (Hypothesen) auf ihre Bewährung zu testen.

Wie sich die Gesamtstichprobe von 413 Untersuchungseinheiten zusammensetzt und deren

11 Als Total-Grundgesamtheit (Gesamtpopulation) wird die nur den Zahlen nach, nicht jedoch nach ihrer Merkmalsausstattung bekannte Menge bundesdeutscher Erziehungshilfeeinrichtungen und Jugend- und Sozialhilfeverwaltungen bezeichnet. Die aus dieser Menge erhobene Gelegenheitsstichprobe entspricht der Gesamtstichprobe. Die nach verschiedenen Variablen (Trägerschaft, Arbeitsfeld und Größe) gruppierbaren Teilmengen können als eigene Grundgesamtheiten oder (Teil-)Populationen betrachtet werden, aus denen jeweils Teilstichproben entnommen wurden. 
Anteile zu den jeweiligen Grundgesamtheiten verhalten, geht aus nachfolgenden Tabellen hervor. Dabei wird deutlich, dass sich diese Anteile erheblich voneinander unterscheiden.

Tab. 12: Gesamtstichprobe nach Trägerschaft (alle), Art der Dienststelle (öffentl. Träger) und Spitzenverbandszugehörigkeit bzw. Rechtsform (freie Träger)

\begin{tabular}{|c|c|c|c|c|c|c|}
\hline Träger & \multicolumn{2}{|l|}{ öffentlich } & \multicolumn{3}{|c|}{ freigemeinnützig } & privat \\
\hline Differenzierung & $\begin{array}{l}\text { Sozialämter } \\
\text { und } \\
\text { zugeordnete } \\
\text { Dienste }\end{array}$ & $\begin{array}{l}\text { Jugendämter } \\
\text { und } \\
\text { zugeordnete } \\
\text { Dienste }\end{array}$ & $\begin{array}{l}\text { Caritas u. } \\
\text { a. } \\
\text { katholische } \\
\text { Träger }\end{array}$ & $\begin{array}{l}\text { Diakoni- } \\
\text { sches } \\
\text { Werk u. } \\
\text { a. } \\
\text { evangeli- } \\
\text { sche } \\
\text { Träger }\end{array}$ & $\begin{array}{l}\text { AWO, } \\
\text { DPWV, } \\
\text { DRK, } \\
\text { ASB, } \\
\text { gGmbH., } \\
\text { e. V., } \\
\text { Stiftungen }\end{array}$ & $\begin{array}{l}\text { VPK, } \\
\text { GmbH }\end{array}$ \\
\hline $\mathrm{n}(\Sigma=413)$ & 90 & 122 & 24 & 75 & 47 & 55 \\
\hline$\%(\Sigma=100)$ & 21,79 & 29,54 & 5,81 & 18,16 & 11,38 & 13,32 \\
\hline $\mathrm{n} / \%$ & \multicolumn{2}{|l|}{$212 / 51,33$} & \multicolumn{3}{|l|}{$146 / 35,35$} & $\begin{array}{l}55 \quad / \\
13,32\end{array}$ \\
\hline
\end{tabular}

Anmerkungen:

Betr. freigemeinnützige Träger: Während die Zugehörigkeit zur Caritas bzw. zu katholischen Trägern und zum Diakonischen Werk bzw. zu evangelischen Trägern sehr häufig aus der Anschrift klar ersichtlich war, fehlten zumeist entsprechende Hinweise auf Zugehörigkeit zum DPWV oder zu anderen freien Trägergruppen. Diese wurden deshalb in einer Gruppe zusammengefasst. Vereinzelte Fehlzuordnungen sind möglich.

Betr. Sozialämter: Zugeordnet wurden u. a. Amt für Soziales, Sozialdienst für Ältere, Amt für Soziale Integration, Sozialplanung, Soziale Sonderaufgaben, Wohnungs- und Flüchtlingsamt, Amt für Soziale Sicherung, Schuldnerberatung, Betreuungsstelle. Vereinzelte Fehlzuordnungen sind möglich.

Betr. Jugendämter: Zugeordnet wurden u. a. Amt für Jugend und Soziales, Amt für Jugend und Familie, Jugendamt, Dezernat für Jugend/Soziales/Gesundheit, Amt für junge Menschen, Jugendförderung, Leitstelle Kinder und Jugendliche, Fachberatung Kindertagesstätten. Vereinzelte Fehlzuordnungen sind möglich.

Tab. 13: Gesamtstichprobe und Populationsanteile nach Trägerschaft und Arbeitsfeldern

\begin{tabular}{|c|c|c|c|c|}
\hline Träger & öffentlich & & freigemeinnützig & privat \\
\hline Arbeitsfeld & $\begin{array}{l}\text { Sozialamt / } \\
\text { Dienste }\end{array}$ & $\begin{array}{l}\text { Jugendamt / } \\
\text { Dienste }\end{array}$ & $\begin{array}{lr}\text { Hilfen } & \text { zur } \\
\text { Erziehung, Hilfen } \\
\text { für } \\
\text { Volljährige, } \\
\text { Eingliederungshilfe } \\
\text { u. a. }\end{array}$ & $\begin{array}{l}\text { Hilfen } \\
\text { Erziehung, Hilfen } \\
\text { für } \\
\text { Volljährige, } \\
\text { Eingliederungshilfe } \\
\text { u. a. }\end{array}$ \\
\hline $\mathrm{n}_{1-4}(\Sigma=413)$ & 90 & 122 & 146 & 55 \\
\hline$\% \mathrm{n}_{1-4}(\Sigma=100)$ & 21,79 & 29,54 & 35,35 & 13,32 \\
\hline $\mathrm{N}_{1-4}(\Sigma=9883)$ & 2132 & 955 & 6183 & 613 \\
\hline$\% \mathrm{~N}_{1-4}$ & 4,22 & 12,77 & 2,36 & 8,97 \\
\hline
\end{tabular}


Neben dem Kriterium der Trägerschaft (öffentlich/freigemeinnützig/privatgewerblich), der Eingrenzung des Arbeitsfeldes (Erziehungs-/Eingliederungshilfe/Jugend- und Sozialhilfeverwaltung) und der Anzahl der vorhandenen Verwaltungseinheiten und freien Einrichtungen war ein weiterer wichtiger Populationskennwert - wie schon erwähnt nicht ermittelbar, hinsichtlich dessen die Repräsentativität der beteiligten Untersuchungseinheiten hätte beurteilt werden sollen: die Einrichtungsgröße in Form von Platz- und/oder Mitarbeiterzahlen.

Im Unterschied etwa zum Gesundheitswesen, in dem differenziertes und nahezu aktuelles Material über Zahl, Größe und Art der Einrichtungen, der Beschäftigten, der Berufsgruppen usw. verfügbar ist (www.destatis.de,), gibt es - wie Peters (o. J.) betont - in Deutschland kein nationales Register, in dem alle Anbieter von Jugendhilfeleistungen erfasst sind. Angaben über Platzzahlen und Leistungsspektren der Einrichtungen sind über Verbandsanfragen und Länderstatistiken teilweise zugänglich, jedoch nicht in einer vollständigen Zusammenschau.

Die in Tab. 10 zusammengefassten Daten sind somit zwar recht aktuell, differenzieren aber lediglich nach Tageseinrichtungen, sonstigen Einrichtungen und Jugendhilfeverwaltungen sowie grob nach freier und öffentlicher Trägerschaft.

Bezüglich der unabhängigen Variablen der Einrichtungsgröße ließen sich somit keine Rückschlüsse von der Stichprobe auf die Population ableiten, bezüglich des Arbeitsfeldes und der Trägerschaft immerhin solche mit Abstrichen.

\subsection{Verfahren der Stichprobenentnahme}

Die Grundgesamtheiten seien zunächst noch einmal nach ihrem eindeutigsten Kriterium gruppiert und näher charakterisiert - der Trägerschaft.

1. Öffentliche Träger: kommunale Jugendämter, Jugendhilfeverwaltungen von Gemeinden und Gemeindeverbänden ohne Jugendamt, kommunale Sozialämter, Allgemeine Soziale Dienste und sonstige Fachdienste, soweit organisatorisch mit der Jugend- und Sozialhilfeverwaltung verknüpft.

2. Freigemeinnützige Träger: Einrichtungen der freien Wohlfahrtspflege, soweit diese 
Leistungen nach $\S \S 27$ ff., $\S 35 \mathrm{a}$ und $\S 41$ SGB VIII bzw. Hilfe in besonderen Lebenslagen nach BSHG, Abschnitt 3, und/oder sonstige Leistungen der Sozialhilfe erbringen.

3. Privatgewerbliche Träger: Einrichtungen, die überwiegend im Bundesverband privater Träger der freien Kinder-, Jugend- und Sozialhilfe e. V. (VPK) organisiert sind und Leistungen nach $\S \S 27 \mathrm{ff} ., \S 35 \mathrm{a}$ und $\S 41$ SGB VIII erbringen.

Aus diesen Teilpopulationen galt es, den angestrebten Quotierungen entsprechend Stichproben zu entnehmen.

Als einfache Stichprobe mit Zufallswahl ließ sich lediglich die Gruppe der privatgewerblichen Träger mit VPK-Mitgliedschaft einbeziehen. Hier wurde nach einem rollierenden Verfahren jede fünfte (sechste, siebte ...) Adresse aus dem InternetMitgliederverzeichnis des Bundesverbandes entnommen, bis mit 50 von insgesamt 363 Einrichtungen eine Zufallsauswahl von immerhin 13,8\% der Mitgliederschaft erreicht war. Fünf weitere privatwirtschaftliche Einrichtungen kamen aus dem Adressenpool des Deutschen Vereins hinzu, so dass der Anteil der Stichprobe an der Teilpopulation der "Wirtschaftsunternehmen" (55 von 613, s. Tab. 10) 8,97\% betrug.

Auch in Bezug auf die Gruppe der öffentlichen Träger ließ sich eine quantitativ halbwegs befriedigende Auswahl realisieren, wenngleich nicht nach echtem Zufallsprinzip, sondern - wie in Kap. A 5.3.4 erwähnt - auf der Grundlage einer Komplettbefragung aller an QMVeranstaltungen interessierten öffentlichen Träger, die beim Deutschen Verein registriert waren.

Die Einrichtungen und Einzelpersonen des Anschriftenpools wurden bis auf wenige fachfremde Adressaten komplett in die Untersuchung einbezogen, um Auswahleffekte innerhalb dieser Gruppe zu vermeiden. Die Befürchtung mangelnder Repräsentativität aufgrund möglicher Selbstselektion - nur "QM-interessierte" Einrichtungen/Personen sind im Pool enthalten, während "Desinteressierte" oder bereits hinreichend "Sachkundige" sich erst gar nicht über einschlägige Fortbildungsveranstaltungen des DV informiert haben könnten - ist nicht ganz von der Hand zu weisen. Die Folge wäre eine tendenzielle Überschätzung des Anteils der "QM-Aktivisten", mithin eine zu positive Darstellung des tatsächlichen Umsetzungsstandes von QM-Systemen und -instrumenten. 
Der Stichprobenanteil der Jugend- und Sozialhilfeverwaltungen an ihrer jeweiligen Grundgesamtheit beträgt 12,77\% (Jugendhilfe: 122 von 955) bzw. 4,22\% (Sozialhilfe: 90 von 2.132).

Der Stichprobenanteil freigemeinnütziger Träger an der entsprechenden Teilpopulation beläuft sich dagegen nur auf 2,36\% (146 von 6.183). Hinzu kommt die Überrepräsentanz diakonischer Träger. Aufgrund der breiten fachlichen Streuung der Leistungsanbieter dieser Gruppe wird diesem Sachverhalt allerdings nur ein geringer Selektionseffekt beigemessen. Im Rücklauf (s. Kap.4.3.3) wird ohnehin ein ausgewogeneres Verhältnis der freien Trägergruppen zueinander und im Verhältnis zu den öffentlichen Trägern erzielt.

In der Total-Grundgesamtheit von 9.883 Einrichtungen und Diensten sind somit die öffentlichen Träger, insbesondere die Jugendhilfeverwaltungen, über- und die freigemeinnützigen Träger unterrepräsentiert.

\subsection{Rücklauf}

\subsubsection{Gesamtrücklauf}

Von 413 (bzw. 826) versandten oder verteilten Fragebögen kamen 85 (bzw. 102 incl. 17 doppelt vertretenen Einrichtungen, s. Kap. D 4.5) zurück und gingen in die Auswertung ein. Dies entspricht einem Gesamtrücklauf von 20,58\% aller in der Nettostichprobe enthaltenen Einrichtungen und Dienste.

Eine Berechnung der Rücklaufquote auf der Basis aller 826 zugestellten Fragebögen wäre unsinnig, da nur die - unbekannte - Zahl der Einrichtungen mit Qualitätsbeauftragten zwei Bögen in die Auswertung einbringen konnte. 
Tab. 14: Gesamtrücklauf

\begin{tabular}{|l|l|l|l|l|l|}
\hline $\begin{array}{l}\text { Brutto- } \\
\text { Gesamtstich- } \\
\text { probe (vor } \\
\text { Bereinigung) }\end{array}$ & $\begin{array}{l}\text { fachfremde } \\
\text { Adressaten, unzustellbare } \\
\text { Fragebögen }\end{array}$ & $\begin{array}{l}\text { Netto- } \\
\text { Gesamtstich- } \\
\text { probe }\end{array}$ & $\begin{array}{l}\text { nicht } \\
\text { auswertbare } \\
\text { Fragebögen }\end{array}$ & $\begin{array}{l}\text { Rücklauf aus } \\
\text { versch. } \\
\text { Einrichtungen }\end{array}$ & $\begin{array}{l}\text { Gesamtzahl } \\
\text { auswertbarer } \\
\text { Fragebögen }\end{array}$ \\
\hline 441 & 28 & 413 & 0 & 85 & 102 \\
\hline $100 \%$ & $6,35 \%$ & $100 \%$ & $0 \%$ & $20,58 \%$ & - \\
\hline
\end{tabular}

\subsubsection{Rücklauf nach Faktoren}

Der nach Fragebogenversion, Arbeitsfeldern, Einrichtungsgröße, Trägerschaft und Spitzenverbandszugehörigkeit differenzierte Rücklauf ist nachstehenden Tabellen zu entnehmen. Doppelter Rücklauf (Versionen 1 und 2 des Fragebogens) ist ggf. durch Häufigkeitsangaben in Klammern gekennzeichnet; auf die Angabe der Prozentanteile wurde dabei aus Gründen der Übersichtlichkeit verzichtet.

Tab. 15: Rücklauf nach Fragebogenversionen

\begin{tabular}{|ll|l|l|l|l|}
\hline & & Häufigkeit & Prozent & $\begin{array}{l}\text { Gültige Pro- } \\
\text { zente }\end{array}$ & $\begin{array}{l}\text { Kumulierte } \\
\text { Prozente }\end{array}$ \\
\hline Gültig & QB (selbst; Version 1) & 31 & 30,4 & 30,4 & 30,4 \\
& QB (nicht selbst, Vers. 2) & 17 & 16,7 & 16,7 & 47,1 \\
& ohne QB (Version 3) & 54 & 52,9 & 52,9 & 100,0 \\
Gesamt & 102 & 100,0 & 100,0 & \\
\hline
\end{tabular}

Die Zuordnung der Probanden zu den nicht vorab festgelegten Stufen der Faktoren Arbeitsfeld und Einrichtungsgröße wurde nachträglich auf Datenbasis bzw. anhand der Quartilsgrenzen vorgenommen (vgl. Kap. A 5.3.2; in Klammern: doppelter Rücklauf, s. о.). 
Tab. 16: Rücklauf nach Arbeitsfeldern

\begin{tabular}{|ll|l|l|l|l|}
\hline & Häufigkeit & Prozent & $\begin{array}{l}\text { Gültige } \\
\text { Prozente }\end{array}$ & $\begin{array}{l}\text { Kumulierte } \\
\text { Prozente }\end{array}$ \\
\hline Gültig & Jugendamt & $26(2)$ & 30,6 & 31,0 & 31,0 \\
& Sozialamt & $7(0)$ & 8,2 & 8,3 & 39,3 \\
& Jugendhilfeeinrichtung & $34(9)$ & 40,0 & 40,5 & 79,8 \\
& Kita/Fachberatung & $2(1)$ & 2,4 & 2,4 & 82,2 \\
& Altenhilfe/Pflege & $3(0)$ & 3,5 & 3,6 & 85,8 \\
& Internat/Ausbildung/BBW & $5(4)$ & 5,9 & 6,0 & 91,8 \\
& Sonstiges & $7(1)$ & 8,2 & 8,3 & 100,0 \\
Fehlend & keine Angabe & $1(0)$ & 1,2 & 100,0 & \\
Gesamt & & $85(17)$ & 100,0 & & \\
\hline
\end{tabular}

Anmerkung:

$\mathrm{Zu}$ den sonstigen Arbeitsfeldern zählen: Sozialpsychiatrie (2x), Verband (2x), Sozialministerium, Frühe Hilfen, Behindertenhilfe (2x)

Wie aus den Zahlen in Klammern ersichtlich, entfällt über die Hälfte des doppelten Rücklaufs auf Einrichtungen der Jugendhilfe. Generalisierende Aussagen aus anzustellenden Vergleichen zwischen den Gruppen der Qualitätsbeauftragten und Leitungskräfte derselben Einrichtungen sollen deshalb auf diesen Bereich beschränkt werden.

Zugleich fällt die geringe Anzahl vertretener Sozialämter auf. Während auf Seiten der freien Träger einige Arbeitsfelder mit geringem Rücklauf zu einer erweiterten Gruppe der Sonstigen zusammengefasst werden können, sollen die Sozialämter zwar als separate Gruppe untersucht, mögliche Unterschiede jedoch nur mit Vorbehalt interpretiert werden.

Die Rohwerteverteilung der Einrichtungsgröße (operationalisiert durch die Zahl der MitarbeiterInnen) ist in der folgenden Abbildung dargestellt. Zur Stauchung der Y-Achse wurden dabei die Einrichtungen mit mehr als 500 Beschäftigten pauschal mit dem Wert 500 eingegeben. 
Abb. 2: Verteilung der Untersuchungseinheiten im Rücklauf nach Zahl der Beschäftigten

\section{Rücklauf nach Einrichtungsgröße}

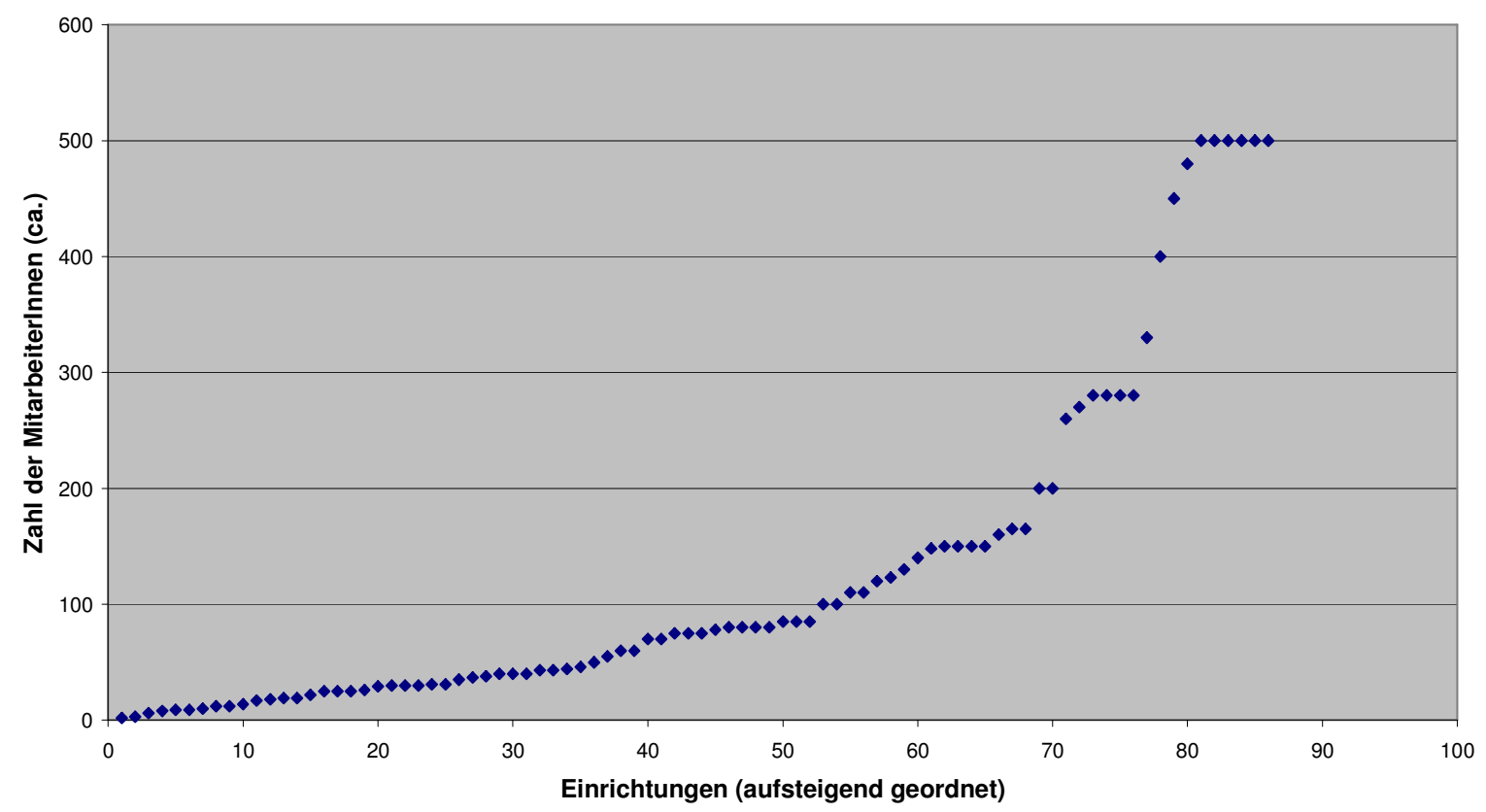

Die kontinuierliche Variable der Einrichtungsgröße wurde auf der Basis des tatsächlichen Rücklaufs auf vier Ordinalstufen reduziert, welche den jeweiligen Quartilen der Verteilung und keinem inhaltlichen Zuordnungskriterium entsprechen: kleinste und kleine Einrichtungen mit bis zu 30 MitarbeiterInnen, mittelgroße Einrichtungen mit 31 bis 75 MitarbeiterInnen, große Einrichtungen mit 76 bis 150 und sehr große mit 151 und mehr MitarbeiterInnen. Abweichungen von den exakten Quartilsgrenzen sind durch nachträgliche Zuordnung von zwei Nachzüglern bedingt. In Klammern: doppelter Rücklauf (s. o.).

Tab. 17: Rücklauf nach Quartilen der Einrichtungsgröße

\begin{tabular}{|ll|l|l|l|l|}
\hline & & Häufigkeit & Prozent & $\begin{array}{l}\text { Gültige Pro- } \\
\text { zente }\end{array}$ & $\begin{array}{l}\text { Kumulierte } \\
\text { Prozente }\end{array}$ \\
\hline Gültig & kleinst/klein & $22(3)$ & 25,9 & 25,9 & 25,9 \\
& mittelgroß & $21(5)$ & 24,7 & 24,7 & 50,6 \\
& groß & $22(3)$ & 25,9 & 25,9 & 76,5 \\
& sehr groß & $20(6)$ & 23,5 & 23,5 & 100,0 \\
Gesamt & & $85(17)$ & 100,0 & 100,0 & \\
\hline
\end{tabular}


Der für die Beantwortung trägerspezifischer Fragestellungen der Studie maßgebliche Rücklauf nach Trägergruppen ist Tab. $18 \mathrm{zu}$ entnehmen (in Klammern: doppelter Rücklauf, s. o.). Wie aus den Zahlen in Klammern ersichtlich ist, entfallen nahezu zwei Drittel des doppelten Rücklaufs auf Einrichtungen freigemeinnütziger Träger. Für später anzustellende Vergleiche zwischen den Gruppen der Qualitätsbeauftragten und Leitungskräften derselben Einrichtungen gelten deshalb die bereits beim Faktor Arbeitsfeld getroffenen Vorbehalte.

Tab. 18: Rücklauf nach Trägergruppen

\begin{tabular}{|ll|l|l|l|l|}
\hline & & Häufigkeit & Prozent & $\begin{array}{l}\text { Gültige Pro- } \\
\text { zente }\end{array}$ & $\begin{array}{l}\text { Kumulierte } \\
\text { Prozente }\end{array}$ \\
\hline Gültig & öffentlich & $35(4)$ & 41,1 & 41,1 & 41,1 \\
& freigemeinnützig & $33(11)$ & 38,9 & 38,9 & 80,0 \\
& privatgewerblich & $17(2)$ & 20,0 & 20,0 & 100,0 \\
Gesamt & & $85(17)$ & 100,0 & 100,0 & \\
\hline
\end{tabular}

Die Zuordnung des Rücklaufs nach Spitzenverbandszugehörigkeit kann nur auf der Basis von 41 Angaben vorgenommen werden; die Mehrheit der befragten Einrichtungen ließ das entsprechende Antwortfeld frei, darunter 31 öffentliche, 8 privatgewerbliche und 5 freie Träger. Für eine differenzierte Betrachtung der abhängigen Variablen nach Spitzenverbandszugehörigkeit erweisen sich die verbleibenden Zellenbesetzungen als zu gering.

Tab. 19: Rücklauf nach Spitzenverbandszugehörigkeit (freie Träger)

\begin{tabular}{|ll|l|l|l|l|}
\hline & Häufigkeit & Prozent & $\begin{array}{l}\text { Gültige Pro- } \\
\text { zente }\end{array}$ & $\begin{array}{l}\text { Kumulierte } \\
\text { Prozente }\end{array}$ \\
\hline Gültig & Caritas & $3(1)$ & 3,5 & 7,3 & 7,3 \\
& Diakonisches Werk & $13(3)$ & 15,3 & 31,7 & 39,0 \\
& DPWV & $8(2)$ & 9,4 & 19,5 & 58,5 \\
& AWO & $3(0)$ & 3,5 & 7,3 & 65,8 \\
& DRK & $1(1)$ & 1,2 & 2,4 & 68,2 \\
& VPK & $9(1)$ & 10,6 & 22,0 & 90,2 \\
& Kommunalverbände & $4(4)$ & 4,7 & 9,8 & 100,0 \\
Fehlend & Gesamt & $41(12)$ & 48,2 & 100,0 & \\
Gesamt & keine Angabe & $44(5)$ & 51,8 & & \\
\hline
\end{tabular}




\subsubsection{Repräsentativität des Rücklaufs}

Ein Aspekt der Repräsentativität bzw. der externen Validität der Befragung bezieht sich auf potenzielle regionale Unterschiede zwischen Population und Stichprobe bzw. Rücklauf. Leider ist die Populationsverteilung mangels zugänglicher Daten nicht bekannt. Überprüfbar ist jedoch die Proportionalität zwischen Stichprobe und Rücklauf. Die NettoGesamtstichprobe von 413 Einheiten enthält Einrichtungen und Dienste aus dem gesamten Bundesgebiet mit folgender Verteilung auf die einzelnen Postleitzahlbereiche: ${ }^{12}$

Tab. 20: Verteilung der Postleitzahlbereiche in Stichprobe und Rücklauf

\begin{tabular}{|l|l|l|l|l|l|l|l|l|l|l|l|}
\hline PLZ & 0 & 1 & 2 & 3 & 4 & 5 & 6 & 7 & 8 & 9 & $\Sigma$ \\
\hline $\begin{array}{l}\mathrm{n} \\
\text { (Stichprobe) })\end{array}$ & 20 & 23 & 50 & 60 & 48 & 50 & 78 & 38 & 30 & 16 & 413 \\
\hline$\%$ & 4,8 & 5,6 & 12,1 & 14,5 & 11,6 & 12,1 & 18,9 & 9,2 & 7,3 & 3,9 & 100 \\
\hline $\begin{array}{l}\mathrm{n} \\
(\text { Rücklauf })\end{array}$ & 3 & 6 & 12 & 14 & 7 & 10 & 14 & 9 & 6 & 4 & 85 \\
\hline$\%$ & 3,5 & 7,1 & 14,1 & 16,5 & 8,2 & 11,8 & 16,5 & 10,6 & 7,1 & 4,7 & 100 \\
\hline $\mathrm{Chi}^{2}$ & 2,$844 ; \mathrm{df}=9 ; \mathrm{p}>.50 ;$ n. s. \\
\hline
\end{tabular}

Als Ergebnis lässt sich konstatieren, dass der Rücklauf in regionaler Hinsicht die Relationen in der Gesamtstichprobe sehr zuverlässig widerspiegelt; die eingetretenen Abweichungen erweisen sich als äußerst gering.

Ein weiterer Gesichtspunkt der Repräsentativität betrifft die Verteilung der Trägergruppen in Population, Stichprobe und Rücklauf. Nur bezüglich dieses Faktors sind - wie erwähnt die Populationskennwerte bekannt, während die Verteilung der Arbeitsfelder und Einrichtungsgrößen weder in der Population, noch in der Stichprobe zu ermitteln war und entsprechende Quoten nur für den Rücklauf berechnet werden können.

12 Die Hauptgeschäftsstelle des Deutschen Vereins befindet sich seit Mitte 2004 in Berlin und lag zuvor in Frankfurt, was aufgrund des Einzugsbereichs die relativ starke Präsenz des Postleitzahlbezirks 6 erklärt (s. Tab. 20). 
Tab. 21: Vergleich der Rücklauf-, Stichproben- und (Teil-)Populationsquoten (in \%)

\begin{tabular}{|l|l|l|l|l|}
\hline & $\begin{array}{l}\text { Rücklaufquote } \\
\text { (Anteil am } \\
\text { Rücklauf von } \\
85)\end{array}$ & $\begin{array}{l}\text { Stichproben- } \\
\text { quote } \\
\text { (Anteil an der } \\
\text { Netto- } \\
\text { Gesamtstich- } \\
\text { probe von } \\
413 \text { ) }\end{array}$ & $\begin{array}{l}\text { Teilpopulati- } \\
\text { onsquote } \\
\text { (Anteil an } \\
\text { Einrichtungen } \\
\text { des Trägers } \\
\text { gemäß Tab. } \\
10)\end{array}$ & $\begin{array}{l}\text { Populations- } \\
\text { quote } \\
\text { (Anteil an der } \\
\text { Total-Grund- } \\
\text { gesamtheit } \\
\text { von 9.883 } \\
\text { Einrich- } \\
\text { tungen) }\end{array}$ \\
\hline $\begin{array}{l}\text { öffentlich (Jugend-/Sozialämter) } \\
\text { Caritas/kathol. Träger }\end{array}$ & 41,1 & 51,3 & 6,87 & 2,15 \\
Diakonisches Werk/evang. Träger & 4,7 & 5,8 & 2,62 & 0,24 \\
Sonstige freigemeinnützige Träger & 16,6 & 18,2 & 4,11 & 0,76 \\
VPK u. a. private Träger & 20,0 & 11,4 & 1,37 & 0,48 \\
Gesamt & 100,0 & 13,3 & 8,97 & 0,56 \\
\hline
\end{tabular}

\section{Anmerkung:}

Die fehlenden Angaben $(n=5)$ zur Spitzenverbandszugehörigkeit der freigemeinnützigen Träger (s. Tab. 19) wurden zur Erzielung von 100\% wie folgt kompensiert: Caritas + 1, Diakonisches Werk + 2, Sonstige + 2 .

Wie schon in Kap. D 4.2 angedeutet, relativiert sich der hohe Stichprobenanteil diakonischer Einrichtungen im Rücklauf; andere freigemeinnützige und vor allem privatgewerbliche Träger sind nunmehr stärker repräsentiert. Insgesamt zeigt sich im Rücklauf ein in Bezug auf die Population ausgewogeneres Verhältnis zwischen öffentlichen und freien Trägern als in der Gesamtstichprobe.

Zur Prüfung der Frage, ob die Hauptträgergruppen (öffentliche, freigemeinnützige, private) in Stichprobe und Rücklauf annähernd die Populationsverhältnisse widerspiegeln, wurde ein Chi-Quadrat-Test auf der Basis der Prozentanteile durchgeführt.

Tab. 22: Prüfung auf Repräsentativität der Netto-Gesamtstichprobe hinsichtlich des Faktors Trägerschaft

\begin{tabular}{|l|l|l|l|}
\hline $\begin{array}{l}\text { Träger / Anteile } \\
\text { (in \%) }\end{array}$ & öffentliche & $\begin{array}{l}\text { freigemeinnützige und } \\
\text { sonstige freie }\end{array}$ & privatgewerbliche \\
\hline beobachtet (o) & 51,33 & 35,35 & 13,32 \\
\hline erwartet (e) & 31,24 & 62,56 & 6,20 \\
\hline $\mathrm{o}-\mathrm{e}$ & 20,09 & $-27,21$ & 7,12 \\
\hline$(\mathrm{o}-\mathrm{e})^{2}$ & 403,61 & 740,38 & 50,69 \\
\hline$(\mathrm{o}-\mathrm{e})^{2}: \mathrm{e}$ & 12,92 & 11,83 & 8,18 \\
\hline $\mathrm{Chi}^{2}=\sum(\mathrm{o}-\mathrm{e})^{2}: \mathrm{e}$ & 32,$93 ; \mathrm{df}=2 ; \mathrm{p} \leq .01 ;$ s. s. & \\
\hline
\end{tabular}


Die prozentualen Anteile der Trägergruppen in der Gesamtstichprobe sind für die TotalGrundgesamtheit nicht repräsentativ. Während öffentliche und privatgewerbliche Träger annähernd doppelt so stark vertreten sind, wie es ihrem Anteil an der Gesamtpopulation entspricht, gilt dies im umgekehrten Sinne für die freigemeinnützigen Träger; ihr Anteil beträgt wenig mehr als die Hälfte des theoretischen Erwartungswertes.

Dieser Effekt war nicht durch sorgfältigere Quotierung $\mathrm{zu}$ vermeiden, da aus den verfügbaren Einrichtungsadressen die Trägerschaft häufig nicht zu ermitteln war und somit erst nach Analyse des Rücklaufs die entsprechenden Anteile berechnet werden konnten.

Es stellt sich im Licht dieser Ergebnisse die Frage, ob eine Zusammenfassung der Jugendund Sozialhilfeverwaltungen mit den Einrichtungen der Erziehungs- und Eingliederungshilfe zu einer Gesamtpopulation überhaupt sinnvoll ist. Im Hinblick auf die formulierten Hypothesen zur Wirksamkeit des Faktors Trägerschaft kommt es ja primär darauf an, etwaige Unterschiede zwischen den Gruppen zu prüfen. Dass unter den gegebenen Umständen die Aussagen über öffentliche Träger auf statistisch solideren Beinen stehen - sprich: auf einem relativ größeren Stichprobenanteil an der Teilpopulation beruhen -, mindert die Bedeutung entsprechender Befunde in keiner Weise.

Während somit die mangelnde Repräsentativität für die Total-Grundgesamtheit aller Einrichtungen und Dienste von geringer praktischer Bedeutung ist, kommt der Frage nach angemessener Repräsentanz des trägerbezogenen Rücklaufs für die Gesamtstichprobe stärkeres Gewicht zu (Zahlen auf eine Dezimale gerundet).

Tab. 23: Prüfung auf Repräsentativität des Rücklaufs für die Gesamtstichprobe hinsichtlich des Faktors Trägerschaft

\begin{tabular}{|l|l|l|l|}
\hline $\begin{array}{l}\text { Träger / Anteile } \\
\text { (in \%) }\end{array}$ & öffentliche & $\begin{array}{l}\text { freigemeinnützige und } \\
\text { sonstige freie }\end{array}$ & privatgewerbliche \\
\hline beobachtet (o) & 41,1 & 38,9 & 20,0 \\
\hline erwartet (e) & 51,3 & 35,4 & 13,3 \\
\hline $\mathrm{o}-\mathrm{e}$ & $-10,2$ & 3,5 & 6,7 \\
\hline$(\mathrm{o}-\mathrm{e})^{2}$ & 104,0 & 12,3 & 44,9 \\
\hline$(\mathrm{o}-\mathrm{e})^{2}: \mathrm{e}$ & 2,0 & 0,3 & 3,4 \\
\hline $\mathrm{Chi}^{2}=\sum(\mathrm{o}-\mathrm{e})^{2}: \mathrm{e}$ & 5,$7 ; \mathrm{df}=2 ; \mathrm{p}>.05 ;$ n. s. \\
\hline
\end{tabular}

Die Abweichungen der Beobachtungs- von den Erwartungswerten sind nicht signifikant auf dem 5\%-Niveau. In Bezug auf den Faktor Trägerschaft ist der Rücklauf somit als 
repräsentativ für die Gesamtstichprobe anzusehen. Allerdings sind unter den öffentlichen Trägern die Sozialämter eindeutig unterrepräsentiert (s. Tab. 16); bei der späteren Interpretation trägerspezifischer Befunde muss dieser Sachverhalt berücksichtigt werden.

Eine analoge Prüfung für die Variablen der Einrichtungsgröße und des Arbeitsfeldes ist wie bereits mehrfach erwähnt - nicht möglich, da die Populationskennwerte unbekannt sind.

\subsubsection{Interkorrelationen der Faktoren im Rücklauf}

Für die Bearbeitung der Untersuchungsfragestellungen nicht unerheblich ist der Sachverhalt, dass die so genannten unabhängigen Variablen (Faktoren) keineswegs sauber voneinander getrennt werden können. Sie interkorrelieren im Rücklauf erheblich und dürften auch in der Grundgesamtheit nicht voneinander unabhängig sein.

Zunächst ist die Rohwerteverteilung für den Zusammenhang zwischen Größe und Trägerschaft kreuztabellarisch dargestellt. Wegen ihres unterschiedlichen Skalenniveaus wurden die "höherwertigen" Daten auf Nominalniveau herabgestuft; als Maß für den Zusammenhang fungiert der Kontingenzkoeffizient.

Tab. 24: Kreuztabelle Einrichtungsgröße x Trägerschaft (Rohwerte)

\begin{tabular}{|l|l|l|l|l|l|}
\hline Träger/Größe & klein/kleinst & mittelgroß & groß & sehr groß & Gesamt \\
\hline öffentlich & 5 & 6 & 16 & 8 & 35 \\
\hline freigemeinnützig & 5 & 13 & 5 & 10 & 33 \\
\hline privatgewerblich & 12 & 2 & 1 & 2 & 17 \\
\hline Gesamt & 22 & 21 & 22 & 20 & 85 \\
\hline
\end{tabular}

Der Kontingenzkoeffizient beträgt .525 und ist hochsignifikant. Allein die Rohwerteinspektion verdeutlicht einen klaren Zusammenhang zwischen der Quartilszugehörigkeit und der Trägerschaft:

○ Unter privatgewerblichen Trägern dominieren kleine und kleinste Einrichtungen. 
o Unter freigemeinnützigen Trägern sind mittelgroße und sehr große Einrichtungen besonders häufig vertreten.

○ Unter öffentlichen Trägern überwiegen große Einrichtungen (76 bis 150 MitarbeiterInnen).

Aufgrund fehlender Populationskennwerte lässt sich nicht feststellen, ob diese Häufigkeitsverteilungen charakteristisch für die jeweiligen Trägergruppen sind. Dies gilt zumindest für die öffentlichen und freigemeinnützigen Träger, während der Sachverhalt überproportional vieler Kleineinrichtungen in privater Trägerschaft den Erwartungen und Vorkenntnissen entspricht.

Für den Zusammenhang zwischen Arbeitsfeld und Einrichtungsgröße ergibt sich im Rücklauf folgendes Bild:

Tab. 25: Kreuztabelle Einrichtungsgröße x Arbeitsfeld (Rohwerte)

\begin{tabular}{|l|l|l|l|l|l|}
\hline Feld/Größe & klein/kleinst & mittelgroß & groß & sehr groß & Gesamt \\
\hline JuHi/SozHi & 5 & 6 & 14 & 8 & 33 \\
\hline Erziehungshilfe & 15 & 7 & 6 & 6 & 34 \\
\hline Sonstige & 2 & 8 & 2 & 5 & 17 \\
\hline Gesamt & 22 & 21 & 22 & 19 & 84 \\
\hline
\end{tabular}

Der Kontingenzkoeffizient beträgt .447 und ist hochsignifikant. Die Rohwerteinspektion erweist, dass öffentliche Jugend- und Sozialhilfeverwaltungen im Sinne der definierten Quartilsgrenzen überwiegend groß sind, während unter den Erziehungshilfeorganisationen die kleinen und kleinsten Einrichtungen dominieren (bedingt durch die Häufung privatgewerblicher Träger in diesem Quartil, s. Tab. 24).

Besonders ausgeprägt ist die Konfundierung der Variablen "Trägerschaft" und "Arbeitsfeld", die sich aus dem öffentlich-rechtlichen Charakter der Jugend- und Sozialhilfeverwaltung und dem sozialpolitischen Subsidiaritätsprinzip (Vorrang freier Träger) ergibt. 
Tab. 26: Kreuztabelle Trägerschaft x Arbeitsfeld (Rohwerte)

\begin{tabular}{|l|l|l|l|l|}
\hline Feld/Träger & öffentlich & freigemeinnützig & privatgewerblich & Gesamt \\
\hline JuHi/SozHi & 32 & 1 & 0 & 33 \\
\hline Erziehungshilfe & 2 & 15 & 17 & 34 \\
\hline Sonstige & 1 & 16 & 0 & 17 \\
\hline Gesamt & 35 & 32 & 17 & 84 \\
\hline
\end{tabular}

Der Kontingenzkoeffizient beträgt hochsignifikante .719. Lässt man den einzigen freigemeinnützigen Träger der Jugend- und Sozialhilfeverwaltung außer Betracht ohnehin vermutlich eine Fehlzuordnung -, ist dieses Arbeitsfeld mit öffentlicher Trägerschaft identisch. Umgekehrt befinden sich die übrigen Arbeitsfelder fest in freigemeinnütziger bzw. privatgewerblicher Hand; öffentliche Leistungsanbieter sind mit nur drei von 51 Einrichtungen vertreten (rund 6\%).

\subsubsection{Mögliche Gründe für geringen Rücklauf}

Die, bezogen auf die Netto-Gesamtstichprobe, erzielte Rücklaufquote von 20,58\% und die absolute Zahl auswertbarer Fragebögen (102 aus 85 verschiedenen Einrichtungen) kann nicht ganz befriedigen, wenngleich sich die Studie damit noch im gewöhnlichen Streubereich der Rücklaufquoten postalischer Befragungen bewegt. Die als Vergleichsstudie herangezogene Untersuchung von Wetzler (2003) - mit wesentlich größerer offizieller Unterstützung durchgeführt - erzielte z. B. nur eine Quote von knapp $16 \%$.

Für das relativ geringe Interesse der Einrichtungen trotz sorgfältiger Vorbereitung der Aktion können eine Reihe von Gründen ins Feld geführt werden. Neben Mutmaßungen und Verweisen auf ähnliche Erfahrungen anderenorts treten dabei explizite Anmerkungen einiger TeilnehmerInnen und Nicht-TeilnehmerInnen. Letztere sind solche, die zwar keinen Fragebogen zurückschickten, jedoch die Aktion kommentierten.

1. Ähnlich wie bei Wetzler (2003, S. 34) in Bezug auf die Behindertenhilfe berichtet, kann vermutet werden, dass manche freien Träger die Erhebung derartiger Daten für politisch zu brisant einschätzten im Hinblick auf anstehende Kostenverhandlungen. Die 
Befürchtung, durch allzu bereitwilliges Sich-in-die Karten-gucken-lassen den auf Leistungsanbieter ausgeübten Druck unbeabsichtigt zu erhöhen, spielt als Widerstand gegen empirische Untersuchungen seit jeher eine beträchtliche Rolle auf Seiten der Praxis.

2. Wiederum ähnlich wie bei Wetzler (a.a.O.) konstatiert, dürften viele Einrichtungen erst wenig nach außen kommunizierbare Aktivitäten zum Qualitätsmanagement vorweisen können und nahmen deshalb erst gar nicht an der Befragung teil (Vermeidung einer Fehlanzeige). Dies impliziert die Schlussfolgerung, dass der Anteil jener Einrichtungen, die über wenige bis gar keine Maßnahmen in Sachen Qualitätsmanagement berichten, aufgrund des Rücklaufs eher unter- als überschätzt wird.

3. Während größere Einrichtungen, die auch in dieser Stichprobe (ähnlich Wetzler, 2003, S. 35f) in Relation zu ihrer absoluten Häufigkeit überrepräsentiert sein dürften (über den Populationskennwert der Einrichtungsgröße liegen keine verbandsübergreifenden Informationen vor), Qualitätsmanagement leichter im Rahmen ihrer Personal- und Finanzressourcen etablieren können, tun sich kleinste und kleine Träger erfahrungsgemäß schwerer (vgl. Gerull, 1998). Überproportional häufig dürften sich solche Klein(st)einrichtungen beteiligt haben, die in Sachen QM relativ rührig sind. Der tatsächliche Abstand zu größeren Einrichtungen wäre tendenziell eher als unterschätzt anzunehmen, was allerdings für die reale Organisationsqualität überhaupt nichts bedeuten mag.

4. Die Frage ist nämlich, ob die - tatsächliche oder vermeintliche - Überrepräsentanz der großen Einrichtungen und Dienste nicht primär damit zusammenhängt, dass Qualitätsmanagement - jedenfalls in der hier abgefragten systematisierten Weise - vor allem ein Instrument ist, das den spezifischen Problemen und größeninduzierten Arbeitsteilungsprozessen solcher Einrichtungen genuin angemessen ist, wohingegen kleinere Institutionen auf eher organische Lösungen ohne formalisierte Strukturen zurückgreifen können.

5. Sieben Vertreter öffentlicher Träger bekundeten in einer telefonischen Rückmeldung oder E-Mail, dass Ihnen die Teilnahme an der Befragung nur möglich sei, wenn zuvor der jeweilige Spitzenverband sein Placet gegeben habe - eine im vorliegenden Kontext 
nicht überwindbare Hürde.

6. Seitens einzelner freier Träger wurde auf Anfrage geäußert, dass sowohl eine gewisse allgemeine Sättigung dem Thema gegenüber vorherrsche, als auch eine konkrete Skepsis, was ihren eigenen Nutzen durch die Aktion anbelange, mitbedingt durch Negativerfahrungen mit Befragungen ähnlicher Art.

7. Vereinzelt wurde eine Teilnahme an der Befragung mit der Begründung abgelehnt, dafür weder personelle Kapazität noch Geld zu haben; einmal wurde die Länge des Fragebogens moniert; die angegebene Bearbeitungszeit von 15 bis 30 Minuten sei unrealistisch.

8. Nicht zuletzt muss allgemein in Rechnung gestellt werden, dass eine derart differenzierte Befragung die Kooperationsbereitschaft von PraktikerInnen auf eine harte Belastungsprobe stellt. Wenngleich mehrere Rückmeldungen die positive Funktion der Fragebogenbearbeitung betonten (Reflexion des eigenen QM-Prozesses, Fokussierung auf unbeachtete Aspekte), dürfte manche/r potenzielle TeilnehmerIn aus Zeitmangel vor dem Bearbeitungsaufwand kapituliert haben.

\subsection{Auswertungsverfahren}

Die Daten aus dem ggf. um Doppelrepräsentanz von Einrichtungen (Version 1 und 2) bereinigten Rücklauf wurden in einen SPSS-Dateneditor eingegeben und mit SPSS, Version 12.0, teilweise auch mit Microsoft Excel ausgewertet. Einzelne Verrechnungen bereits aggregierter Daten (darunter mehrere Chi-Quadrat-Tests auf Basis von Prozentwerten, s. Tab. 20, 22 und 23) wurden manuell vorgenommen.

Zum Zwecke des multiplen Mittelwertsvergleichs der Stichproben unter den verschiedenen Faktorstufen wurden Varianzanalysen durchgeführt, soweit die Anwendungsbedingungen erfüllt waren (Intervallskalenniveau der abhängigen Variable, Varianzhomogenität, hinreichende Zellenbesetzungen). Etwaige Differenzen sollten anschließend mittels t-Tests für unabhängige, ggf. abhängige Stichproben geprüft werden. 
UV 1: Arbeitsfeld (Stufen: Jugendhilfeeinrichtung, Jugend-/Sozialhilfeverwaltung, ggf. Sonstiges);

○ UV 2: Einrichtungsgröße (Stufen: sehr groß, groß, mittelgroß, klein/kleinst);

○ UV 3: Trägerschaft (Stufen: öffentlich, freigemeinnützig, privatgewerblich);

○ UV 4: QB-Geschlecht (Stufen: weiblich, männlich);

○ UV 5: QB-Alter (Stufen: bis 30, 31-40, 41-50, 51 und älter);

- AV 4 (1 bis n): Maßnahmen zur Qualitätsentwicklung (Likert-Skala);

○ AV 6 (1 bis n): QB-Zweckmäßigkeit (Likert-Skala);

- AV 7 (1 bis n): QB-Konfliktpotenziale (Likert-Skala);

- $\quad \operatorname{AV} 8$ (1 bis n): QB-Rollenerwartungen/-wahrnehmung (Likert-Skala);

- AV 9 (1 bis n): QB-Tätigkeiten (Likert-Skala).

Zwecks Bildung annähernd gleicher Zellenbesetzungen wurden für die varianzanalytische Auswertung die schwächer repräsentierten Arbeitsfelder zu einer Gruppe der Sonstigen zusammengefasst.

Für die Darstellung und Signifikanzprüfung nominal- und ordinalskalierter Variablen kamen Häufigkeits- und Kreuztabellen bzw. Chi-Quadrat-Tests und nonparametrische Korrelationsmaße in Betracht. Dies gilt auch für Likert-skalierte Variablen, für die eine varianzanalytische Auswertung mangels ausreichender Zellenbesetzung nicht in Frage kam; zusätzlich konnten in diesen Fällen Mittelwertsvergleiche angestellt werden.

○ UV 1: Arbeitsfeld;

- UV 2: Einrichtungsgröße;

○ UV 3: Trägerschaft;

U UV 4: QB-Geschlecht;

○ UV 5: QB-Alter;

- AV 1 (1 bis n): QM-Organisation, Programmatik, Instrumente (Nominal- und Ordinalskalen);

- AV 2: Beteiligung der MitarbeiterInnen (Ordinalskala);

- AV 3: Qualifizierung der MitarbeiterInnen (Ordinalskala);

○ AV 5 (1 bis n): QB-Merkmale (Nominal- und Ordinalskalen);

○ AV 6 (1 bis n): QB-Zweckmäßigkeit (Likert-Skala);

- AV 7 (1 bis n): QB-Konfliktpotenziale (Likert-Skala);

○ AV 8 (1 bis n): QB-Rollenerwartungen/-wahrnehmung (Likert-Skala);

○ AV 9 (1 bis n): QB-Tätigkeiten (Likert-Skala). 


\subsection{Behandlung von mehrfach vertretenen Einrichtungen}

Einrichtungen mit Qualitätsbeauftragten wurden für den Fall einer Beantwortung der Versionen 1 und 2 durch verschiedene MitarbeiterInnen darum gebeten, die Bögen zusammen in dem beigefügten (adressierten, jedoch unfrankierten) Umschlag zurückzusenden, um eine Identifizierung der Datenpaare zu ermöglichen. Leider machten nur 17 Einrichtungen (entsprechend 20\% des Rücklaufs) davon Gebrauch, darunter auch einige unvollständig ausgefüllte Exemplare. Diese 17 Fragebögen wurden bei der Auswertung der Struktur-Items (Trägerschaft, Größe, Arbeitsfeld, Qualitätsmanagement) berücksichtigt, indem nur die Angaben des/der Qualitätsbeauftragten (Version 1) verwendet wurden, um Doppelrepräsentanz der betreffenden Einrichtungen auszuschließen.

Die möglichen Vergleichsberechnungen zwischen Version 1 und 2 wurden nur für die QB bezogenen Items vorgenommen und gesondert dargestellt, um Urteilsabweichungen zwischen Qualitätsbeauftragten und Leitungskräften zu ermitteln. Diese Vergleiche stehen zwar statistisch auf recht schwachen Füßen (teilweise $\mathrm{n}=12$ ), sind aber dennoch informativ. Naturgemäß lassen die Vergleiche nur Aussagen über Unterschiede zwischen Gruppen und keine Rückschlüsse darauf zu, wie häufig oder stark das Instrument des/der QB innerhalb einer Einrichtung unterschiedlich beurteilt wird von StelleninhaberInnen und leitenden KollegInnen, die Erfahrungen damit haben, ohne das Amt selbst auszuüben.

In die Bewertung von Maßnahmen der Qualitätsentwicklung gingen dagegen alle 102 Fragebögen ein, da hier primär das an einer möglichst großen Stichprobe ermittelte Urteil sozialer Fachkräfte gefragt war und weniger die potenziellen Zusammenhänge mit Einrichtungsgröße, Trägerschaft und Arbeitsfeld; diesbezüglich war die im Rücklauf erzielte Zellenbesetzung zu gering, um verlässliche Differenzierungen zu ermöglichen. Dabei muss auch berücksichtigt werden, dass bei diesem Untersuchungsaspekt Einstellungen im Zentrum standen und nicht - wie bei den Fragen zu Strukturelementen des Qualitätsmanagements - Fakten, die bezüglich ihres Vorhanden- oder Nichtvorhandenseins zu bewerten waren. 


\subsection{Ergebnisse}

Die exakten Formulierungen der Fragebogen-Items und im Text erwähnte, aber nicht dargestellte Ergebnistabellen sind dem Anhang (Kap. G) zu entnehmen. Im Folgenden sind die Fragen und vorgegebenen Antwortmöglichkeiten nur dann wortgetreu aufgeführt, wenn es für das Verständnis erforderlich ist; ansonsten sind längere Formulierungen sinnvoll abgekürzt, um eine tabellarische Übersicht zu ermöglichen. Häufigkeitsangaben in Klammern beziehen sich auf doppelten Rücklauf (s. Kap. D 4.5).

\subsubsection{Organisation des Qualitätsmanagements}

\subsubsection{Aufbau- und Ablaufbeschreibung}

Über $80 \%$ der Einrichtungen in der Stichprobe verfügen über eine funktionale Beschreibung ihrer Organisation; knapp 10\% arbeiten daran und rund 5\% planen entsprechende Aktivitäten. Ergänzend ist anzumerken, dass die zwei Einrichtungen ohne Organigramm zur Gruppe der kleinen/kleinsten Einrichtungen gehören.

Tab. 27: Aufbau- und Ablaufbeschreibung (Organigramm)

\begin{tabular}{|ll|l|l|l|l|}
\hline & & Häufigkeit & Prozent & $\begin{array}{l}\text { Gültige } \\
\text { Prozente }\end{array}$ & $\begin{array}{l}\text { Kumulierte } \\
\text { Prozente }\end{array}$ \\
\hline Gültig & vorhanden & $71(14)$ & 83,5 & 83,5 & 83,5 \\
& in Arbeit & $8(1)$ & 9,4 & 9,4 & 92,9 \\
& geplant & $4(1)$ & 4,7 & 4,7 & 97,6 \\
& nicht vorhanden & $2(0)$ & 2,4 & 2,4 & 100,0 \\
& Gesamt & $85(16)$ & 100,0 & 100,0 & \\
Fehlend & keine Angabe & $(1)$ & & & \\
Gesamt & & $85(17)$ & & & \\
\hline
\end{tabular}




\subsubsection{QM-System}

Rund zwei Drittel der Einrichtungen verfügen zumindest in Ansätzen über ein QMSystem; ein weiteres Sechstel hegt Einführungspläne. Unter dem Zehntel ohne QMSystem, das auch keine Einführung vorsieht, sind alle Größen vertreten, schwerpunktmäßig jedoch die kleinsten Einrichtungen.

Tab. 28: QM-System

\begin{tabular}{|ll|l|l|l|l|}
\hline & & Häufigkeit & Prozent & $\begin{array}{l}\text { Gültige } \\
\text { Prozente }\end{array}$ & $\begin{array}{l}\text { Kumulierte } \\
\text { Prozente }\end{array}$ \\
\hline Gültig & vorhanden & $28(9)$ & 32,9 & 35,9 & 35,9 \\
& in Ansätzen & $27(4)$ & 31,8 & 34,6 & 70,5 \\
& geplant & $14(1)$ & 16,5 & 17,9 & 88,5 \\
& nicht vorhanden & $9(2)$ & 10,6 & 11,5 & 100,0 \\
& Gesamt & $78(16)$ & 91,8 & 100,0 & \\
Fehlend & keine Angabe & $7(1)$ & 8,2 & & \\
Gesamt & & $85(17)$ & 100,0 & & \\
\hline
\end{tabular}

Vorstehende Gesamtübersicht verdeckt jedoch erhebliche Unterschiede zwischen den verschiedenen Faktorstufen. Die folgenden Tabellen liefern ein wesentlich differenzierteres Bild (fehlende Angaben sind nicht ausgewiesen).

Tab. 29: QM-System nach Arbeitsfeldern

\begin{tabular}{|l|l|l|l|l|l|}
\hline & vorhanden & in Arbeit & geplant & weder noch & Gesamt \\
\hline Jugendamt & 3 & 8 & 5 & 6 & 22 \\
\hline Sozialamt & 0 & 3 & 1 & 3 & 7 \\
\hline Erziehungshilfe & 13 & 11 & 7 & 0 & 31 \\
\hline Sonstiges & 12 & 4 & 1 & 0 & 17 \\
\hline Gesamt & 28 & 26 & 14 & 9 & 77 \\
\hline
\end{tabular}

Die unterschiedliche Umsetzung systematischen Qualitätsmanagements in verschiedenen Arbeitsfeldern ist hochsignifikant ( $\mathrm{p}=.000)$; Erziehungshilfeeinrichtungen und sonstige, überwiegend in freier Trägerschaft befindliche Organisationen verfügen mehrheitlich über 
ein entwickeltes System. Jugend- und mehr noch Sozialämter befinden sich erheblich im Rückstand. Diese Formulierung insinuiert allerdings einen (durchaus fraglichen) Konsens darüber, dass ein entwickeltes QM-System eine Art anzustrebenden Soll-Zustands darstellt.

Betrachtet man die arbeitsfeldbezogenen Mittelwerte - ungeachtet des eher ordinalen Skalenniveaus -, so ergibt sich folgende Rangordnung in der Umsetzung von QM (SD = 1,00):

1. Sonstige Einrichtungen (u. a. Schule/Berufsbildungswerk, Altenpflege; $M=1,35$ ),

2. Erziehungshilfeeinrichtungen $(M=1,81)$,

3. Jugendämter $(M=2,64)$,

4. Sozialämter $(M=3,00)$.

Aufgrund der teilweise sehr geringen Stichprobengrößen ist nur die Differenz zwischen Erziehungshilfeeinrichtungen und Jugendämtern signifikant.

Wegen der Konfundierung der Variablen ergibt sich für den Faktor Trägerschaft zwangsläufig ein analoges Ergebnis.

Tab. 30: QM-System nach Trägerschaft

\begin{tabular}{|l|l|l|l|l|l|}
\hline & vorhanden & in Arbeit & geplant & weder noch & Gesamt \\
\hline öffentlich & 4 & 12 & 6 & 9 & 31 \\
\hline freigemeinnützig & 19 & 9 & 3 & 0 & 31 \\
\hline privatgewerbl. & 5 & 6 & 5 & 0 & 16 \\
\hline Gesamt & 28 & 27 & 14 & 9 & 78 \\
\hline
\end{tabular}

Betrachtet man die trägerbezogenen Mittelwerte - wiederum ungeachtet des eher ordinalen Skalenniveaus -, so ergibt sich folgende Rangordnung in der Umsetzung von QM:

1. freigemeinnützige Träger $(M=1,48)$,

2. privatgewerbliche Träger $(\mathrm{M}=2,00)$,

3. öffentliche Träger $(M=2,65)$. 
Die Differenzen sind signifikant.

Im Sinne der in Kap. A 5.2.1 formulierten Hypothese unerwartet fällt die Verteilung nach Größenquartilen aus. Sieht man von der relativ hohen Zahl von fünf großen Einrichtungen ohne QM-System und entsprechende Pläne ab, sind fast alle Zellen proportional besetzt. Erwartungsgemäß ist der Anteil der Einrichtungen mit entwickeltem QM-System in sehr großen Organisationen am höchsten; insgesamt ergibt sich jedoch kein signifikanter Zusammenhang zwischen dem Faktor der Größe und dem Stand der Umsetzung von QM.

Tab. 31: QM-System nach Einrichtungsgröße

\begin{tabular}{|l|l|l|l|l|l|}
\hline & vorhanden & in Arbeit & geplant & weder noch & Gesamt \\
\hline klein/kleinst & 5 & 9 & 5 & 1 & 20 \\
\hline mittelgroß & 9 & 4 & 4 & 2 & 19 \\
\hline groß & 4 & 7 & 3 & 5 & 19 \\
\hline sehr groß & 10 & 7 & 2 & 1 & 20 \\
\hline Gesamt & 28 & 27 & 14 & 9 & 78 \\
\hline
\end{tabular}

Betrachtet man auch hier die größenbezogenen Mittelwerte - ungeachtet des eher ordinalen Skalenniveaus -, so ergibt sich folgende Rangordnung in der Umsetzung von QM:

1. sehr große Einrichtungen $(M=1,70)$,

2. mittelgroße Einrichtungen $(M=1,95)$,

3. kleine/kleinste Einrichtungen $(M=2,10)$,

4. große Einrichtungen $(\mathrm{M}=2,47)$.

Signifikant auf dem 5\%-Niveau sind die Differenzen zwischen kleinen/kleinsten und sehr großen sowie großen und sehr großen Einrichtungen. In diesen Zahlen kommt allerdings der überproportional hohe Anteil öffentlicher Träger am dritten Größenquartil zum Ausdruck. Da diese Trägergruppe mehrheitlich noch nicht über ein QM-System verfügt, wird ein tatsächlich vorhandener Größeneffekt - zumindest im Bereich der Erziehungshilfen - überdeckt. 


\subsubsection{Art des QM-Systems}

Fehlende Angaben in der Tabelle entfallen naturgemäß vorwiegend auf jene Einrichtungen, die kein QM-System haben oder sich erst in der Planungsphase befinden. Unter den abgegebenen Nennungen dominiert die ISO 9000 ff. mit gut einem Viertel, von dem wiederum ein Drittel zertifiziert ist oder eine Zertifizierung anstrebt. Das EFQMModell wird von einem Fünftel der Einrichtungen als Referenzsystem genannt, ServAs (Service Assessment) als komprimierter Ableger beider dominierenden Systeme nur einmal.

In etwa gleichem Maße wie ISO 9000 ff. werden einrichtungsintern entwickelte und eklektische Ansätze verwendet, so dass von einer Trisektion der Systeme in der Praxis gesprochen werden kann: ISO 9000 ff., organisationsspezifische Eigen- und Mischsysteme und EFQM.

Tab. 32: Art des QM-Systems (Mehrfachnennungen möglich; in Klammern Doppelrücklauf)

\begin{tabular}{|ll|l|l|l|l|}
\hline & & Häufigkeit & Prozent & $\begin{array}{l}\text { Gültige } \\
\text { Prozente }\end{array}$ & $\begin{array}{l}\text { Kumulierte } \\
\text { Prozente }\end{array}$ \\
\hline Gültig & EFQM & $16(3)$ & 20,5 & 20,5 & 20,5 \\
& ISO 9000 ff. & $22(8)$ & 28,2 & 28,2 & 48,7 \\
& Zertifizierung/geplant & $7(4)$ & 9,0 & 9,0 & 57,7 \\
& ServAs & $1(0)$ & 1,3 & 1,3 & 59,0 \\
& Verbandssystem & $3(0)$ & 3,8 & 3,8 & 62,8 \\
& "Marke Eigenbau" & $16(5)$ & 20,5 & 20,5 & 83,3 \\
& Mischsystem & $8(4)$ & 10,3 & 10,3 & 93,6 \\
& Mitglied Kooperative & $1(0)$ & 1,3 & 1,3 & 94,9 \\
& Sonstiges & $4(0)$ & 5,1 & 5,1 & 100,0 \\
Gesamt & keine Angabe & $78(24)$ & 100,0 & 100,0 & \\
& $28(2)$ & & & \\
\hline
\end{tabular}

Anmerkungen:

An Verbandssystemen werden genannt: 2 x PQ-Sys, 1 x AWO. An sonstigen Systemen kommen vor: je $1 \mathrm{x}$ QDS, CAF, Diakoniesiegel Pflege.

Der Qualitäts-Check PQ-Sys® umfasst eine Prüfung anhand verschiedener von der PARITÄTISCHEN Gesellschaft für Qualität mbH (PQ) entwickelter Evaluationsbögen. CAF (Common Assessment Framework) ist ein gemeinsames europäisches Qualitätsbewertungssystem für die öffentlichen Verwaltungen, ein im Vergleich zum TQM- und EFQM-Modell stark vereinfachtes System zur Selbstbewertung als Einstieg in Qualitätsmanagement. QDS steht für Quality Data Service Gmbh und ist ein System zur Datenaufbereitung in verschiedenen Arbeitsfeldern, also kein Qualitätskonzept im hier verstandenen Sinne.

Wie (hier nicht dargestellte) Kreuztabellierungen ausweisen, sind Zusammenhänge 
zwischen der QM-Systemorientierung und den unabhängigen Variablen sämtlich signifikant $(\alpha=.05)$ :

○ In Jugend- und Sozialämtern, die bereits über ein QM-System verfügen, dominiert neben Eigenentwicklungen (6 Nennungen) und Mischsystemen (4) die Orientierung am EFQM-Modell (8), während die ISO (2) kaum eine Rolle spielt.

○ In der Erziehungshilfe überwiegen insgesamt zwar die Eigenentwicklungen (13), jedoch kommt der ISO (9) ein wesentlich höherer Stellenwert zu als EFQM (4).

○ Unter den sonstigen Arbeitsfeldern befinden sich alle Einrichtungen mit erfolgter oder angestrebter Zertifizierung nach ISO 9001 (Berufsbildungswerke, Behindertenhilfe).

○ Analoge Befunde ergeben sich für den Faktor Trägerschaft: ISO ist eine Domäne der freien Träger, EFQM eher der öffentlichen; privatgewerbliche Einrichtungen bevorzugen Eigenentwicklungen.

○ Wegen der schon mehrfach erwähnten Häufung der Jugendämter im dritten Größenquartil sind die Befunde beim Faktor Einrichtungsgröße weniger klar, jedoch signifikant $(\mathrm{p}=.048)$ : Eigenentwicklungen sind eher für kleine Einrichtungen charakteristisch, an ISO orientierte Systeme eher für sehr große.

\subsubsection{Programmatik}

\subsubsection{Grundsätze zur Qualitätspolitik}

Knapp die Hälfte der in der Stichprobe vertretenen Einrichtungen verfügt über schriftlich fixierte und bekannt gegebene Grundsätze zur Qualitätspolitik; fast ebenso viele arbeiten daran oder planen, dies zu tun. Rund 12\% der Einrichtungen hegen keine entsprechenden Ambitionen. 
Tab. 33: Grundsätze zur Qualitätspolitik

\begin{tabular}{|ll|l|l|l|l|}
\hline & & Häufigkeit & Prozent & $\begin{array}{l}\text { Gültige } \\
\text { Prozente }\end{array}$ & $\begin{array}{l}\text { Kumulierte } \\
\text { Prozente }\end{array}$ \\
\hline Gültig & schriftlich fixiert & $39(6)$ & 45,9 & 45,9 & 45,9 \\
& in Arbeit & $25(8)$ & 29,4 & 29,4 & 75,3 \\
& geplant & $11(3)$ & 12,9 & 12,9 & 88,2 \\
& nicht geplant & $10(0)$ & 11,8 & 11,8 & 100,0 \\
Gesamt & & $85(17)$ & 100,0 & 100,0 & \\
\hline
\end{tabular}

Zusammenhänge mit den UV erweisen sich nur in einem Fall als signifikant: Öffentliche Träger bzw. Jugend- und Sozialämter haben seltener Grundsätze zur Qualitätspolitik formuliert als Erziehungshilfeeinrichtungen freier Träger.

\subsubsection{Grundsätze zur Führung}

Ein Siebtel der Einrichtungen beabsichtigt nicht, Grundsätze zur Führung zu fixieren; ein weiteres Siebtel befindet sich in der Planungsphase, während rund ein Viertel mit dem Arbeitsprozess begonnen hat. Annähernd die Hälfte verfügt bereits über entsprechende Richtlinien.

Tab. 34: Grundsätze zur Führung

\begin{tabular}{|ll|l|l|l|l|}
\hline & & Häufigkeit & Prozent & $\begin{array}{l}\text { Gültige } \\
\text { Prozente }\end{array}$ & $\begin{array}{l}\text { Kumulierte } \\
\text { Prozente }\end{array}$ \\
\hline Gültig & schriftlich fixiert & $40(10)$ & 47,1 & 47,1 & 47,1 \\
& in Arbeit & $20(2)$ & 23,5 & 23,5 & 70,6 \\
& geplant & $13(3)$ & 15,3 & 15,3 & 85,9 \\
& nicht geplant & $12(1)$ & 14,1 & 14,1 & 100,0 \\
Gesamt & & $85(16)$ & 100,0 & 100,0 & \\
Fehlend & keine Angabe & $(1)$ & & & \\
Gesamt & & $85(17)$ & & & \\
\hline
\end{tabular}

Zusammenhänge mit UV: Fehlanzeige. 


\subsubsection{Leitbild für die Gesamteinrichtung}

Über zwei Drittel der vertretenen Organisationen verfügen über ein schriftlich fixiertes und bekannt gegebenes Leitbild für die Gesamteinrichtung, wie auch immer - partizipativ oder "per Erlass" - dieses entwickelt wurde (danach wurde leider nicht gefragt). Ein weiteres Fünftel steht im Prozess oder plant entsprechende Aktivitäten. Rund jede zehnte Einrichtung äußert keinerlei Ambitionen.

Tab. 35: Leitbild für die Gesamteinrichtung

\begin{tabular}{|ll|l|l|l|l|}
\hline & & Häufigkeit & Prozent & $\begin{array}{l}\text { Gültige } \\
\text { Prozente }\end{array}$ & $\begin{array}{l}\text { Kumulierte } \\
\text { Prozente }\end{array}$ \\
\hline Gültig & schriftlich fixiert & $59(15)$ & 69,4 & 69,4 & 69,4 \\
& in Arbeit & $9(2)$ & 10,6 & 10,6 & 80,0 \\
& geplant & $8(0)$ & 9,4 & 9,4 & 89,4 \\
& nicht geplant & $9(0)$ & 10,6 & 10,6 & 100,0 \\
Gesamt & & $85(17)$ & 100,0 & 100,0 & \\
\hline
\end{tabular}

Als bemerkenswert kann ergänzt werden, dass bei insgesamt nicht signifikantem Zusammenhang zwischen UV und Leitbildentwicklung die sieben befragten Sozialämter in diesem Punkt "die Nase vorn" haben.

\subsubsection{Instrumente}

\subsubsection{Qualitäts(management)-Handbuch}

Ein Viertel der Einrichtungen sieht keine Handbucherstellung vor; ein weiteres Viertel hat einschlägige Pläne. 20\% arbeiten am Handbuch und knapp 30\% verfügen bereits darüber. 
Tab. 36: QM-Handbuch

\begin{tabular}{|ll|l|l|l|l|}
\hline & & Häufigkeit & Prozent & $\begin{array}{l}\text { Gültige } \\
\text { Prozente }\end{array}$ & $\begin{array}{l}\text { Kumulierte } \\
\text { Prozente }\end{array}$ \\
\hline Gültig & schriftlich fixiert & $25(8)$ & 29,4 & 29,4 & 29,4 \\
& in Arbeit & $17(4)$ & 20,0 & 20,0 & 49,4 \\
& geplant & $21(2)$ & 24,7 & 24,7 & 74,1 \\
& nicht geplant & $22(3)$ & 25,9 & 25,9 & 100,0 \\
Gesamt & & $85(17)$ & 100,0 & 100,0 & \\
\hline
\end{tabular}

QM-Handbücher spielen bei freigemeinnützigen Trägern - und hier vor allem im Arbeitsfeld Sonstige (speziell in Schule/Berufsbildungswerk) - die signifikant größte Rolle, während öffentliche und mehr noch privatgewerbliche Träger seltener davon Gebrauch machen. Auch ein Zusammenhang mit der Einrichtungsgröße ist evident. Wegen des überproportionalen Anteils der Jugendämter - die seltener mit Handbüchern arbeiten unter den großen Einrichtungen beträgt die Korrelation jedoch nur -.269 (signifikant, negatives Vorzeichen polungsbedingt). Für den Bereich der Erziehungshilfen ist ein wesentlich stärkerer Zusammenhang zwischen Einrichtungsgröße und Einsatz von QMHandbüchern zu unterstellen.

\subsubsection{Qualitätszirkel}

In über 70\% der Einrichtungen wird das Instrument Qualitätszirkel (QZ) bereits praktiziert, mit leichtem Übergewicht in Form speziell eingeführter, Bereiche und/oder Hierarchie übergreifender Arbeitsgruppen. Häufig anzutreffen sind aber auch Zirkel auf der Ebene natürlicher Organisationseinheiten (Teams, Abteilungen), die im Rahmen der betriebsüblichen Besprechungskontingente anstehende Qualitätsfragen bearbeiten. Von beiden Möglichkeiten zugleich wird nur selten Gebrauch gemacht. Knapp ein Fünftel der Einrichtungen befindet sich in der Planungsphase; nur rund 8\% erwägen keine Einführung von Qualitätszirkelarbeit. 
Tab. 37: Qualitätszirkel

\begin{tabular}{|ll|l|l|l|l|}
\hline & & Häufigkeit & Prozent & $\begin{array}{l}\text { Gültige } \\
\text { Prozente }\end{array}$ & $\begin{array}{l}\text { Kumulierte } \\
\text { Prozente }\end{array}$ \\
\hline Gültig & auf Teamebene & $24(7)$ & 28,2 & 28,2 & 28,2 \\
& als spezielle Gruppe & $35(6)$ & 41,2 & 41,2 & 69,4 \\
& sowohl als auch & $3(1)$ & 3,5 & 3,5 & 72,9 \\
& geplant & $16(2)$ & 18,8 & 18,8 & 91,7 \\
& nicht geplant & $7(1)$ & 8,3 & 8,3 & 100,0 \\
Gesamt & & $85(17)$ & 100,0 & 100,0 & \\
\hline
\end{tabular}

Zwischen der Einsatzhäufigkeit von QZ und der Anwendung eines QM-Systems besteht keine Korrelation (Kendall's Tau-b = .009). Die Existenz eines QZ lässt somit keine Rückschlüsse auf den Umsetzungsstand des QM zu.

Mit den UV ergeben sich keine signifikanten Zusammenhänge; in allen vertretenen Arbeitsfeldern, Trägergruppen und Einrichtungsgrößen sind die Antwortkategorien annähernd proportional besetzt. Statistisch bedeutsam ist dagegen ein relativer Vorsprung jener Einrichtungen, die sich nicht an einem der prominenten Referenzsysteme (EFQM, ISO 9000 ff.) orientieren, sondern organisationsspezifische, gemischte oder sonstige QMModelle präferieren: in fast allen sind QZ bereits installiert (Kreuztabelle Systemorientierung x QZ: Chi-Quadrat $=18,474, \mathrm{df}=10, \mathrm{p}=.047)$. Auch mit dem Grad der Mitarbeiterbeteiligung zeigt sich eine auf dem 5\%-Niveau signifikante Korrelation von .211 (Kendall’s Tau-b).

\subsubsection{Vorschlagswesen und Ideenmanagement}

Drei Viertel der Einrichtungen haben mindestens Pläne, ein betriebliches Vorschlagswesen zu etablieren; vorhanden und systematisch angewandt ist ein solches Ideenmanagement allerdings erst bei einem Sechstel. 27\% sehen offenbar keinen Handlungsbedarf in dieser Richtung. 
In der Untersuchung von Wetzler (2003, S. 59f) wurde von 47,1\% der befragten Wohneinrichtungen der Behindertenhilfe (Ende 2001) angegeben, über ein Vorschlags- und Verbesserungswesen zu verfügen; 10,8\% planten den Einsatz; 42,1\% verneinten dies.

Tab. 38: Vorschlagswesen/Ideenmanagement

\begin{tabular}{|ll|l|l|l|l|}
\hline & & Häufigkeit & Prozent & $\begin{array}{l}\text { Gültige } \\
\text { Prozente }\end{array}$ & $\begin{array}{l}\text { Kumulierte } \\
\text { Prozente }\end{array}$ \\
\hline Gültig & vorhanden & $14(7)$ & 16,5 & 16,5 & 16,5 \\
& in Ansätzen & $35(4)$ & 41,2 & 41,2 & 57,7 \\
& geplant & $13(2)$ & 15,3 & 15,3 & 73,0 \\
& nicht geplant & $23(4)$ & 27,0 & 27,0 & 100,0 \\
Gesamt & & & 100,0 & 100,0 & \\
\hline
\end{tabular}

Bemerkenswert ist, dass bei insgesamt nicht signifikanten Unterschieden zwischen den diversen Faktorstufen die Sozialämter - wie schon beim Leitbild - den relativ höchsten Umsetzungsstand vermelden. Dies gilt gleichermaßen für die nachfolgend erörterten Instrumente des Beschwerdemanagements und des Personalentwicklungskonzepts.

\subsubsection{Beschwerdemanagement}

Die Zahlen gleichen sehr den Angaben zum Vorschlagswesen; der Anteil inaktiver Einrichtungen ist mit rund einem Fünftel etwas geringer.

In der Untersuchung von Wetzler (2003, S. 59) wird zur Verbreitung eines Beschwerdemanagements folgende Antwortverteilung angegeben: $45 \%$ ja, $17 \%$ geplant, $38 \%$ nein.

Tab. 39: Beschwerdemanagement

\begin{tabular}{|ll|l|l|l|l|}
\hline & & Häufigkeit & Prozent & $\begin{array}{l}\text { Gültige } \\
\text { Prozente }\end{array}$ & $\begin{array}{l}\text { Kumulierte } \\
\text { Prozente }\end{array}$ \\
\hline Gültig & vorhanden & $12(7)$ & 14,1 & 14,1 & 14,1 \\
& in Ansätzen & $38(7)$ & 44,7 & 44,7 & 58,8 \\
& geplant & $17(2)$ & 20,0 & 20,0 & 78,8 \\
& nicht geplant & $18(1)$ & 21,2 & 21,2 & 100,0 \\
Gesamt & & & 100,0 & 100,0 & \\
\hline
\end{tabular}


Sehr große Einrichtungen verfügen häufiger über ein Beschwerdemanagement als andere Organisationsgrößen. Im Übrigen wird auf die Anmerkung zu Tab. 38 verwiesen.

\subsubsection{Personalentwicklungskonzept}

Über $90 \%$ der Einrichtungen befassen sich zumindest auf Planungsebene mit Personalentwicklungskonzepten. Allerdings verfügt erst ein Sechstel über ein Konzept, das auch systematisch angewandt wird. Unter den Einrichtungen ohne diesbezügliche Ambitionen (rund 8\%) überwiegen kleine und kleinste.

Tab. 40: Personalentwicklungskonzept

\begin{tabular}{|ll|l|l|l|l|}
\hline & & Häufigkeit & Prozent & $\begin{array}{l}\text { Gültige } \\
\text { Prozente }\end{array}$ & $\begin{array}{l}\text { Kumulierte } \\
\text { Prozente }\end{array}$ \\
\hline Gültig & vorhanden & $14(5)$ & 16,5 & 16,9 & 16,9 \\
& in Ansätzen & $42(8)$ & 49,4 & 50,6 & 67,5 \\
& geplant & $20(2)$ & 23,5 & 24,1 & 91,6 \\
& nicht geplant & $7(1)$ & 8,2 & 8,4 & 100,0 \\
& Gesamt & $83(16)$ & 97,6 & 100,0 & \\
Fehlend & keine Angabe & $2(1)$ & 2,4 & & \\
Gesamt & & $85(17)$ & 100,0 & & \\
\hline
\end{tabular}

Zusammenhänge mit UV bestehen nicht; auch hier ist jedoch auf die Anmerkung zu Tab. 38 hinzuweisen.

\subsubsection{Evaluation}

Fast alle Einrichtungen geben an, Evaluationsverfahren einzusetzen oder dies zu planen. Ein Viertel praktiziert den Einsatz bereits regel- und routinemäßig, knapp die Hälfte bei besonderen Anlässen. 
Tab. 41: Einsatz von Evaluationsverfahren

\begin{tabular}{|ll|l|l|l|l|}
\hline & & Häufigkeit & Prozent & $\begin{array}{l}\text { Gültige } \\
\text { Prozente }\end{array}$ & $\begin{array}{l}\text { Kumulierte } \\
\text { Prozente }\end{array}$ \\
\hline Gültig & regelmäßig eingesetzt & $20(6)$ & 23,5 & 24,4 & 24,4 \\
& bei besond. Anlässen & $37(8)$ & 43,5 & 45,2 & 69,6 \\
& geplant & $23(3)$ & 27,1 & 28,0 & 97,6 \\
& nicht geplant & $2(0)$ & 2,4 & 2,4 & 100,0 \\
& Gesamt & $82(17)$ & 96,5 & 100,0 & \\
Fehlend & keine Angabe & $3(0)$ & 3,5 & & \\
Gesamt & & $85(17)$ & 100,0 & & \\
\hline
\end{tabular}

Differenzen zwischen Einrichtungen unterschiedlicher Faktorstufen sind minimal und in keinem Fall signifikant.

\subsubsection{Controlling}

Das gemeinhin stark betriebswirtschaftlich konnotierte Thema des Controllings erfreut sich in den Einrichtungen der Stichprobe gleichfalls großer Prominenz. Nur jede zehnte Einrichtung hat keine entsprechenden Pläne; über die Hälfte verfügt über Ansätze, während rund ein Viertel Controllingverfahren bereits systematisch anwendet.

Tab. 42: Einsatz von Controllingverfahren

\begin{tabular}{|ll|l|l|l|l|}
\hline & Häufigkeit & Prozent & $\begin{array}{l}\text { Gültige } \\
\text { Prozente }\end{array}$ & $\begin{array}{l}\text { Kumulierte } \\
\text { Prozente }\end{array}$ \\
\hline Gültig & vorhanden & $19(11)$ & 22,4 & 23,5 & 23,5 \\
& in Ansätzen & $45(5)$ & 52,9 & 55,5 & 79,0 \\
& geplant & $9(0)$ & 10,6 & 11,1 & 90,1 \\
& nicht geplant & $8(1)$ & 9,4 & 9,9 & 100,0 \\
& Gesamt & $81(17)$ & 95,3 & 100,0 & \\
Fehlend & keine Angabe & $4(0)$ & 4,7 & & \\
Gesamt & & $85(17)$ & 100,0 & & \\
\hline
\end{tabular}


Von den insgesamt nicht signifikanten Zusammenhängen mit UV ist der relativ hohe Umsetzungsstand in den befragten Sozialämtern und in sehr großen Einrichtungen am auffälligsten.

\subsubsection{Beteiligung der MitarbeiterInnen}

Über ein Viertel der befragten Einrichtungen gibt an, die Mitarbeiterschaft umfassend am QM-Prozess zu beteiligen; punktuell gilt dies für ein weiteres Drittel. Zur Sache der Leitung oder einzelner MitarbeiterInnen macht immerhin rund ein Fünftel das Thema.

Tab. 43: Beteiligung der MitarbeiterInnen am QM-Prozess

\begin{tabular}{|ll|l|l|l|l|}
\hline & & Häufigkeit & Prozent & $\begin{array}{l}\text { Gültige } \\
\text { Prozente }\end{array}$ & $\begin{array}{l}\text { Kumulierte } \\
\text { Prozente }\end{array}$ \\
\hline Gülttig & umfassend alle & $24(9)$ & 28,2 & 28,2 & 28,2 \\
& punktuell alle & $30(4)$ & 35,3 & 35,3 & 63,5 \\
& nur einzelne MA & $13(3)$ & 15,3 & 15,3 & 78,8 \\
& nur Leitung & $3(0)$ & 3,5 & 3,5 & 82,3 \\
& entfällt/nicht begonnen & $15(0)$ & 17,7 & 17,7 & 100,0 \\
& Gesamt & $85(16)$ & 100,0 & 100,0 & \\
Fehlend & keine Angabe & $(1)$ & & & \\
Gesamt & & $85(17)$ & & & \\
\hline
\end{tabular}

Naturgemäß entfallen die Angaben zur Beteiligung der MitarbeiterInnen am QM-Prozess ausschließlich auf solche Einrichtungen, die bereits über ein QM-System verfügen oder dessen Entwicklung planen. Geprüft wurde deshalb auch die Frage nach einem möglichen Zusammenhang zwischen Beteiligungsgrad und Art dieses Systems. 
Tab. 44: Kreuztabelle Beteiligung x Systemorientierung (Rohwerte)

\begin{tabular}{|l|l|l|l|l|l|l|}
\hline & umfassend & $\begin{array}{l}\text { punktuell } \\
\text { alle }\end{array}$ & $\begin{array}{l}\text { einzelne } \\
\text { MA }\end{array}$ & $\begin{array}{l}\text { nur } \\
\text { Leitung }\end{array}$ & $\begin{array}{l}\text { noch nicht } \\
\text { begonnen }\end{array}$ & Gesamt \\
\hline EFQM & 3 & 8 & 4 & 0 & 1 & 16 \\
\hline ISO & 12 & 8 & 2 & 0 & 0 & 22 \\
\hline Zertifizierung & 5 & 2 & 0 & 0 & 0 & 7 \\
\hline Eigenbau & 3 & 8 & 4 & 0 & 1 & 16 \\
\hline Mischsystem & 2 & 2 & 3 & 0 & 1 & 8 \\
\hline Sonstige & 1 & 5 & 0 & 2 & 1 & 9 \\
\hline Gesamt & 26 & 33 & 13 & 2 & 4 & 78 \\
\hline
\end{tabular}

○ Von 22 ISO-orientierten Einrichtungen geben 12 an, die Mitarbeiterschaft umfassend $\mathrm{zu}$ beteiligen, acht weitere sprechen von punktueller Beteiligung aller und nur zwei haben lediglich einzelne MitarbeiterInnen involviert.

○ In EFQM-orientierten Einrichtungen und solchen mit intern entwickelten oder gemischten Systemen dominiert bislang eher die punktuelle Beteiligung der Belegschaft oder einzelner Personen.

Die Unterschiede sind hochsignifikant.

Signifikant auf dem 5\%-Niveau sind des Weiteren ein höherer Beteiligungsgrad in freigemeinnützigen Einrichtungen (Mittelwert 1,8) gegenüber öffentlichen und privatgewerblichen Trägern (je 2,9), analog in Erziehungshilfe- und sonstigen Einrichtungen (2,3 und 2,0) gegenüber Jugend- und Sozialämtern (2,9 und 2,7). In den meisten größeren Organisationen ist der Beteiligungsgrad $(2,0$ bis 2,6) höher als in kleinen und kleinsten $(2,8)$, allerdings ohne Differenzierung nach Arbeitsfeldern nicht signifikant.

\subsubsection{Qualifizierung der MitarbeiterInnen}

Aufgrund von Mehrfachnennungen ergibt sich in der Tabelle eine Gesamtzahl von 105 Antworten. Unter den praktizierten Veranstaltungen zur Qualifizierung der Akteure in Sachen QM dominieren mit rund 28\% spezifische Fortbildungen für Qualitätsbeauftragte, 
Leitungskräfte und MultiplikatorInnen, zusätzlich richten sich etwa 8\% speziell an QB. Basisveranstaltungen für die gesamte Belegschaft oder bestimmte Abteilungen werden von einem knappen Viertel angegeben. In rund 9\% der Einrichtungen finden keine Fortbildungsaktivitäten statt.

Tab. 45: QM-Fortbildungen und Veranstaltungen (Mehrfachnennungen möglich)

\begin{tabular}{|ll|l|l|l|l|}
\hline & & Häufigkeit & Prozent & $\begin{array}{l}\text { Gültige } \\
\text { Prozente }\end{array}$ & $\begin{array}{l}\text { Kumulierte } \\
\text { Prozente }\end{array}$ \\
\hline Gültig & alle MA kontinuierlich-systematisch & $11(4)$ & 10,5 & 10,5 & 10,5 \\
& spez. QB/Leitung/Multiplikatoren & $29(7)$ & 27,6 & 27,6 & 38,1 \\
& spez. Qualitätsbeauftragte/r & $8(4)$ & 7,6 & 7,6 & 45,7 \\
& Basisveranstaltung für alle & $17(6)$ & 16,2 & 16,2 & 61,9 \\
& Basisveranst. für best. Abteilungen & $8(1)$ & 7,6 & 7,6 & 69,5 \\
& einzelne Angebote o. syst. Zus.hang & $23(2)$ & 21,9 & 21,9 & 91,4 \\
& keine Fortbildungsaktivität in QM & $9(1)$ & 8,6 & 8,6 & 100,0 \\
Gesamt & & $105(25)$ & 100,0 & 100,0 & \\
\hline
\end{tabular}

Auch hinsichtlich QM bezogener Qualifizierungsmaßnahmen entfallen naturgemäß die meisten Angaben auf Einrichtungen, die über ein QM-System verfügen oder in der Planung stehen. Geprüft wurde deshalb auch hier die Frage nach einem möglichen Zusammenhang zwischen Qualifizierungsgrad und Art des vorhandenen Systems. Zwar zeigt die Kreuztabellierung überproportional kontinuierlich-systematische Qualifizierungen der Belegschaft in ISO-orientierten Einrichtungen, während allgemein Maßnahmen für einzelne Gruppen dominieren, doch sind die Unterschiede nicht signifikant ( $\mathrm{p}=.077)$.

Tab. 46: Kreuztabelle Qualifizierung x Systemorientierung (Rohwerte, Mehrfachnennungen möglich)

\begin{tabular}{|l|l|l|l|l|l|l|l|l|}
\hline & $\begin{array}{l}\text { system. } \\
\text { alle }\end{array}$ & $\begin{array}{l}\text { spez. } \\
\text { einzeln }\end{array}$ & $\begin{array}{l}\text { spez. } \\
\text { QB }\end{array}$ & $\begin{array}{l}\text { Basis } \\
\text { für alle }\end{array}$ & $\begin{array}{l}\text { Basis } \\
\text { einzelne }\end{array}$ & unsyst. & keine & Gesamt \\
\hline EFQM & 0 & 4 & 2 & 4 & 2 & 5 & 0 & 17 \\
\hline ISO & 6 & 9 & 5 & 7 & 2 & 2 & 2 & 33 \\
\hline Zertifizierg. & 3 & 7 & 0 & 1 & 0 & 0 & 0 & 11 \\
\hline Eigenbau & 1 & 8 & 0 & 2 & 0 & 7 & 0 & 18 \\
\hline Mischsystem & 1 & 3 & 1 & 0 & 0 & 4 & 1 & 10 \\
\hline Sonstige & 0 & 4 & 3 & 0 & 0 & 2 & 0 & 9 \\
\hline Gesamt & 11 & 35 & 11 & 14 & 4 & 20 & 3 & 98 \\
\hline
\end{tabular}

Signifikante Zusammenhänge mit UV zeigen sich in gleicher Weise wie beim 
Beteiligungsgrad: freigemeinnützige Einrichtungen qualifizieren am intensivsten (Mittelwert 2,8), öffentliche Träger $(4,4)$ bzw. Jugend- und Sozialämter $(4,2$ und 5,6) sowie kleine Einrichtungen $(4,3)$ eher basal, unsystematisch oder gar nicht.

\subsubsection{Signifikante Interkorrelationen der Ordinalvariablen}

Fast alle Items zu Organisation, Programmatik, Instrumenten, Beteiligung und Qualifizierung im Rahmen des Qualitätsmanagements interkorrelieren erwartungsgemäß mehr oder minder stark. Aus der Matrix von $n=19$ Variablen - entsprechend $n \times(n-1) / 2=$ 171 Koeffizienten - sind nachfolgend 51 signifikante Werte zusammengefasst.

Tab. 47: Signifikante Korrelationen zwischen Ordinalvariablen der Studie

\begin{tabular}{|c|c|c|c|}
\hline korrelierte Variablen & $\mathrm{n}$ & $\begin{array}{l}\text { Korrelations- } \\
\text { koeffizient } \\
\text { (Kendall's tau) }\end{array}$ & $\begin{array}{l}\text { Signifikanz } \\
\text { (zweiseitig, } \alpha=.05 \\
=\text { s.; } \alpha=.01=\text { s.s.) }\end{array}$ \\
\hline Einrichtungsgröße x QM-Handbuch & 102 & -.269 & .001 s.s. \\
\hline Einrichtungsgröße x QB & 102 & -.205 & $.015 \mathrm{~s}$. \\
\hline Einrichtungsgröße x Controlling & 98 & -.175 & $.045 \mathrm{~s}$ \\
\hline Einrichtungsgröße x Organigramm & 101 & -.277 & .002 s.s. \\
\hline QM-System x QM-Handbuch & 94 & .696 & .000 s.s. \\
\hline QM-System x QB & 94 & .417 & .000 s.s. \\
\hline QM-System x Qualitätspolitik & 102 & .652 & .000 s.s. \\
\hline QM-System x Führungsgrundsätze & 93 & .292 & .001 s.s. \\
\hline QM-System x Controlling & 90 & .206 & $.026 \mathrm{~s}$. \\
\hline QM-System x Beteiligung & 101 & .410 & .000 s.s. \\
\hline QM-System x Qualifizierung & 101 & .347 & .000 s.s. \\
\hline QM-Handbuch x QB & 102 & .457 & .000 s.s. \\
\hline QM-Handbuch x Qualitätspolitik & 102 & .511 & .000 s.s. \\
\hline QM-Handbuch x Führungsgrundsätze & 101 & .256 & .003 s.s. \\
\hline QM-Handbuch x Controlling & 98 & .300 & .001 s.s. \\
\hline QM-Handbuch x Beteiligung & 101 & .453 & .000 s.s. \\
\hline QM-Handbuch x Qualifizierung & 101 & .468 & .000 s.s. \\
\hline QB x Vorschlagswesen & 102 & .278 & .001 s.s. \\
\hline QB x Beschwerdemanagement & 102 & .249 & .003 s.s. \\
\hline QB x Qualitätspolitik & 102 & .203 & $.018 \mathrm{~s}$. \\
\hline QB x Leitbild & 102 & .304 & .001 s.s. \\
\hline QB x Controlling & 98 & .348 & .000 s.s. \\
\hline Vorschlagswesen x Beschwerdeman. & 102 & .270 & .001 s.s. \\
\hline Vorschlagswesen x PE-Konzept & 99 & .331 & .000 s.s. \\
\hline Vorschlagswesen x Führungsgrunds. & 101 & .282 & .001 s.s. \\
\hline Vorschlagswesen x Leitbild & 102 & .176 & $.045 \mathrm{~s}$. \\
\hline Beschwerdeman. x PE-Konzept & 99 & .296 & .001 s.s. \\
\hline Beschwerdeman. x Führungsgrund. & 101 & .190 & $.027 \mathrm{~s}$. \\
\hline Beschwerdeman. x Leitbild & 102 & .278 & .002 s.s. \\
\hline Beschwerdeman. x Controlling & 98 & .368 & .000 s.s. \\
\hline PE-Konzept x Führungsgrundsätze & 98 & .310 & .000 s.s. \\
\hline PE-Konzept x Evaluation & 97 & .257 & .004 s.s. \\
\hline
\end{tabular}




\begin{tabular}{|l|l|l|l|}
\hline PE-Konzept x Controlling & 96 & .232 & .010 s.s. \\
\hline PE-Konzept x Beteiligung & 98 & .205 & .018 s. \\
\hline PE-Konzept x Qualifizierung & 98 & .175 & .037 s. \\
\hline PE-Konzept x Organigramm & 98 & .204 & .028 s. \\
\hline Qualitätspolitik x Führungsgrunds. & 101 & .450 & .000 s.s. \\
\hline Qualitätspolitik x Controlling & 98 & .177 & .046 s. \\
\hline Qualitätspolitik x Beteiligung & 101 & .378 & .000 s.s. \\
\hline Qualitätspolitik x Qualifizierung & 101 & .368 & .000 s.s. \\
\hline Führungsgrundsätze x Leitbild & 101 & .229 & .010 s. \\
\hline Führungsgrundsätze x Beteiligung & 100 & .266 & .002 s.s. \\
\hline Leitbild x Controlling & 98 & .373 & .000 s.s. \\
\hline Leitbild x Beteiligung & 101 & .381 & .000 s.s. \\
\hline Leitbild x Qualifizierung & 101 & .212 & .012 s. \\
\hline Evaluation x Controlling & 98 & .213 & .018 s. \\
\hline Evaluation x Beteiligung & 98 & .249 & .004 s.s. \\
\hline Evaluation x Qualifizierung & 98 & .185 & .029 s. \\
\hline Controlling x Beteiligung & 97 & .440 & .000 s.s. \\
\hline Controlling x Qualifizierung & 97 & .398 & .000 s.s. \\
\hline Beteiligung x Qualifizierung & 101 & .479 & .000 s.s. \\
\hline
\end{tabular}

Da angesichts der Vielzahl der Interkorrelationen auch mit zufälligen Signifikanzen gerechnet werden muss, sollten nur die größeren Koeffizienten interpretiert werden. Auf eine detaillierte Betrachtung an dieser Stelle wird jedoch verzichtet.

Besonders deutlich ist der Zusammenhang zwischen QM-System, QM-Handbuch und Qualitätspolitik: Einrichtungen mit einem QM-System verfügen zumeist auch über ein QM-Handbuch und Grundsätze zur Qualitätspolitik.

Wie die tabellarische Übersicht noch einmal zeigt, besteht zwar ein signifikanter Zusammenhang zwischen der Einrichtungsgröße (die übrigen UV sind sämtlich nominalskaliert und hier nicht verrechnet) und dem Einsatz mehrerer Instrumente des Qualitätsmanagements, allerdings nicht mit dem Einsatz von QM-Systemen an sich (Korrelation -.120, in Tab. 47 nicht aufgeführt; negative Vorzeichen beruhen auf der unterschiedlichen Polung der verwendeten Skalen). Ein genereller Effekt im Sinne zunehmender Anwendungshäufigkeit bei steigender MitarbeiterInnenzahl besteht demnach nicht, sondern wird erst bei der getrennten Betrachtung der Arbeitsfelder und Trägergruppen deutlich, und zwar bei freigemeinnützigen Einrichtungen der Erziehungshilfe. 


\subsubsection{Maßnahmen zur Qualitätsentwicklung}

\subsubsection{Nutzenbewertung}

Bereits in Kap. D 4.5 wurde darauf hingewiesen, dass in die Bewertung von Maßnahmen der Qualitätsentwicklung alle 102 Fragebögen aus dem Rücklauf eingingen, da hier primär das an einer möglichst großen Stichprobe zu ermittelnde Urteil sozialer Fachkräfte gefragt war. Ungeachtet dessen wird im Folgenden auch erwähnt, wenn sich signifikante Einflüsse des Arbeitsfeldes, der Einrichtungsgröße oder der Trägerschaft in differenzierten Bewertungen der Fachkräfte niederschlagen.

Vorgegeben wurden Maßnahmen und Vorkehrungen zur Optimierung der Arbeit bzw. zur Qualifizierung der Leistungsprozesse und Akteure, die im Bereich sozialer Dienstleistungen fachlich en vogue sind. Auf die Frage "Wie schätzen Sie jeweils den praktischen Nutzen dieser Maßnahmen ein?" sollte nach folgender Skala differenziert werden:

$0=$ kann ich nicht beurteilen - Maßnahme wird bei uns nicht praktiziert

$1=\operatorname{sehr}$ hoch $2=$ hoch $3=$ eher hoch als gering $4=$ eher gering als hoch $5=$ gering $6=$ sehr gering

Skalenstufe 0 wurde separat nach Häufigkeit ausgewertet und bei der Mittelwertsberechnung als "missing data" behandelt. Anderenfalls hätte sich ein Artefakt ergeben: Maßnahmen, die von vielen Einrichtungen nicht praktiziert werden, erhielten einen extrem "verrückten" Mittelwert.

Angegeben sind jeweils die Stichprobenumfänge (n), die Mittelwerte (M), Standardabweichungen (SD) und Mittelwert-Rangplätze (R), und zwar in der Reihenfolge der Itempräsentation. In der letzten Spalte der Tab. 48 ist der Anteil jener Einrichtungen aufgeführt, welche die Maßnahme nicht praktizieren und deshalb keine Angabe machten (k. A. in \%). Die Zahlen zu Unterschiedsprüfungen im Hinblick auf die relevanten Faktoren dieser Studie werden anschließend erörtert. 
Tab. 48: Nutzenbewertung von Maßnahmen zur Qualitätsentwicklung

\begin{tabular}{|c|c|c|c|c|c|}
\hline Item & $\mathrm{n}$ & $\mathrm{M}$ & SD & $\mathrm{R}$ & $\%$ k. A. \\
\hline $\begin{array}{l}\text { innerbetriebliche Fortbildung durch interne } \\
\text { Leitungs- und Beratungskräfte }\end{array}$ & 87 & 2,54 & 0,962 & 24 & 14,1 \\
\hline $\begin{array}{l}\text { innerbetriebliche Fortbildung durch externe } \\
\text { Beratungskräfte ("Inhouse"-Seminare) }\end{array}$ & 88 & 2,28 & 1,184 & 11 & 14,1 \\
\hline außerbetriebliche Fort- und Weiterbildung & 100 & 2,33 & 0,975 & 14 & 1,2 \\
\hline Team-/Gruppensupervision & 87 & 2,15 & 1,234 & 6 & 15,3 \\
\hline $\begin{array}{l}\text { sonstige Teamentwicklungsmaßnahmen (z. B. } \\
\text { Moderation, Video-Interaktions-Begleitung) }\end{array}$ & 69 & 2,38 & 1,086 & 18 & 32,9 \\
\hline Einzelsupervision und Coaching & 82 & 2,28 & 1,298 & 11 & 20,0 \\
\hline $\begin{array}{l}\text { arbeitsfeldspezifische Zusatzausbildung (z. B. } \\
\text { Heilpädagogik, Sozialtherapie) }\end{array}$ & 81 & 2,36 & 1,165 & 16 & 20,0 \\
\hline $\begin{array}{l}\text { Fachberatung durch Interne (z. B. eigene } \\
\text { Fachdienste) }\end{array}$ & 72 & 2,53 & 1,061 & 23 & 29,4 \\
\hline $\begin{array}{l}\text { Fachberatung durch Externe (z. B. } \\
\text { PsychologInnen, ÄrztInnen) }\end{array}$ & 77 & 2,70 & 1,148 & 26 & 25,9 \\
\hline systematische kollegiale Beratung & 92 & 2,18 & 1,037 & 7 & 9,4 \\
\hline systematische Planungsverfahren & 82 & 2,01 & 1,048 & 3 & 18,8 \\
\hline interne Fallreflexion & 92 & 1,79 & 0,859 & 1 & 9,4 \\
\hline $\begin{array}{l}\text { Maßnahmen zur gezielten } \\
\text { Einbeziehung/Partizipation der } \\
\text { "KundInnenen"/KlientInnen }\end{array}$ & 90 & 2,30 & 1,136 & 13 & 11,8 \\
\hline $\begin{array}{l}\text { Maßnahmen zur Pflege der Zusammenarbeit mit } \\
\text { Externen (Kooperationspartnerschaften) }\end{array}$ & 89 & 2,37 & 1,059 & 17 & 9,4 \\
\hline $\begin{array}{l}\text { Konzeptionsentwicklung: Umsetzung und } \\
\text { Innovation }\end{array}$ & 98 & 1,82 & 0,829 & 2 & 3,5 \\
\hline $\begin{array}{l}\text { Beschreibung und fachgerechte Gestaltung von } \\
\text { Schlüsselprozessen }\end{array}$ & 91 & 2,01 & 0,937 & 3 & 10,6 \\
\hline Kundenbefragungen (KlientInnen) & 77 & 2,52 & 1,242 & 22 & 24,7 \\
\hline Kundenbefragungen (Kooperationspartner) & 59 & 2,76 & 1,394 & 28 & 41,2 \\
\hline Mitarbeiterbefragungen & 70 & 2,46 & 1,236 & 20 & 29,4 \\
\hline Selbstevaluationsprojekte & 64 & 2,70 & 1,150 & 26 & 34,1 \\
\hline $\begin{array}{l}\text { Selbstbewertung mittels standardisierter } \\
\text { Fragebögen }\end{array}$ & 56 & 2,89 & 1,231 & 30 & 44,7 \\
\hline $\begin{array}{l}\text { interne Audits durch Leitung und/oder beauftragte } \\
\text { KollegInnen }\end{array}$ & 56 & 2,82 & 1,539 & 29 & 45,9 \\
\hline $\begin{array}{l}\text { externe Audits im Rahmen einer } \\
\text { Zertifizierung/Qualitätsprüfung }\end{array}$ & 42 & 3,19 & 1,811 & 32 & 60,0 \\
\hline $\begin{array}{l}\text { Fremdevaluation durch beauftragte Dritte (externe } \\
\text { ExpertInnen) }\end{array}$ & 43 & 3,60 & 1,591 & 33 & 61,2 \\
\hline Qualitätszirkel (kontinuierlich arbeitend) & 74 & 2,34 & 1,296 & 15 & 28,2 \\
\hline Projektgruppen (temporär arbeitend) & 91 & 2,21 & 0,995 & 8 & 11,8 \\
\hline $\begin{array}{l}\text { regelmäßige Mitarbeiter- } \\
\text { /Personal(entwicklungs)gespräche }\end{array}$ & 89 & 2,13 & 1,227 & 5 & 12,9 \\
\hline $\begin{array}{l}\text { kriterienorientierte Personalbeurteilung } \\
\text { (systematisches Feedback) }\end{array}$ & 54 & 2,57 & 1,561 & 25 & 45,9 \\
\hline $\begin{array}{l}\text { Einbeziehung der "KundInnen"-Perspektive in die } \\
\text { Prozessgestaltung (z. B. mittels } \\
\text { "Kundenpfadanalyse") }\end{array}$ & 54 & 2,89 & 1,586 & 30 & 47,1 \\
\hline $\begin{array}{l}\text { Erarbeitung verbindlicher Qualitätsstandards als } \\
\text { "Muss"- oder "Soll"-Werte }\end{array}$ & 77 & 2,21 & 1,030 & 8 & 24,7 \\
\hline $\begin{array}{l}\text { Erhebung und Kommunikation von finanziellen } \\
\text { und nichtfinanziellen Kennzahlen }\end{array}$ & 70 & 2,46 & 1,099 & 20 & 34,1 \\
\hline $\begin{array}{l}\text { regelmäßige und systematische Berichterstattung } \\
\text { (z. B. Jahres-, Qualitätsbericht) }\end{array}$ & 85 & 2,25 & 1,184 & 10 & 16,5 \\
\hline zielorientierte Führung, "Kontraktmanagement" & 70 & 2,41 & 1,210 & 19 & 32,9 \\
\hline
\end{tabular}


Die Ergebnisse wurden teilweise bereits in Kap. D 1.3.2 verarbeitet und in einen Vergleichszusammenhang mit anderen Einrichtungen und Fachkräften der Erziehungshilfe gestellt. Hier kommt es zunächst darauf an, die an einer erweiterten Stichprobe aus sozialen Handlungsfeldern ermittelten Prioritäten darzustellen. Ergänzend ist aufschlussreich, wie groß die jeweiligen Anteile jener Einrichtungen sind, in denen die angesprochenen Maßnahmen nicht praktiziert werden, zum Teil auch gar nicht bekannt sind.

Abgesehen vom relativ deutlich abgeschlagenen letzten Rangplatz (Fremdevaluation durch beauftragte Dritte: Mittelwert 3,6) ordnen sich alle übrigen Items kontinuierlich nach je geringen Mittelwertsdifferenzen. Zwischen Rangplatz 8 und 25 etwa liegt eine Distanz von nur 0,36 Skalenpunkten.

Die Favoriten - hier begrenzt auf die ersten sieben Ränge - lauten:

1. interne Fallreflexion,

2. Konzeptionsentwicklung: Umsetzung und Innovation,

3. Beschreibung und fachgerechte Gestaltung von Schlüsselprozessen,

4. systematische Planungsverfahren,

5. regelmäßige Mitarbeiter-/Personal(entwicklungs)gespräche,

6. Team-/Gruppensupervision,

7. systematische kollegiale Beratung.

Zunehmend am schlechtesten weg in der Bewertung kommen die folgenden Maßnahmen:

1. kriterienorientierte Personalbeurteilung (systematisches Feedback),

2. Selbstevaluationsprojekte,

3. Fachberatung durch Externe (z. B. PsychologInnen, ÄrztInnen),

4. Kundenbefragungen (Kooperationspartner),

5. interne Audits durch Leitung und/oder beauftragte KollegInnen,

6. Einbeziehung der KundInnen-Perspektive in die Prozessgestaltung (z. B. mittels Kundenpfadanalyse),

7. Selbstbewertung mittels standardisierter Fragebögen,

8. externe Audits im Rahmen einer Zertifizierung/Qualitätsprüfung,

9. Fremdevaluation durch beauftragte Dritte (externe ExpertInnen). 


\subsubsection{Zusammenhänge zwischen unabhängigen Variablen und Nutzenbewertung}

Faktor Trägerschaft:

- Zwischen freigemeinnützigen und privatgewerblichen Trägern auftretende Mittelwertsdifferenzen sind sämtlich nicht signifikant, wohl aber zahlreiche Unterschiede zu öffentlichen Trägern.

○ Bis auf Mitarbeiterbefragungen (Mittelwert 2,6 vs. 2,8; n. s.) bewerten öffentliche Träger die vorgegebenen Maßnahmen zur Qualitätsentwicklung durchgängig weniger positiv.

- Von 33 Items weisen 12 signifikante Mittelwertsdifferenzen $(\alpha=.05)$ zwischen den beiden Hauptgruppen der öffentlichen und freigemeinnützigen Träger auf (gerundete Werte): außerbetriebliche Fort- und Weiterbildung (2,6 vs. 2,1), Beschreibung und fachgerechte Gestaltung von Schlüsselprozessen (2,5 vs. 1,7), Erhebung von Kennzahlen (3,0 vs. 2,0), arbeitsfeldspezifische Zusatzausbildung (3,0 vs. 2,3), Fachberatung durch Externe (3,3 vs. 2,5), interne Fallreflexion (2,2 vs. 1,6), Konzeptionsentwicklung (2,2 vs. 1,7), Projektgruppen (2,9 vs. 1,8), kriterienorientierte Personalbeurteilung (3,5 vs. 2,1), sonstige Teamentwicklungsmaßnahmen (2,8 vs. 2,1), Maßnahmen zur Kundenpartizipation $(2,8$ vs. 2,0) und Kundenpfadanalyse (4,1 vs. $2,4)$.

Faktor Arbeitsfeld:

Wegen der bereits mehrfach erwähnten Konfundierung der Variablen Trägerschaft und Arbeitsfeld wird auf die Angabe signifikanter Unterschiede zwischen Jugend- und Sozialämtern einerseits und Erziehungshilfeeinrichtungen andererseits verzichtet, da sie mit den zuvor beschriebenen Differenzen weitgehend zusammenfallen. Die übrigen Arbeitsfelder sind in der Stichprobe so gering vertreten $(n=1$ bis 9$)$, dass hier nur die eindrucksvollsten Mittelwertsunterschiede ohne Angabe von Zahlen Erwähnung finden:

- Innerbetriebliche Fortbildung durch Interne steht beim Arbeitsfeld Berufsbildung/Schule deutlich höher im Kurs als bei allen anderen. 
- Maßnahmen zur gezielten Kundenpartizipation, Konzeptionsentwicklung, Schlüsselprozessbeschreibung/-gestaltung und Klientenbefragungen werden allseits, besonders im Feld Berufsbildung/Schule, hoch geschätzt, deutlich weniger jedoch in Sozialämtern.

- Zwischen Berufsbildung/Schule und Sozialamt bestehen ähnlich hohe und gleichgerichtete Unterschiede auch in der Bewertung von Projektgruppen, Mitarbeitergesprächen und Kennzahlen.

Faktor Einrichtungsgröße:

○ In sehr großen Einrichtungen wird im Vergleich zu den übrigen Größenquartilen Maßnahmen zur gezielten Kundenpartizipation (1,7 vs. 2,3 bis 2,7) sowie Klientenbefragungen ( 2,0 vs. 2,5 bis 3,2$)$ besonderer Nutzen zugeschrieben.

\section{Faktor Geschlecht:}

Geschlechtsspezifische Nutzenbewertungen sind nur in Form eines Gruppenvergleichs zwischen weiblichen und männlichen Qualitätsbeauftragten überprüfbar, da nicht generell nach dem Geschlecht gefragt wurde. Die Anzahl abgegebener Urteile zu den einzelnen Items beträgt minimal 4 und maximal 15; signifikante Differenzen sollen deshalb nur mit Vorsicht interpretiert werden.

- Interne Fortbildung durch Interne: weiblich 2,8 / männlich 2,0, t =2,364, df $=27, \mathrm{p}=$ .026 ;

○ Fremdevaluation: 2,0 / 4,1, $\mathrm{t}=-2,819, \mathrm{df}=10, \mathrm{p}=.018$;

○ Projektgruppen: $1,6 / 2,3, \mathrm{t}=-2,370, \mathrm{df}=25, \mathrm{p}=.026$;

- Kontraktmanagement: $1,0 / 2,7, \mathrm{t}=-3,343, \mathrm{df}=13, \mathrm{p}=.005$. 


\subsubsection{Qualitäts(management)beauftragte}

\subsubsection{Institutionalisierung des Instruments}

Von 85 Einrichtungen des Gesamtrücklaufs verfügt rund ein Drittel über die Funktionsstelle des/der QB; alle davon waren zum Berichtszeitpunkt besetzt. Gleich groß ist die Fraktion derer, die noch nicht über eine Funktionsstelle für QB diskutiert haben.

Da sich die Anteile der noch Unentschiedenen und derjenigen, die das Instrument nicht benutzen wollen, die Waage halten, kann konstatiert werden: Von den rund 65\% $(n=55)$ der Einrichtungen, die eine Funktionsstelle QB überhaupt schon diskutiert haben, entschied sich über die Hälfte (29) dafür und ein Fünftel dagegen (13).

Tab. 49: Funktionsstelle Qualitätsbeauftragte/r

\begin{tabular}{|ll|l|l|l|l|}
\hline & & Häufigkeit & Prozent & $\begin{array}{l}\text { Gültige } \\
\text { Prozente }\end{array}$ & $\begin{array}{l}\text { Kumulierte } \\
\text { Prozente }\end{array}$ \\
\hline Gültig & Stelle besetzt & $29(12)$ & 34,1 & 34,1 & 34,1 \\
& vorhanden, aber unbesetzt & $0(0)$ & 0,0 & 0,0 & 34,1 \\
& noch nicht entschieden & $13(2)$ & 15,3 & 15,3 & 49,4 \\
& dagegen entschieden & $13(1)$ & 15,3 & 15,3 & 64,7 \\
& noch nicht diskutiert & $30(2)$ & 35,3 & 35,3 & 100,0 \\
Gesamt & $85(17)$ & 100,0 & 100,0 & \\
\hline
\end{tabular}

Differenzierungen nach Faktorstufen folgen in Kap. 4.6.8.3.

\subsubsection{Entscheidungsgründe gegen die Funktionsstelle QB}

Von besonderem Interesse ist in diesem Zusammenhang die Frage, warum 56 von 85 Einrichtungen und Diensten das Thema QB noch nicht diskutiert bzw. sich noch nicht oder bereits dagegen entschieden haben. 
Auf die Frage "Wenn Sie in Ihrer Einrichtung keine/n Qualitätsbeauftragte/n haben und auch keine/n vorsehen: Welche Gründe haben Sie dazu bewogen?" verteilen sich die Antworten wie folgt (Anzahl der Nennungen = n, Anteil an der Gesamtzahl der Nennungen in \%):

Tab.50: Entscheidungsgründe gegen die Funktionsstelle des/der Qualitätsbeauftragten (Mehrfachnennungen möglich)

\begin{tabular}{|l|l|l|}
\hline Item & $\mathrm{n}$ & $\%$ \\
\hline$\circ$ & 1 & 1,0 \\
\hline $\begin{array}{l}\text { QB wir haben das Instrument wieder abgeschafft, weil... } \\
\text { wird. }\end{array}$ & 24 & 24,5 \\
\hline$\circ \quad$ Wir haben die Funktion anders organisiert und benannt. & 2 & 2,1 \\
\hline$\circ \quad$ Wir haben niemand, der sich für diese Funktion interessiert. & 1 & 1,0 \\
\hline$\circ \quad$ Wir halten das Instrument QB nicht für zweckmäßig. & 7 & 7,2 \\
\hline $\begin{array}{l}\text { QM bezogene Aufgaben werden nach Bedarf und Situation } \\
\text { von unterschiedlichen Personen wahrgenommen. }\end{array}$ & 24 & 24,5 \\
\hline$\circ \quad$ Befürworter des Instruments QB konnten sich nicht durchsetzen. & 1 & 1,0 \\
\hline$\circ \quad$ Wir wollen keine Spezialisten, sondern Qualitätswissen breit streuen. & 17 & 17,5 \\
\hline$\circ \quad$ Wir greifen bedarfsweise lieber auf externe Unterstützung zurück. & 13 & 13,4 \\
\hline$\circ \quad$ Wir fürchten, das Qualitätsthema würde beim QB "abgeladen". & 5 & 5,2 \\
\hline$\circ \quad$ Wir fürchten eine Aushöhlung der Leitungsverantwortung. & 0 & 0,0 \\
\hline$\circ \quad$ Wir glauben, dass QB ein konfliktreicher und undankbarer Job ist. & 2 & 2,1 \\
\hline Gesamt & 97 & 100,0 \\
\hline$\circ \quad$ Sonstige Gründe & 10 & \\
\hline Gesamt incl. sonstige Gründe & 107 & \\
\hline
\end{tabular}

Anmerkungen:

Abgeschafft wurde das Instrument 1x mit der Begründung: neuer Bürgermeister, neue Mehrheiten, Abbruch aller Entwicklungen.

Als sonstige Gründe wurden je 1x genannt:
QM ist Querschnittsaufgabe, keine Spezialaufgabe.
Keine personellen Ressourcen, Funktion wird "mitgemacht", soweit es geht.
Funktion wird z. Zt. von einer Projektleitung ausgeübt, bis Entscheidung fällt.
QM ist noch kein ausgereiftes Thema vor Ort, wird vom Landesverband wahrgenommen.
Wir sind eine kleine, sehr kommunikative und überschaubare Einrichtung.
Wird von der Jugendamtsdirektorin nicht gewünscht.

Unter den angegebenen Gründen dominieren die Aussagen

- QB wird nicht benötigt, weil Funktion von der Leitung mit übernommen wird.

- QM bezogene Aufgaben werden nach Bedarf und Situation von unterschiedlichen Personen wahrgenommen.

- Wir wollen keine Spezialisten, sondern Qualitätswissen breit streuen.

- Wir greifen bedarfsweise lieber auf externe Unterstützung zurück. 
Diese Argumente machen rund $80 \%$ aller Nennungen aus (ohne sonstige Gründe). Zusammenhänge mit den UV sind aufschlussreich und werden hier ohne tabellarische Einzeldarstellung zusammengefasst:

1. In kleinen/kleinsten Einrichtungen übernimmt in der Regel die Leitung entsprechende Funktionen oder andere Personen üben sie je nach Situation und Bedarf aus; auch die Absicht, keine Spezialisten auszubilden, sondern Qualitätswissen breit zu streuen und bei Bedarf lieber auf Externe zurückzugreifen, geht überwiegend auf das Konto der Einrichtungen im ersten Größenquartil.

2. Die übrigen Argumente verteilen sich uncharakteristisch über alle Einrichtungsgrößen.

3. Sehr große Einrichtungen machen insgesamt seltener von den genannten Argumenten Gebrauch, da sie mehrheitlich über Funktionsstellen für QB verfügen.

\subsubsection{Zusammenhänge zwischen unabhängigen Variablen und Funktionsstelle QB}

Abweichungen zwischen der Anzahl von 29 eingerichteten und besetzten Funktionsstellen (s. Tab. 49) und der teilweise höheren Gesamtzahl der Antworten in nachfolgenden Tabellen ergeben sich daraus, dass zwei entsprechend qualifizierte TeilnehmerInnen bereits als QB antworteten, obwohl ihre Stelle noch nicht offiziell eingerichtet war. Niedrigere Zahlen als 29 beruhen auf fehlenden Angaben.

QB sind zum Untersuchungszeitpunkt offenbar eine Domäne der freigemeinnützigen Einrichtungen. Bei öffentlichen Trägern überwiegt die Zahl derer, die das Thema noch gar nicht diskutiert haben; allerdings spricht sich von den befragten Einrichtungen nur eine dagegen aus. Unter den 13 Organisationen, die sich insgesamt gegen QB entschieden, dominieren (kleine) privatgewerbliche Träger.

Eine separate Analyse nach Spitzenverbandszugehörigkeit wird nicht tabellarisch aufgeführt, da 87,5\% der Zellen erwartete Häufigkeitswerte kleiner 5 aufweisen. Die Mehrzahl der freien Einrichtungen, in denen das Thema noch nicht diskutiert wurde, gehört jedoch dem Paritätischen an. 
Tab. 51: Funktionsstelle QB nach Trägerschaft

\begin{tabular}{|l|l|l|l|l|l|}
\hline $\begin{array}{l}\text { Träger } / \text { QB- } \\
\text { Stelle }\end{array}$ & vorhanden & $\begin{array}{l}\text { noch nicht } \\
\text { entschieden }\end{array}$ & $\begin{array}{l}\text { dagegen } \\
\text { entschieden }\end{array}$ & $\begin{array}{l}\text { noch nicht } \\
\text { diskutiert }\end{array}$ & Gesamt \\
\hline offentlich & 6 & 7 & 1 & 21 & 35 \\
\hline freigemeinnützig & 21 & 2 & 2 & 8 & 33 \\
\hline privatgewerbl. & 2 & 4 & 10 & 1 & 17 \\
\hline Gesamt & 29 & 13 & 13 & 30 & 85 \\
\hline Signifikanz & \multicolumn{6}{l}{ Chi-Quadrat $=54,776 ; \mathrm{df}=6 ; \mathrm{p}=.000 ;$ s. s. } \\
\hline Konting.koeff. & \multicolumn{6}{l}{626} & & \\
\hline
\end{tabular}

Erwartungsgemäß spiegeln sich die zuvor genannten Zusammenhänge auch im Faktor Arbeitsfeld wider. Erziehungshilfeeinrichtungen geben aufgrund der träger- (und größenspezifischen) Unterschiede ein uneinheitliches Bild ab. Unter den Sonstigen fallen die Berufsbildungswerke besonders ins Gewicht.

Tab. 52: Funktionsstelle QB nach Arbeitsfeldern

\begin{tabular}{|l|l|l|l|l|l|}
\hline $\begin{array}{l}\text { Arbeitsfeld / } \\
\text { QB-Stelle }\end{array}$ & vorhanden & $\begin{array}{l}\text { noch nicht } \\
\text { entschieden }\end{array}$ & $\begin{array}{l}\text { dagegen } \\
\text { entschieden }\end{array}$ & $\begin{array}{l}\text { noch nicht } \\
\text { diskutiert }\end{array}$ & Gesamt \\
\hline Jugendamt & 3 & 5 & 1 & 17 & 26 \\
\hline Sozialamt & 1 & 2 & 0 & 4 & 7 \\
\hline JuHi-Einrichtung & 12 & 6 & 11 & 5 & 34 \\
\hline Sonstige & 12 & 0 & 1 & 4 & 17 \\
\hline Gesamt & 28 & 13 & 13 & 30 & 84 \\
\hline Signifikanz & Chi-Quadrat 38,173; df $=9 ; \mathrm{p}=.000 ;$ s. s. \\
\hline Konting.koeff. & .559 &
\end{tabular}

Die insgesamt nicht signifikanten, partiell jedoch auffälligen Zusammenhänge zwischen Einrichtungsgröße und Funktionsstelle QB betreffen den relativ hohen Ablehnungsgrad in kleinen und mittelgroßen Organisationen und die häufigste Nutzung des Instruments in großen und sehr großen Einrichtungen.

Tab. 53: Funktionsstelle QB nach Einrichtungsgröße

\begin{tabular}{|l|l|l|l|l|l|}
\hline $\begin{array}{l}\text { Größe / QB- } \\
\text { Stelle }\end{array}$ & vorhanden & $\begin{array}{l}\text { noch nicht } \\
\text { entschieden }\end{array}$ & $\begin{array}{l}\text { dagegen } \\
\text { entschieden }\end{array}$ & $\begin{array}{l}\text { noch nicht } \\
\text { diskutiert }\end{array}$ & Gesamt \\
\hline klein/kleinst & 4 & 4 & 7 & 7 & 22 \\
\hline mittelgroß & 6 & 3 & 4 & 8 & 21 \\
\hline groß & 8 & 3 & 0 & 11 & 22 \\
\hline sehr groß & 11 & 3 & 2 & 4 & 20 \\
\hline Gesamt & 29 & 13 & 13 & 30 & 85 \\
\hline Signifikanz & Chi-Quadrat $=15,238 ; \mathrm{df}=9 ; \mathrm{p}=.085 ;$ n. $\mathrm{s}$. \\
\hline Kont.koeff. & .390 &
\end{tabular}


Ergänzend von Interesse ist die Frage nach Zusammenhängen zwischen Anwendungshäufigkeit des Instruments QB und Systemorientierung des Qualitätsmanagements. Erwartet wird eine Dominanz ISO-orientierter Einrichtungen unter denen mit QB, da nur die ISO das Instrument obligatorisch fordert.

Tab. 54: Funktionsstelle QB nach QM-System

\begin{tabular}{|l|l|l|l|l|l|}
\hline QM-System & QB vorhanden & $\begin{array}{l}\text { noch nicht } \\
\text { entschieden }\end{array}$ & $\begin{array}{l}\text { dagegen } \\
\text { entschieden }\end{array}$ & $\begin{array}{l}\text { noch nicht } \\
\text { diskutiert }\end{array}$ & Gesamt \\
\hline EFQM & 5 & 7 & 2 & 3 & 17 \\
\hline ISO 9000 ff. & 16 & 1 & 1 & 3 & 21 \\
\hline Zertifizierung & 9 & 0 & 0 & 0 & 9 \\
\hline "Eigenbau" & 5 & 5 & 2 & 7 & 19 \\
\hline Mischsystem & 1 & 1 & 0 & 2 & 4 \\
\hline Sonstiges & 0 & 0 & 2 & 0 & 2 \\
\hline Gesamt & 36 & 7 & 15 & 72 \\
\hline Signifikanz & Chi-Quadrat $=46,999 ; \mathrm{df}=15 ; \mathrm{p}=.000$, s.s. \\
\hline Konting.koeff. & .628 &
\end{tabular}

Anmerkung:

Infolge Mehrfachnennungen summieren sich die Angaben auf mehr als die QB-Anzahl in der Stichprobe.

Die erwartete Häufung von QB in ISO-orientierten Einrichtungen ist damit bestätigt.

Eine Inspektion der Interkorrelationstabelle in Kap. D 4.6.6 sowie entsprechender Kreuztabellen zeigt gleichermaßen, dass die meisten Instrumente des Qualitätsmanagements in Einrichtungen mit QB signifikant häufiger eingesetzt werden als in solchen ohne QB. Auch das Ausmaß der Mitarbeiterbeteiligung am QM-Prozess sowie der systematischen Qualifizierung der Gesamtbelegschaft oder einzelner Gruppen korreliert hochsignifikant positiv mit dem Einsatz von QB (Kontingenzkoeffizienten .519 und .583).

\subsubsection{Strukturmerkmale der Funktionsstelle QB}

Zusammenhänge zwischen Strukturmerkmalen und UV werden erwähnt, soweit sie nach Inspektion der Kreuztabellen bedeutsam erscheinen. Auf eine gesonderte Darstellung wird verzichtet, ebenso auf Signifikanzberechnungen, zumal die Mehrzahl der Fälle sich auf ein bis zwei Antwortkategorien konzentriert und viele Zellen unbesetzt bleiben.

Sofern nicht bereits in Kap. D 2.1.2/2.1.3 erwähnt, werden Vergleichsbefunde angespro- 
chen aus der Untersuchung von Wetzler (2003), die 2001 in Wohneinrichtungen der Behindertenhilfe durchgeführt wurde.

Zwei Drittel der QB sind für die jeweilige Gesamteinrichtung zuständig. Logischerweise ist das restliche Drittel in stark dezentralisierten und in der Regel größeren Einrichtungen tätig.

Tab. 55: Zuständigkeitsbereich des/der QB

\begin{tabular}{|ll|l|l|l|l|}
\hline & & Häufigkeit & Prozent & $\begin{array}{l}\text { Gültige } \\
\text { Prozente }\end{array}$ & $\begin{array}{l}\text { Kumulierte } \\
\text { Prozente }\end{array}$ \\
\hline Gültig & Gesamteinrichtung & 20 & 66,7 & 66,7 & 66,7 \\
& Teileinrichtung & 2 & 6,7 & 6,7 & 73,4 \\
& Fachbereich/Abteilung & 8 & 26,6 & 26,6 & 100,0 \\
Gesamt & & 30 & 100,0 & 100,0 & \\
\hline
\end{tabular}

Nahezu jede/r zweite QB bekleidet eine mehr als Dreiviertel- bis Vollstelle (28,9 bis 38,5 Wochenstunden), ein weiteres Drittel wird projektabhängig in unterschiedlichem Maße freigestellt. Nur $10 \%$ haben eine Teilzeitstelle mit weniger als 28,9 Wochenstunden inne.

In der Untersuchung von Wetzler (2003, S. 66) beträgt der Anteil der Dreiviertel- bis Vollstellen 32,7\% und ist insbesondere bei größeren Einrichtungen anzutreffen. Teilzeitstellen mit weniger als der Hälfte der Wochenstunden machen insgesamt rund $56 \%$ aus.

Tab. 56: Umfang der Freistellung (in Arbeitsstunden/Woche bzw. Stellenanteil)

\begin{tabular}{|ll|l|l|l|l|}
\hline & & Häufigkeit & Prozent & $\begin{array}{l}\text { Gültige } \\
\text { Prozente }\end{array}$ & $\begin{array}{l}\text { Kumulierte } \\
\text { Prozente }\end{array}$ \\
\hline Gültig & projektabhängig & 10 & 33,3 & 33,3 & 33,3 \\
& bis Viertel-Stelle & 3 & 10,0 & 10,0 & 43,3 \\
& bis halbe Stelle & 2 & 6,7 & 6,7 & 50,0 \\
& bis Dreiviertel-Stelle & 1 & 3,3 & 3,3 & 53,3 \\
& bis Vollstelle & 14 & 46,7 & 46,7 & 100,0 \\
Gesamt & & 30 & 100,0 & 100,0 & \\
\hline
\end{tabular}


Während über die Hälfte der QB ihre Freistellung nur durch interne Umverteilung von Ressourcen erhält, werden rund $40 \%$ voll durch Neueinstellungen/Neubesetzungen kompensiert. Diesbezüglich ist ein klares Alles-oder-Nichts-Prinzip erkennbar.

Wetzler (2003, S. 72) beziffert den Anteil der Einrichtungen mit interner Umverteilung von Ressourcen mit 40\%. 60\% verneinten die Frage, ob zur Schaffung der QB-Stelle personelle Ressourcen in anderen Bereichen eingespart worden seien.

Tab. 57: Ausgleich im Stellenplan

\begin{tabular}{|ll|l|l|l|l|}
\hline & & Häufigkeit & Prozent & $\begin{array}{l}\text { Gültige } \\
\text { Prozente }\end{array}$ & $\begin{array}{l}\text { Kumulierte } \\
\text { Prozente }\end{array}$ \\
\hline Gültig & voller Ausgleich & 11 & 39,3 & 39,3 & 39,3 \\
& teilweiser Ausgleich & 1 & 3,6 & 3,6 & 42,9 \\
& kein Ausgleich & 16 & 57,1 & 57,1 & 100,0 \\
Gesamt & & 28 & 100,0 & 100,0 & \\
\hline
\end{tabular}

Die unbefristete Stelle dominiert deutlich. Unter den befristeten Lösungen ist ein fester Zeitrahmen häufiger anzutreffen als eine prozessabhängige Befristung.

Auch bei Wetzler (2003, S. 71) ist die dauerhafte Stellenbesetzung die Regel (75,5\%).

Tab. 58: Befristung der QB-Stelle

\begin{tabular}{|ll|l|l|l|l|}
\hline & & Häufigkeit & Prozent & $\begin{array}{l}\text { Gültige } \\
\text { Prozente }\end{array}$ & $\begin{array}{l}\text { Kumulierte } \\
\text { Prozente }\end{array}$ \\
\hline Gültig & unbefristet & 24 & 82,8 & 82,8 & 82,8 \\
& $\begin{array}{l}\text { befristet bis Abschluss } \\
\text { QM-Implementation }\end{array}$ & 2 & 6,9 & 6,9 & 89,7 \\
& befristet auf ... Jahre & 3 & 10,3 & 10,3 & 100,0 \\
Gesamt & 29 & 100,0 & 100,0 & \\
\hline
\end{tabular}

Die Besetzung der Funktionsstelle QB ist offenbar eine Domäne der Einrichtungsleitung, und zwar überwiegend ohne vorherige interne Ausschreibung. Nur 10\% der Stellen wurden durch Belegschaftswahl oder durch Neueinstellung nach Ausschreibung besetzt. Sonstige Verfahren wurden in $10 \%$ der Einrichtungen angewandt, jedoch nicht näher expliziert. 
Wetzler (2003, S. 77) teilt ganz ähnliche Zahlen für den Behindertenbereich mit. Mit zunehmender Einrichtungsgröße steigt der Anteil der Leitungsentscheidungen von 83,3 auf $100 \%$.

Tab. 59: Stellenbesetzungsverfahren

\begin{tabular}{|c|l|l|l|l|}
\hline & Häufigkeit & Prozent & $\begin{array}{l}\text { Gültige } \\
\text { Prozente }\end{array}$ & $\begin{array}{l}\text { Kumulierte } \\
\text { Prozente }\end{array}$ \\
\hline $\begin{array}{c}\text { Gültig } \begin{array}{c}\text { intern: Leitungsbeschluss ohne } \\
\text { Ausschreibung }\end{array} \\
\begin{array}{c}\text { intern: Leitungsbeschluss nach } \\
\text { Ausschreibung }\end{array}\end{array}$ & 17 & 56,7 & 56,7 & 56,7 \\
$\begin{array}{c}\text { intern: Belegschaftswahl } \\
\text { nach Ausschreibung }\end{array}$ & 2 & 23,3 & 23,3 & 80,0 \\
$\begin{array}{c}\text { extern: Leitungsbeschluss ohne } \\
\text { Ausschreibung }\end{array}$ & 0 & 6,7 & 6,7 & 86,7 \\
$\begin{array}{c}\text { extern: Leitungsbeschluss nach } \\
\text { Ausschreibung }\end{array}$ & 1 & 0,0 & 0,0 & 86,7 \\
$\quad \begin{array}{l}\text { sonstiges Verfahren } \\
\text { Gesamt }\end{array}$ & 3 & 3,3 & 3,3 & 90,0 \\
\hline
\end{tabular}

Die Funktionsstelle QB ist ein modernes Produkt. Der quantitative Höhepunkt der Stellenbesetzungen entfällt auf den Zeitraum 2001/2002. Immerhin zwei QB wurden bereits vor 1995 berufen, seinerzeit eine echte Pionieraufgabe.

Tab. 60: Erstbesetzung der Stelle

\begin{tabular}{|ll|l|l|l|l|}
\hline & & Häufigkeit & Prozent & $\begin{array}{l}\text { Gültige } \\
\text { Prozente }\end{array}$ & $\begin{array}{l}\text { Kumulierte } \\
\text { Prozente }\end{array}$ \\
\hline Gültig & vor 1995 & 2 & 6,9 & 6,9 & 6,9 \\
& $1995 / 1996$ & 0 & 0,0 & 0,0 & 6,9 \\
& $1997 / 1998$ & 3 & 10,4 & 10,4 & 17,3 \\
& $1999 / 2000$ & 4 & 13,8 & 13,8 & 31,1 \\
& $2001 / 2002$ & 13 & 44,8 & 44,8 & 75,9 \\
& $2003 / 2004$ & 7 & 24,1 & 24,1 & 100,0 \\
Gesamt & weiß nicht & 0 & 0,0 & 0,0 & \\
\hline
\end{tabular}


Rund 70\% der QB verfügen über schriftliche Stellenbeschreibungen und/oder im Organigramm explizit geklärte Kompetenzen. Der Rest verteilt sich gleichermaßen auf teilweise bzw. nicht explizit geklärte Verhältnisse.

Tab. 61: Klärung der Kompetenzen

\begin{tabular}{|c|c|c|c|c|c|}
\hline & & Häufigkeit & Prozent & $\begin{array}{l}\text { Gültige } \\
\text { Prozente }\end{array}$ & $\begin{array}{l}\text { Kumulierte } \\
\text { Prozente }\end{array}$ \\
\hline \multirow[t]{3}{*}{ Gültig } & explizit geklärt & 18 & 69,2 & 69,2 & 69,2 \\
\hline & teilweise explizit geklärt & 4 & 15,4 & 15,4 & 84,6 \\
\hline & nicht explizit geklärt & 4 & 15,4 & 15,4 & 100,0 \\
\hline Gesamt & & 26 & 100,0 & 100,0 & \\
\hline
\end{tabular}

Bei der Itemkonstruktion und Prätestung der Frage nach der Art der Kompetenzen wurde übersehen, dass zwischen den Antwortstufen "nur Beratungskompetenz" und "reine Stabsstelle" kein plausibler inhaltlicher Unterschied besteht. Etwa die Hälfte der 23 antwortenden QB entfällt auf diese kombinierte Kategorie. Auch die übrigen Kategorien sind nicht logisch trennscharf und können zusammengezogen werden. Insgesamt verteilen sich die QB mit reiner Beratungsfunktion und diejenigen mit zusätzlicher Umsetzungskompetenz nahezu hälftig.

Tab. 62: Art der Kompetenzen

\begin{tabular}{|cl|l|l|l|l|}
\hline & Häufigkeit & Prozent & $\begin{array}{l}\text { Gültige } \\
\text { Prozente }\end{array}$ & $\begin{array}{l}\text { Kumulierte } \\
\text { Prozente }\end{array}$ \\
\hline Gültig & nur Beratungskompetenz & 4 & 17,4 & 17,4 & 17,4 \\
& $\begin{array}{l}\text { Umsetzungskompetenz } \\
\text { im Rahmen von } \\
\text { Leitungsbeschlüssen }\end{array}$ & 4 & 17,4 & 17,4 & 34,8 \\
& $\begin{array}{l}\text { Beratungs- und } \\
\text { Umsetzungskompetenz }\end{array}$ & 8 & 34,8 & 34,8 & 69,6 \\
& $\begin{array}{l}\text { reine Stabsstelle ohne } \\
\text { Umsetzungskompetenz }\end{array}$ & 7 & 30,4 & 30,4 & 100,0 \\
Gesamt & 23 & 100,0 & 100,0 & \\
\hline
\end{tabular}




\subsubsection{Personen- und Ausbildungsmerkmale von Qualitätsbeauftragten}

Die Funktionsstelle des/der QB ist in der Stichprobe geschlechtsparitätisch besetzt.

Tab. 63: Geschlechtsverteilung

\begin{tabular}{|ll|l|l|l|l|}
\hline & Häufigkeit & Prozent & $\begin{array}{l}\text { Gültige } \\
\text { Prozente }\end{array}$ & $\begin{array}{l}\text { Kumulierte } \\
\text { Prozente }\end{array}$ \\
\hline Gültig & weiblich & 16 & 51,6 & 51,6 & 51,6 \\
männlich & 15 & 48,4 & 48,4 & 100,0 \\
Gesamt & & 31 & 100,0 & 100,0 & \\
\hline
\end{tabular}

QB sind mehrheitlich über 30 Jahre alt; ein knappes Drittel zählt zu den Älteren über 50.

Tab. 64: Altersverteilung

\begin{tabular}{|ll|l|l|l|l|}
\hline & Häufigkeit & Prozent & $\begin{array}{l}\text { Gültige } \\
\text { Prozente }\end{array}$ & $\begin{array}{l}\text { Kumulierte } \\
\text { Prozente }\end{array}$ \\
\hline Gültig & bis 30 Jahre & 2 & 6,5 & 6,5 & 6,5 \\
& 31 bis 40 Jahre & 11 & 35,5 & 35,5 & 42,0 \\
& 41 bis 50 Jahre & 9 & 29,0 & 29,0 & 71,0 \\
& 51 Jahre und älter & 9 & 29,0 & 29,0 & 100,0 \\
Gesamt & & 31 & 100,0 & 100,0 & \\
\hline
\end{tabular}

Die Kreuztabellierung zeigt, dass männliche QB zumeist den oberen Altersgruppen angehören, während die Jüngeren überwiegend weiblichen Geschlechts sind.

Tab. 65: Altersverteilung x Geschlecht (Kreuztabelle)

\begin{tabular}{|ll|l|l|l|l|}
\hline & weiblich & männlich & $\begin{array}{l}\text { Gültige } \\
\text { Prozente }\end{array}$ & $\begin{array}{l}\text { Kumulierte } \\
\text { Prozente }\end{array}$ \\
\hline Gültig & bis 30 Jahre & 2 & 0 & 6,5 & 6,5 \\
& 31 bis 40 Jahre & 7 & 4 & 35,5 & 42,0 \\
& 41 bis 50 Jahre & 5 & 4 & 29,0 & 71,0 \\
& 51 Jahre und älter & 2 & 7 & 29,0 & 100,0 \\
Gesamt & 16 & 15 & 100,0 & \\
\hline
\end{tabular}


QB in der Jugend- und Sozialhilfe sind weit überwiegend Personen mit mindestens Fachhochschulabschluss. Nur rund ein Achtel fällt nicht unter diese Kategorie.

In der Untersuchung von Wetzler (2003, S. 65) ist der Anteil der Hochschul- und Fachhochschulabsolventen mit zusammen $71,3 \%$ etwas geringer.

Tab. 66: Formale Qualifikation

\begin{tabular}{|ll|l|l|l|l|}
\hline & Häufigkeit & Prozent & $\begin{array}{l}\text { Gültige } \\
\text { Prozente }\end{array}$ & $\begin{array}{l}\text { Kumulierte } \\
\text { Prozente }\end{array}$ \\
\hline Gültig & Hochschule & 10 & 32,3 & 32,3 & 32,3 \\
& Fachhochschule & 16 & 51,6 & 51,6 & 83,9 \\
& Fach(ober)schule & 2 & 6,5 & 6,5 & 90,4 \\
& Sonstiger Abschluss & 3 & 9,7 & 9,7 & 100,0 \\
Gesamt & & 31 & 100,0 & 100,0 & \\
\hline
\end{tabular}

QB verfügen mehrheitlich über Leitungserfahrung mindestens auf der Ebene von Gruppenbzw. Hausleitungen. Rund 39\% waren zuletzt als BereichsleiterInnen tätig, weitere $10 \%$ in Stabsfunktionen aktiv. Nur insgesamt ein Fünftel kommt aus Gruppendienst, Verwaltung und sonstigen Arbeitsbereichen.

Wetzler (2003, S. 69) gibt den Anteil der aus Leitung und Bereichsleitung rekrutierten QB mit 43,6\% an, hinzu kommen je 17,6\% Gruppenleitungen und Fachdienste.

Tab. 67: Letzter beruflicher Hintergrund vor Funktionsübernahme

\begin{tabular}{|ll|l|l|l|l|}
\hline & Häufigkeit & Prozent & $\begin{array}{l}\text { Gültige } \\
\text { Prozente }\end{array}$ & $\begin{array}{l}\text { Kumulierte } \\
\text { Prozente }\end{array}$ \\
\hline Gültig & Leitung/Vorstand & 3 & 9,7 & 9,7 & 9,7 \\
& Bereichsleitung & 12 & 38,7 & 38,7 & 48,4 \\
& Fachberatung/Stab & 3 & 9,7 & 9,7 & 58,1 \\
& Gruppen-/Hausleitung & 7 & 22,6 & 22,6 & 80,6 \\
& Gruppendienst o. Ä. & 3 & 9,7 & 9,7 & 90,3 \\
& Verwaltung & 1 & 3,2 & 3,2 & 93,5 \\
& Sonstiges & 2 & 6,5 & 6,5 & 100,0 \\
Gesamt & \multicolumn{2}{|c|}{100,0} & 100,0 & \\
\hline
\end{tabular}


Ergänzt werden können Befunde aus (hier nicht dargestellten) Kreuztabellierungen mit den Variablen Geschlecht und Alter.

○ Männliche QB, mehrheitlich älteren Jahrgängen zugehörig, rekrutieren sich vorwiegend aus Bereichsleitungen (10); von ihnen behielten fünf ihre Funktion auch nach Übernahme der QB-Stelle bei; drei wechselten auf eine Vollstelle.

○ Vergleichbare Zahlen liegen bezüglich der vormaligen Gruppen- oder Hausleitungen vor: Sechs von sieben sind Frauen, von denen drei ihre Funktion weiter ausüben, während die übrigen eine Vollstelle als QB einnehmen.

- Ansonsten wechselten weibliche QB aus verschiedenen Funktionsbereichen auf die QB-Stelle und nehmen diese überwiegend in Vollzeit (8) wahr, während Männer in 11 von 15 Fällen eine Kombinationslösung praktizieren.

Der in der nächsten Tabelle angegebene Anteil Vollzeit beschäftigter QB weicht mit 40\% etwas ab von den knapp 47\% aus Tab. 56; möglicherweise gibt es einen Anteil von QB, der zwischen 28,9 und 38,5 Wochenstunden freigestellt ist, aber eben nicht im Umfang einer vollen Stelle.

Tab. 68: Sonstige derzeitige Tätigkeit

\begin{tabular}{|ll|l|l|l|l|}
\hline & & Häufigkeit & Prozent & $\begin{array}{l}\text { Gültige } \\
\text { Prozente }\end{array}$ & $\begin{array}{l}\text { Kumulierte } \\
\text { Prozente }\end{array}$ \\
\hline Gültig & entfällt, Vollstelle QB & 12 & 40,0 & 40,0 & 40,0 \\
& Bereichsleitung & 5 & 16,7 & 16,7 & 56,7 \\
& Gruppen-/Hausleitung & 4 & 13,3 & 13,3 & 70,0 \\
& Fachberatung/Stab & 3 & 10,0 & 10,0 & 80,0 \\
& Leitung/Vorstand & 1 & 3,3 & 3,3 & 83,3 \\
& Sonstiges & 5 & 16,7 & 16,7 & 100,0 \\
Gesamt & & 30 & 100,0 & 100,0 & \\
\hline
\end{tabular}

Personalunion von Einrichtungsleitung und QB kommt nur einmal vor, Kombinationen mit nachgeordneten Leitungs- und Beratungsfunktionen sind häufiger anzutreffen (Anteil 
40\%). $\mathrm{Zu}$ den sonstigen Tätigkeiten wurde angegeben: Dozent, kommissarische Leitung, stellvertretende Leitung, Betreuerin, selbständig (neben Einrichtungsleitung), Projektmanagement.

Leider antworteten nur 22 QB auf alle Aspekte der Frage nach funktionsbezogener Ausbildung, die hier aus Gründen besserer Übersichtlichkeit dreigeteilt dargestellt wird (Tab. 69 bis 71). Die Möglichkeit der Mehrfachnennung führte offenbar zu Irritationen. Unter den abgegebenen Antworten dominieren extern angebotene Weiterbildungen mit zertifiziertem Abschluss. Immerhin rund ein Drittel der QB hat sich allein autodidaktisch weitergebildet.

Tab. 69: Funktionsbezogene Ausbildung als Qualitätsbeauftragter

\begin{tabular}{|cl|l|l|l|l|}
\hline & Häufigkeit & Prozent & $\begin{array}{l}\text { Gültige } \\
\text { Prozente }\end{array}$ & $\begin{array}{l}\text { Kumulierte } \\
\text { Prozente }\end{array}$ \\
\hline Gültig & nur autodidaktisch & 7 & 31,8 & 31,8 & 31,8 \\
& $\begin{array}{l}\text { träger- oder } \\
\text { verbandsspezifisch mit } \\
\text { formalem Abschluss }\end{array}$ & 3 & 13,6 & 13,6 & 45,4 \\
& $\begin{array}{l}\text { zertifizierte } \\
\text { Weiterbildung durch } \\
\text { Externe (z. B. DGQ) }\end{array}$ & 12 & 54,5 & 54,5 & 100,0 \\
Gesamt & 22 & 100,0 & 100,0 & \\
\hline
\end{tabular}

Funktionsbezogene Ausbildungen von Qualitätsbeauftragten haben mehrheitlich die Normenreihe der ISO 9000 zum Schwerpunkt (zwei Drittel). Gut ein Fünftel verfügt über eine EFQM-spezifische Weiterbildung.

Tab. 70: Inhaltlicher Schwerpunkt der funktionsbezogenen Ausbildung

\begin{tabular}{|ll|l|l|l|l|}
\hline & & Häufigkeit & Prozent & $\begin{array}{l}\text { Gültige } \\
\text { Prozente }\end{array}$ & $\begin{array}{l}\text { Kumulierte } \\
\text { Prozente }\end{array}$ \\
\hline Gültig & TQM & 2 & 8,7 & 8,7 & 8,7 \\
& EFQM & 5 & 21,7 & 21,7 & 30,4 \\
& ISO 9000 ff. & 15 & 65,2 & 65,2 & 95,6 \\
& EFQM + ISO & 1 & 4,4 & 4,4 & 100,0 \\
Gesamt & & 23 & 100,0 & 100,0 & \\
\hline
\end{tabular}


Die Antwortkategorien zum dritten Teil der Frage sind nicht trennscharf - ein Mangel, der durch die Möglichkeit der Mehrfachnennung nicht kompensiert wurde. Fast alle QB der Stichprobe haben eine spezifische, also auf ihre bevorstehende oder bereits wahrgenommene Aufgabe abgestimmte, Weiterbildung erfahren. Gut die Hälfte davon leistete diese berufsbegleitend $a b$.

Tab. 71: Art und Weise der funktionsbezogenen Ausbildung

\begin{tabular}{|r|l|l|l|l|}
\hline & Häufigkeit & Prozent & $\begin{array}{l}\text { Gültige } \\
\text { Prozente }\end{array}$ & $\begin{array}{l}\text { Kumulierte } \\
\text { Prozente }\end{array}$ \\
\hline Gültig spezifisch für QB & 3 & 13,6 & 13,6 & 13,6 \\
unspezifisch ohne Schwerpunkt & 0 & 0,0 & 0,0 & 13,6 \\
Ausbildung vor Funktionsübernahme & 2 & 9,1 & 9,1 & 22,7 \\
Ausbildung on the job & 5 & 22,7 & 22,7 & 45,4 \\
QB-spezifisch on the job & 6 & 27,3 & 27,3 & 72,7 \\
QB-spez. vor Funktionsübernahme & 6 & 27,3 & 27,3 & 100,0 \\
Gesamt & 22 & 100,0 & 100,0 & \\
\hline
\end{tabular}

\subsubsection{Tätigkeiten von Qualitätsbeauftragten}

Auf die Instruktion "Bitte geben Sie ungefähr an, wie häufig Sie die folgenden Aufgaben ausüben." wurden folgende Durchschnittsantworten auf der nachstehenden sechsstufigen Skala gegeben $($ Stichprobenumfang $=\mathrm{n}$, Mittelwerte $=\mathrm{M}$, Standardabweichungen $=\mathrm{SD}$, Rangordnung $=\mathrm{R}$ ); die Items sind nach Rängen geordnet.

\footnotetext{
1 = täglich/fast täglich $\quad 2=$ wöchentlich bis 14-täglich $\quad 3=$ monatlich $\quad 4$ = quartalsmäßig $5=1-3 x$ jährlich $\quad 6=$ noch seltener/bislang gar nicht/nicht mehr
} 
Tab. 72: Tätigkeiten von Qualitätsbeauftragten (nach abnehmender Häufigkeit geordnet)

\begin{tabular}{|l|l|l|l|l|}
\hline Item & $\mathrm{n}$ & $\mathrm{M}$ & $\mathrm{SD}$ & $\mathrm{R}$ \\
\hline Bearbeitung sonstiger qualitätsrelevanter Dokumente & 29 & 2,41 & 1,296 & 1 \\
\hline Durchführung/Koordination Qualitätsprojekte/-maßnahmen & 29 & 2,76 & 1,244 & 2 \\
\hline Mitarbeit am Qualitäts(management)handbuch & 29 & 2,76 & 1,746 & 2 \\
\hline Sonstige Aktivitäten & 13 & 2,85 & 1,676 & 4 \\
\hline Pflege/Fortschreibung des Qualitäts(management)handbuchs & 29 & 3,07 & 1,668 & 5 \\
\hline Planung von Qualitätsprojekten/-maßnahmen & 27 & 3,07 & 1,107 & 6 \\
\hline mündl./schriftl. Berichterstattung über Maßnahmen & 29 & 3,21 & 1,320 & 7 \\
\hline Sichtung qualitätsrelevanter Fachliteratur & 29 & 3,21 & 1,292 & 7 \\
\hline Beratung von Führungskräften bei Qualitätsfragen & 29 & 3,48 & 1,639 & 9 \\
\hline Beratung von Fachkräften bei Qualitätsfragen & 29 & 3,69 & 1,514 & 10 \\
\hline Anschaffung/Erstellung von Informationsmaterialien & 27 & 3,74 & 1,403 & 11 \\
\hline Bearbeitung von Vorschlägen, Anregungen und Beschwerd. & 29 & 4,10 & 1,319 & 12 \\
\hline Unterstützung/Durchführung von Evaluationsmaßnahmen & 29 & 4,17 & 1,466 & 13 \\
\hline Mitarbeit in einer Steuerungsgruppe "Qualïät" & 27 & 4,22 & 1,601 & 14 \\
\hline Moderation von/Mitarbeit in internen Qualitätszirkeln & 29 & 4,31 & 1,815 & 15 \\
\hline Leitung einer Steuerungsgruppe "Qualität" & 29 & 4,34 & 1,738 & 16 \\
\hline Aktionen zur Werbung/Motivierung für das Qualitätsthema & 29 & 4,59 & 1,296 & 17 \\
\hline Vorbereitung interner Audits & 29 & 4,62 & 1,522 & 18 \\
\hline ReferentIn/Mitarbeit bei internen Fortbildungsveranstalt. & 29 & 4,76 & 1,057 & 19 \\
\hline Durchführung interner Audits & 29 & 4,90 & 1,235 & 20 \\
\hline Moderation von/Mitarbeit in externen Qualitätszirkeln & 29 & 5,03 & 1,349 & 21 \\
\hline eigene externe Fort- oder Weiterbildung als QB & 29 & 5,03 & 0,823 & 21 \\
\hline Außenrepräsentanz bei QM bezogenen Anlässen & 28 & 5,14 & 0,848 & 23 \\
\hline Mitarbeit am Qualitäts-/Jahresbericht & 29 & 5,34 & 0,484 & 24 \\
\hline ReferentIn/Mitarbeit bei externen Fortbildungsveranstalt. & 29 & 5,41 & 0,867 & 25 \\
\hline Vorbereitung interner Fortbildungen mit FremdreferentInnen & 29 & 5,52 & 0,738 & 26 \\
\hline Vorbereitung externer Audits & 29 & 5,59 & 0,825 & 27 \\
\hline Erstellen von Beiträgen für Fachpublikationen & 29 & 5,83 & 0,602 & 28 \\
\hline
\end{tabular}

Die mit relativ deutlichem Abstand (0,35 Skalenpunkte) häufigste Aktivität von QB ist die Bearbeitung "sonstiger qualitätsrelevanter Dokumente". Daneben stehen weitere Aufgaben der Dokumentation und Information sowie Planungs- und Beratungsfunktionen auf vorderen Rängen.

Die Standardabweichungen, mehr noch die Häufigkeitsverteilungen enthüllen die große Varianz der Antworten. Während die meisten Items relativ hohe durchschnittliche Abweichungen vom jeweiligen Mittelwert in der Größenordnung von 1,1 bis 1,8 Skalenpunkten aufweisen, streuen die Mitarbeit am Qualitäts-/Jahresbericht (SD = 0,484), das Erstellen von Beiträgen für Fachpublikationen $(\mathrm{SD}=0,602)$ sowie die auf Fortbildung und externe Audits bezogenen Items gering. Es handelt sich dabei ausschließlich um Tätigkeiten, die nur zu bestimmten und im Jahresverlauf relativ selten vorkommenden Anlässen ausgeübt werden, so dass die eingeschränkte Varianz einen Effekt der objektiven Ereignishäufigkeit bzw. der Skalenkonstruktion darstellt. 
Dass sich unter der gemeinsamen Bezeichnung QB überaus differente Tätigkeitsprofile befinden, wird besonders augenfällig durch jene Items belegt, die über die gesamte Skalenbreite streuen: Evaluationsmaßnahmen, Durchführung von Projekten, Mitarbeit/Pflege QM-Handbuch, Beratung von Fach- und Führungskräften, Sichtung von Fachliteratur, Erstellung/Anschaffung von Informationsmaterial, Vorbereitung interner Audits.

Zusammenhänge mit UV wurden nur für Variablen mit ausreichender Zellenbesetzung überprüft. Für die meisten Tätigkeiten kann zusammenfassend festgestellt werden, dass sie von Einrichtungen zunehmender Größe signifikant häufiger ausgeführt werden als von kleinen und in Erziehungshilfeeinrichtungen häufiger als in Jugendämtern.

Einflüsse der Variablen Geschlecht und Alter zeigen sich in folgender Weise:

○ Männliche QB beschäftigen sich häufiger mit der Arbeit am QM-Handbuch $(\mathrm{p}=.044)$ und dessen Fortschreibung/Pflege $(\mathrm{p}=.019)$.

$\circ$ Dies gilt in gleicher Weise für die älteren Jahrgänge $(\mathrm{p}=.038$ und .035$)$, unter denen die Männer dominieren (s. Tab. 65).

- Die Ergebnisse lassen offen, ob die häufigere Befassung mit dem QM-Handbuch eher geschlechts-, alters- oder wechselwirkungsbedingt ist.

\subsubsection{Typisierung von Tätigkeiten}

Zur Prüfung der Frage, ob sich die Vielzahl der tätigkeitsbezogenen Daten auf wenigen

Dimensionen abbilden und dadurch typologisch verdichten lässt, wurde eine Faktorenanalyse nach der Hauptkomponentenmethode durchgeführt.

Folgende 6-Faktorenlösung mit ca. 80\%-iger Varianzaufklärung erwies sich als relativ gut interpretierbar, auch im Hinblick auf die in Kap. D 3.2/3.3 erörterten Befunde zur Rollenwahrnehmung und -erwartung von Qualitätsbeauftragten bzw. Leitungskräften (zu Kommunalitäten, Varianz- und Komponentenmatrix s. Anhang): 
Tab. 73: Ergebnisse der Hauptkomponentenanalyse von QB-Tätigkeiten (Faktoren 1 bis 6)

\begin{tabular}{|l|l|l|l|}
\hline Komponente & Eigenwert & erklärte Varianz (in \%) & kumul. Varianz (in \%) \\
\hline 1 & 9,086 & 33,652 & 33,652 \\
\hline 2 & 3,520 & 13,036 & 46,689 \\
\hline 3 & 3,443 & 12,753 & 59,442 \\
\hline 4 & 1,981 & 7,338 & 66,780 \\
\hline 5 & 1,798 & 6,660 & 73,440 \\
\hline 6 & 1,660 & 6,147 & 79,587 \\
\hline
\end{tabular}

Die einzelnen Faktoren können auf der Basis der jeweils am höchsten ladenden Items wie folgt charakterisiert werden:

- Faktor 1 (teilweise sehr hohe Ladungen zwischen .845 für Beratung von Fachkräften und .389 für Bearbeitung von Vorschlägen usw. auf allen Items, ausgenommen drei Audit bezogene Items und eigene Fortbildung: .022 bis .223):

Der erste Faktor deckt die gesamte Palette einrichtungs- und mitarbeiterorientierter Qualitätsaktivitäten ab mit Ausnahme von Auditierung und eigener externer Fortbildung als QB. Angesprochen sind allgemeine, primär einrichtungsintern ausgerichtete Unterstützungsfunktionen mit multiplen Schwerpunkten (Beratung, Fortbildung, Moderation, Dokumentation, Information, Projekte). Charakterisiert werden kann dieser Typus QB-spezifischer Tätigkeit als Experte.

- Faktor 2 (hohe Ladungen zwischen .715 und .508 auf Audit bezogenen Items und Planung von Projekten; negative Ladung von -.541 auf Mitarbeit Steuerungsgruppe):

Der zweite Faktor entspricht einem stärker spezialisierten, mit Auditierungs-, Planungs- und fachlichen Repräsentations- und Berichterstattungsfunktionen beauftragten Typus von QB: Auditor/Controller.

- Faktor 3 (vorwiegend durch hohe Negativladungen charakterisiert: -.725 Außenrepräsentanz, -.608 Mitarbeit bei externer Fortbildung, -.563 Vorbereitung interner Fortbildung mit externen ReferentInnen, -.594 eigene Fortbildung; positive Ladungen mittlerer Stärke auf Audit bezogenen Items sowie auf Bearbeitung sonstiger Dokumente/.432 und Bearbeitung von Vorschlägen usw./.430): 
Der dritte Faktor enthält vorwiegend intern ausgerichtete Dienstleistungs- und Sachbearbeitungsaufgaben vorbereitender, dokumentierender und berichtender Art. Wegen der negativen Ladungen auf Items, die im weiteren Sinne fachliche Profilierung und Mulitplikatorenfunktion verkörpern, entspricht der zuordenbare Typus am ehesten der - relativ subaltern akzentuierten - Rolle als Sekretär/Assistent.

○ Faktor 4 (negative Ladungen zwischen -.571 und -.374 auf eigene Fortbildung und Projekte bezogenen Items, positive Ladungen um .470 auf Moderation/Mitarbeit externe Qualitätszirkel, Durchführung interner Audits und Beiträge für Fachpublikationen):

Der vierte Faktor - schon deutlich weniger Varianz erklärend (s. Tab. 73) - ist kaum $\mathrm{zu}$ interpretieren. Die nicht klar konturierte Komponentenmatrix entspricht einer Mischfunktion mit den Schwerpunkten Auditieren, Publizieren, extern Moderieren. Diese eher mit hoher Qualifikation assoziierten Tätigkeiten kontrastieren mit den negativ geladenen Items, die sich auf eigene Fortbildung und Projekte beziehen. Spekuliert werden kann, ob es sich um einen bereits hochqualifizierten, auf eigene Fortbildung nicht mehr angewiesenen Typus von QB mit weitgehender Freistellung vom operativen Tagesgeschäft und sporadisch-spezieller Aktivität im Bereich Audits und externe Qualitätszirkel handelt. Typus "Edelpraktiker"?

- Faktor 5 (hohe Ladung auf Mitarbeit Qualitäts-/Jahresbericht/.786, Erstellung von Informationsmaterialien/.438; Moderation/Mitarbeit interne Qualitätszirkel/.325 und Moderation/Mitarbeit externe QZ/.321 laden gleichfalls positiv; fast alle anderen Ladungen sind nicht substantiell):

Die hohe Ladung auf dem Item "Qualitätsbericht", dazu die mittleren Ladungen auf Items mit im weiteren Sinne ebenfalls kommunikationsbezogenen Aufgaben charakterisieren den fünften Faktor als sehr speziell. Der zugeordnete Tätigkeitsbereich und die diesem entsprechende Rolle des/der QB lassen sich typisieren als Kommunikator/Moderator.

- Faktor 6 (hohe Ladung von .724 auf Evaluationsmaßnahmen, bis auf Vorbereitung externer Audits/.447 keine weiteren substantiellen Ladungen): 
Von allen Faktoren der gewählten Extraktionsmethode lässt sich der sechste am eindeutigsten charakterisieren. Die Vorbereitung, Unterstützung und/oder Durchführung von Evaluationsmaßnahmen, zu denen auch externe Audits gezählt werden können, kennzeichnen den entsprechenden Aufgaben- bzw. Rollentypus als Evaluator.

\subsubsection{Bewertung der Zweckmäßigkeit}

Auf die Frage "Für wie zweckmäßig halten Sie das Instrument $Q B$ in sozialen Einrichtungen und Diensten?" wurden von QB und Leitungskräften aus Einrichtungen mit QB nachstehende Antworten abgegeben.

Tab. 74: Zweckmäßigkeit des Instruments Qualitätsbeauftragte/r im Selbst- (S) und Fremdbild (F)

\begin{tabular}{|ll|l|l|l|l|l|l|}
\hline \multirow{2}{*}{} & & \multicolumn{2}{l|}{ Häufigkeit } & \multicolumn{2}{l|}{ Gültige Prozente } & \multicolumn{2}{l|}{ Kum. Prozente } \\
\cline { 3 - 8 } & S & F & S & F & S & F \\
\hline Gültig & (noch) nicht beurteilbar & 2 & 0 & 6,5 & 0,0 & 6,5 & 0,0 \\
& sehr zweckmäßig & 7 & 8 & 22,6 & 57,2 & 29,1 & 57,2 \\
& zweckmäßig & 17 & 3 & 54,8 & 21,4 & 83,9 & 78,6 \\
& eher zweckmäßig & 5 & 3 & 16,1 & 21,4 & 100,0 & 100,0 \\
& eher unzweckmäßig & 0 & 0 & 0,0 & 0,0 & & \\
& unzweckmäßig & 0 & 0 & 0,0 & 0,0 & & \\
Gesamt & sehr unzweckmäßig & 0 & 0 & 0,0 & 0,0 & & \\
& & 31 & 14 & 100,0 & 100,0 & & \\
\end{tabular}

Eine statistische Prüfung der Häufigkeitsverteilungen mittels Chi-Quadrat-Test ergab eine signifikante Differenz auf dem 5\%-Niveau ( $\mathrm{p}=.037$, einseitig): Leitungskräfte urteilen tendenziell positiver über das Instrument QB als die Beauftragten selbst.

Auf die Frage "Welche Gruppe profitiert Ihrer Meinung nach am meisten von dem Instrument $Q B$ ?" antworteten QB ( $=31)$ und Leitungskräfte $(\mathrm{n}=14)$ aus Einrichtungen 
mit QB wie folgt:

Tab. 75: Profiteure des Instruments Qualitätsbeauftragte/r im Selbst- (S) und Fremdbild (F)

\begin{tabular}{|c|c|c|c|c|c|c|c|}
\hline & \multicolumn{2}{|c|}{ Häufigkeit } & \multicolumn{2}{|c|}{ Gültige Prozente } & \multicolumn{2}{|c|}{ Kum. Prozente } \\
\hline & & $\mathrm{S}$ & $\mathrm{F}$ & $\mathrm{S}$ & F & $\mathrm{S}$ & $\mathrm{F}$ \\
\hline \multirow[t]{6}{*}{ Gültig } & keine & 0 & 0 & 0,0 & 0,0 & 0,0 & 0,0 \\
\hline & MitarbeiterInnen & 3 & 2 & 9,7 & 14,3 & 9,7 & 14,3 \\
\hline & KlientInnen/Kunden & 4 & 2 & 12,9 & 14,3 & 22,6 & 28,6 \\
\hline & Leitung & 5 & 0 & 16,1 & 0,0 & 38,7 & 28,6 \\
\hline & QB selbst & 0 & 0 & 0,0 & 0,0 & 38,7 & 28,6 \\
\hline & alle mehr oder weniger & 19 & 10 & 61,3 & 71,4 & 100,0 & 100,0 \\
\hline Gesamt & & 31 & 14 & 100,0 & 100,0 & & \\
\hline
\end{tabular}

Eine statistische Prüfung der Häufigkeitsverteilungen mittels Chi-Quadrat-Test ergab keine signifikanten Differenzen auf dem 5\%-Niveau ( $\mathrm{p}=.456$, zweiseitig).

Auf die Frage "Hat sich aufgrund gemachter Erfahrungen Ihre Einstellung zur Zweckmäßigkeit des Instruments $Q B$ gegenüber früher geändert?" wurden von QB und Leitungskräften aus Einrichtungen mit QB folgende Antworten abgegeben:

Tab. 76: Änderungen der Zweckmäßigkeitsbewertung von Qualitätsbeauftragten im Selbst- (S) und Fremdbild (F)

\begin{tabular}{|c|c|c|c|c|c|c|c|}
\hline & \multicolumn{2}{|c|}{ Häufigkeit } & \multicolumn{2}{|c|}{ Gülttige Prozente } & \multicolumn{2}{|c|}{ Kum. Prozente } \\
\hline & & $\mathrm{S}$ & $\mathrm{F}$ & $\mathrm{S}$ & $\mathrm{F}$ & $\mathrm{S}$ & $\mathrm{F}$ \\
\hline \multirow[t]{6}{*}{ Gültig } & ja, viel skeptischer & 3 & 1 & 10,0 & 7,1 & 10,0 & 7,1 \\
\hline & ja, ein wenig skeptischer & 2 & 1 & 6,7 & 7,1 & 16,7 & 14,2 \\
\hline & nein, nicht geändert & 7 & 4 & 23,3 & 28,6 & 40,0 & 42,8 \\
\hline & ja, etwas zuversichtlicher & 5 & 4 & 16,7 & 28,6 & 56,7 & 71,4 \\
\hline & ja, viel zuversichtlicher & 12 & 4 & 40,0 & 28,6 & 96,7 & 100,0 \\
\hline & weiß ich nicht (mehr) & 1 & 0 & 3,3 & 0,0 & 100,0 & \\
\hline Gesamt & & 30 & 14 & 100,0 & 100,0 & & \\
\hline
\end{tabular}


Eine statistische Prüfung der Häufigkeitsverteilungen mittels Chi-Quadrat-Test ergab keine signifikanten Differenzen auf dem 5\%-Niveau ( $\mathrm{p}=.893$, zweiseitig).

Die Ermittlung möglicher Zusammenhänge zwischen Zweckmäßigkeitsurteil und UV scheiterte an $\mathrm{zu}$ geringen Zellenbesetzungen. Lediglich bezüglich des dichotomen Merkmals Geschlecht lässt sich konstatieren, dass Männer und Frauen die Zweckmäßigkeit des Instruments QB annähernd gleich einschätzen.

\subsubsection{Konfliktpotenziale in der Praxis}

QB und Leitungskräfte aus Einrichtungen mit QB wurden gefragt: "Wie stark sind folgende Konfliktpotenziale, welche sich für die Rolle des/der QB ergeben können, auch in Ihrer Einrichtung spürbar?"

$\mathrm{Zu}$ antworten war auf einer sechsstufigen Skala:

$1=\operatorname{sehr}$ stark $2=$ stark $3=$ eher stark $4=$ eher schwach $5=$ schwach $6=$ kein Thema/kommt nicht vor.

Nachstehende Darstellung komprimiert die Ergebnisse (angegeben sind Stichprobenumfang $=\mathrm{n}$; Rangplätze $=\mathrm{R}$, bezogen auf die Stärke des wahrgenommenen Konfliktpotenzials; Ergebnisse der Signifikanzprüfung mittels Chi-Quadrat-Test auf der Basis der Antwortverteilungen $=\mathrm{p}$; Kontingenzkoeffizient $=\mathrm{C}$ ):

Tab. 77: Konfliktpotenziale von Qualitätsbeauftragten im Selbst- (S) und Fremdbild (F)

\begin{tabular}{|l|l|l|l|l|l|l|}
\hline \multirow{2}{*}{ Item } & \multicolumn{2}{l|}{$\mathrm{n}$} & $\mathrm{R}$ & $\mathrm{p}$ & $\mathrm{C}$ \\
\cline { 2 - 6 } & $\mathrm{S}$ & $\mathrm{F}$ & $\mathrm{S}$ & $\mathrm{F}$ & \\
\hline $\begin{array}{l}\text { mangelhafte Unterstüt- } \\
\text { zung des/der QB durch } \\
\text { Leitung }\end{array}$ & 29 & 15 & 3 & 9 & .436 & .315 \\
\hline $\begin{array}{l}\text { Konkurrenz (offen/latent) } \\
\text { zwischen Leitung und } \\
\text { QB }\end{array}$ & 29 & 14 & 8 & 13 & .205 & .379 \\
\hline $\begin{array}{l}\text { QB als reine Formalität } \\
\text { (Alibifunktion) ohne Ein- } \\
\text { fluss }\end{array}$ & 29 & 14 & 6 & 13 & $.053 \mathrm{s.}$ & .422 \\
\hline $\begin{array}{l}\text { unklare Rollenerwartun- } \\
\text { gen, Rollenunsicherheit }\end{array}$ & 29 & 14 & 5 & 3 & & .401 \\
\hline
\end{tabular}




\begin{tabular}{|c|c|c|c|c|c|c|}
\hline $\begin{array}{l}\text { unrealistische/überzogene } \\
\text { Erwartungshaltung an } \\
\text { QB }\end{array}$ & 29 & 14 & 10 & 2 & .259 & .363 \\
\hline $\begin{array}{l}\text { Leitung entledigt sich der } \\
\text { eigenen Verantwortung } \\
\text { für Qualität }\end{array}$ & 29 & 14 & 6 & 3 & .388 & .329 \\
\hline $\begin{array}{l}\text { zu geringe Freistellung } \\
\text { von anderen Aufgaben }\end{array}$ & 29 & 14 & 11 & 8 & .803 & .191 \\
\hline $\begin{array}{l}\text { zu geringe } \\
\text { Kompetenzen/Gestal- } \\
\text { tungsmöglichkeiten }\end{array}$ & 29 & 13 & 9 & 5 & .938 & .137 \\
\hline $\begin{array}{l}\text { Akzeptanzprobleme in } \\
\text { der Belegschaft }\end{array}$ & 29 & 14 & 12 & 6 & .635 & .195 \\
\hline $\begin{array}{l}\text { QM wird von Leitung als } \\
\text { unverbindliche Spielwie- } \\
\text { se betrachtet }\end{array}$ & 28 & 14 & 13 & 11 & .259 & .334 \\
\hline $\begin{array}{l}\text { heimliche Leitung durch } \\
\text { QB bei schwacher Füh- } \\
\text { rung }\end{array}$ & 29 & 14 & 14 & 12 & .776 & .199 \\
\hline $\begin{array}{l}\text { skeptische/schwer zu mo- } \\
\text { tivierende Mitarbeiter- } \\
\text { schaft }\end{array}$ & 29 & 14 & 2 & 1 & $.100 \mathrm{~s}$. & .391 \\
\hline $\begin{array}{l}\text { QB als Einzelkämpfer an } \\
\text { mehreren Fronten }\end{array}$ & 29 & 14 & 1 & 6 & $.088 \mathrm{~s}$. & .398 \\
\hline $\begin{array}{l}\text { Sonstiges } \\
\text { Konfliktpotenzial (nicht } \\
\text { expliziert) }\end{array}$ & 26 & 13 & 4 & 10 & .175 & .374 \\
\hline
\end{tabular}

Anmerkung:

Die angegebenen Signifikanzwerte beziehen sich auf zweiseitige Fragestellungen und müssen im Hinblick auf die gerichteten Hypothesen der Studie halbiert werden. Dadurch ergeben sich drei Werte unterhalb der Signifikanzschwelle $(\alpha=.05)$.

Eine Inspektion der Mittelwerte (s. Anhang) zeigt, dass bis auf die nahezu gleich bewerteten Items "unrealistische/überzogene Erwartungshaltung an QB" und "Akzeptanzprobleme in der Belegschaft" alle vorgegebenen Konfliktpotenziale, wenn auch in unterschiedlichem Maße, stärker seitens der QB betont werden. Während im Chi-QuadratTest (s. Tab. 77) nur die Items "QB als reine Formalität (Alibifunktion)", "QB als Einzelkämpfer an mehreren Fronten" sowie "skeptische/schwer zu motivierende Mitarbeiterschaft" signifikante Unterschiede auf dem 5\%-Niveau aufweisen, sind beim t-Test der Mittelwertsdifferenzen auch die Items "mangelnde Unterstützung durch Leitungsebene", "Konkurrenz zwischen Leitung und QB" und "sonstiges Konfliktpotenzial" bei einseitiger Fragestellung signifikant. 
Nicht alle signifikanten Differenzen spiegeln sich auch in den Rangordnungen deutlich wider. Dabei ist jedoch zu berücksichtigen, dass die kleine Stichprobe der Leitungskräfte zwangsläufig zu sehr geringen Zellenbesetzungen führte und häufig Werte von Null verarbeitet werden mussten. Die Signifikanzen sind daher nur mit Vorsicht zu interpretieren. Maßgeblich ist die Gleichrichtung der Bewertungen von 12 der 14 Items, die ein wesentlich stärkeres Konfliktempfinden auf Seiten der QB belegt.

Die spezifische Information der Rangordnungen besteht darin, dass unterschiedliche Gewichtungen der Konfliktpotenziale deutlich werden. Nicht nur werden diese durchweg stärker von den Rollenakteuren selbst wahrgenommen als von Leitungskräften (die überwiegend an der Rollenbesetzung mitgewirkt haben dürften). Vielmehr unterscheidet sich auch der Stellenwert, der den einzelnen Konfliktbereichen zugemessen wird.

Darüber hinaus sind folgende Zusammenhänge zwischen UV und wahrgenommenen Konfliktpotenzialen signifikant:

○ QB öffentlicher Träger äußern im Vergleich mit QB freigemeinnütziger Träger stärkeres Empfinden von Konkurrenz zur Leitung (Mittelwert 3,3 vs. 4,9; $\mathrm{p}=.013$ ), unklaren Rollenerwartungen (3,4 vs. 4,6; $\mathrm{p}=.035)$, unrealistischer Erwartungshaltung (3,7 vs. 4,$9 ; \mathrm{p}=.018)$, Verantwortungsentledigung seitens Leitung $(3,3$ vs. 4,8; $\mathrm{p}=$ .023), QM als Spielwiese (3,5 vs. 5,2; $\mathrm{p}=.005)$ und QB als Einzelkämpfer (3,1 vs. 4,3; $\mathrm{p}=.035)$.

- QB in kleinen/kleinsten Einrichtungen geben im Vergleich mit QB sehr großer Organisationen geringe Freistellung $(3,0$ vs. 5,5; p = .004) häufiger als Konfliktpotenzial an.

- Akzeptanzprobleme in der Belegschaft werden von männlichen QB stärker als von weiblichen empfunden ( 4,2 vs. 5,$3 ; \mathrm{p}=.026)$. 


\subsection{Prüfung der Hypothesen dieser Studie}

\subsubsection{Hypothesen zur Ausgestaltung des Qualitätsmanagements}

- Hypothese 1:

Freie Träger verfügen über ein signifikant elaborierteres Qualitätsmanagement als öffentliche Träger; Stand der Umsetzung und/oder Vielfalt des Instrumenteneinsatzes sind weiter entwickelt.

Ergebnis:

Freie und öffentliche Träger unterscheiden sich in der Umsetzung systematischen Qualitätsmanagements und in der Vielfalt des Instrumenteneinsatzes signifikant $(\mathrm{C}=$ .508 , Kendall's tau $=-.214$, Vorzeichen polungsbedingt). Von 47 freien Einrichtungen verfügen rund 51\% über ein QM-System, fast ein weiteres Drittel arbeitet daran und der Rest hegt entsprechende Pläne. Von 31 Organisationen in öffentlicher Trägerschaft vermelden nur vier $(12,9 \%)$ ein vorhandenes QM-System, immerhin arbeiten $39 \%$ daran und ein weiteres Fünftel plant; aber fast $30 \%$ sind diesbezüglich inaktiv.

Die hypothetische Vorannahme eines relativen Vorsprungs der freien Träger in Sachen systematischen Qualitätsmanagements vor den öffentlichen Trägern kann als bewährt angesehen werden. Ein Urteil über die tatsächliche Qualität der betreffenden Organisationen ist damit jedoch nicht verbunden.

- Hypothese 2:

Einrichtungen der Kinder-, Jugend- und Sozialhilfe weisen ein signifikant elaborierteres Qualitätsmanagement auf als Einrichtungen und Dienste der Jugendund Sozialhilfeverwaltung.

Ergebnis:

Wegen der starken Konfundierung der UV Trägerschaft und Arbeitsfeld sind die Ergebnisse trivial und gehen nicht über die Aussagen zur Hypothese 1 hinaus. Zwischen den in der Studie eindeutig unterscheidbaren Arbeitsfeldern der Jugend- und Sozialhilfeverwaltungen einerseits und den leistungserbringenden Einrichtungen andererseits gibt es hochsignifikante Differenzen in Bezug auf Umsetzungsstand und 
Ausgestaltung des Qualitätsmanagements. Von 48 leistungserbringenden freien Einrichtungen verfügen 52\% über ein QM-System; der Rest arbeitet daran oder plant, dies zu tun. Von 29 Jugend- und Sozialhilfeverwaltungen haben erst 10\% (sämtlich Jugendämter) ein QM-System, fast ein Drittel hegt keine entsprechenden Pläne.

Die Hypothese wird empirisch in vollem Umfang bestätigt.

Hypothese 3:

Innerhalb der Gruppe öffentlicher Verwaltungen weisen Jugendämter eine signifikant stärkere Aktivität in Sachen QM auf als Sozialämter.

Ergebnis:

Ungeachtet der für solide Aussagen zu kleinen Stichprobe der Sozialämter, zeigt die Inspektion der Rohdaten eine Tendenz im Sinne der Hypothese. Von 22 Jugendämtern verfügen drei über ein QM-System, weitere acht arbeiten daran, eines steht in der Planung und sechs berichten keinerlei Ambitionen. Von sieben Sozialämtern hat noch keines ein QM-System, drei arbeiten daran, eines plant und drei melden Fehlanzeige.

Über die Hypothese kann nicht auf verlässlicher statistischer Grundlage entschieden werden.

- Hypothese 4:

Einrichtungen und Dienste der Kinder-, Jugend- und Sozialhilfe weisen ein umso elaborierteres Qualitätsmanagement auf, je größer sie sind.

Ergebnis:

Die Größe der Einrichtungen und Dienste - operationalisiert als Zahl der beschäftigten MitarbeiterInnen und differenziert nach Quartilen - korreliert zwar mit der Existenz eines QM-Systems $(\mathrm{C}=.362$, Kendall's tau = -.120, Vorzeichen polungsbedingt $)$, aber nicht signifikant für die Gesamtpopulation. Ein stärkerer Zusammenhang wird durch die Überrepräsentanz öffentlicher Träger ohne QM-System unter den Einrichtungen des dritten Größenquartils verhindert: Während alle freien Träger zumindest ein QMSystem planen, entfallen von neun Einrichtungen ohne QM-System allein fünf auf die Organisationen mit 76 bis 150 MitarbeiterInnen, sämtlich in öffentlicher Trägerschaft. 
Die Hypothese bewährt sich nur im Hinblick auf Einrichtungen in freier Trägerschaft.

Bezogen auf die Grundgesamtheit aller Einrichtungen und Dienste kann die Nullhypothese aufrechterhalten werden.

\subsubsection{Hypothesen zum Einsatz von Qualitätsbeauftragten:}

- Hypothese 5:

Das Instrument QB wird in sehr großen (Mitarbeiterzahl > 150) und großen Einrichtungen (Mitarbeiterzahl > 75) signifikant häufiger verwendet als in mittelgroßen (Mitarbeiterzahl > 30) und in diesen häufiger als in kleinen und kleinsten (Mitarbeiterzahl < 31) Einrichtungen. M. a. W., die Verwendungshäufigkeit steigt mit der Mitarbeiterzahl (linear?) an.

Ergebnis:

Die Anteile der Einrichtungen mit QB in den verschiedenen Größenquartilen steigt nahezu linear an $(18 \%, 29 \%, 36 \%, 55 \%)$; fast analog verhält es sich mit den Entscheidungen gegen die Funktionsstelle (32\%, 19\%, 0\%, 10\%). Der Kontingenzkoeffizient beträgt .390 und ist signifikant ( $\mathrm{p}=.047$, einseitig).

Somit kann die Hypothese als bewährt angesehen werden: Das Instrument QB wird mit zunehmender Einrichtungsgröße immer häufiger angewandt.

- Hypothese 6:

Das Konfliktpotenzial der Funktionsstelle QB bzw. wahrgenommene Hindernisse in der Rollenausübung werden von QB selbst stärker eingeschätzt als von Leitungskräften aus Einrichtungen mit QB.

Ergebnis:

Je nach statistischem Testverfahren zeigen mindestens drei der 14 Items auf dem gewählten 5\%-Niveau einen signifikanten Unterschied in der Bewertung: "QB als reine Formalität (Alibifunktion) ohne Einfluss", "QB als Einzelkämpfer an mehreren Fronten" und "skeptische/schwer zu motivierende Mitarbeiterschaft" differieren im 
Sinne stärkerer Konfliktwahrnehmung auf Seiten der QB. Auch die über alle Items ermittelte Anzahl der Antworten in den Kategorien "sehr stark" (QB: 9, Leitung 0) und "stark" (QB: 27, Leitung: 8) weist in die hypothetisch formulierte Richtung.

Da unter der Nullhypothese keinerlei Bewertungsunterschiede auftreten sollten, kann die Hypothese als bewährt angesehen werden: Zwischen QB und Leitungskräften aus Einrichtungen mit QB werden die in der Rollenausübung liegenden Konfliktpotenziale in der Stichprobe signifikant, wenngleich nicht durchgängig über alle Items, unterschiedlich beurteilt. QB äußern sich im Durchschnitt konfliktbetroffener.

- Hypothese 7:

Leitungskräfte aus Einrichtungen mit QB beurteilen die Zweckmäßigkeit der Funktionsstelle signifikant positiver als QB selbst.

Ergebnis:

Die Begründungen zu dieser und der vorherigen Hypothese sind (psycho)logisch eng miteinander verknüpft. Insofern überrascht nicht, dass sich auch die Ergebnisse gleichen. Der ermittelte Kontingenzkoeffizient beträgt .365 und ist mit $p=.037$ (einseitig) signifikant. Die Antwortverteilung auf die Zweckmäßigkeitsfrage weist für beide Gruppen ausschließlich Werte auf den positiven Skalenstufen auf, jedoch mit tendenziell günstigerer Beurteilung durch die Leitungskräfte: sehr zweckmäßig (QB: 22,6\%, Leitung: 57,2\%), zweckmäßig (QB: 54,8\%, Leitung: 21,4\%), eher zweckmäßig (QB: 16,1\%, Leitung: 21,4\%).

Auch hier kann die Hypothese als bewährt betrachtet werden: Von Leitungskräften der Einrichtungen mit QB wird die Zweckmäßigkeit der Funktionsstelle signifikant größer beurteilt als von QB selbst - insgesamt jedoch von beiden Gruppen eindeutig positiv. 


\subsection{Zusammenfassung und Diskussion der Ergebnisse}

\subsubsection{Vorbemerkungen}

In Anbetracht der im Rahmen dieser Untersuchung nicht zu bewältigenden Stichprobenprobleme und der überwiegend unbekannten Populationskennwerte reduziert sich die Grundgesamtheit, über die im Wege statistischer Inferenz verallgemeinerbare Aussagen gemacht werden können, auf freie Einrichtungen der Erziehungshilfe einerseits und öffentliche Jugendhilfeverwaltungen andererseits.

Feinere Differenzierungen nach Arbeitsfeld, Trägerschaft oder Einrichtungsgröße lassen sich auf der Basis des Rücklaufs nur vereinzelt treffen. Aussagen über Zusammenhänge zwischen abhängigen und unabhängigen Variablen sind überdies rein korrelativer Natur.

\subsubsection{Fragenkomplex "Umsetzung von Qualitätsmanagement"}

- Qualitätsmanagement steht bei fast $90 \%$ der befragten Einrichtungen auf der Agenda; in systematisierter Form wird es jedoch erst von gut einem Drittel praktiziert.

○ Während öffentliche Träger bzw. Jugend- und Sozialämter einen deutlich geringeren Umsetzungsstand aufweisen, besteht mit dem Faktor Einrichtungsgröße kein signifikanter Zusammenhang. Tendenziell deutet sich jedoch der hypothetisch angenommene Einfluss an, wonach mit zunehmender Organisationsgröße der Anteil der Einrichtungen mit entwickeltem QM-System ansteigt. Unter den aus dem Erwartungsrahmen fallenden großen Einrichtungen befinden sich nämlich ausnahmslos Jugendämter; dies wirkt sich im dritten Größenquartil unverhältnismäßig stark aus und überlagert den für Erziehungshilfeeinrichtungen beobachtbaren Zusammenhang zwischen Größe und QM.

- Organisationsspezifische Eigenentwicklungen und Mischformen, ISO $9000 \mathrm{ff}$. und das EFQM-Excellence-Modell sind in fast 90\% der Fälle das QM-System der Wahl; den Rest bilden überwiegend Verbandssysteme. 
Während Qualitätsbeauftragte mehrheitlich über eine ISO-orientierte Ausbildung verfügen, wird in der Praxis der Einrichtungen relativ häufig auch das anspruchsvollere EFQM-Modell als Referenzsystem verwendet. Als prominentestes QM-System dominiert die ISO jedoch das externe Weiterbildungsangebot und dürfte für viele Leistungsanbieter schon deshalb interessant sein, weil die Option einer Zertifizierung nach ISO - wenn auch von den meisten Einrichtungen derzeit nicht vorgesehen - vielfach mit einem potenziellen Marktvorteil assoziiert wird.

- Sowohl in Bezug auf die Organisation des Qualitätsmanagements, als auch hinsichtlich der qualitätsbezogenen Programmatik - Skeptiker sprechen auch von "QualitätsRhetorik" - verhält sich ein relativ konstanter Anteil von gut 10\% der Einrichtungen abstinent. Ungeachtet des tendenziell beobachtbaren Zusammenhangs zwischen Größe und QM weist die Tatsache, dass dies auch in großen und sehr großen Einrichtungen vorkommt, auf die Kontext- und Interessenabhängigkeit derartiger Entscheidungen hin. Schließlich sind es nicht die Einrichtungen, welche sich ein QM-System zulegen oder nicht, sondern konkrete Personen mit unterschiedlichen Erfahrungen und Präferenzen und bisweilen ausgeprägtem Widerwillen gegen MainstreamEntwicklungen im Managementbereich.

- Jeweils annähernd doppelt so hoch ist der Anteil der Einrichtungen, die den Einsatz der Instrumente QM-Handbuch, Qualitätsbeauftragte, Vorschlagswesen und Beschwerdemanagement nicht in Erwägung ziehen. Über 70\%, darunter überproportional viele sehr große Einrichtungen, praktizieren jedoch diese Instrumente oder planen zumindest ihren Einsatz.

○ Qualitätszirkel sind in drei von vier Einrichtungen in unterschiedlicher Form vorhanden. Der Befund, dass in EFQM- und ISO-orientierten Organisationen ein beträchtlicher Anteil erst mit der Planung befasst ist oder noch gar keine QZ in Erwägung zieht (drei ISO-Einrichtungen), dürfte eher mit anderen Prioritäten (z. B. Durchführung einer umfassenden Selbstbewertung, Handbucherstellung, Vorbereitung auf Zertifizierung) zusammenhängen als mit einer systembedingten Ablehnung des Instruments. Die Nullkorrelation zwischen QZ und QM spricht jedenfalls dafür, dass Qualitätszirkelarbeit unabhängig vom jeweiligen Entwicklungsstand eines systematischen Qualitätsmanagements praktiziert wird - trotz oder auch wegen der 
explizit qualitätsbezogenen Benennung des Instruments. Während die meisten Strukturelemente eines QM mehr oder minder stark interkorrelieren, scheint Qualitätszirkeln ein Sonderstatus zu eignen. Möglicherweise werden sie von vielen Einrichtungen nicht nur als ein, wenn auch wichtiges, Element systematischen Qualitätsmanagements betrachtet, sondern als Surrogat für QM schlechthin, das weitere Maßnahmen (zumindest vorerst) verzichtbar macht.

- Personalentwicklungskonzepte, Evaluation und Controlling beschäftigen über $90 \%$ der Einrichtungen mindestens auf der Planungsebene, sind aber überwiegend - nämlich jeweils knapp zur Hälfte - erst in Ansätzen realisiert.

Mutmaßlich im Zusammenhang mit den populär gewordenen Kunden- und Mitarbeiterbefragungen scheint sich vor allem Evaluation zu einem zentralen Thema des Qualitätsmanagements entwickelt zu haben. Damit zeichnet sich ein Trend ab, wie er im Schulbereich (PISA-Studie!) besonders deutlich wird: der Versuch, Praxis zu verbessern, indem Output- und Outcome-Variablen gemessen und - so jedenfalls die gute Absicht - für die Steuerung von Input- und Prozessvariablen genutzt werden.

- Elemente des Controllings sind ohnehin traditioneller Bestandteil der Geschäftsführung bzw. Verwaltungsleitung sozialer Einrichtungen und Dienste; relativ neu sind einschlägige Anforderungen im Bereich der öffentlichen Verwaltung.

- Der relativ hohe Umsetzungsstand von Leitbildentwicklung, Vorschlags- und Beschwerdemanagement sowie Personalentwicklungs-Konzeptionen in Sozialämtern ist angesichts der Fallzahl von nur sieben kaum interpretationswürdig. Immerhin sprechen diese Befunde dafür, dass Sozialämter keineswegs durchgängig im Hintertreffen sind, ihre QM-Aktivitäten aber möglicherweise auf wenige, für besonders relevant gehaltene Bereiche fokussieren (wollen).

- In der Fachliteratur besteht Einigkeit darüber, dass für eine erfolgreiche Implementation von QM-Systemen die partizipative Einbindung der Mitarbeiterschaft von zentraler Bedeutung ist. Strittig dürfte sein, ob dies in der Praxis heißt, die Fachkräfte "in jede Sackgasse [mitzunehmen, P. G.], in die sich die Leitung begibt ..." (von Spiegel, 2000, S. 154). Ob somit eine umfassende oder punktuelle Beteiligung der 
gesamten Belegschaft anzustreben ist, kann nicht dogmatisch entschieden werden. Dass eine solche Beteiligung immerhin von rund zwei Dritteln der befragten Einrichtungen angegeben wird, während nur ein knappes Fünftel QM bislang allein zur Sache der Leitung oder einzelner MitarbeiterInnen macht, ist in jedem Falle positiv zu bewerten.

Der Anteil der Einrichtungen, in denen eine mögliche Beteiligung entfällt, da noch kein QM-Prozess begonnen wurde, liegt im Übrigen mit knapp 18\% (s. Tab. 43) deutlich höher als der angegebene Anteil von gut $11 \%$ jener Einrichtungen, die kein QM-System einzuführen beabsichtigen (s. Tab. 28). Ungeachtet der Möglichkeit von Messfehlern kann diese Differenz bedeuten, dass einige Einrichtungsleitungen sich bereits mit der Planung von QM befassen, jedoch noch nicht initiativ in Sachen Mitarbeiterbeteiligung geworden sind.

○ Überraschend ist der ermittelte Zusammenhang zwischen Beteiligungsgrad und Wahl eines bestimmten QM-Referenzsystems. Nicht das explizit als TQM-System ausgewiesene EFQM Excellence Modell oder intern entwickelte Eigensysteme, sondern die ISO dominiert in jenen Einrichtungen, die den höchsten Beteiligungsgrad angeben.

Bedeutet dies, dass die ISO besonders partizipationsorientiert ist oder mit Vorliebe von Organisationen mit hoher Mitarbeiterbeteiligung als Referenzsystem gewählt wird? Derartige Schlussfolgerungen sind des nur korrelativen Zusammenhangs zwischen allen gemessenen Variablen wegen unzulässig. Die Frage, ob die verglichenen Einrichtungen im Hinblick auf maßgebliche Einflussfaktoren und Störvariablen äquivalent sind (Ceteris-Paribus-Distributionibus-Bedingungen) und die Untersuchung somit intern valide ist (Hager, 2004, S. 25), kann nämlich gar nicht beantwortet werden. Möglicherweise legt auch die intensive Befassung mit bestimmten QMSystemen ein spezifisches, systemkonformes Verständnis von "Beteiligung" und "umfassend" nahe, jedenfalls bei den befragten Qualitätsbeauftragten und Leitungskräften.

Sollte tatsächlich eine besonders hohe Mitarbeiterbeteiligung für die ISO charakteristisch sein, so kämen als mögliche Gründe u. a. folgende in Betracht: 
1. Nur die ISO fordert obligatorisch die Dokumentation des QM-Systems in einem zentralen Handbuch. Soll dieses nicht nur der Selbstdarstellung der Einrichtung dienen, sondern praktische Steuerungsrelevanz erlangen, ist zu seiner Erarbeitung eine disziplinierte Kooperation mit allen prozessbeteiligten Personen unerlässlich. Hinzu kommt die potenziell kommunikations- und transparenzförderliche Funktion eines solchen Handbuchs (vgl. Kap. C 2.2.2).

2. Die ISO erfordert wegen ihrer relativ technisch anmutenden OriginalBegrifflichkeit ein hohes Maß an Überzeugungs- und Übersetzungsarbeit (s. Kap. C 1.4.1), um die Mitarbeiterschaft "ins Boot zu bekommen". Hohe Beteiligung hieße unter diesen Umständen auch systembedingt hoher Aufwand.

3. Die Implementierung eines QM-Systems nach ISO impliziert zumeist die Option einer Zertifizierung und ist somit auf die Erfüllung standardisierter Mindestanforderungen ausgerichtet. Dies kann bedeuten, dass der selbstgewählte Zeitrahmen in solchen Einrichtungen mit hohem Grad an Verbindlichkeit gesteckt und mit großem Ressourceneinsatz, sprich: Mitarbeiterbeteiligung, ausgefüllt wird.

Ungeachtet dieser oder weiterer Gründe sprechen die Ergebnisse der Befragung dafür, dass die ISO 9000 ff. bei verständiger Anwendung ebenso gut für soziale Handlungsfelder geeignet ist wie andere Systeme auch, unter dem gemeinhin für besonders wichtig gehaltenen Aspekt der Mitarbeiterpartizipation allem Anschein nach sogar besonders gut.

Maßgeblich für die Bewertung des Beteiligungsgrades muss aber in jedem Falle auch dessen Nachhaltigkeit sein: Projektmäßig forcierte temporäre Höchstbeteiligung aller kann langfristig fatal sein, wenn nach Erreichen des Etappenziels erschöpfte MitarbeiterInnen alsbald wieder "business as usual" praktizieren. Die Frage nach dem Beteiligungsgrad führt deshalb ins Zentrum des Implementierungsproblems (s. Kap. B $1.8)$.

- Die Angaben zur Qualifizierung der Mitarbeiterschaft enthüllen, dass umfassende Beteiligung keineswegs bedeutet, alle MitarbeiterInnen kontinuierlich und systematisch zu Qualitäts-ExpertInnen auszubilden. Anderenfalls wäre die Diskrepanz 
zwischen den entsprechenden Angaben (28,2\% "umfassend" zu 10,5\% "alle kontinuierlich", s. Tab. 43 und 45) nicht zu erklären. Vielmehr kann davon ausgegangen werden, dass umfassende Beteiligung sich selektiv auf jene Aufgaben und Rollen bezieht, die aus Sicht der befragten Qualitätsbeauftragten und Leitungskräfte das jeweilige Handlungsfeld der MitarbeiterInnen kennzeichnen.

$\mathrm{Zu}$ vermuten ist, dass sich bei einer Befragung von Fachkräften an der betrieblichen Basis andere Gewichtungen ergäben. Eine tendenziell kritischere Einschätzung des tatsächlichen Beteiligungsgrades wäre dabei nicht überraschend, aber keineswegs zwangsläufig. Berücksichtigt man die relativ geringe Bereitschaft vieler MitarbeiterInnen, sich selbst in spezielle QM-Aktivitäten einzubringen (s. Kap. D 1.2.2), könnte sich durchaus ein Befragungsergebnis einstellen, das eher als Wunsch zu interpretieren wäre, von allzu viel Qualitätsmanagement verschont zu bleiben.

\subsubsection{Fragenkomplex "Qualitätsentwicklungsmaßnahmen"}

- Da die vorgegebene Auswahl von Verfahren ein breites Spektrum vermeintlich oder tatsächlich qualitätsentwickelnder Instrumente repräsentiert, verwundert nicht, dass fast allen eine zumindest tendenziell positive Bewertung zukommt (Linksschiefe der Verteilung). Als Favoriten kristallisieren sich heraus: interne Fallreflexion und Konzeptionsentwicklung (Umsetzung und Innovation).

- Bei den schlechter bewerteten Maßnahmen handelt es sich mit Ausnahme der externen Fachberatung (k. A.: 25,9\%) um solche mit den zugleich meisten Fehlanzeigen. Maßnahmen, die von 34,1\% (Selbstevaluation) bis 61,2\% (Fremdevalaution) nicht beurteilt werden, weil sie in der Einrichtung nicht stattfinden und/oder nicht hinreichend bekannt sind, schneiden auch in der Bewertung durch die übrigen Organisationen relativ schlecht ab.

Bereits an anderer Stelle (s. Kap. D 1.4.3) war konstatiert worden, dass Fachkräfte der Sozialen Arbeit offenbar die größte Bedeutung für die Qualifizierung der Leistungsprozesse und Akteure eher klassischen Maßnahmen zumessen, die auch schon vor Aufkommen der Qualitätsdebatte fachlich en vogue waren. 
Bei aller Wertschätzung auch jenen neuen oder wiederentdeckten Instrumenten gegenüber, die im Zuge eines Qualitätsmanagements thematisiert werden, drückt sich darin mutmaßlich auch die Überzeugung aus, dass die Maßstäbe eigener Fachlichkeit und "handwerklicher" Professionalität nicht durch Methodenimporte aus anderen Handlungsfeldern ersetzt werden können oder sollten.

○ Zum Faktor Trägerschaft lässt sich feststellen, dass öffentliche Träger tendenziell zu allen vorgegebenen Maßnahmen der Qualitätsentwicklung weniger positive Bewertungen abgeben als freie Träger; in Einzelfällen sind privatgewerbliche Träger von dieser Tendenz ausgenommen.

Diese - in der Regel kleineren - Einrichtungen geben u. a. in den Items interne Audits, Kennzahlen, Selbstbewertung mittels Fragebögen und Kontraktmanagement deutlich kritischere Bewertungen ab als die freigemeinnützigen Träger. Der Charakter dieser Maßnahmen entspricht wohl auch kaum den organisationsspezifischen Anforderungen kleiner, überschaubarer Einheiten, in denen alle Mitglieder kurze Kommunikationswege zueinander haben und die zur Organisationsbeschreibung benötigten Datenmengen gering sind.

- In der größeren Zurückhaltung der Jugend- und Sozialhilfeverwaltungen in puncto Nutzenbewertung könnten latente Interessenkonflikte zum Ausdruck kommen: Öffentliche Kostenträger sehen sich in Entgeltverhandlungen immer wieder Forderungen der leistungserbringenden Einrichtungen gegenüber, Qualitätsentwicklungsmaßnahmen in die Vergütungen einzukalkulieren, darunter nicht selten Maßnahmen, die in den Behörden selbst keineswegs zum Standard gehören. Seitens der Leistungs-"Anbieter" zu versuchen, durch relativ hohe Nutzenzuschreibungen möglichst viele Support-Prozesse von den "Nachfragern" finanziert zu bekommen, käme als handlungsleitendes Motiv durchaus in Betracht.

- Zum Faktor Arbeitsfeld ist ergänzend anzumerken, dass im Bereich Berufsbildungswerk/Schule die tendenziell positivsten Bewertungen geäußert werden, seitens der befragten Sozialämter dagegen die skeptischsten. Ein Teil der Varianz zwischen öffentlichen und freien Trägern geht offenbar zu Lasten der wenigen teilnehmenden Sozialämter. Ob diese Tendenz charakteristisch ist für die öffentlichen 
Dienste der Sozialhilfe und ihre stärker verfahrensrechtlich orientierten Binnenprozesse, kann aufgrund des schwachen Rücklaufs von nur sieben Fragebögen nicht beurteilt werden.

- Der Faktor Einrichtungsgröße hat offenbar nur geringen Einfluss. Dabei entbehrt es gewiss nicht der Logik, dass sehr große Organisationen gezielte Maßnahmen zur Partizipation ihrer KundInnen und entsprechende Befragungen für sehr nützlich halten - jedenfalls aus der Sicht von Personen mit zentralen Beratungs- und Steuerungsaufgaben, die ab einer bestimmten Einrichtungsgröße nur noch eingeschränkten Klientenkontakt haben.

\subsubsection{Fragenkomplex "Qualitätsbeauftragte/r"}

- Die Frage der Institutionalisierung von Qualitätsbeauftragten muss differenziert beantwortet werden, da sich zwischen Trägergruppen bzw. Arbeitsfeldern und Einrichtungsgrößen erhebliche Unterschiede zeigen. Es sind vor allem die freigemeinnützigen Träger (Erziehungshilfe und Sonstige), die mit zunehmender Größe von dem Instrument Gebrauch machen. In Jugend- und Sozialämtern wird das Thema überwiegend noch gar nicht diskutiert, während in den zumeist kleinen privatgewerblichen Einrichtungen mehrheitlich eine Entscheidung dagegen getroffen wurde.

- Bemerkenswert sind die hochsignifikanten Interkorrelationen zwischen QBInstitutionalisierung und dem Einsatz vieler Instrumente des QM sowie dem Ausmaß der Beteiligung und systematischen Qualifizierung der MitarbeiterInnen. Dies spricht dafür, dass - ungeachtet der Legitimität vorgebrachter Einwände gegen die Funktionsstelle (s. Kap. D 4.6.8.2) - in Einrichtungen mit QB keine spezialistische Abwälzung QM-bezogener Aufgaben stattfindet, sondern deren Basis eher verbreitert wird.

Zur strukturellen Ausgestaltung der Funktionsstelle lässt sich konstatieren:

○ QB sind in der Regel für ihre Gesamteinrichtung zuständig; lediglich in großen und 
dezentralen Einrichtungen gibt es zum Teil mehrere Beauftragte.

- Sofern nicht projektabhängige Lösungen gewählt werden, sind QB mehrheitlich mit mehr als Dreiviertel ihrer Wochenarbeitszeit für ihre Aufgaben freigestellt, und zwar weit überwiegend (rund 83\%) unbefristet. Die nur in einem Fünftel der Einrichtungen praktizierte Teilzeit-Freistellung mit festem Stundenvolumen dürfte dem Umstand geschuldet sein, dass eine punktgenaue Fixierung des bedarfsgerechten Arbeitsaufwandes in der Praxis schwierig ist und eine plausible Kontingentierung von Dienstzeiten voraussetzt.

- Die hohe Zahl nicht temporär, sondern dauerhaft eingerichteter QB-Funktionsstellen spricht - wie auch Wetzler (2003, S. 71) betont - für die Nachhaltigkeit der eingesetzten Ressourcen und Qualitätspolitik. Ebenso wie im Behindertenbereich scheint auch in anderen sozialen Handlungsfeldern eine neue Berufsgruppe heranzuwachsen, die neben primär fachlichen Aufgaben ein kontinuierliches Qualitätsmanagement gewährleisten soll.

○ Allerdings geht diese Entwicklung in mehr als der Hälfte der Fälle mit einer internen Umverteilung der verfügbaren Ressourcen einher, m. a. W.: es wird an anderen Stellen gespart. Nur in gut $40 \%$ der befragten Einrichtungen erfolgt ein voller Ausgleich durch Neueinstellung.

- Dass die Stellenbesetzung in fast allen Einrichtungen aufgrund von Leitungsentscheidungen zustande kommt, spricht einerseits für die strategische Bedeutung, die dem Qualitätsmanagement zugemessen wird und diese Entscheidung zu einer originär unternehmerischen macht. Andererseits kommt Qualitätsbeauftragten gerade in sozialen Einrichtungen und Diensten eine besondere Moderatoren- und Vermittlungsrolle zu, die der breiten Akzeptanz bedarf. Insofern spricht vieles dafür, in der Frage der Stellenbesetzung problemsensibel vorzugehen und eine konsensuelle Lösung mit der Belegschaft anzustreben.

- Rund 70\% der befragten QB berichten, dass ihre Kompetenzen in Stellenbeschreibungen und/oder im Organigramm explizit geklärt seien. Jeweils etwa die Hälfte der QB ist mit reinen Beratungsfunktionen betraut oder verfügt zusätzlich 
über Umsetzungskompetenzen. Ob es sich bei den je ca. 15\% der Fälle mit nicht oder nur teilweise explizit geklärten Kompetenzen um wenigstens mündlich abgesprochene, relativ offen und flexibel gehandhabte Aktionsbereiche handelt oder um ein ständig virulentes Konfliktfeld, geht aus den Daten nicht hervor.

Folgende Personen- und Ausbildungsmerkmale sind für die befragten QB charakteristisch:

○ Die Funktionsstellen verteilen sich annähernd gleich auf beide Geschlechter.

Angesichts immer noch vorhandener Männerdominanz in exponierten Funktionen (auch in der Stichprobe entfallen die meisten Einrichtungs- und Bereichsleitungen auf Männer) kann dies als Indiz gewertet werden, dass die QB-Stelle von Frauen als eine Möglichkeit wahrgenommen wird, innerbetrieblich auf- oder umzusteigen.

○ Altersmäßig dominieren die über 40-Jährigen. Dies spricht sowohl dafür, dass für die Funktion mehrheitlich ein erhebliches Maß an fachlicher Erfahrung und betrieblicher Insider-Kenntnis gewünscht wird, als auch für eine besondere Attraktivität der Aufgabe und der damit verbundenen Perspektiven gerade für die "reiferen" Jahrgänge.

Als eine solche Perspektive kann vermutlich auch die Entlastung von reinen Leitungsaufgaben angesehen werden; denn immerhin 71\% der Akteure wechselten aus Funktionen des oberen oder mittleren Managements in die Funktion des/der QB, während nur ein Drittel weiterhin Leitungsaufgaben bekleidet.

○ Über die Hälfte der QB hat ein Fachhochschulstudium abgeschlossen, ein weiteres Drittel verfügt über einen Hochschulabschluss.

- Eine überwiegend berufsbegleitende, in einem Viertel der Fälle schon vor Funktionsübernahme genossene, Qualifizierung für ihre Aufgabe weisen fast alle QB auf. Dabei dominieren mit zwei Dritteln externe Weiterbildungen mit dem inhaltlichen Schwerpunkt ISO $9000 \mathrm{ff}$. 
Tätigkeitsmerkmale:

- Inspiziert man die Antwortverteilungen auf die Frage nach der Häufigkeit bestimmter Tätigkeiten von QB, so ergibt sich ein buntes Bild. Die große Varianz bei relativ geringer Zellenbesetzung der Antwortkategorie "täglich/fast täglich" - bei 28 Items insgesamt nur 34 mal angekreuzt, davon allein 21 mal bei "Mitarbeit/Pflege QMHandbuch" und "Bearbeitung sonstiger qualitätsrelevanter Dokumente" - unterstreicht die Vielfalt der Anforderungen, die sich kaum zu einem charakteristischen Tätigkeitsprofil verdichten lassen, zumal angesichts unterschiedlicher Freistellungen. Viele der vorderen Plätze werden von Aufgaben belegt, die gemeinhin eher als "bürokratisch" betrachtet werden: Bearbeitung von Dokumenten, Mitarbeit am QMHandbuch, Berichterstattung, Sichtung von Fachliteratur.

- Allerdings lassen die Antworten aufgrund der Skalencharakteristik keine Rückschlüsse darauf zu, welcher subjektive Stellenwert und zeitliche Aufwand mit den jeweiligen Tätigkeiten verknüpft ist.

So ist z. B. die Erstellung eines Qualitäts-/Jahresberichts naturgemäß nur einmal im Jahr vonnöten, mag aber mit erheblichem Aufwand und hohem Maß subjektiver Bedeutung und Befriedigung einhergehen. In der Rangordnung der häufigsten Aktivitäten belegt dieses Item gleichwohl nur einen hinteren Platz.

○ Männliche und ältere QB beschäftigen sich signifikant häufiger als Frauen und jüngere QB mit Tätigkeiten rund um das QM-Handbuch. Dieser Effekt geht vorwiegend auf das männliche Konto, da vornehmlich Männer zu den älteren Jahrgängen gehören. Die Annahme einer "maskulinen Präferenz für Arbeiten am Schreibtisch und Teil-Rückzug aus dem operativen Tagesgeschäft" wäre angesichts der kleinen Stichprobe maliziös überzogen; bemerkenswert ist jedoch, dass Männer mehrheitlich (11 von 15) kombinierte Lösungen praktizieren, während Frauen (8 von 15) eine Vollstelle als QB bekleiden. Stellt das Instrument für Männer eher eine Maßnahme des Job Enrichments und für Frauen eher eine Um- und Aufstiegsmöglichkeit dar?

- Die größte Standardabweichung $(\mathrm{SD}=1,815)$ zeigt sich bei der Moderation von/Mitarbeit in internen Qualitätszirkeln, gefolgt von der Mitarbeit am QMHandbuch $(\mathrm{SD}=1,746)$ und der Leitung einer Steuerungsgruppe Qualität $(\mathrm{SD}=$ 
1,738). Diesbezüglich liegt die Annahme nahe, dass nicht in allen Einrichtungen mit QB entsprechende Instrumente oder Gremien etabliert sind. Dadurch fallen die möglichen Antworten zwangsläufig weniger homogen aus als solche auf Tätigkeiten, die quasi ubiquitär sind (z. B. Bearbeitung von Vorschlägen, Bearbeitung von sonstigen Dokumenten, Sichtung Fachliteratur).

○ Neben der Häufigkeit bestimmter Tätigkeiten erscheint die Frage interessant, ob sich charakteristische Zusammenhänge zwischen einzelnen Items herauskristallisieren, die als funktionale Einheiten oder Rollentypen interpretiert werden können. Wie sich faktorenanalytisch zeigen lässt, entsprechen die gefundenen Komponenten recht gut den hypostasierten Mustern von Rollenwahrnehmungen und -erwartungen, die in Kap. D 3.2 beschrieben wurden.

Bis auf relativ schwer operationalisierbare Tätigkeiten, die auf der Ebene von Vertrauensbildung, Motivierung, Betriebsklima und Interessenvertretung angesiedelt sind und sich $\mathrm{zu}$ den Rollentypen Promotor und Delegierte/r verdichten lassen, enthalten die in dieser Studie verwendeten Items zu Tätigkeiten von QB offenbar alle Aspekte, die bei der Konstruktion der übrigen Typen formulierungsleitend waren. Die Typen Controller und Moderator treten allerdings nicht trennscharf in Erscheinung.

Trotzdem kommt der durchgeführten Faktorenanalyse eine konfirmatorische Funktion zu: Die aufgrund bestimmter Vorannahmen und Stellenbeschreibungen konstruierten Typen lassen sich in der untersuchten Stichprobe empirisch recht gut bestätigen. Zusätzlich kristallisiert sich ein spezieller Typus Evaluator heraus, der nicht in den Funktionen Auditor und Controller aufzugehen scheint.

\section{Zweckmäßigkeit des Instruments:}

- Fast alle befragten QB und alle Leitungskräfte, die sich zu dieser Frage äußerten, attestieren dem Instrument, zumindest "eher zweckmäßig" zu sein; signifikant günstiger urteilen dabei die Leitungskräfte. Von ihnen vergeben rund $57 \%$ die Note "sehr zweckmäßig". 
- Im Hinblick darauf, welche Gruppe am meisten von dem Instrument profitiert, sind sich QB und Leitungskräfte weitgehend einig: alle mehr oder weniger. Während allerdings fünf von 31 QB der Meinung sind, es sei die Leitung, die am meisten profitiere, gibt von 14 Leitungskräften niemand diese Antwort. Beide Gruppen benennen darüber hinaus mit jeweils gut 10\% MitarbeiterInnen und KlientInnen als Hauptprofiteure.

○ Einstellungsänderungen in der Beurteilung der Zweckmäßigkeit aufgrund gemachter Erfahrungen zeigen sich bei beiden Gruppen in ähnlicher Richtung. Zwar verteilen sich die Antworten auf alle Skalenstufen, es überwiegt jedoch wachsende Zuversicht.

Konfliktpotenziale und Argumente gegen die Funktionsstelle:

- Drei Items weisen signifikante Bewertungsunterschiede zwischen QB und Leitung im Sinne stärker empfundenen Konfliktpotenzials seitens der QB auf - QB als Formalität, QB als Einzelkämpfer an mehreren Fronten und skeptische/schwer zu motivierende Mitarbeiterschaft. Weitere Items zeigen tendenziell die gleiche Richtung an.

- QB öffentlicher Träger äußern bei sechs von 14 Items signifikant stärkeres Konfliktpotenzial als QB freigemeinnütziger Träger. Ohne die Einzelergebnisse überbewerten zu wollen (geringe Zellenbesetzungen), kann insgesamt ein größeres Konfliktpotenzial der Funktionsstelle bei öffentlichen Trägern konstatiert werden.

- Geringe Freistellung ist häufiger ein Problem in kleinen als in sehr großen Einrichtungen, in denen QB durchweg eine Vollstelle bekleiden und teilweise sogar mehrere Funktionsstellen unterhalten werden.

- Insgesamt muss jedoch betont werden, dass die Akteure - QB selbst und Leitungen, die mit ihnen zusammenarbeiten - das Instrument als längst nicht so problematisch empfinden, wie Skeptiker befürchten (s. Kap. C 2.2.4). Aufschlussreich wäre in diesem Zusammenhang das Votum der betroffenen Belegschaften sowie anderer Kooperationspartner. Insofern verweist die Studie auf Anschluss-Forschungsbedarf. 
- Die Zahl von 107 Nennungen auf die Frage nach Entscheidungsgründen gegen die Funktionsstelle des/der Qualitätsbeauftragten spricht dafür, dass sich zu dieser Frage nicht nur VertreterInnen jener 13 Einrichtungen geäußert haben, die definitiv keine/n QB wollen. Die Antworten sind deshalb auch ein Hinweis darauf, warum Leitungskräfte erst gar nicht initiativ werden, das Thema in ihren Einrichtungen zur Diskussion zu stellen.

Dabei dominiert auf Seiten der kleinen/kleinsten Einrichtungen die Überzeugung, dass die Funktion von der Leitung mit übernommen oder bedarfs- und situationsweise von unterschiedlichen Personen wahrgenommen werden kann. Aber auch der bewusste Verzicht auf die Etablierung einer neuen "Spezial-Disziplin", bedarfsweise Inanspruchnahme von Externen und die Betonung des Querschnittscharakters von Qualitätsmanagement spielen eine bedeutende Rolle, wiederum zumeist von Einrichtungen des ersten Größenquartils geäußert. 


\section{Resümee: Folgerungen für die Praxis}

Hauptanliegen dieser Untersuchung und der darin einbezogenen Ergebnisse aus Vor- und Vergleichsstudien ist die Bestandsaufnahme zur Umsetzung, Ausgestaltung und Nutzenbewertung eines Qualitätsmanagements in sozialen Arbeitsfeldern. Die aktuellen Befunde stützen sich auf differenzierte Angaben aus 85 Organisationen unterschiedlicher Größe, vornehmlich aus den Bereichen öffentliche Jugendhilfeverwaltung und Erziehungshilfeeinrichtungen in freier Trägerschaft. Nur diesbezüglich sollen an dieser Stelle Folgerungen gezogen werden, wenngleich einige Befunde aus zahlenmäßig gering vertretenen Bereichen interessant und möglicherweise auch repräsentativ sind. Sie finden an dieser Stelle jedoch keine Erwähnung mehr.

Von unmittelbarer Bedeutung für die Praxis erscheinen folgende Befunde bzw. abgeleitete Schlussfolgerungen:

○ Qualitätsmanagement trifft als Fach- und Fortbildungsthema vorwiegend auf offene Ohren, jedoch in stärkerem Maße bei Leitungskräften als bei MitarbeiterInnen der Basis, deren Bereitschaft zur aktiven Funktionsübernahme denn auch begrenzt ist. Die naive, wenngleich gut gemeinte Absicht, QM zu propagieren, indem die Belegschaft kollektiv zu "Qualitäts-Managern" ernannt wird, verändert nicht grundlegende Aufgaben und Zuständigkeiten: Qualitätsmanagement als systematische Kontextsteuerung ist ungeachtet der Notwendigkeit, die entscheidenden Botschaften und Methoden mittels geeignetem Informations- und Beteiligungsmanagement "in die Köpfe" aller Organisationsmitglieder zu befördern, genuine Leitungsaufgabe.

○ Dementsprechend ist auch die im Vergleich zu Leitungskräften größere Zurückhaltung der MitarbeiterInnen $\mathrm{zu}$ bewerten, was die proklamierten Wirkungen eines QM anbetrifft. Wer als PraktikerIn im Feld der Sozialen Arbeit den Wandel von Managementtheorien und fachlichen Konzepten am eigenen Leibe erlebt - StaubBernasconi (1996, zit. nach Spreyermann, 1996, S. 4) spricht von 15 neuen Paradigmen in der deutschsprachigen Sozialarbeit der letzten 40 Jahre -, ist legitimerweise skeptischer als deren professionelle Promotoren und MultiplikatorInnen. Wichtiger als qualitätsrhetorische Euphorie ist deshalb die im Sinne eines kontinuierlichen Optimierungsprozesses vollzogene, zumeist unspektakulär kleinschrittige Umsetzung 
spürbarer Verbesserungen im beruflichen Alltag, die vor allem auch eingebettet sind in eine glaubwürdig vermittelte Einbeziehung der obersten Leitungsebene.

○ Die bemerkenswert homogene Nutzeneinschätzung qualitätsverbessernder Maßnahmen durch soziale Fach- und Leitungskräfte und die Präferenz für eher klassische, fachnahe Formen wie Fallreflexion, Konzeptionsentwicklung, Supervision u. a. m. fügen sich gleichfalls in diese Argumentationsrichtung. Solange solche Formen professioneller Qualifizierung der Strukturen, Prozesse und Akteure nicht integraler Bestandteil einer qualitätsorientierten Praxis sind, kann die relative Abwertung "moderner" Methoden aus dem Arsenal des QM nicht verwundern. Die namentlich in den einbezogenen Vorstudien zum Ausdruck kommende Wertschätzung der Fachkräfte für kollegiale Kooperation ("Ziehen an einem Strang"), für Klarheit über die Kriterien fachlich guter Arbeit und bedarfsgerechte personelle Ausstattung spricht dafür, dass es Qualitätsversprechen weniger "hinter dem nächsten Berg", als vielmehr "vor der eigenen Haustür" einzulösen gilt. Dabei sind diese Kriterien bruchfrei mit zentralen Anliegen eines seriösen Qualitätsmanagements in Einklang zu bringen: Teamgeist zu fördern, Maßstäbe für gute Arbeit zu explizieren, strukturelle Voraussetzungen zu gewährleisten und unsachgerechte Varianz im beruflichen Handeln zu reduzieren.

- Vergleicht man die Befunde zur Nutzenbewertung mit der Umsetzung bestimmter Instrumente des QM, so kommt man nicht umhin, gewisse Unstimmigkeiten festzustellen. Während sich z. B. die Bedeutung von Personalentwicklungsgesprächen sowohl im hohen Anteil geplanter, ansatzweise oder systematisch angewandter Konzepte als auch in hoher Nutzenbewertung ausdrückt, korrespondiert der fast von allen Einrichtungen zumindest geplante Einsatz von Evaluationsverfahren keineswegs mit entsprechender Wertschätzung zuordenbarer Maßnahmen. Befragungen von KlientInnen, KooperationspartnerInnen und MitarbeiterInnen, Selbst- und Fremdevaluationsprojekte tauchen in dieser Reihenfolge erst auf hinteren Rangplätzen auf. Wie schon die zuvor erörterten Befunde spricht auch dies dafür, dass in der Praxis der Sozialen Arbeit manche QM-Aktivität weniger aus Nutzenüberzeugung, denn als Zugeständnis an den managerialistischen Zeitgeist erfolgt. Evaluation als bestes Beispiel hierfür ist "in", ihr professioneller Nutzwert indes erst mangelhaft erwiesen (vgl. Pollitt, 2000, S. 62). 
○ Ähnlich verhält es sich mit dem prominenten Instrument des Qualitätszirkels, in nahezu drei Vierteln aller befragten Einrichtungen praktiziert und von den meisten übrigen geplant. In der Rangreihe der Qualitätsentwicklungsmaßnahmen sogar hinter temporären Projektgruppen platziert (Rang 15 vs. 8, Mittelwerte 2,34 vs. 2,21, nicht signifikant), weist das Instrument keinerlei bedeutsame Korrelation mit anderen QMElementen auf. Lediglich mit dem Grad der Mitarbeiterbeteiligung am QM-Prozess besteht ein knapp signifikanter Zusammenhang (.211), der angesichts der genuinen Funktion von QZ als Plattform qualitätsorientierter Partizipation eher überraschend niedrig ausfällt. Dass Qualitätszirkel eingerichtet sind oder nicht, lässt jedenfalls keinen Rückschluss darauf zu, ob eine Einrichtung über ein systematisches QM verfügt, programmatische Leitlinien ausformuliert hat, weitere Instrumente einsetzt, ihre Mitarbeiter qualifiziert oder dies jeweils unterlässt. Handelt es sich demnach um ein völlig losgelöstes Instrument, das sowohl integraler Bestandteil eines systematischen QM sein kann als auch Alibi dafür, dass weitere Maßnahmen nicht erforderlich seien?

○ Die Funktionsstelle des/der Qualitätsbeauftragten scheint sich zu einem neuen und nachhaltigen Betätigungsfeld für erfahrene MitarbeiterInnen mit Fachhochschul- oder Hochschulabschluss entwickelt zu haben. Namentlich in größeren Einrichtungen freier Träger sind es häufig Kräfte des mittleren Managements (Bereichs-, Haus- und Gruppenleitungen), die auf eine Vollstelle wechseln oder unter Beibehaltung ihrer bisherigen Funktion eine kombinierte Lösung praktizieren.

Die Rollenwahrnehmungen und -erwartungen sind bis auf unterschiedlich pointierte Einzelaspekte recht homogen. Wenngleich RolleninhaberInnen spezifische Konfliktpotenziale stärker zum Ausdruck bringen als die kooperierenden Leitungskräfte, sind sich beide Gruppen in ihrem grundsätzlichen Zweckmäßigkeitsurteil weitgehend einig. Dies spricht dafür, dass die QB-Stelle zumindest aus Sicht der Akteure und Entscheidungsträger funktional ist: Die einen erfreuen sich überwiegend ihres neuen Jobs, die anderen sind froh, bestimmte Aktivitäten gebündelt und delegiert zu haben. Offen bleibt zwar im Rahmen dieser Studie, ob sich die hypostasierte Interessengemeinschaft auch in der Wahrnehmung von MitarbeiterInnen und KundInnen widerspiegelt. Die Stelle als solche scheint jedoch dort, wo sie eingerichtet und nicht von vornherein als unnötig betrachtet wird (vornehmlich in kleinen Organisationen), weniger problematisch zu sein als von Skeptikern befürchtet. 
Immerhin korreliert die Besetzung der Funktionsstelle positiv mit einer Reihe anderer Qualitätsaktivitäten im Feld und indiziert somit eine gewisse Breitenwirkung.

○ Neben dieser "Rehabilitierung" des in der Fachliteratur umstrittenen Instruments QB ist noch ein weiterer Befund $\mathrm{zu}$ vermelden, der die bisweilen polarisierte Debatte zwischen QM-Befürwortern und Skeptikern zu versachlichen geeignet ist - die Rolle der ISO 9000 ff. in Einrichtungen namentlich der Erziehungshilfe. Wiederum unter dem Vorbehalt, dass die hier berücksichtigten Befunde auf Angaben von Qualitätsbeauftragten und Leitungskräften beruhen und Basis-MitarbeiterInnen in der Fachliteratur mitunter eher als "ISO-Geschädigte" zitiert werden (z. B. bei Speck, 2002, S. 11), sammelt die ISO in dieser Studie Punkte vor allem in Sachen Mitarbeiterbeteiligung. Mögliche Gründe dafür wurden erörtert.

In jedem Fall scheint das Urteil gerechtfertigt, dass die ISO sich als Orientierungsrahmen oder Grundlage für ein systematisches QM nicht schlechter eignet als andere Systeme auch und sie nicht ohne Grund von vielen Einrichtungen, namentlich größerer und in freier Trägerschaft befindlicher, als Referenzmodell gewählt wird. Dass in öffentlichen Einrichtungen und Diensten von den prominenten Universalkonzepten das EFQM-System die wesentlich größere Rolle spielt als die ISO, könnte damit zusammenhängen, dass es bereits speziell auf öffentliche Verwaltungen zugeschnittene Selbstbewertungsverfahren auf Basis des EFQM-Modells gibt (z. B. CAF, Saatweber, 2003), während die ISO noch keine diesbezügliche Spezifizierung erfahren hat.

Im Übrigen sei an die primär deskriptive, populationsbeschreibende Funktion der Studie erinnert. Auch ohne unmittelbar praxisrelevante Folgerungen aufzudrängen, dürfte es für die Einrichtungen der Jugend- und Sozialhilfe aufschlussreich sein, sich vergleichend zu positionieren, sei es, um Aktivitäten mit Verweis auf die "Durchschnittskonkurrenz" zu forcieren, sei es, um vermeintlichem Handlungsdruck im Bewusstsein "guter Gesellschaft" begründet zu widerstehen. 


\section{E Zusammenfassung und Ausblick}

\section{Qualitätsmanagement und Organisationsforschung}

\subsection{Vorbemerkungen}

Die in Kap. A 3.2 formulierten Fragestellungen dieser Untersuchung haben - jedenfalls war das die erklärte Absicht - die inhaltliche Gliederung dieser Arbeit und der darin erörterten literaturanalytischen und empirischen Befunde strukturiert. Wenngleich nicht immer explizit auf den Wortlaut dieser Fragestellungen bezogen, stellt die Kapitelabfolge somit den Versuch dar, Zusammenhänge herzustellen und nach Möglichkeit Antworten zu geben. Sie hier zusammenfassen zu wollen, heißt zwangsläufig, komplexe Sachverhalte zu verkürzen und für besonders wesentlich gehaltene Aspekte auszuwählen.

\subsection{Kontext, Grundlagen und thematische Schnittmengen}

Nach einer komprimierten Beschreibung der vor allem ökonomischen und sozialpolitischen Bedingungsfaktoren für die Aktualität und Herkunft des Qualitätsdiskurses im Humandienstleistungsbereich werden genuin fachliche Anknüpfungspunkte der Debatte erörtert.

Bibliometrisch wird belegt, dass im Unterschied zum sozialwissenschaftlichen Bereich die Psychologie sich des Themas "Qualitätsmanagement" bislang kaum angenommen hat. Allerdings zeigt sich, dass auch die sozialwissenschaftliche Befassung mit Qualität und deren Management überwiegend darin besteht, die in anderen - namentlich ingenieur-, arbeits- und wirtschaftswissenschaftlichen - Disziplinen entwickelten Konzepte zu rezipieren und im Kontext veränderter Rahmenbedingungen zu diskutieren.

Ausgehend von einer kurzen Darstellung des bundesdeutschen Systems sozialer Sicherung und der Rolle freier Träger, werden Tendenzen einer Ökonomisierung des Sozialen und Bemühungen um eine Modernisierung der öffentlichen Verwaltungen nachgezeichnet, 
bevor das Qualitätsthema in der Jugend- und Sozialhilfe rechtlich verortet wird. Dem Kinder- und Jugendhilfegesetz (SGB VIII) werden im Vergleich zu anderen Sozialgesetzen die relativ größten Potenziale für eine fachpolitisch offensive Verarbeitung des Qualitätsthemas attestiert, weil nicht das administrativ-kontrollierende Motiv der Prüfung, sondern der Impuls fachlicher Qualitätsentwicklung im Vordergrund steht.

Methodologische Überlegungen zum literaturanalytischen und empirischen Teil der Studie beschließen den ersten Abschnitt.

Ein geschichtlicher Abriss des Qualitätsmanagements und eine Übersicht zu dessen Grundbegriffen, Modellen, Techniken, Kontext- und Implementierungskonzepten bildet anschließend den Ausgangspunkt für eine Darstellung ausgewählter Bereiche, in denen sich Qualitätsmanagement und Organisationsforschung überschneiden bzw. praktisch identische Fragestellungen bearbeiten. Konzepte der traditionell interdisziplinär ausgerichteten nordamerikanischen Organisationsforschung (Change Management, Human Resource Management, Learning Organization, Empowerment), arbeitswissenschaftliche, organisationspsychologische und betriebssoziologische Ansätze (Organisationsdiagnose und -analyse, Organisations- und Personalentwicklung, Mitarbeiterbeteiligung, Evaluation) und betriebswirtschaftlich orientierte Instrumente (Controlling, Balanced Scorecard) werden exemplarisch beschrieben.

$\mathrm{Zu}$ konstatieren ist, dass es der sich unter Anleihen bei verschiedenen Wissenschaftsfeldern relativ wildwüchsig in der industriellen Praxis entwickelnden "Qualitätsbewegung" ebenso an einem einheitlichen konzeptuellen Rahmen ermangelt wie den zumeist in einzelwissenschaftlichen Paradigmen verhafteten Beiträgen der Organisationsforschung. Eine Absorption aller wesentlichen Bestandteile des Qualitätsmanagements durch etablierte akademische Disziplinen bzw. das Aufgehen spezifischer Beiträge in übergreifenden Diskursen wird jedoch für wahrscheinlicher gehalten als die Herausbildung einer "Qualitätswissenschaft". Der gegenwärtige Zustand einer möglichen und sinnvollen Integration wird allerdings als fragmentarisch bezeichnet. 


\section{Qualitätsmanagement in sozialen Handlungsfeldern}

\subsection{Konzepte und Instrumente}

Bezug nehmend auf Schwachstellen eines Qualitäts- und Managementverständnisses technischer Provenienz in sozialen Handlungsfeldern, werden $u$ a. die Kontextgebundenheit und der Konstruktcharakter sozialer Dienstleistungsqualität betont. Dieser Besonderheiten wegen wird kein Versuch unternommen, verschiedenste Qualitäts(management)konzepte nach einem einheitlichen Kriterienraster zu beschreiben und zu bewerten. Stattdessen werden die in der Fachliteratur diskutierten Ansätze nur formal systematisiert. Die prominentesten Konzepte mit universellem Anspruch (ISO 9000 ff., TQM, KVP, EFQM, Integriertes QM) werden in Grundzügen dargestellt und kritisch erörtert.

Anschließend werden im Rahmen dieser Konzepte oder unabhängig davon häufig verwendete Instrumente in der Organisationspraxis (Leitbild, Handbuch, Qualitätsbeauftragte, Qualitätszirkel, Benchmarking, Prozess-, Ideen-, Beschwerde- und Wissensmanagement), die fast sämtlich auch im empirischen Teil der Studie eine Rolle spielen, unter Einbezug veröffentlichter Erfahrungsberichte beschrieben. Hingewiesen wird auf die Bedeutung des organisationsstrukturellen und -kulturellen Kontextes, in den die jeweiligen Praktiken einzubetten sind, um nachhaltig positive Effekte zu erzielen.

In einem Resümee werden Essentials eines sozialwirtschaftlichen Qualitätsmanagements diskutiert. Bemühungen um perfektionierte Strukturen der Verhaltenskontrolle werden für dysfunktional gehalten. Wesentliche Elemente werden in der Betonung des Verantwortungsaspekts und im Empowerment der MitarbeiterInnen gesehen.

\subsection{Empirische Befunde zum QM in der Jugend- und Sozialhilfe}

\subsubsection{Einstellung zum Qualitätsmanagement}

Befragungen von MitarbeiterInnen und Leitungskräften zeigen, dass das in seiner wesentlichen Dynamik und Begrifflichkeit aus dem Wirtschaftsbereich in soziale 
Handlungsfelder exportierte Thema "Qualitätsmanagement" in der sozialen Fachpraxis auf positive Resonanz gestoßen ist. Besonders auf Leitungsebene dominiert eine wohlwollende und aufgeschlossene Haltung, gepaart mit hoher Bereitschaft, sich fortzubilden und einschlägige Funktionen zu übernehmen.

Auch unter den MitarbeiterInnen findet sich mehrheitlich eine positive Einstellung, die sich jedoch eher allgemein in Zustimmung $\mathrm{zu}$ entsprechenden Aktivitäten der Einrichtungen artikuliert und mit relativer Zurückhaltung einhergeht, sich selbst zu exponieren. Auch zeigt sich eine bedeutende Gruppe von eher skeptisch Abwartenden und Unentschiedenen, was die erhofften Effekte von Qualitätsmanagement anbelangt. Für eine Polarisierung der Belegschaften im Sinne von Befürwortern und Ablehnern gibt es jedoch keine Anhaltspunkte.

Belege für eine Einstellungsänderung im Verlauf der Qualitätsdiskussion - bezogen auf den Zeitraum 1997 bis 2003 - fanden sich nicht, wohl aber für die Bedeutung einrichtungsspezifischer Kontexte und Strategien, welche den jeweiligen Umgang mit dem Thema moderieren (Ausgangssituation, Präferenzen und Rolle der Leitung, Informationsmanagement, Resonanz in der Belegschaft).

\subsubsection{Ausgestaltung des Qualitätsmanagements}

Seit dem bisherigen Höhepunkt der fachpublizistischen Auseinandersetzung mit dem Thema in 1998 (vgl. Abb. 1) ist Qualitätsmanagement in steigendem Maße Sache der PraktikerInnen in Einrichtungen und Diensten geworden und steht in 2004 bei fast $90 \%$ der befragten Organisationen auf der Agenda; in systematisierter Form wird es jedoch erst von gut einem Drittel praktiziert. Allerdings sind die Unterschiede zwischen den Arbeitsfeldern, Trägergruppen und Einrichtungsgrößen erheblich: Die maßgeblich im Rücklauf vertretenen freien Erziehungshilfeeinrichtungen wenden mit zunehmender Größe fast alle ein QM-System an oder arbeiten daran; von den öffentlichen Jugend- und Sozialhilfeverwaltungen ist jede zweite diesbezüglich inaktiv oder erst im Planungsstadium.

Was die konzeptionelle Orientierung QM-bezogener Aktivitäten anbelangt, sind organisationsspezifische Eigenentwicklungen und Mischformen, ISO 9000 ff. und das 
EFQM-Excellence-Modell in fast 90\% der Fälle das System der Wahl. Während sich öffentliche Träger vornehmlich an EFQM oder Eigenentwicklungen ausrichten, stellt die ISO 9000 ff. nach organisationsspezifischen Ansätzen das bevorzugte System freier Träger dar, zumal der sehr großen Einrichtungen.

Eine umfassende oder punktuelle Beteiligung der gesamten Belegschaft am QM-Prozess wird von knapp zwei Dritteln der befragten Einrichtungen angegeben, während etwa ein Fünftel QM bislang allein zur Sache der Leitung oder einzelner MitarbeiterInnen gemacht hat. In rund $18 \%$ der Fälle ist Fehlanzeige $\mathrm{zu}$ vermelden. Freigemeinnützige und insbesondere größere Einrichtungen weisen den relativ höchsten, öffentliche und kleine privatgewerbliche Organisationen den relativ geringsten Beteiligungsgrad auf.

Gleichlautende Befunde zeigen sich bezüglich der Qualifizierung der Mitarbeiterschaft, bei der im Übrigen spezifische Fortbildungen für Qualitätsbeauftragte, Leitungskräfte und MultiplikatorInnen, Einzelangebote ohne systematischen Zusammenhang und obligatorische Basisveranstaltungen dominieren.

Relativ deutlich ist ein Zusammenhang zwischen Beteiligungsgrad und Wahl eines bestimmten QM-Referenzkonzepts. Nicht das explizit als TQM-System ausgewiesene EFQM Excellence Modell oder intern entwickelte Ansätze, sondern die ISO dominiert in jenen Einrichtungen, die den höchsten Beteiligungsgrad angeben. Ungeachtet der als möglich erörterten Gründe (s. Kap. D 4.8.2) spricht dies dafür, dass die bei Fachkräften der Sozialen Arbeit bisweilen auf starke Vorbehalte stoßende ISO 9000 ff. bei verständiger Anwendung ebenso gut für soziale Handlungsfelder geeignet ist wie andere Systeme auch.

In der Anwendung bestimmter Instrumente und Organisationsformen des Qualitätsmanagements zeigen sich unterschiedliche Entwicklungen in den einbezogenen Studien seit 1998. Während die Systematisierung des Qualitätsmanagements im Allgemeinen und der Einsatz von Qualitätszirkeln, Evaluationsverfahren, Handbüchern, Organigrammen und Leitbildern im Besonderen deutliche Wachstumsraten verzeichnen, hat sich der jeweilige Anteil der Jugendhilfe-Einrichtungen, die sich zertifizieren lassen wollen (etwa jede zehnte) oder eine/n Qualitätsbeauftragte/n ernennen wollen (etwa jede zweite), seither kaum verändert. Für Wohneinrichtungen der Behindertenhilfe werden höhere Anteile berichtet: rund 20\% bzw. 60\% (Wetzler, 2003). 


\subsubsection{Nutzenbewertung von Maßnahmen zur Qualitätsentwicklung}

In der Einschätzung des praktischen Nutzens, der sich aus der Anwendung bestimmter Maßnahmen und Vorkehrungen zur Qualifizierung der Leistungsprozesse und Akteure ergibt bzw. ergeben kann, sind sich soziale Fachkräfte bemerkenswert einig. Zwar lassen sich unter der Vielzahl thematisierter Instrumente einige finden, die von Angehörigen verschiedener Arbeitsfelder oder Organisationsgrößen different beurteilt werden - zumeist mit erschließbarer Plausibilität. Die relativ größten Differenzen zeigen sich dabei zwischen den Arbeitsfeldern Schule/Berufsbildungswerk und Sozialamt, die allerdings beide nur mit kleinen Anteilen in der Stichprobe vertreten sind.

Insgesamt ergibt sich eine Präferenz für fachnahe Formen der methodischen Qualifizierung "on the job": interne Fallreflexion, Konzeptionsentwicklung, außerbetriebliche Fortbildung, kollegiale Beratung. Hinzu kommen Maßnahmen zur Verbesserung der internen und externen Kooperation sowie - quasi als strukturqualitative Voraussetzung eine bedarfsgerechte personelle Ausstattung. Den mehr dem Jargon und Arsenal des Qualitätsmanagements zuzurechnenden Methoden Selbst- und Fremdevaluation, interne und externe Audits wird deutlich geringere Wertschätzung entgegengebracht.

Analog zur relativ stärkeren Umsetzung systematischen Qualitätsmanagements in Einrichtungen freier Träger ist hier eine allgemein günstigere Nutzeneinschätzung der abgefragten Maßnahmen und Vorkehrungen anzutreffen als bei öffentlichen Trägern. Wenngleich ein Teil der Varianz zu Lasten der sich besonders skeptisch äußernden Sozialämter geht, drückt sich hierin - bei weitgehend ähnlicher Bewertungsrichtung größere Zurückhaltung in Jugend- und Sozialhilfeverwaltungen aus, was die Optimierungsressourcen der praktischen Arbeit durch Maßnahmen des Qualitätsmanagements anbelangt.

\subsubsection{Einsatz von Qualitätsbeauftragten}

Etwa ein Drittel der befragten Einrichtungen und Dienste verfügt über die Funktionsstelle eines/r Qualitätsbeauftragten. Zusammenhänge mit den Faktoren Arbeitsfeld/Trägerschaft und Einrichtungsgröße sind dabei deutlich: Öffentliche Träger und kleine Einrichtungen 
machen viel seltener von dem Instrument Gebrauch. Argumentativ überwiegen weniger Zweifel an der grundsätzlichen Zweckmäßigkeit, als vielmehr Präferenzen für "unspezialistische" Lösungen. Die Interkorrelationen zwischen Beteiligungs- und Qualifizierungsgrad der Mitarbeiterschaft und Institutionalisierung des Instruments QB sprechen jedoch dafür, dass Befürchtungen, das Thema QM werde in der Praxis auf Spezialisten abgewälzt, nicht begründet sind.

Der Modaltypus von QB ist unabhängig vom Geschlecht seit 2001/2002 für die Gesamteinrichtung zuständig, von anderen Verpflichtungen freigestellt, durch interne Ressourcenumverteilung finanziert, unbefristet durch Leitungsbeschluss ernannt, verfügt über explizit geklärte Beratungs- und/oder Umsetzungskompetenzen, ist mittleren, sofern männlich auch höheren Alters, FachhochschulabsolventIn, kommt aus dem mittleren Management, hat eine QM-Ausbildung mit dem Schwerpunkt ISO 9000 ff. abgeschlossen und ist vor allem mit Dokumentations-, Projekt-, Informations- und Beratungstätigkeiten beschäftigt.

Qualitätsbeauftragte und mehr noch Einrichtungsleitungen attestieren der Funktionsstelle hohe Zweckmäßigkeit. Als Profiteure werden mehr oder weniger alle Organisationsmitglieder und KundInnen betrachtet. Rollenerwartungen und wahrnehmungen sind punktuell different, stimmen aber insgesamt recht gut überein. Qualitätsbeauftragte selber nehmen jedoch in stärkerem Maße Konfliktpotenziale in der Praxis wahr als Einrichtungsleitungen.

\section{Ausblick: Zur Zukunft des Qualitätsmanagements}

Bezogen auf US-amerikanische Verhältnisse, spricht DuBrin (2002, S. 296) vom möglichen Ende der "Qualitätsbewegung", ohne allerdings die Bedeutung von Qualität an sich zu relativieren:

"Although the term TQM has fallen into almost disuse, and many people consider the quality movement to have passed, the quest for high quality remains an important thrust." 
Auch Hackman \& Wageman (2000, S. 44f) entwerfen eine eher düstere Perspektive des TQM-Ansatzes, ungeachtet der Nützlichkeit vieler seiner Ideen. Die Autoren sehen drei Trends, die gleichermaßen dazu beitragen, dass TQM wahrscheinlich an Prominenz und Popularität verlieren werde:

Trend 1: Rhetorik obsiegt über Substanz; die TQM-Rhetorik erweist sich als konsistent mit dem managerialistischen Zeitgeist und der Vorliebe für Organisationslösungen, die den Beigeschmack der Rationalität besitzen. Das, was viele Organisationen aktuell implementieren, sei jedoch nicht mehr als eine blasse oder in hohem Maße verdrehte Version der von den Begründern des TQM formulierten Prinzipien.

Trend 2: Verlust der konzeptionellen Spezifität. TQM verliert als populäres und allseits akzeptiertes Programm sein unterscheidbares Profil, weil nahezu alle Interventionen zur Organisationsentwicklung unter dem TQM-Banner subsumiert werden.

Trend 3: Forschung erfüllt nicht die notwendige Korrekturfunktion für TQM. Die überwiegend anekdotischen und simplifizierenden Evaluationsstudien in der Fachliteratur seien eher dazu geeignet, TQM politisch zu promoten oder in Frage zu stellen, als Wissen über die Mechanismen und Effekte von TQM-Praktiken zu begründen.

Ist die Qualitätsbewegung - hierzulande (noch) nicht so bezeichnet, aber mit der allfälligen TQM-Rhetorik weitgehend identisch - auch in Deutschland vor ihrem Ende? Und wenn ja, ist dies ihrem überschätzten Problemlösepotenzial, der Ablösung durch eine neue, noch attraktivere Management-Mode oder dem Aufgehen in einer übergreifenden Organisationstheorie zuzurechnen?

Für alle Annahmen finden sich Indizien.

Zunächst einmal scheint sich die nüchterne Auffassung durchzusetzen, dass QM kein Allheilmittel darstellt, das nach der Devise "viel hilft viel" für alle Wechselfälle organisationalen Lebens geeignet ist.

"Im Verlauf der Qualitätsdebatte in der Sozialen Arbeit haben sich die Meinungen und Einstellungen zu diesem Thema verändert. Mit wachsender Erfahrung sind sowohl überhöhte Erwartungen als auch dramatisierende Befürchtungen vor diesem ungewohnten Arbeitsfeld in realitätsgerechte Bahnen geraten" (Meinhold \& Matul, 2003, S. 11). 
Der sich in Japan abzeichnende Wandel der vorherrschenden Management-Philosophie in Richtung eines "environmentalism" (Hamada, 2000, s. Kap. B 2.3.2), für den die wachsende Bedeutung biologischer statt ingenieurwissenschaftlicher Analogien kennzeichnend ist, wird vermutlich auch in den USA und Europa Richtung bestimmend werden. Die konstruktivistische Kritik am traditionellen Qualitätsdiskurs, popularisierte Konzepte der lernenden und "empowernden" Organisation und ein potenziell wieder erstarkendes Selbstbewusstsein der sozialen Berufe, die den unter der Formel "Managerialismus versus Professionalismus" ausgetragenen Richtungsstreit als Entwicklungsanreiz für die eigene Professionalisierung nutzen können (vgl. Sommerfeld \& Haller, 2003, S. 87), deuten auf ein Ende des "Faszinosums Qualitätsmanagement".

Hierfür spricht auch die gebetsmühlenartig wiederholte Betonung, dass "quality improvement"-Maßnahmen in eine allgemeine Kultur der Offenheit usw. "eingebettet" sein müssten, um ihre volle und nachhaltige Wirksamkeit entfalten zu können. Diese rituell anmutende Beschwörung des organisationskulturellen Kontextes nimmt den meisten Instrumenten des Qualitätsmanagements ihre zuvor errichtete rationale Aura, wenn man die Auffassung teilt, dass Organisationskulturen "nicht konstruierbar", also planmäßig herstellbar seien. Damit nähern sich die Organisationsdiagnostiker und -analytiker, auch ohne dezidiert konstruktivistische Positionen einzunehmen, den postmodernen Kritikern des Qualitätsdiskurses erheblich an. Der alte und unausrottbare technokratische Traum von der Beherrschbarkeit aller Leistungsprozesse stößt offenbar wieder einmal hart an die Grenzen der Realität.

Kühl (2000, S. 60) weist am Beispiel des Konzepts der Lernenden Organisation nach, dass an die Stelle dieses Traums von der rationalen Organisation zunehmend die einleuchtende, aber nicht weniger ignorante Vorstellung eines rational steuerbaren Organisationswandels getreten sei. Der Autor kondensiert aus der Fachliteratur zum geplanten Organisationswandel sieben Prinzipien, die den in Veränderung befindlichen Organisationen eine gewisse Stabilität bieten sollen ( $S$. 66 ff.):

1. klare Zielsetzungen.

2. Identifikation der Mitarbeiter mit dem Veränderungsprozess.

3. Mitarbeiter als zentrale Ressource.

4. Kommunikation und Überzeugungsarbeit.

5. Selbstorganisation (statt Reagieren nach Vorschrift).

6. Ressourcen für Veränderungen.

7. Wandel als permanenter Prozess (Lernen).

Kühl beschreibt sowohl die Zweckmäßigkeit dieser Prinzipien als auch ihre jeweiligen Schattenseiten, z. B. die innovationshemmende Funktion zu enger Zielpräzisierungen oder zu stark produktbezogener Identifika- 
tion, und betont den dilemmatischen Charakter entsprechender Strategien. Am Beispiel von selbstorganisierten Arbeitsgruppen, die genau jene Strukturen und Hierarchien reproduzierten, die vormals fremdgesteuert waren, wird ein weiteres Dilemma illustriert. Auch das Lean Management, das in Zeiten stabiler Umweltbedingungen (Märkte) große Erfolge erzielt habe, sei problematisch, weil durch Abschmelzen der "Fettpolster" (Puffer) Reserven verloren gingen, die für Innovation genutzt werden und das System weniger störanfällig machen können.

Der bereits mehrfach angesprochene Disput zwischen eher "universalistisch" argumentierenden Qualitätsmanagern, welche mit zeitgeistig aufgeladenen Konzepten globale Unternehmensfragen zu beantworten suchen und eher "adaptationistischen" Organisationstheoretikern, die auf Kontexte und Kontingenzen verweisen, ist allerdings keine Erkenntnis, die sozialwissenschaftlichen Kritikern moderner Management-Rezepte vorbehalten blieb. So wird z. B. in einem überwiegend von Ingenieurwissenschaftlern verfassten Buch zum Qualitätsmanagement (Eversheim,1997, S. 185) betont, dass in Unternehmen, für die eine konsequente Kundenorientierung mit einer entsprechenden Dienstleistungsmentalität aller MitarbeiterInnen elementare Existenzvoraussetzung und nicht der Lösungsweg ist, Qualitätsmanagement nach industriellem Vorbild eher hemmende als fördernde Wirkung habe und je nach Branche sehr unterschiedliche Zielausrichtungen vorhanden seien.

Während im Bereich öffentlicher Organisationen (Bundes- und Landesbehörden, Stadtverwaltungen, kommunale Einrichtungen etc.) unter Qualitätsmanagement in erster Linie eine stärkere Bürgerorientierung durch neu gestaltete Dienstleistungsstrukturen und Organisationsabläufe verstanden werde, stehe bei technischen Dienstleistern (Kundendienst, Ersatzteilhandel, Handwerk etc.) der Aufbau von Kundenproblemlösungsstrategien durch die Kopplung von Produkt- und Servicequalität im Vordergrund. Bei Dienstleistungsverbünden (Güterverkehrszentren, Systemdienstleister, Dienstleistungszentren etc.) gehe es um ein übergeordnetes gemeinsames QM-System durch die Vernetzung von speziellen Managementfunktionen rechtlich selbständiger, einzelner Organisationen (a.a.O.).

Diese Aufzählung kann exemplarisch ergänzt werden: Dezentralisierte soziale Dienstleister (Heimverbünde, Bildungsträger, karitative Dienste etc.), zumal wenn sie auf gemeinsamer normativer Basis agieren (wollen), versprechen sich eine Verringerung von Zentrifugalkräften und internen Reibungsverlusten sowie eine professionellere Nutzerorientierung. Nicht oder gering formalisierte soziale Dienstleistungsorganisationen (Projekte, Selbsthilfegruppen, Kleinsteinrichtungen etc.) erhoffen sich eine transparente Beschreibung ihrer Arbeitsprozesse und Gewinne aus maßvoller Strukturierung ihres oft urwüchsig chaotischen Handlungsfeldes. 
So different somit die Erwartungen an Qualitätsmanagement sind, so unterschiedlich ist auch das Ausmaß dessen, was sich eine Organisation an QM zumuten will und sollte. QM ist kein distinktes Phänomen, sondern eher ein Konglomerat aus selbst entwickelten oder sich einverleibten Konzepten, Praktiken, Instrumenten und Techniken. Auch die einschlägigen sozialrechtlichen Vorgaben sind in der Regel durch ein hohes Maß inhaltlicher Unverbindlichkeit charakterisiert, solange nur irgendwo das Label "Qualitätsmanagement" erscheint.

"Generell ist auch noch mal zu bekräftigen, dass es bei der Qualitätsentwicklung weniger auf geniale Ideen ankommt als darauf, einfache und wichtige Dinge mit einer gewissen Hartnäckigkeit immer wieder gut zu machen und dem natürlichen Wegdriften jeder Organisation von Zielnormen hartnäckig und langfristig entgegenzusteuern" (Lambach, 2004, S. 8).

Vieles spricht dafür, dass sich die Befassung mit solchen "ewigen" Fragen der Organisationspraxis, wie sie in Kap. C 3. sinngemäß ähnlich formuliert worden sind, in absehbarer Zeit nicht mehr, zumindest nicht mehr so dezidiert, unter dem Etikett "QM" abspielt und auch der Qualitätsbegriff selbst an Aufmerksamkeitswert verlieren wird. Schon berichtet Weick (2000, S. 171), es sei in Gesprächen über Qualität nicht ungewöhnlich, Leute sagen zu hören: "Oh, we are already doing that, we just don't call it quality."

In diesem Sinne sollten auch die Erörterungen, Bewertungen und Befunddarstellungen in dieser Arbeit betrachtet werden - nicht als Plädoyer für oder wider Qualitätsmanagement, sondern als Beschreibung einer vorgefundenen Lage, in der sich Organisationen in unterschiedlichem Ausmaß aus einem umfangreichen Fundus verfügbarer konzeptioneller und methodischer Hilfsmittel bedienen (können), um gute Arbeit und gute Dienste zu leisten.

Ob im Zuge der Qualitätsdebatte die "eher als behäbig, etwas altlinks und schmuddlig bekannte Dame Soziale Arbeit" (B. Müller, 2000, S. 135) tatsächlich "an Tempo gewinnt" oder sich lediglich "ein völlig neues Outfit zulegt", um sich zu ändern, "damit sich möglichst wenig ändert" (a.a.O.), ist damit noch nicht gewährleistet und bleibt als Thema auf der Diskurs-Agenda. 


\section{F Quellenverzeichnis}

1. Aamodt, M. G. (1996): Applied Industrial/Organizational Psychology. 2. Aufl., Brooks/Cole, Pacific Grove

2. Achberger, C., Wittig-Koppe, H. (1998): Projekt "Kompaß". Der Leitbildprozeß im Paritätischen. In: Der Paritätische Wohlfahrtsverband/Landesverband Schleswig-Holstein: Qualitätssicherung - aber klar!? Workshop-Unterlagen der Tagung vom 16./17.6.1998 in Schleswig-Pulverholz

3. Ackermann, S. (2003): Qualität entwickeln - in kleinen Einrichtungen. Das GAB-Verfahren. Grundmodell des Qualitätsentwicklungsprozesses. Manuskriptdruck, München

4. Alber, J. (1998): Der deutsche Sozialstaat im Lichte international vergleichender Daten. In: Leviathan, 26 (2)

5. Amann, R., Straus, F. (2000): Teamkonferenz als Schlüsselprozess. In: Schröer u. a. (Hrsg.): Qualitätsmanagement in der Praxis. Lambertus, Freiburg, S. 135-147

6. Amt für Soziale Dienste der Landeshauptstadt Kiel (2001): Ziele, Strukturen, Standards. Manuskriptdruck, Stand 04.12.2001

7. Antoni, C. H. (1999): Konzepte der Mitarbeiterbeteiligung: Delegation und Partizipation. In: Hoyos, C. G., Frey, D. (Hrsg.): Arbeits- und Organisationspsychologie. PVU, Weinheim, S. 569-583

8. Argyris, C. (1992): On organizational learning. Blackwell, Cambridge/MA

9. Argyris, C. (1998): Empowerment - nur eine Illusion? In: Harvard Business Manager 6, S. 9-16. [Orig. erschienen 1998: The emperor's new clothes. In: Harvard Business Review 76, S. 98-105]

10. Argyris, C., Schön, D. A. (1978): Organizational learning: A theory of action perspective. Addison Wesley, Reading/MA

11. Arnold, U. (1998): Besonderheiten der Dienstleistungsproduktion. In: Arnold, U., Maelicke, B. (Hrsg.): Lehrbuch der Sozialwirtschaft. Nomos, Baden-Baden, S. 257-276

12. Arnold, U., Maelicke, B. (Hrsg.) (1998): Lehrbuch der Sozialwirtschaft. Nomos, Baden-Baden, S. $257-$ 276

13. Backhaus-Maul, H. (1998): Sozialpolitische Entwicklungslinien in Deutschland. In: Arnold, U., Maelicke, B. (Hrsg.): Lehrbuch der Sozialwirtschaft. Nomos, Baden-Baden, S. 25- 45

14. Badelt, C. (Hrsg.) (1997): Handbuch der Nonprofit Organisation. Strukturen und Management. SchäfferPoeschel, Stuttgart

15. Bauer, C. (1996): Qualitätsmanagement. In: AFET (Hrsg.): Organisations- und Personalentwicklung für Träger der öffentlichen und freien Jugendhilfe. Sonderveröffentlichung 1, S. 15-33

16. Bergbauer, A. K. (1998): Unternehmensqualität ist messbar. QZ 43, S. 689-692

17. Berger, G. (1998): Die SIESTA-Qualitätsdiagnose als erster Schritt einer Qualitätsentwicklung über das bereits Erreichte hinaus. In: Blonski, H. (Hrsg.): Qualitätsmanagement in der Altenpflege. Kunz, Hagen, S. 47-93

18. Bergius, R. (1998): Konstruktionismus, sozialer. In: Häcker, H., Stapf, K. H. (Hrsg.): Dorsch Psychologisches Wörterbuch. 13., überarb. u. erw. Aufl., Huber, Bern et al., S. 456

19. Bergius, R. (1998a): Kontingenz-Modell der Gruppeneffektivität. In: Häcker, H., Stapf, K. H. (Hrsg.): Dorsch Psychologisches Wörterbuch. 13., überarb. u. erw. Aufl., Huber, Bern et al., S. 458

20. Bergius, R. (1998b): Symbolischer Interaktionismus. In: Häcker, H., Stapf, K. H. (Hrsg.): Dorsch Psychologisches Wörterbuch. 13., überarb. u. erw. Aufl., Huber, Bern et al., S. $851 \mathrm{f}$

21. Berner, F., Leisering, L. (2003): Sozialreform "von unten" - Neue Wissenssysteme in der kommunalen Sozialhilfeverwaltung - Ergebnisse einer bundesweiten Erhebung. In: Nachrichtendienst des Deutschen Vereins, 5, S. 186-193

22. BETTAL Quality Consultancy, Fraunhofer-Institut für Arbeitswirtschaft und Organisation IAO, Gemeinschaftsprojekt - Handbuch zur Qualitätssicherung", Pilota - Mobiles Fortbildungsteam Altenhilfe (Hrsg.) (1998): Selbstbewertungssystem für stationäre Einrichtungen der Altenhilfe. Stuttgart/Frankfurt

23. Beywl, W. (1999): Praxisnahe Evaluation als Instrument der Qualitätsentwicklung in Einrichtungen und Diensten der Jugendhilfe. In: Evangelischer Erziehungsverband e. V. (Hrsg.): Qualitätsentwicklungsvereinbarung nach $\S 78$ b KJHG. Begründung - Umsetzung - Fortschreibung. EREV-Schriftenreihe 2, S. 134-138

24. Beywl, W., Schepp-Winter, E. (2000): Zielgeführte Evaluation von Programmen. In: Bundesministerium für Familie, Senioren, Frauen und Jugend (Hrsg.): Materialien zur Qualitätssicherung in der Kinder- und Jugendhilfe. Qs 29, Köln

25. Biebricher, M. (2003): Das Konzept Selbstbewertung des Qualitätsmanagements in der Jugendhilfe (SQJ). In: Boeßenecker et al. (Hrsg.): Qualitätskonzepte in der Sozialen Arbeit. Eine Orientierung für Ausbildung, Studium und Praxis. Beltz/Votum, Weinheim, S. 141-151 
26. Bläsing (2001): Total Quality Management (Experten-Interview). In: Kämmer, K., Hennecke, M., Trapp, F., Bruns-Waigand, W. (2001): Qualitätsverfahren im Überblick. Der Weg zum besten System. Vincentz, Hannover, S. 125-126

27. Bleicher, K. (1994): Normatives Management. Politik, Verfassung und Philosophie des Unternehmens. Campus, Frankfurt/New York

28. Bleicher, K. (1996): Das Konzept Integriertes Management. 3. Aufl., Campus, Frankfurt/New York

29. Blickle, G. (2004): Zur Ethik der Arbeit in Organisationen. In: Schuler, H. (Hrsg.): Lehrbuch Organisationspsychologie. 3., vollst. überarb. u. erw. Aufl., Huber, Bern, S. 143-154

30. Bobzien, M., Nüßle, W. Stark, W. (1995): Qualitätsmanagement in gesundheitsbezogenen Einrichtungen und Projekten. Verein zur Förderung der Selbsthilfe und Selbstorganisation, München

31. Bobzien, M., Stark, W., Straus, F. (1996): Qualitätsmanagement. Sandmann, Alling

32. Böckels, L. (2002): Haftungs- und Strafrechtsrisiken kann man durch Qualitätsmanagement minimieren. In: Theorie und Praxis der Sozialen Arbeit 6, S. 452-455

33. Boeßenecker, K.-H. (2001): Kooperation, Konkurrenzdruck und Qualitätssicherung in der kommunalen Sozialpolitik. In: Schädler, J., Schwarte, N., Trube, A. (Hrsg.) (2001): Der Stand der Kunst. Qualitätsmanagement Sozialer Dienste. Votum, Münster, S. 97-111

34. Boeßenecker, K.-H., Vilain, M., Biebricher, M., Buckley, A., Markert, A. (Hrsg.) (2003): Qualitätskonzepte in der Sozialen Arbeit. Eine Orientierung für Ausbildung, Studium und Praxis. Beltz/Votum, Weinheim

35. Bofinger, P. (2003): Grundzüge der Volkswirtschaftslehre. Pearson, München

36. Boxberg, O. (2003): Unternehmergeist gesucht. Die Theorie der lernenden Organisation. In: Diakonie Impulse 4, S. 7-9

37. Brater, M., Maurus, A. (1997): Das GAB-Verfahren zur Qualitätssicherung und Qualitätsentwicklung in pädagogischen und sozialen Einrichtungen. GAB-Gesellschaft für Ausbildungsforschung und Berufsentwicklung GbR, Bodenseestr. 5, 81241 München

38. Bretzke, W.-R. (1995): Zertifizierung von Qualitätsmanagementsystemen in Dienstleistungsunternehmen. In: Bruhn, M., Stauss, B. (Hrsg.): Dienstleistungsqualität. Konzepte - Methoden - Erfahrungen. 2. Aufl., Gabler, Wiesbaden, S. 401-427

39. Bruhn, M. (2003): Qualitätsmanagement für Dienstleistungen - Grundlagen, Konzepte, Methoden. Berlin, 4. Aufl., Springer, Berlin/Heidelberg

40. Bumbacher, U., Kaufmann, G. (1998): Qualität und Qualitätsmanagement. In: Verbands-Management 1, S. $8-17$

41. Bundesverband Evangelische Behindertenhilfe e. V. (BEB), Fachverband Psychiatrie in der Caritas (PiC) (Hrsg.) (1998): Pro Psychiatrie Qualität PPQ - Handbuch zur Qualitätsentwicklung in der Sozialpsychiatrie. Diakonie-Verlag, Reutlingen

42. Bundesverband privater Anbieter sozialer Dienstleistungen (bpa) (1998): Qualität auf dem Prüfstand. In: bpa-Magazin 3, Bonn

43. Bungard, W., Antoni, C. (2004): Gruppenorientierte Interventionstechniken. In: Schuler, H. (2004): Lehrbuch Organisationspsychologie. 3., vollst. überarb. u. erw. Aufl., Huber, Bern et al., S. 439-473

44. Bungard, W., Holling, H., Schultz-Gambard, J. (1996): Methoden der Arbeits- und Organisationspsychologie. Beltz, Weinheim

45. Burdett, J. O. (1994): TQM and Re-Engineering: The battle for the organization of tomorrow. In: The TQM Magazine 6 (2), S. 7-13

46. Burmeister, J. (1997): Benchmarking statt Zertifikate. In: BMFSFJ (Hrsg.): Materialien zur Qualitätssicherung in der Kinder- und Jugendhilfe. Qs 7, S. 26-35

47. Burmeister, J. (1999): Qualitätsmanagement in der Heimerziehung durch den Vergleich mit anderen ein Plädoyer für ein pragmatisches Verfahren. In: Forum Erziehungshilfen 1, S. 47-53

48. Burmeister, J. (2000): Benchmarking - Darstellung des Verfahrens. In: Merchel, J. (Hrsg.): Qualitätsentwicklung in Einrichtungen und Diensten der Erziehungshilfe. IgfH-Eigenverlag, Frankfurt, S. 77-86

49. Burmeister, J., C. Lehnerer (1996): Qualitätsmanagement in der Jugendverbandsarbeit. Qualitätsmanagement am Beispiel der Zentralstelle Jugendhaus Düsseldorf e. V. in Abgrenzung zu ISO 9000 ff. In: BMFSFJ (Hrsg.): Materialien zur Qualitätssicherung in der Kinder- und Jugendhilfe. Qs 4

50. Büssing, A. (2004): Organisationsdiagnose. In: Schuler, H. (Hrsg.): Lehrbuch Organisationspsychologie. 3., vollst. überarb. u. erw. Aufl., Huber, Bern et al., S. 557-599

51. Büssing, A., Glaser, J. (2003): Dienstleistungsqualität und Qualität des Arbeitslebens im Krankenhaus. Hogrefe, Göttingen

52. Calliess, C. (2004): Verfassungsbund oder "Superstaat Europa"? Perspektiven nach der EU-Erweiterung. In: Georgia Augusta, Wissenschaftsmagazin der Georg-August-Universität Göttingen, 3, S. 19-25

53. Cameron, K. S., Barnett, C. K. (2000): Organizational Climate as a Cultural Variable: An Empirical Investigation of Quality Culture, Processes, and Outcomes. In: Cole, R. E., Scott, W. R. (Hrsg.): The 
Quality Movement and Organizational Theory. Sage, Thousand Oaks, S. 271-294

54. Campbell, D. T., Stanley, J. C. (1973): Experimentelle und quasi-experimentelle Anordnungen in der Unterrichtsforschung. In: Ingenkamp, K. (Hrsg.): Handbuch der Unterrichtsforschung. Beltz, Weinheim, S. $99-193$

55. Cascio, W. F. (1992): Managing human resources: Productivity, quality of work life, profits. 3. Aufl., McGrawHill, New York

56. Cassel, M. (2004): Qualitätsmanagement nach ISO 9001:2000. Hanser, München

57. Cole, R. E., Scott, W. R. (Hrsg.) (2000): The Quality Movement and Organizational Theory. Sage, Thousand Oaks

58. Cook, T. D., Campbell, D. T. (1979): Quasi-Experimentation. Design and analysis issues for field settings. Rand McNally, Chicago

59. Crom, S., France, H. (1996): Teamwork brings breakthrough improvements in quality and climate. In: Quality Progress 29 (3), S. 39-42

60. Cummings, T., Blumberg, M. (1987): Advanced manufacturing technology and work design. In: Wall et al. (Hrsg.): The human side of advanced-manufacturing technology. Chichester, Wiley, S. 37-60

61. Czichos, R. (1990): Change Management. Reinhardt, München/Basel

62. Dachler, P. Wilpert, B. (1978): Conceptual dimensions and boundaries of participation in organizations: A critical evaluation. Administrative Science Quarterly, 23, S. 1-39

63. Dahlberg, G., Moss, P., Pence, A. (1999): Beyond Quality in Early Childhood Education and Care: Postmodern Perspectives. Falmer Press, London

64. Daumenlang, K., Palm, W. (1997): Qualitätsmanagement. In: Hauser, A., Neubarth, R., Obermair, W. (Hrsg.): Management-Praxis. Handbuch soziale Dienstleistungen. Luchterhand, Neuwied/Kriftel/ Berlin, S. $355-378$

65. Dean, J. W., Bowen, D. E. (2000): Management Theory and Total Quality: Improving Research and Practice Through Theory Development. In: Cole, R. E., Scott, W. R. (Hrsg.): The Quality Movement and Organizational Theory. Sage, Thousand Oaks, S. 3-21. [Original erschienen in: Academy of Management Review 19(3), 1994]

66. DEGEMED (Hrsg.) (1998): QM-Handbuch für Einrichtungen der Rehabilitation. Bonn

67. Deming. W. E. (1986): Out of the Crisis. Center of Advanced Engineering Study, MIT, Cambridge

68. Depner, R., Trube, A. (2001): Der Wandel der Gesellschaft und die Qualitätsdebatte im Sozialsektor. In: Neue Praxis 3, S. 217-238

69. Der Paritätische Wohlfahrtsverband/Landesverband Hamburg e. V. (1996): Qualitätshandbuch stationäre Altenhilfe. Leistungsstandards und Qualitätsmanagement.

70. Der Paritätische Wohlfahrtsverband/Landesverband Hamburg e. V. (o. J.): Qualitätsoffensive in Altenund Pflegeheimen. Gütegemeinschaft Pflege

71. Dettling, W. (2000): Der Wohlfahrtsstaat am Ende? Die Bürgergesellschaft als Antwort auf die neue soziale und demokratische Frage. In: Teuber et al. (Hrsg.): Qualität durch Partizipation und Empowerment - Einmischungen in die Qualitätsdebatte. dgvt-Verlag, Tübingen, S. 27-33

72. Deutsche Bundesregierung (2003): Common Assessment Framework (CAF): Verbesserung der Organisation durch Selbstbewertung: Ein Gemeinsames Europäisches Qualitätsbewertungssystem. Berlin

73. Deutscher Bundestag (2001): Gesetz zur Qualitätssicherung und zur Stärkung des Verbraucherschutzes in der Pflege (Pflege-Qualitätssicherungsgesetz - PQsG)

74. Deutsches Institut für Normung (1992): Qualitätsmanagement und Elemente eines Qualitätssicherungssystems. Leitfaden für Dienstleistungen (DIN ISO 9004-2). Beuth, Berlin

75. Deutsches Institut für Normung (1994): Normen zum Qualitätsmanagement und zur Qualitätssicherung/QM-Darlegung DIN EN ISO 9000 ff. Beuth, Berlin

76. Deutsches Institut für Normung (2000): Qualitätsmanagementsysteme - Forderungen. Deutsche Rohübersetzung zu ISO/FDIS 9001:2000-09. Beuth, Berlin

77. Diakonisches Werk Hamburg (2000): Landesrahmenhandbuch Hamburg Diakonie-Siegel Pflege

78. Dietzfelbinger, M., Haid-Loh, A. (Hrsg.) (1998): Qualitätsentwicklung - eine Option für Güte. Qualitätsmanagement in Psychologischen Beratungsstellen evangelischer Träger. Band 1 und 2, ezi-Eigenverlag, Berlin

79. Donabedian, A. (1966): Evaluating the quality of medical care. In: Millbank Memorial Fund Quarterly. No. 2, USA, S. 166-206

80. Doyle, K. (1992): Who’s Killing Total Quality? In: Incentive 166 (8), S. 12-19

81. Drever, J., Fröhlich, W. D. (1968): Wörterbuch zur Psychologie. dtv, München

82. DuBrin, A. J. (2002): Fundamentals of Organizational Behavior. South Western College Publishing, Cincinnati

83. Dudenredaktion (2000). Duden - Das Fremdwörterbuch. 7., neu bearb. u. erw. Aufl., Mannheim 
84. Dumaine, B. (1994): Mr. learning organization. Fortune, October 17, S. 147-157

85. Easton, G. S., Jarrell, S. L. (2000): The Effects of Total Quality Management on Corporate Performance. In: Cole, R. E., Scott, W. R. (Hrsg.) (2000): The Quality Movement and Organizational Theory. Sage, Thousand Oaks, S. 237 ff. [Original erschienen in: Journal of Business, 71 (2), 1998, S. 253-307]

86. EFQM (1998): Die Leistung steigern mit dem EFQM-Modell für Business Excellence. Brüssel

87. EFQM (1998a): Selbstbewertung 1998/Richtlinien für den Öffentlichen Sektor. Brüssel

88. Ehrhart, K. J. (o. J.): Vorwort. In: Ministerium für Wirtschaft und Mittelstand, Technologie und Verkehr des Landes Nordrhein-Westfalen (Hrsg.): Qualitätsmanagement in NRW. Düsseldorf, S. 4-5

89. Eiff, von, W. (2000): Das Elend des Benchmarking. In: Das Krankenhaus 2, S. 94-97

90. Eisenreich, T. (2000): Balanced Scorecard - ein maßgeschneidertes Managementinstrument für die Jugendhilfe. In: Gerull, P. (Hrsg.): Hand- und Werkbuch Soziales Qualitätsmanagement. EREV-Schriftenreihe, Sonderausgabe März, Kap. 4, S. 186-197

91. Emery, C. R., Summers, T. P., Surak, J. G. (1996): The role of organizational climate in the implementation of total quality management. In: Journal of Managerial Issues 8 (4), S. 484-496

92. Engel, M., Flösser, G., Gensink, G. (1996): Qualitätsentwicklung in der Dienstleistungsgesellschaft Perspektiven für die Soziale Arbeit. In: Heiner, M. (Hrsg.): Qualitätsentwicklung durch Evaluation. Lambertus, Freiburg, S. 48-67

93. Engelhardt, H. D. (1999): Wozu nützt Sozialmanagement? In: Gemeinsam leben 1, S. 4-11

94. Erickson, T. J. (1992): Beyond TQM: Creating the High Performance Business. In: Management Review 5, S. 58-60

95. Etzioni, A. (1994): Jenseits des Egoismusprinzips. Stuttgart

96. Eversheim, W. (Hrsg.) (1997): Qualitätsmanagement für Dienstleister. Grundlagen - Selbstanalyse Umsetzungshilfen. Springer, Berlin/Heidelberg

97. Ewers, M., Schaeffer, D. (1998): Qualitätsentwicklung in der Pflege. Entwicklung und Stand der bundesdeutschen Diskussion sowie künftig zu bearbeitende Herausforderungen. In: Diakonisches Werk der Ev. Kirche von Westfalen (Hrsg.): Qualitätsentwicklung in der Pflege und Qualitätssicherung nach $§ 80$ SGB XI. Aktueller Stand und Perspektiven. Friesenring 32/34, 48147 Münster, S. 5-21

98. Fiedler, F. E. (1964): A contingency model of leadership effectiveness. In: Berkowitz , L. (Hrsg.): Advances in experimental social psychology, Bd. 1, Academic Press, New York

99. Fiene, M., Kirchner, H., Ollenschläger, G. (2001): Aktuelle Diskussionen zur Realisierung von Qualitätsmanagement im deutschen Gesundheitswesen. In: psychomed 13 (3), S. 132-137

100.Flösser, G. (2000): EFQM-Modell der European Foundation for Quality Management. Darstellung des Verfahrens. In: Merchel, J. (Hrsg.): Qualitätsentwicklung in Einrichtungen und Diensten der Erziehungshilfe. IgfH-Eigenverlag, Frankfurt, S. 161-168

101.Flösser, G., Otto, H.-U. (Hrsg.) (1992): Sozialmanagement oder Management des Sozialen. Böllert KT, Bielefeld

102.Foucault, M. (1976): Überwachen und Strafen. Frankfurt

103.Fourali, C. (1999): Quality assurance in psychotherapy and counselling. In: Intern. Journal of Psychotherapy 4 (2), S. 161-177

104.Freigang, W.: Benchmarking: Kommentierung und Bewertung des Verfahrens für die Erziehungshilfe. In: Merchel, J. (Hrsg.): Qualitätsentwicklung in Einrichtungen und Diensten der Erziehungshilfe. IgfHEigenverlag, Frankfurt, S. 97-109

105.Frerichs, F., Leichsenring, K., Naegele, G., Reichert, M., Stadler-Vida, M. (2003): Qualität Sozialer Dienste in Deutschland und Österreich. LIT Verlag, Münster

106.Frey, D., Brodbeck, F. C., Schulz-Hardt, S. (1999): Ideenfindung und Innovation. In: Hoyos, C. G., Frey, D. (Hrsg.): Arbeits- und Organisationspsychologie. PVU, Weinheim, S. 122-136

107.Frey, D., Schulz-Hardt, S. (Hrsg.) (2000): Vom Vorschlagswesen zum Ideenmanagement. Hogrefe, Göttingen

108.Frey, K. (1997): Qualitätsmanagement-Systeme im Vergleich. Die Qualitätsnorm ISO 9000 ff.. In: BMFSFJ (Hrsg.): Materialien zur Qualitätssicherung in der Kinder- und Jugendhilfe. Qs 9, S. 16-20

109.Frey, K. (1997a): Qualitätsmanagement-Systeme im Vergleich. Empfehlung für die Jugend- und Erwachsenenbildung. In: BMFSFJ (Hrsg.): Materialien zur Qualitätssicherung in der Kinder- und Jugendhilfe. Qs 9, S. 31-36

110.Friedag, H. R.: Mit der Zeit gehen ... Ein neuer Ansatz für die betriebliche Praxis: Die Balanced Scorecard. http://home.t-online.de/home

111.Frieling, E., Sonntag, K. (1999): Lehrbuch Arbeitspsychologie. 2., vollst. überarb. u. erw. Aufl., Huber, Bern

112.Fuchsberg, G. (1992): Total Quality is Termed Only Partial Success. In: The Wall Street Journal (October 1), B1

113.Fukuyama, F. (1995): Konfuzius und Marktwirtschaft. Der Konflikt der Kulturen. München 
114.GAB - Gesellschaft für Ausbildungsforschung und Berufsentwicklung (2003): Qualität entwickeln - in kleinen Einrichtungen. Leitfaden, Kap. 10 "Der Qualitätsordner"

115.Garvin, D. A. (1984): What does "product quality" really mean? In: Sloan Management Review 1, S. 2543

116.Garvin, D. A. (1986): Quality problems, policies, and attitudes in the United States and Japan: An exploratory study. In: Academy of Management Journal 29, S. 653-673

117.Gebert, D. (2004): Organisationsentwicklung. In: Schuler, H. (Hrsg.): Lehrbuch Organisationspsychologie, Huber, Bern, S. 601-616

118.Gebert, D., Rosenstiel, L., v. (2002): Organisationspsychologie. 5., aktual. u. erw. Aufl., Kohlhammer, Stuttgart

119.Geiger, W. (1998): Qualitätslehre. Einführung, Systematik, Terminologie. 3. Aufl., Vieweg, Braunschweig/Wiesbaden

120.Gerull, P. (1997): Qualitätsmanagement in Einrichtungen der Erziehungshilfe. Einführung und praktische Anregungen für Fach- und Führungskräfte der Kinder- und Jugendhilfe. EREV-Schriftenreihe 3

121.Gerull, P. (1998): Qualitätsmanagement in der Praxis der Erziehungshilfe. Auswertungsbericht über die Fragebogenaktion zur Qualitätsentwicklung in den EREV-Mitgliedseinrichtungen (August 1998). In: Evangelische Jugendhilfe 5, S. 276-284

122.Gerull, P. (1998a): Qualitätsentwicklung in Einrichtungen und Diensten der Erziehungshilfe. In: Evangelische Jugendhilfe 4, S. 237-238

123.Gerull, P. (1999): Qualitätsmanagement in sozialen Handlungsfeldern - Überblick und aktueller Diskussionsstand. In: Gemeinsam leben 1, S. 12-16

124.Gerull, P. (1999a): Ansätze interner und externer Qualitätsbewertung. In: Evangelische Jugendhilfe 4, S. 228-234

125.Gerull, P. (1999b): Zertifizierung von Qualitätsmanagementsystemen in der Kinder- und Jugendhilfe immer noch nicht vom Tisch!? In: Evangelische Jugendhilfe 2, S. 89-95

126.Gerull, P. (2000a): Selbstbewertung des Qualitätsmanagements in der Jugendhilfe (SQ-J). Revisionsfassung SQ-J 1. Eigenverlag, Hessisch Oldendorf

127.Gerull, P. (2000b): Zum praktischen Stellenwert von Managementkonzepten und -instrumenten im Allgemeinen und Qualitätsbewertungsverfahren im Besonderen. In: Evangelische Jugendhilfe 5, S. 262-275

128.Gerull, P. (2001): Qualitätsmanagement light. Beiträge zur ressourcenschonenden Professionalisierung. Votum, Münster

129.Gerull, P. (2004): Qualitätsmanagement sozialer Dienstleistungen. 2. überarb. u. erg. Aufl., CD-ROM, Eigenverlag, Hessisch Oldendorf

130.Gerull, P. (Hrsg.) (2000): Hand- und Werkbuch Soziales Qualitätsmanagement. EREV-Schriftenreihe, Sonderausgabe März, Hannover

131.Giebing, H. (1991): Qualitätssicherung in den Niederlanden: eine Philosophie für die Praxis. In: Die Schwester, Der Pfleger. Dezember-Ausgabe, S. 1109

132.Glover, J. (1993): Achieving the Organizational Change Necessary for Successful TQM. In: International Journal of Quality \& Reliability Measurement 10 (6), S. 47-64

133.Grant, R. M., Shani, R., Krishnan, R. (1994): TQM's challenge to management theory and practice. In: Sloan Management Review 35, S. 25-35

134.Greif, S. (1998): Organisationsentwicklung (OE). In: Häcker, H. \& Stapf, K. H. (Hrsg): Dorsch Psychologisches Wörterbuch, 13., überarb. u. erw. Aufl., Huber, Bern, S. 600-601

135.Greif, S. (1998a): Organisationskultur. In: Häcker, H. \& Stapf, K. H. (Hrsg.): Dorsch Psychologisches Wörterbuch, 13., überarb. u. erw. Aufl., Huber, Bern, S. 601

136.Greif, S. (1998b): Personalentwicklung. In: Häcker, H. \& Stapf, K. H. (Hrsg): Dorsch Psychologisches Wörterbuch, 13., überarb. u. erw. Aufl., Huber, Bern, S. 623-624

137.Greif, S. (1998c): Management. In: Häcker, H. \& Stapf, K. H. (Hrsg): Dorsch Psychologisches Wörterbuch, 13., überarb. u. erw. Aufl., Huber, Bern, S. 515

138.Greif, S. (2004): Geschichte der Organisationspsychologie. In: Schuler, H. (Hrsg.): Lehrbuch Organisationspsychologie. 3., vollst. überarb. u. erw. Aufl., Huber, Bern, S. 21-57

139.Griffin, R. W. (1988): A longitudinal assessment of the consequencies of quality circles in an industrial setting. In: Academy of Management Journal, 31, S. 338-358

140.Grönroos, C. (1984): A Service Quality Model and its Marketing Implications. In: European Journal of Marketing, 18 (4), S. 36-44

141.Groß, P. (1983): Die Verheißungen der Dienstleistungsgesellschaft. Westdeutscher Verlag, Opladen

142.Häcker, H., Stapf, K. H. (Hrsg.) (1998): Dorsch Psychologisches Wörterbuch. 13. überarb. u. erw. Aufl., Huber, Bern et al.

143.Hackman, J. R., Wageman, R. (2000): Total Quality Management: Empirical, Conceptual, and Practical 
Issues. In: Cole, R. E., Scott, W. R. (Hrsg.): The Quality Movement and Organizational Theory. Sage, Thousand Oaks, S. 23-48. [Original erschienen in: Administrative Science Quarterly 40, 1995, S. 309342]

144.Hager, W. (1987): Grundlagen einer Versuchsplanung zur Prüfung empirischer Hypothesen der Psychologie. In: Lüer, G. (Hrsg.): Allgemeine Experimentelle Psychologie. Fischer, Stuttgart, S. 43-264

145.Hager, W. (2004): Testplanung zur statistischen Prüfung psychologischer Hypothesen. Hogrefe, Göttingen

146.Hager, W., Spies, K. (1991): Versuchsdurchführung und Versuchsbericht. Ein Leitfaden. Hogrefe, Göttingen

147.Haisch, W. (1998): GBM Benutzerhandbuch. Programmodul für das Qualitätsmanagement in der Betreuung nach dem Verfahren des GBM. BEB (Hrsg.), Stuttgart

148.Halfar, B. (1996a): Benchmarking statt Zertifikate. In: Socialmanagement 1, S. 25-27

149.Halfar, B. (1996b): Qualitätssicherung durch datenbankgestützte Betriebsvergleiche. In: Maelicke, B. (Hrsg.): Qualitätsmanagement in sozialen Betrieben und Unternehmen. Nomos, Baden-Baden, S. 111123

150.Halfar, B. (2000): Steuerung in der Sozialarbeit durch quantitatives und qualitatives Controlling. In: König, J., Oerthel, C., Puch, H.-J. (Hrsg.): Qualitätsmanagement und Informationstechnologien im Sozialmarkt. Schulz, Starnberg, S. 67-78

151.Hamada, T. (2000): Quality as a Cultural Concept: Messages and Metamessages. In: Cole, R. E., Scott, W. R. (Hrsg.): The Quality Movement and Organizational Theory. Sage, Thousand Oaks, S. 295-312

152.Harnach-Beck, V. (1997): Informationsgewinnung durch Fachkräfte des Jugendamtes. Professionelle Datenermittlung als Aspekt des Qualitätsmanagements. In: Kindheit und Entwicklung 1, S. 31-39

153.Harris, C. R., Purdy, R. L. (1998): The role of participative management in the implementation of total quality management programs. In: International Journal of Technology Management 16, S. 466-478

154.Harris, R., Klie, T. Ramin, E. (1995): Heime zum Leben: Wege zur bewohnerorientierten Qualitätssicherung. Vincentz, Hannover

155.Hartmann, H. (2001): Benchmarking in der kommunalen Sozialhilfe. Zeitschrift für das Fürsorgewesen 6, S. 121-129

156.Haselbacher, M., Prax, F., Vogt, U., Wuksch, D. (2001): Prozessmanagement. Cicero Consulting GmbH/DQS/ÖQS, Klagenfurt/Frankfurt/Wien

157.Hauser, A., Neubarth, R., Obermair, W. (Hrsg.) (1997): Management-Praxis. Handbuch soziale Dienstleistungen. Luchterhand, Neuwied/Kriftel/Berlin

158.Heine, J. (1995): Prozessorientierte Methodik zur Einführung eines Qualitätsmanagementsystems. In: Berichte aus der Produktionstechnik 5, Aachen

159.Heiner, M. (1996): Ziel- und kriterienbezogenes Qualitätsmanagement in der sozialen Arbeit. Vom Katalogisieren der Aktivitäten zur Reflexion von Qualitätskriterien. In: Merchel, J., Schrapper, C. (Hrsg.): Neue Steuerung. Tendenzen der Organisationsentwicklung in der Sozialverwaltung. Votum, Münster, S. 210-230

160.Heiner, M. (1996a): Evaluation zwischen Qualifizierung, Qualitätsentwicklung und Qualitätssicherung. In: Heiner, M. (Hrsg.): Qualitätsentwicklung durch Evaluation. Lambertus, Freiburg, S. 20-47

161.Heiner, M. (1998): Lernende Organisation und Experimentierende Evaluation. Verheißungen Lernender Organisationen. In: Heiner, M. (Hrsg.): Experimentierende Evaluation. Juventa, Weinheim/München, S. 11-53

162.Heiner, M. (Hrsg.) (1998): Experimentierende Evaluation. Juventa, Weinheim/München

163.Heinze, R., Schmid, J., Strünck, C. (1997): Zur politischen Ökonomie der sozialen Dienstleistungsproduktion. Der Wandel der Wohlfahrtsverbände und die Konjunkturen der Theoriebildung. In: Kölner Zeitschrift für Soziologie 49, S. 242-271

164.Hekele, K. (1999): Qualitätssteuerung sozialer Arbeit. Wie kann man mit einem zielorientierten, ressourcenorientierten Selbststeuerungsverfahren arbeiten? In: Kröger, R. (Hrsg.): Leistung, Entgelt und Qualitätsentwicklung in der Jugendhilfe. Arbeitshilfen mit Musterbeispielen zur Umsetzung der $\S \S 78 \mathrm{a}-$ g SGB VIII. Luchterhand, Neuwied/Kriftel, S. 207-236

165.Hellerich, G., White, D. (2003): Empowerment - Eine Auseinandersetzung aus postmoderner Sicht. In: Soziale Arbeit 7-8, S. 36-41

166.Hellriegel, D., Slocum, J. W., Woodman, R. W. (1995): Organizational Behavior. 7. Aufl., West Publishing Company, St. Paul

167.Hemker, B. (2003): Beschwerdesteuerung: Möglichkeiten und Grenzen. In: Forum Erziehungshilfen 4, S. 212-220

168.Hentze, J., Kammel, A, Schwager, M (2000): Ideenmanagement als kontinuierlicher Verbesserungsprozess. In: Frey, D., Schulz-Hardt, S. (Hrsg.): Vom Vorschlagswesen zum Ideenmanagement. Hogrefe, Göttingen 
169.Herrmannstorfer, U. (1999): "Wege zur Qualität" - Der Arbeitsansatz der anthroposophisch orientierten Einrichtungen der Heilpädagogik und Sozialtherapie (Schweiz). In: Peterander, F., Speck, O. (Hrsg.): Qualitätsmanagement in sozialen Einrichtungen. Reinhardt, München/Basel, S. 146-156

170.Hiller, P. (2003): Professioneller Umgang mit Beschwerden - ein beschwerlicher Weg. In: Forum Erziehungshilfen 4, S. 196-200

171.Hirschman, A. O. (1974): Abwanderung und Widerspruch. Reaktionen auf Leistungsabfall bei Unternehmen, Organisationen und Staaten. Tübingen

172.Ho, S. K. M. (1999): TQM and Organizational Change. In: Intern. Journal of Organizational Analysis 7 (2), S. 169-181

173.Hoerner/Vitinius (1997): Heiße Luft in neuen Schläuchen. Eichborn, Frankfurt

174.Hollerith, E. (1995a): Organisatorische Aspekte des Aufbaus von QM. Qualitätsmanagement in der Ev. Heimstiftung Pfalz. Q 2, St. Klara-Kloster-Weg 7, 67346 Speyer

175.Hollerith, E. (1995b): Qualitätssicherung und Qualitätsförderung als Aufgaben des Trägers von sozialen Einrichtungen. Teil I: Grundlegende Überlegungen zum Qualitätsmanagement. In: Evangelische Jugendhilfe 5

176.Hollerith, E. (1996): Qualitätssicherung und Qualitätsförderung als Aufgaben des Trägers von sozialen Einrichtungen. Teil II: Einführung und Aufbau eines Qualitätsmanagements am Beispiel der Ev. Heimstiftung Pfalz. In: Evangelische Jugendhilfe 1,S. 37-43.

177.Holling, H., Kanning, U. P. (2004): Theorien der Organisationspsychologie. In: Schuler, H. (Hrsg.): Lehrbuch Organisationspsychologie. Huber, Bern et al., S. 59-87

178.Holling, H., Liepmann, D. (2004): Personalentwicklung. In: Schuler, H. (Hrsg.): Lehrbuch Organisationspsychologie. 3., vollst. überarb. u. erw. Aufl., Huber, Bern et al., S. 345-383

179.Horak, C. (1995): Controlling in Nonprofit-Organisationen. Erfolgsfaktoren und Instrumente. Deutscher Universitäts Verlag, Wiesbaden

180.Horbach, A. (2000): Strategien zur Umsetzung von Total Quality Management bei Non-ProfitDienstleistern. Techn. Univ. Chemnitz, Dissertation

181.Horvath, P. u. a. (1997): Neues Verwaltungsmanagement. Raabe, Düsseldorf

182.Hoyos, C. Graf, Frey., D. (Hrsg.) (1999): Arbeits- und Organisationspsychologie. Ein Lehrbuch. PVU, Weinheim

183.Hron, J., Lauche, K., Schultz-Gambard, J. (2000): Training im Qualitätsmanagement: Eine Interventionsstudie zur Vermittlung von Qualitätswissen und handlungsleitenden Kognitionen. In: Zeitschrift für Arbeits- und Organisationspsychologie 4, S. 192-201

184.Hunter, J. E., Schmidt, F. L. (1990): Methods of meta-analysis. Sage, Newbury Park

185.Hütte, M. (1998): Qualitätssicherung in der Jugendhilfe - Chance zur verbesserten Legitimation vergesellschafteter Kosten oder der Weg in eine technokratische Pädagogik. In: Forum Erziehungshilfen 2, S. 116-121

186.Ichniowski, C., Shaw, K. (2000): Quality Improvement Practices and Innovative HRM Practices. In: Cole, R. E., Scott, W. R. (Hrsg.): The Quality Movement and Organizational Theory. Sage, Thousand Oaks, S. 347-365

187.Imai, M. (1994): Kaizen - Der Schlüssel zum Erfolg der Japaner im Wettbewerb. Ullstein, Frankfurt

188.Initiative Qualitätssicherung NRW (o. J.): www.iqsnrw.de

189.Johannesson, R., Ritchie, J. R. (1997): An employee survey measuring total quality management practices and culture: Development and Culture. In: Group and Organization Management 22, S. 414444

190.Jordan, E., Reismann, H. (1998): Qualitätssicherung und Verwaltungsmodernisierung in der Jugendhilfe. Votum, Münster

191.Kaboolian, L. (2000): Quality Comes to the Public Sector. In: Cole, R. E., Scott, W. R. (Hrsg.): The Quality Movement and Organizational Theory. Sage, Thousand Oaks, S. 131-153

192.Kamiske, G. H., Brauer, J.-P. (1996): ABC des Qualitätsmanagements. 3. Aufl., Hanser, München/Wien

193.Kämmer, K., Hennecke, M., Trapp, F., Bruns-Waigand, W. (2001): Qualitätsverfahren im Überblick. Der Weg zum besten System. Vincentz, Hannover

194.Kaplan, R. S., Norton, D. P. (1997): Balanced Scorecard. Schäffer-Poeschel, Stuttgart

195.Kendrick, J. J. (1993): TQM: Is it Forging Ahead or Falling Behind? In: Quality 32 (5), S. 13

196.Keupp, H. (1998): Von der fürsorglichen Belagerung zum Empowerment: Perspektiven einer demokratischen Wohlfahrtsgesellschaft. Gemeindepsychologie Rundbrief 1, S. 20-37

197.Keupp, H. (2000): Die Suche nach der Qualität Sozialer Arbeit im Spannungsfeld von Markt, Staat und Bürgergesellschaft. In: König, J., Oerthel, C., Puch, H.-J. (Hrsg.): Qualitätsmanagement und Informationstechnologien im Sozialmarkt. Schulz, Starnberg, S. 27-49

198.Keupp, H. (2003): Von der fürsorglichen Belagerung zum Empowerment: Ideen für eine zivilgesellschaftlich angeregte Sozialpolitik. In: Sozialpädagogisches Institut im SOS-Kinderdorf e. V. 
(Hrsg.): Die Gesellschaft umbauen. SPI-Schriftenreihe, Autorenband 7, Eigenverlag, München, S. 67-99 199.Kieser, A., Kubicek, H. (1992): Organisation. 4. Aufl., DeGruyter, Berlin

200.Kim, D. H. (1989): Toward Learning Organizations: Integrating Total Quality Control and Systems Thinking. Unveröffentl. Manuskript Nr. 3037-89-BPS, Sloan School of Management, MIT

201.Kirstein, H. (2000): Das neue EFQM Excellence Modell für das Jahr 2000 http://www.deutscheefqm.de/efqm/modell 2000-1.html.

202.Kirstein, H. (o. J.): http://www.deutscheefqm.de/iso9000/iso2000.html

203.Kirstein, H. (o. J.): Was ich schon immer über die Balanced Scorecard wissen wollte. http://www.deutsche-efqm.de/efqm/balanscore

204.Klatetzki, T. (1996): Qualitätsmanagement in der Jugendhilfe. In: Maelicke, B. (Hrsg.): Qualitätsmanagement in sozialen Betrieben und Unternehmen. Nomos, Baden-Baden, S. 55-63

205.Klatetzki, T. (1998): Qualitäten der Organisation. In: Merchel, J. (Hrsg.): Qualität in der Jugendhilfe. Votum, Münster, S. 61-75

206.Klaus, P. G. (1991): Die Qualität von Bedienungsinteraktionen. In: Bruhn, M., Stauss, B. (Hrsg.): Dienstleistungsqualität. Konzepte, Methoden, Erfahrungen. Gabler, Wiesbaden, S. 247-266

207.Knorr, F. (1999): "The witch doctors" - Management-Gurus im Kreuzfeuer der Kritik. In: Theorie und Praxis der Sozialen Arbeit 6, S. 227-230

208.Knorr, F., Halfar, B. (2000): Qualitätsmanagement in der Sozialarbeit. Walhalla, Regensburg

209.Knouse, S. B., Smith, A., Smith, P. (2001): Book Review: Cole, R. E., Scott, W. R. (Hrsg.): The Quality Movement and Organizational Theory. Sage, Thousand Oaks. In: Personnel Psychology 54 (3), S. 757761

210.Koch, C. (2003): Balanced Scorecard (BSC). In: Boeßenecker et al. (Hrsg.): Qualitätskonzepte in der Sozialen Arbeit. Eine Orientierung für Ausbildung, Studium und Praxis. Beltz/Votum, Weinheim/Basel/Berlin, S. 15-22

211.Koch, J. (2003): Integrierte Erziehungshilfen im Sozialraum - Erfahrungen aus dem Modellverbund INTEGRA. In: Zentralblatt für Jugendrecht 11, S. 423-432

212.Koch. J. (2000): Interne Evaluation: Kommentierung und Bewertung des Verfahrens für die Erziehungshilfe. In: Merchel, J. (Hrsg.): Qualitätsentwicklung in Einrichtungen und Diensten der Erziehungshilfe. IgfH-Eigenverlag, Frankfurt, S. 146-160

213.Kochan, T. A., Rubinstein, S. (2000): Human Resource Policies and Quality: From Quality Circles to Organizational Transformation. In: Cole, R. E., Scott, W. R. (Hrsg.): The Quality Movement and Organizational Theory. Sage, Thousand Oaks, S. 387-399

214.Kolhoff, L. (2003): Qualität im Personalmanagement - Neue Anforderungen an soziale Einrichtungen. In: Theorie und Praxis der Sozialen Arbeit 1, S. 55-60

215.Kommerell, T. (2000): Pflegequalität und ihre Sicherung. In Schäffler, A., Menche, N., Bazlen, U., Kommerell, T.: Pflege heute. Lehrbuch und Atlas für Pflegeberufe. Urban \& Fischer, München/Jena

216.Kommunale Gemeinschaftsstelle für Verwaltungsvereinfachung (KGSt) (1993): Das neue Steuerungsmodell. Bericht 5, Köln

217.Kommunale Gemeinschaftsstelle für Verwaltungsvereinfachung (KGSt) (1994): Outputorientierte Steuerung in der Jugendhilfe. Bericht 9, Köln

218.Kommunale Gemeinschaftsstelle für Verwaltungsvereinfachung (KGSt) (1995): Qualitätsmanagement. Bericht 6, Köln

219.Kommunale Gemeinschaftsstelle für Verwaltungsvereinfachung (KGSt) (1998): Kontraktmanagement zwischen öffentlichen und freien Trägern am Beispiel der ambulanten Hilfen zur Erziehung. Überarbeiteter Berichtsentwurf vom 21.09.1998

220.König, J. (2000): Einführung in die Selbstevaluation. Ein Leitfaden zur Bewertung der Praxis Sozialer Arbeit. Lambertus, Freiburg

221.Köpp, C., Neumann, S. (2002): Die Qualitätsdebatte in der Sozialpädagogik. Literaturbericht und Bibliographie. Arbeitspapier II-01 des Zentrums für sozialpädagogische Forschung der Universität Trier. www.uni-trier.de

222.Kottmann, B. (2002): Stellenbeschreibung Qualitätsbeauftragte. In: Die Schwester/Der Pfleger 3, S. 191194

223.Krause, H.-U. (2003): Kritik und Beteiligung: Horizonte stationärer erzieherischer Hilfen. In: Forum Erziehungshilfen 4, S. 207-211

224.Krenz, A. (2001): Qualitätssicherung in Kindertagesstätten. Kieler Instrumentarium für Elementarpädagogik und Leistungsqualität - K. I. E. L. Reinhardt, München

225.Kronberger Kreis für Qualitätsentwicklung in Kindertageseinrichtungen (1998): Qualität im Dialog entwickeln. Wie Tageseinrichtungen für Kinder besser werden. Kallmeyer'sche Verlagsbuchhandlung, Seelze

226.Kühl, S. (2000): Das Regenmacher-Phänomen. Widersprüche und Aberglaube im Konzept der lernenden 
Organisation. Campus, Frankfurt

227.Kühn, D. (1995): Neue Steuerungsmodelle der Sozialverwaltung - Chancen und Gefahren. In: Neue Praxis 4, S. 340-348

228.Kurzlechner, C. (1999): Qualitätssicherung in der Sozialen Arbeit unter Berücksichtigung berufsethischer Prinzipien am Beispiel Schuldnerberatung. Eigenverlag, Waisenhausstr. 76, 80636 München

229.Lachnit, G. (2003): Vom Leitbild zur pädagogischen Zielvereinbarung. In: Jugendhilfe 41 (4), S. 178191

230.Lambach, R. (2004): Qualitätsmanagement in der Praxis - Erfahrungen aus einer Einrichtung. In: Blickpunkt Jugendhilfe, Heft 2, www.projekt-petra.de

231.Lang, H. (2002): Beschwerdemanagement. Vortrag zur 3. Internationalen Tagung "Qualitätsmanagement in der öffentlichen Verwaltung", 11.-12.06.2002, München

232.Lang, R. (o. J.): Evaluationsforschung. In: Denzin-v. Broich-Oppert, U. et al. (Hrsg.): Qualität - Standards, Messung, Überprüfung, Sicherung. Dokumentation einer Vortragsreihe der Sozialpädagogischen Fortbildungsstätte Haus am Rupenhorn, März 1996 bis September 1997. Am Rupenhorn 5, 14055 Berlin, S. 48-55

233.Langnickel, H. (2003): Das EFQM-Modell für Excellence - Der Europäische Qualitätspreis. In: Boeßenecker et al. (Hrsg.): Qualitätskonzepte in der Sozialen Arbeit. Eine Orientierung für Ausbildung, Studium und Praxis. Beltz/Votum, Weinheim, S. 38-47

234.Leana, C. R. (1987): Power relinquishment versus power sharing: Theoretical clarification and empirical comparison of delegation and participation. In: Journal of Applied Psychology 72, S. 228-233

235.Lechner, K., Egger, A., Schauer, R. (1989): Einführung in die allgemeine Betriebswirtschaftslehre. 12. Aufl., Wien

236.Lemme, M., Ochs, M. (1998): Qualitätssicherung in der Jugendhilfe - Ein Beispiel. In: Brunner et al. (Hrsg.): Soziale Einrichtungen bewerten. Theorie und Praxis der Qualitätssicherung. Lambertus, Freiburg, S. 156-176

237.Levine, D. I., Shaw, K. (2000): The Incentives of Quality and the Quality of Incentives: Quality Improvement and Incentive Pay for Frontline Workers. In: Cole, R. E., Scott, W. R. (Hrsg.): The Quality Movement and Organizational Theory. Sage, Thousand Oaks, S. 367-386

238.Link, J., Schöpflin, E., Gruhler, S. (1999): Kooperative Qualitätsentwicklung öffentlicher und freier Träger der Jugendhilfe. Das Qualitätsmanagement-Modell der Region Heilbronn-Franken. EREVSchriftenreihe, Sonderausgabe Juli

239.Lo, V. H. Y., Sculli, D. (1998): Role of the management representative in quality management projects. In: International Journal of Continuing Engineering Education and Life-Long Learning, Vol. 8, Ns. 1/2, p. $76-88$

240.Loh, M. (1999a): Integriertes Qualitätsmanagement und die Bedeutung von Leitbildern. In: Fachverband Ev. Erziehungshilfe Berlin-Brandenburg e. V./Projektgruppe "fairhandeln" (Hrsg.): fairhandeln. Dialoge, Suchprozesse, Wege der Qualitätsentwicklung. Berlin, S. 67-75

241.Loh, M. (1999b): Integriertes Qualitätsmanagement und Führung. In: Fachverband Ev. Erziehungshilfe Berlin-Brandenburg e. V./Projektgruppe "fairhandeln" (Hrsg.): fairhandeln. Dialoge, Suchprozesse, Wege der Qualitätsentwicklung. Berlin, S. 76-82

242.Löwenhaupt, S. (1999): Benchmarking: Ein Verfahren zur Qualitätsentwicklung in der Jugendhilfe. In: Jugendhilfe 5, S. 272-278

243.Luhmann, N. (1987): Soziale Systeme. Grundriß einer allgemeinen Theorie. Frankfurt

244.Luhmann, N., Schorr, K. E. (1982): Das Technologiedefizit in der Erziehung und die Pädagogik. In: dies. (Hrsg.): Zwischen Technologie und Selbstreferenz. Fragen an die Pädagogik. Frankfurt, S. 11-40

245.Luthans, F., Rubach, M. J., Marsnik, P. (1995): Going beyond total quality: The characteristics, techniques, and measures of learning organizations. In: Intern. Journal of Organizational Analysis 3 (1), S. 24-44

246.Maaser, W. (2002): Qualität und Ethos - Normative Aspekte der Qualitätssicherung von sozialen Dienstleistungen. In: Theorie und Praxis der Sozialen Arbeit 2, S. 135-141

247.Maßß, V. (1999): Qualitätsmanagement in der Jugendhilfe. AFET-Nachrichten 3, S. 19-22

248. Maelicke, B. (1989): Management in sozialen Organisationen. Ganzheitliche und sozialökologische Organisationsentwicklung für Non-Profit-Unternehmen. In: Blätter der Wohlfahrtspflege, 136 (3), S. 6770

249.Maelicke, B. (1998): Controlling. In: Arnold, U., Maelicke, B. (Hrsg.): Lehrbuch der Sozialwirtschaft. Nomos, Baden-Baden, S. 590-598

250.Maelicke, B. (Hrsg.) (1996): Qualitätsmanagement in sozialen Betrieben und Unternehmen. Nomos, Baden-Baden

251.Malorny, C. (1996): TQM umsetzen. Der Weg zur Business Excellence. Schäffer-Poeschel, Stuttgart 252.Mankiw, N. G. (2004): Grundzüge der Volkswirtschaftslehre. 3., überarb. Aufl., Schaeffer-Poeschel, 
Stuttgart

253.Masing, W. (1990): Handbuch der Qualitätssicherung. Hanser, München

254.Matul, C. (1998): Controlling in der Sozialen Arbeit. In: Soziale Arbeit. Schweizerischer Berufsverband SBS, 17, S. 4-11

255.Maurus, A. (o. J.): Leitbildarbeit an der Berufsschule für Elektroinstallationstechnik und Elektromechanik. www.Leitbildarbeit_BS_Elektroinstallation

256.McGovern, K., Oberste-Ufer, R., Rohrmann, A., Schädler, J., Schwarte, N. (2000): Arbeitshilfe zur Qualitätsentwicklung für Familienunterstützende und Familienentlastende Dienste. Ein Beitrag zur Qualitätssicherung und Qualitätsentwicklung in Offenen Hilfen. Siegen

257.Mclaughlin, J. E, Herr, U. (1999): Individual learning in the context of complex change: A study of management representatives in organizations registered to ISO 9000. Dissertation Abstracts International Section A: Humanities \& Social Sciences 60 (3A), S. 639

258. Meinhold, M. (1996): Qualitätssicherung und Qualitätsmanagement in der Sozialen Arbeit. Lambertus, Freiburg

259. Meinhold, M. (1999): Die Arbeit am Handbuch zum Qualitätsmanagement. Ein Werkstattbericht. In: Soziale Arbeit 9, S. 290-296

260.Meinhold, M., Matul, C. (2003): Qualitätsmanagement aus der Sicht von Sozialarbeit und Ökonomie. Nomos, Baden-Baden

261.Merchel, J. (1998): Zwischen Effizienzsteigerung, fachlicher Weiterentwicklung und Technokratisierung: Zum sozialpolitischen und fachpolitischen Kontext der Qualitätsdebatte in der Jugendhilfe. In: Merchel, J. (Hrsg.): Qualität in der Jugendhilfe. Kriterien und Bewertungsmöglichkeiten. Votum, Münster, S. 20-42

262.Merchel, J. (2000): Zwischen Hoffnung auf Qualifizierung und Abwehrreflex: Zum Umgang mit dem Qualitätsthema in der Jugendhilfe. In: Helmke, A., Hornstein, W., Terhart, E. (Hrsg.): Qualität und Qualitätssicherung im Bildungsbereich. 41. Beiheft der Zeitschrift für Pädagogik. Weinheim/Basel, S. 161-183

263.Merchel, J. (2001): Qualitätsmanagement in der Sozialen Arbeit. Ein Lehr- und Arbeitsbuch. Votum, Münster

264.Metzen, H. (1994): Schlankheitskur für den Staat. Lean Management in der öffentlichen Verwaltung, Campus, Frankfurt

265.Meyer, A., Mattmüller, R. (1987): Qualität von Dienstleistungen. Entwurf eines praxisorientierten Qualitätsmodells. In: Marketing ZFP, 9 (3), S. 187-195

266.Meyer, A., Westerbarkey, P. (1995): Bedeutung der Kundenbeteiligung für die Qualitätspolitik von Dienstleistungsunternehmen. In: Bruhn, M., Stauss, B. (Hrsg.): Dienstleistungsmarketing. Gabler, Wiesbaden, S. 81-103

267.Moore, S. T., Kelly, M. J. (1996): Quality Now: Moving Human Services Organizations toward a Consumer Orientation to Service Quality. In: Social Work 41 (1), S. 33-41

268. Moran, E. T. \& Volkwein, J. F. (1992): The cultural approach of the formation of organizational climate. In: Human Relations, 45, S. 19-47

269.Moser, K. (2004): Planung und Durchführung organisationspsychologischer Untersuchungen. In: Schuler, H. (2004): Lehrbuch Organisationspsychologie. 3., vollst. überarb. u. erw. Aufl., Huber, Bern et al., S. 89-119

270.Muchinsky, P. M. (2000): Psychology Applied to Work. 6. Aufl., Wadsworth/Thomson Learning, Belmont

271.Müller, B. (1996): Sinnvolle Produktorientierung in der Jugendhilfe. Ein Beitrag zum Verhältnis von Ökonomie und Ethik des Sozialen. In: Denzin-v. Broich-Oppert et al. (Hrsg.): Qualität - Standards, Messung, Überprüfung, Sicherung. Dokumentation einer Vortragsreihe der Sozialpädagogischen Fortbildungsstätte Haus am Rupenhorn, März 1996 bis September 1997. Am Rupenhorn 5, 14055 Berlin, S. 72-78

272.Müller, B. (1996a): Qualitätsprodukt Jugendhilfe. Kritische Thesen und praktische Vorschläge. Lambertus, Freiburg (2. Aufl. 1998)

273.Müller, B. (2000): Welche Unternehmensphilosophie braucht die Jugendhilfe? Normative Aspekte der Qualitätsdiskussion und Folgerungen für die Praxis. In: Evangelische Jugendhilfe 3, S. 135-142

274.Müller, J. (2002): Das soziale Kapital - Grundlage für ein Zusammenleben in weltweiter Solidarität. In: König, J., Oerthel, C., Puch, H.-J. (Hrsg.): Soziale Organisationen gestalten. Fachlichkeit in neuen Strukturen - ConSozial 2001. Schulz, Starnberg

275.Müller, J. F. W. (2002): Qualitätsmanagement-Handbuch ambulante, teilstationäre und stationäre Altenpflege. 2., überarb. Aufl., Potsdam

276.Müller, J. F. W. (2004): Organisationsentwicklung und Personalentwicklung im Qualitätsmanagement der Einrichtungen des Sozial- und Gesundheitswesens am Beispiel Altenhilfe. Hampp, München 
277.Müller-Kohlenberg, H. (1997): Evaluation von sozialpädagogischen Maßnahmen aus unterschiedlicher Perspektive: Die Sicht der Träger, der Programmanager/-innen und der Nutzer/-innen. In: BMFSFJ (Hrsg.): Evaluation der sozialpädagogischen Praxis. Materialien zur Qualitätssicherung in der Kinderund Jugendhilfe. Qs 11, S. 8-20

278.Müller-Kohlenberg, H., Münstermann, K. (Hrsg.) (2000): Qualität von Humandienstleistungen: Evaluation und Qualitätsmanagement in Sozialer Arbeit und Gesundheitswesen. Leske u. Budrich, Opladen

279.Mummendey, H. D. (1995): Die Fragebogen-Methode. 2. korr. Aufl., Hogrefe, Göttingen

280.Münder, J. u. a. (2002): Frankfurter Lehr- und Praxiskommentar zum KJHG. Münster 2002

281.Nerdinger, F. W. (1994): Zur Psychologie der Dienstleistung. Schäffer-Poeschel, Stuttgart

282.North, K. (1999): Wissensorientierte Unternehmensführung - Wertschöpfung durch Wissen. 2., erw. Aufl., Gabler, Wiesbaden

283.Nüßle, W. (1999): Qualitätsbeauftragte - Motor und Mentor der Qualitätsentwicklung. In: Peterander, F., Speck, O. (Hrsg.): Qualitätsmanagement in sozialen Einrichtungen. Reinhardt, München/Basel, S. 106118

284.Offermann, C. (2001): Anwendung der DIN ISO 9000 ff. in der Altenpflege. In: Kämmer, K., Hennecke, M., Trapp, F., Bruns-Waigand, W. (2001): Qualitätsverfahren im Überblick. Der Weg zum besten System. Vincentz, Hannover

285.Offermann, C. (2001a): Qualitätsentwicklung in der Altenhilfe - Möglichkeiten und Chancen. In: König, J., Oerthel, C., Puch, H.-J. (Hrsg.): Qualitätsmanagement und Informationstechnologien - ConSozial 2000. Schulz, Starnberg, S. 109-120

286.Oppen, M. (1993): Neue "Produktions"konzepte für öffentliche Dienstleistungen - Krankenkassen zwischen Markt und Staat. Berlin

287.Ostner, I. (2004): Europäisierung als Amerikanisierung des Sozialen. Vom alten zum neuen Europäischen Sozialmodell. In: Georgia Augusta, Wissenschaftsmagazin der Georg-August Universität Göttingen, 3, S. 48-54

288.Paeger, A., Ortega-Suhrkamp, E., Hofmann, P. (1998): AMIQ - Asklepios-Modell für Integriertes Qualitätsmanagement. In: Haake, D., Kugler, J., Lippert, H. (Hrsg.): Der leitende Arzt in der Krankenhausorganisation. Spitta, Balingen

289.Parasuraman, A., Zeithaml, V. A., Berry, L. L. (1985): A Conceptual Model of Service Quality and its Implications for Future Research. In: Journal of Marketing, 49, S. 41-50

290.Paritätische Gesellschaft für Qualität mbH (1998): Qualitätsentwicklung als zentrale Aufgabe des Paritätischen Wohlfahrtsverbandes und seiner Mitgliedsorganisationen. Rahmenkonzeption. Saarbrücken

291.Patton, M. Q. (1998): Die Entdeckung des Prozessnutzens. Erwünschtes und unerwünschtes Lernen durch Evaluation. In: Heiner, M. (Hrsg.) (1998): Experimentierende Evaluation. Ansätze zur Entwicklung lernender Organisationen. Juventa, Weinheim, S. 55-66

292.Peschke, M. A. (2003): Wissensmanagement wird zu einem zentralen Erfolgsfaktor in der Sozialwirtschaft. In: Theorie und Praxis der Sozialen Arbeit 6, S. 31-36

293.Peterander, F., Speck, O. (Hrsg.) (1999): Qualitätsmanagement in sozialen Einrichtungen. Reinhardt, München/Basel. (2., völlig neu bearb. Aufl. 2004)

294.Peters, F., Dörfer, K., Schollmeyer, K. (o. J.): Forschungsprojekt Qualitätsentwicklung in der Jugendhilfe. www.fh.erfurt.de/so/projekte/qualitaet/ergebnisse/ergebnisse.html

295.Peters, T. J., Waterman, R. H. (1982): In search of excellence. Harper \& Row, New York

296.Pfeifer, T. (1996): Qualitätsmanagement. Strategien, Methoden, Techniken. Hanser, München

297.Pollitt, C. (2000): Qualitätsmanagement und Evaluation in Europa: Strategien der Innovation oder der Legitimation? In: Müller-Kohlenberg, H., Münstermann, K. (Hrsg.): Qualität von Humandienstleistungen: Evaluation und Qualitätsmanagement in Sozialer Arbeit und Gesundheitswesen. Leske u. Budrich, Opladen, S. 59-76

298.Probst, G., Raub, S., Romhardt, K. (1999): Wissen managen. Wie Unternehmen ihre wertvollste Ressource optimal nutzen. 3. Aufl., Frankfurt

299.Projektgruppe WANJA (2000): Handbuch zum Wirksamkeitsdialog in der Offenen Kinder- und Jugendarbeit. Votum, Münster

300.Prölß, R. (1999): Qualität in der Jugendhilfe. Ein Plädoyer zur Beendigung einer irreführenden Debatte. In: Jugend, Beruf, Gesellschaft. Zeitschrift für Jugendsozialarbeit 2, S. 106-113

301.Radtke, P. (1999): Leitfaden zur Excellence. Das Berliner TQM-Umsetzungsmodell. Hanser, München/Wien

302.Rappe-Giesecke, K. (2003): Kleine Schritte auf dem Weg. Lerndende Organisation und Führungsalltag Techniken und Haltung. In: Diakonie Impulse 4, S. 10-12

303.Reck-Hog, U., Klink, G. (1998): Kundenorientierte Pflege - Prüfung von ambulanten Pflegediensten durch die TÜV Rheinland-Gruppe. In: Blonski, H. (Hrsg.): Qualitätsmanagement in der Altenpflege. Hagen, S. $171 \mathrm{ff}$. 
304.Regus, M. (2001): Qualitätssicherung im Kontext wettbewerbsorientierter Gesundheitspolitik. In: Schädler, J., Schwarte, N., Trube, A. (Hrsg.) (2001): Der Stand der Kunst. Qualitätsmanagement Sozialer Dienste. Votum, Münster, S. 68-96

305.Reichers, A. E., Schneider, B. (1990): The Role of Climate and Culture in Productivity. In: Schneider, B. (Hrsg.): Organizational Climate and Culture. Jossey-Bass, San Francisco

306.Ringwald, P. (2000): Leitbilder = Bilder der Leitung. In: Gerull, P. (Hrsg.): Hand- und Werkbuch Soziales Qualitätsmanagement. EREV-Schriftenreihe, Sonderausgabe März, Hannover, S. 4:11-14

307.Roes, M., François-Kettner, H., Schmälzle, G., Lehmann, T. (2000): MUM - ein Qualitätsprogramm zum Anfassen. Huber, Bern

308.Rosenstiel, L., v. (2000): Grundlagen der Organisationspsychologie. Basiswissen und Anwendungshinweise. 4., überarb. u. erw. Aufl., Schäffer-Poeschel, Stuttgart

309.Rüegsegger, R. (1986): Die Geschichte der Angewandten Psychologie 1900-1940. Huber, Bern et al.

310.Rugor, R., Studzinski, von, G. (2003): Qualitätsmanagement nach der ISO Norm. Eine Praxisanleitung für MitarbeiterInnen in sozialen Einrichtungen. Beltz, Weinheim

311.Saatweber, V. S. (2003): Qualitätsmanagement: Sich selber prüfen. In: Sozialwirtschaft aktuell 21, S. 3

312.Sanders, J. R. (Hrsg.) (1999): Handbuch der Evaluationsstandards. Leske u. Budrich, Opladen

313.Schädler, J. (2001): Qualitätsentwicklung und Qualitätssicherung - Plädoyer für ein professionsnahes Konzept in der Sozialen Arbeit. In: Schädler, J., Schwarte, N., Trube, A. (Hrsg.): Der Stand der Kunst. Qualitätsmanagement Sozialer Dienste. Votum, Münster, S. 13-38

314.Schiepek, G., Bauer, P. (1998): Produktion und Beurteilung von "Qualität" in psychosozialen Einrichtungen. In: Brunner, E. J., Bauer, P., Volkmar, S. (Hrsg.): Soziale Einrichtungen bewerten. Theorie und Praxis der Qualitätssicherung. Lambertus, Freiburg, S. 16-53

315.Schild, W. (1996): Qualitätssicherung in der Sozialen Arbeit. In: Sozialarbeit 7, S. 15-21

316.Schild, W. (1999): Der systemische Charakter der Praxis der Sozialen Arbeit als Hintergrund für die Wahl von speziellen Konzepten und Vorgehensweisen zur Qualitätssicherung und -entwicklung. In: Verein zur Förderung der Sozialen Arbeit als akademische Disziplin (Hrsg.): Entwicklung adäquater Qualitätsstandards in der Sozialen Arbeit. Forum Soziale Arbeit. Beiheft 2, Bern, S. 69-86

317.Schlottke, P. F. (1998): Modell. In: Häcker, H., Stapf, K. H. (Hrsg.): Dorsch Psychologisches Wörterbuch.13. überarb. u. erw. Aufl., Huber, Bern et al., S. 543-544

318.Schmid, J. (o. J.): Wohlfahrtsstaaten im Vergleich: Bestandsaufnahme und aktuelle Diskussion. www.uni-tuebingen.de/pol/schmwiv.pdf

319.Schmidt-Grunert, M. (1997): Die BWL-isierung/Ökonomisierung der sozialen Arbeit. In: EREV (Hrsg.): Leistung und Qualität in der Jugendhilfe. Dokumentation des Bundesfachkongresses 22.-24.4.97 in Bremen. EREV-Schriftenreihe 2, S. 105-123

320.Schnoor, H. (2003): Chancen und Probleme in der Arbeit von Qualitätszirkeln. Ergebnisse eines Schulbegleitforschungsprojekts. In: Evangelische Jugendhilfe 2, S. 73-81

321.Schoch, D. (2000): Vereinbarungen zur qualitätsorientierten Arbeit bei der Hilfe in Einrichtungen. Neuregelungen der $\S \S 93$ ff. BSHG ab 1.1.1999. In: Zeitschrift für das Fürsorgewesen 1, S. 1-13

322.Scholl, W. (2004): Grundkonzepte der Organisation. In: Schuler, H. (2004): Lehrbuch Organisationspsychologie. 3., vollst. überarb. u. erw. Aufl., Huber, Bern et al., S. 515-556

323.Schöpflin, E. (2000): Aspekte eines normativen Controllings in Jugendhilfeeinrichtungen. In: Gerull, P. (Hrsg.) (2000): Hand- und Werkbuch Soziales Qualitätsmanagement. EREV-Schriftenreihe, Sonderausgabe März, Hannover, Kap. 4, S. 182-184

324.Schramm, D. (1994): Qualitätskontrolle in "Sozialen Einrichtungen"/Nonprofit-Organisationen, Sozialmagazin 3, S. 22-28

325.Schreyer-Schubert, A., Hanselmann, P. G., Friz, A. (2000): Leitfaden für Qualitätsbeauftragte. In: Bundesministerium für Familie, Senioren, Frauen und Jugend (Hrsg.): Materialien zur Qualitätssicherung in der Kinder- und Jugendhilfe. Qs 28

326.Schröer, H., Schwarzmann, B., Stark, W., Straus, F. (2000): Qualitätsmanagement in der Praxis. Lambertus, Freiburg

327.Schruth, P. (2003): Wettbewerb in der Jugendhilfe aus europäischer Perspektive. In: EREVSchriftenreihe 2, S. 92-98

328.Schubert, H.-J., Zink, K. J. (Hrsg.) (2001): Qualitätsmanagement im Gesundheits- und Sozialwesen. 2. Aufl., Luchterhand, Neuwied/Kriftel

329.Schuler, H. (2004): Lehrbuch Organisationspsychologie. 3., vollst. überarb. u. erw. Aufl., Huber, Bern et al.

330.Schulin, B., Igl, G. (2002): Sozialrecht. 7. Aufl., Düsseldorf

331.Schultz-Gambard, J., Lauche, K., Hron, J. (1999): Qualitätssicherung und Qualitätsmanagement. In: Hoyos, C. Graf, Frey, D. (Hrsg.): Arbeits- und Organisationspsychologie. Psychologie Verlags Union, Weinheim, S. 91-104 
332.Schwarte, N., Oberste-Ufer, R. (1997): LEWO: Lebensqualität in Wohnstätten für erwachsene Menschen mit geistiger Behinderung. Ein Instrument zur Qualitätsentwicklung. Lebenshilfe-Verlag Marburg

333.Schwartz, R, Davis, S. (1981): Matching Corporate Culture and Business Strategy. In: Organizational Dynamics 10, S. 30-48

334.Schwarz, M. (2000): Qualitätsmanagement und die Zukunft der Psychologie. Ängste, Aspekte, Perspektiven. http://idq.de/zukunft.pdf

335.Schwarz, P., Purtschert, R., Giroud, C. (1999): Das Freiburger Management-Modell für NonprofitOrganisationen. Haupt, Bern

336.Scott, W. R., Cole, R. E. (2000): Introduction: The Quality Movement and Organization Theory. In: Cole, R. E., Scott, W. R. (Hrsg.): The Quality Movement and Organizational Theory. Sage, Thousand Oaks, S. xiii-xxix

337.Seghezzi, H. D. (1994): Qualitätsmanagement. Schäffer-Pöschel, Stuttgart

338.Seghezzi, H. D. (1996): Integriertes Qualitätsmanagement. Das St. Galler Konzept. Hanser, München/Wien

339.Seghezzi, H. D. (2003): Integriertes Qualitätsmanagement. Das St. Galler Konzept. 2., vollst. überarb. u. erw. Aufl., Hanser, München/Wien

340.Seidel, W., Stauss, B. (2002): Beschwerdemanagement: Personalpolitische Konsequenzen für Dienstleistungsunternehmen. In: Hansen, W., Kamiske, G. F. (Hrsg.): Qualitätsmanagement im Dienstleistungsbereich. Symposion, Düsseldorf, S. 131-142

341.Seits, M., Tews, B. (1999): Der Pflege-TÜV. In: Häusliche Pflege 9, Hannover

342.Senge, P. M. (1990): The Fifth Discipline: The Art and Practice of the Learning Organization. Doubleday, New York

343.Senge, P. M. (1992): Building learning organizations. In: The Journal for Quality and Participation 3, S. 30-38

344.Senge, P. M., Kleiner, A., Roberts, C., Ross, R. B., Smith, B. J. (1994): The fifth discipline fieldbook. Doubleday, New York

345.Severing, E. (1999): Genese und gegenwärtiger Stand der Qualitätssicherung in der beruflichen Bildung. In: Bildung und Erziehung 2, S. 143-156

346.Shapiro, E. C. (1998): Trendsurfen in den Chefetagen. Unternehmensführung jenseits der ManagementModen. Heyne, München

347.Shiba, S., Walden, D. (2001): Four practical revolutions in management. Systems for creating unique organizational capability. Productivity Press, Portland/Oregon

348.Simon, H. (1999): Wunsch-Wissen. In: Manager-Magazin 11, S. 307-308

349.Simon, H. A. (1960): The new science of management decision. Harper, New York

350.Sitkin, S. B., Sutcliffe, K. M., Schroeder, R. G. (1994): Distinguishing control from learning in total quality management: A contingency perspective. In: Academy of Management Review 19, S. 537-564

351.Sommerfeld, P., Haller, D. (2003): Professionelles Handeln und Management. In: Neue Praxis 1, S. 6189

352.Sörgel, G., Merkl, U. (2000): Von der Schwachstelle zur Schnittstelle - Zusammenarbeit von Leitung und Qualitätsbeauftragten. In: Schröer u. a. (Hrsg.): Qualitätsmanagement in der Praxis. Lambertus, Freiburg, S. 97-105

353.Späth, K. (1999): Erwartungen an die neuen Regelungen der $§ 78$ a-g KJHG. In: Jugendwohl 2, S. 5969

354.Speck, O. (1999): Marktgesteuerte Qualität - eine neue Sozialphilosophie? In: Peterander, F., Speck, O. (Hrsg.): Qualitätsmanagement in sozialen Einrichtungen. Reinhardt, München/Basel, S. 15-30

355.Speck, O. (2002): Ökonomisierung sozialer Qualität. Ein beunruhigender Aspekt der Qualitätsentwicklung. In: Rieder-Aigner (Hrsg.): Zukunftshandbuch Kindertageseinrichtungen. Walhalla, Regensburg/Berlin, Kap. I.7

356.Spiegel, von, H. (1994): Perspektiven der Selbstevaluation. In: Qualitätsentwicklung bei knapper werdenden Ressourcen. Materialien aus der Arbeit der Diakonischen Akademie. Berlin

357.Spiegel, von, H. (1997): Perspektiven der Selbstevaluation. In: BMFSFJ (Hrsg.): Materialien zur Qualitätssicherung in der Kinder- und Jugendhilfe. Qs 11, S. 32-48

358.Spiegel, von, H. (2000): Wie muss Qualitätsentwicklung/Qualitätsmanagement praktisch gestaltet werden, um für AdressatInnen und MitarbeiterInnen einen erkennbaren Nutzen zu stiften? In: Evangelische Jugendhilfe 3, S. 147-157

359.Spiegel, von, H. (2002): Qualität selbst bestimmen. Das Konzept "Qualitätsentwicklung, Qualitätssicherung und Selbstevaluation in der Kinder- und Jugendarbeit". In: Deutsche Jugend 6, S. 256-264

360.Spiegel, von, H. (Hrsg.) (2000a): Jugendarbeit mit Erfolg. Votum, Münster 
361.Spiegel, von, H., Sturzenhecker, B., Deinet, U. (2002): Qualitätsstandards Offener Jugendarbeit selbst bestimmen oder übernehmen? Die Modelle "QQS" (Qualitätsentwicklung/Selbstevaluation) und "WANJA""(Selbstbewertung) im Vergleich. In: Deutsche Jugend 6, S. 247-255

362.Spindler, H. (2001): Benchmarking und Sozialhilfe passen nicht zusammen. Zeitschrift für das Fürsorgewesen 7, S. 145-152

363.Spreyermann, C. (1996): Qualität mit Selbstevaluation entwickeln. In: DrogenMagazin - Zeitschrift für Suchtfragen. Basel, S. 3-6

364.Stadt Dormagen (Hrsg.) (2001): Dormagener Qualitätskatalog der Jugendhilfe. Leske und Budrich, Opladen

365.Stark, W. (1996): Empowerment. Neue Handlungskompetenzen in der psychosozialen Praxis. Lambertus, Freiburg

366.Stark, W. (2000): "It don't mean a thing, i fit ain’t got that swing" - Zukünftige Herausforderungen für Qualitätsmanagement im Kontext anderer Entwicklungen im Sozial- und Gesundheitsbereich. In: Schröer, H. et al. (Hrsg.): Qualitätsmanagement in der Praxis. Lambertus, Freiburg

367. Statistisches Bundesamt Deutschland (2004): Statistik der Kinder- und Jugendhilfe, Teil III.2

368.Staub-Bernasconi, S. (1996): Gegenstandsbestimmung Sozialer Arbeit als Voraussetzung für die Förderung der Sozialen Arbeit als Disziplin und Profession. In: Verein zur Förderung der Sozialen Arbeit als akademische Disziplin. Symposium Soziale Arbeit. Edition Soziothek, Köniz

369.Staufenbiel, T. (1999): Personalentwicklung. In: Hoyos, C. G., Frey, D. (Hrsg.): Arbeits- und Organisationspsychologie. PVU, Weinheim, S. 510-525

370.Stauss, B., Seidel, W. (1996): Beschwerdemanagement. Fehler vermeiden - Leistung verbessern Kunden binden. Hanser, München/Wien

371.Stauss, B., Seidel, W. (2002): Beschwerdemanagement: Kundenbeziehungen erfolgreich managen durch customer care. Hanser, München/Wien

372.Straus, F. (1998): Partizipatives Qualitätsmanagement als Erweiterung praxisorientierter Evaluationskonzepte. In: Heiner, M. (Hrsg.): Experimentierende Evaluation. Ansätze zur Entwicklung lernender Organisationen. Juventa, Weinheim, S. 67-92

373.Struck, N. (1995): Jugendhilfeplanung tut not! In: Blätter der Wohlfahrtspflege 11+12, S. 284-288

374.Struck, N. (2000): EFQM-Modell der European Foundation for Quality Management. Kommentierung und Bewertung des Verfahrens für die Erziehungshilfe. In: Merchel, J. (Hrsg.): Qualitätsentwicklung in Einrichtungen und Diensten der Erziehungshilfe. IgfH-Eigenverlag, Frankfurt, S. 188-192

375.Struck, N. (2003): Qualitätsentwicklung und Beschwerdemanagement - eine Zwischenbilanz und eine Empfehlung. In: Forum Erziehungshilfen 4, S. 201-206

376.Sturzenhecker, B. (2000): Grenzen von Planung in der Offenen Jugendarbeit. In: v. Spiegel, H. (Hrsg.): Jugendarbeit mit Erfolg. Votum, Münster, S. $159 \mathrm{ff}$.

377.Sutcliffe, K. M., Sitkin, S. B., Browning, L. D. (2000): Tailoring Process Management to Situational Requirements: Beyond the Control and Exploration Dichotomy. In: Cole, R. E., Scott, W. R. (Hrsg.): The Quality Movement and Organizational Theory. Sage, Thousand Oaks, S. 315-330

378.Tata, J., Prasad, S., Thorn, R. (1999): The Influence Of Organizational Structure On The Effectiveness Of TQM Programs. In: Journal of Managerial Issues 11 (4), S. 440-453

379.Teuber, K., Stiemert-Strecker, S., Seckinger, M (Hrsg.) (2000): Qualität durch Partizipation und Empowerment - Einmischungen in die Qualitätsdebatte. dgvt-Verlag, Tübingen

380.Theden, P., Colsman, H. (1996): Qualitätstechniken. Hanser, München

381.Thom, N. (1991): Betriebliches Vorschlagswesen. Ein Instrument der Betriebsführung. Lang, Bern

382.Thorwart, D. (2000): Vom Motor des Prozesses zur Koordinatorin - Rolle und Erfahrung einer Qualitätsbeauftragten. In: Schröer, H. et al. (Hrsg.): Qualitätsmanagement in der Praxis. Lambertus, Freiburg, S. 91-96

383.Tietze, W., Schuster, K. M., Grenner, K., Rossbach, H.-G. (2001): Die Kindergarten-Skala. Revidierte Fassung (KES-R). 2. Aufl., Luchterhand, Neuwied/Kriftel, Berlin

384.Tietze, W., Schuster, K. M., Rossbach, H.-G. (1997): Kindergarteneinschätzskala (KES). Luchterhand, Neuwied/Kriftel/Berlin

385.Titz, K., Paratsch, F. (2001): Unser Ziel: Ihre Zufriedenheit. Gemeinsam mit den Bewohnern die Qualität verbessern. In: Altenheim 10, S. 26-29

386.Tornow, H. (1999): Benchmarking als Methode der Qualitätsentwicklung. e/1/s Bergische Diakonie Aprath, Manuskriptdruck

387.Trost, M., Marquart, P. (2002): Lörracher Qualitätskonzept für die Krankenhaus-Sozialarbeit Akutkrankenhaus. Überarb. u. erw. Auflage. Eigenverlag, Lörrach/Essen

388.Trube, A., Regus, M., Depner, R. (2001): Fach- und nutzerorientiertes Qualitätsmanagement für soziale Dienste. In: Schädler u. a. (Hrsg.): Der Stand der Kunst. Votum, Münster, S. 227-233

389.Türk, K. (1989): Neuere Entwicklungen der Organisationsforschung. Ein Trend Report. Enke, Stuttgart 
390.Ulich, E. (2001): Arbeitspsychologie. 5., vollst. überarb. u. erw. Aufl., Schäffer-Poeschel, Stuttgart

391.Verband katholischer Einrichtungen und Dienste für lern- und geistigbehinderte Menschen e. V. (VKELG)(Hrsg.) (1996): System der Leistungsbeschreibung, Qualitätsbeschreibung, Qualitätsprüfung und Entgeltberechnung (SYLQUE) - Manual I (Wohneinrichtungen der Behindertenhilfe). Lambertus, Freiburg

392.Vilain, M. (2003): Ergebnisse des Projektes "Qualität in der Sozialen Arbeit": Das Konzept QUI. In: Boeßenecker, K.-H. et al. (Hrsg.): Qualitätskonzepte in der Sozialen Arbeit. Eine Orientierung für Ausbildung, Studium und Praxis. Beltz/Votum, Weinheim, S. 175-196

393.Vogt, P. (2000): EFQM-Modell der European Foundation for Quality Management. Praxiserfahrungen im Umgang mit dem Verfahren. In: Merchel, J. (Hrsg.): Qualitätsentwicklung in Einrichtungen und Diensten der Erziehungshilfe. IgfH-Eigenverlag, Frankfurt, S. 169-187

394.Volz, F.-R. (2000): Mythos Qualität? Thesen zu einer fälligen Mythenkritik. In: Deutscher Berufsverband für Sozialarbeit, Sozialpädagogik und Heilpädagogik e. V./Landesverband BadenWürttemberg (Hrsg.): Mythos Qualität. Erfahrungsberichte aus der Sozialen Arbeit. Verlag für Wissenschaft und Bildung, Berlin, S. 167-177

395.Wabnitz, R. J. (1999): Qualitätsentwicklung als gesetzliche Aufgabe. In: Evangelischer Erziehungsverband (Hrsg.): Qualitätsentwicklungsvereinbarung nach § 78b KJHG. EREV-Schriftenreihe 2, S. $15-26$

396. Walker, A. (2001): Thesaurus of Psychological Index Terms. 9. Aufl., APA, Washington D. C.

397.Wall, T. D., Cordery, J. L., Clegg, C. W. (2002): Empowerment, Performance, and Operational Uncertainty: A Theoretical Integration. In: Applied Psychology: An International Review 51 (1), S. 146169

398.Wanke, H.-J. (2003): Qualitäts-Check. In: Boeßenecker; K.-H. et al. (Hrsg.): Qualitätskonzepte in der Sozialen Arbeit. Beltz/Votum, Weinheim, S. 130-140

399.Weick, K. E. (1995): Sensemaking in organizations. Sage, Thousand Oaks

400.Weick, K. E. (2000): Quality Improvement: A Sensemaking Perspective. In: Cole, R. E., Scott, W. R. (Hrsg.): The Quality Movement and Organizational Theory. Sage, Thousand Oaks, S. 155-172

401.Weinert, A. B. (1998): Organisationspsychologie. Ein Lehrbuch. 4., vollst. überarb. u. erw. Aufl., PVU, Weinheim

402.Wendt, W. R. (1999): Berechtigte Erwartungen. In: Socialmanagement 5, S. 12-15

403.Westermann, R. (2000): Wissenschaftstheorie und Experimentalmethodik. Hogrefe, Göttingen

404.Wetzler, R. (2003): Qualitätsmanagement in Wohneinrichtungen der Behindertenhilfe. Eine empirische Bestandsaufnahme. Lambertus, Freiburg

405.Whalen, M. J., Rahim, M. A. (1994): Common barriers to implementation and development of a TQM program. In: Industrial Management 36 (2), S. 19-21

406.Wienand, M. (1999): Sozialsystem und soziale Arbeit in der Bundesrepublik Deutschland. 2. Aufl., Eigenverlag des Deutschen Vereins für öffentliche und private Fürsorge, Frankfurt

407.Wieneke, W.: Controlling in Diakonischen Einrichtungen und Werken. In: Diakonisches Werk der EKD (Hrsg.): Leitfaden zur wirtschaftlichen Führung diakonischer Einrichtungen und Werke. Verlagswerk der Diakonie, 1993, S. 395-426

408.Wiesner, R. (2001): Rechtliche Grundlagen der Erziehungshilfen. In: Birtsch, V., Münstermann, K., Trede, W. (Hrsg.): Handbuch Erziehungshilfen. Votum, Münster, S. 329-352

409.Wilpert, B. (2004): Organisation und Umwelt. In: Schuler, H. (2004): Lehrbuch Organisationspsychologie. 3., vollst. überarb. u. erw. Aufl., Huber, Bern et al., S. 641-659

410.Winter, S. G. (2000): Organizing for Continuous Improvement: Evolutionary Theory Meets the Quality Revolution. In: Cole, R. E., Scott, W. R. (Hrsg.): The Quality Movement and Organizational Theory. Sage, Thousand Oaks, S. 49-64. [Original erschienen in: Baum \& Singh (Hrsg.) (1994): Evolutionary Dynamics of Organization. Oxford University Press]

411.Wiswede, G. (2000): Einführung in die Wirtschaftspsychologie. 3., überarb. u. erw. Aufl., Reinhardt, München/Basel

412.Wöhe, G. (2002): Einführung in die Allgemeine Betriebswirtschaftslehre. 21., neu bearb. Aufl., Vahlen, München

413.Wohlfahrt, N. (o. J. ): Ökonomisierung der sozialen Arbeit als Auslöser von QM-Strategien. http://www.efh-bochum.de/homepages/wohlfahrt

414.Wolters, L., Albrecht, C., Schwabe, B. (1995): Qualitätssicherung/Qualitätsmanagement im Handwerksunternehmen - eine wichtige Strategie zur Unternehmenssicherung.Technologie-TransferRing Handwerk NRW

415.Womack, J. P., Jones, D. T., Ross, D. (1992): Die zweite Revolution in der Autoindustrie. Frankfurt/New York

416.Wottawa, H., Thierau, H. (1998): Evaluation. 2., überarb. Aufl., Huber, Bern et al. 
417.Wunderer, R. (1998): Beurteilung des Modells der Europäischen Gesellschaft für Qualitätsmanagement (EFQM) und dessen Weiterentwicklung zu einem umfassenden Business Excellence Modell. In: Qualitätsmanagement an der Schwelle zum 21. Jahrhundert. München, S. 53-67

418.www.achim-trube.de

419.www.artset-lqw.de

420.www.bagso.de

421.www.caf-netzwerk.de

422.www.destatis.de

423.www.deutsche-efqm.de/inhseiten/efqmmodell.html

424.www.fh-erfurt.de/so/projekte/qualitaet

425.www.freyakademie.ch

426.www.kda.de

427.www.ktq.de

428.www.lsw.nrw.de

429. www.psychologie.uni-mannheim.de/psycho1/info

430.www.ziegenhorn.com/de/jugend

431. Yasuda, Y. (1994): Mitarbeiterkreativität in Japan. So nutzt Toyota das betriebliche Vorschlagswesen. Verlag Moderne Industrie, Landsberg

432.Zeithaml, V., Parasuraman, A., Berry, L. L. (1992): Qualitätsservice. Frankfurt

433.Ziesche, U. (2001): Qualitätswerkstatt Kita. Konflikte in Kindertageseinrichtungen. Luchterhand, Neuwied

434.Zink, K. (1995): TQM als integratives Managementkonzept. München/Wien

435.Zollondz, H.-D. (2001): Lexikon Qualitätsmanagement. Oldenbourg, München/Wien

436.Zollondz, H.-D. (2002): Grundlagen Qualitätsmanagement. Einführung in Geschichte, Begriffe, Systeme und Konzepte. Oldenbourg, München/Wien 


\section{G Lebenslauf}

Klaus-Peter Gerull

Dipl.-Psychologe
31840 Hessisch Oldendorf, Falkenweg 9

Tel. 05152/6787, p.gerull@t-online.de

Persönliche Daten

Geburtstag $\quad 10.02 .1949$

Geburtsort Wülfrath/Kreis Mettmann

Familienstd. verheiratet mit Siegrun Gerull, Lehrerin, drei volljährige Kinder

Ausbildung

1955 - 1959 Grundschule Wülfrath

1959 - 1965 Realschule Wülfrath (Abschluss: Mittlere Reife)

1965 - 1967 Gymnasium Mettmann (Abschluss: Abitur)

1967 - 1968 Studium der Philosophie und Publizistik an der Universität Göttingen

1968 - 1974 Studium der Psychologie in Göttingen (Abschluss: Diplom, Note 1)

Diplom-Arbeit: Komparative Evaluation zweier Formen des

Neurophysiologiepraktikums für Mediziner und Analyse des Zusammenhangs von

Intelligenz-, Vorkenntnis- und Leistungsvariablen

2003 - 2004 Promotionsstudium an der Universität Göttingen, Sozialwissenschaftliche Fakultät

Fort- und Weiterbildung

$1974 \quad$ Verhaltenstherapie

1975 - 1980 Gesprächstherapie

1985 Sozialmanagement

$1993 \quad$ Organisationsentwicklung

1993 Rechnungswesen

seit 1994 Qualitätsmanagement

Berufspraxis

1974 - 1976 wiss. Angestellter am Physiologischen Institut der Universität Göttingen

1977 - 1981 freie psychologische Gemeinschaftspraxis in Hameln

1978 - 1983 freie Tätigkeit als Sachverständiger für Familiengericht Hameln

1979 - 1980 Teilzeitbeschäftigung als Psychologe im Jugendhof Porta Westfalica

1979 - 1994 freier Mitarbeiter der Zentralstelle für Psychologische Information und

Dokumentation an der Universität Trier

1981 - 1984 Erziehungsleiter im Jugendhof Porta Westfalica

1984 - 1995 Heimleiter im Jugendhof Porta Westfalica

1996 Hausmann

seit 1997 freie Beratungs-, Referenten- und Autorentätigkeit

1998 - 2000 Projektleiter „Qualitätsentwicklung in Einrichtungen und Diensten der

Erziehungshilfe" beim Ev. Erziehungsverband (EREV) 